\title{
INVENTORY OF PUBLIC WATER SUPPLIES IN ARKANSAS
}

By Nancy T. Baker, Carolyn A. Manning, and Elizabeth A. Beavers

\section{U.S. GEOLOGICAL SURVEY}

Open-File Report 91-247

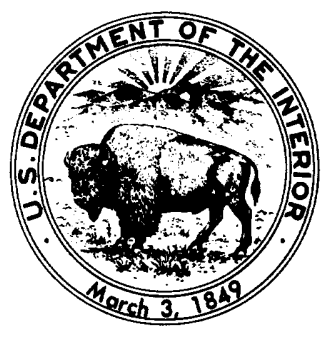

Prepared in cooperation with the

ARKANSAS SOIL AND WATER CONSERVATION COMMISSION

Little Rock, Arkansas 


\section{U.S. DEPARTMENT OF THE INTERIOR}

MANUEL LUJAN, JR., Secretary

U.S. GEOLOGICAL SURVEY

Dallas L. Peck, Director

For additional information write to:

District Chief

U.S. Geological Survey 2301 Federal Office Bldg. 700 West Capitol

Little Rock, Arkansas 72201
Copies of this report can be purchased from:

U.S. Geological Survey Books and Open-File Reports Federal Center, Bldg. 810

Box 25425

Denver, Colorado 80225 


\section{CONTENTS}

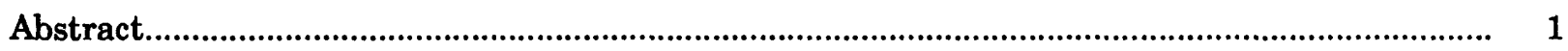

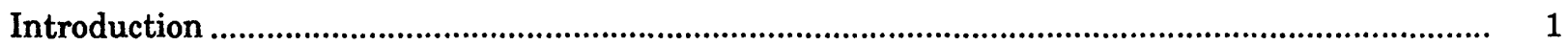

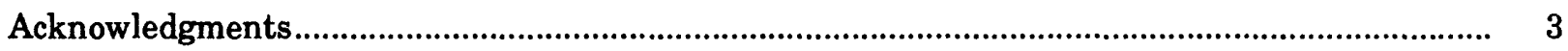

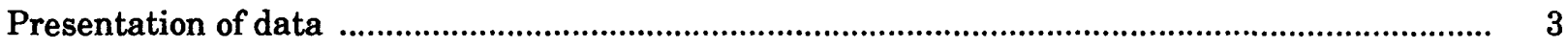

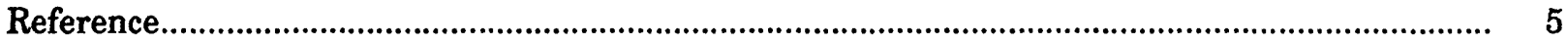

Appendix A. Alphabetical list of water supply system names and associated water-user ID's...... A-1

Appendix B. Hydrologic unit codes and basin names for Arkansas ........................................ B-1

\section{ILLUSTRATIONS}

Figure 1. Map showing location of public water-supply systems in Arkansas

2. Map showing location of surface-water withdrawal sites and water-quality sampling sites

\section{TABLES}

Table 1. Description of public water supplies in Arkansas

2. General description of public-supply withdrawal sites in Arkansas

3. Supplemental public-supply well information for sites investigated by the U.S.

Geological Survey

4. Supplemental public-supply ground-water quality information for sites investigated by the U.S. Geological Survey.

5. Supplemental surface-water-quality information for sites near public-supply intakes... 154 


\section{CONVERSION FACTORS}

Multiply

acre

acre-foot (acre-ft)

foot (ft)

million gallon per day (Mgal/d)
By

4,047

1,233

0.3048

3,785
To obtain

square meter

cubic meter

meter

cubic meter per day

Temperature in degrees Fahrenheit as follows:

$$
{ }^{\circ} \mathrm{F}=1.8 \times{ }^{\circ} \mathrm{C}+32
$$




\title{
INVENTORY OF PUBLIC WATER SUPPLIES IN ARKANSAS
}

\author{
By Nancy T. Baker, Carolyn A. Manning, and Elizabeth A. Beavers
}

\begin{abstract}
This report contains information for 697 public water-supply systems in Arkansas. Ground water is the source of supply for $\mathbf{4 3 5}$ of these water systems, and surface water is the source of supply for 72 systems. Ground and surface water is the source of supply for 6 systems, and 184 systems purchase their water from other public suppliers. About 943,000 people are served by ground-water supplies, and 1,136,000 people are served by surface-water supplies. Public supply includes water withdrawn by public and private water suppliers and delivered to users that do not supply their own water. Public suppliers report water withdrawals and related data to the Arkansas Soil and Water Conservation Commission. The U.S. Geological Survey works in cooperation with the Arkansas Soil and Water Conservation Commission in the collection, management, and storage of data for all categories of water use. Information in this document is obtained from Arkansas Soil and Water Conservation Commission reports and from field investigations conducted by the U.S. Geological Survey.
\end{abstract}

\section{INTRODUCTION}

Public water supply in Arkansas includes water withdrawn for all uses by public and private water suppliers and delivered to users that do not supply their own water. Water suppliers provide water for a variety of uses, such as domestic, commercial, thermoelectric power, industrial, and public water use. Information for 697 public-supply systems was obtained from water withdrawal information reported to the Arkansas Soil and Water Conservation Commission (ASWCC) for 1989. The U.S. Geological Survey (USGS) works in cooperation with the ASWCC in the collection, management, and storage of data for all categories of water use. The information contained in this report includes data reported to the ASWCC by each public supplier, and supplemental well data (ground-water sites) and selected water-quality data collected by the USGS for a limited number of sites. The USGS also assisted in the determination of source aquifers for some ground-water supplied systems. Estimates of population served by each public supply system were obtained from Arkansas Department of Health records (H. E. Seifert, Arkansas Department of Health, 1990, written commun.).

Of the 697 public-supply systems reporting water use, ground water is the source of supply for 435 of these systems, and surface water is the source of supply for 72 systems. Ground and surface water is the source of supply for six systems. Of the systems reporting, 184 systems purchase their water from other public suppliers. About 943,000 people are served by groundwater supplies, and 1,136,000 people are served by surface-water supplies.

Every effort has been made to include information for all public water suppliers that serve over 25 people in the State of Arkansas (fig. 1). The amount and quality of water-use data and related information presented in this document for each public water supplier is dependent upon the information reported by the individual supplier to the ASWCC. Supplemental well information (for ground-water sites) and water-quality information is provided for those sites where the USGS has conducted field investigations. 


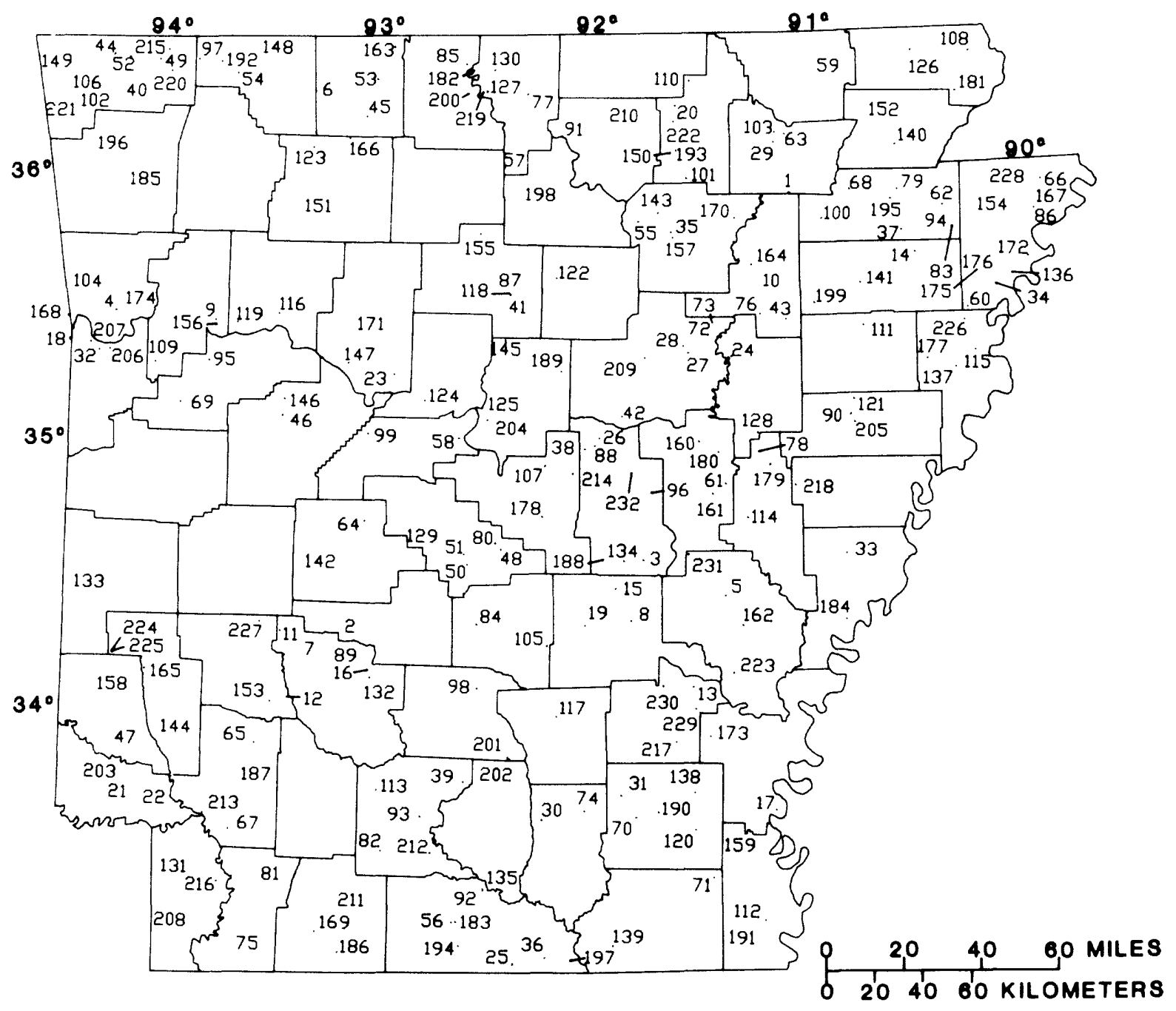

Figure 1a.--Location of public water-supply systems in Arkansas. Map numbers refer to water systems listed in appendix $A$. 


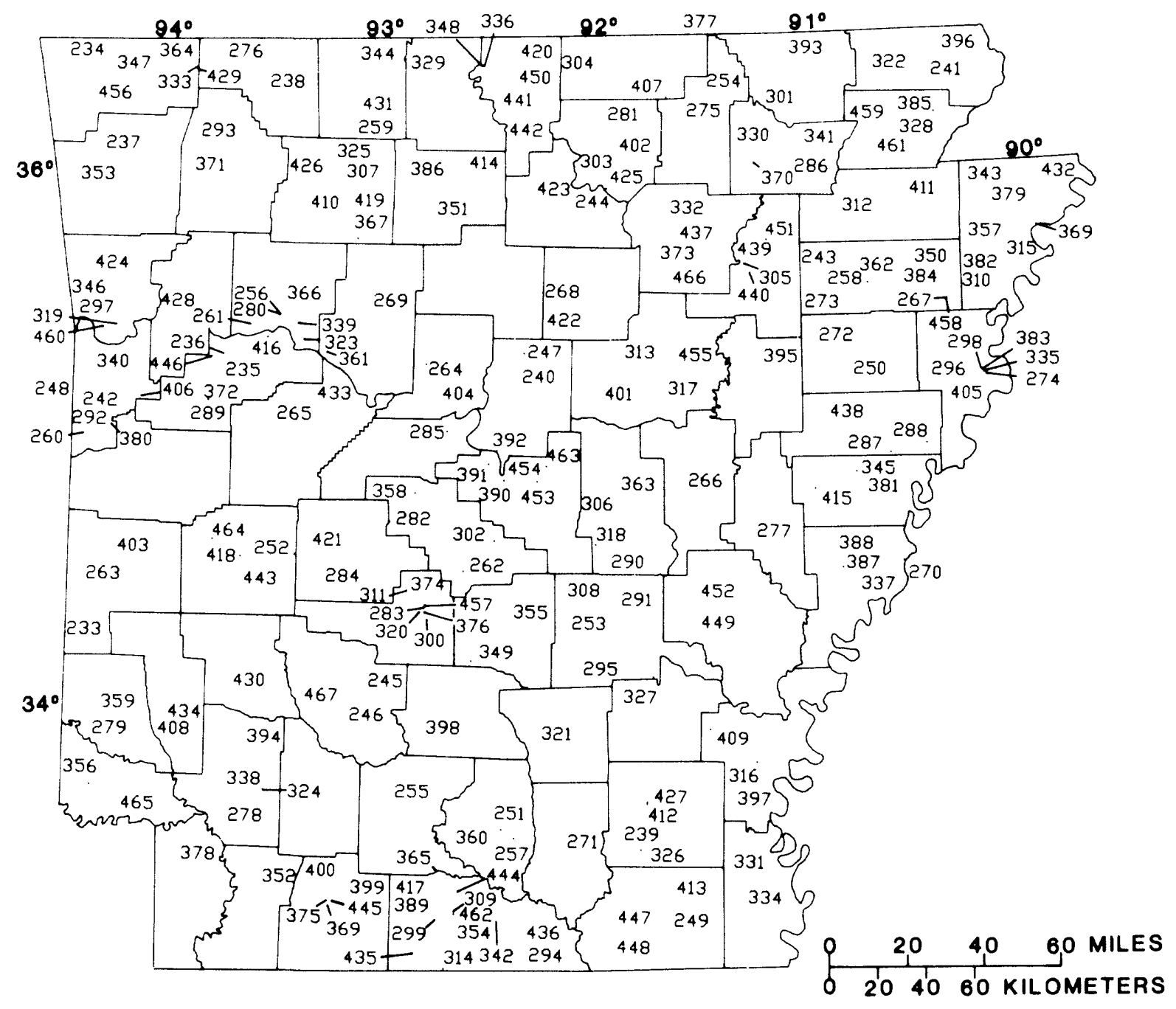

Figure 1b.--Location of public water-supply systems in Arkansas. Map numbers refer to water systems listed in appendix $A$. 


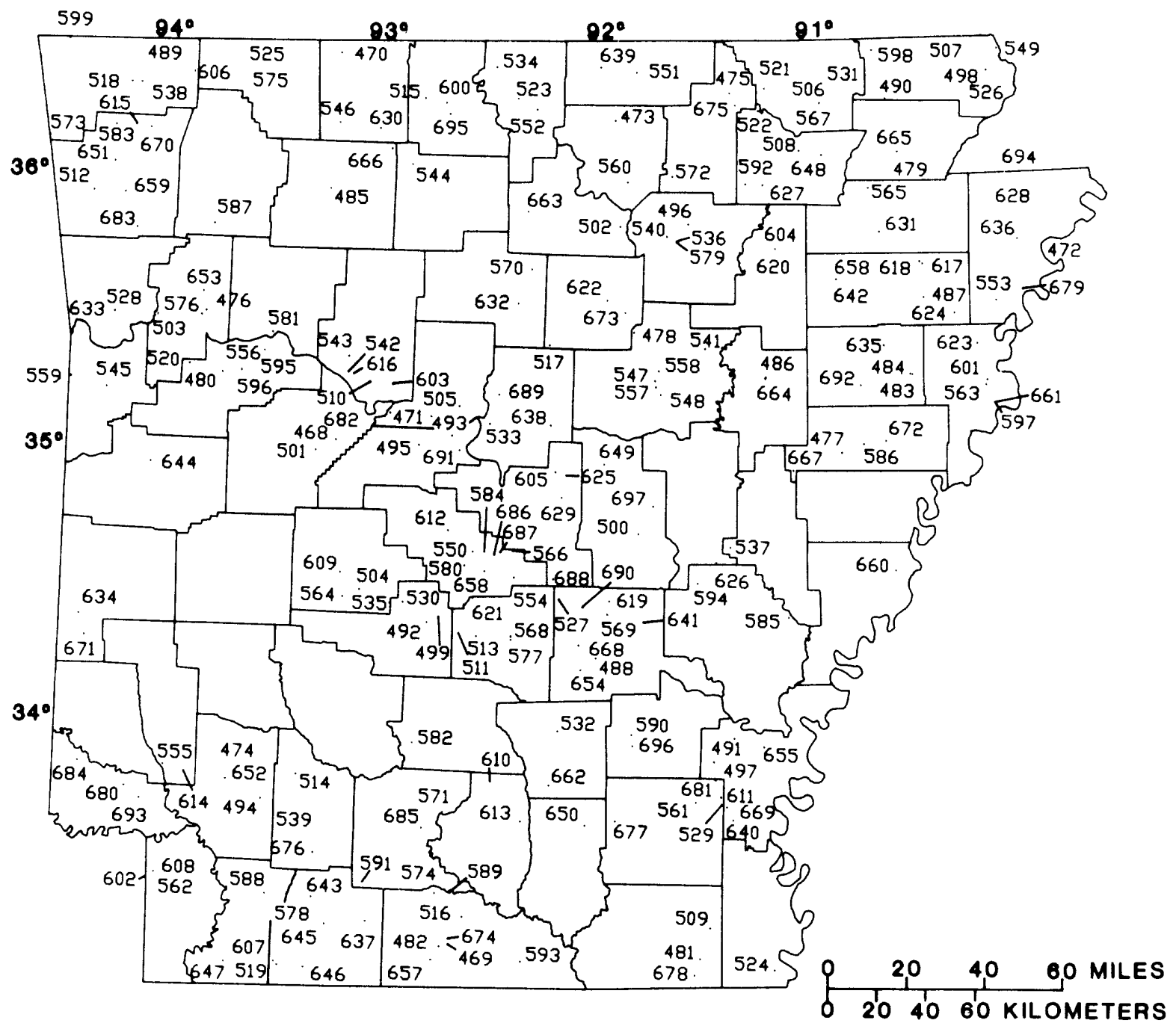

Figure 1c.--Location of public water-supply systems in Arkansas. Map numbers refer to water systems listed in appendix $A$. 


\section{ACKNOWLEDGMENTS}

The authors express appreciation to Mr. Harold Seifert, Director, Division of Engineering, Arkansas Department of Health for providing the initial list and addresses of public suppliers in Arkansas, and for providing estimates of population served by each system.

\section{PRESENTATION OF DATA}

The information contained in this report is presented in five tables (located at end of report). The first two tables contain water-use and related data that were reported to the ASWCC for 1989 (tables 1 and 2). Table 3 contains supplemental well data collected by the USGS, and tables 4 and 5 contain supplemental water-quality data collected by the USGS.

Water-use data collected by the ASWCC are stored in the USGS's Site-Specific Water-Use Data System (SSWUDS). SSWUDS data are available for each public water-supply system, and in most cases, SSWUDS data also are available for the individual withdrawal points associated with each system. Table 1 provides basic system information such as: water-user ID number, water system name and address, county of operation, source of water (ground or surface water) and name of source (lake or stream name for surface water or primary source aquifer for ground water), 1989 withdrawal amounts, local population served, total population served, water-user ID numbers to whom water is sold, and the water-user ID numbers from which water is purchased. Population served data were obtained from Arkansas Department of Health records because population served data reported to the ASWCC does not adequately differentiate local population served by other water suppliers who purchase water from that system. Table 2 provides a general description of the withdrawal sites associated with each system and includes the following information: water-user ID number, withdrawal site ID number, water system name, latitude and longitude of withdrawal site, hydrologic unit code, source of water (ground or surface water) and name of source (lake or stream name for surface water or primary source aquifer for ground water), pump horsepower, pump power source, pump type, well diameter and depth (for ground water), and a local descriptive identifier. Water-user ID numbers and the associated water system name are listed alphabetically in appendix $\mathrm{A}$ and hydrologic unit codes are listed in appendix $B$.

In addition to water-use data stored in SSWUDS, the USGS stores ground water data in the Ground-Water Site Inventory (GWSI) data system, and water quality data in the Quality of Water (QW) data system for a limited number of sites where the USGS has conducted field investigations (tables 3-5). Although the site ID is the primary identifier between the SSWUDS, GWSI, and QW databases, the SSWUDS water-user ID and water system name have been included in tables 3 and 4 to aid in ease of comparison between the water-use data and the supplemental data. Table 3 provides supplemental data for a limited number of public-supply wells that have been investigated by the USGS. The information includes: the principal source aquifer geohydrologic unit, the altitude of land surface, the depth of the well, depth to the first opening, casing diameter, water level and date measured, discharge of the well, specific capacity of the well, and types of well logs available. Table 4 contains supplemental waterquality data for public supply wells and table 5 contains supplemental water-quality data for surface-water sites that are near public supply intakes. Figure 2 shows site locations of the surface-water quality stations listed in table 5. The U.S. Environmental Protection Agency's (1988) recommended Secondary Maximum Contaminant Levels (SMCLs) for drinking water are given below. 


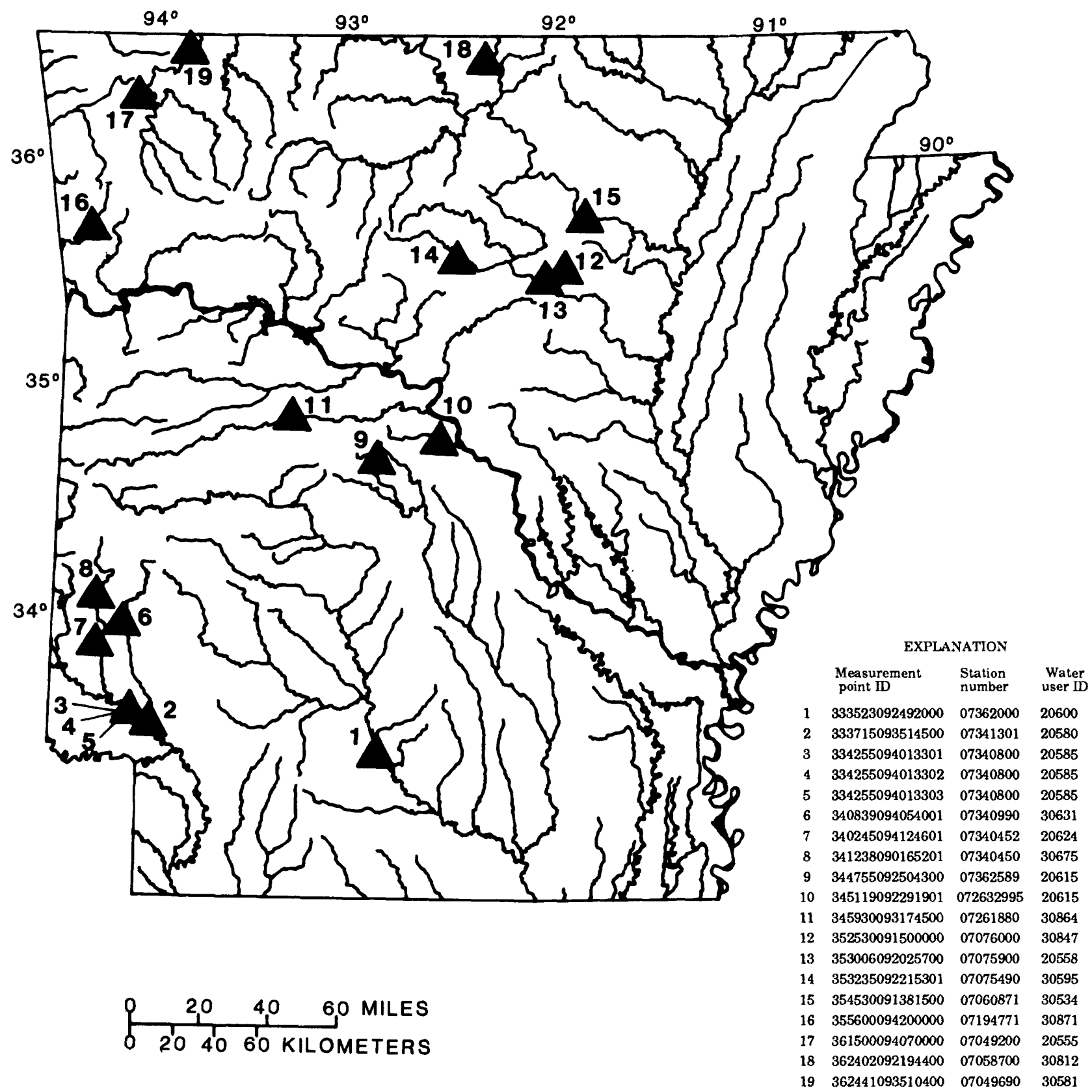

Figure 2.--Location of surface-water withdrawal sites and water-quality sampling sites. 
Constituent or

physical property
Secondary maximum

contaminant level

(milligrams per liter,

except where noted)
Chloride

250

Color (platinum-cobalt units)

Dissolved solids

Fluoride

Iron

pH (standard units)
15

500

2.0

0.3

6.5-8.5

The U.S. Environmental Protection Agency has established SMCLs for contaminants that can adversely affect the odor or appearance of water and result in the discontinuation of use of the water. SMCLs are not health based and are not Federally enforceable rather are intended as guidelines for the States.

The following example can be used to illustrate the procedure for cross referencing information from tables 1-4 and the appendices. Appendix A contains an alphabetical listing of all public water-supply facilities in Arkansas. Appendix A also includes a map identification number (to be used with fig. 1) and a water-user ID. The water-user ID is used to cross reference tables 1 and 2. For example: to obtain information about the Huttig Waterworks, one could look at appendix A and find that the map ID is 294 (located on map 2 of fig. 1) and that the water-user ID is 30724 . The entries in table 1 are arranged in numerical order, so system information for water-user 30724 is found by simply scanning the table for the appropriate water-user ID. If withdrawal-site data are desired, table 2 is referenced by using the wateruser ID. Table 2 is also arranged in numerical order by the water-user ID; however, there may be several withdrawal sites associated with a single water-user ID. Huttig Waterworks has two withdrawal sites. Each withdrawal site has a unique site ID. Hydrologic unit codes (table 2) and their associated basin names are given in appendix B. Supplemental well information (table 3) and supplemental ground-water quality information (table 4) are referenced using the wateruser ID and the site ID. Supplemental well information is available for both Huttig Waterworks sites, but supplemental ground-water quality information is only available for one site.

\section{REFERENCE}

U.S. Environmental Protection Agency, 1988, Secondary maximum contaminant levels (section 143.3 of part 143, National secondary drinking-water regulations): U.S. Code of Federal Regulations, Title 40, Parts 100 to 149, revised as of July 1, 1988, p. 608. 


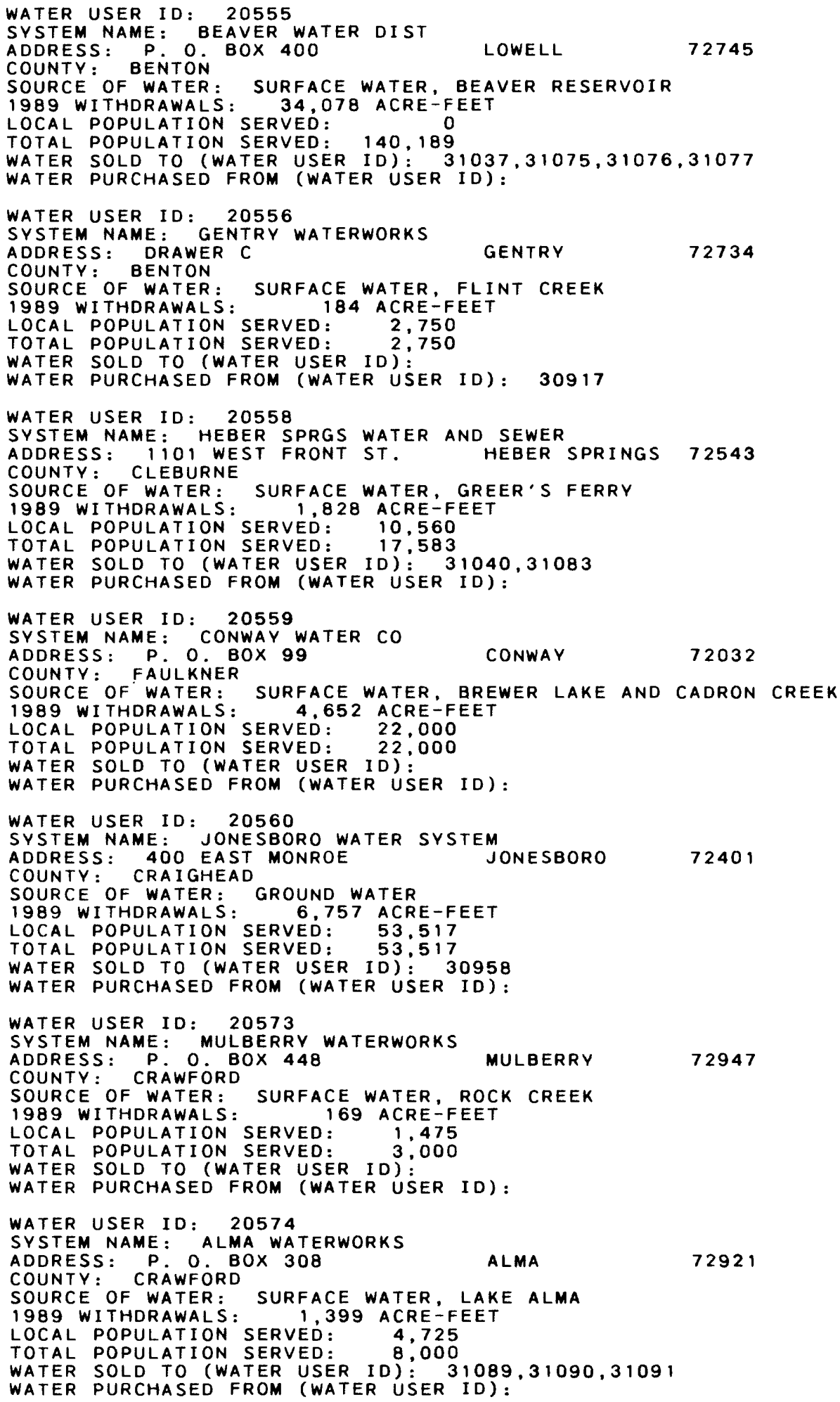


Table 1.--Description of public water supplies in Arkansas--Continued

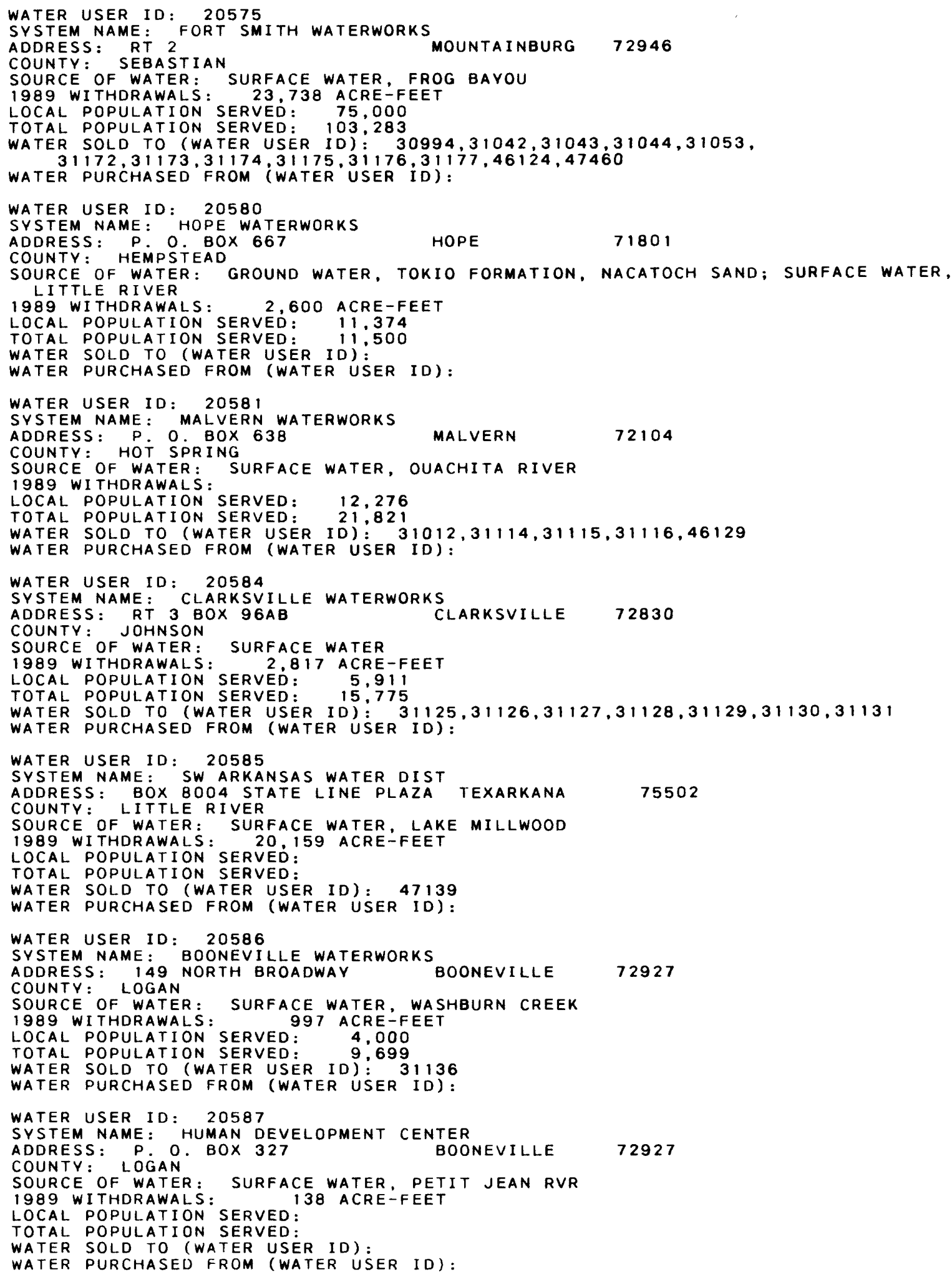


Table 1.--Description of public water supplies in Arkansas--Continued

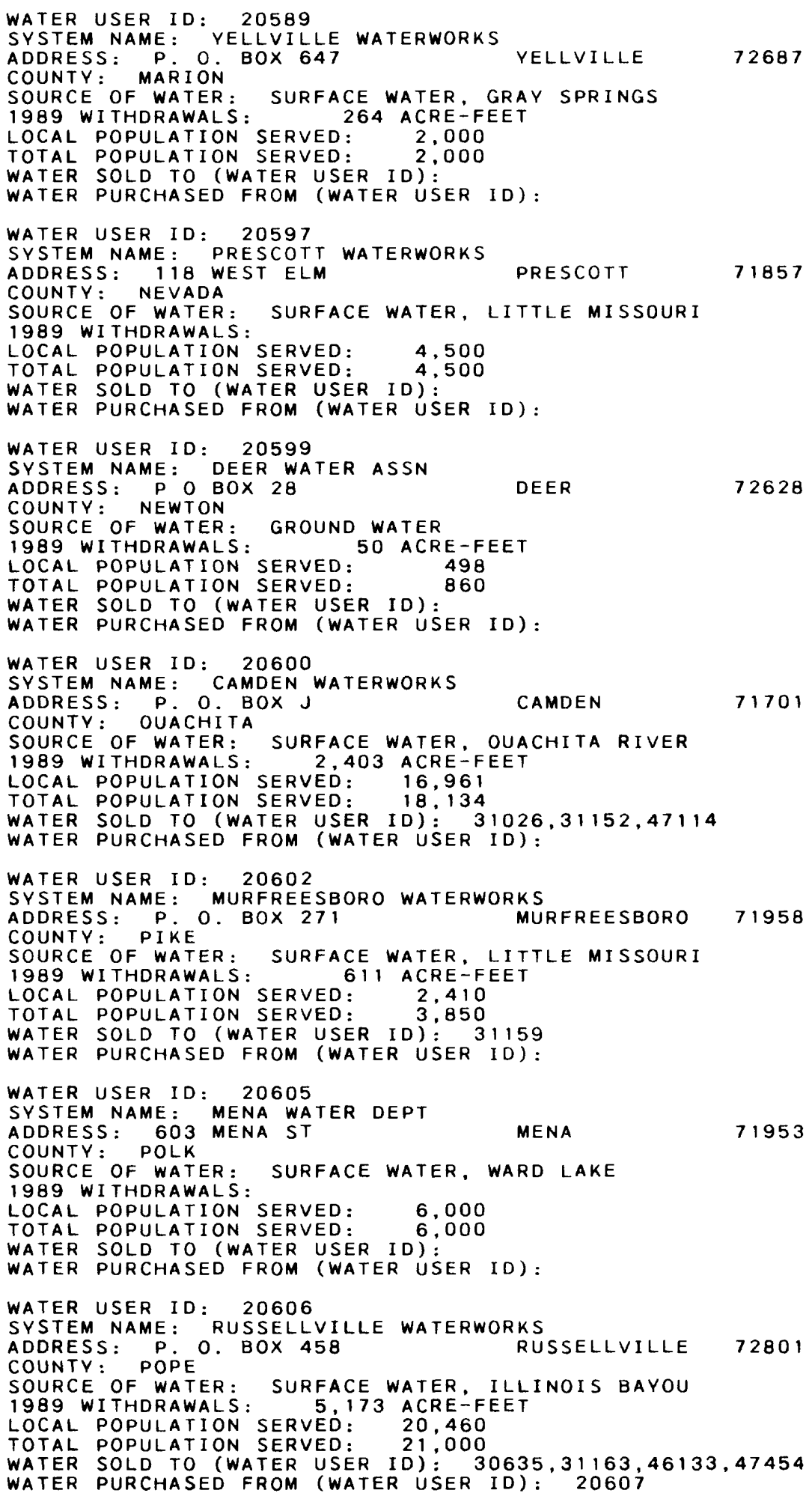


Table 1.--Description of public water supplies in Arkansas--Continued

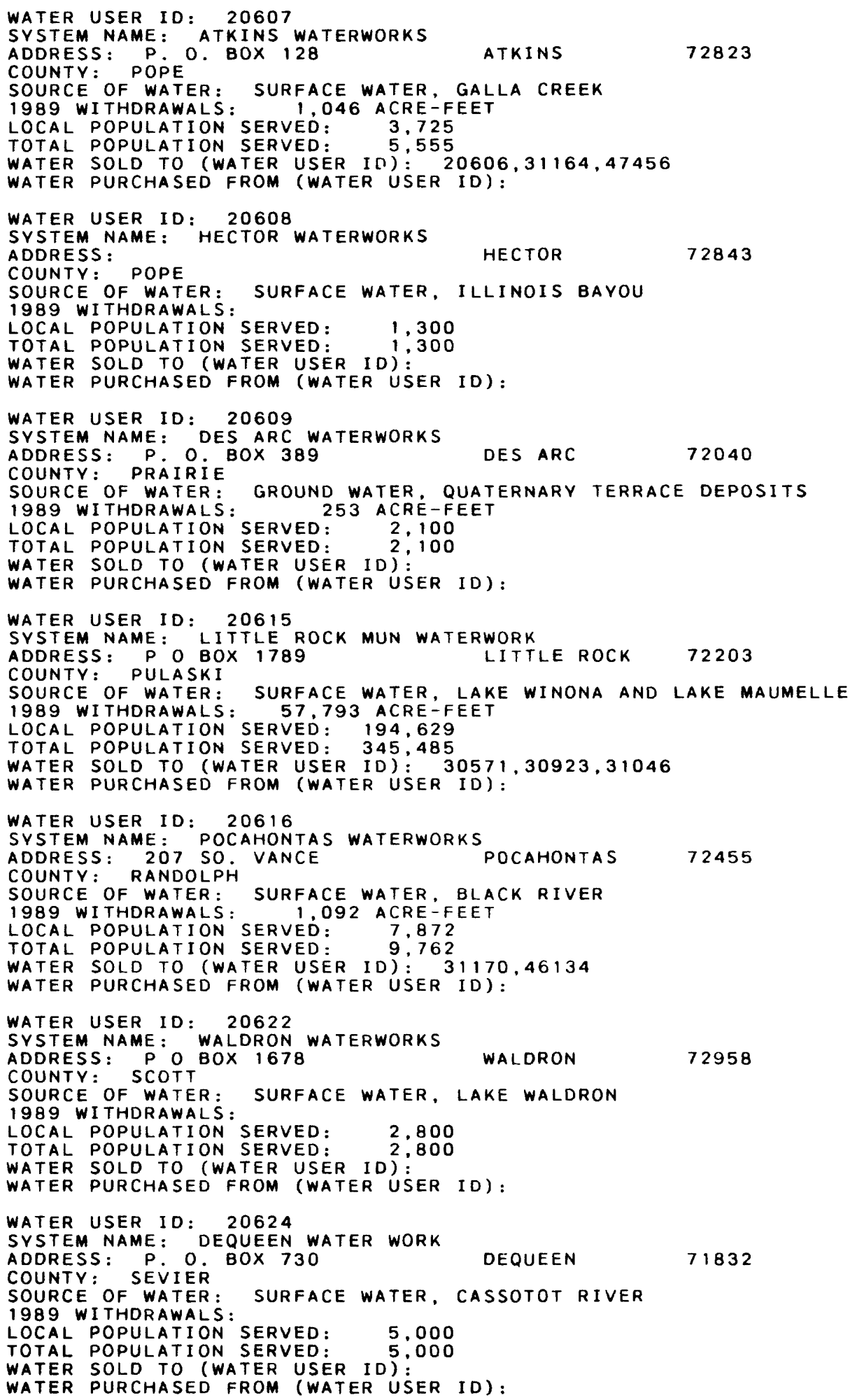


Table 1.--Description of public water supplies in Arkansas--Continued

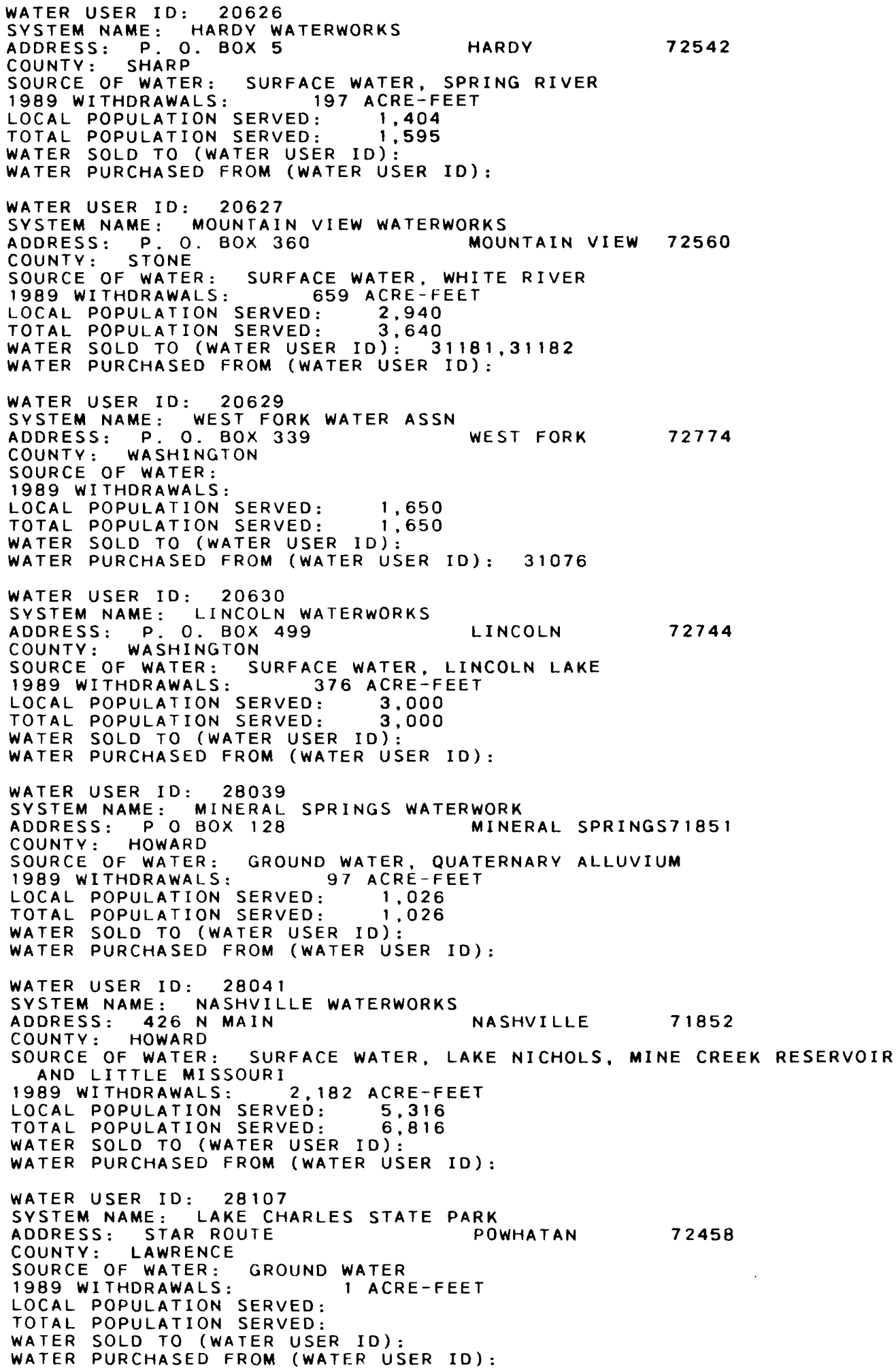


Table 1.--Description of public water supplies in Arkansas--Continued

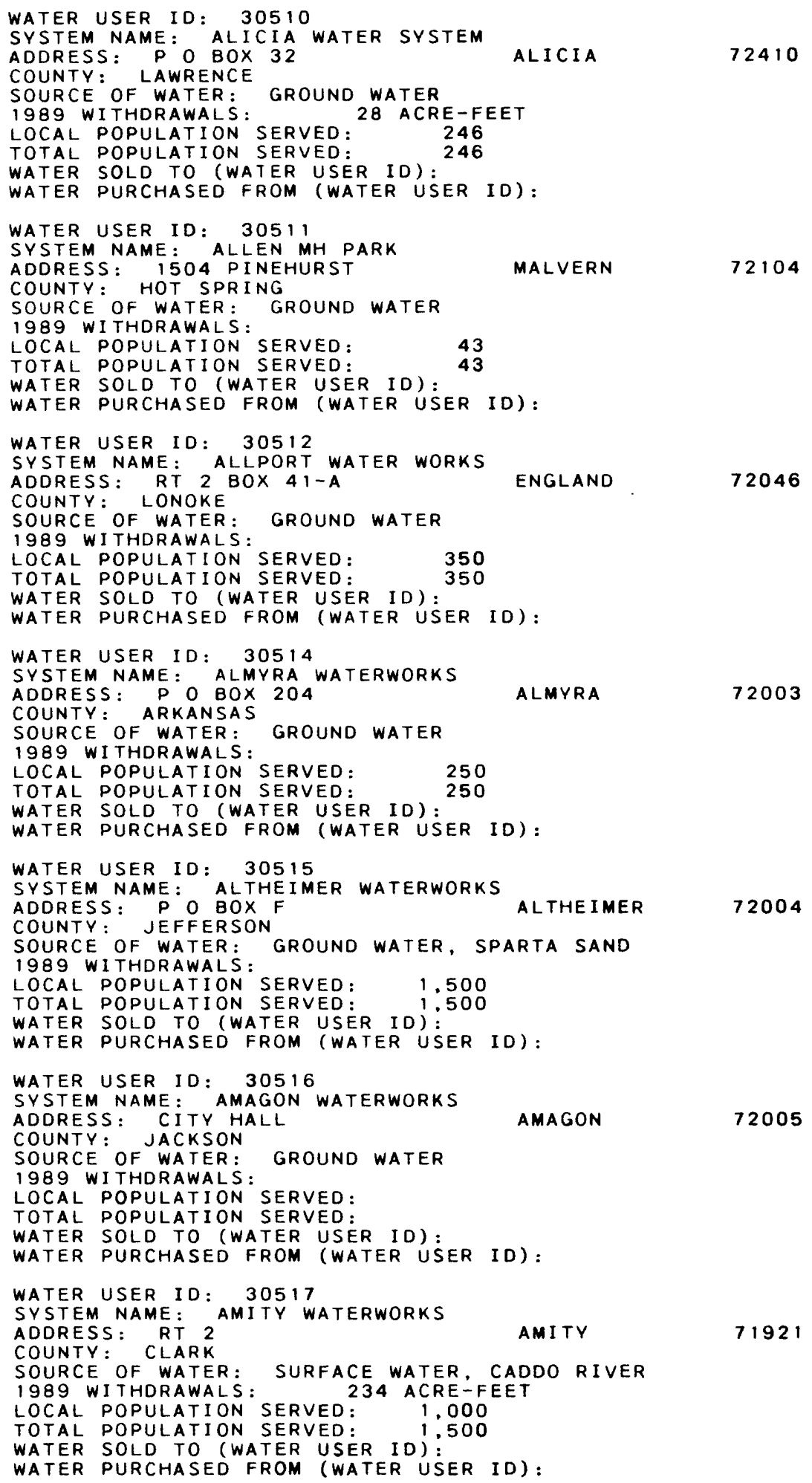


Table 1.--Description of public water supplies in Arkansas--Continued

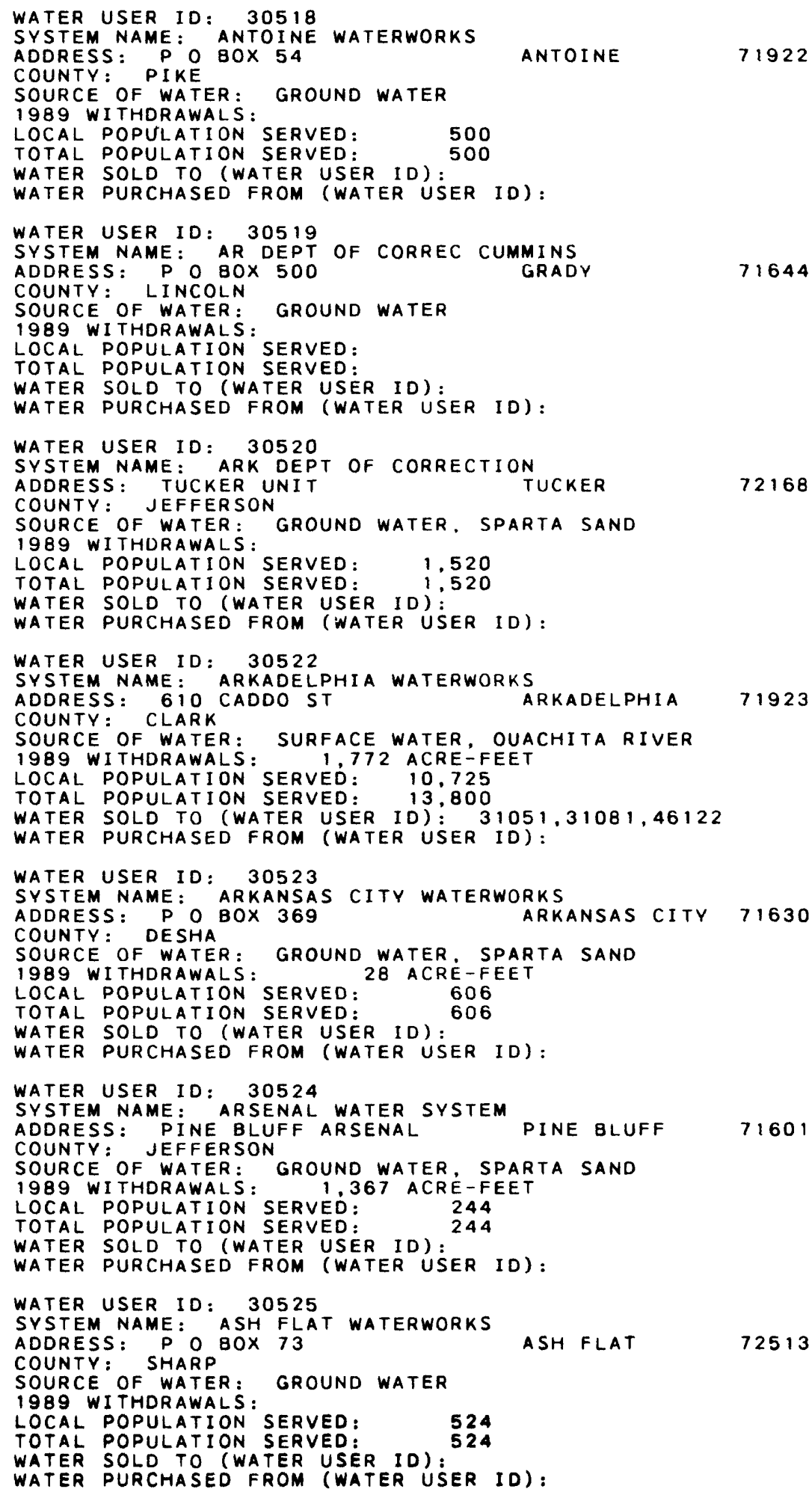


Table 1.--Description of public' water supplies in Arkansas--Continued

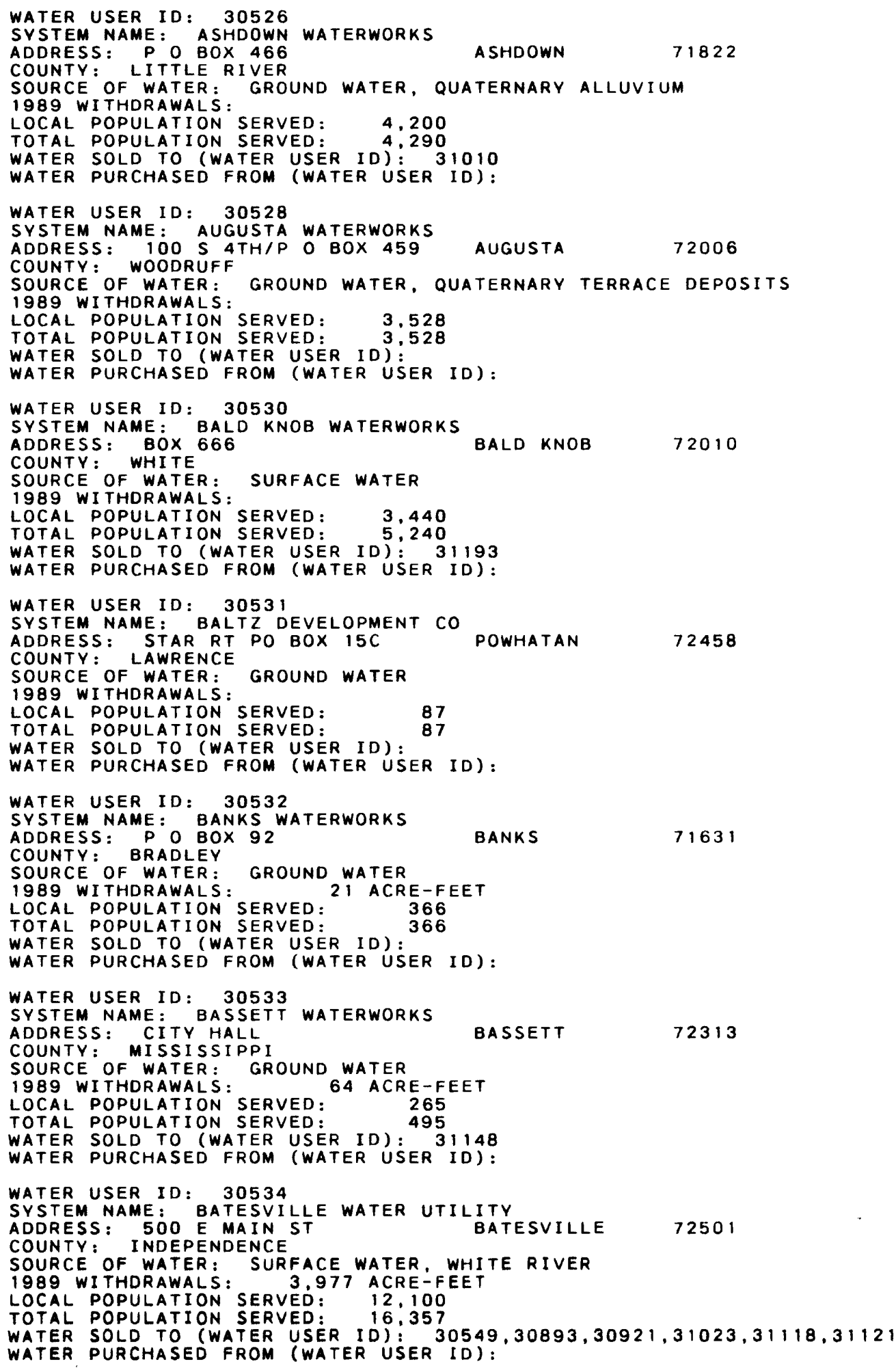


Table 1.--Description of public water supplies in Arkansas--Continued

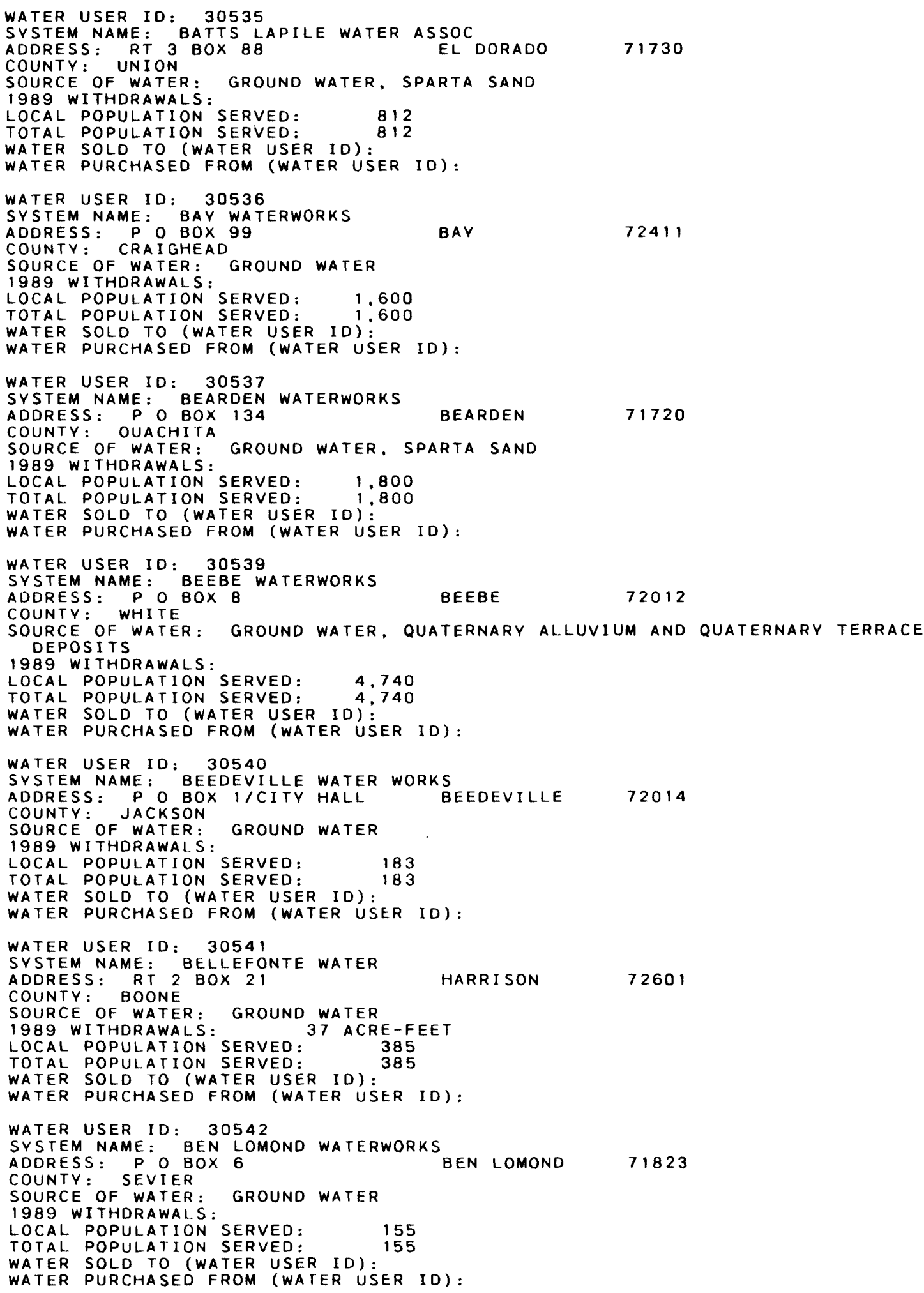


Table 1.--Description of public water supplies in Arkansas--Continued

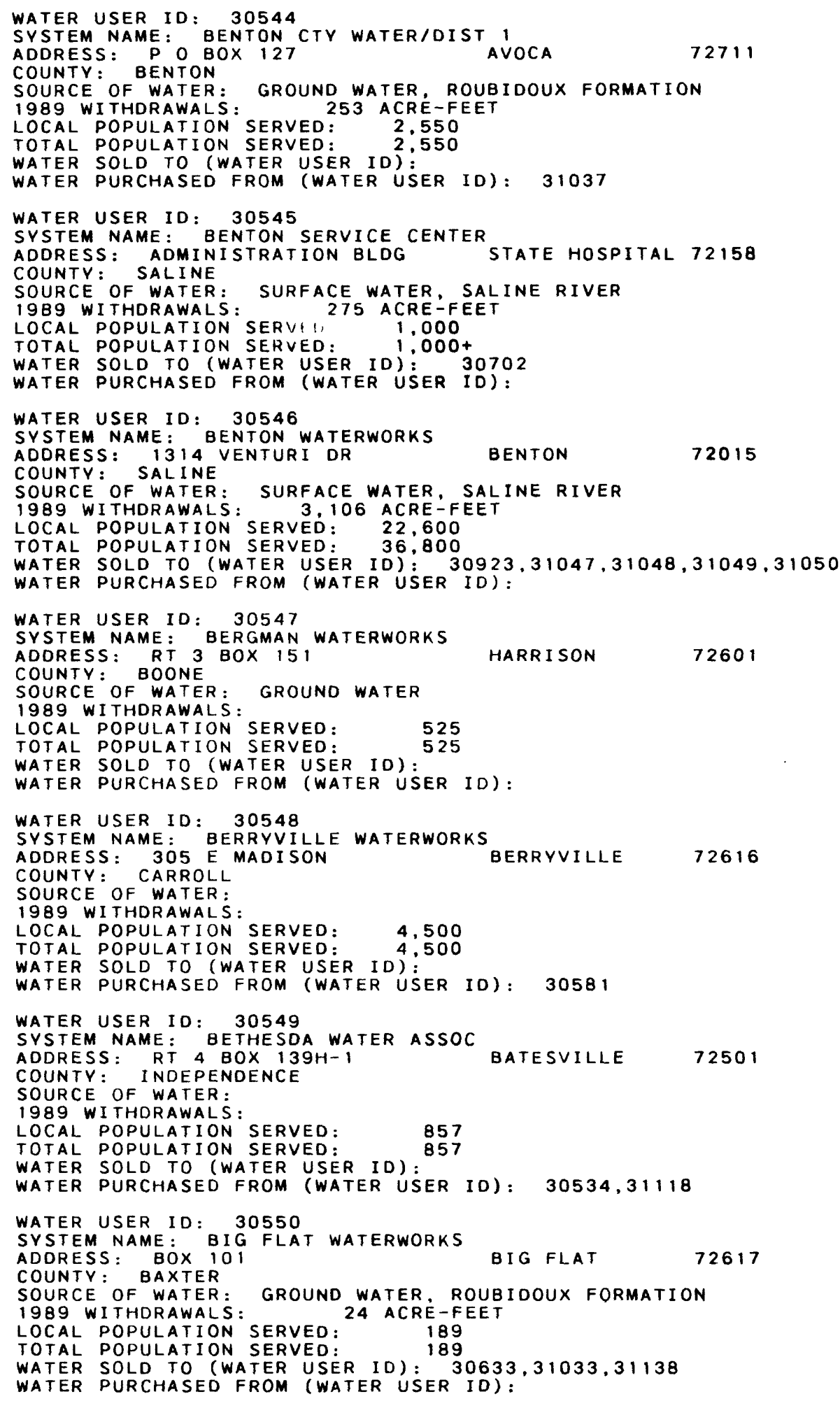


Table 1.--Description of public water supplies in Arkansas--Continued

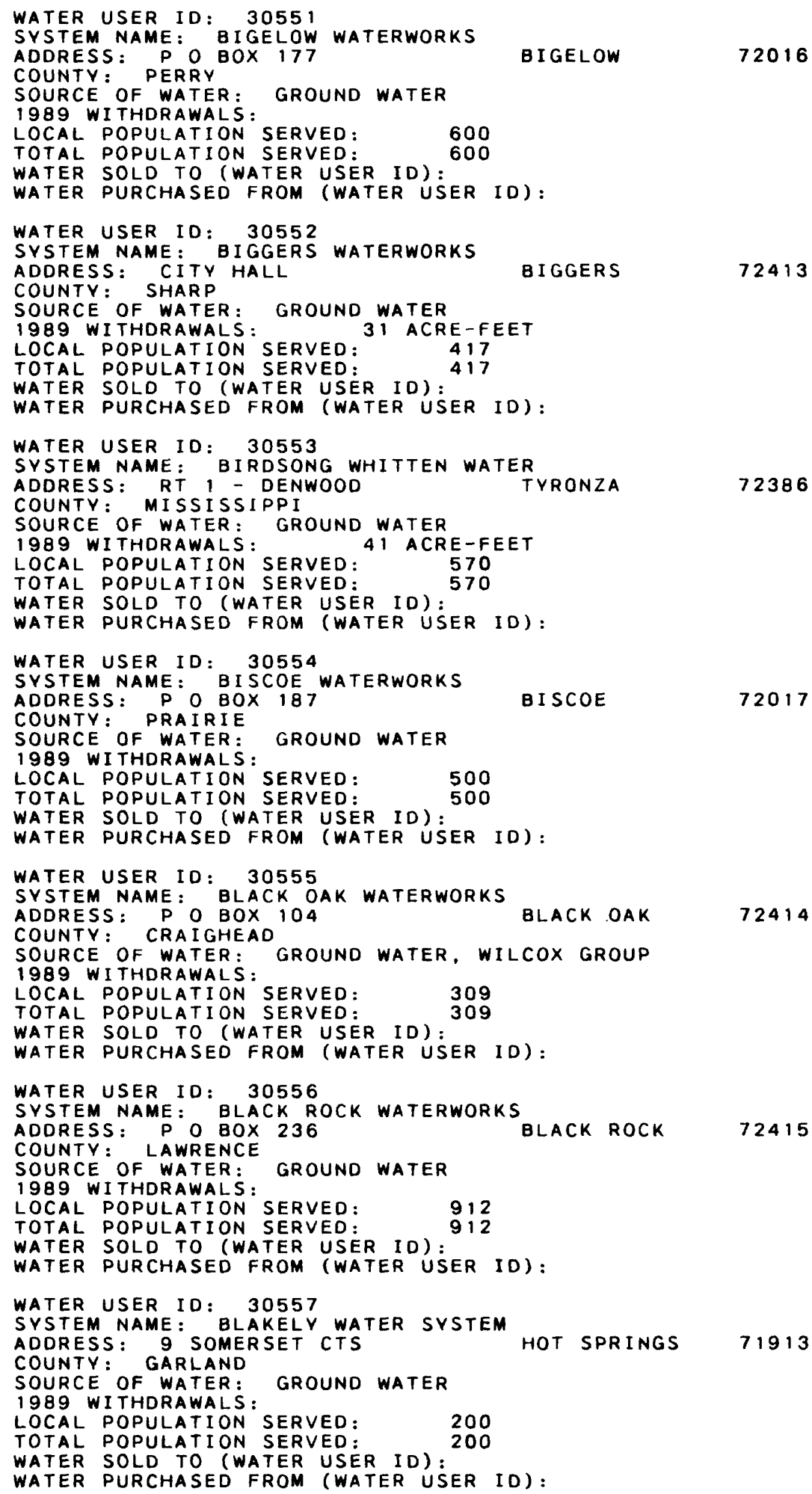


Table 1.--Description of public water supplies in Arkansas--Continued

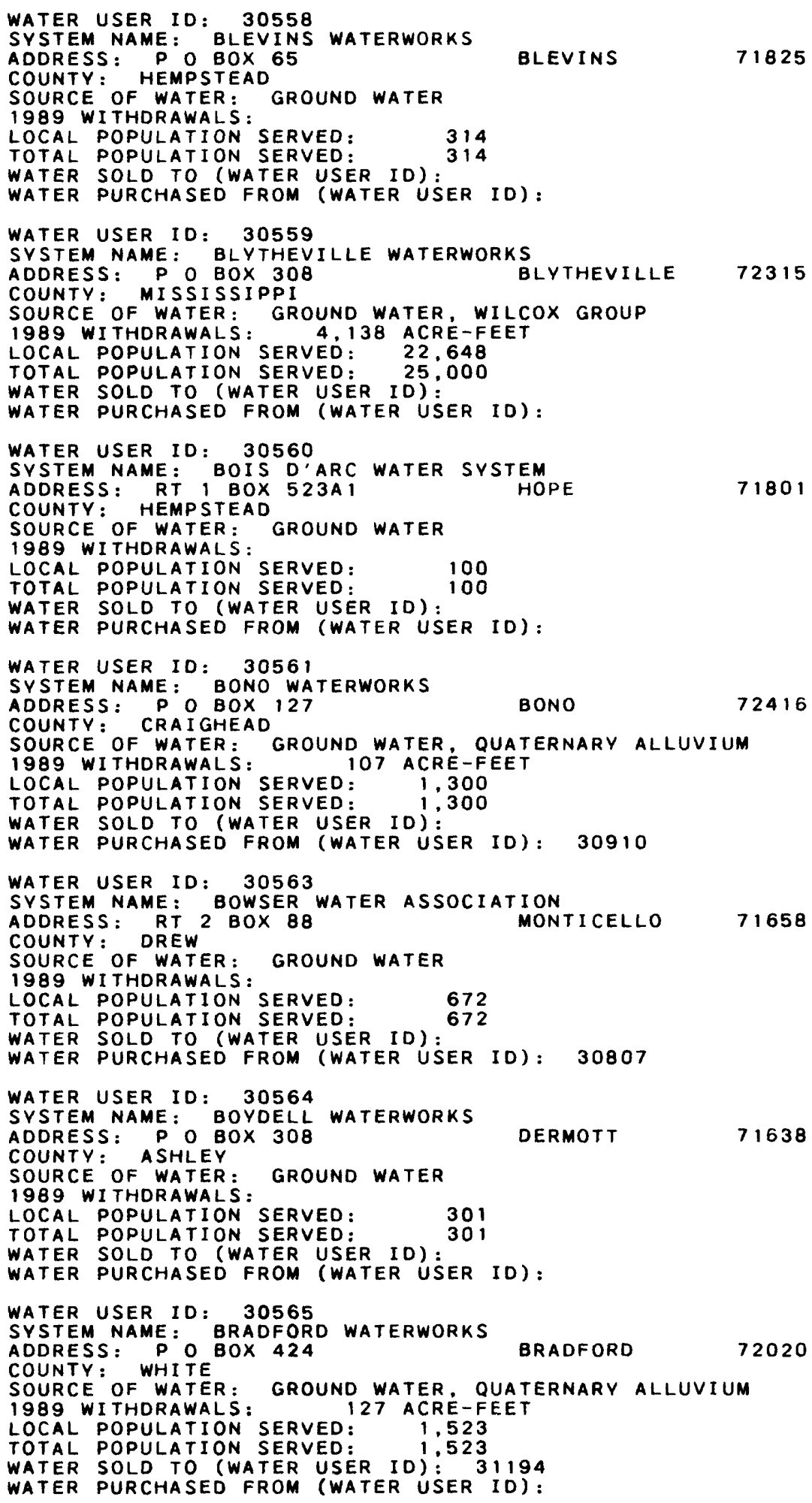


Table 1.--Description of public water supplies in Arkansas--Continued

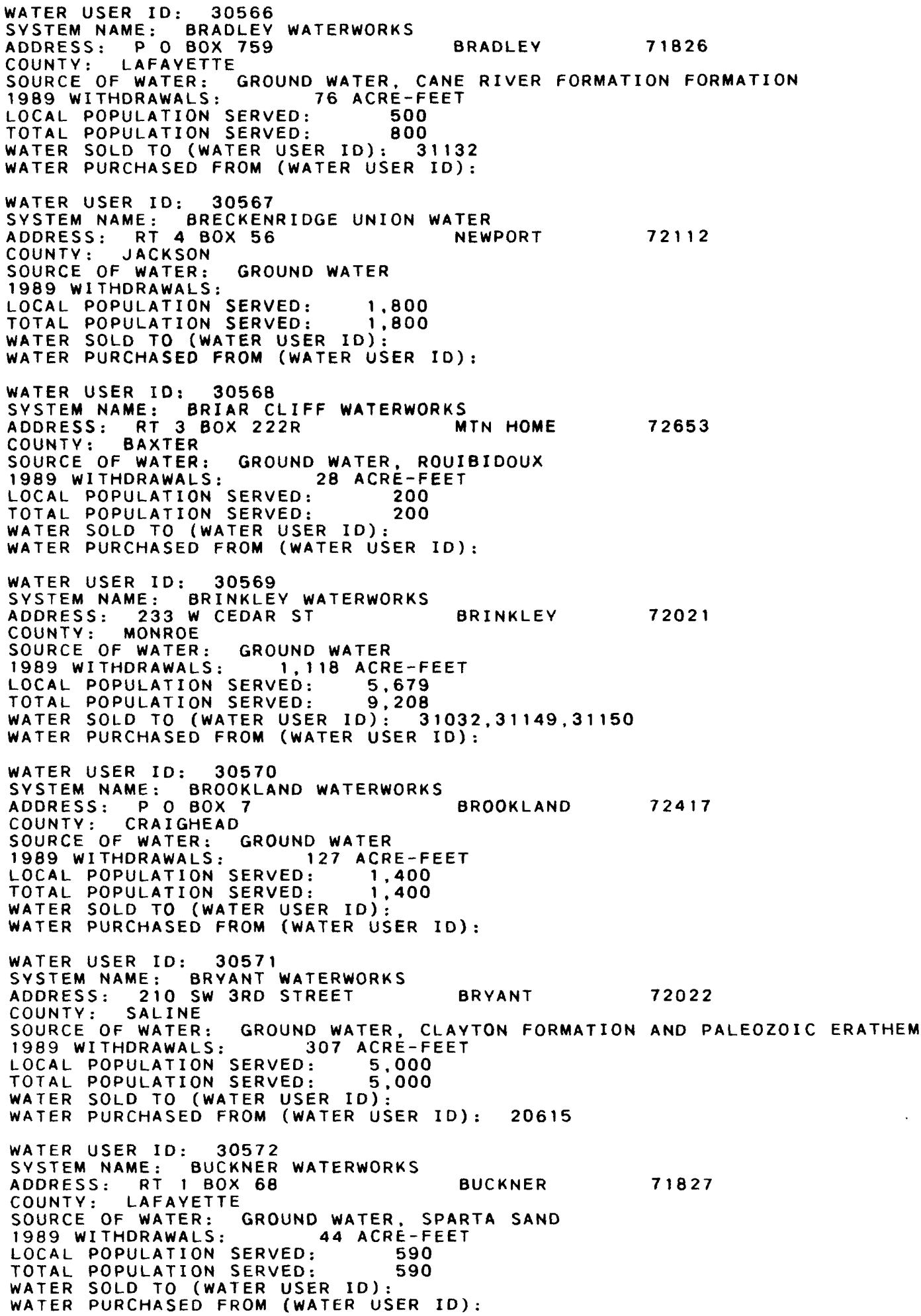


Table 1.--Description of public water supplies in Arkansas--Continued

WATER USER ID: 30573

SYSTEM NAME: BULL SHOALS WATER

ADDRESS: $P$ O BOX 390

COUNTY: MARION

SOURCE OF WATER: GROUND WATER, ROUBIDOUX FORMATION

1989 WI THDRAWALS : 147 ACRE-FEET

LOCAL POPULATION SERVED: 1,884

TOTAL POPULATION SERVED: 1, 1884

WATER SOLD TO (WATER USER ID):

WATER PURCHASED FROM (WATER USER ID):

WATER USER ID: $\quad 30574$

SYSTEM NAME: BURDETTE WATERWORKS

ADDRESS: P O BOX 112

COUNTY: MISSISSIPPI

SOURCE OF WATER: GROUND WATER, WILCOX GROUP

1989 WITHDRAWALS: 135 ACRE-FEET

LOCAL POPULATION SERVED: 910

TOTAL POPULATION SERVED:

WATER PURCHASED FROM (WATER USER ID):

WATER USER ID: $\quad 30575$

SYSTEM NAME: CABOT WATERWORKS

ADDRESS: P O BOX 1113

CABOT

72023

COUNTY: LONOKE

SOURCE OF WATER: GROUND WATER

1989 WITHDRAWALS: 690 ACRE-FEET

LOCAL POPULATION SERVED: 9,120

TOTAL POPULATION SERVED: 9.870

WATER SOLD TO (WATER USER ID):

WATER PURCHASED FROM (WATER USER ID): 30730

WATER USER ID: $\quad 30576$

SYSTEM NAME: CALICO ROCK WATERWORKS

ADDRESS: $P$ : 0 BOX 380

COUNTY: IZARD

SOURCE OF WATER: GROUND WATER

1989 WITHDRAWALS: 151 ACRE-FEET

LOCAL POPULATION SERVED: 1,550

TOTAL POPULATION SERVED: 1,550

WATER SOLD TO (WATER USER ID):

WATER PURCHASED FROM (WATER USER ID):

WATER USER ID: $\quad 30577$

SYSTEM NAME: CALION WATER WORKS

ADDRESS: P O BOX 405

COUNTY: UNION

SOURCE OF WATER: GROUND WATER, SPARTA SAND

1989 WITHDRAWALS : 71 ACRE-FEET

LOCAL POPULATION SERVED: 920

TOTAL POPULATION SERVED: 920

WATER SOLD TO (WATER USER ID)

WATER PURCHASED FROM (WATER USER ID) :

WATER USER ID: 30579

SYSTEM NAME: CARAWAY WATERWORKS

ADDRESS: CITY HALL/P O BOX 549

COUNTY: CRAIGHEAD

CARAWAY

72419

SOURCE OF WATER: GROUND WATER

1989 WITHDRAWALS

LOCAL POPULATION SERVED: 1.305

TOTAL POPULATION SERVED: 1,988

WATER SOLD TO (WATER USER ID): 31087

WATER PURCHASED FROM (WATER USER ID):

WATER USER ID: $\quad 30580$

SYSTEM NAME: CARLISLE WATERWORKS

ADDRESS: P O BOX 11 CITY HALL

COUNTY: LONOKE

SOURCE OF WATER: GROUND WATER, QUATERNARY ALLUVIUM

1989 WITHDRAWALS: $\quad 441$ ACRE-FEET

LOCAL POPULATION SERVED: 1,600

TOTAL POPULATION SERVED: 1.600

WATER SOLD TO (WATER USER ID)

WATER PURCHASED FROM (WATER USER ID) : 
Table 1.--Description of public water supplies in Arkansas--Continued

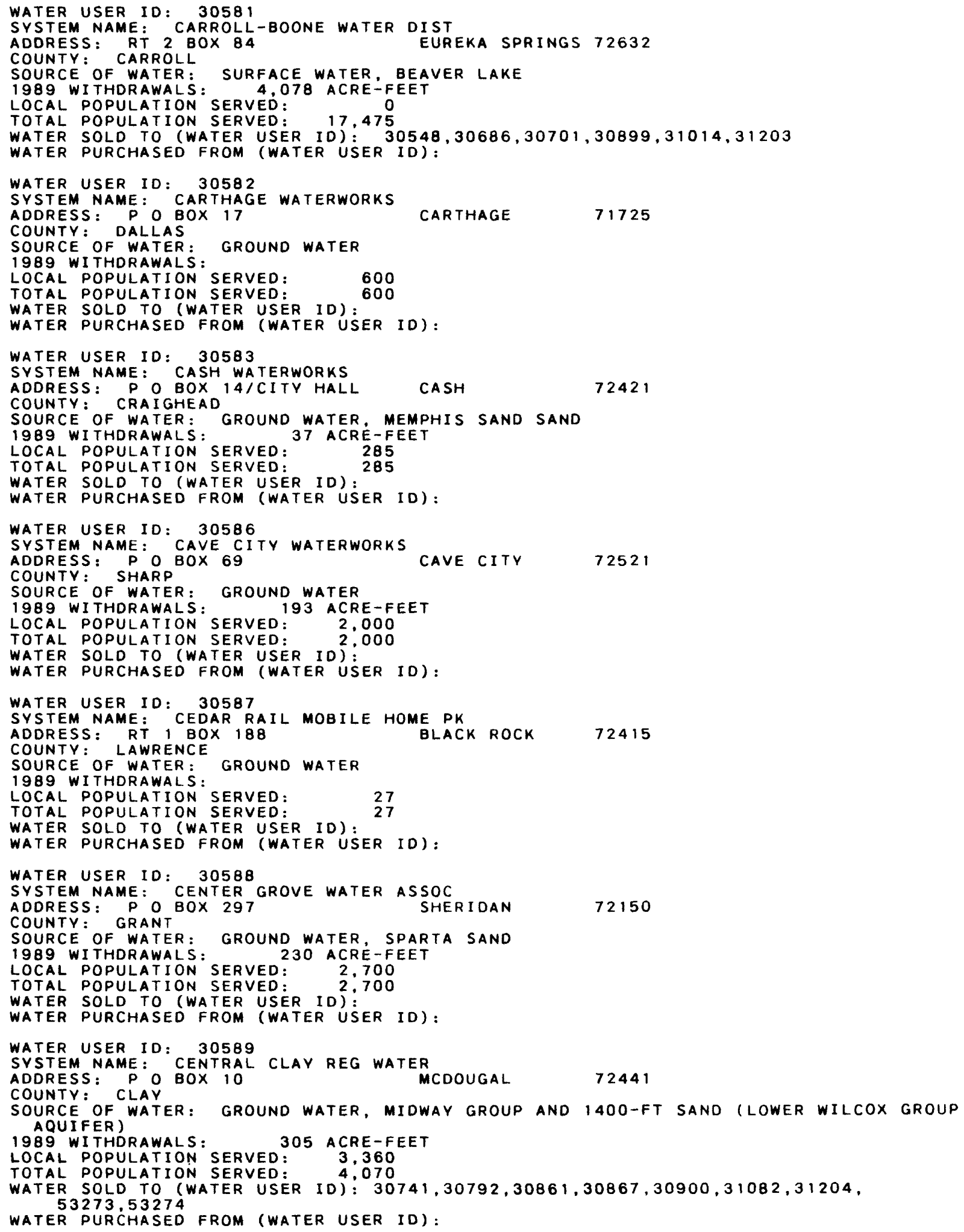


Table 1.--Description of public water supplies in Arkansas--Continued

WATER USER ID: 30590

SYSTEM NAME: CHARLESTON WATERWORKS

ADDRESS: $P$ : 0 BOX 426

ADDRESS: POO BOX
COUNTY: FRANKLIN

SOURCE OF WATER: SURFACE WATER, CHARLESTON LAKE

72933

1989 WI THDRAWALS :

LOCAL POPULATION SERVED: 2,163

TOTAL POPULATION SERVED: 2,163

WATER SOLD TO (WATER USER ID):

WATER PURCHASED FROM (WATER USER ID):

WATER USER ID: 30591

SYSTEM NAME: CHERRY VALLEY WATERWORKS

ADDRESS: $P$ O BOX 233 CHERRY VALLEY 72324

COUNTY: CROSS

SOURCE OF WATER: GROUND WATER, MEMPHIS SAND SAND

1989 WI THDRAWALS: 73 ACRE-FEET

LOCAL POPULATION SERVED: $\quad 729$

TOTAL POPULATION SERVED:
WATER SOLD TO (WATER USER ID):

WATER PURCHASED FROM (WATER USER ID):

WATER USER ID: 30592

SYSTEM NAME: CHIDESTER WATER WORKS

ADDRES: OP O BOX

SOURCE OF WATER: GROUND WATER, SPARTA SAND

1989 WI THDRAWALS:

LOCAL POPULATION SERVED:

TOTAL POPULATION SERVED:

WATER SOLD TO (WATER USER ID):

WATER PURCHASED FROM (WATER USER ID):

WATER USER ID: 30593

SYSTEM NAME: CLARENDON WATERWORKS

ADDRESS: 270 MADISON ST

COUNTY: MONROE

SOURCE OF WATER: GROUND WATER, SPARTA SAND

1989 WITHDRAWALS: 355 ACRE-FEET

LOCAL POPULATION SERVED: 2,361

TOTAL POPULATION SERVED: 2,361

WATER SOLD TO (WATER USER ID):

WATER PURCHASED FROM (WATER USER ID):

WATER USER ID: 30595

SYSTEM NAME: CLINTON WATERWORKS

ADDRESS: P O BOX 277

COUNTY: VAN BUREN

SOURCE OF WATER: SURFACE WATER, GREER'S FERRY

1989 WITHDRAWALS: 1,043 ACRE-FEET

LOCAL POPULATION SERVED: 5,100

TOTAL POPULATION SERVED: 9,500

WATER SOLD TO (WATER USER ID) : 31188,31189,31190,31191,31192

WATER PURCHASED FROM (WATER USER ID):

WATER USER ID: $\quad 30596$

SYSTEM NAME: COLLINS WATER ASSOC

ADDRESS: GENERAL DELIVERY ASSOC COLLINS

COUNTY: DREW

PARTA SAND

SOURCE OF WATER:

1989 WITHDRAWALS

$\begin{array}{ll}\text { LOCAL POPULATION SERVED: } & 250 \\ \text { TOTAL POPULATION SERVED: } & \mathbf{2 5 0}\end{array}$

WATER SOLD TO (WATER USER ID):

WATER PURCHASED FROM (WATER USER ID) :

WATER USER ID: 30597

SYSTEM NAME: COLT WATER ASSOCIATION

ADDRESS:

COUNTY: ST FRANCIS

SOURCE Gr WATER: GROUND WATER

1989 WITHDRAWALS: 94 ACRE-FEET

LOCAL POPULATION SERVED:

TOTAL POPULATION SERVED:

WATER SOLD TO (WATER USER ID):

WATER PURCHASED FROM (WATER USER ID): 
Table 1.--Description of public water supplies in Arkansas--Continued

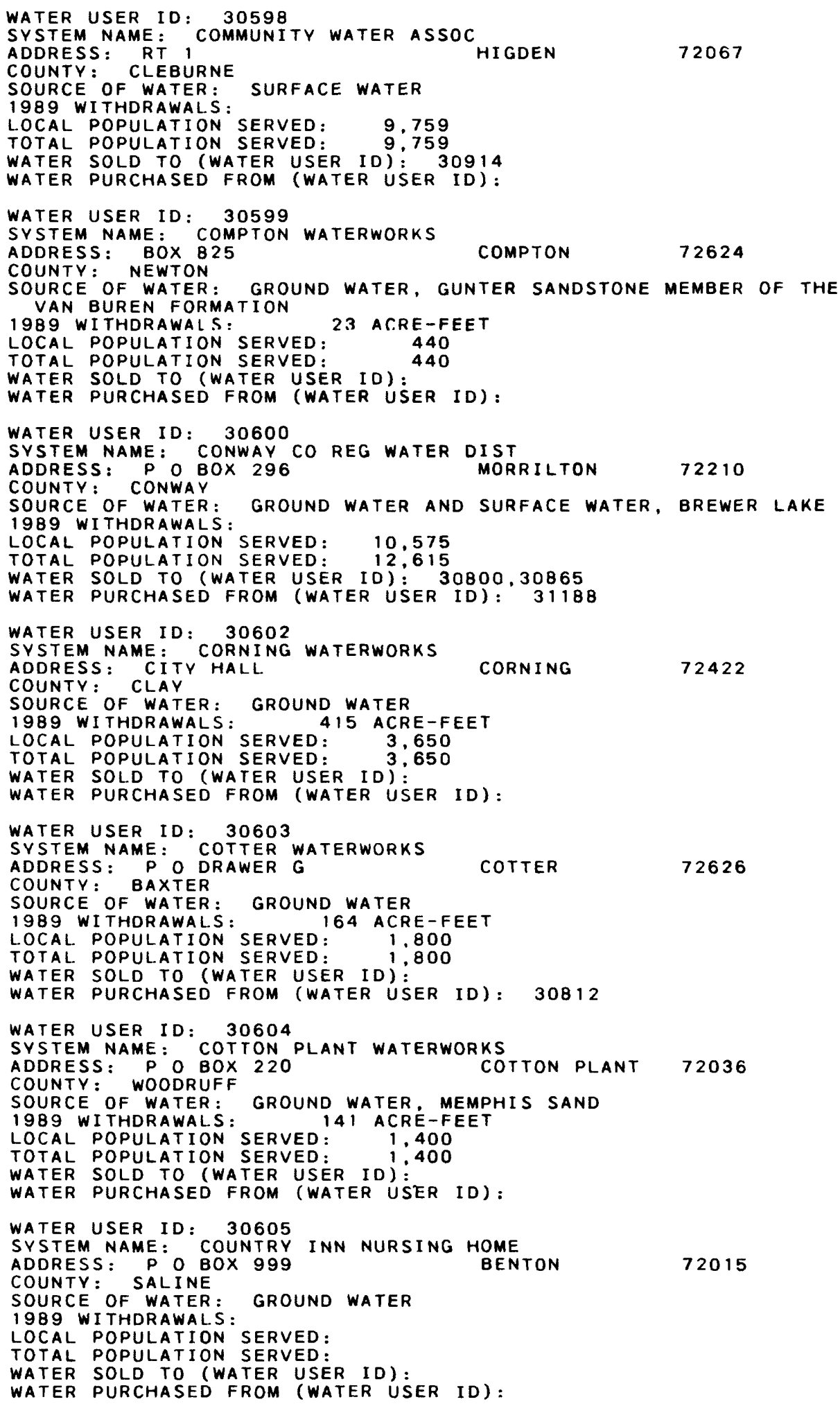


Table 1.--Description of public water supplies in Arkansas--Continued

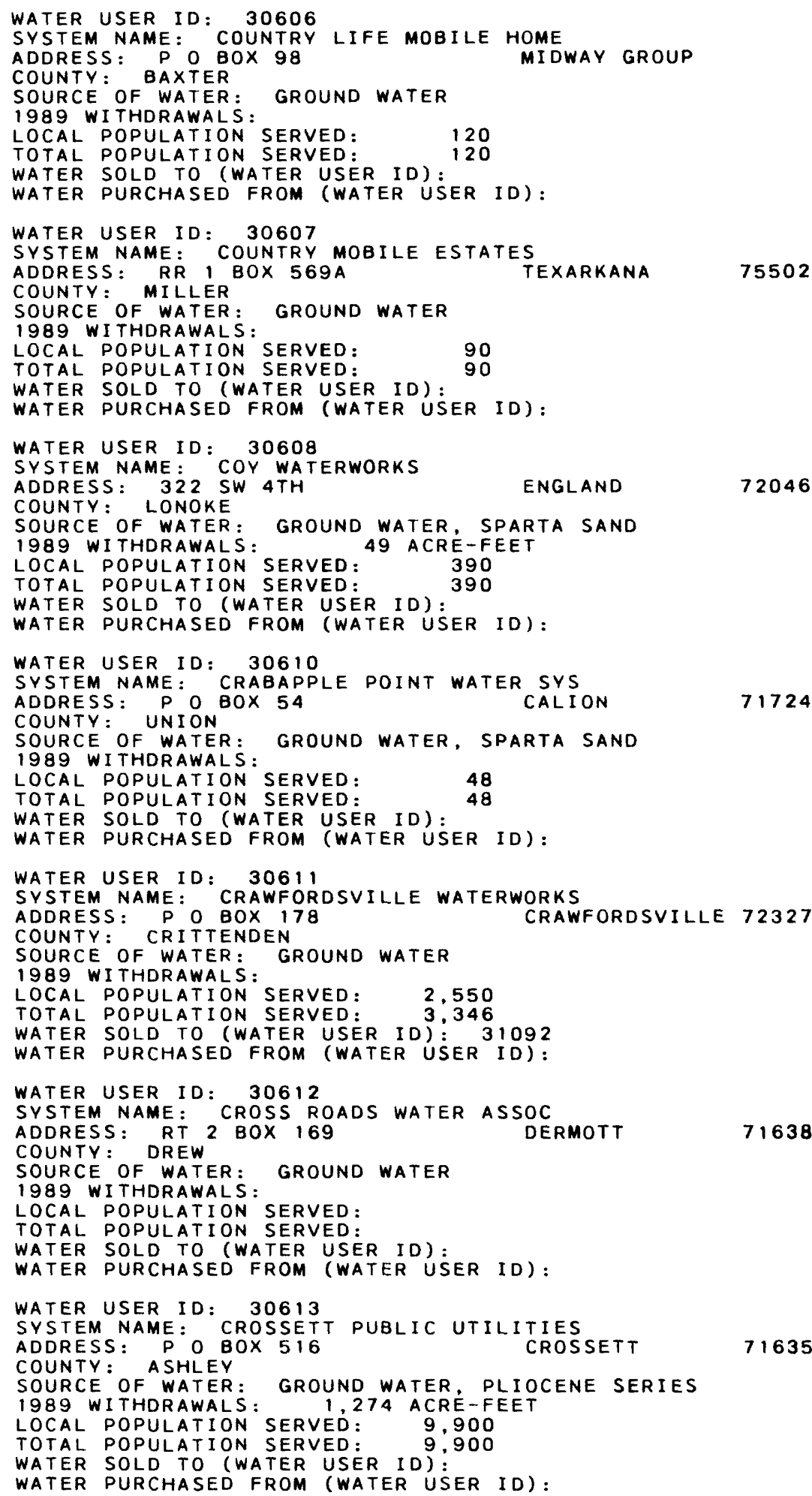


Table 1.--Description of public water supplies in Arkansas--Continued

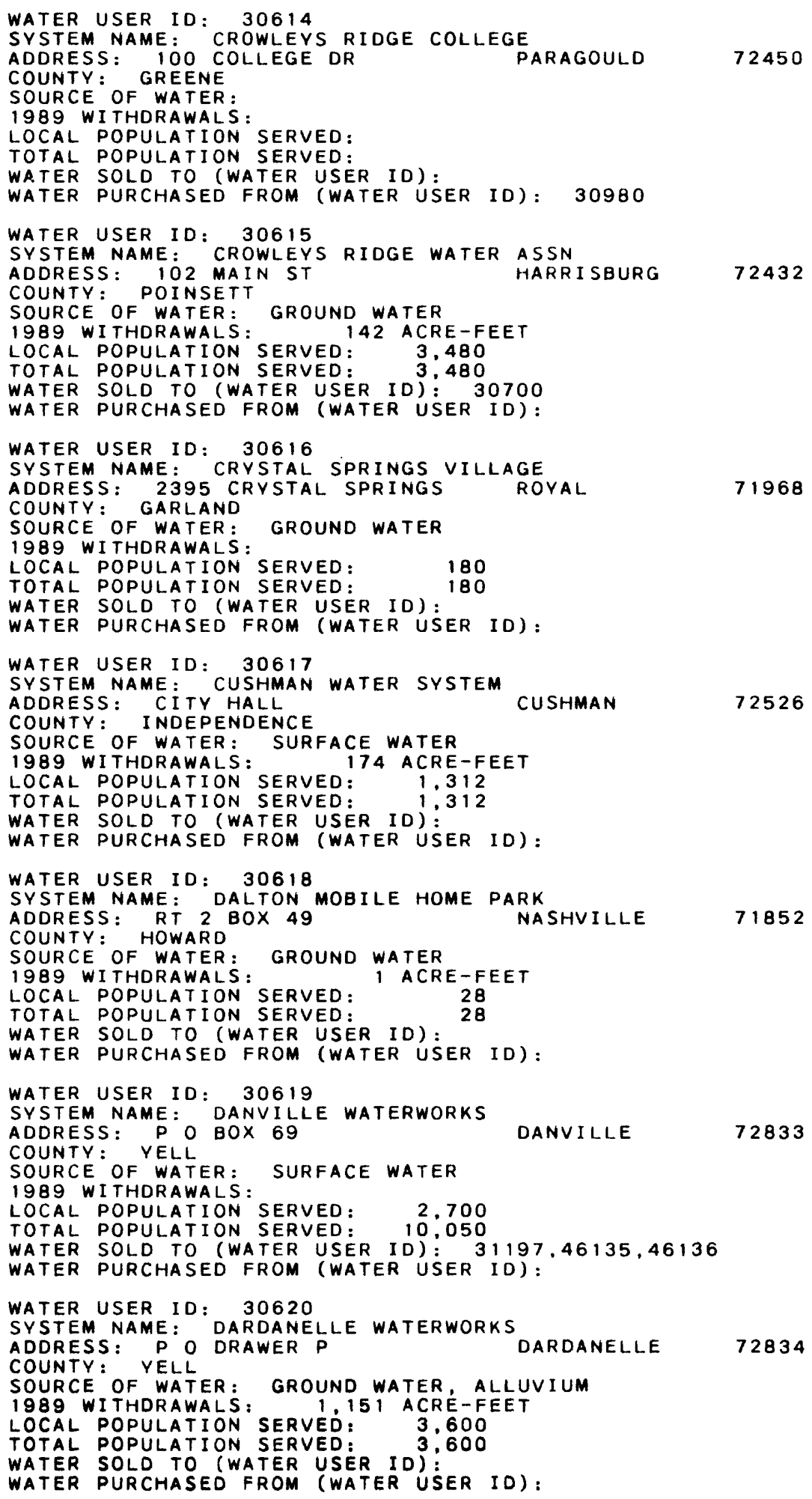


Table 1.--Description of public water supplies in Arkansas--Continued

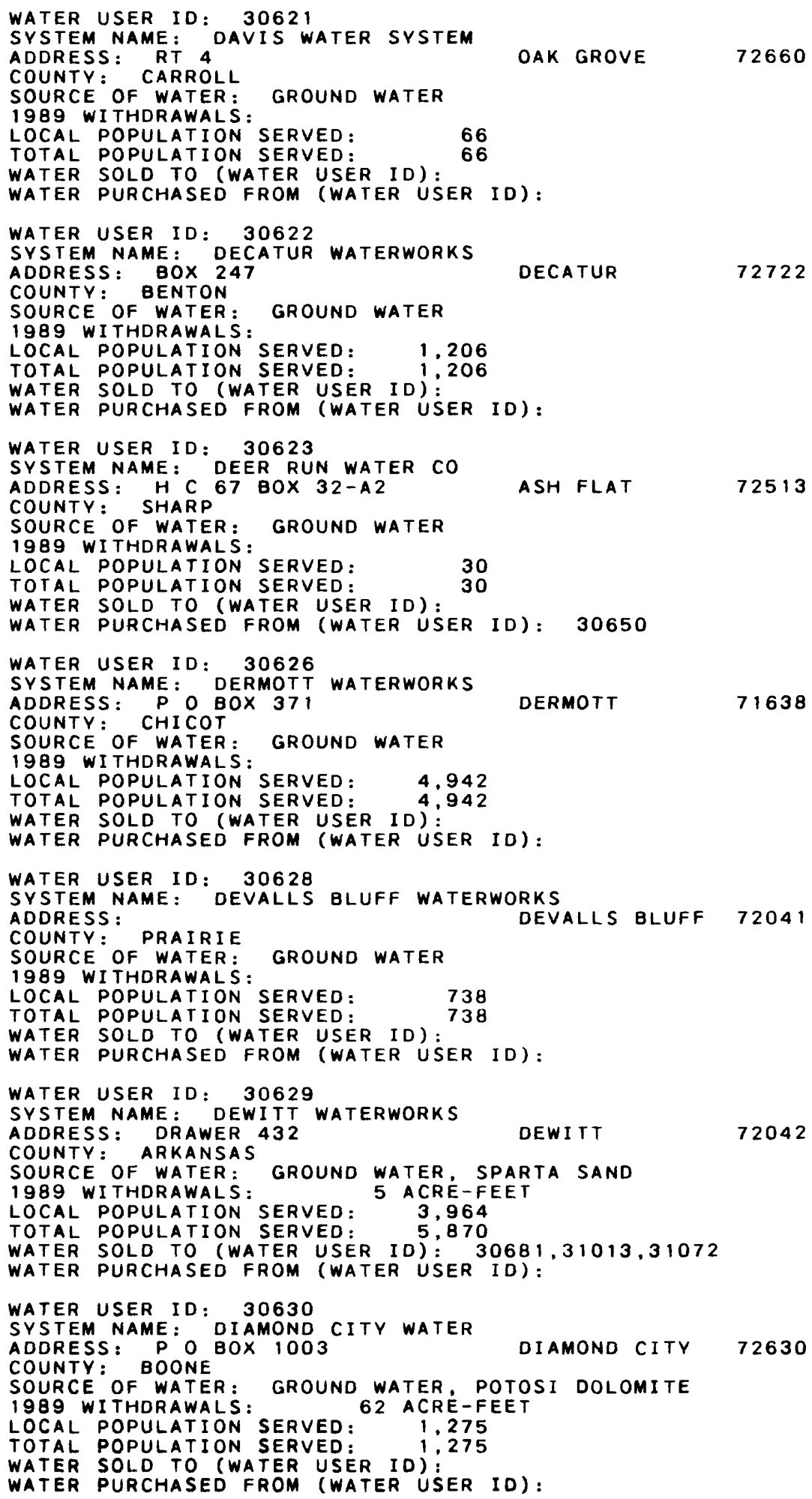


Table 1.--Description of public water supplies in Arkansas--Continued

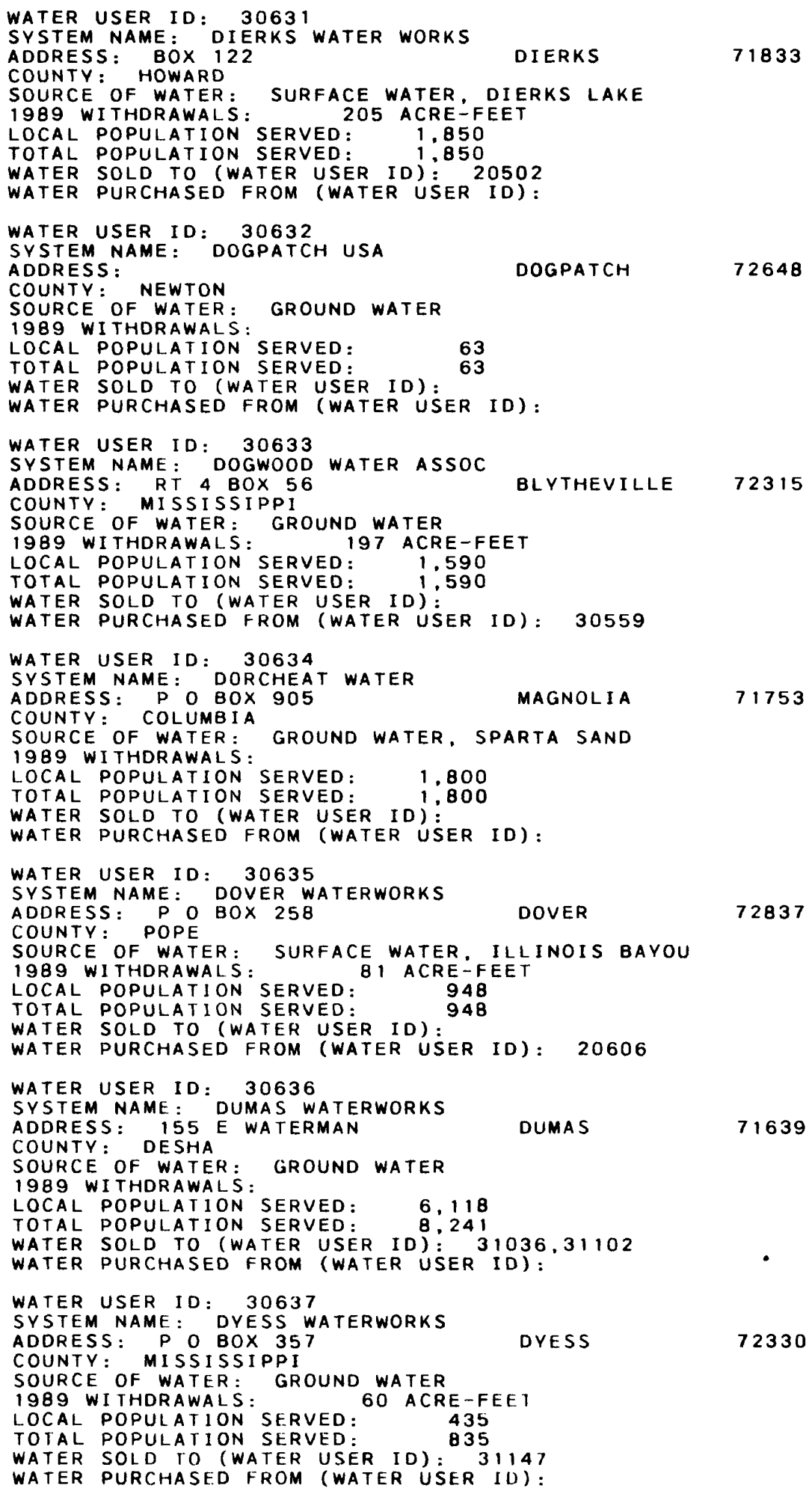


Table 1.--Description of public water supplies in Arkansas--Continued

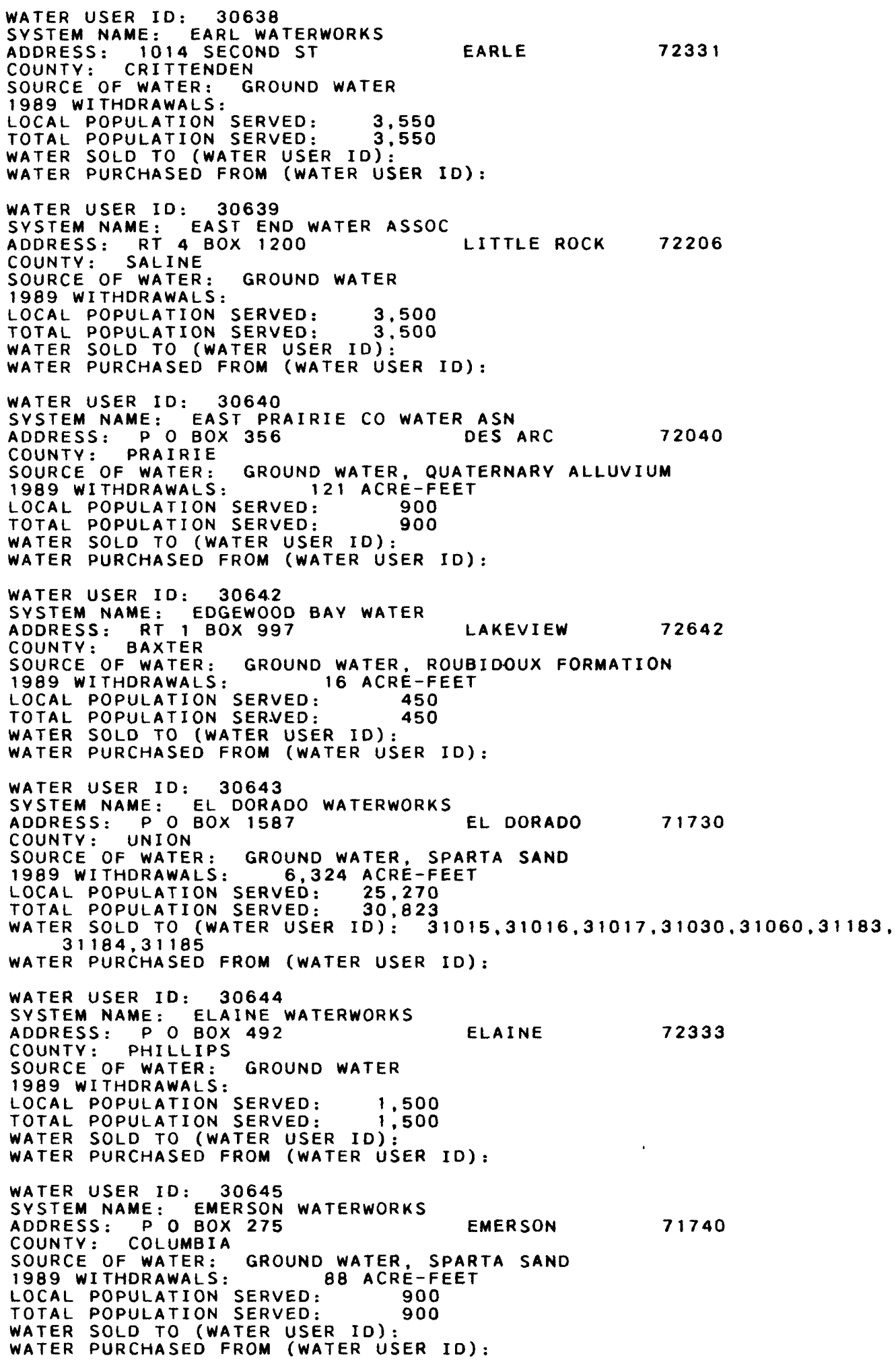

ELAINE $\quad \mathbf{7 2 3 3 3}$

71740 
Table 1.--Description of public water supplies in Arkansas--Continued

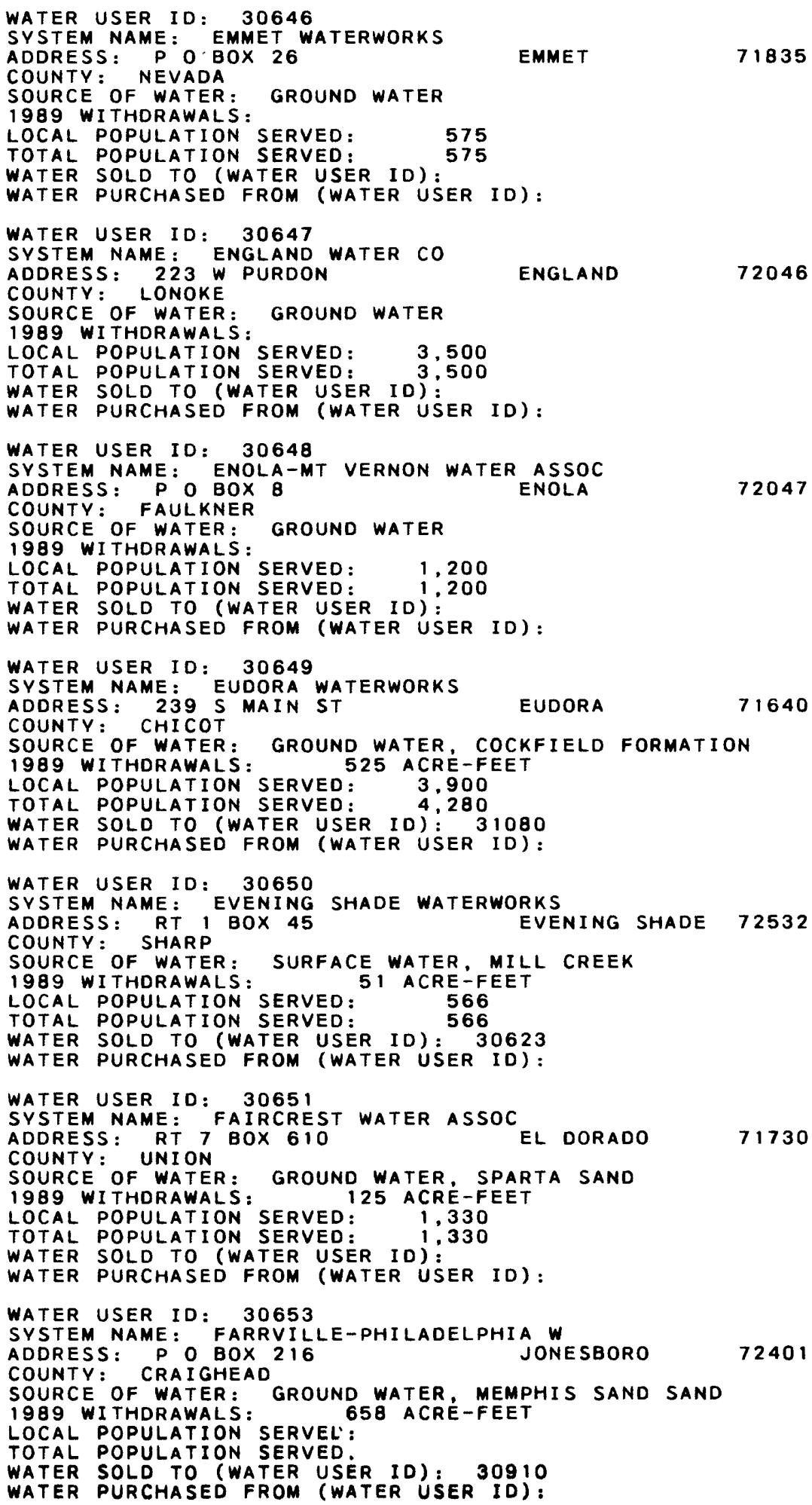


Table 1.--Description of public water supplies in Arkansas--Continued

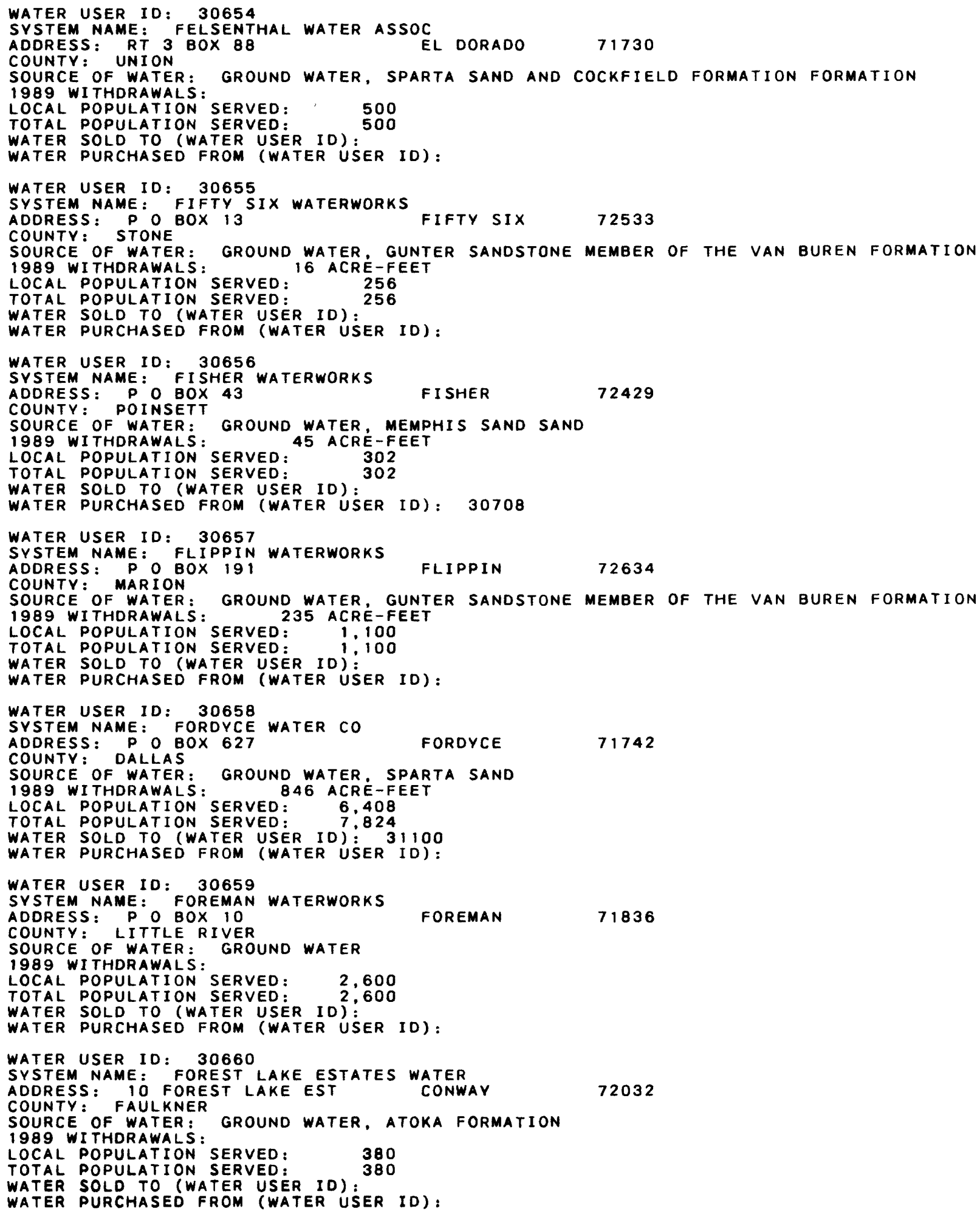


Table 1.--Description of public water supplies in Arkansas--Continued

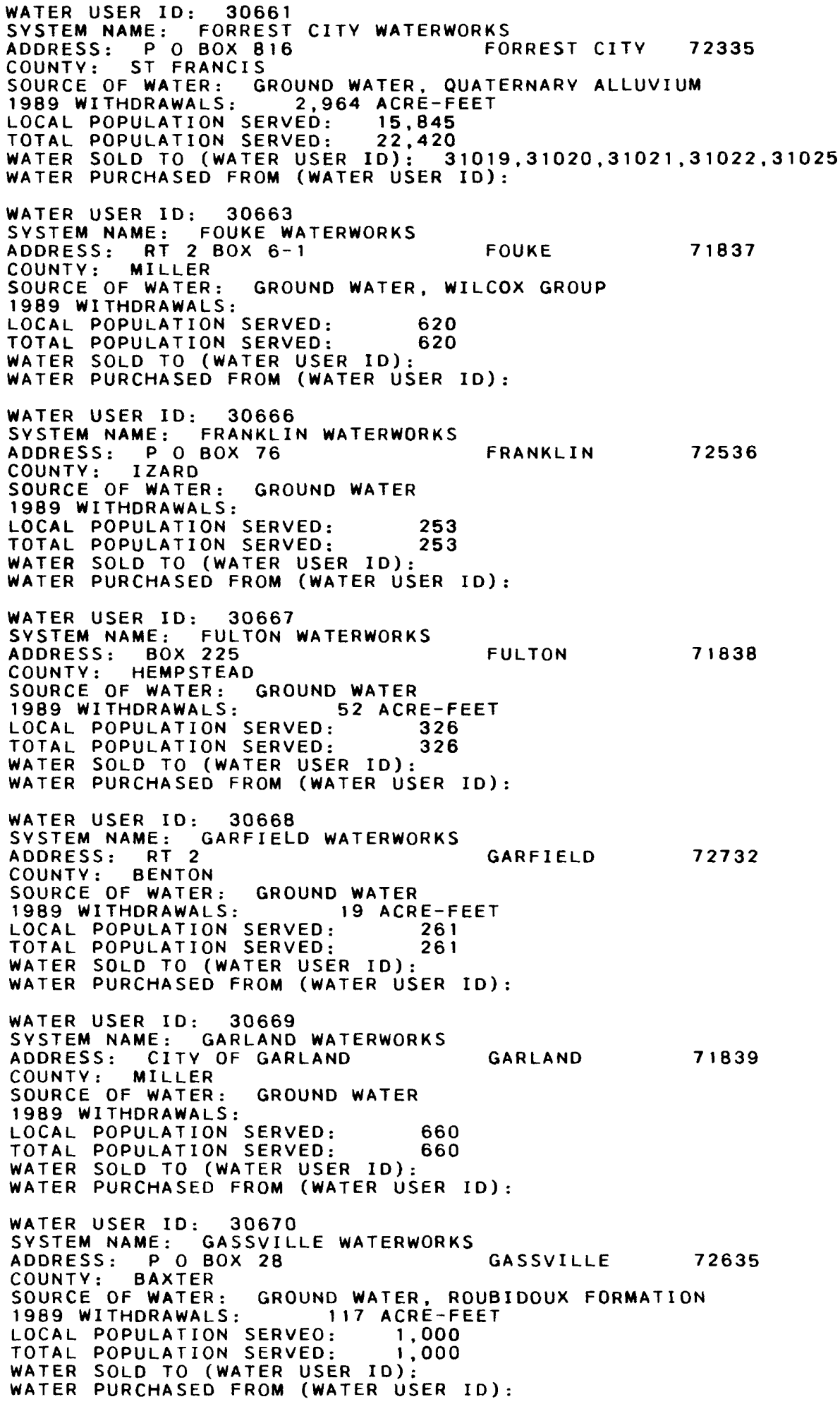


Table 1.--Description of public water supplies in Arkansas--Continued

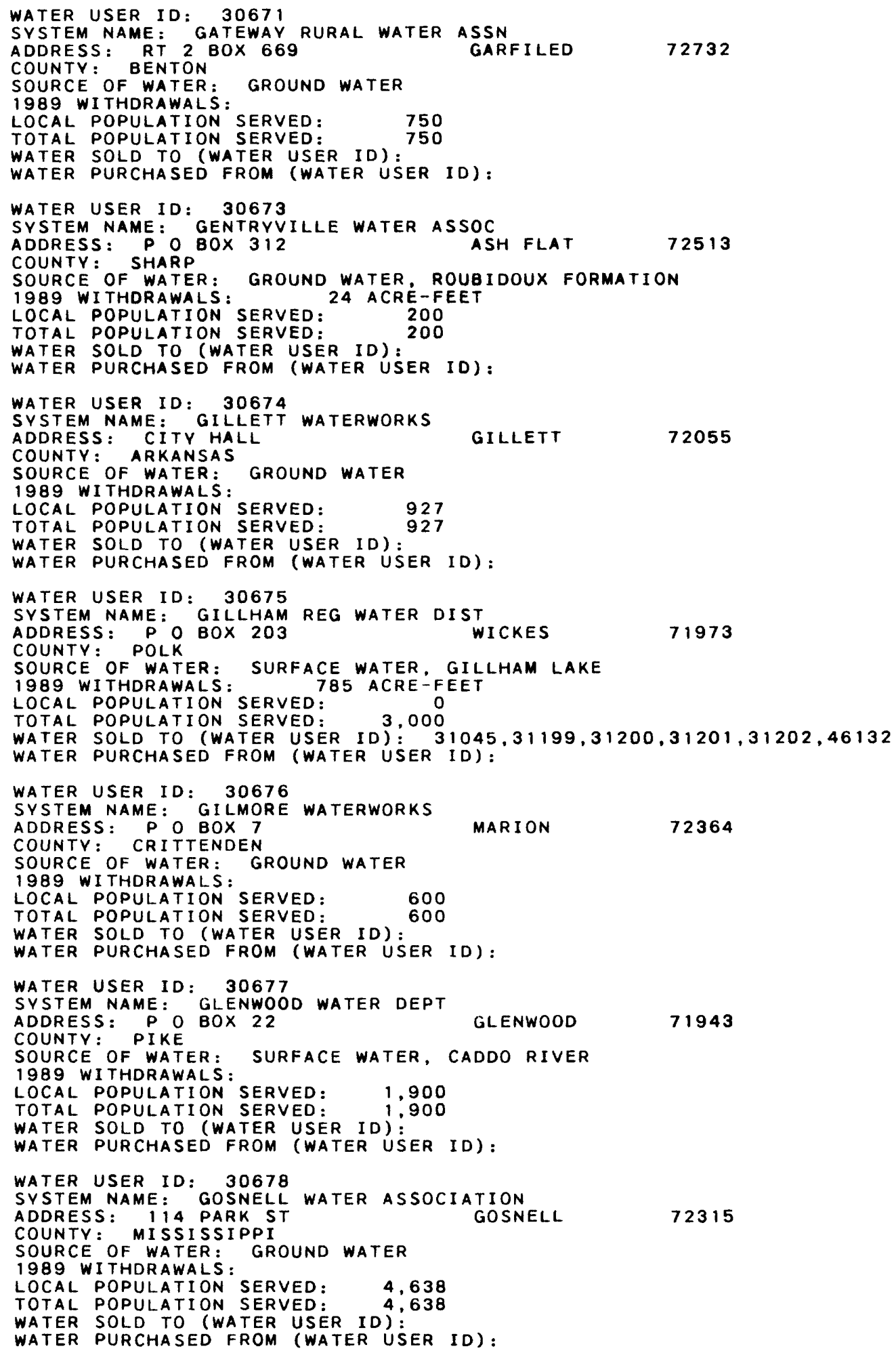


Table 1.--Description of public water supplies in Arkansas--Continued

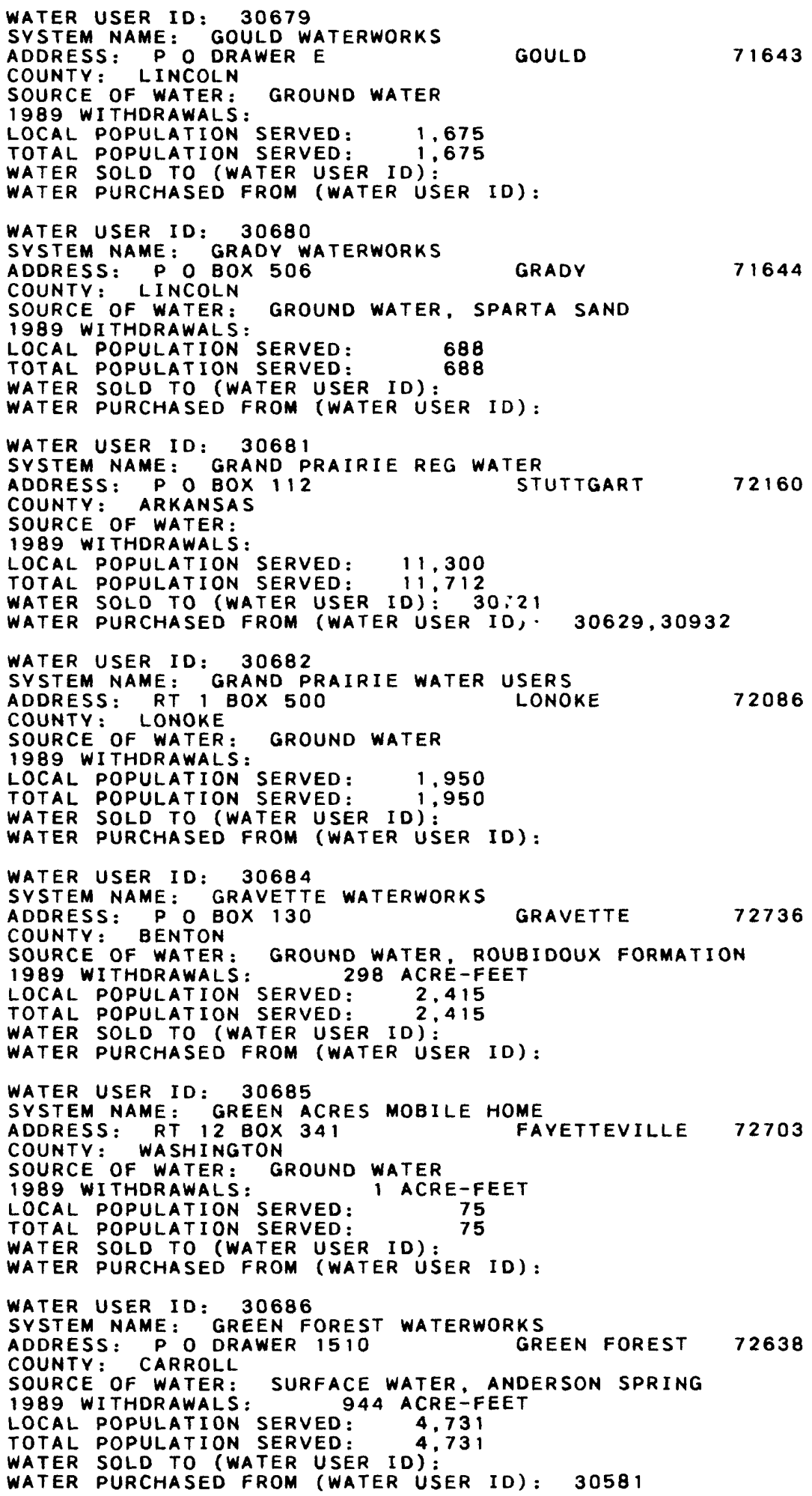


Table 1.--Description of public water supplies in Arkansas--Continued

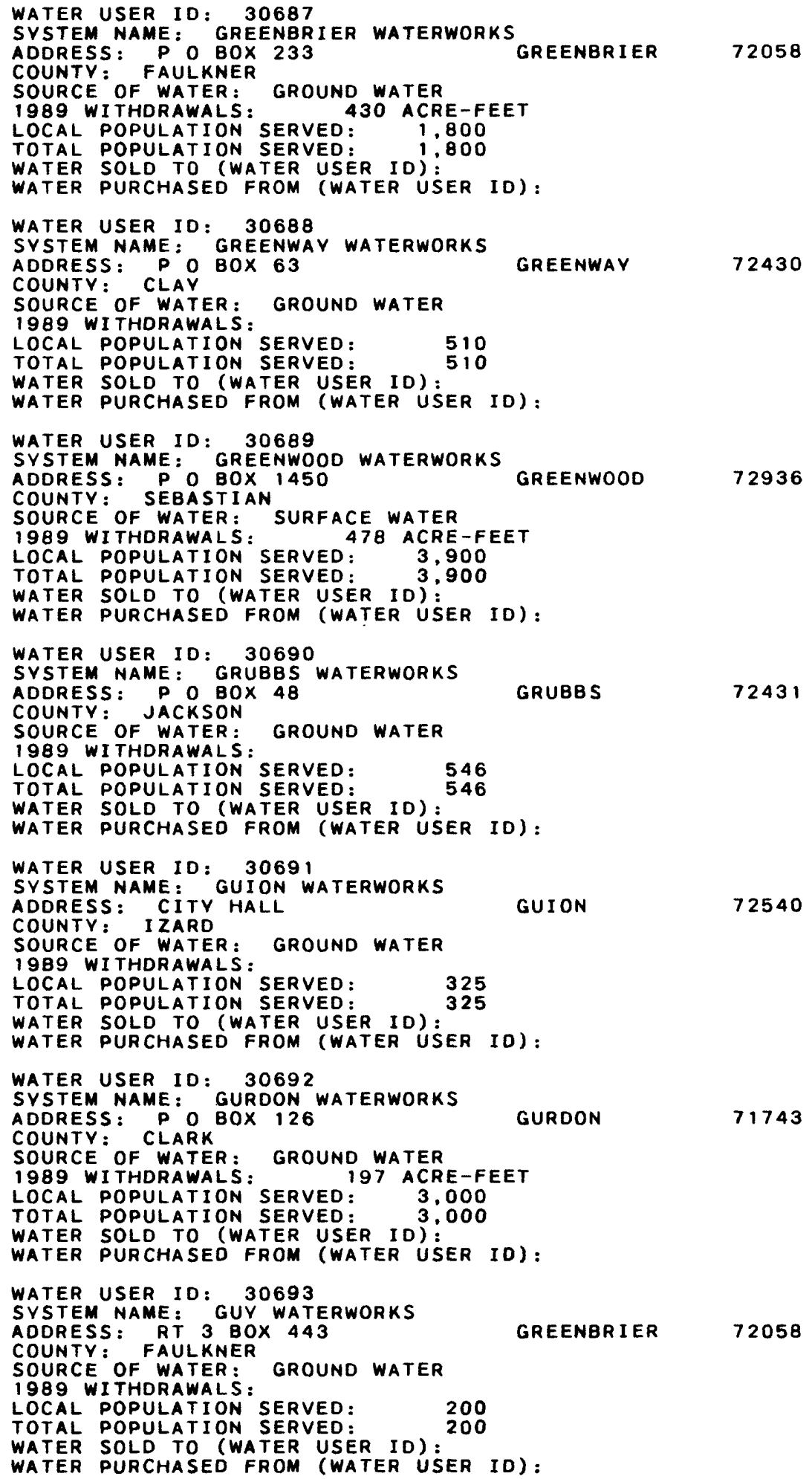


Table 1.--Description of public water supplies in Arkansas--Continued

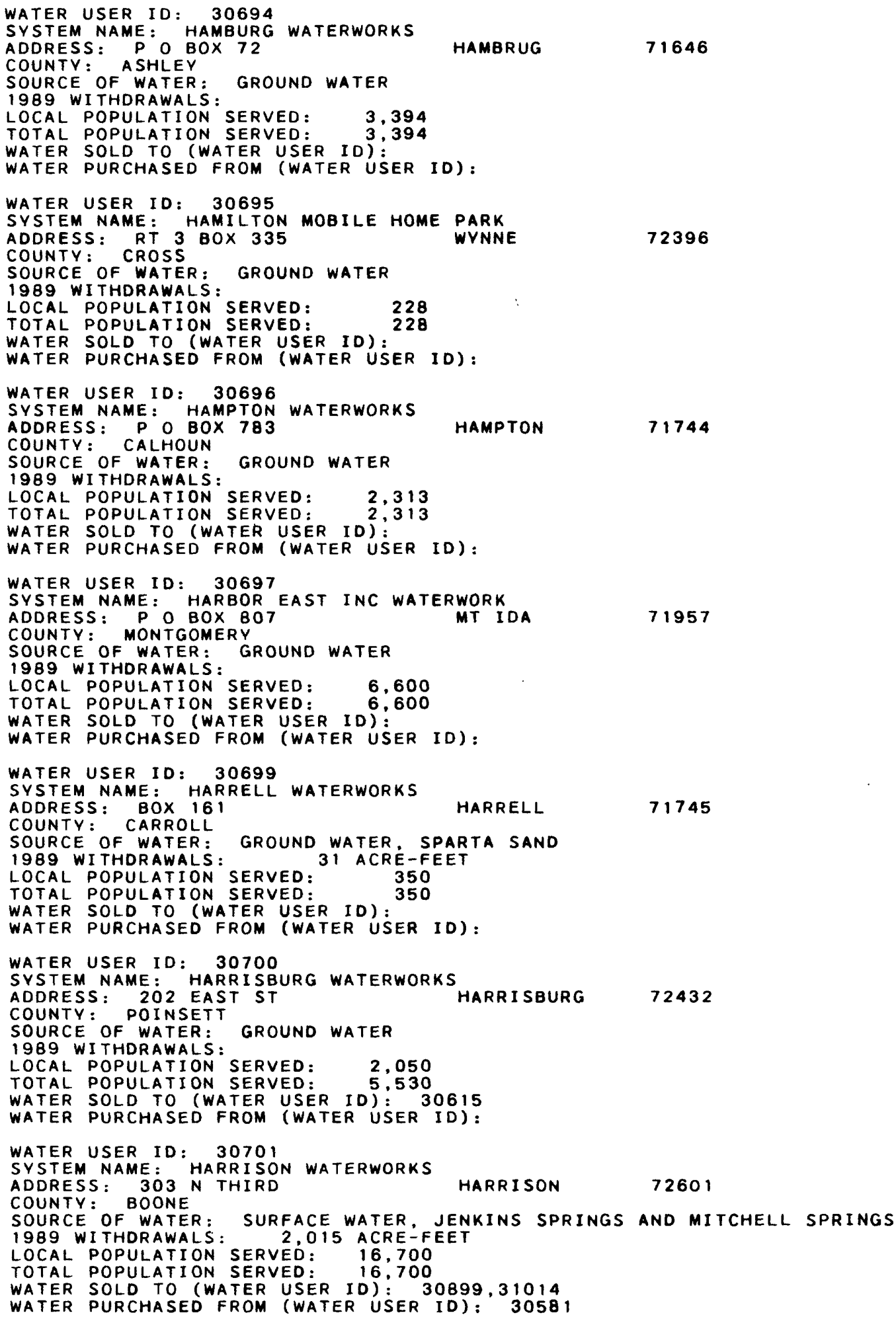


Table 1.--Description of public water supplies in Arkansas--Continued

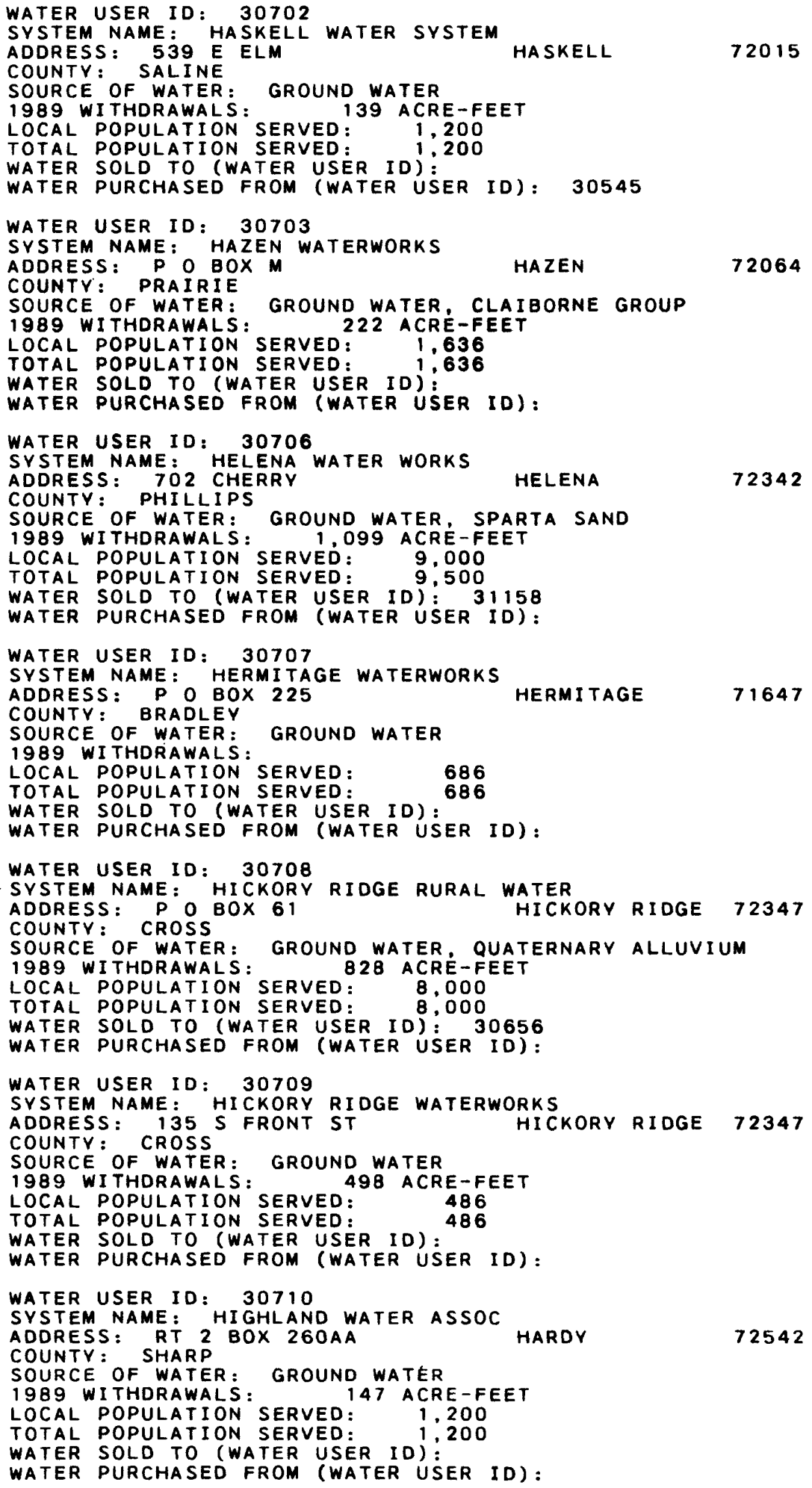


Table 1.--Description of public water supplies in Arkansas--Continued

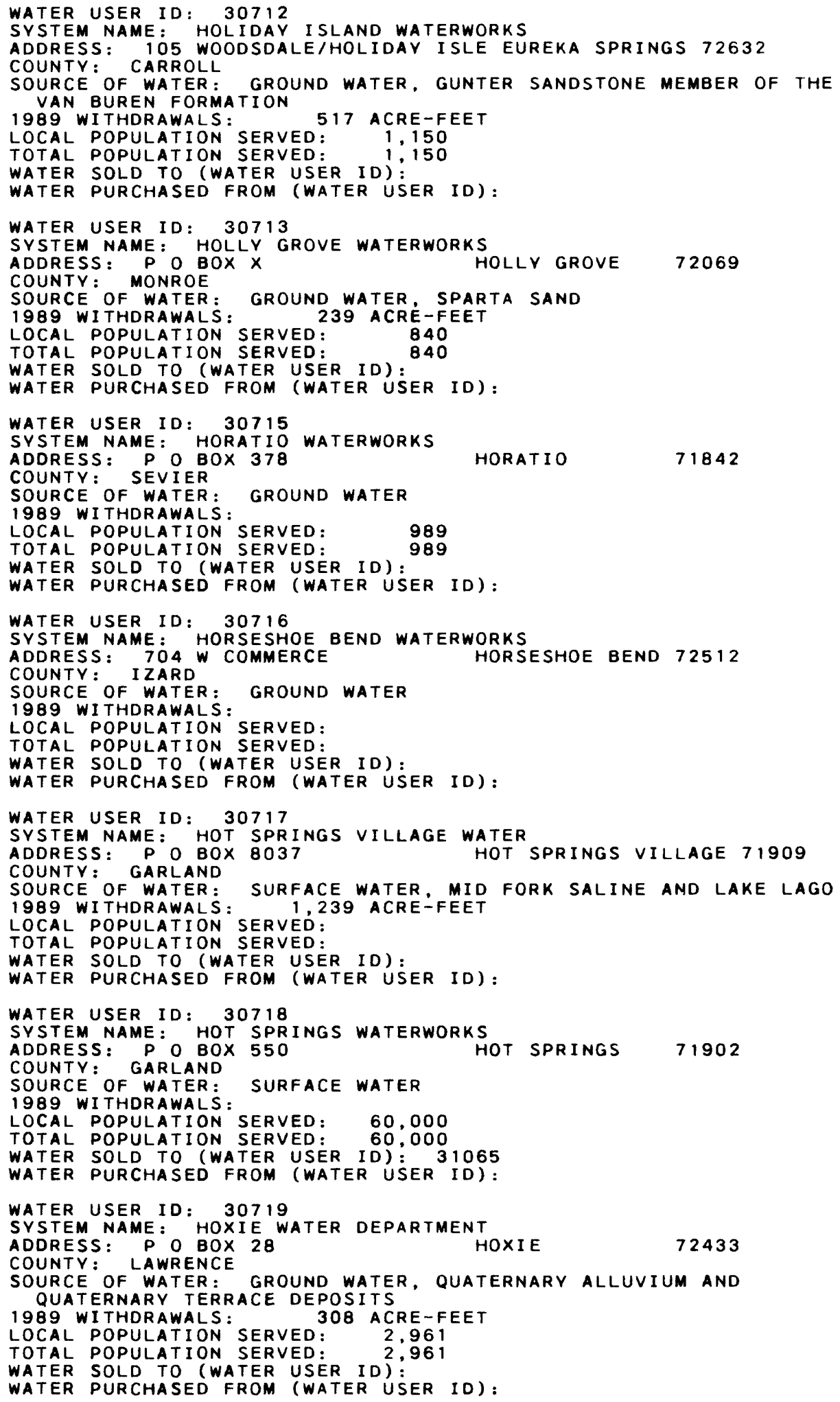


Table 1.--Description of public water supplies in Arkansas--Continued

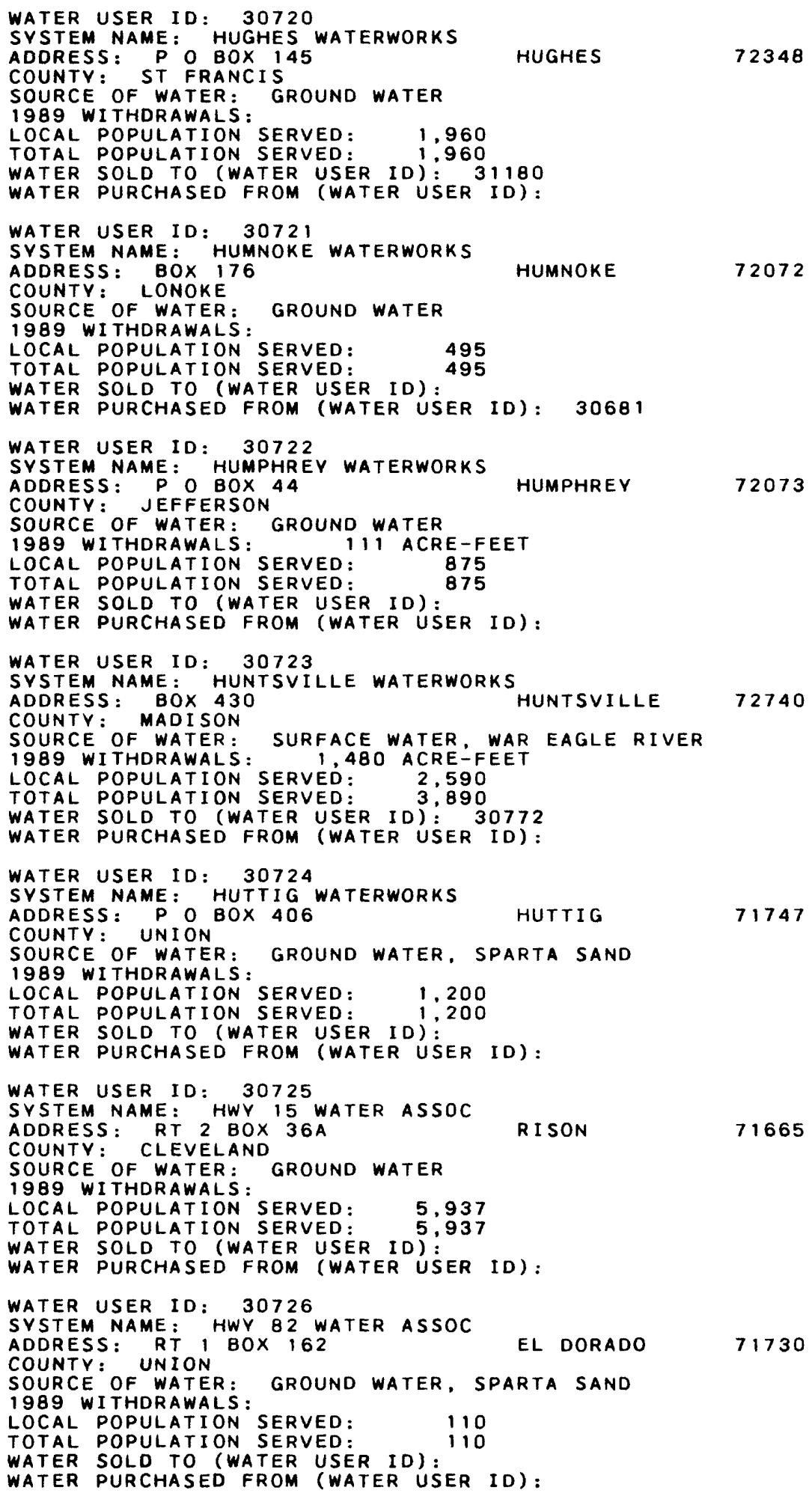


Table 1.--Description of public water supplies in Arkansas--Continued

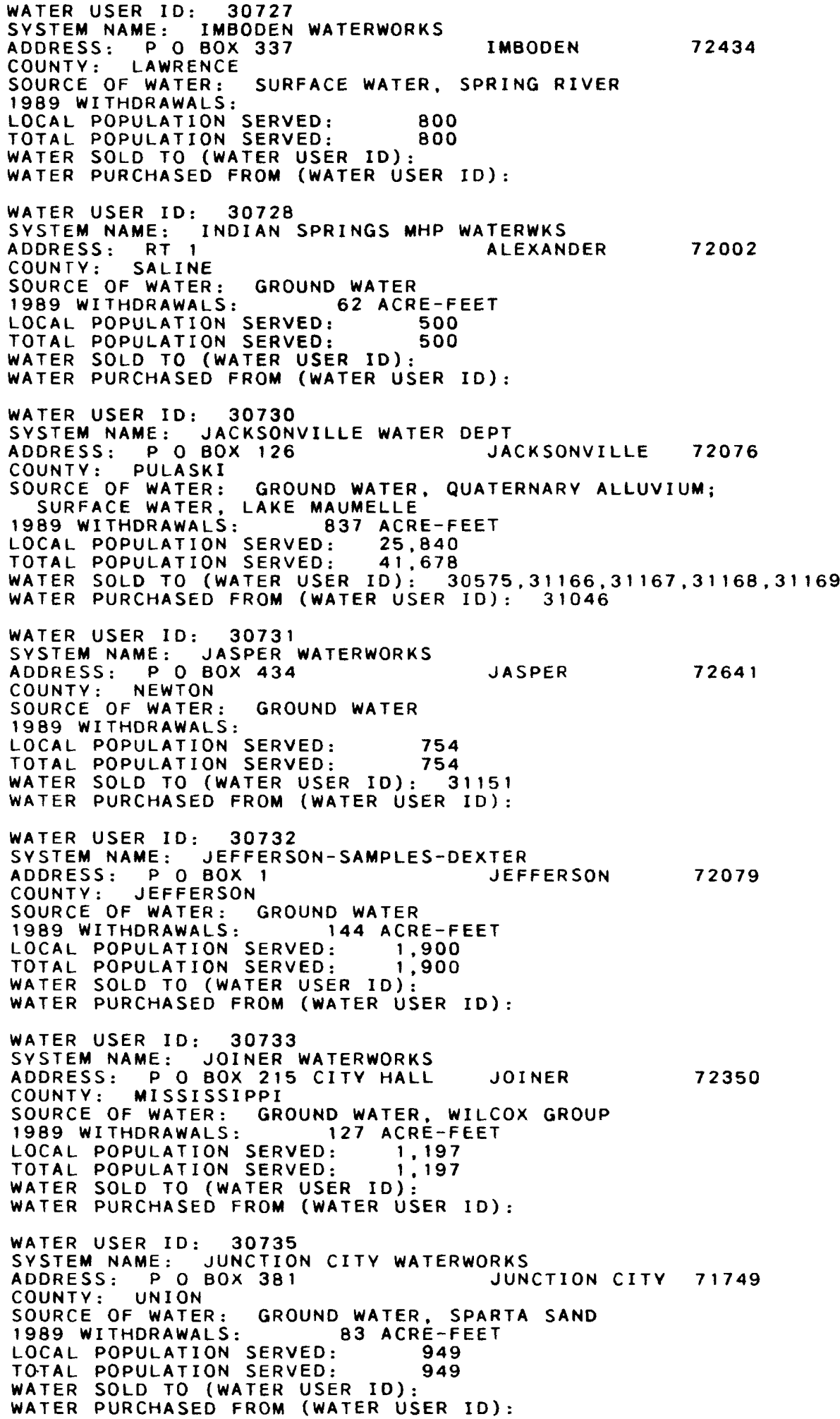


Table 1.--Description of public water supplies in Arkansas--Continued

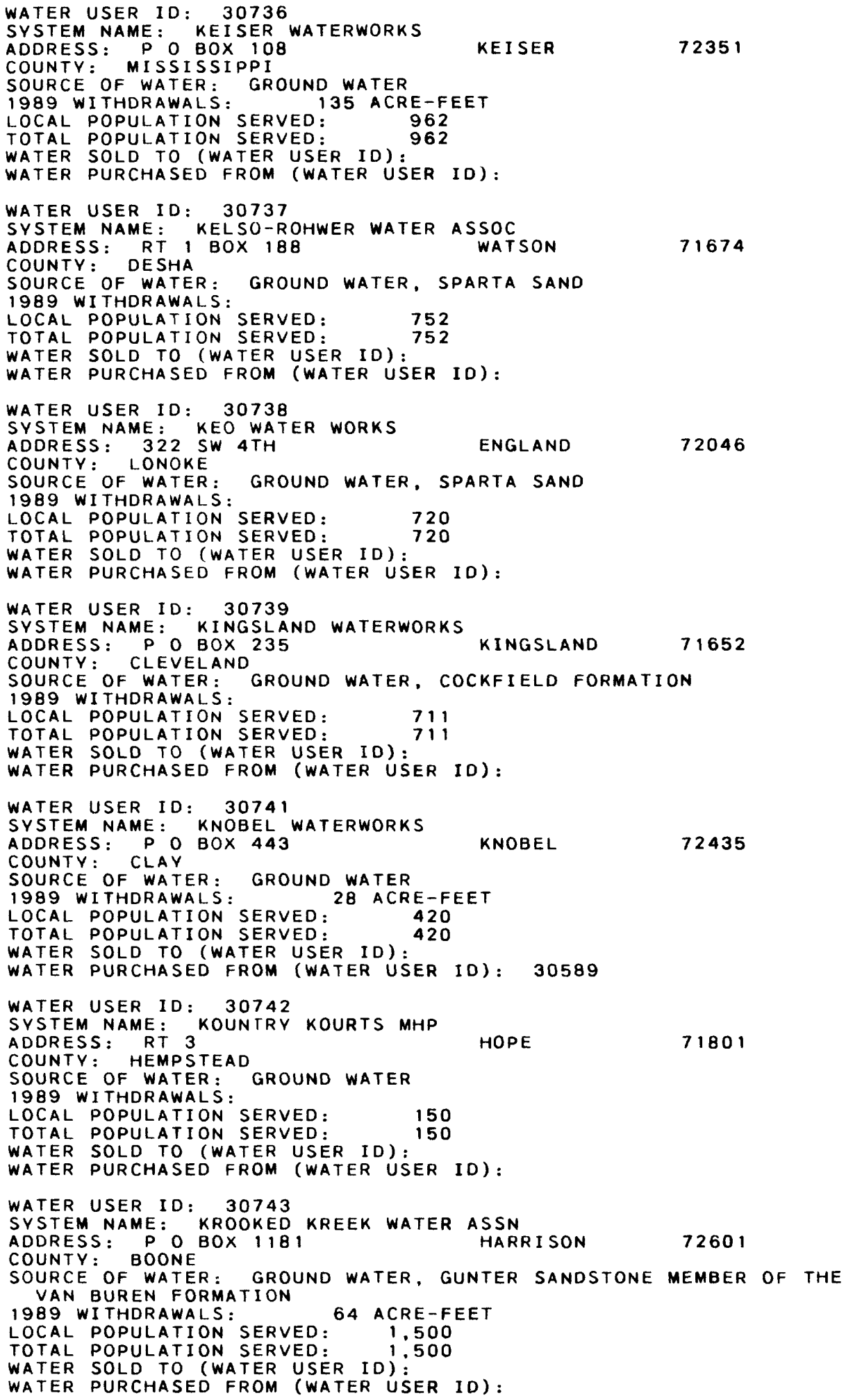


Table 1.--Description of public water supplies in Arkansas--Continued

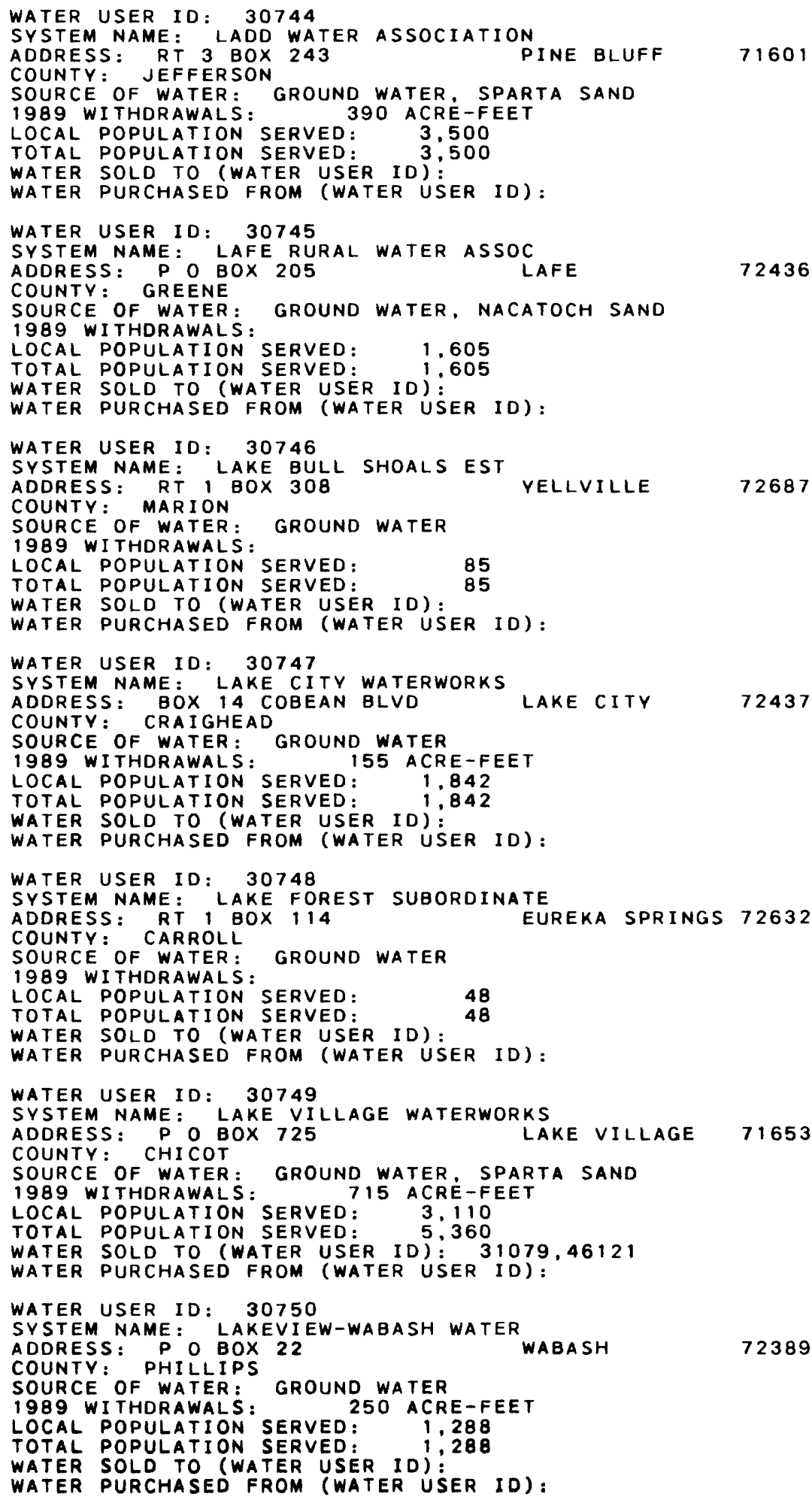


Table 1.--Description of public water supplies in Arkansas--Continued

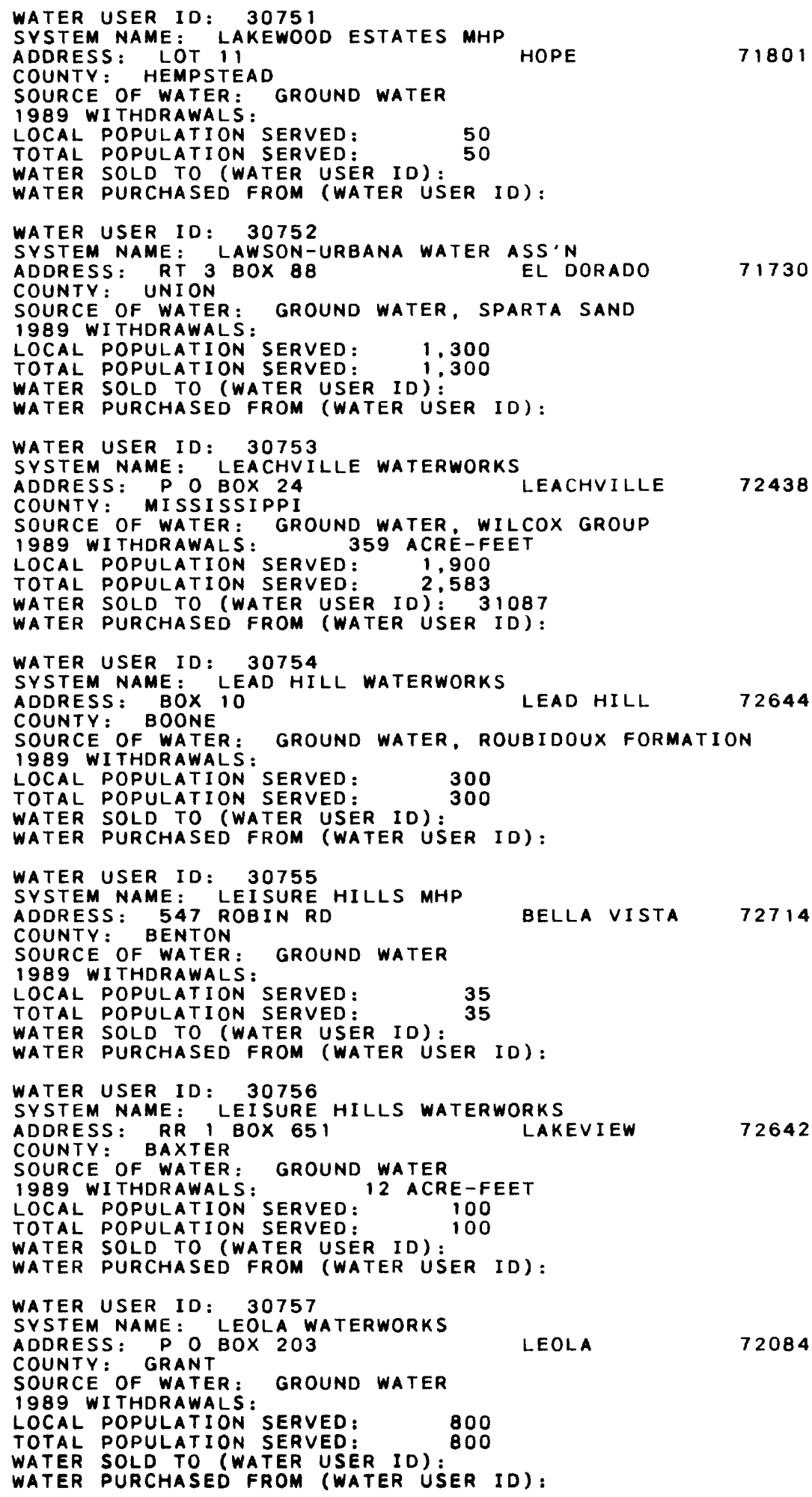


Table 1.--Description of public water supplies in Arkansas--Continued

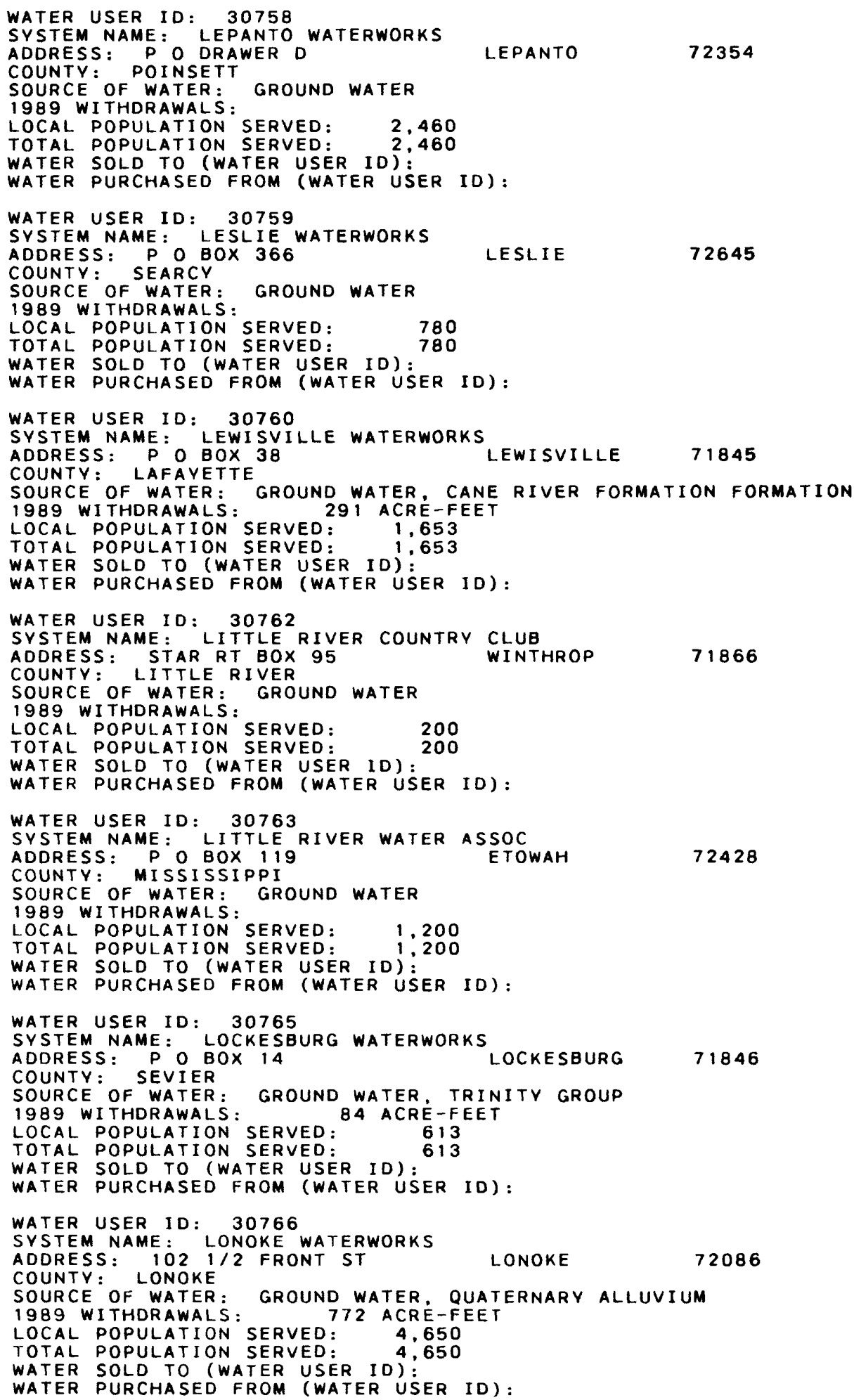


Table 1.--Description of public water supplies in Arkansas--Continued

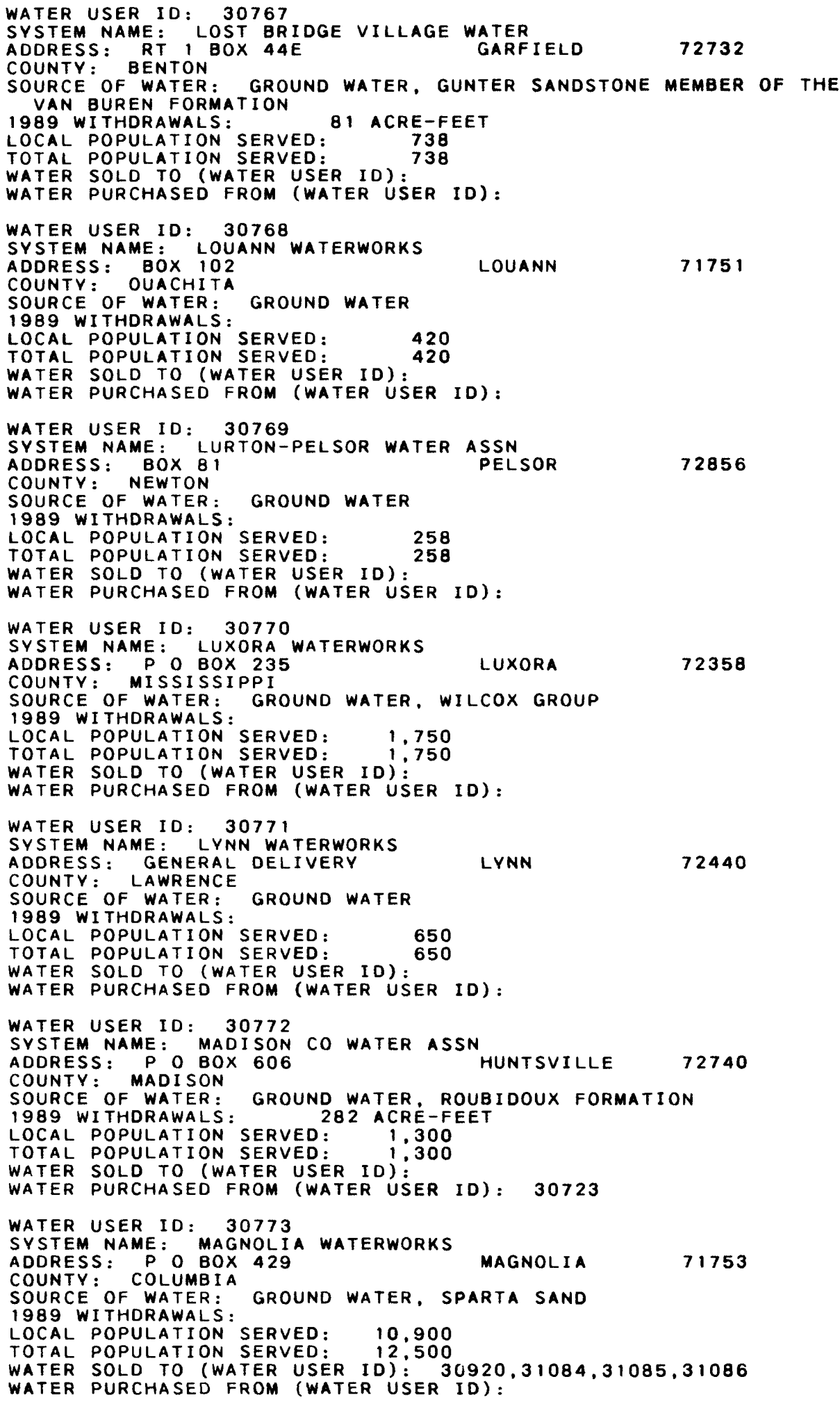


Table 1.--Description of public water supplies in Arkansas--Continued

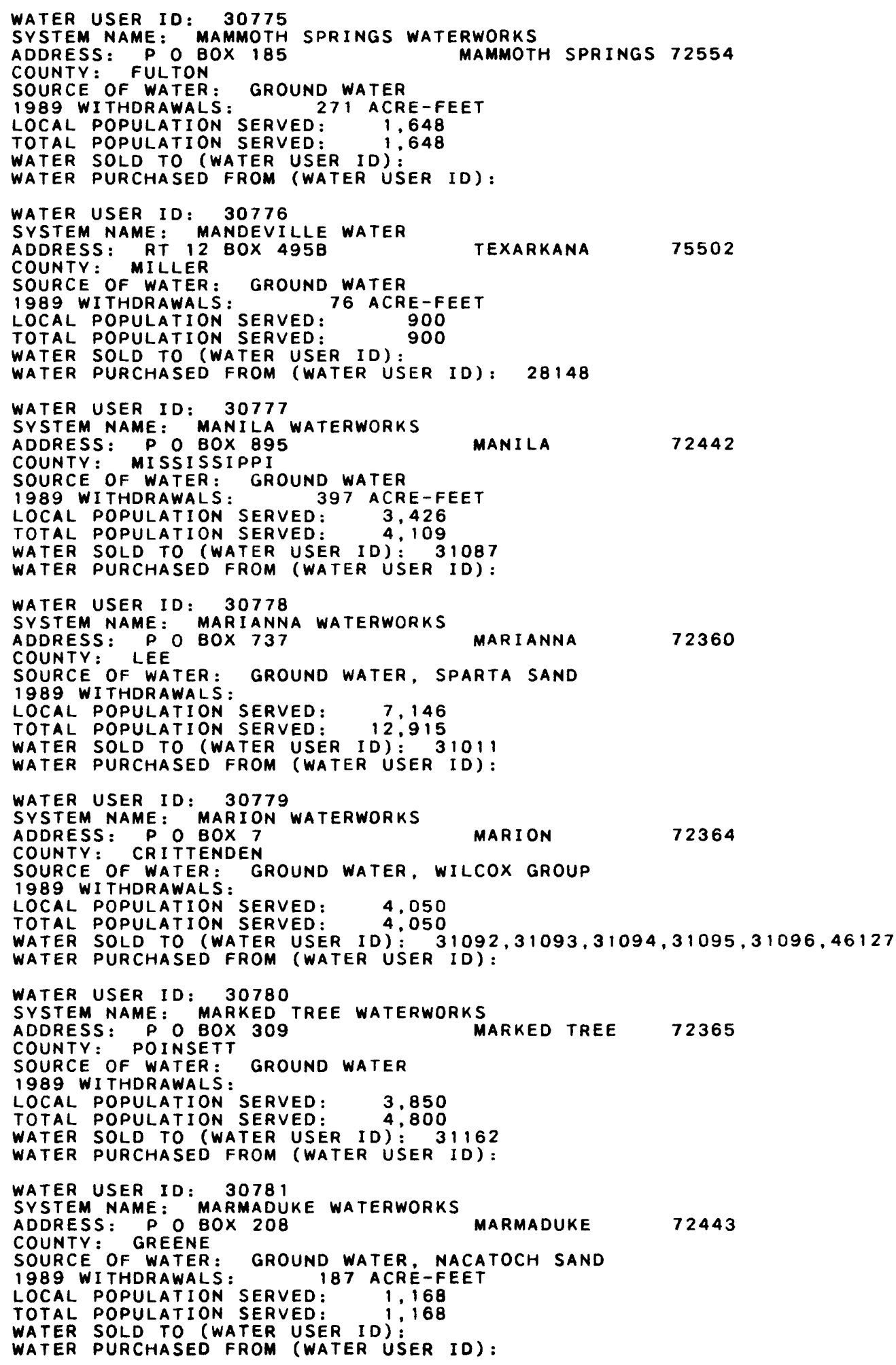


Table 1.--Description of public water supplies in Arkansas--Continued

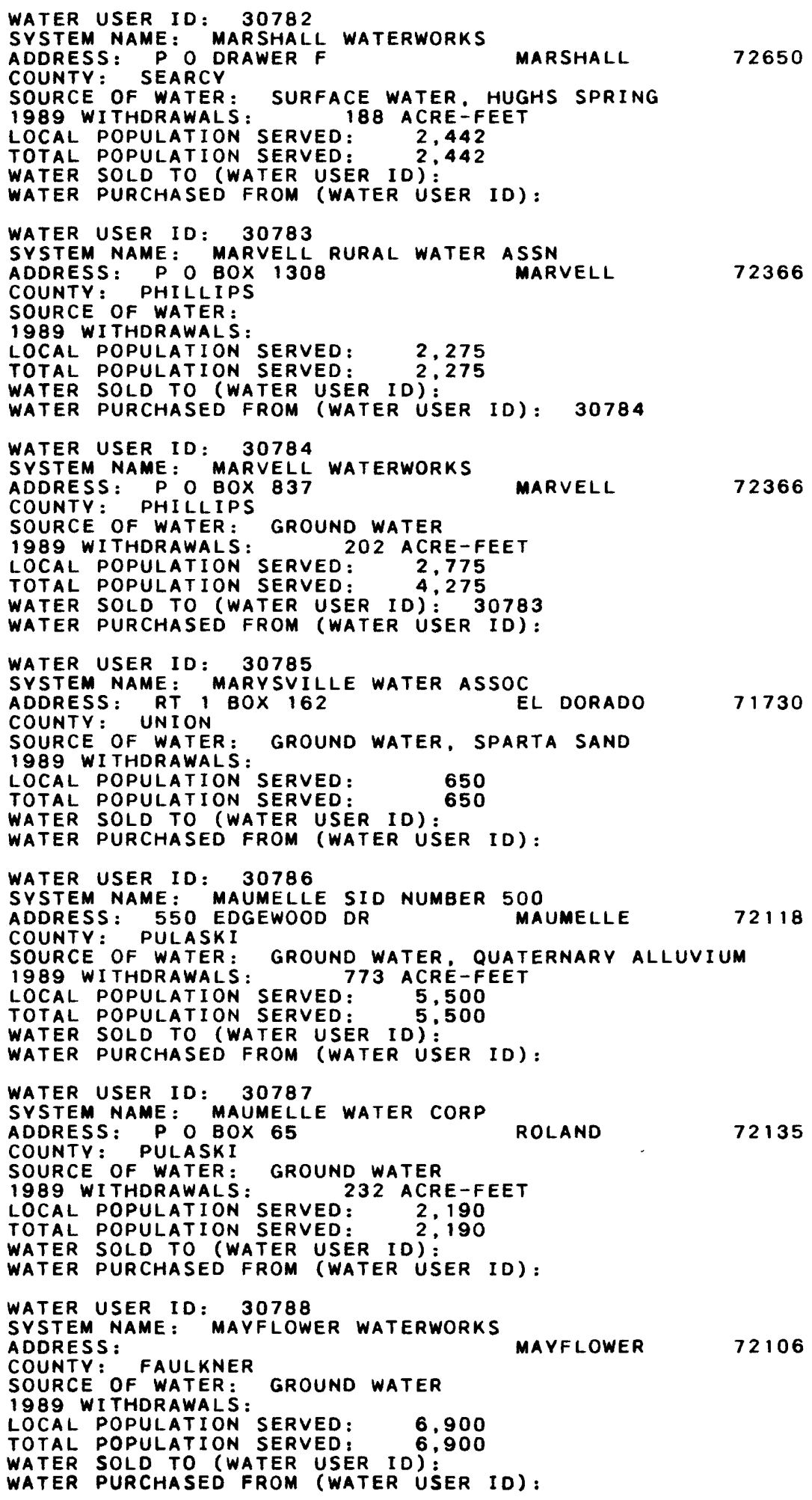


Table 1.--Description of public water supplies in Arkansas--Continued

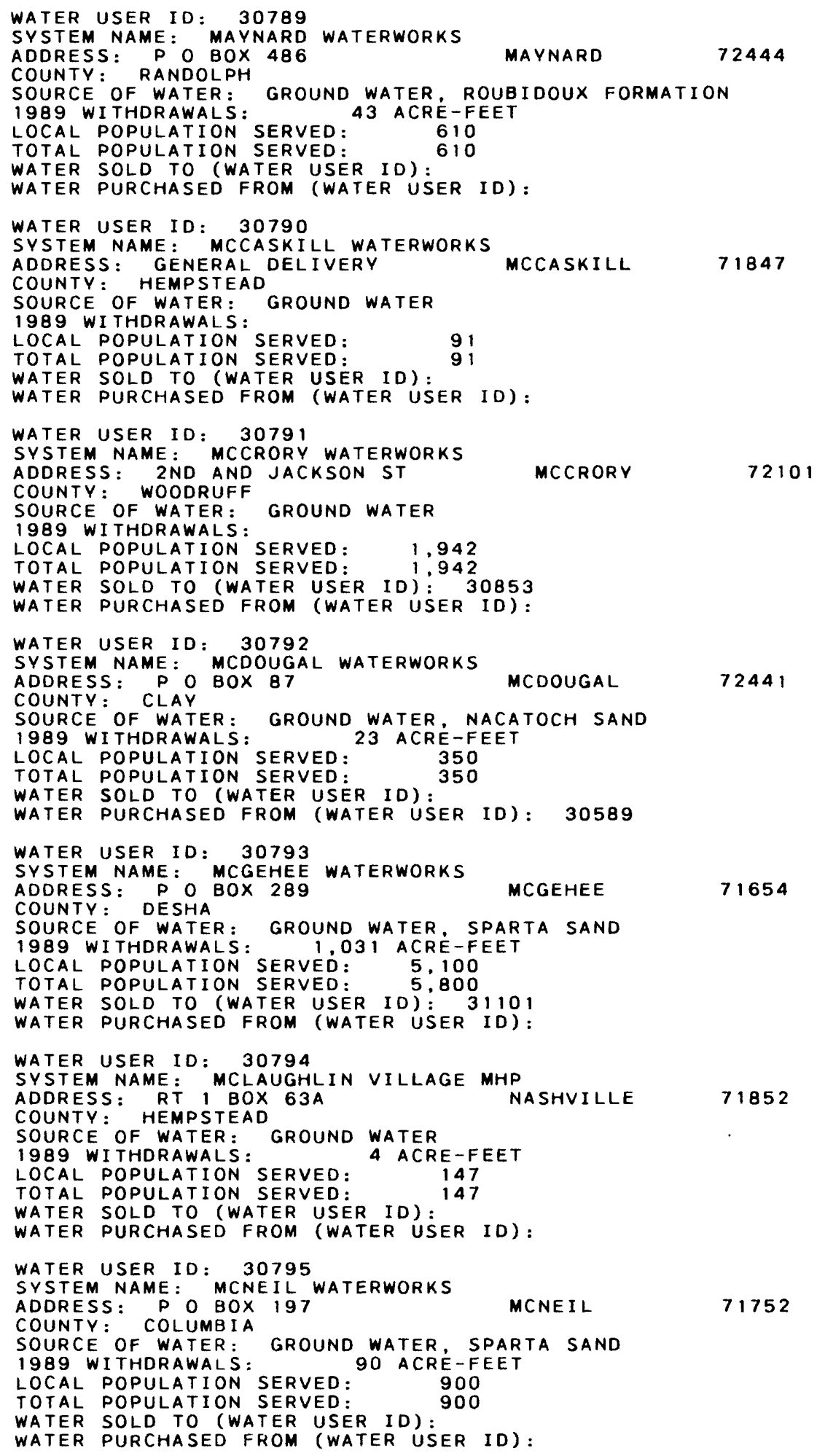


Table 1.--Description of public water supplies in Arkansas--Continued

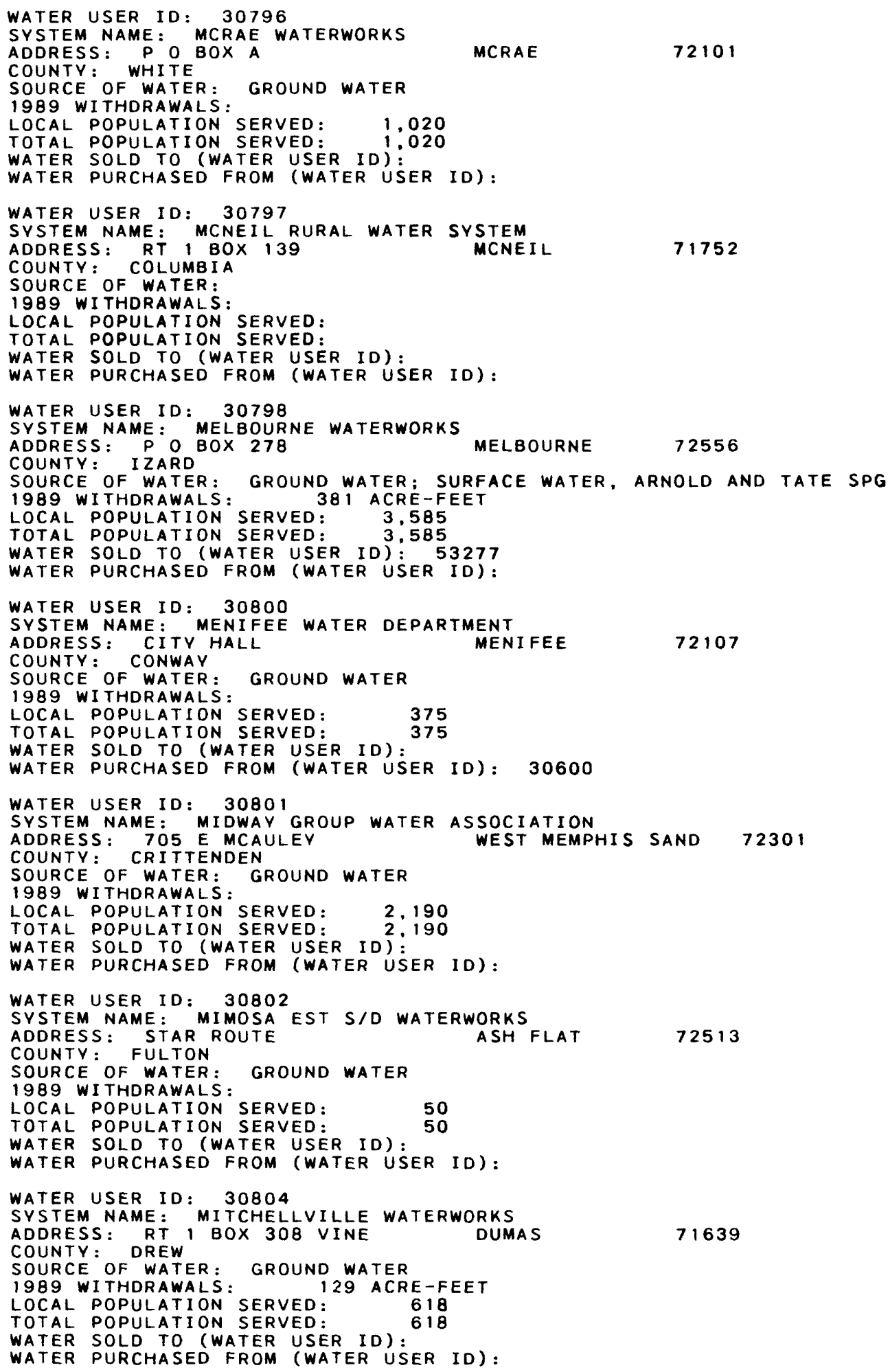


Table 1.--Description of public water supplies in Arkansas--Continued

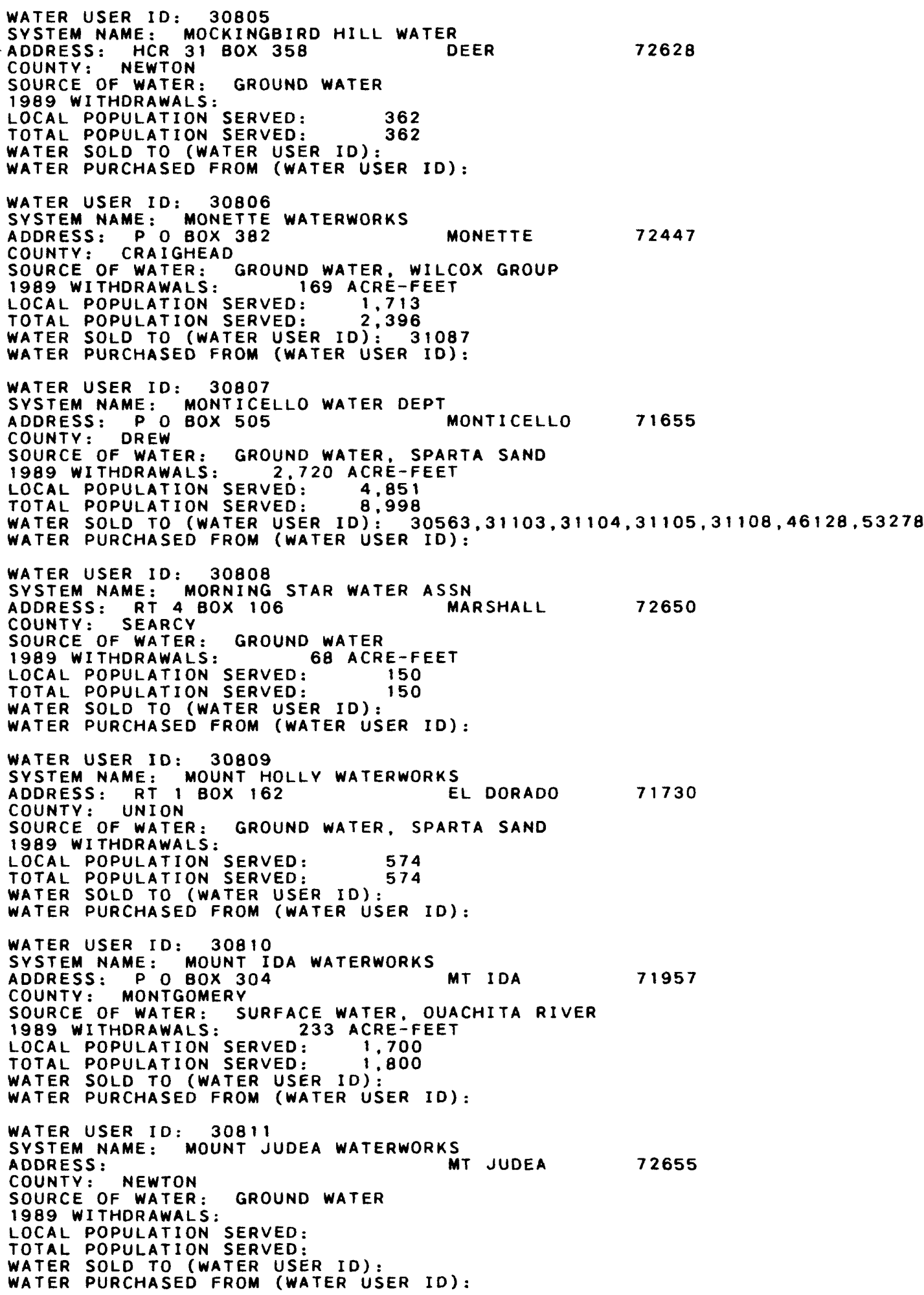


Table 1.--Description of public water supplies in Arkansas--Continued

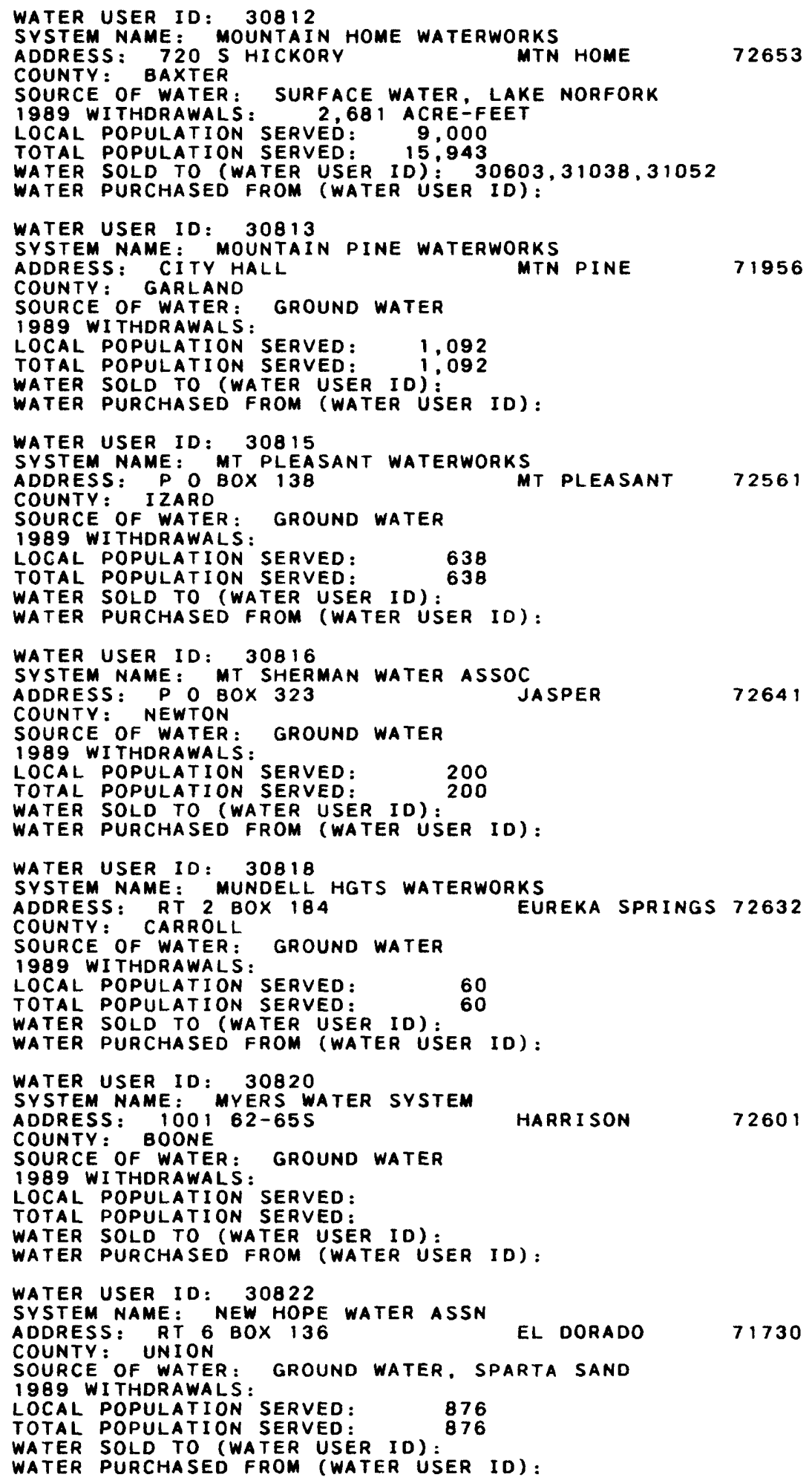


Table 1.--Description of public water supplies in Arkansas--Continued

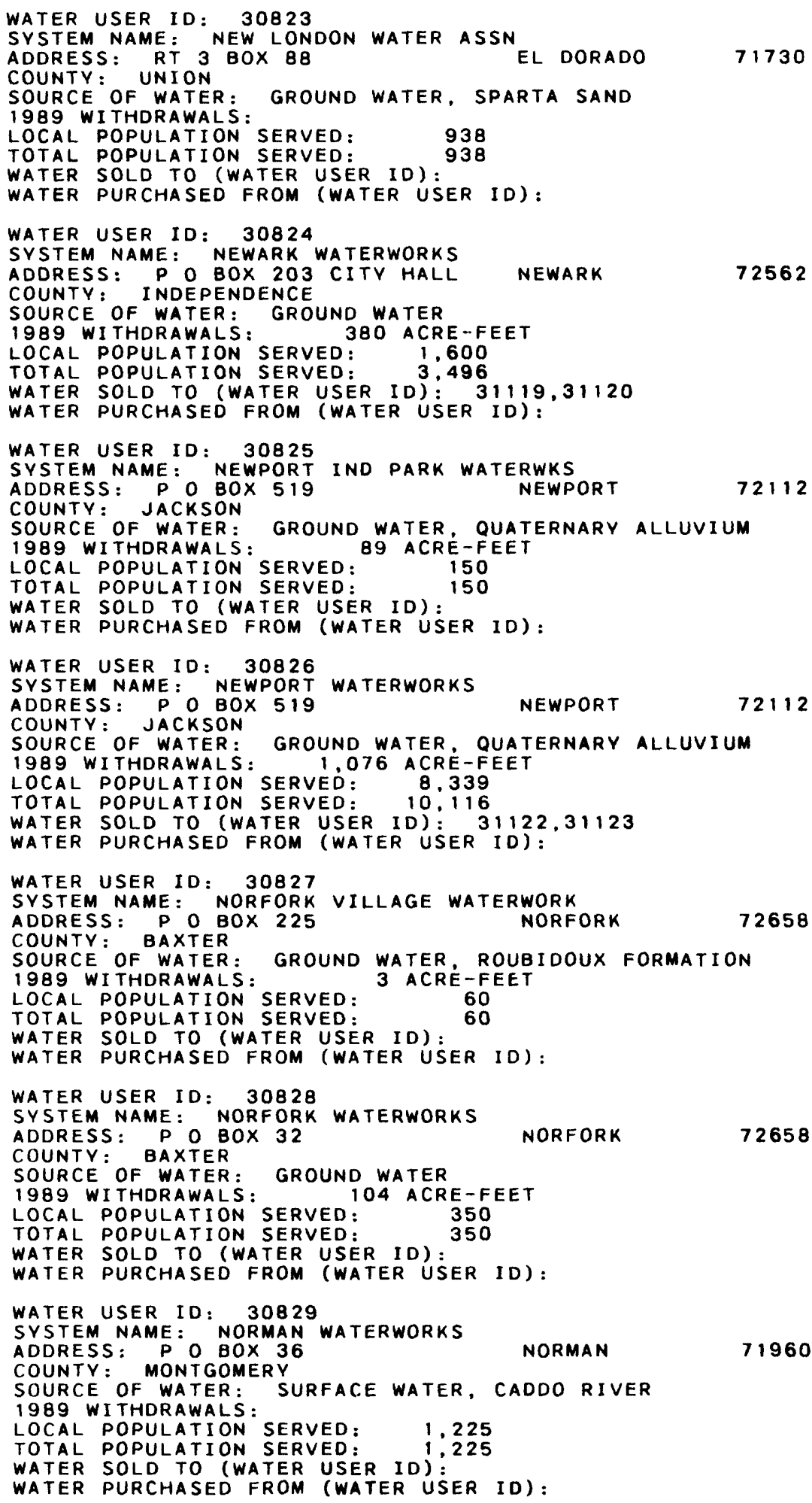


Table 1.--Description of public water supplies in Arkansas--Continued

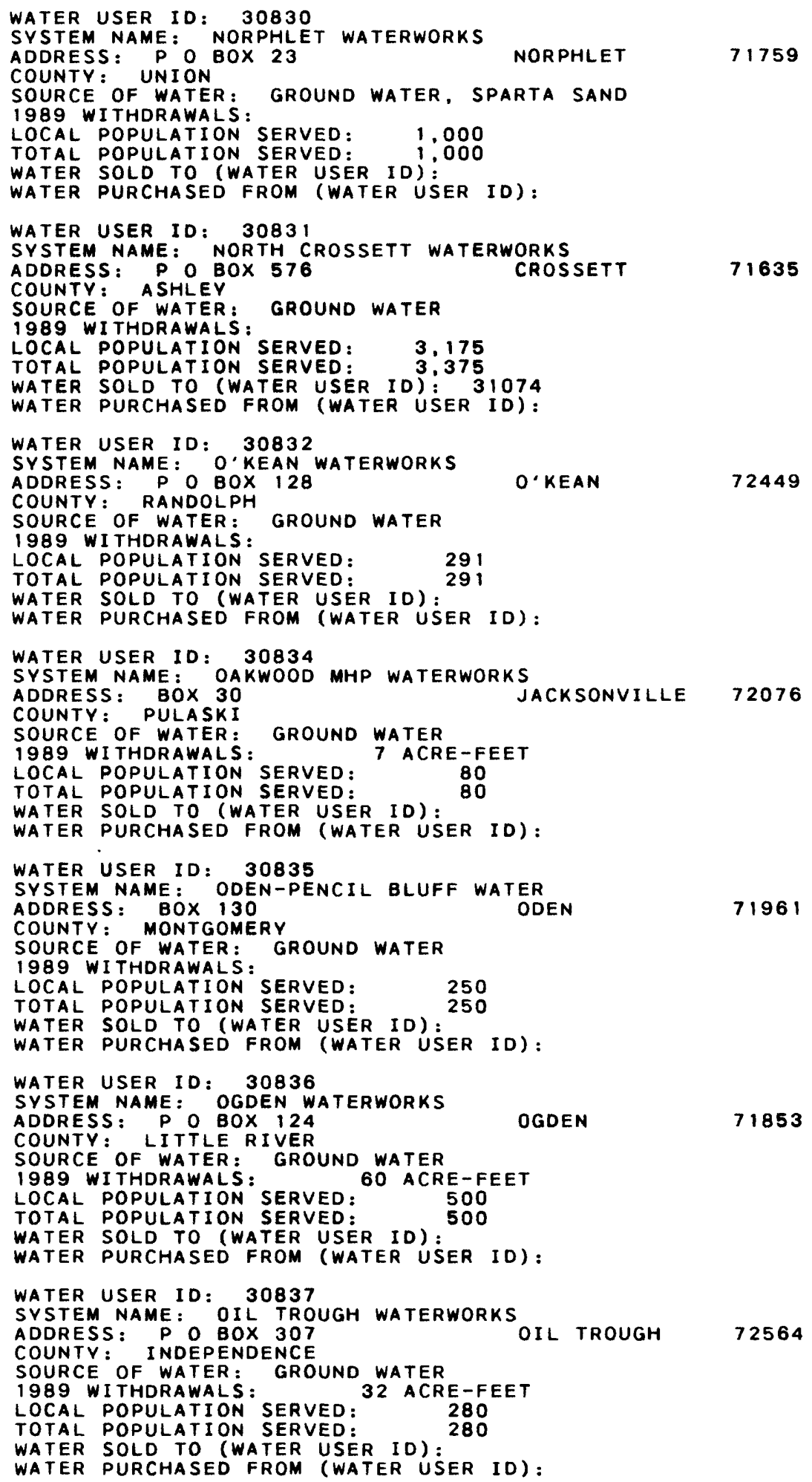


Table 1.--Description of public water supplies in Arkansas--Continued

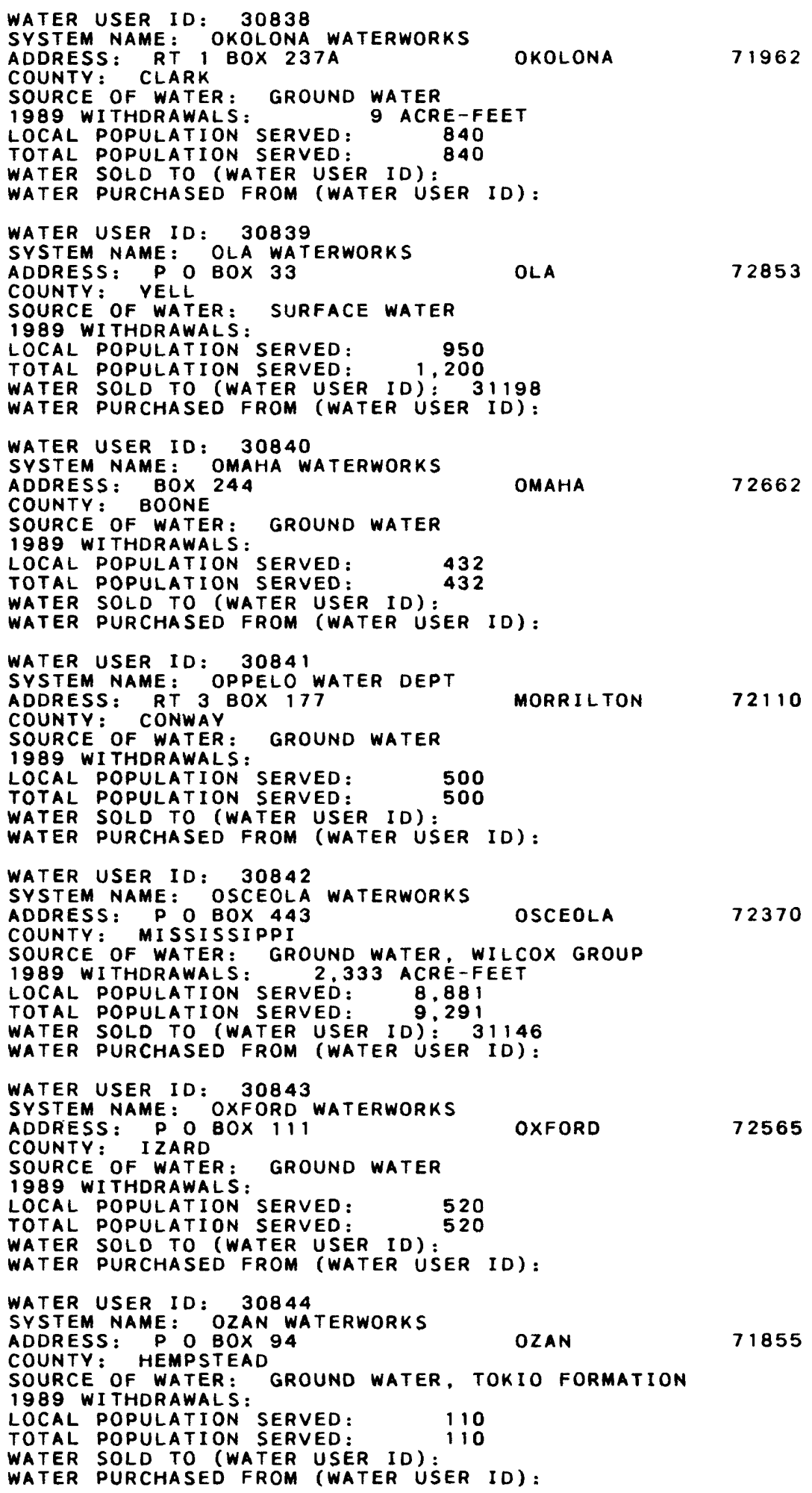


Table 1.--Description of public water supplies in Arkansas--Continued

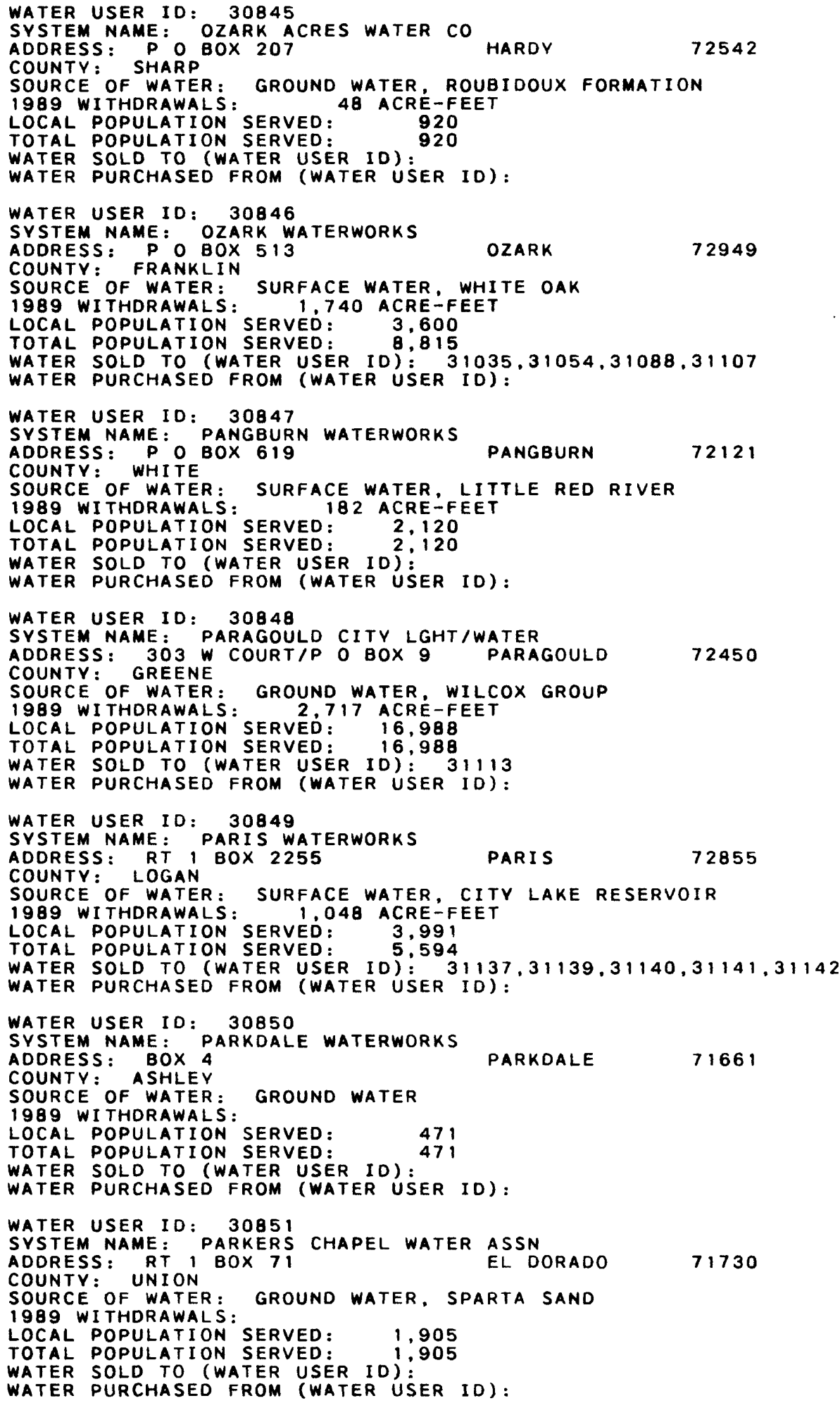


Table 1.--Description of public water supplies in Arkansas--Continued

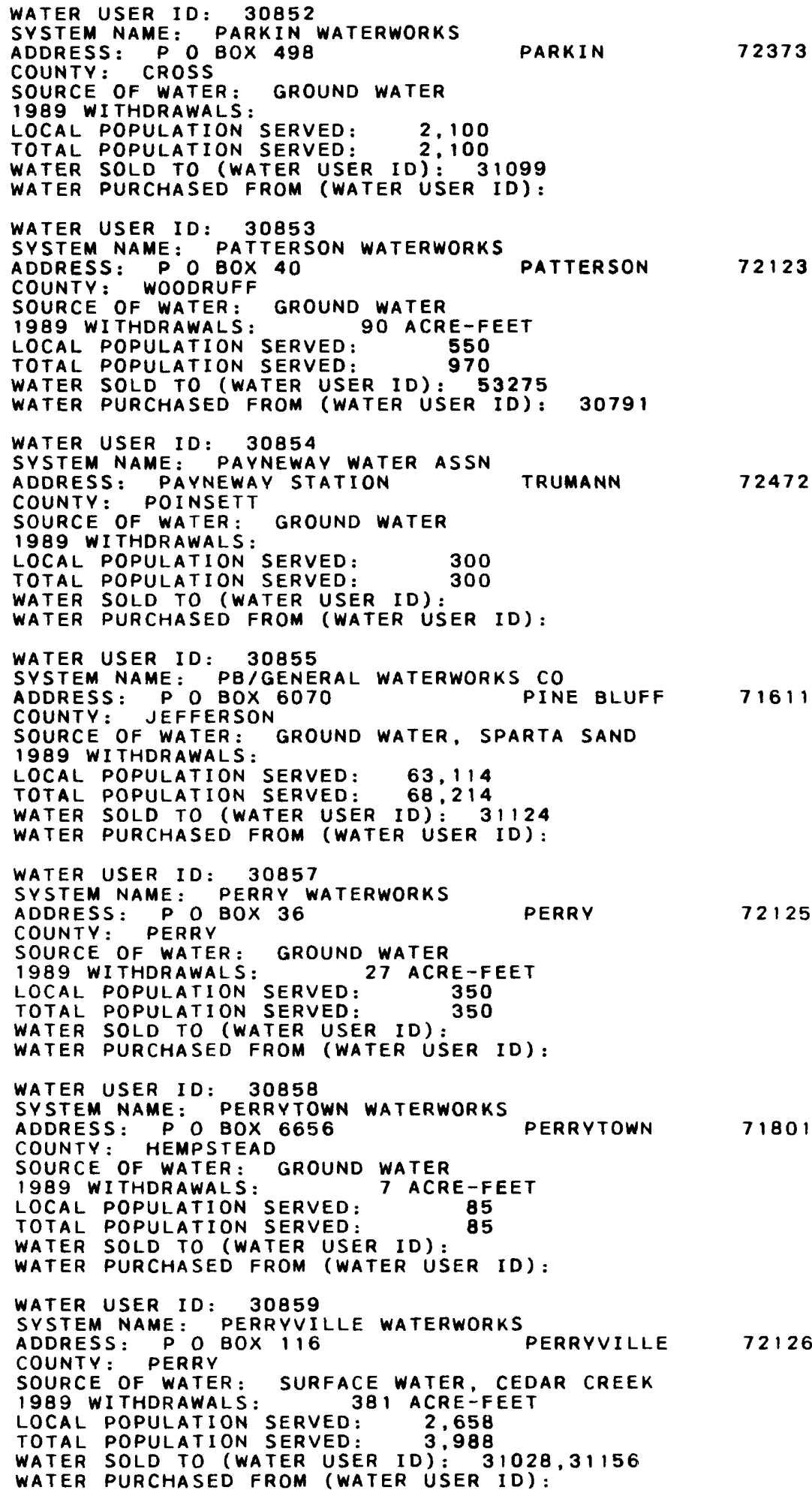


Table 1.--Description of public water supplies in Arkansas--Continued

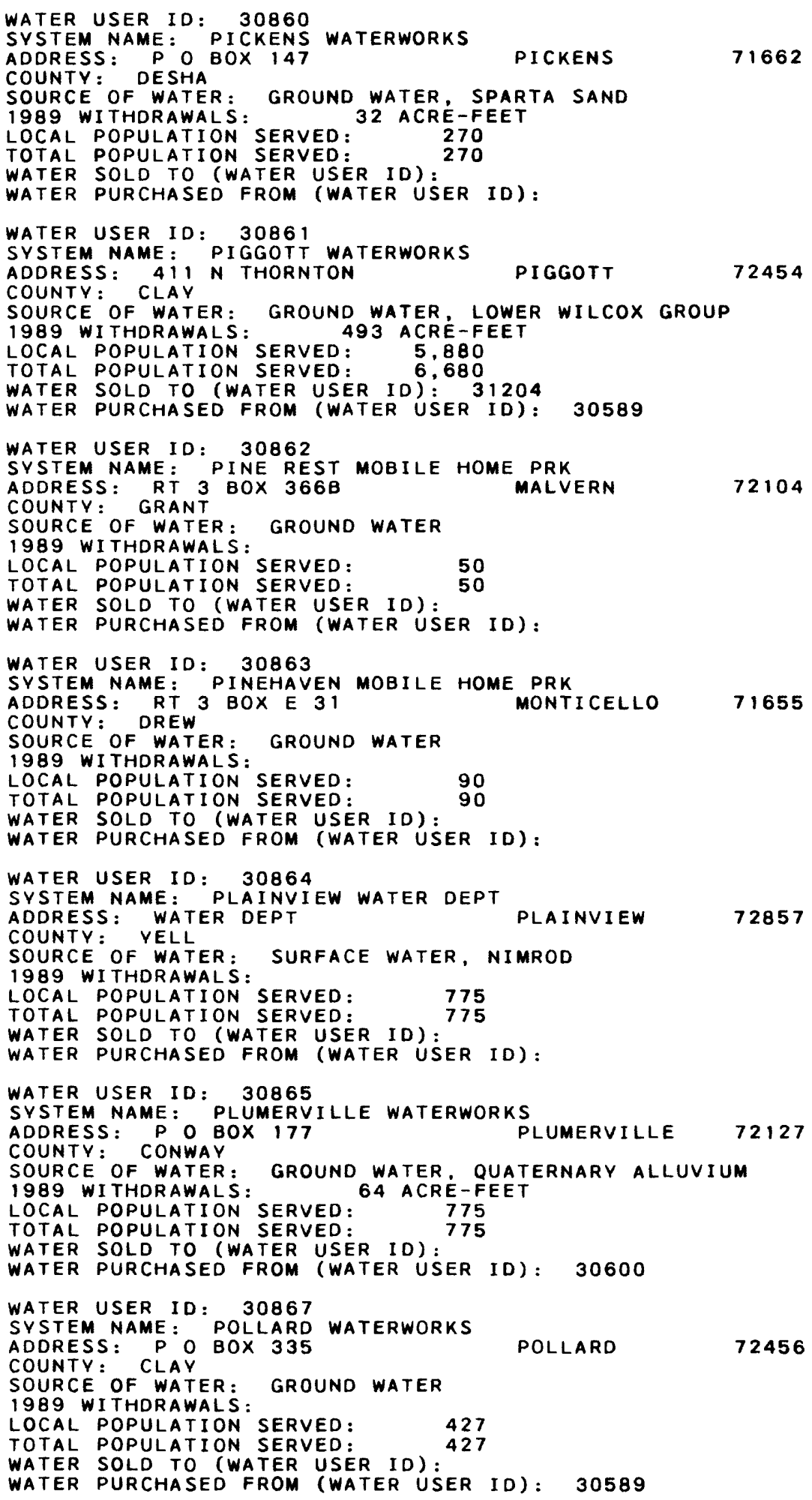


Table 1.--Description of public water supplies in Arkansas--Continued

WATER USER ID: 30868

SYSTEM NAME: PORTIA WATERWORKS

ADDRESS: CITY HALL

COUNTY: LAWRENCE

PORTIA $\quad 72457$

SOURCE OF WATER:

LOCAL POPULATION SERVED.

TOTAL POPULATION SERVED:

38 ACRE-FEET

480

WATER SOLD TO (WATER USER ID):

WATER PURCHASED FROM (WATER USER ID):

WATER USER ID: $\quad 30869$

SYSTEM NAME: PORTLAND WATERWORKS

ADDRESS: P O BOX 226

PORTLAND $\quad 71663$

COUNTY: ASHLEY

SOURCE OF WATER: GROUND WATER, COCKFIELD FORMATION

1989 WITHDRAWALS: 155 ACRE-FEET

LOCAL POPULATION SERVED: 701

TOTAL POPULATION SERVED: 1.301

WATER SOLD TO (WATER USER ID): 31073

WATER PURCHASED FROM (WATER USER ID):

WATER USER ID: 30870

SYSTEM NAME: POYEN WATERWORKS

ADDRESS: $P$ O BOX 184

POYEN

72128

COUNTY: GRANT

SOURCE OF WATER: GROUND WATER

1989 WITHDRAWALS

LOCAL POPULATION SERVED: $\quad 400$

TOTAL POPULATION SERVED: 400

WATER SOLD TO (WATER USER ID):

WATER PURCHASED FROM (WATER USER ID):

WATER USER ID: 30871

SYSTEM NAME: PRAIRIE GROVE WATERWORKS

ADDRESS: $P$ O BOX 255

COUNTY: WASHINGTON

SOURCE OF WATER: SURFACE WATER, PRAIRIE GROVE

1989 WITHDRAWALS: 310 ACRE-FEET

LOCAL POPULATION SERVED: 3,033

TOTAL POPULATION SERVED: $\quad 3,033$

WATER SOLD TO (WATER USER ID):

WATER PURCHASED FROM (WATER USER ID):

WATER USER ID: 30872

SYSTEM NAME: PRATTSVILLE WATERWORKS

ADDRESS: $P$ O BOX 27

COUNTY: GRANT

SOURCE OF WATER: GROUND WATER, SPARTA SAND

1989 WITHDRAWALS : 38 ACRE-FEET

LOCAL POPULATION SERVED: 600

TOTAL POPULATION SERVED: 1,100

WATER SOLD TO (WATER USER ID): 31112

WATER PURCHASED FROM (WATER USER ID) :

WATER USER ID: 30874

SYSTEM NAME: PYATT WATERWORKS

ADDRESS: P O BOX 107 PYATT

PYATT $\mathbf{7 2 6 7 2}$

COUNTY: MARION

1989 WITHDRAWALS: 592 ACRE-FEET

LOCAL POPULATION SERVED: 300

TOTAL POPULATION SERVED: 300

WATER SOLD TO (WATER USER ID):

WATER PURCHASED FROM (WATER USER ID):

WATER USER ID: 30875

SYSTEM NAME: CHEROKEE VILLAGE WATER

ADDRESS: P O BOX 600

CHEROKEE VLG

72525

SOURCE OF WATER: SURFACE WATER, ROARING SPRINGS

1989 WITHDRAWALS:

LOCAL POPULATION SERVED:

TOTAL POPULATION SERVED

WATER SOLD TO (WATER USER ID)

WATER PURCHASED FROM (WATER USER ID) : 
Table 1.--Description of public water supplies in Arkansas--Continued

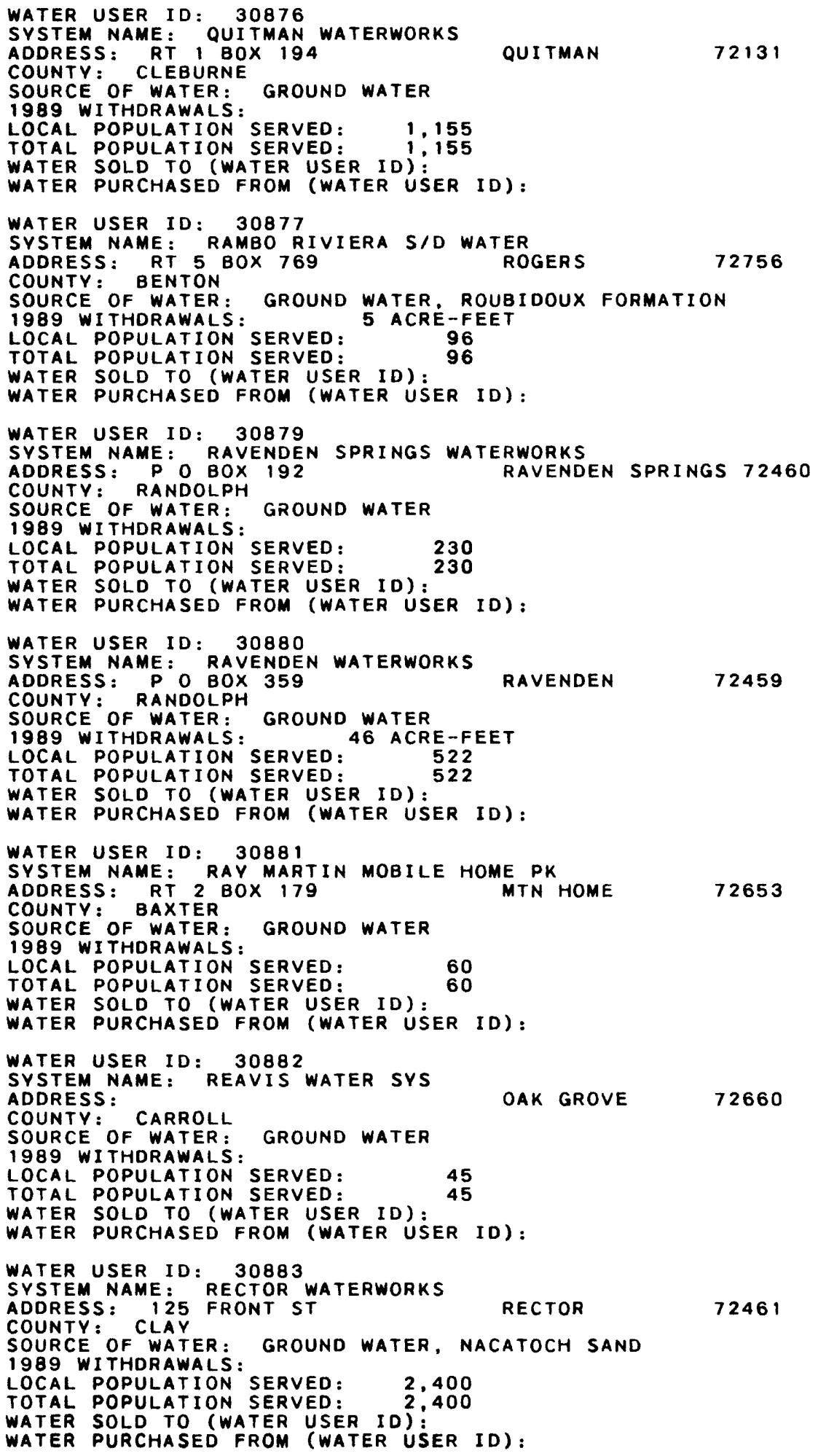


Table 1.--Description of public water supplies in Arkansas--Continued

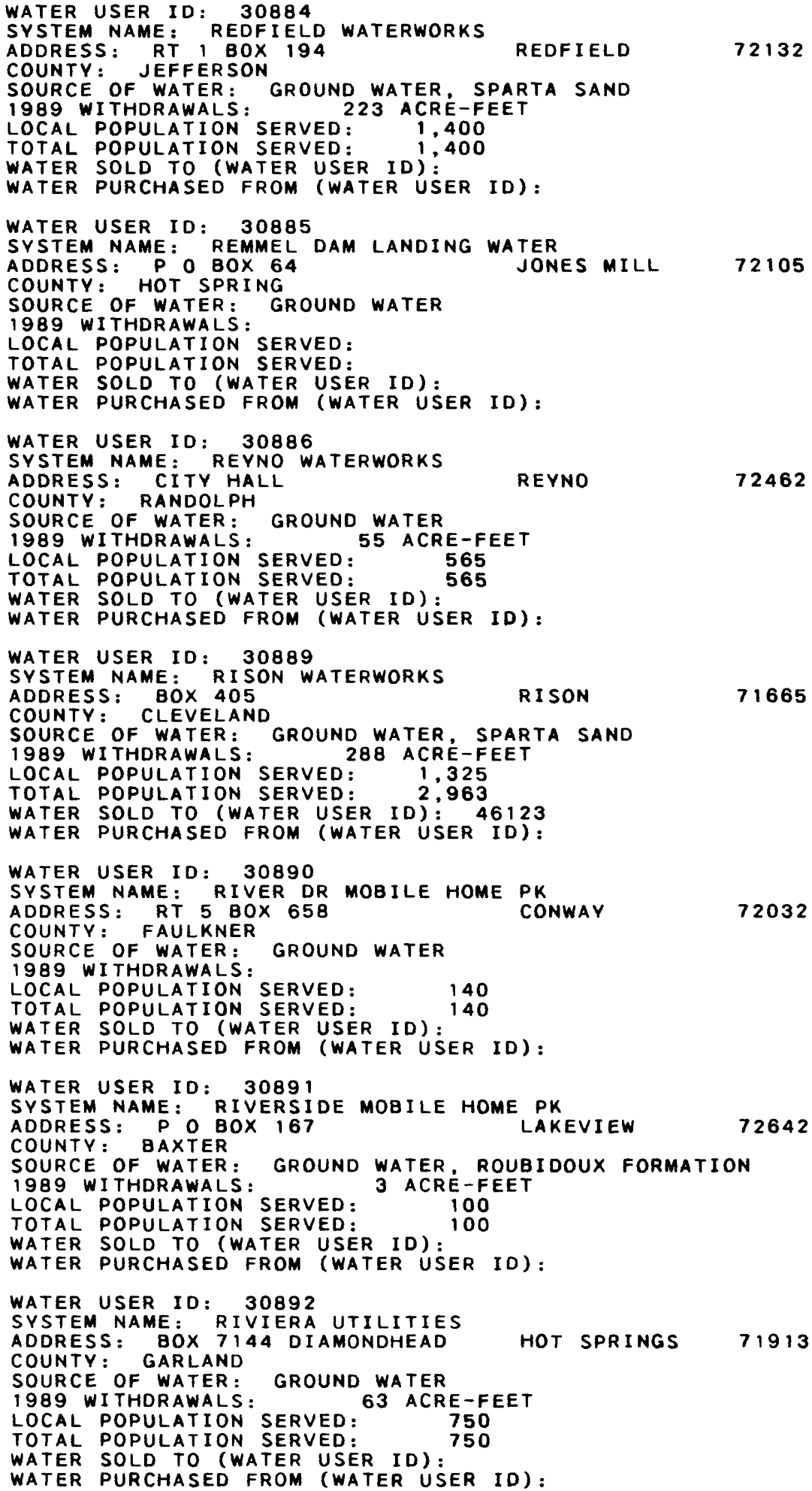


Table 1.--Description of public water supplies in Arkansas--Continued

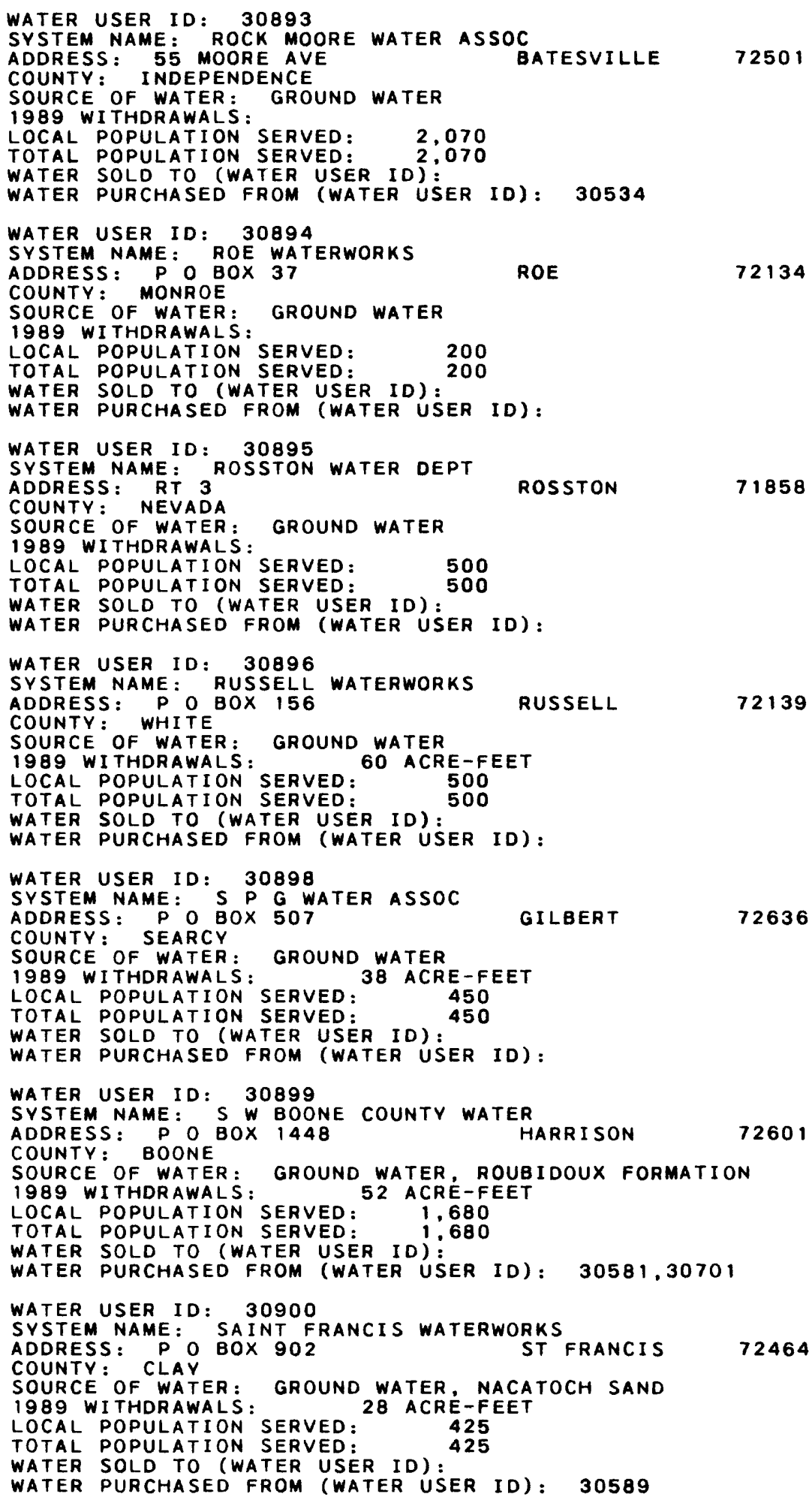


Table 1.--Description of public water supplies in Arkansas--Continued

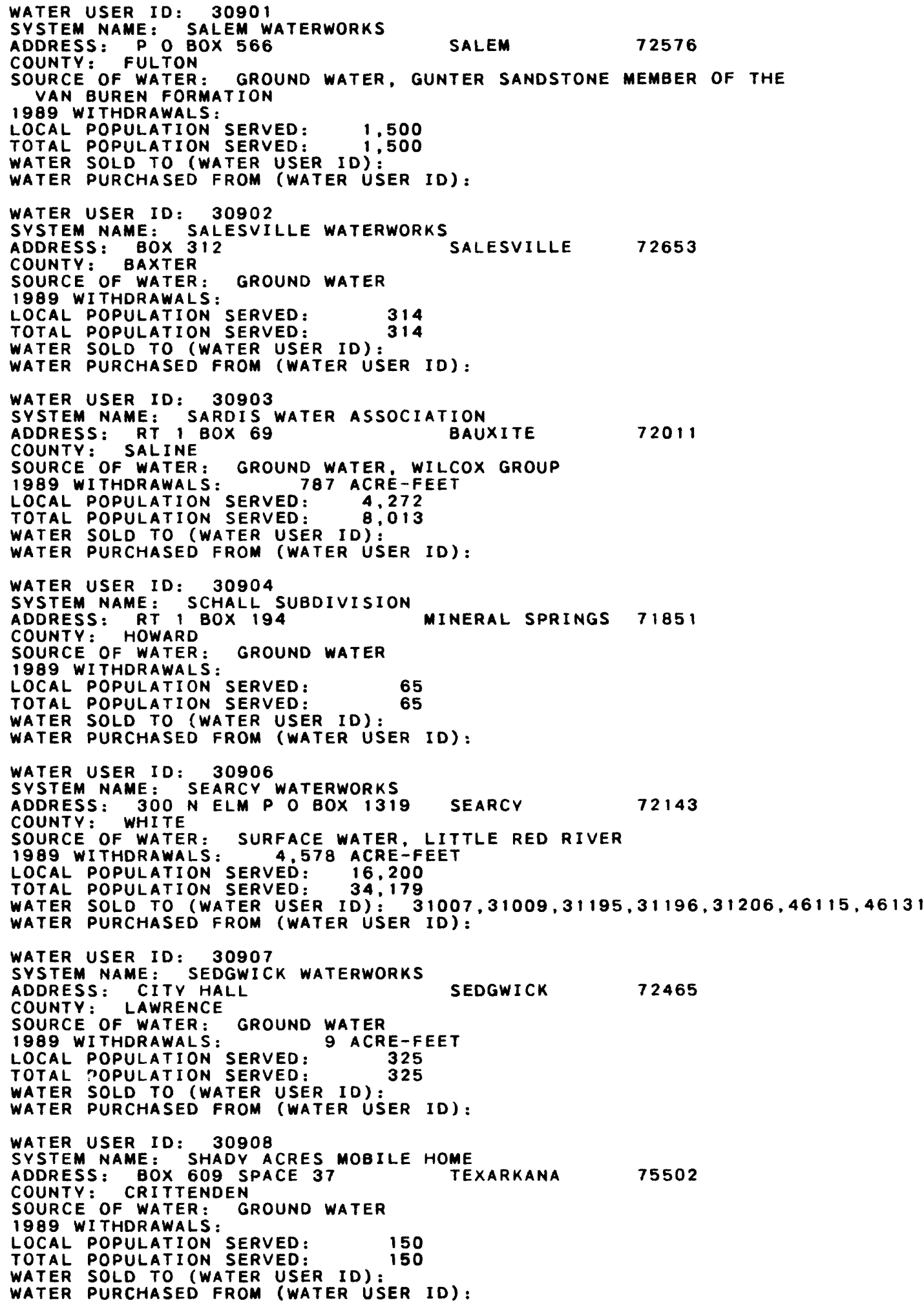


Table 1.--Description of public water supplies in Arkansas--Continued

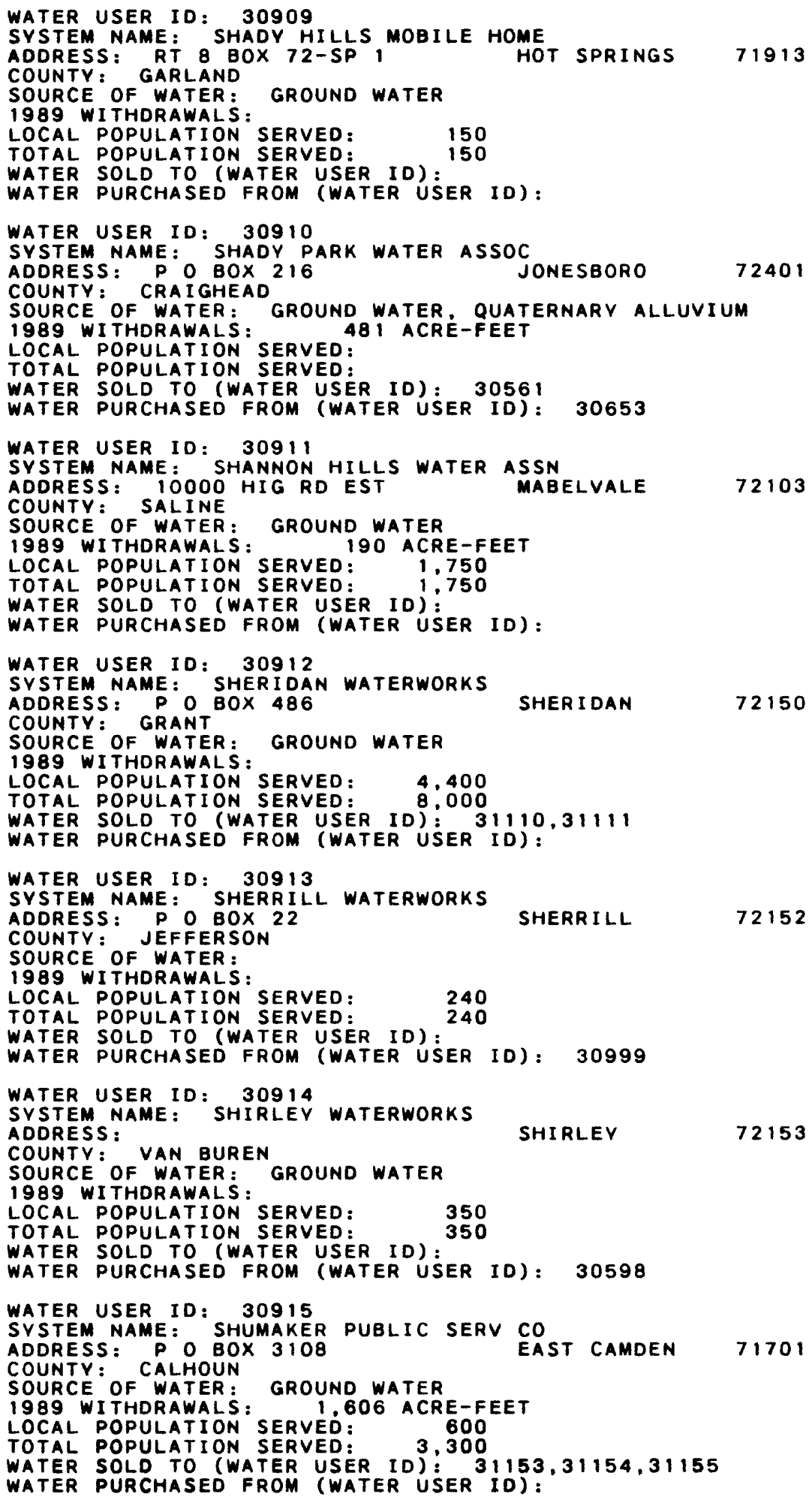


Table 1.--Description of public water supplies in Arkansas--Continued

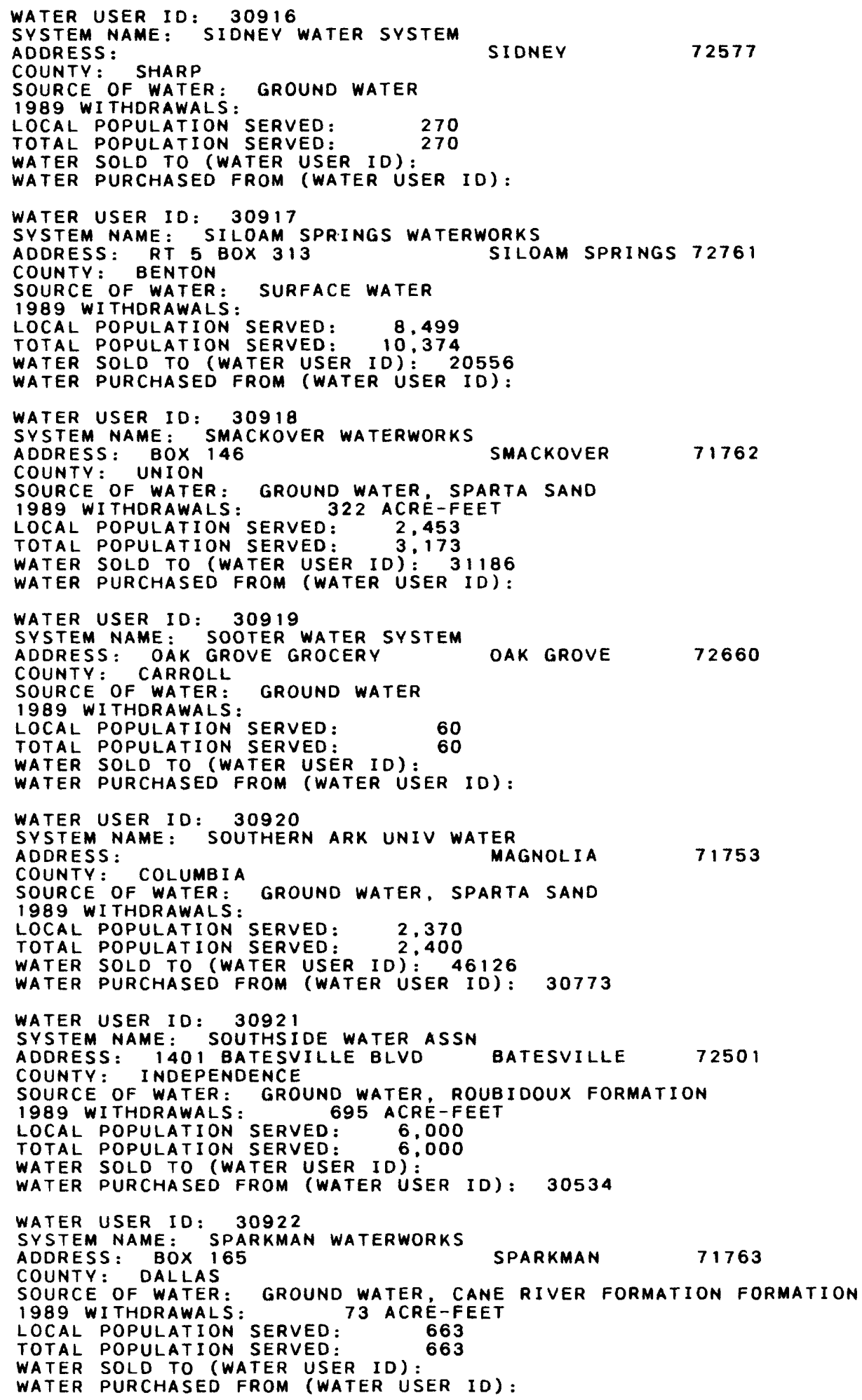


Table 1.--Description of public water supplies in Arkansas--Continued

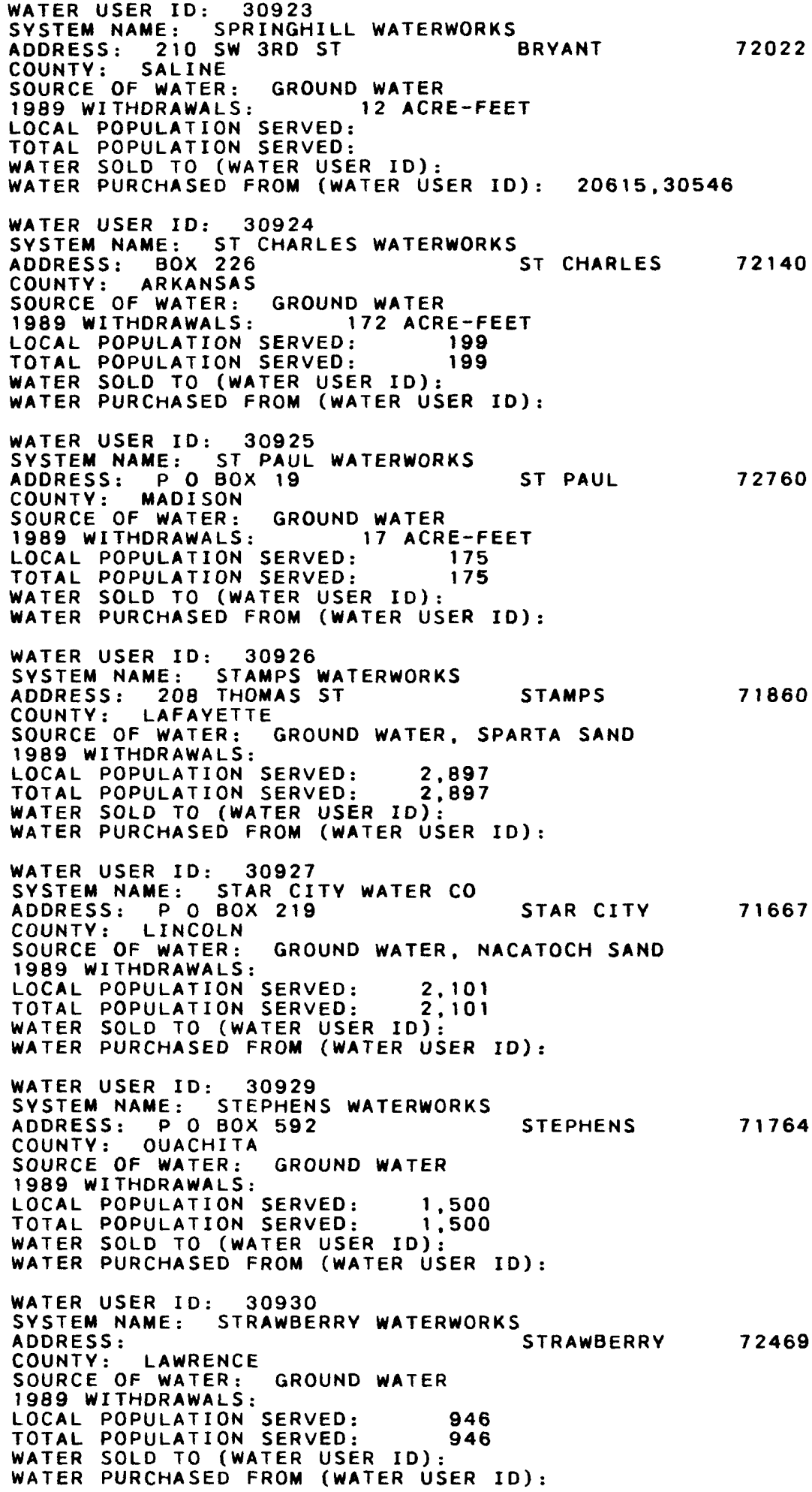

COUNTY: LAWRENCE

SOURCE OF WATER: GROUND WATER

1989 WITHDRAWALS :

LOCAL POPULATION SERVED: $\quad 946$

TOTAL POPULATION SERVED: 946

WATER SOLD TO (WATER USER ID):

WATER PURCHASED FROM (WATER USER ID) : 
Table 1.--Description of public water supplies in Arkansas--Continued

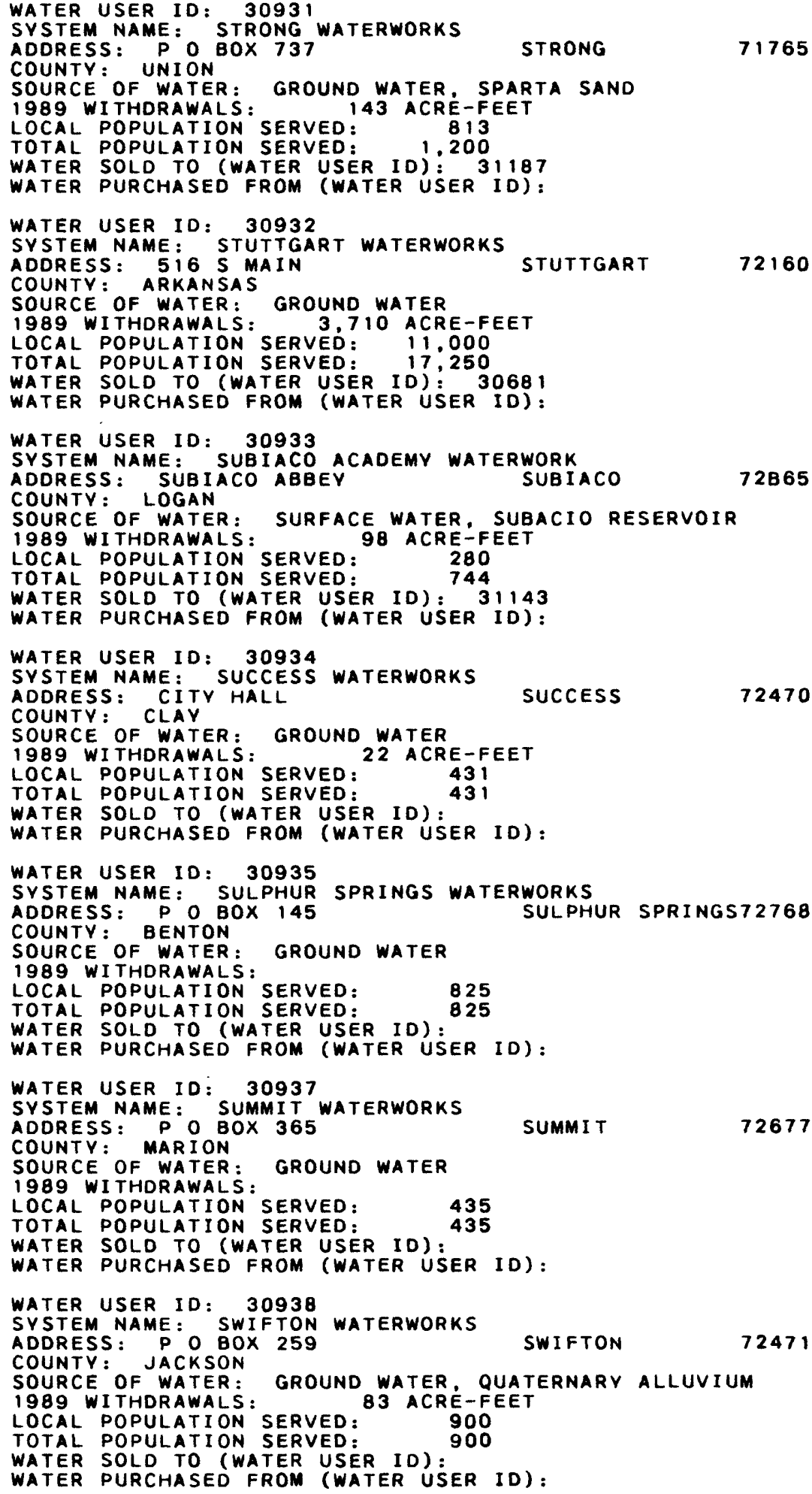


Table 1.--Description of public water supplies in Arkansas--Continued

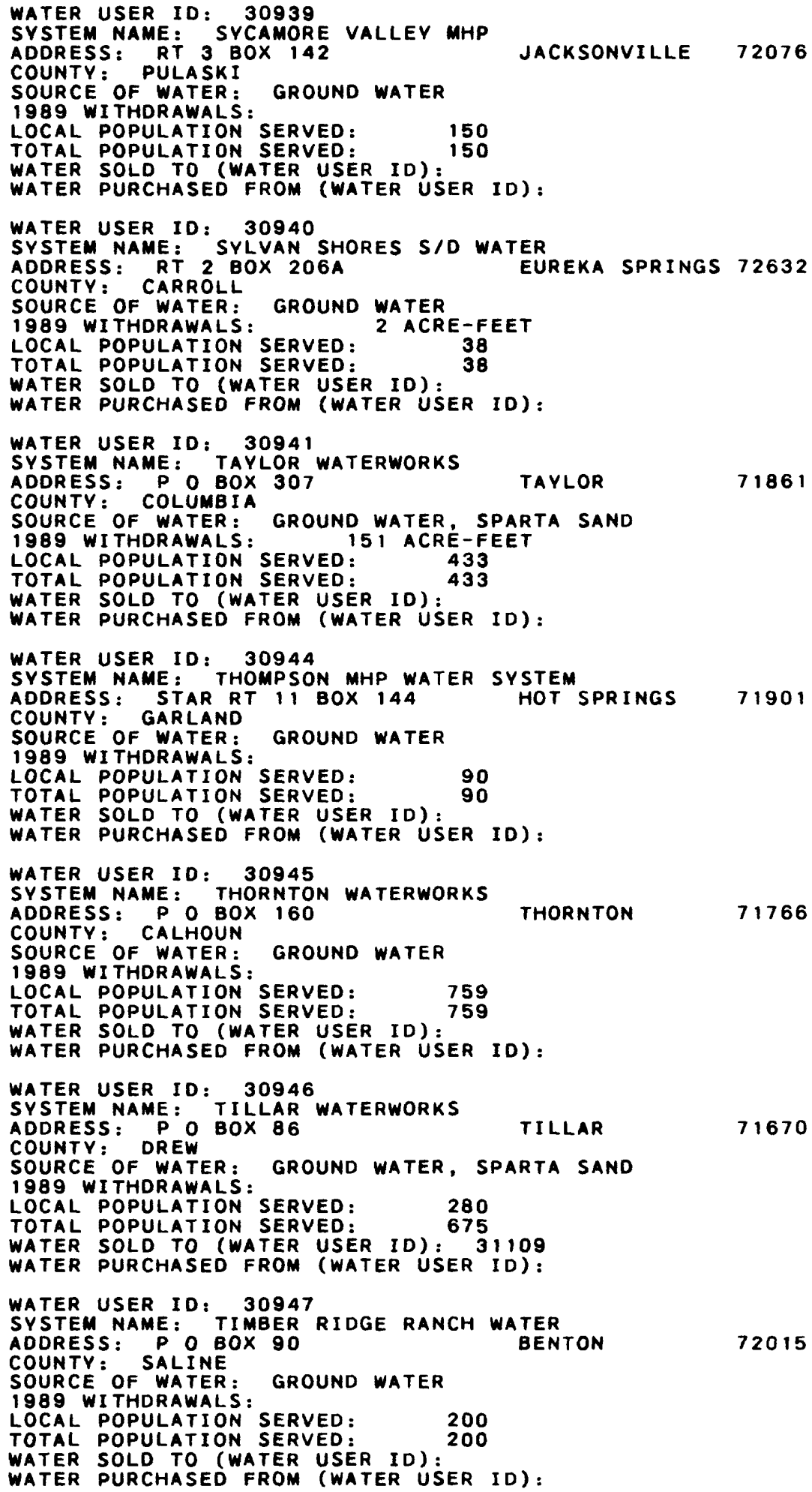


Table 1.--Description of public water supplies in Arkansas--Continued

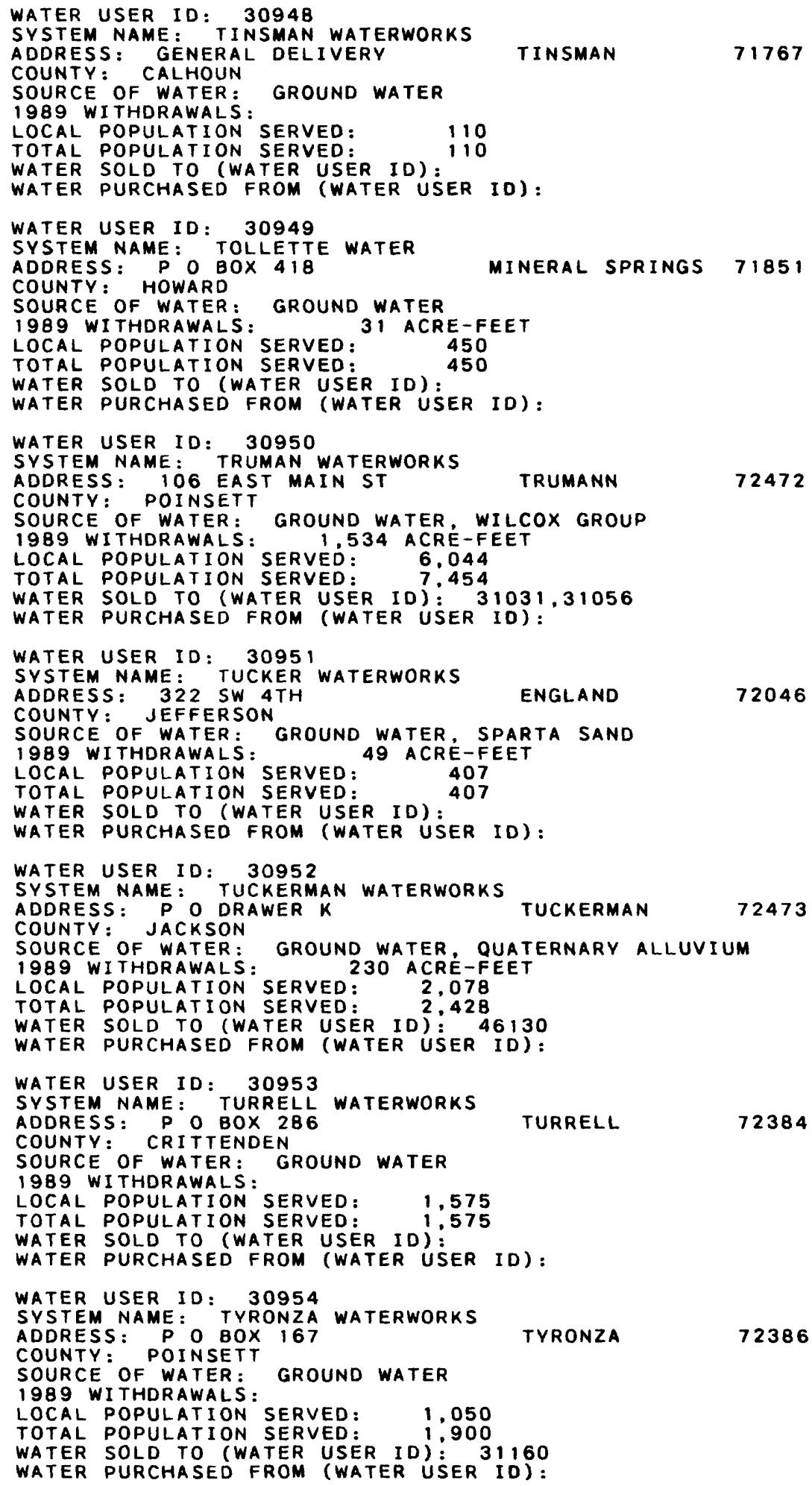


Table 1.--Description of public water supplies in Arkansas--Continued

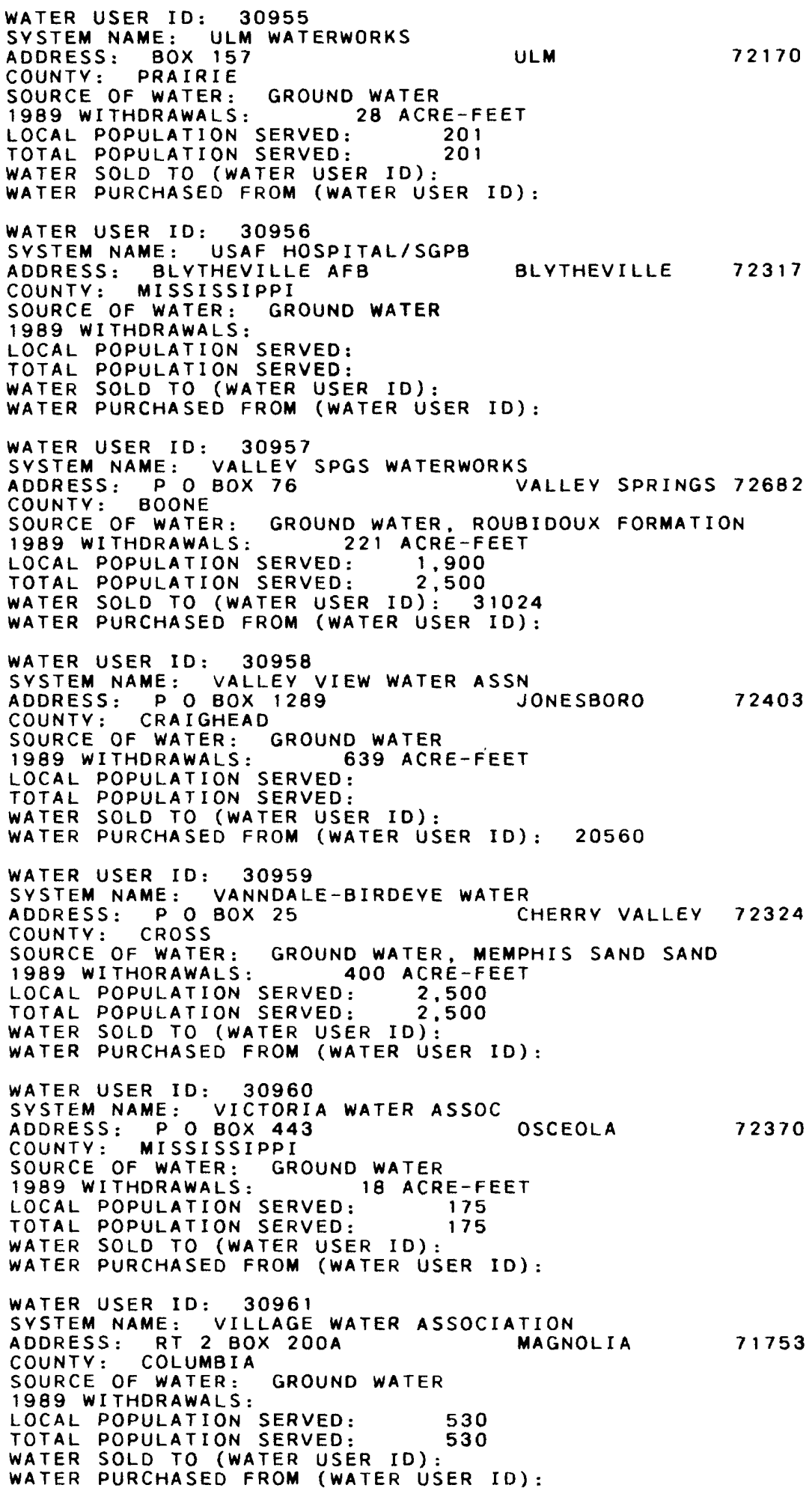


Table 1.--Description of public water supplies in Arkansas--Continued

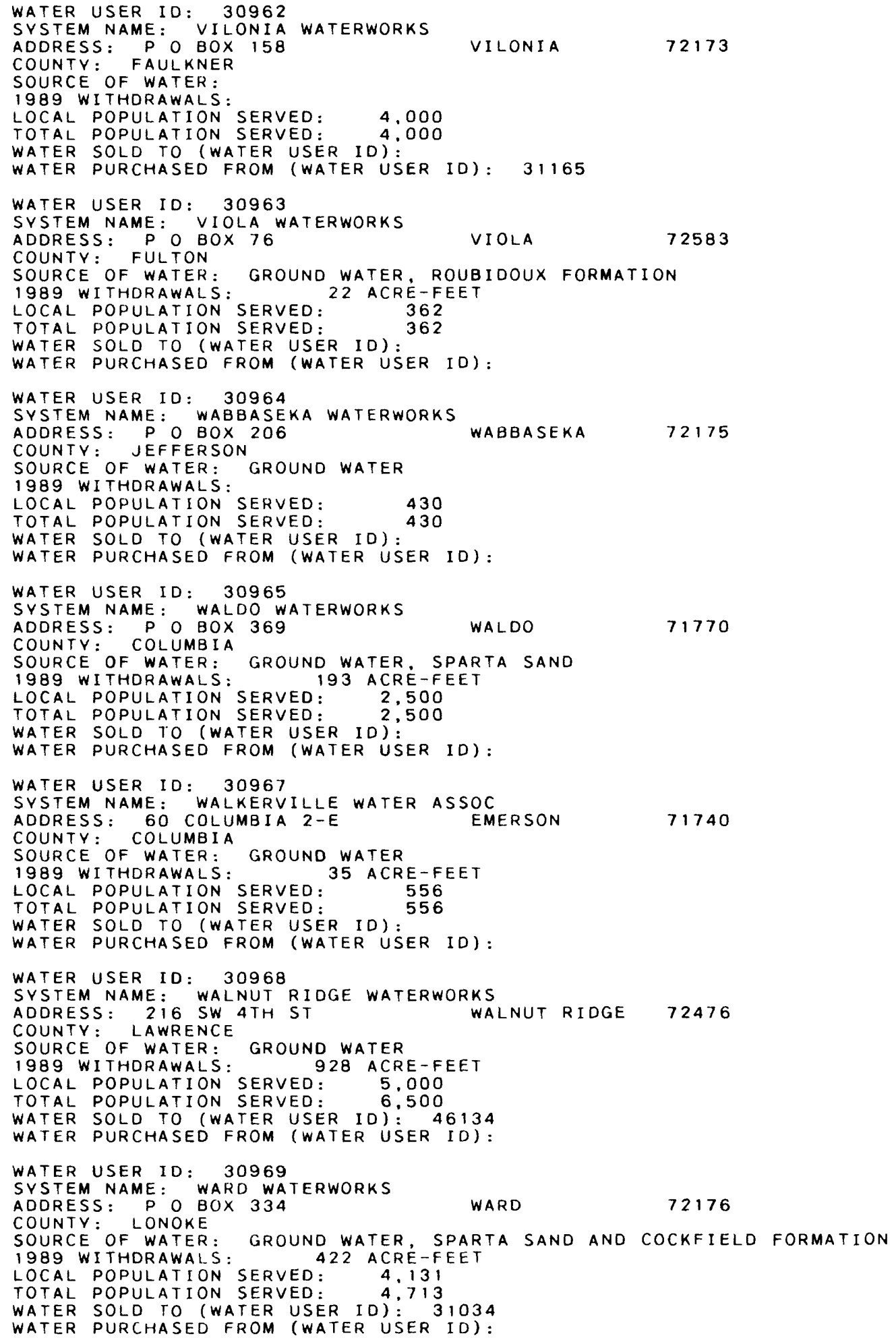


Table 1.--Description of public water supplies in Arkansas--Continued

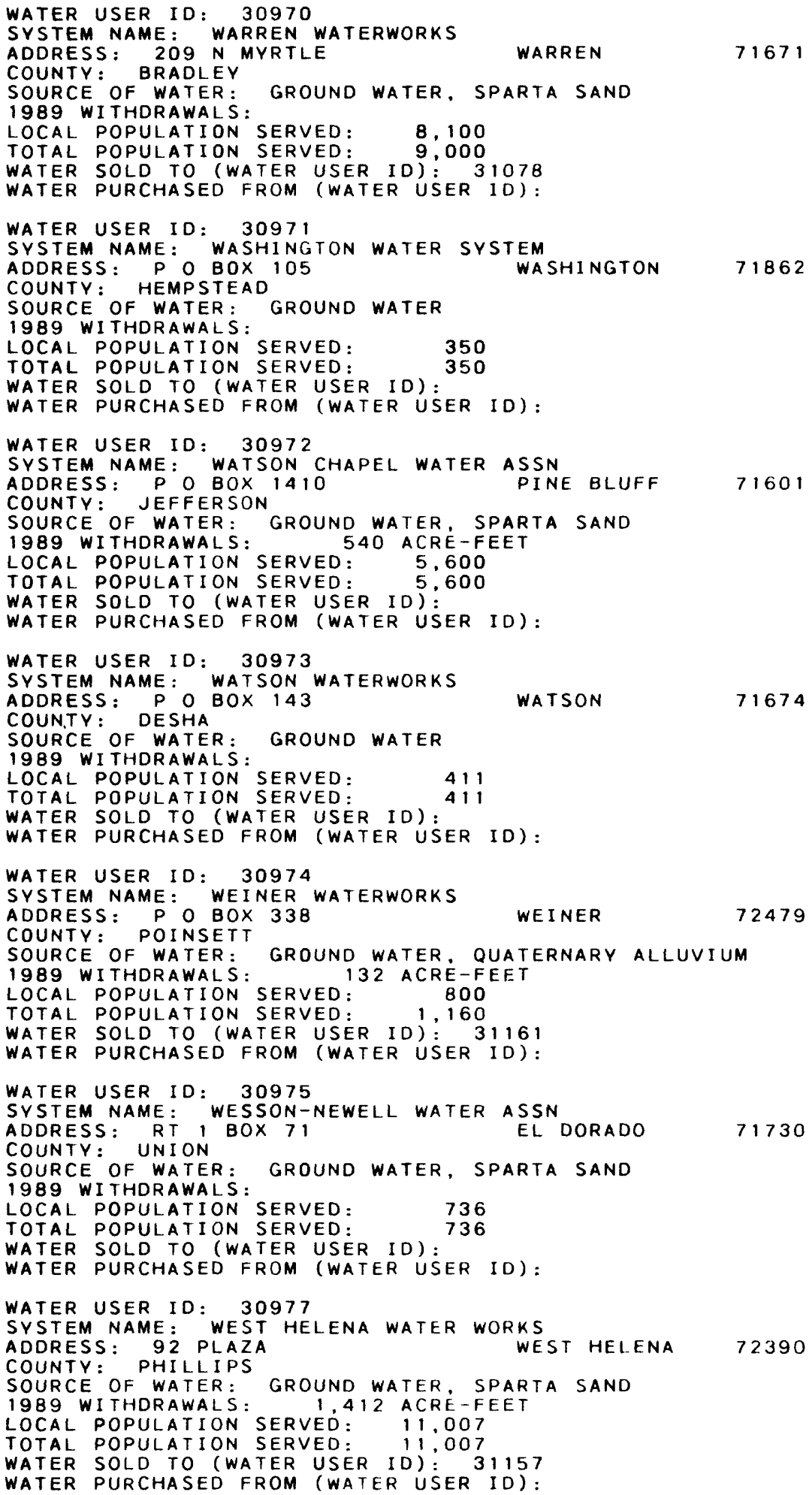


Table 1.--Description of public water supplies in Arkansas--Continued

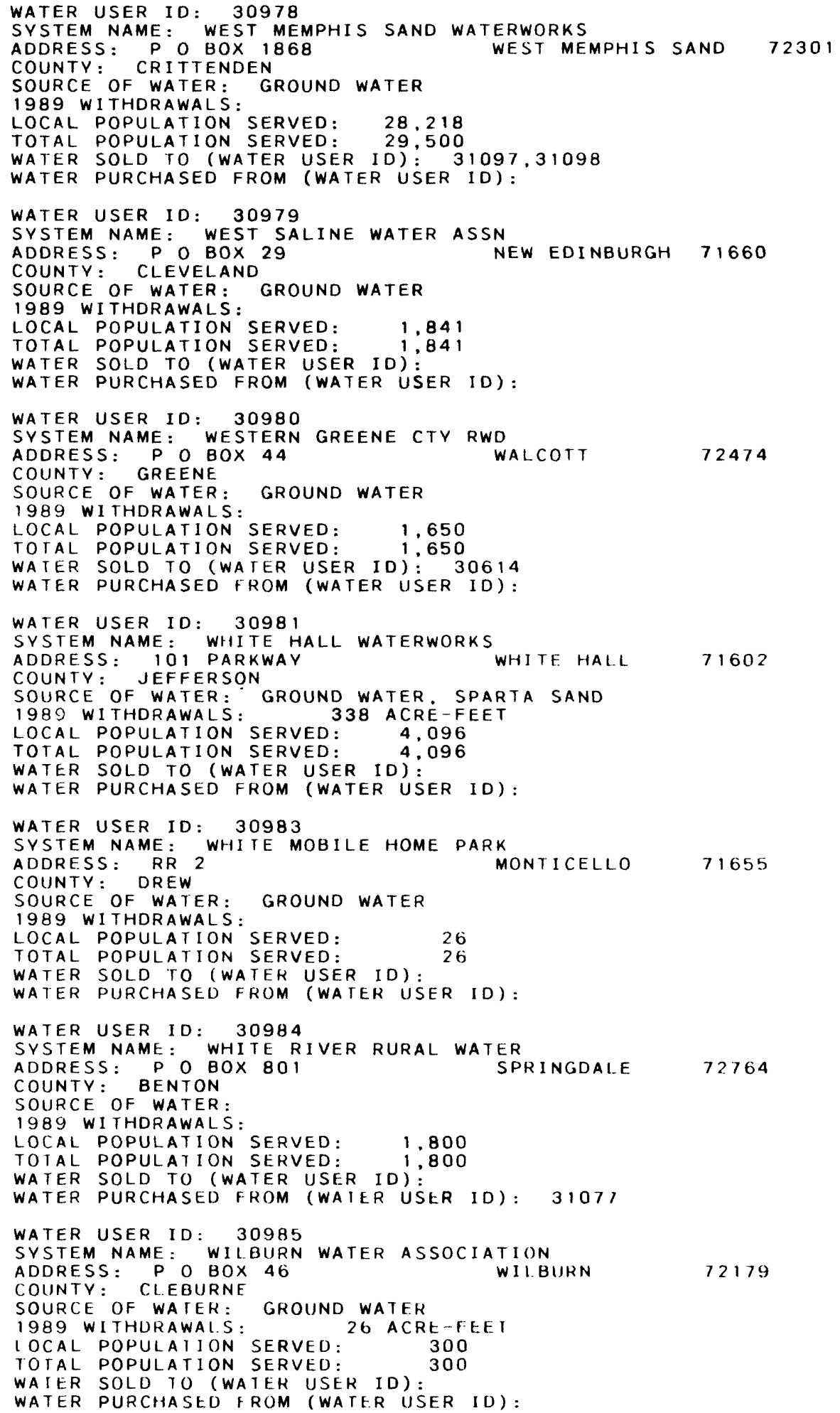


Table 1.--Description of public water supplies in Arkansas--Continued

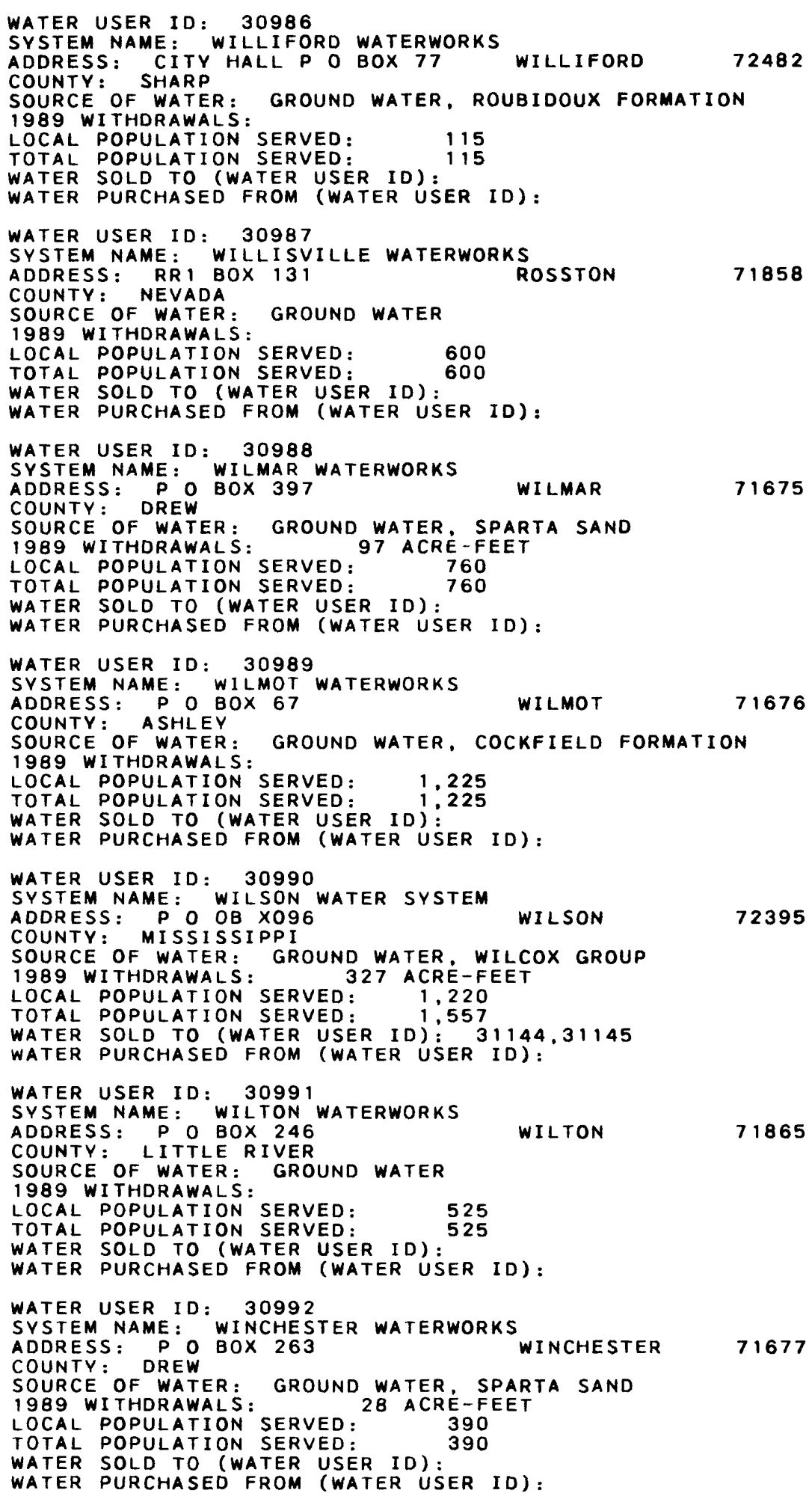


Table 1.--Description of public water supplies in Arkansas--Continued

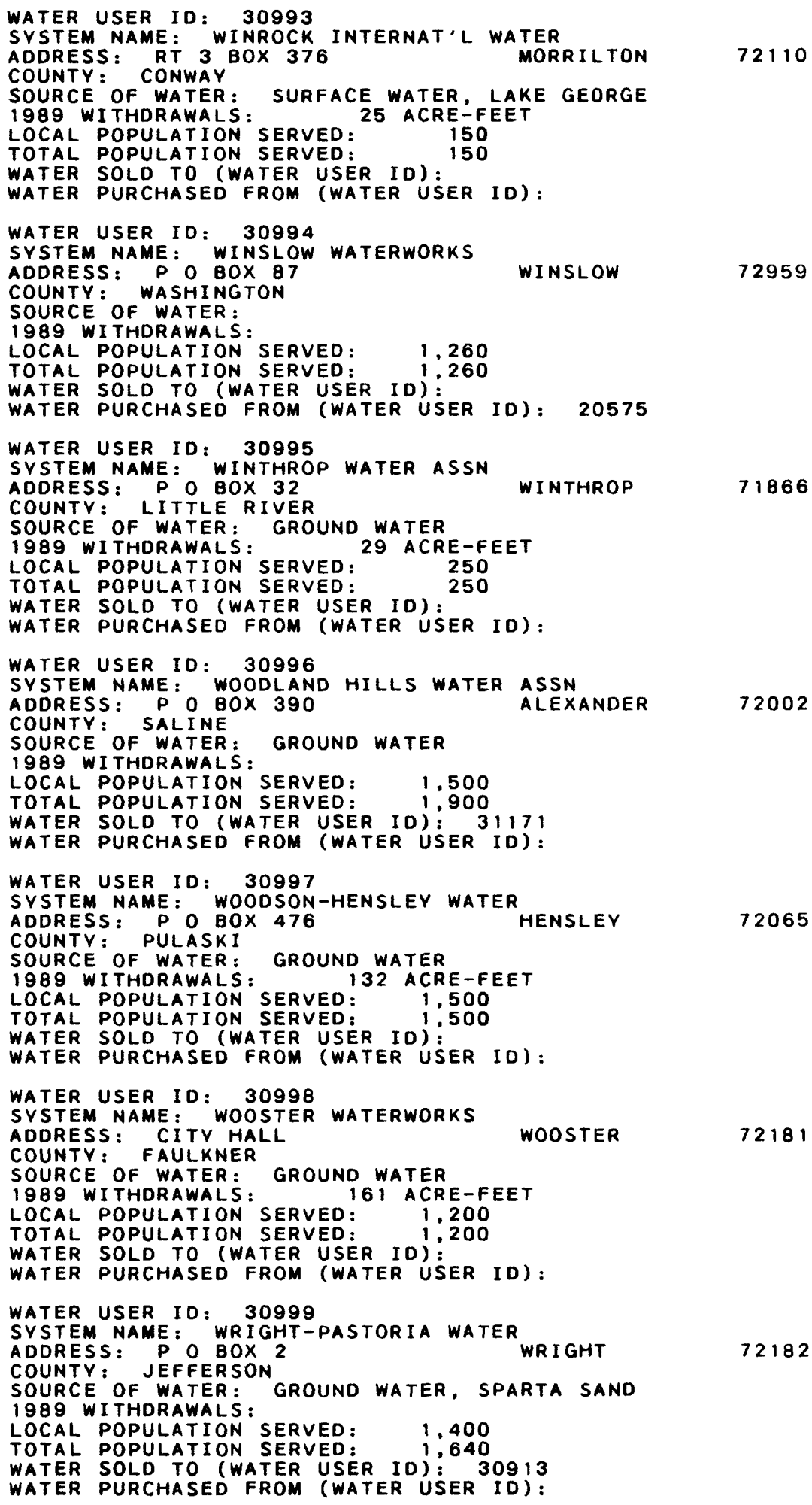


Table 1.--Description of public water supplies in Arkansas--Continued

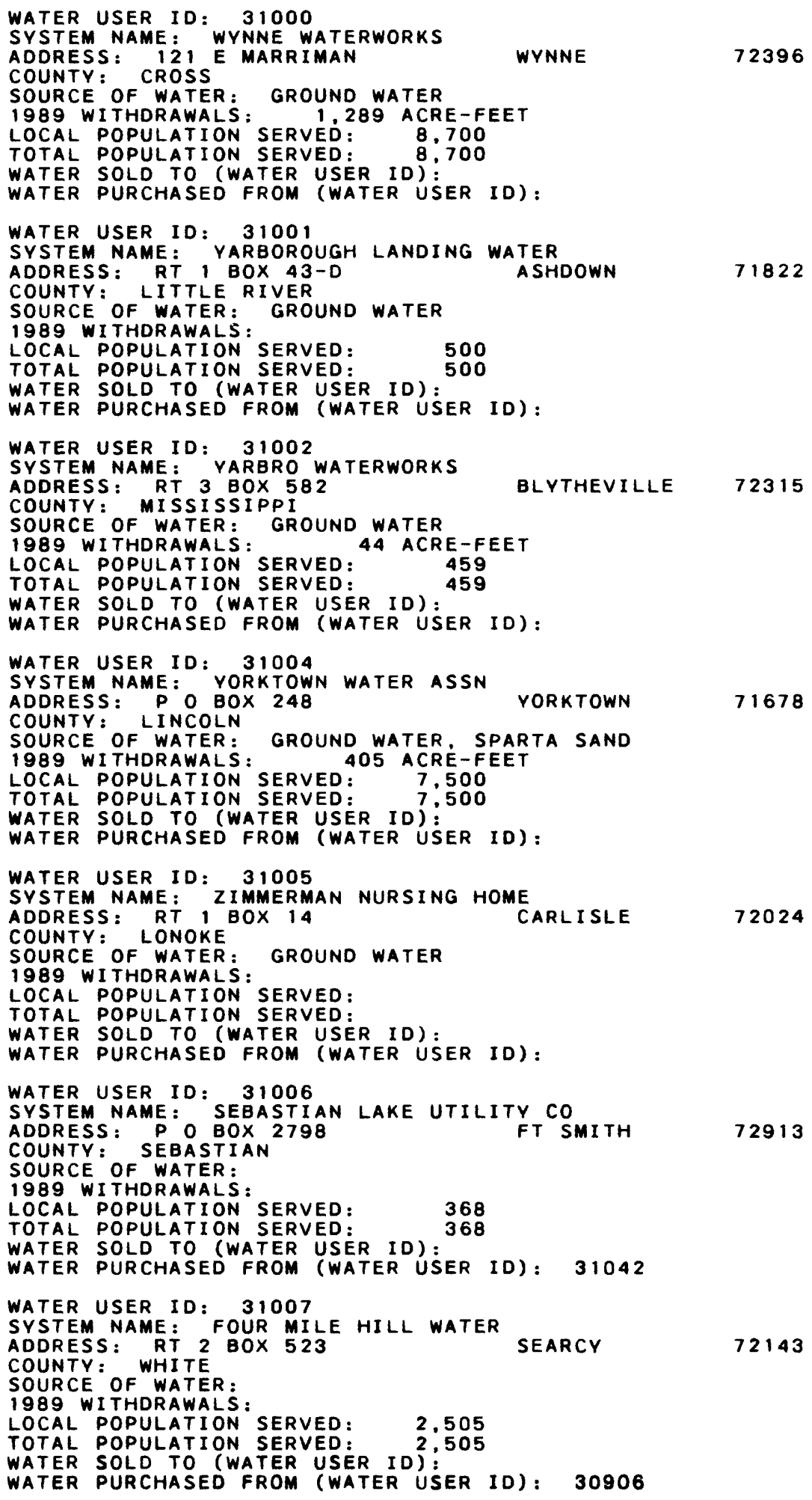


Table 1.--Description of public water supplies in Arkansas--Continued

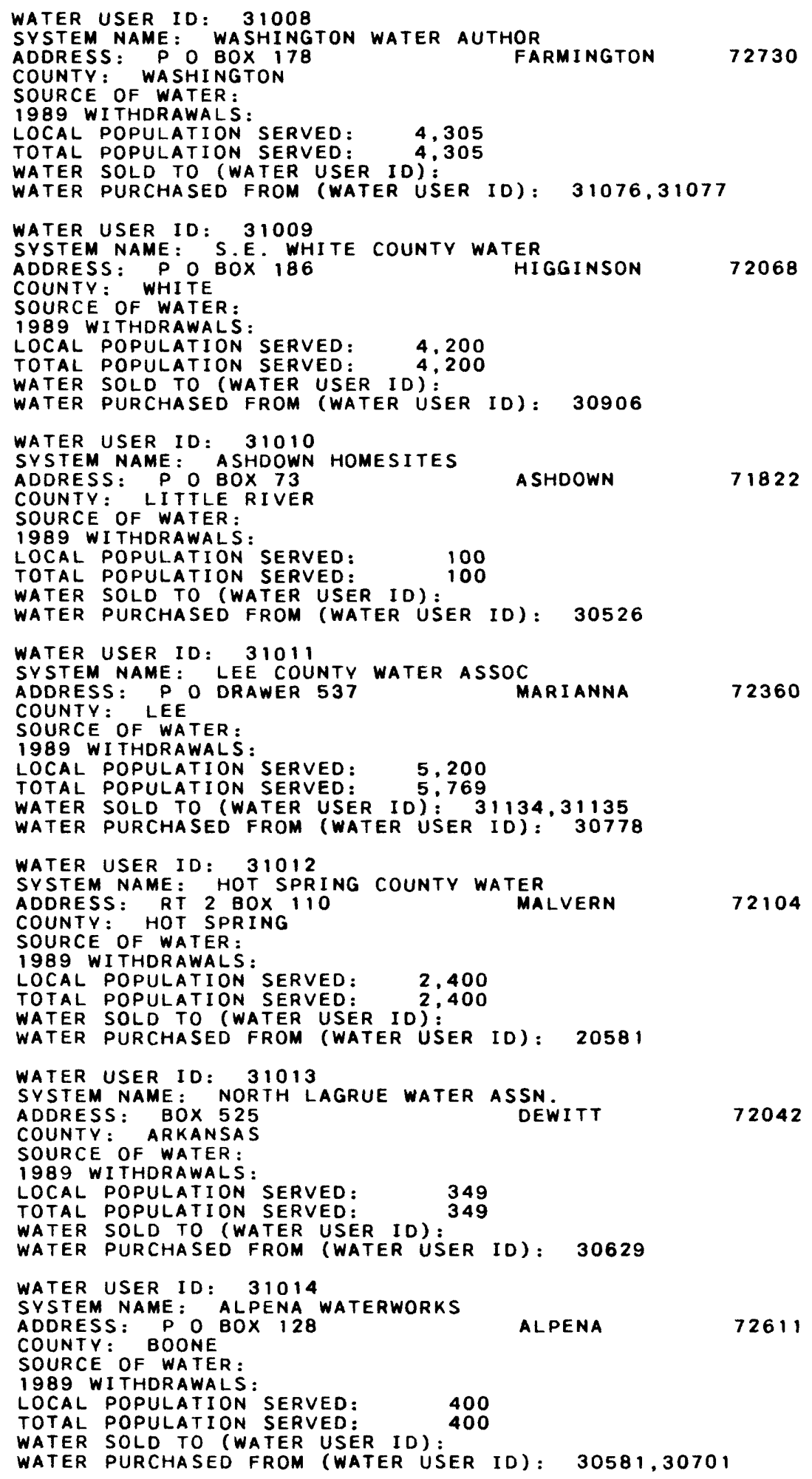


Table 1.--Description of public water supplies in Arkansas--Continued

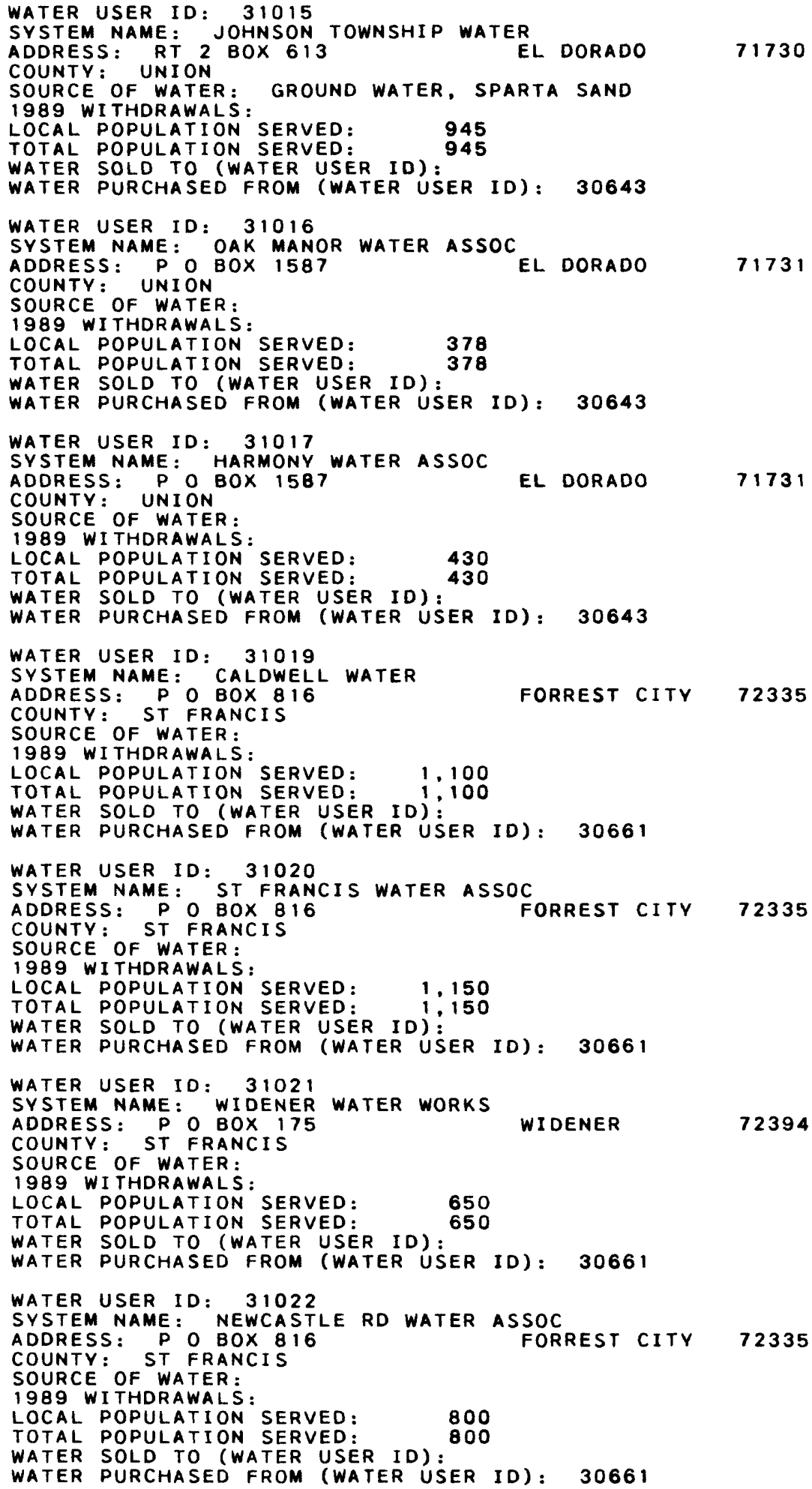


Table 1.--Description of public water supplies in Arkansas--Continued

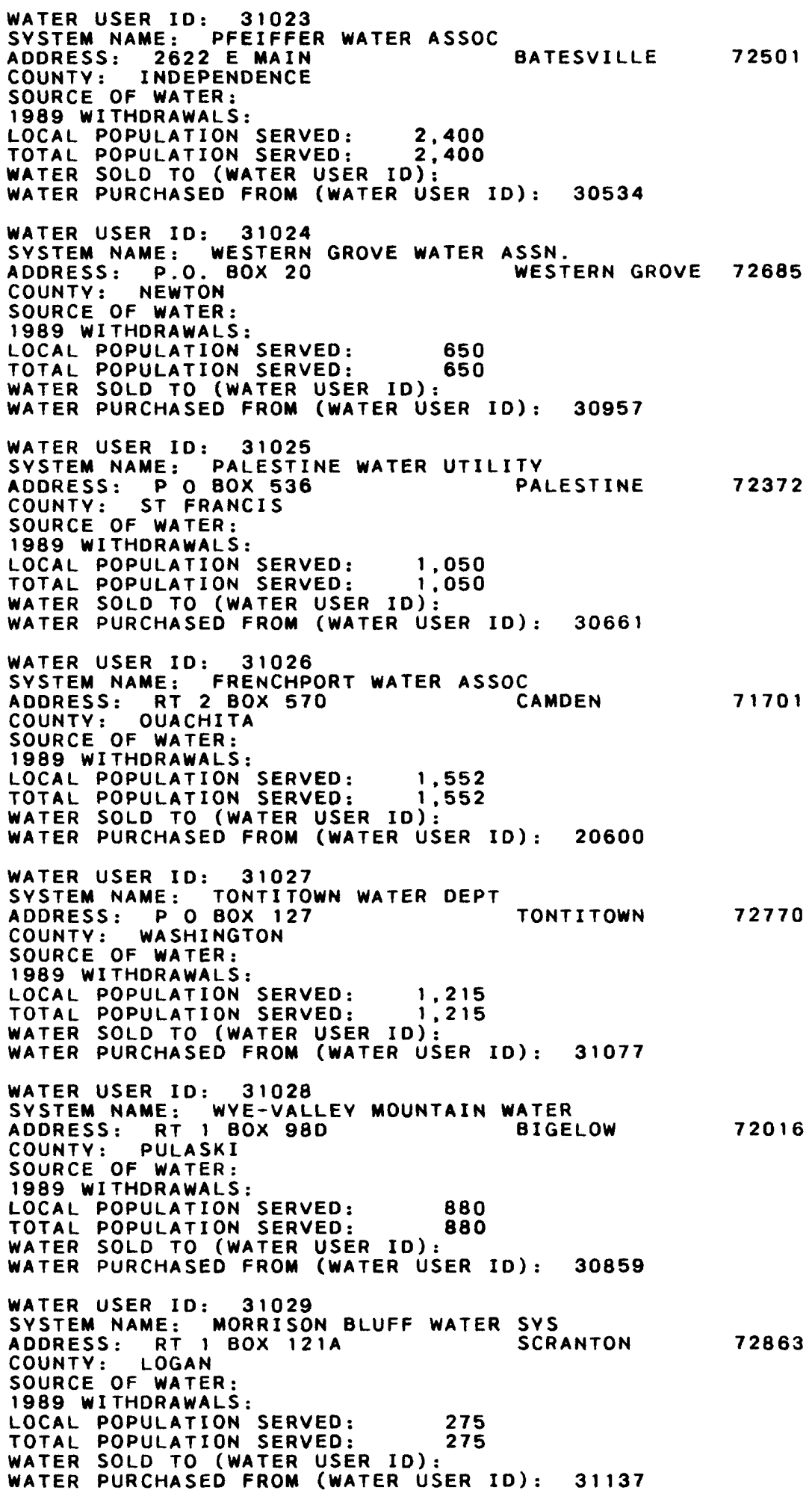


Table 1.--Description of public water supplies in Arkansas--Continued

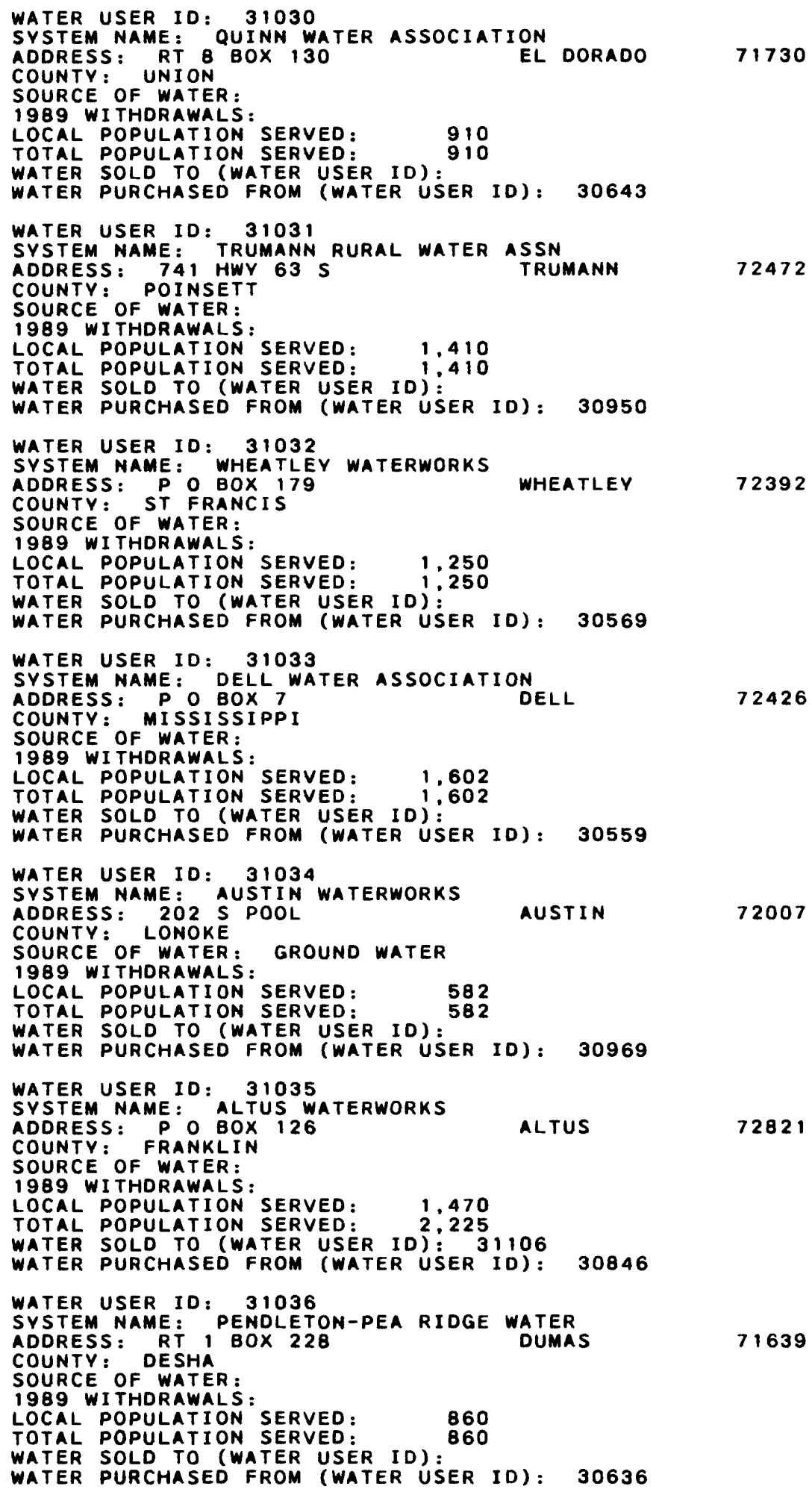


Table 1.--Description of public water supplies in Arkansas--Continued

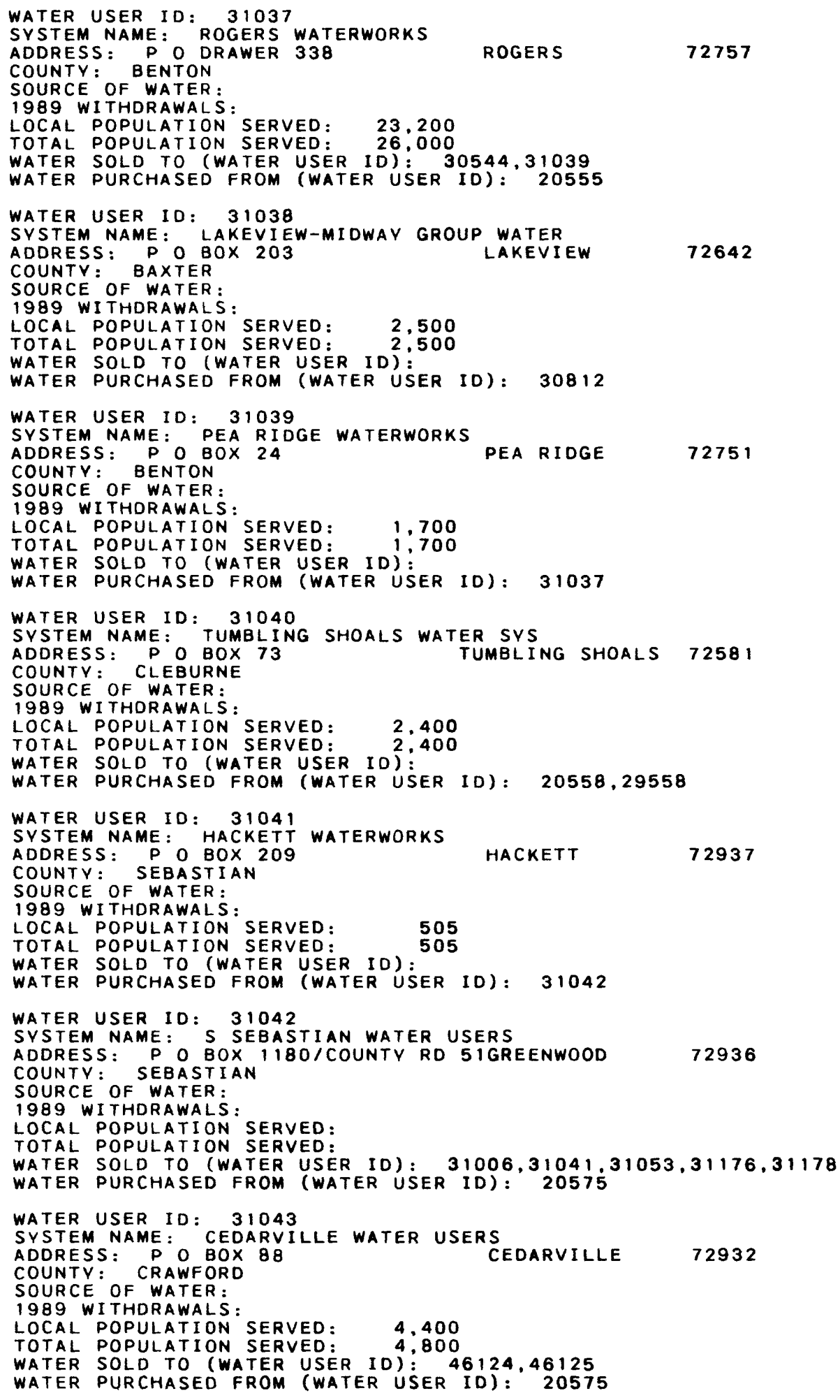


Table 1.--Description of public water supplies in Arkansas--Continued

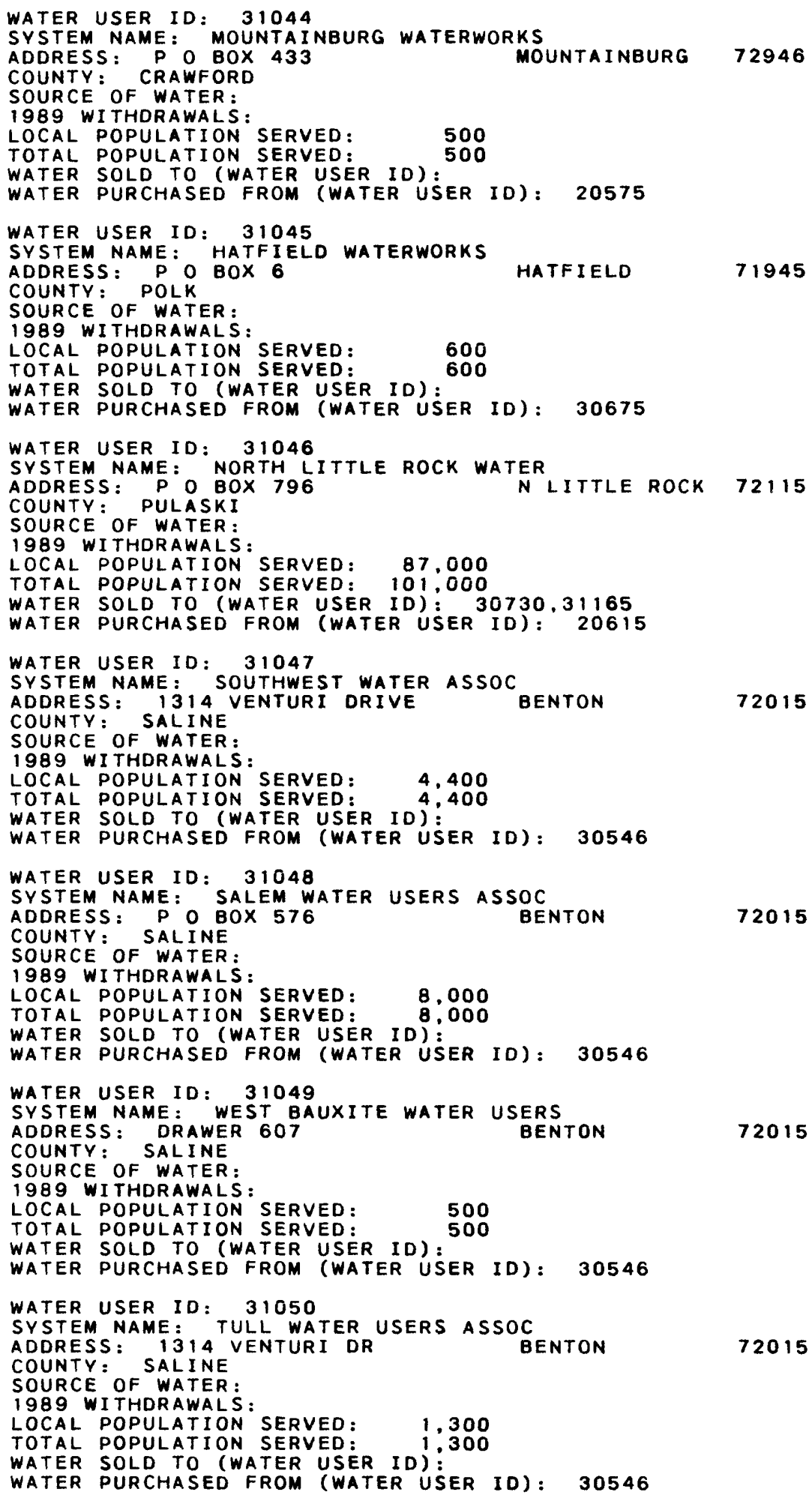


Table 1.--Description of public water supplies in Arkansas--Continued

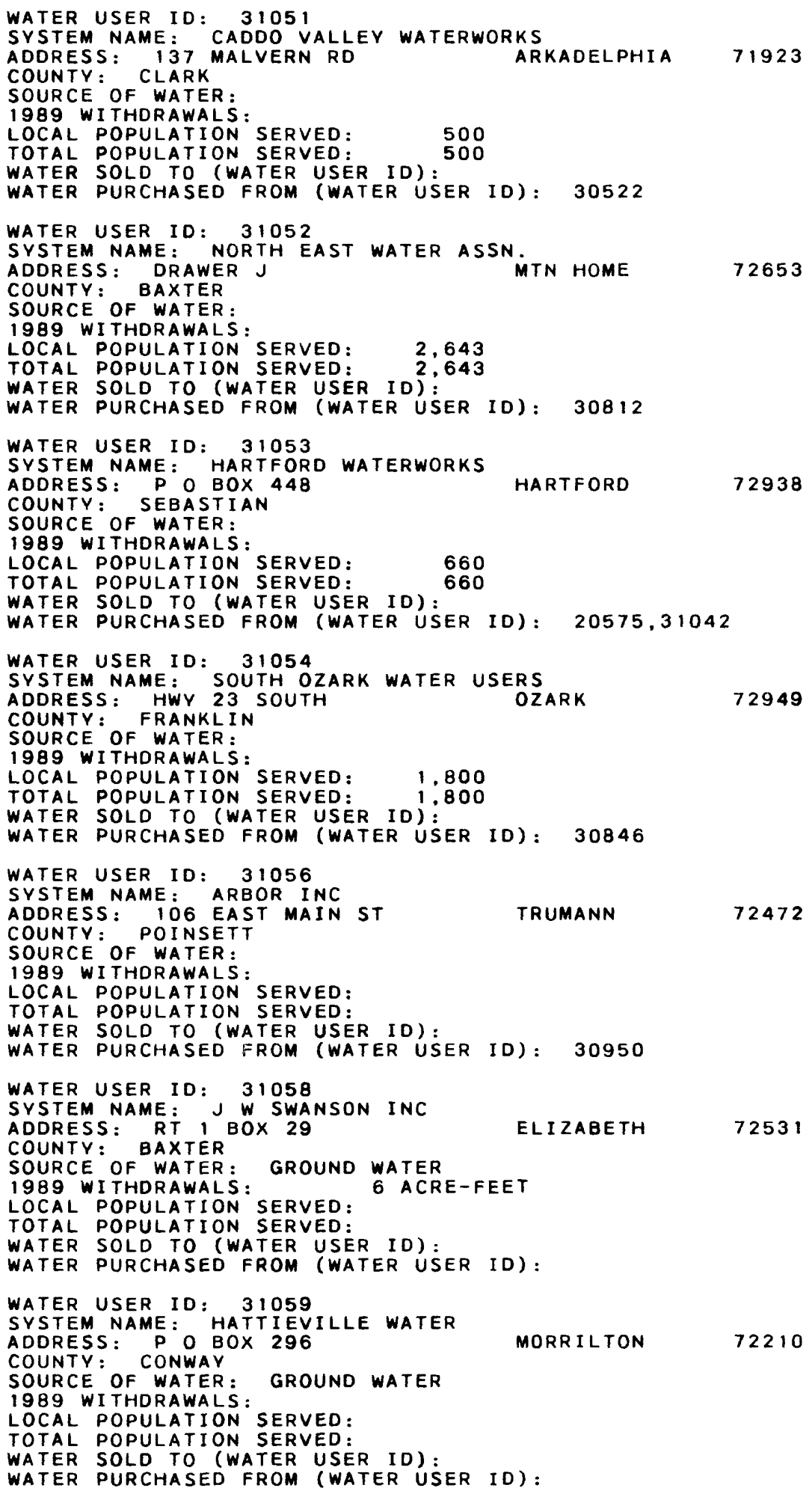


Table 1.--Description of public water supplies in Arkansas--Continued

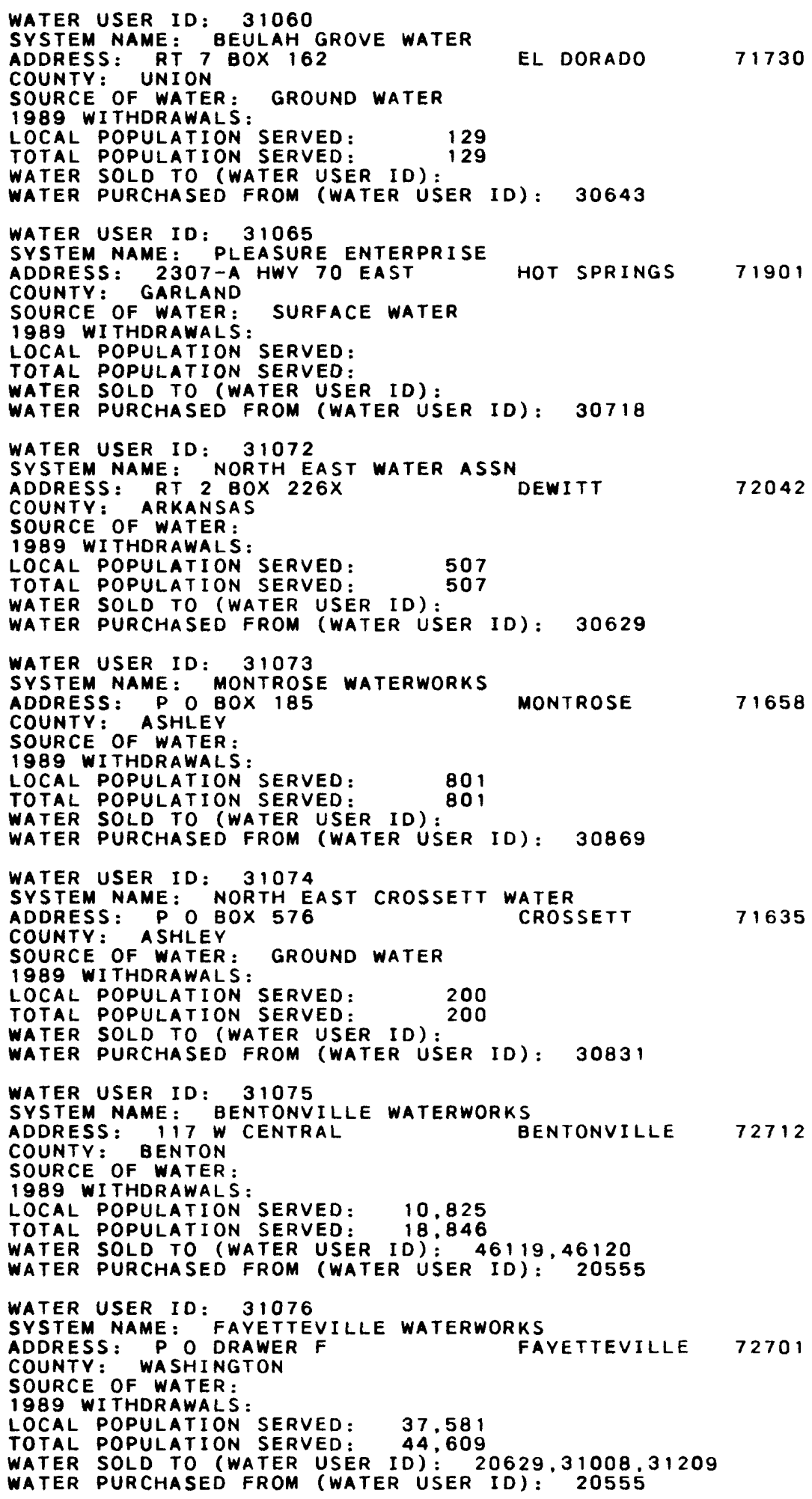


Table 1.--Description of public water supplies in Arkansas--Continued

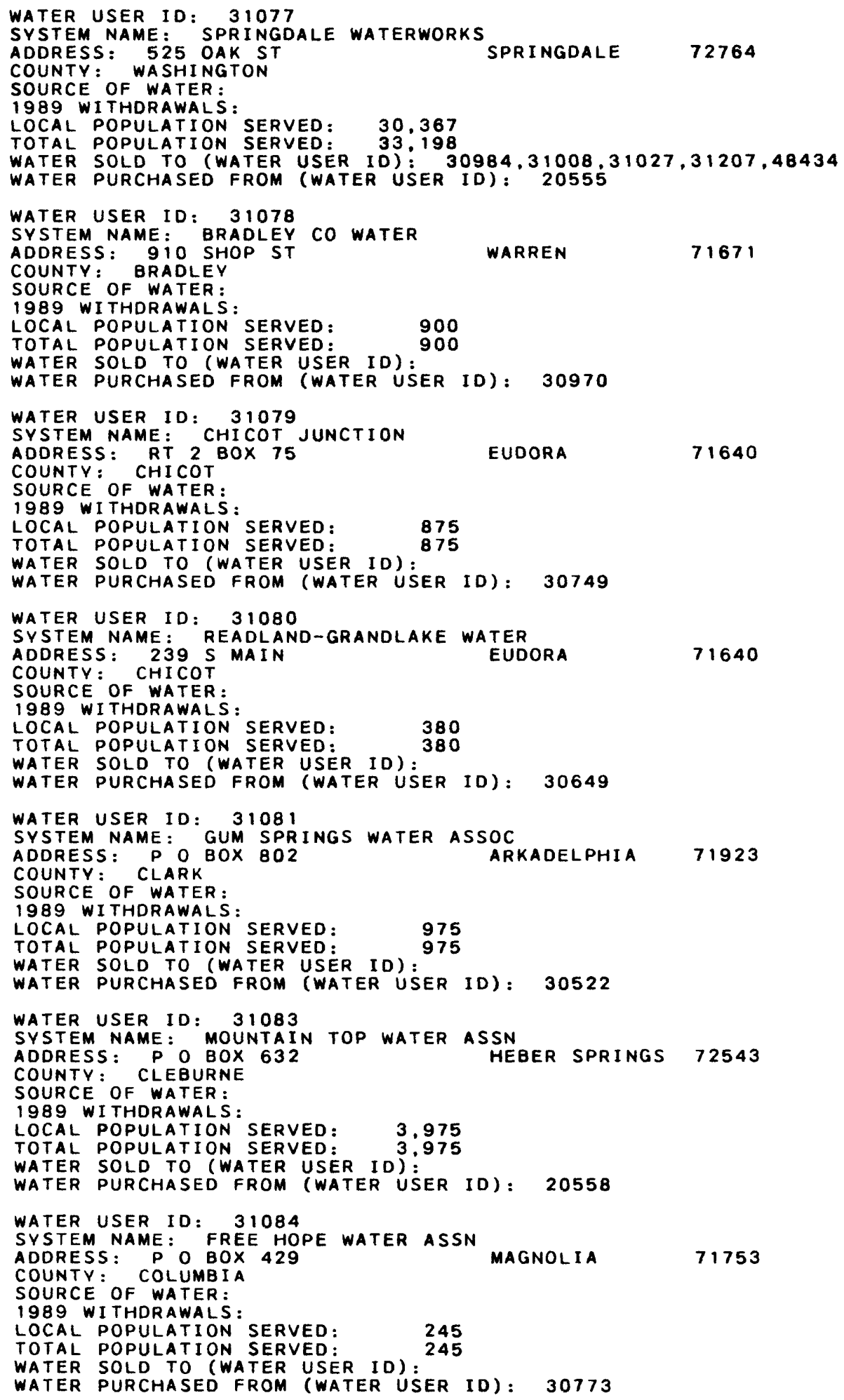


Table 1.--Description of public water supplies in Arkansas--Continued

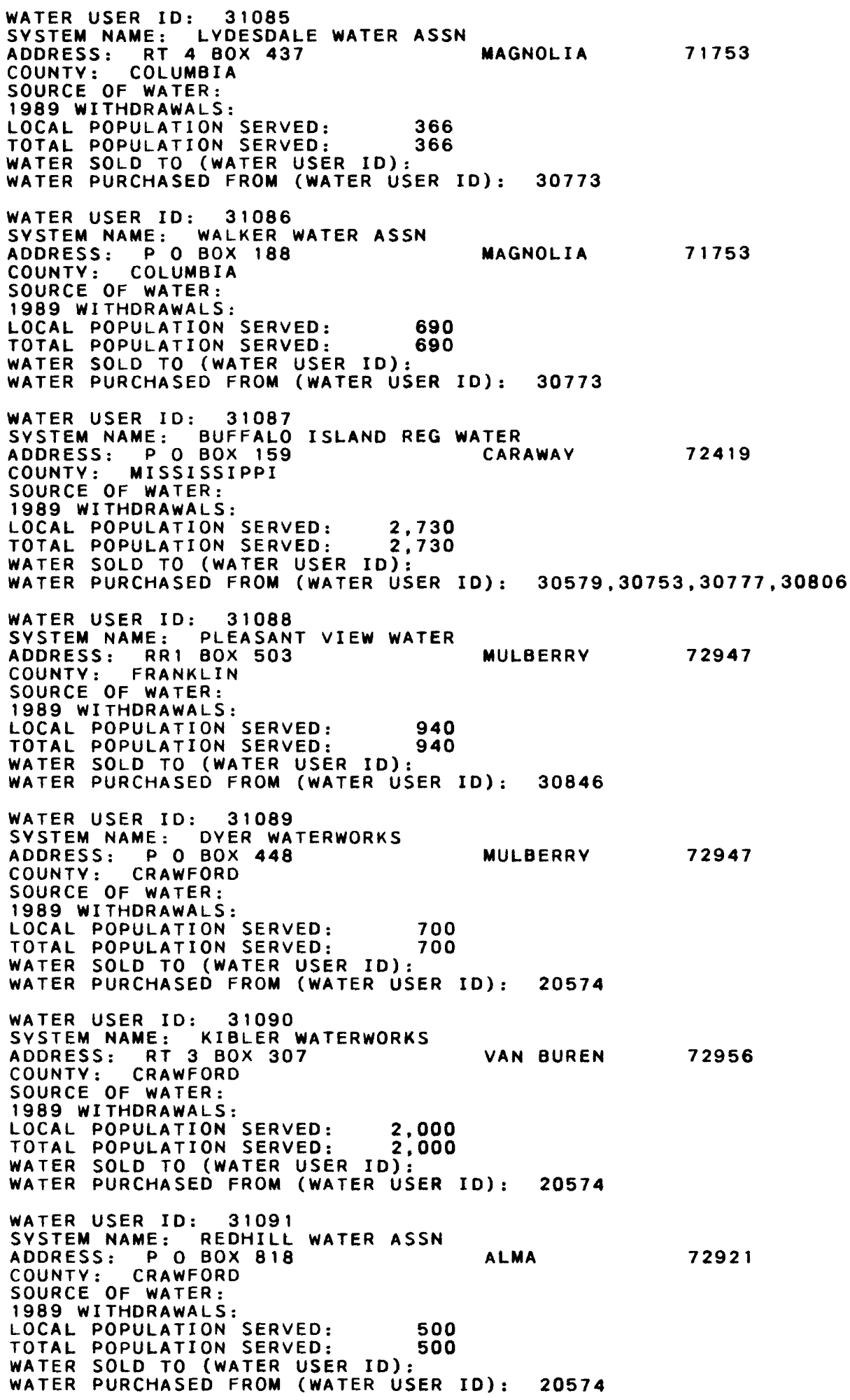


Table 1.--Description of public water supplies in Arkansas--Continued

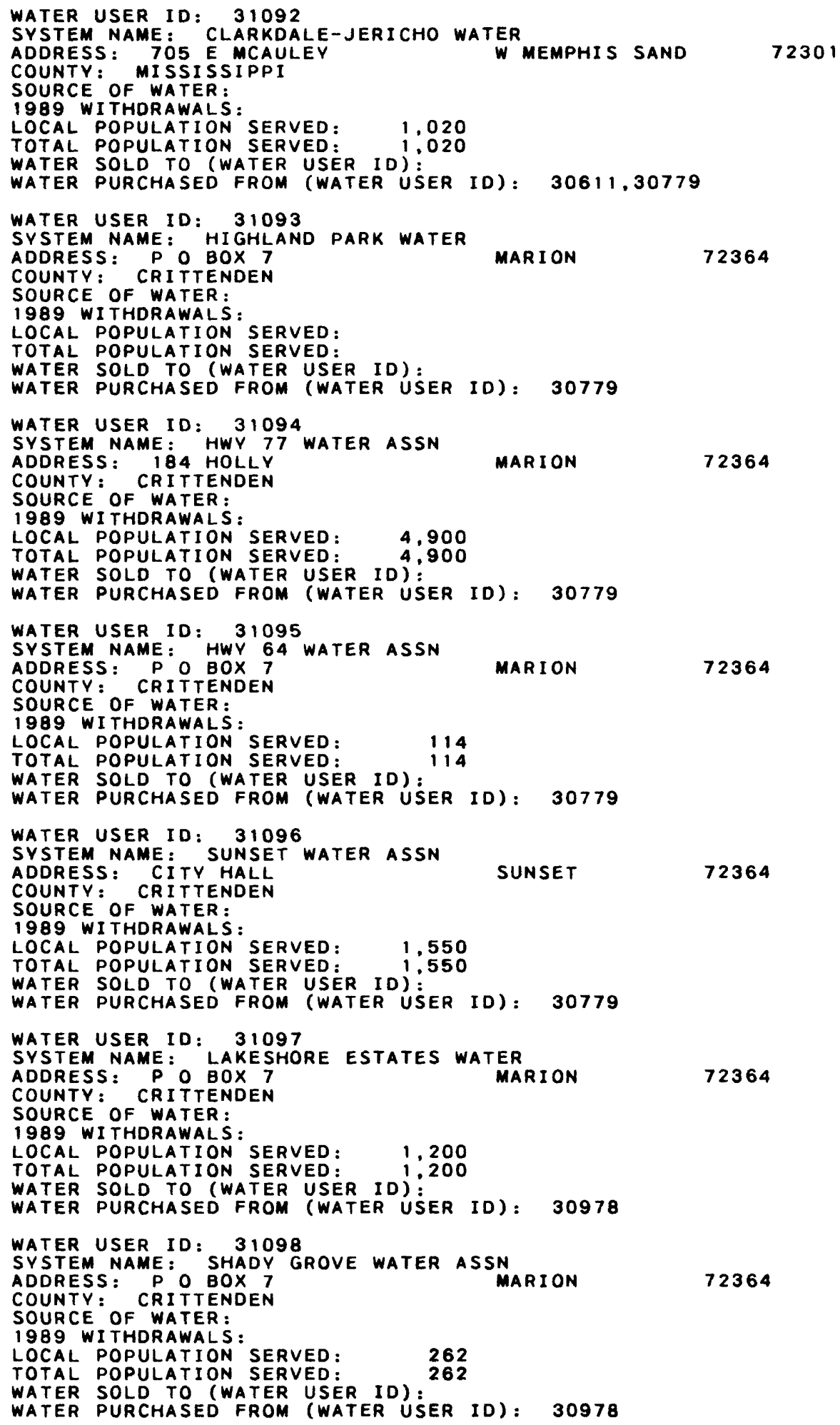


Table 1.--Description of public water supplies in Arkansas--Continued

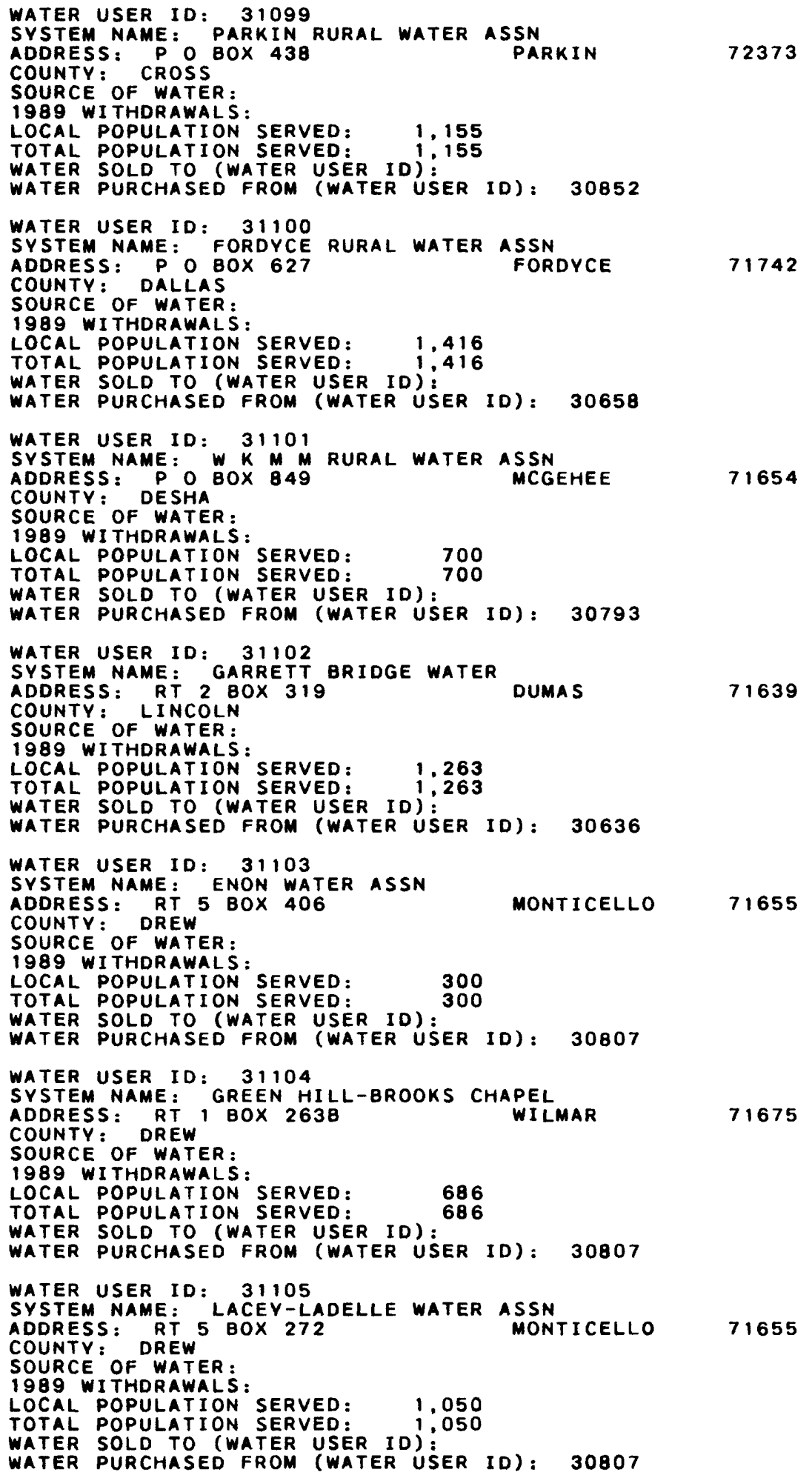


Table 1.--Description of public water supplies in Arkansas--Continued

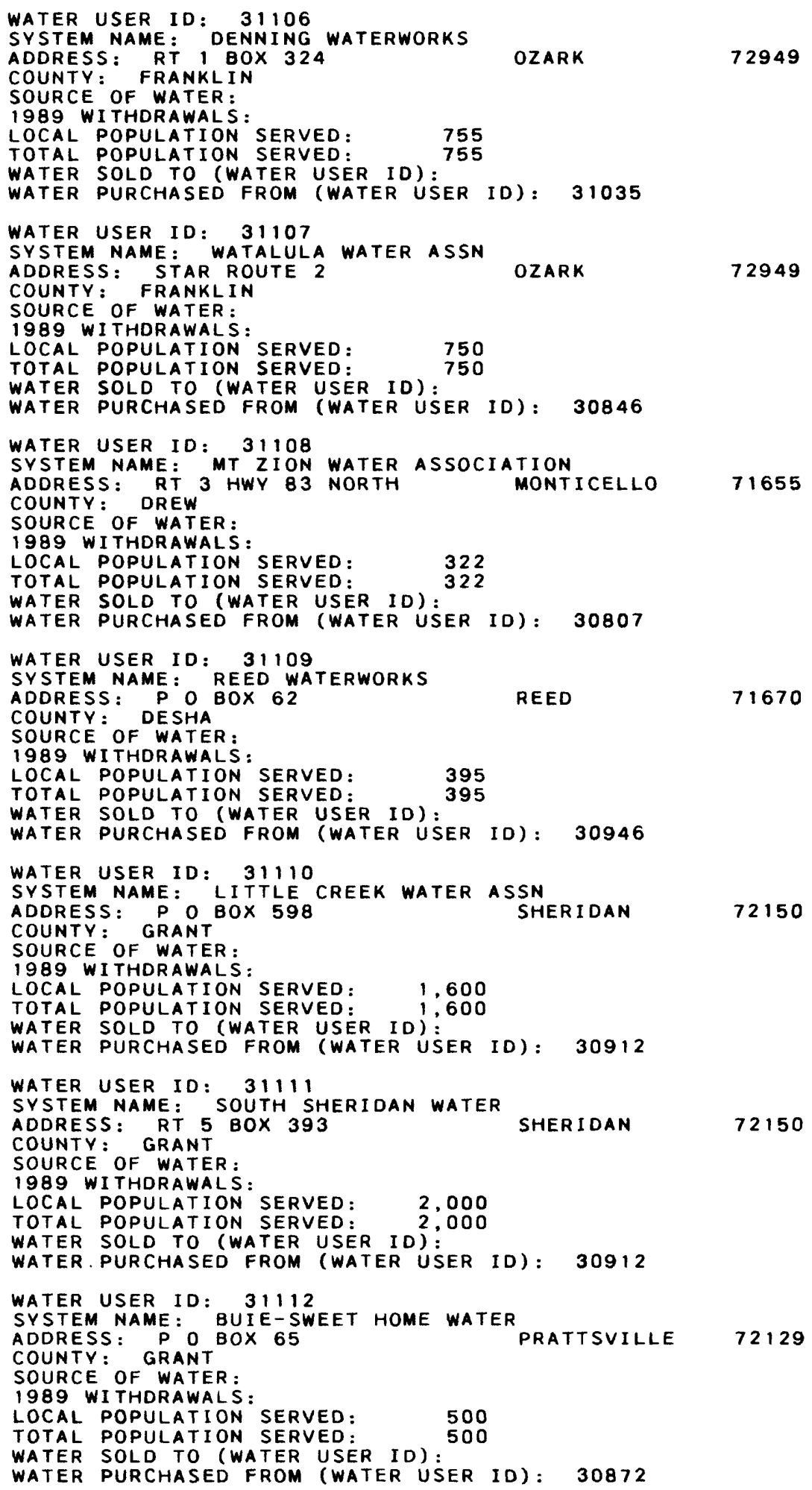


Table 1.--Description of public water supplies in Arkansas--Continued

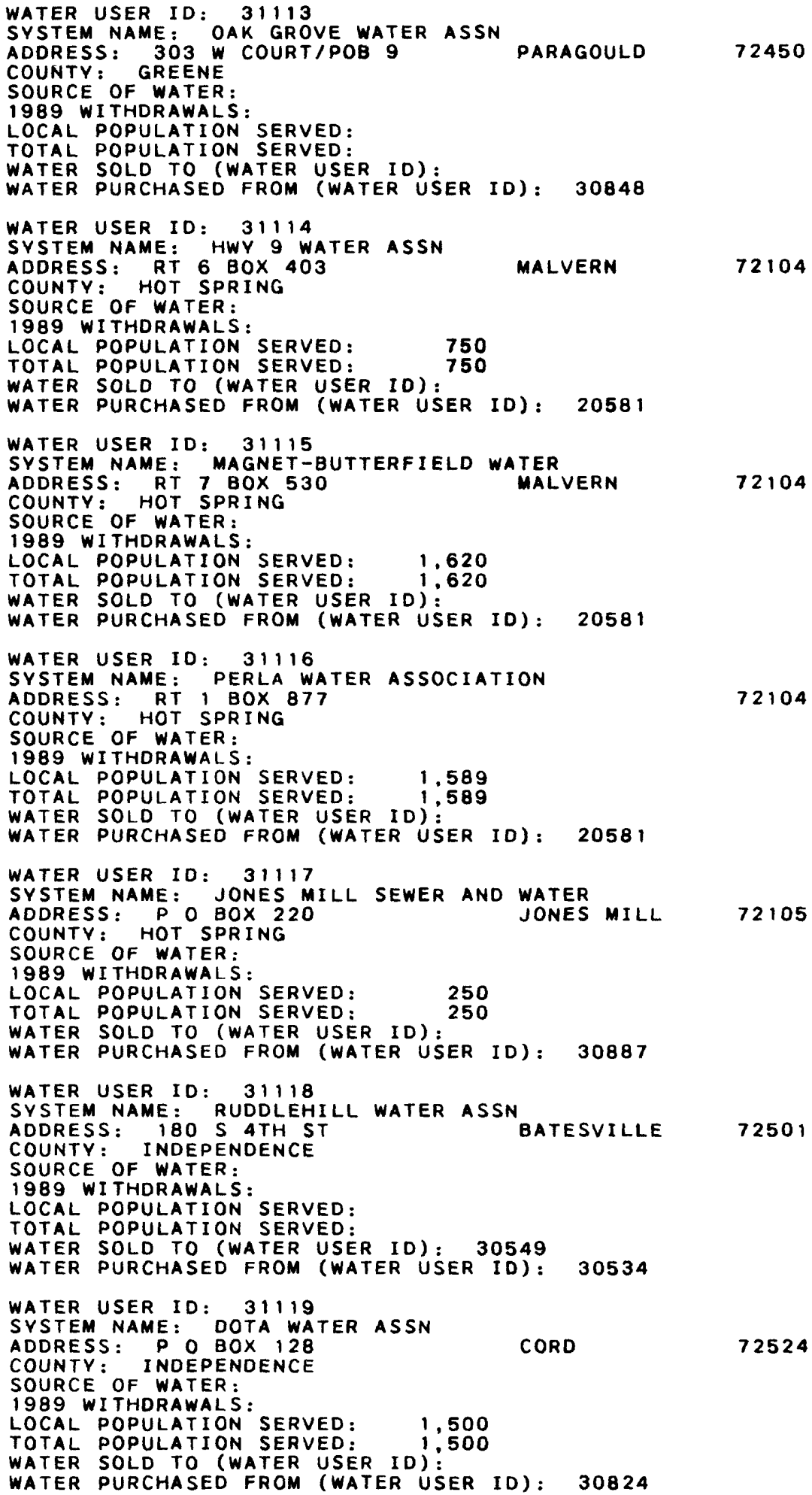


Table 1.--Description of public water supplies in Arkansas--Continued

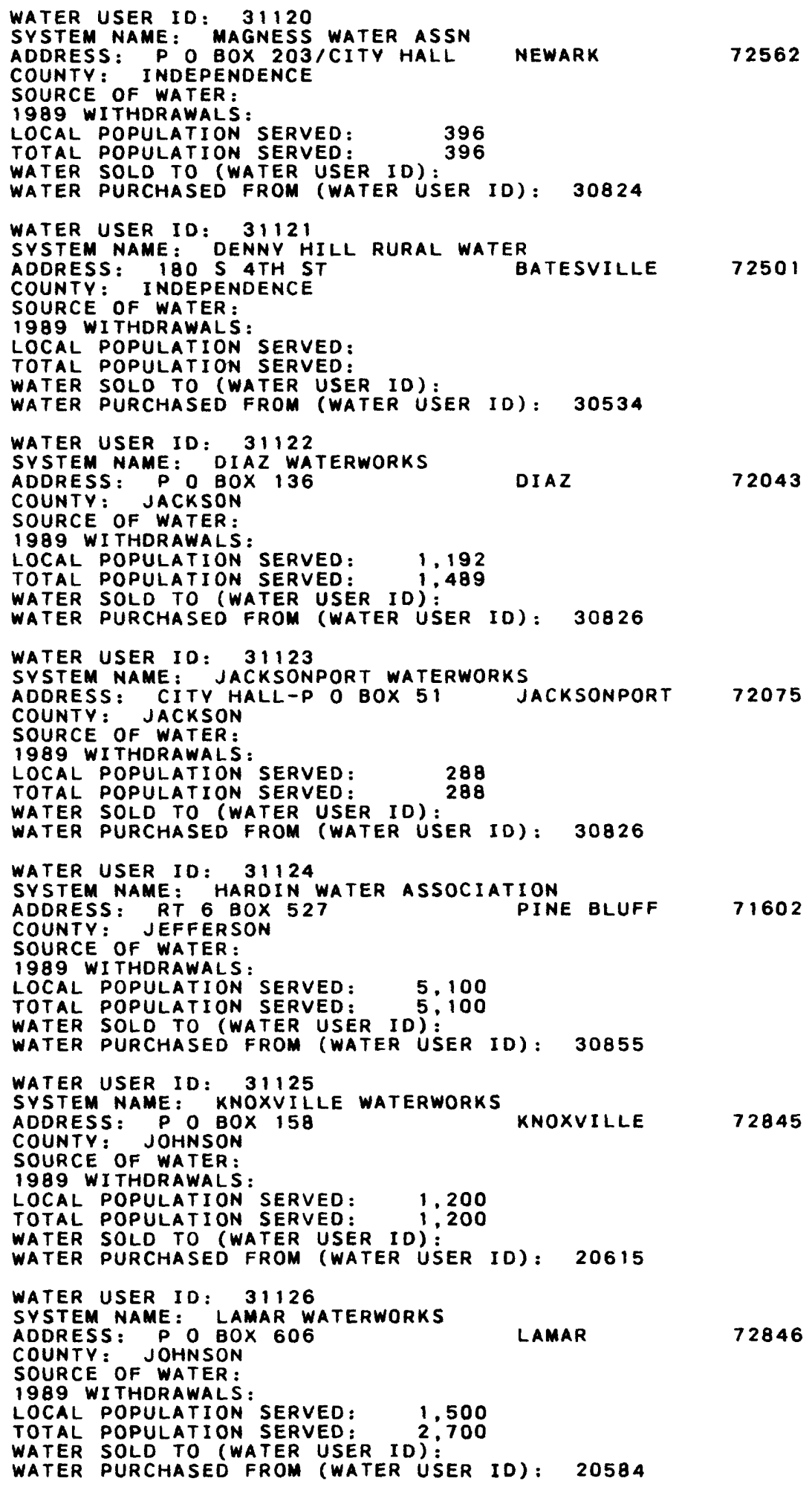


Table 1.--Description of public water supplies in Arkansas--Continued

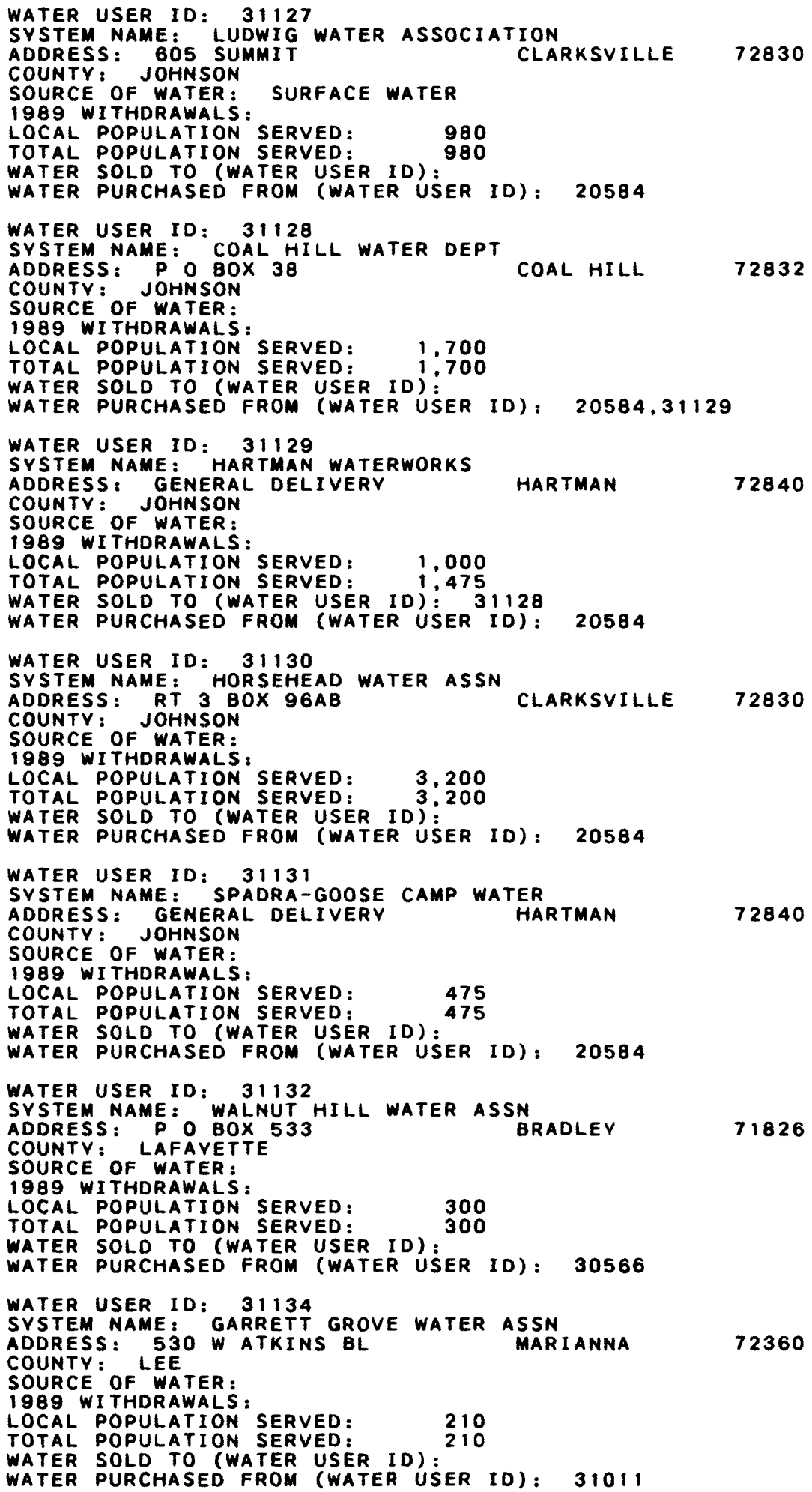


Table 1.--Description of public water supplies in Arkansas--Continued

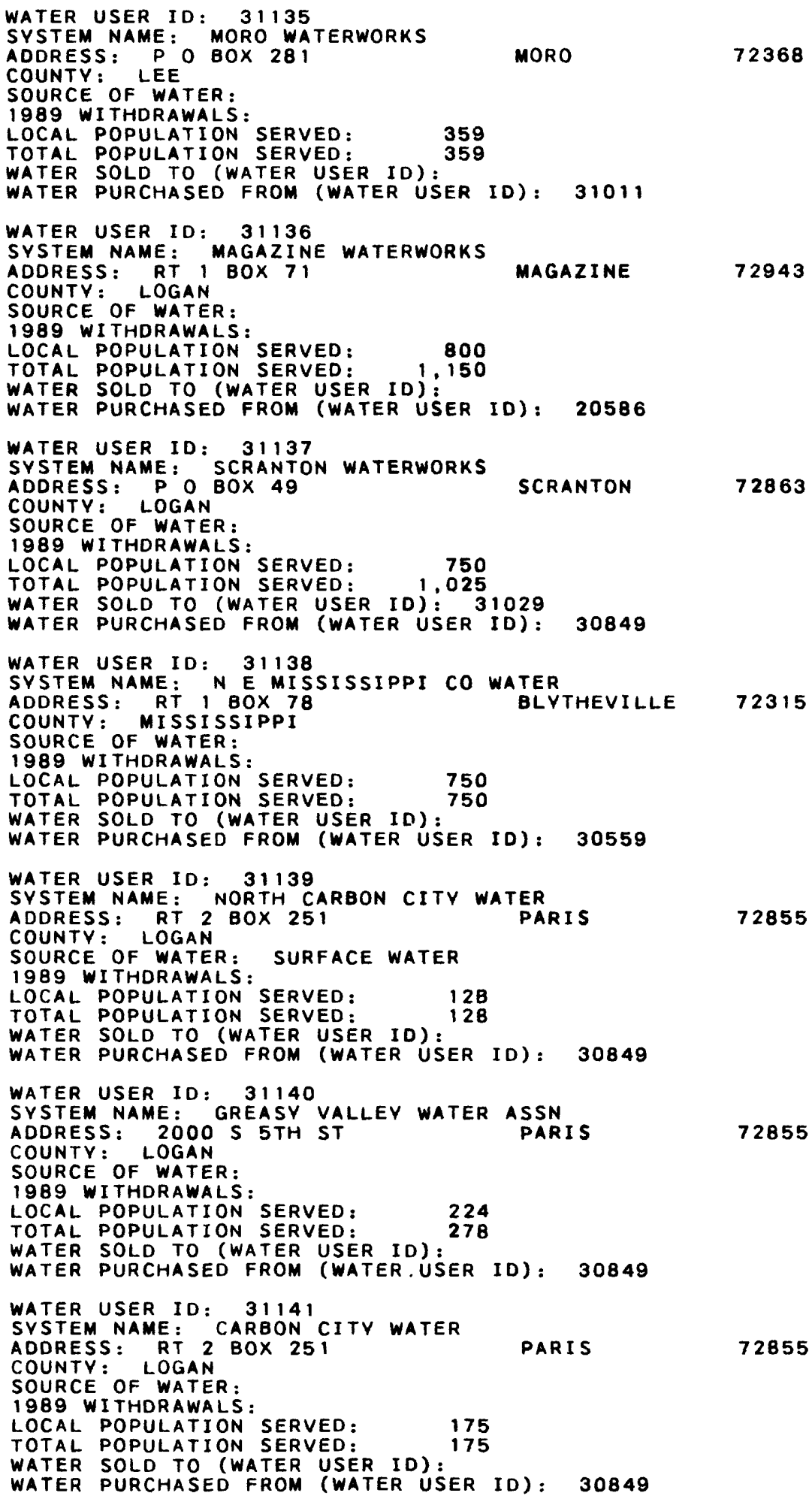


Table 1.--Description of public water supplies in Arkansas--Continued

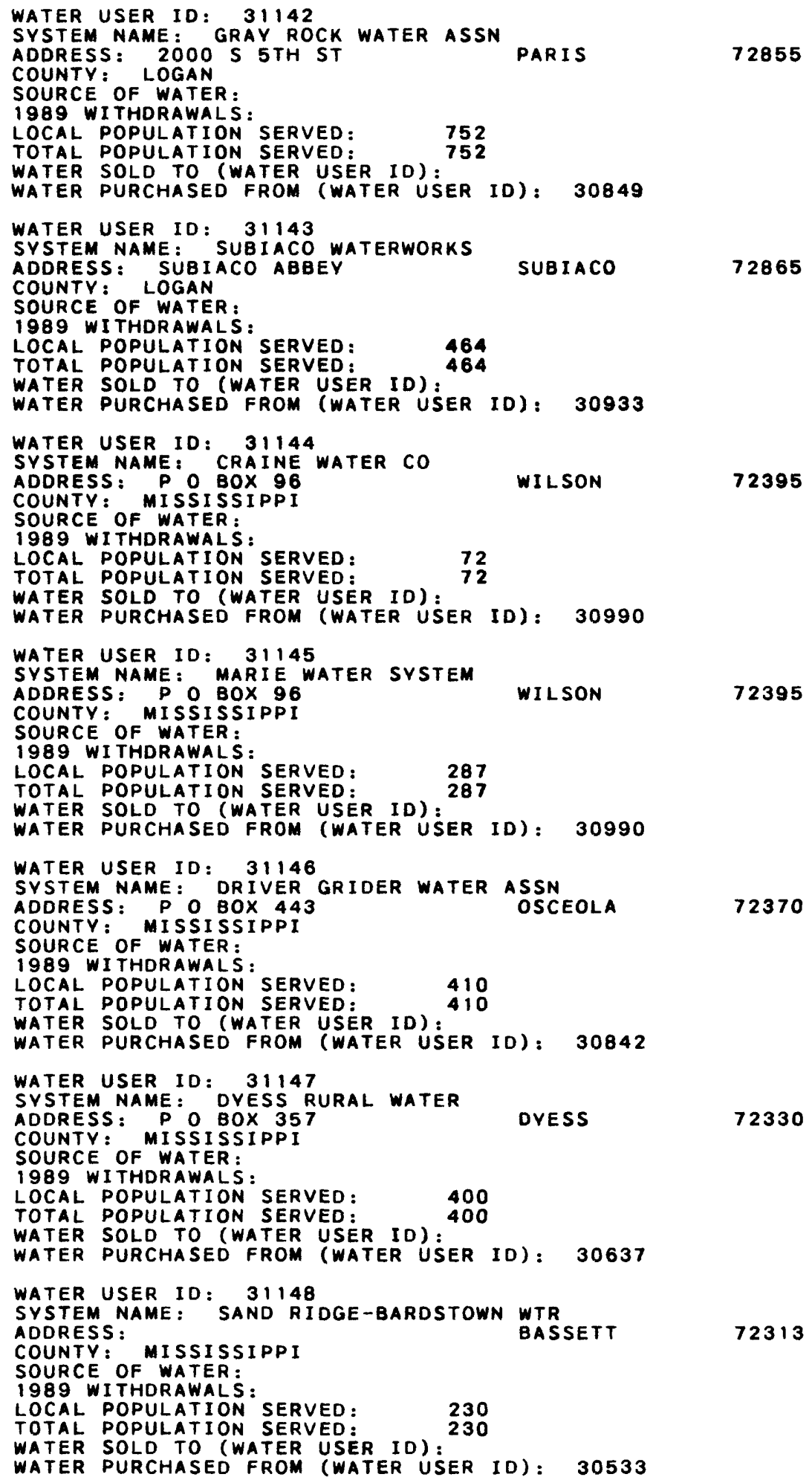


Table 1.--Description of public water supplies in Arkansas--Continued

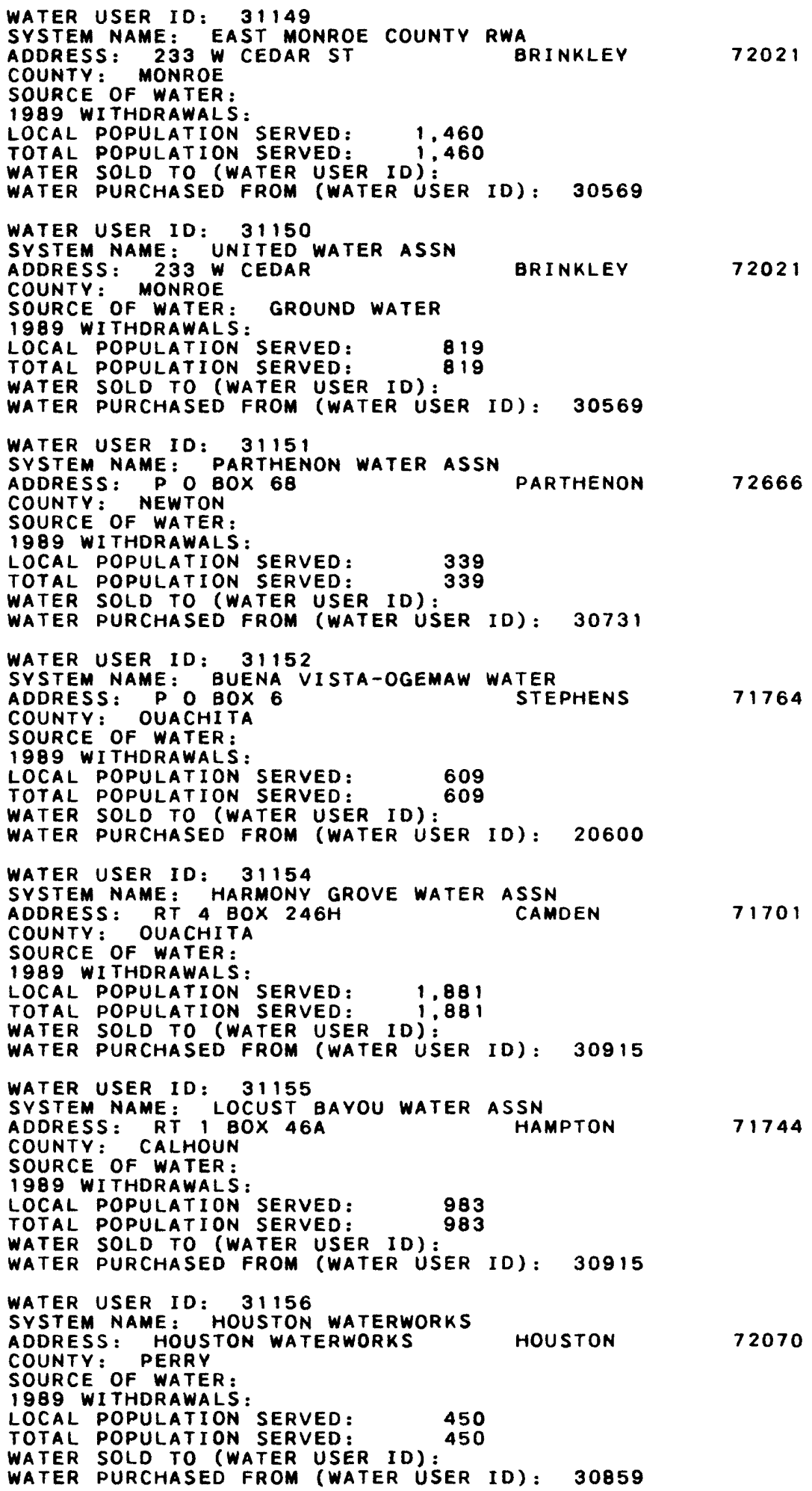


Table 1.--Description of public water supplies in Arkansas--Continued

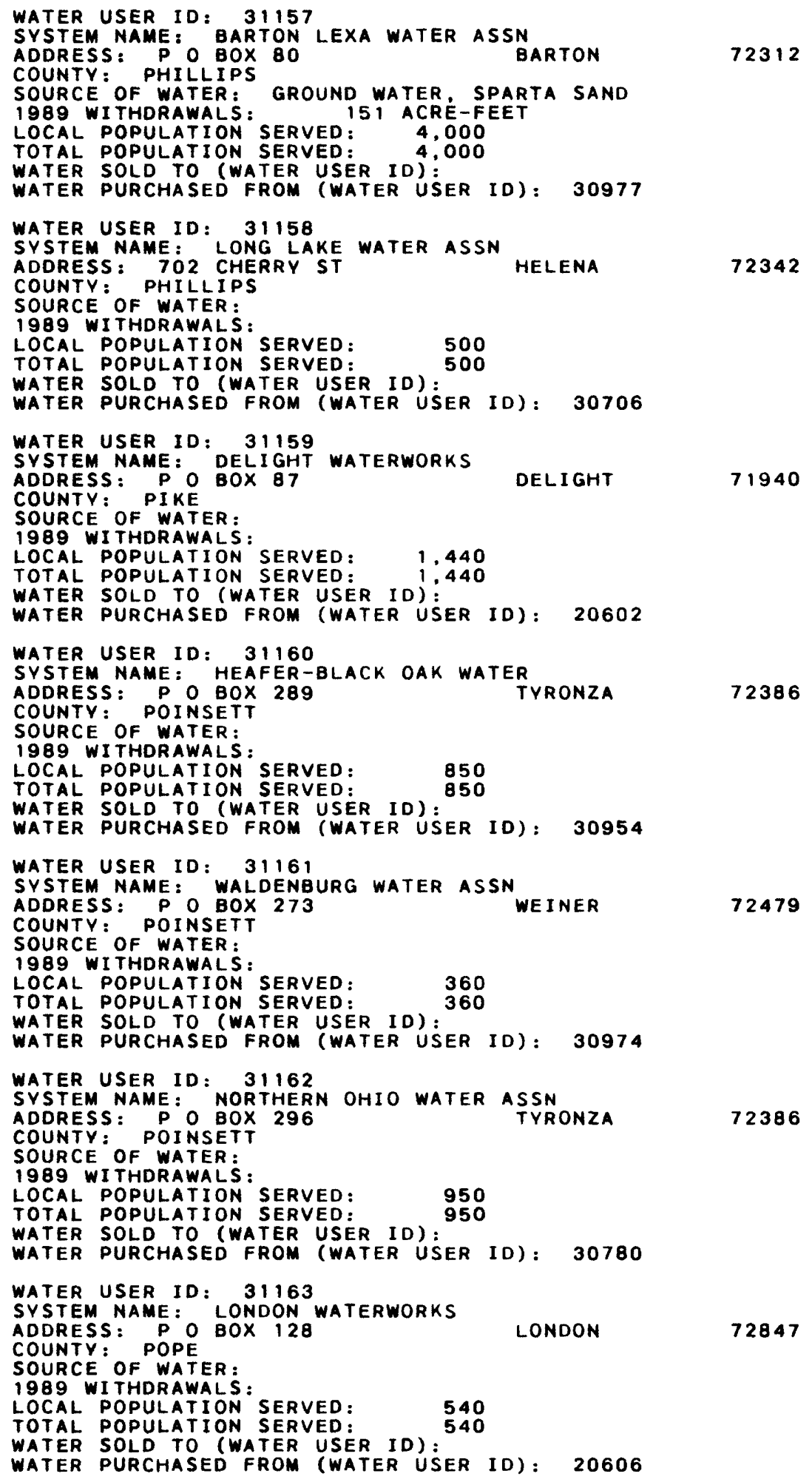


Table 1.--Description of public water supplies in Arkansas--Continued

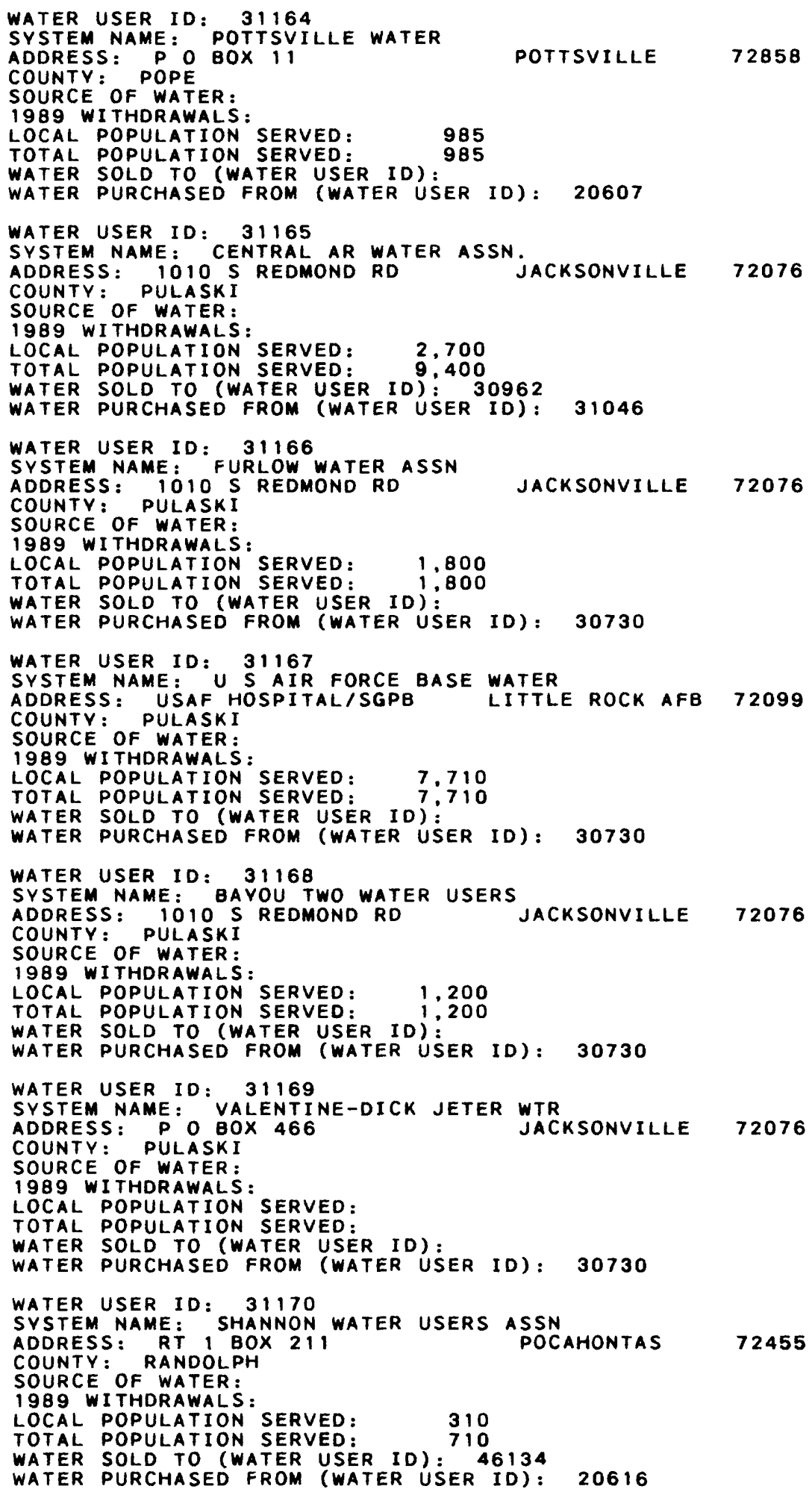


Table 1.--Description of public water supplies in Arkansas--Continued

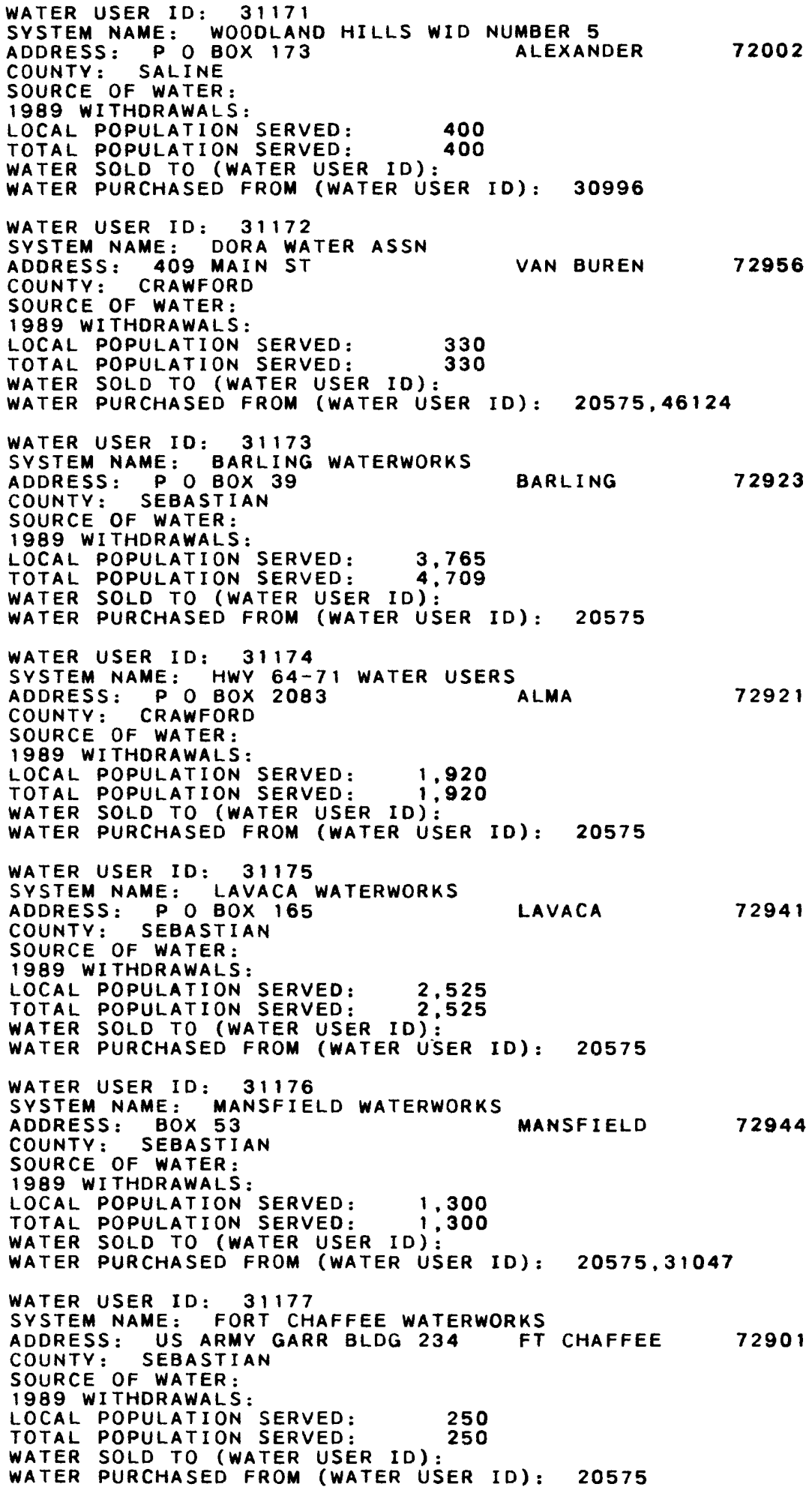

WATER SOLD TO (WATER USER ID)
WATER PURCHASED FROM (WATER USER ID): 20575 
Table 1.--Description of public water supplies in Arkansas--Continued

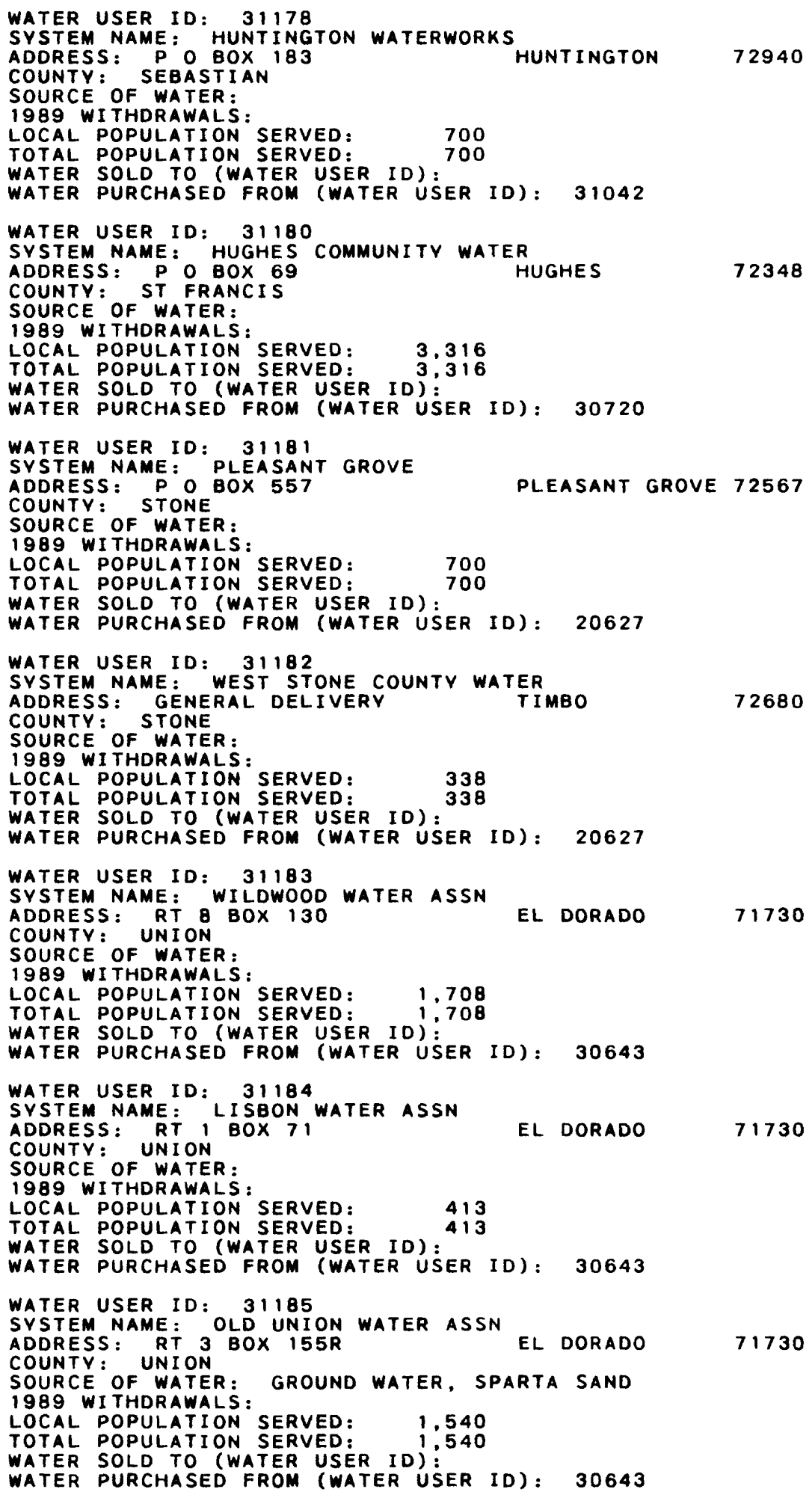


Table 1.--Description of public water supplies in Arkansas--Continued

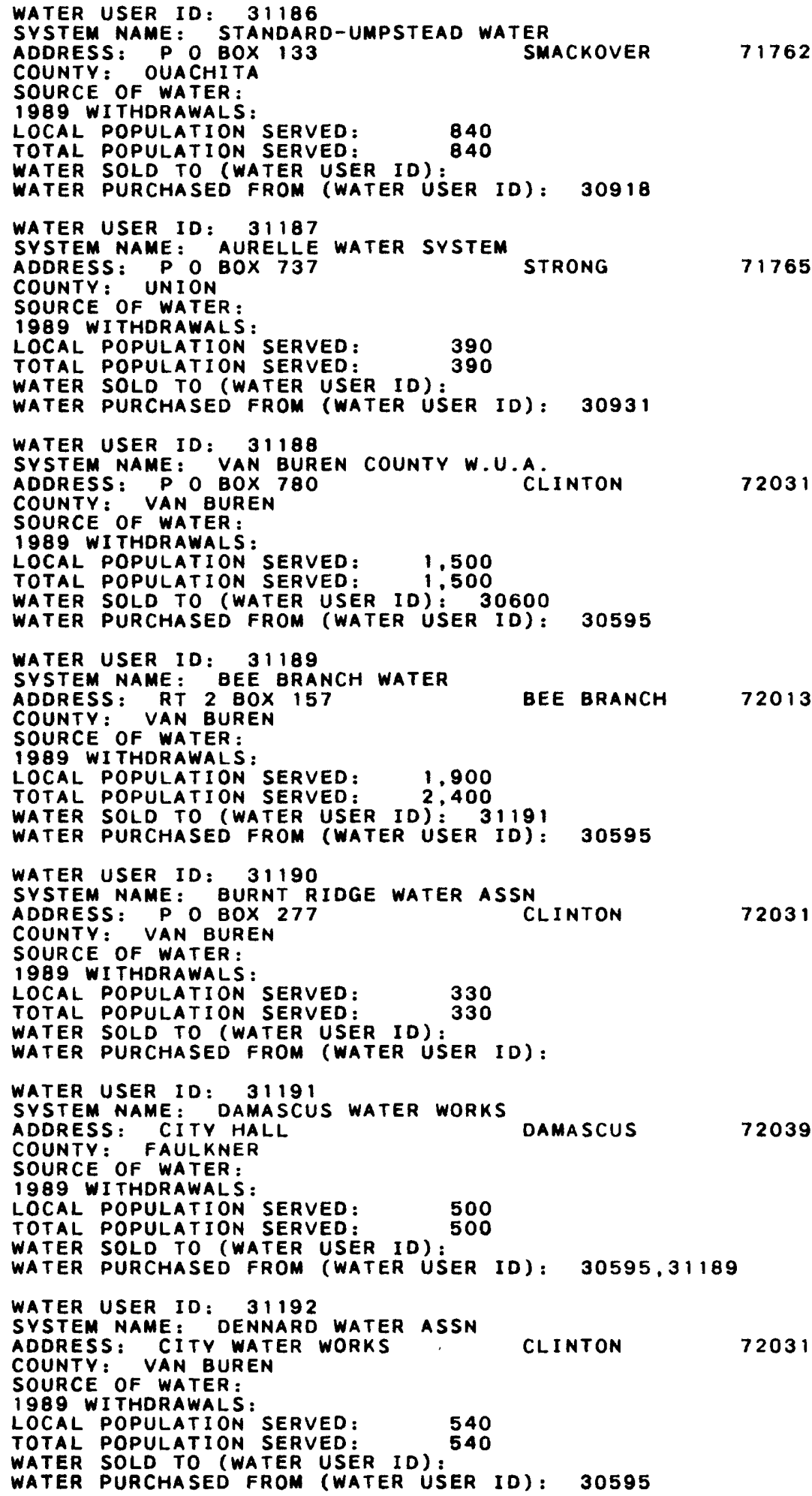


Table 1.--Description of public water supplies in Arkansas--Continued

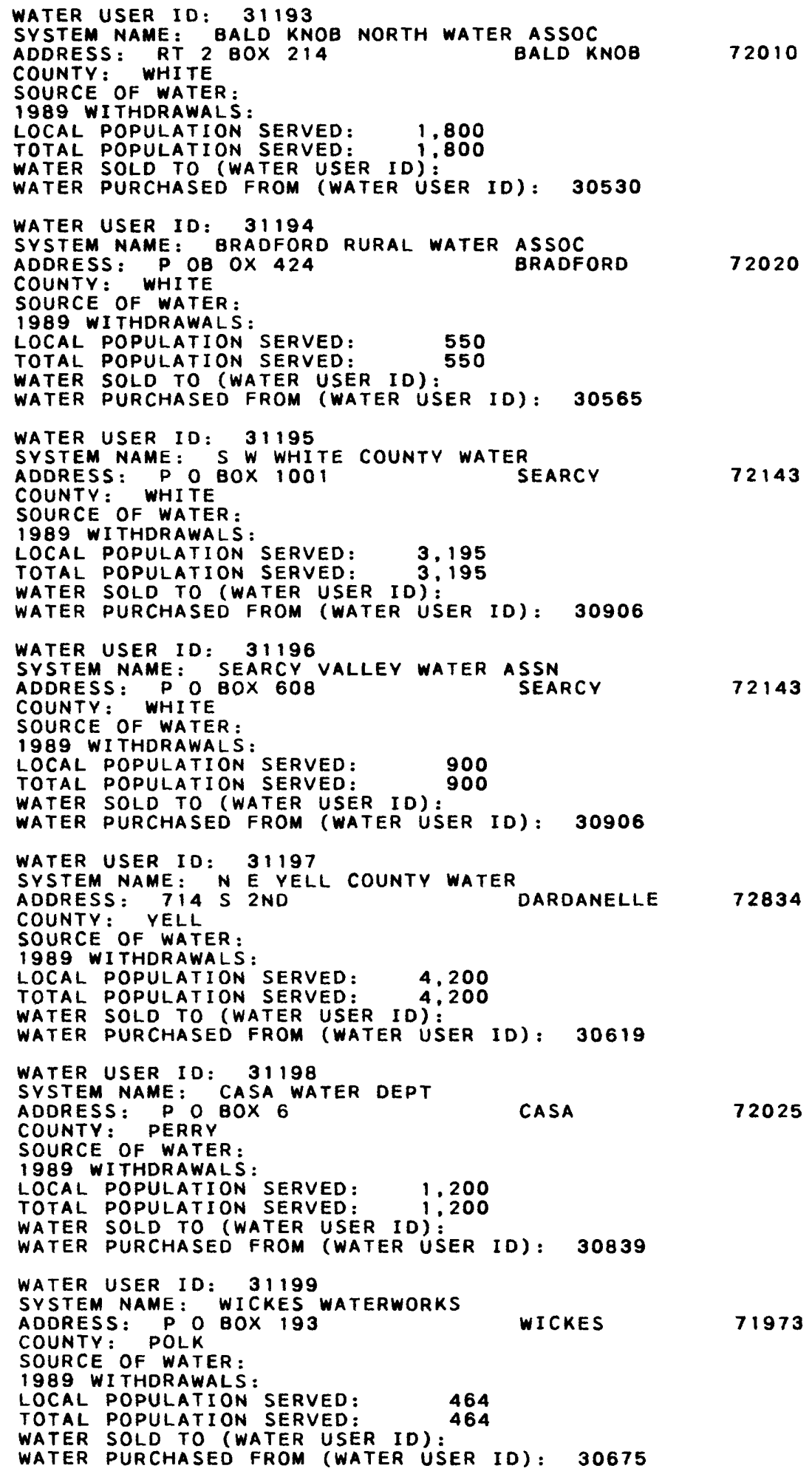


Table 1.--Description of public water supplies in Arkansas--Continued

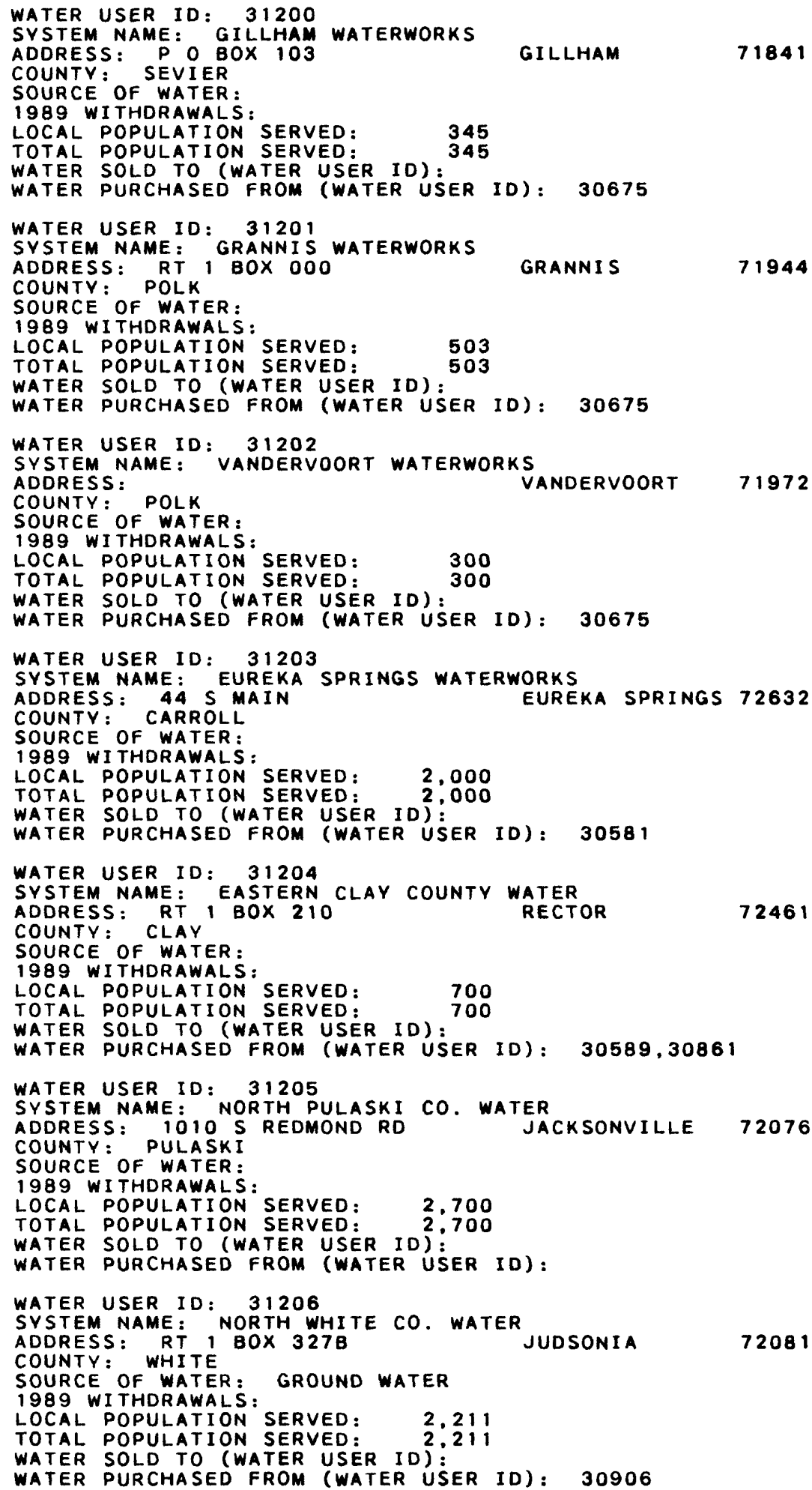


Table 1.--Description of public water supplies in Arkansas--Continued

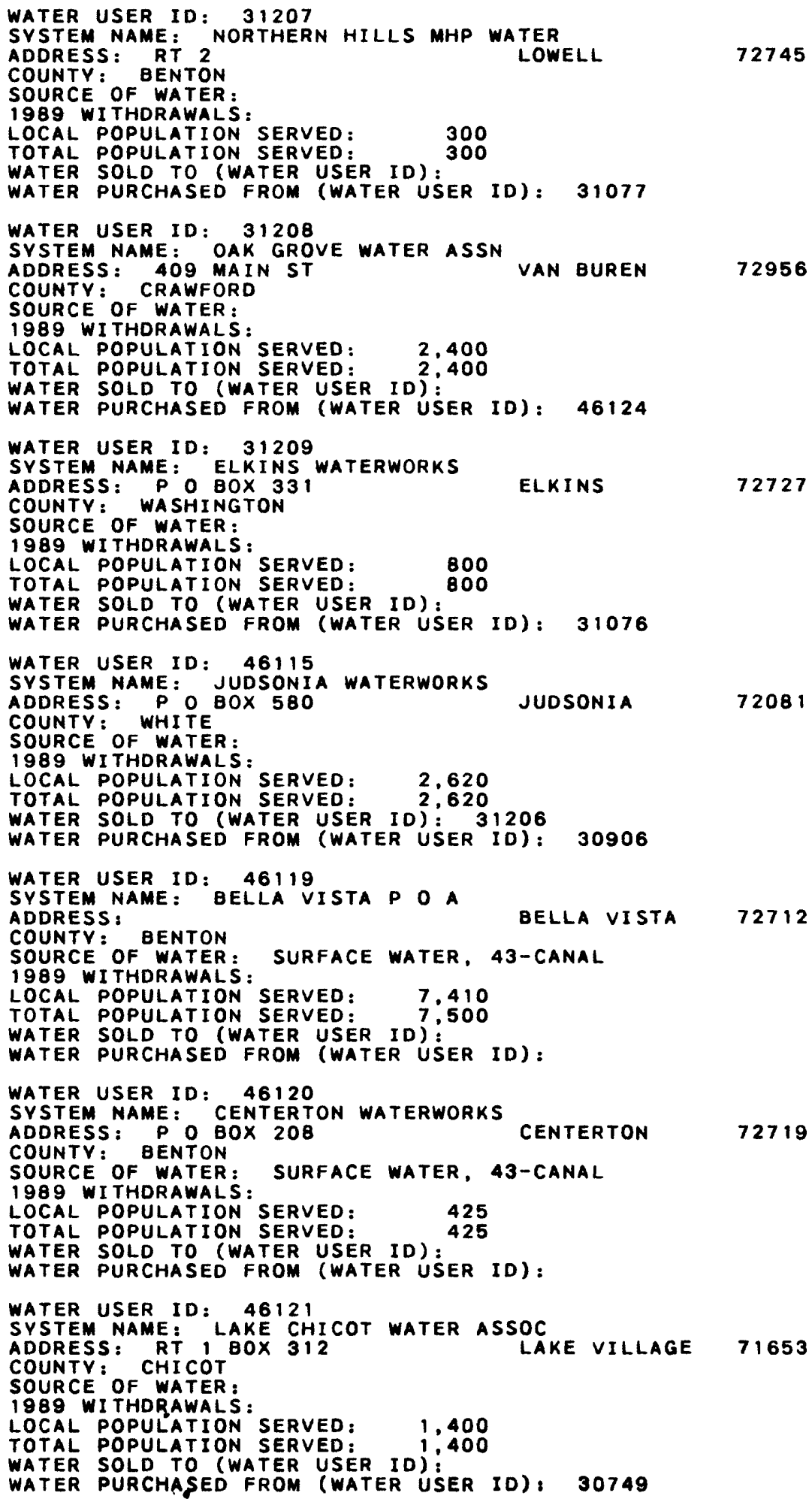


Table 1.--Description of public water supplies in Arkansas--Continued

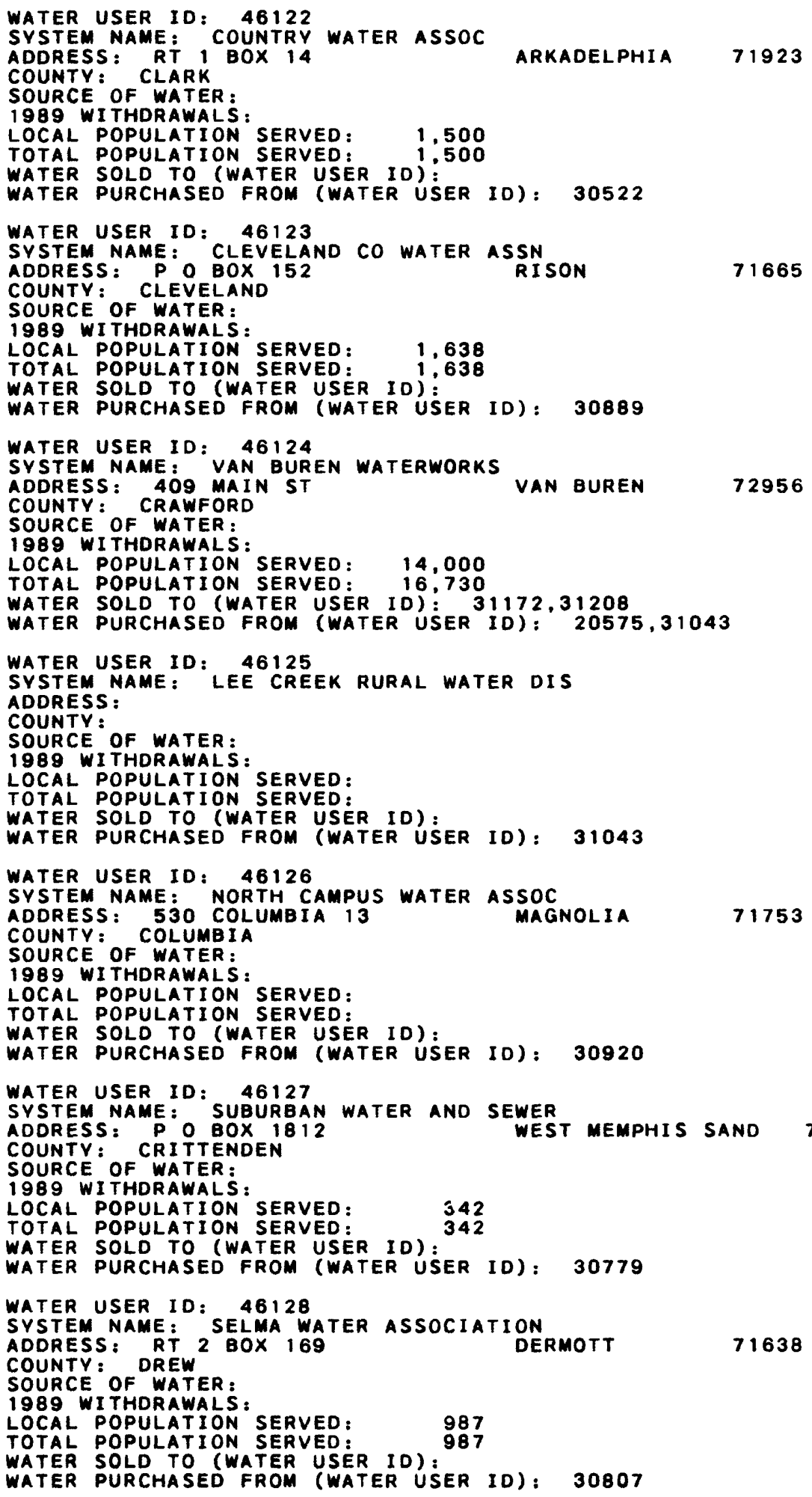


Table 1.--Description of public water supplies in Arkansas--Continued

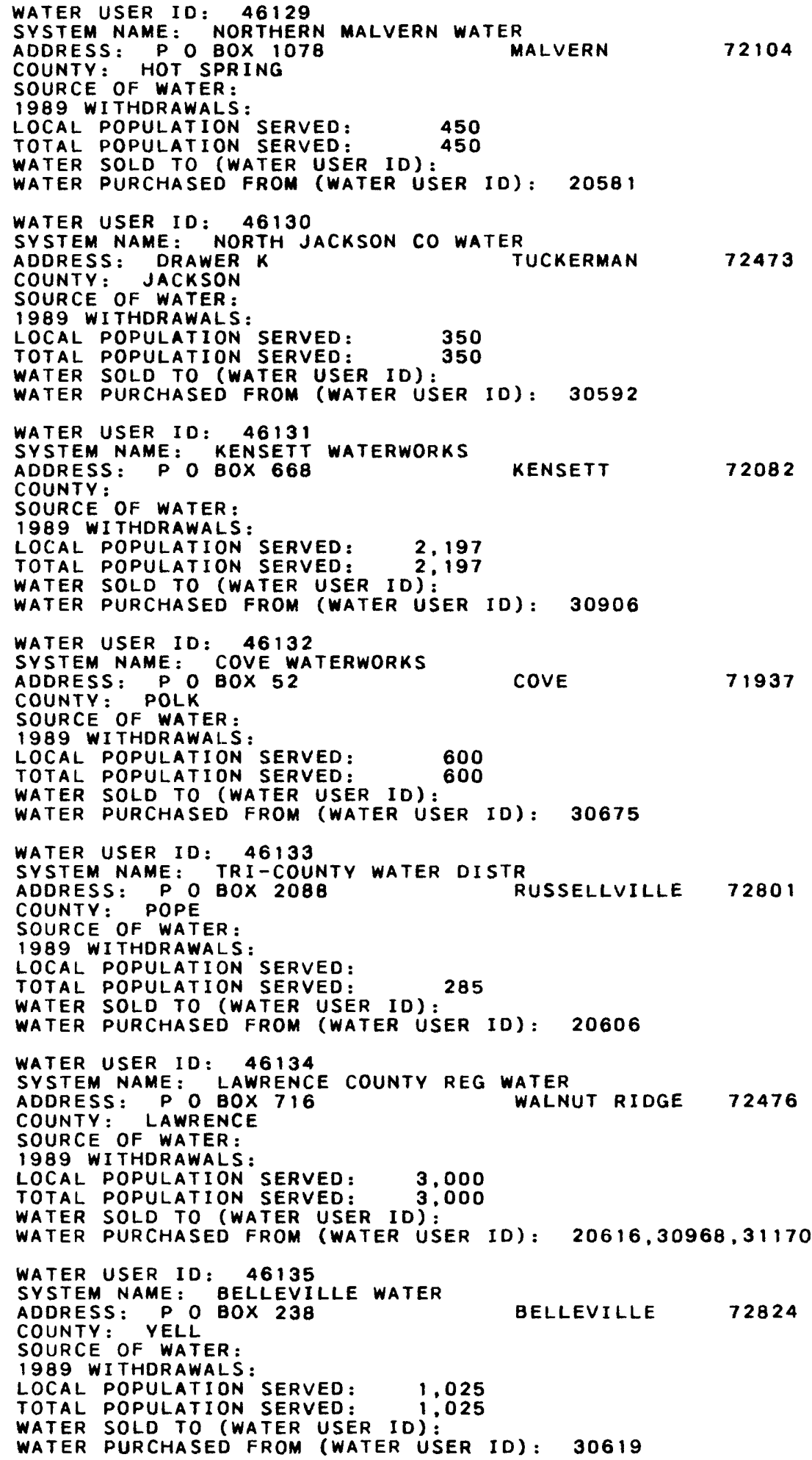


Table 1.--Description of public water supplies in Arkansas--Continued

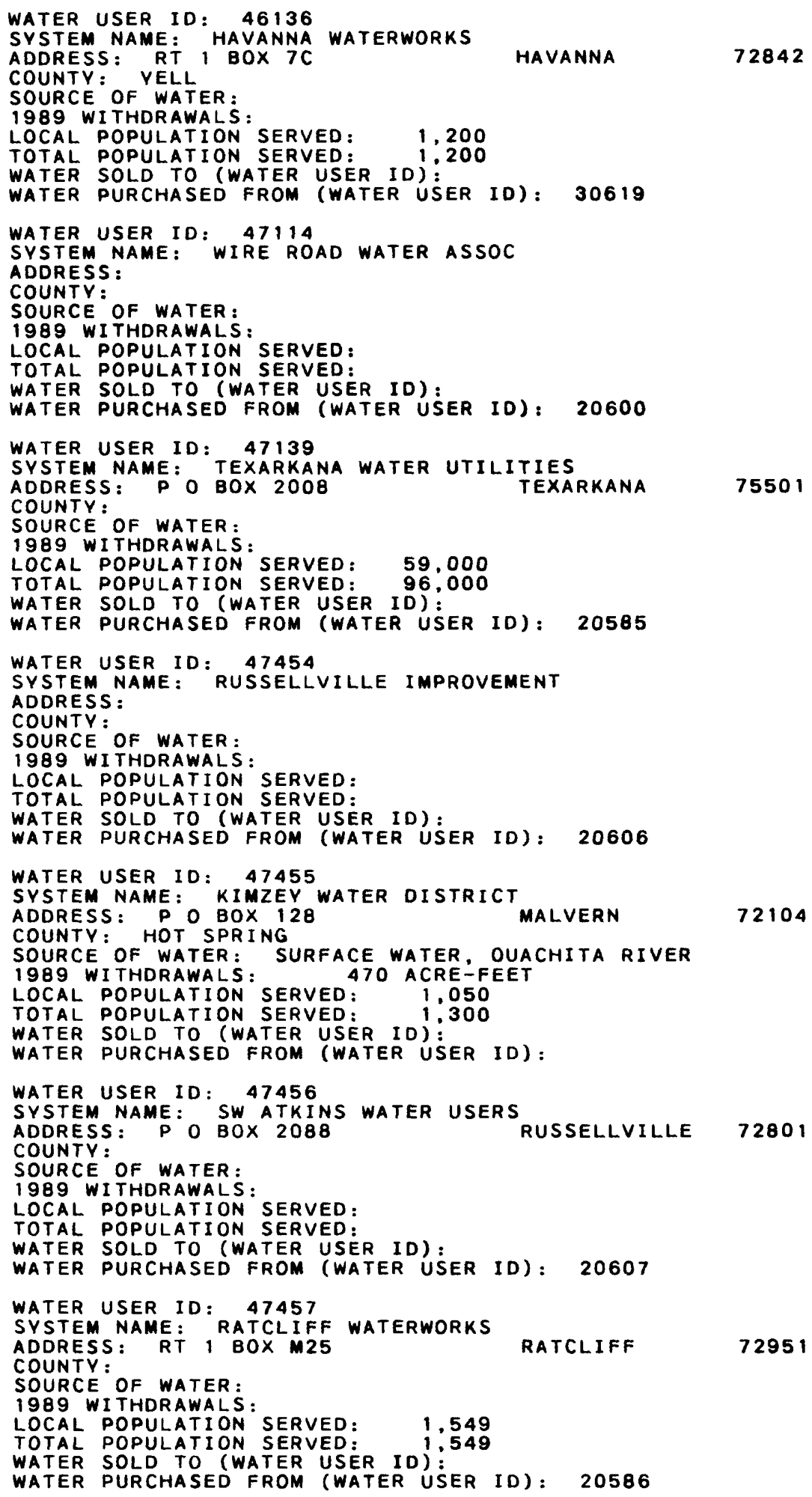


Table 1.--Description of public water supplies in Arkansas--Continued

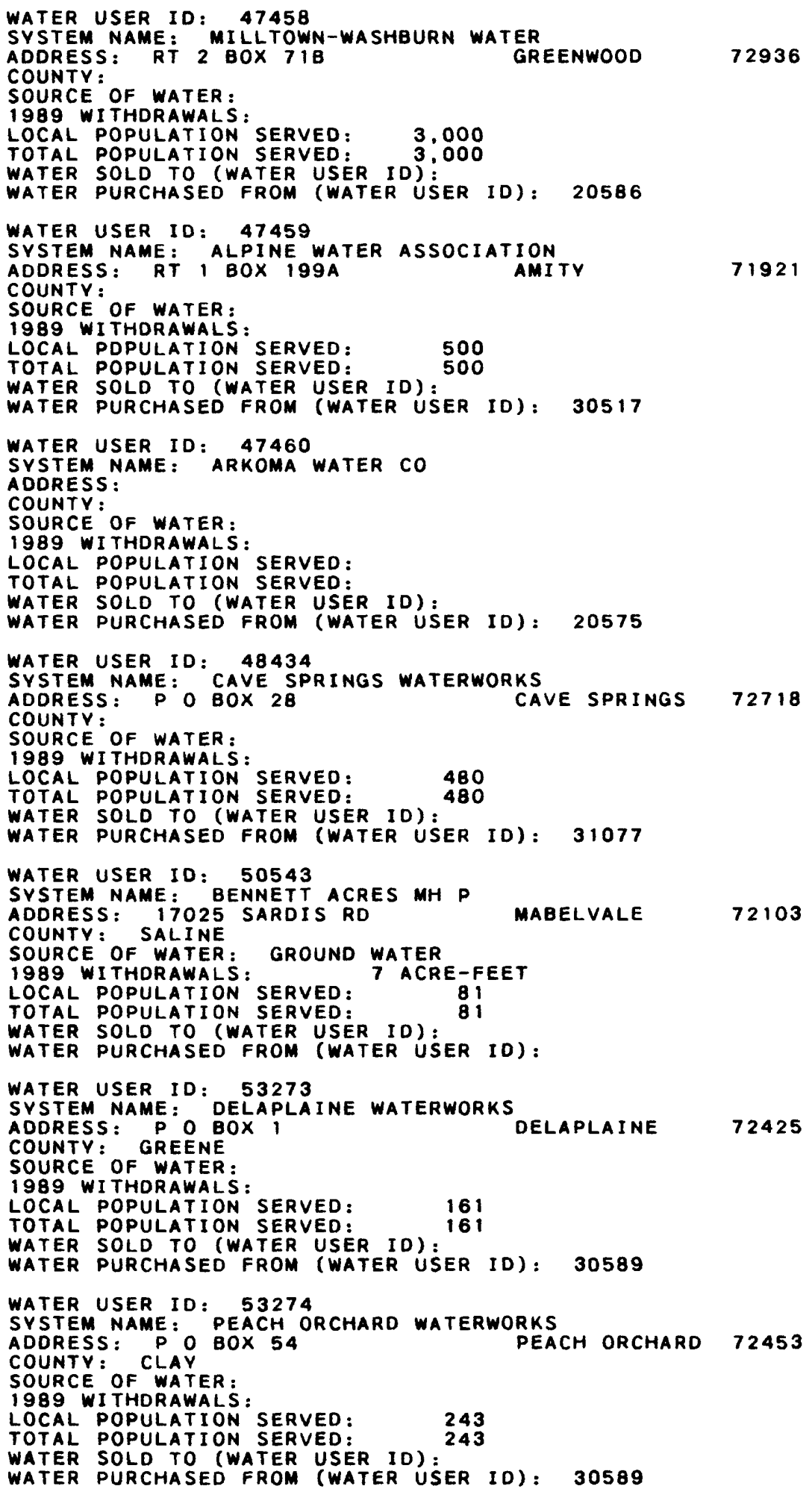


Table 1.--Description of public water supplies in Arkansas--Continued

WATER USER ID: 53275
SYSTEM NAME: WEST WOODRUFF WATER DIST

ADDRESS: $P$ O BOX 40

PATTERSON

72123

COUNTY: WOODRUFF

SOURCE OF WATER:

1989 WITHDRAWALS:

LOCAL POPULATION SERVED: 420

TOTAL POPULATION SERVED:

WATER SOLD TO (WATER USER ID):

WATER PURCHASED FROM (WATER USER ID): 30853

WATER USER ID: 53277

SYSTEM NAME: IZARD CO RURAL WATER ASSN

ADDRESS: P O BOX 278 MELBOURNE

COUNTY: IZARD

SOURCE OF WATER:

1989 WITHDRAWALS.

LOCAL POPULATION SERVED: 225

TOTAL POPULATION SERVED: 225

WATER SOLD TO (WATER USER ID)

WATER PURCHASED FROM (WATER USER ID): 30798

WATER USER ID: 53278

SYSTEM NAME: BARKADA WATER ASSOCIATION

ADDRESS: $P$ BOX 6 WILMAR

71675

COUNTY: OREW

IOB9 WI THDRAWALS

LOCAL POPULATION SERVED:

TOTAL POPULATION SERVED:

WATER SOLD TO (WATER USER ID)

WATER PURCHASED FROM (WATER USER IO): 30807 
Table 2.--General description of public-supply

[ELC, electricity; GRV, gravity; STP, stationary;

\begin{tabular}{|c|c|c|c|c|c|}
\hline $\begin{array}{c}\text { Water } \\
\text { user } \\
\text { I0 } \\
\end{array}$ & $\begin{array}{c}\text { Site } \\
\text { ID } \\
\end{array}$ & Water system name & $\begin{array}{r}\text { Lati- } \\
\text { tude } \\
\end{array}$ & $\begin{array}{c}\text { Longi- } \\
\text { tude }\end{array}$ & $\begin{array}{l}\text { Hydro- } \\
\text { logic } \\
\text { unit } \\
\text { code }\end{array}$ \\
\hline $\begin{array}{l}20555 \\
20556 \\
20558 \\
20559 \\
20559\end{array}$ & $\begin{array}{l}361500094070000 \\
361452094291400 \\
353006092025700 \\
351440092354000 \\
350652092312701\end{array}$ & $\begin{array}{l}\text { BEAVER WATER DISTRICT } \\
\text { GENTRY WATERWORKS } \\
\text { HEBER SPRINGS WATER AND SEWER } \\
\text { CONWAY WATER CO } \\
\text { CONWAY WATER CO }\end{array}$ & $\begin{array}{l}361500 \\
361452 \\
353006 \\
351440 \\
350652\end{array}$ & $\begin{array}{l}0940700 \\
0942914 \\
0920257 \\
0923540 \\
0923127\end{array}$ & $\begin{array}{l}11010001 \\
11110103 \\
11010014 \\
11110203 \\
11110203\end{array}$ \\
\hline $\begin{array}{l}20560 \\
20560 \\
20560 \\
20560 \\
20560\end{array}$ & $\begin{array}{l}354844090394501 \\
355019090375301 \\
354929090391801 \\
354929090392201 \\
355037090422701\end{array}$ & $\begin{array}{l}\text { JONESBORO WATER SYSTEM } \\
\text { JONESBORO WATER SYSTEM } \\
\text { JONESBORO WATER SYSTEM } \\
\text { JONESBORO WATER SYSTEM } \\
\text { JONESBORO WATER SYSTEM }\end{array}$ & $\begin{array}{l}354844 \\
355019 \\
354929 \\
354929 \\
355037\end{array}$ & $\begin{array}{l}0903945 \\
0903753 \\
0903918 \\
0903922 \\
0904227\end{array}$ & $\begin{array}{l}08020203 \\
08020203 \\
08020203 \\
08020203 \\
08020302\end{array}$ \\
\hline $\begin{array}{l}20560 \\
20560 \\
20560 \\
20560 \\
20560\end{array}$ & $\begin{array}{l}355041090422301 \\
355107090422801 \\
354839090403301 \\
354929090391802 \\
354929090392204\end{array}$ & $\begin{array}{l}\text { JONESBORO WATER SYSTEM } \\
\text { JONESBORO WATER SYSTEM } \\
\text { JONESBORO WATER SYSTEM } \\
\text { JONESBORO WATER SYSTEM } \\
\text { JONESBORO WATER SYSTEM. }\end{array}$ & $\begin{array}{c}355041 \\
355107 \\
354836 \\
-- \\
--\end{array}$ & $\begin{array}{c}0904223 \\
0904228 \\
0903953 \\
-- \\
--\end{array}$ & $\begin{array}{l}08020302 \\
08020302 \\
08020203 \\
08020203 \\
08020203\end{array}$ \\
\hline $\begin{array}{l}20560 \\
20560 \\
20560 \\
20560 \\
20560\end{array}$ & $\begin{array}{l}355037090422702 \\
355116090422901 \\
354915090391501 \\
354915090391502 \\
354915090391503\end{array}$ & $\begin{array}{l}\text { JONESBORO WATER SYSTEM } \\
\text { JONESBORO WATER SYSTEM } \\
\text { JONESBORO WATER SYSTEM } \\
\text { JONESBORO WATER SYSTEM } \\
\text { JONESBORO WATER SYSTEM }\end{array}$ & $\begin{array}{c}-- \\
355116 \\
-- \\
-- \\
--\end{array}$ & $\begin{array}{c}-- \\
0904229 \\
-- \\
-- \\
--\end{array}$ & $\begin{array}{l}08020302 \\
08020302 \\
08020302 \\
08020302 \\
08020302\end{array}$ \\
\hline $\begin{array}{l}20573 \\
20574 \\
20575 \\
20580 \\
20580\end{array}$ & $\begin{array}{r}353213094042000 \\
352950094131701 \\
3540150940715 \\
334008093361701 \\
334007093355601\end{array}$ & $\begin{array}{l}\text { MULBERRY WATERWORKS } \\
\text { ALMA WATERWORKS } \\
\text { FORT SWITH WATERWORKS } \\
\text { HOPE WATERWORKS } \\
\text { HOPE WATERWORKS }\end{array}$ & $\begin{array}{l}353213 \\
352950 \\
354015 \\
334008 \\
334007\end{array}$ & $\begin{array}{l}0940420 \\
0941317 \\
0940715 \\
0933617 \\
0933556\end{array}$ & $\begin{array}{l}11110201 \\
11110201 \\
11110104 \\
11140201 \\
11140201\end{array}$ \\
\hline $\begin{array}{l}20580 \\
20580 \\
20580 \\
20580 \\
20580\end{array}$ & $\begin{array}{l}334011093353701 \\
334358093370101 \\
334336093380601 \\
334403093364601 \\
334405093363801\end{array}$ & $\begin{array}{l}\text { HOPE WATERWORKS } \\
\text { HOPE WATERWORKS } \\
\text { HOPE WATERWORKS } \\
\text { HOPE WATERWORKS } \\
\text { HOPE WATERWORKS }\end{array}$ & $\begin{array}{l}334011 \\
334410 \\
334336 \\
334403 \\
334407\end{array}$ & $\begin{array}{l}0933537 \\
0933820 \\
0933806 \\
0933646 \\
0933635\end{array}$ & $\begin{array}{l}11140201 \\
11140201 \\
11140201 \\
11140201 \\
11140201\end{array}$ \\
\hline $\begin{array}{l}20580 \\
20580 \\
20580 \\
20580 \\
20581\end{array}$ & $\begin{array}{r}334412093362001 \\
334331093381901 \\
334337093380001 \\
3337150935145 \\
341718092441501\end{array}$ & $\begin{array}{l}\text { HOPE WATERWORKS } \\
\text { HOPE WATERWORKS } \\
\text { HOPE WATERWORKS } \\
\text { HOPE WATERWORKS } \\
\text { MALVERN WATERWORKS }\end{array}$ & $\begin{array}{l}334413 \\
334331 \\
334341 \\
333720 \\
341718\end{array}$ & $\begin{array}{l}0933615 \\
0933819 \\
0933902 \\
0935135 \\
0924415\end{array}$ & $\begin{array}{l}11140201 \\
11140201 \\
11140201 \\
11140201 \\
08040102\end{array}$ \\
\hline $\begin{array}{l}20581 \\
20584 \\
20585 \\
20585 \\
20585\end{array}$ & $\begin{array}{r}342045092492401 \\
3529000932730 \\
334255094013301 \\
334255094013302 \\
334255094013303\end{array}$ & $\begin{array}{l}\text { MALVERN WATERWORKS } \\
\text { CLARKSVILLE WATERWORKS } \\
\text { SW ARKANSAS WATER DISTRICT } \\
\text { SW ARKANSAS WATER DISTRICT } \\
\text { SW ARKANSAS WATER DISTRICT }\end{array}$ & $\begin{array}{c}342045 \\
352900 \\
334255 \\
-- \\
--\end{array}$ & $\begin{array}{c}0924924 \\
0932730 \\
0940133 \\
-- \\
--\end{array}$ & $\begin{array}{l}08040102 \\
11110202 \\
11140109 \\
11140109 \\
11140109\end{array}$ \\
\hline $\begin{array}{l}20586 \\
20587 \\
20587 \\
20589 \\
20597\end{array}$ & $\begin{array}{r}3509000940345 \\
3505150932545 \\
35053009350301 \\
361445092412401 \\
335110093180501\end{array}$ & $\begin{array}{l}\text { BOONEVILLE WATERWORKS } \\
\text { HUMAN DEVELOPMENT CENTER } \\
\text { HUMAN DEVELOPMENT CENTER } \\
\text { YELLVILLE WATERWORKS } \\
\text { PRESCOTT WATERWORKS }\end{array}$ & $\begin{array}{l}350900 \\
350515 \\
350530 \\
361445 \\
335110\end{array}$ & $\begin{array}{l}0940345 \\
0932545 \\
093503 \\
0924124 \\
0931805\end{array}$ & $\begin{array}{l}11110204 \\
11110204 \\
11110204 \\
11010003 \\
08040103\end{array}$ \\
\hline $\begin{array}{l}20599 \\
20600 \\
20602 \\
20605 \\
20606\end{array}$ & $\begin{array}{r}3549450931445 \\
333523092492000 \\
340512093422401 \\
343526094145000 \\
352020093085001\end{array}$ & $\begin{array}{l}\text { DEER WATER ASSOC } \\
\text { CAMDEN WATERWORKS } \\
\text { MURFREESBORO WATERWORKS } \\
\text { MENA WATER DEPT } \\
\text { RUSSELLVILLE WATERWORKS }\end{array}$ & $\begin{array}{l}354945 \\
333552 \\
340512 \\
343526 \\
352020\end{array}$ & $\begin{array}{l}0931445 \\
0924918 \\
0934224 \\
0941450 \\
0930850\end{array}$ & $\begin{array}{l}11110202 \\
08040102 \\
08040103 \\
08040101 \\
11110202\end{array}$ \\
\hline $\begin{array}{l}20606 \\
20607 \\
20608 \\
20609 \\
20615\end{array}$ & $\begin{array}{r}352018093085101 \\
351456092570001 \\
3528150925830 \\
345838091295001 \\
344755092504300\end{array}$ & $\begin{array}{l}\text { RUSSELLVILLE WATERWORKS } \\
\text { ATKINS WATERWORKS } \\
\text { HECTOR WATERWORKS } \\
\text { DES ARC WATERWORKS } \\
\text { LITTLE ROCK WUNICIPAL WATERWORKS }\end{array}$ & $\begin{array}{l}352018 \\
351456 \\
352815 \\
345838 \\
344755\end{array}$ & $\begin{array}{l}0930851 \\
0925700 \\
0925830 \\
0913115 \\
0925043\end{array}$ & $\begin{array}{l}11110202 \\
11110203 \\
11110202 \\
08020301 \\
08040203\end{array}$ \\
\hline $\begin{array}{l}20615 \\
20616 \\
20622 \\
20624 \\
20626\end{array}$ & $\begin{array}{l}345119092291901 \\
361534090580601 \\
355630094030000 \\
340245094124601 \\
361900091290001\end{array}$ & $\begin{array}{l}\text { LITTLE ROCK MUNICIPAL WATERWORKS } \\
\text { POCAHONTAS WAIERWORKS } \\
\text { WALDRON WATERWORKS } \\
\text { OEQUEEN WATERWORKS } \\
\text { HARDY WATERWORKS }\end{array}$ & $\begin{array}{c}345119 \\
361534 \\
355630 \\
340245\end{array}$ & $\begin{array}{l}0922919 \\
0905806 \\
0940300 \\
0941246\end{array}$ & $\begin{array}{l}11110207 \\
11010009 \\
11110105 \\
11140109 \\
11010010\end{array}$ \\
\hline
\end{tabular}


withdrawal sites in Arkansas

OTH, other: --, no data available]

\begin{tabular}{|c|c|c|c|c|c|c|c|}
\hline $\begin{array}{c}\text { Source } \\
\text { type }\end{array}$ & Source & $\begin{array}{l}\text { Pump } \\
\text { Horse- } \\
\text { power }\end{array}$ & $\begin{array}{l}\text { lescriot } \\
\text { Power } \\
\text { source }\end{array}$ & $\begin{array}{l}\text { Pon } \\
\text { Pump } \\
\text { tyoe }\end{array}$ & $\begin{array}{r}\text { Well } \\
\text { dia- } \\
\text { meter } \\
\text { Cinches }\end{array}$ & $\begin{array}{c}\text { Depth } \\
\text { of } \\
\text { well } 1 \\
\text { (feet) }\end{array}$ & Lecal descriotive ID \\
\hline $\begin{array}{l}\text { SURFACE } \\
\text { SURFACE } \\
\text { SURFACE } \\
\text { SURFACE } \\
\text { SURFACE }\end{array}$ & $\begin{array}{l}\text { BEAVER RES } \\
\text { FLINT CREEK } \\
\text { GREER'S FERRY } \\
\text { BREWER LAKE } \\
\text { CADRON CREEK }\end{array}$ & $\begin{array}{l}650 \\
25 \\
100 \\
125 \\
75\end{array}$ & $\begin{array}{l}\text { ELC } \\
\text { ELC } \\
\text { ELC } \\
\text { ELC } \\
\text { ELC }\end{array}$ & $\begin{array}{l}\text { STP } \\
\text { STP } \\
-- \\
\text { STP } \\
\text { STP }\end{array}$ & $\begin{array}{l}-- \\
-- \\
-- \\
36 \\
16\end{array}$ & $\begin{array}{l}-- \\
-- \\
-- \\
--\end{array}$ & $\begin{array}{lc} & -- \\
& -- \\
\text { 1ST } & -- \\
\text { 2NDTAKE } & \\
\text { INTAKE / IDLE }\end{array}$ \\
\hline $\begin{array}{l}\text { GROUND } \\
\text { GROUND } \\
\text { GROUND } \\
\text { GROUND } \\
\text { GROUND }\end{array}$ & $\begin{array}{l}\text { CLAIBORNE GROUP } \\
\text { MEMPHIS AQUIFER } \\
\text { QUATERNARY ALLUVIUM } \\
\text { MEMPHIS AQUIFER } \\
\text { CLAIBORNE GROUP }\end{array}$ & $\begin{array}{l}60 \\
50 \\
40 \\
60 \\
50\end{array}$ & $\begin{array}{l}\text { ELC } \\
\text { ELC } \\
\text { ELC } \\
\text { ELC } \\
\text { ELC }\end{array}$ & $\begin{array}{l}\text { STP } \\
\text { STP } \\
\text { STP } \\
\text { STP } \\
\text { STP }\end{array}$ & $\begin{array}{r}10 \\
10 \\
8 \\
8 \\
8\end{array}$ & $\begin{array}{l}218 \\
244 \\
216 \\
240 \\
132\end{array}$ & $\begin{array}{l}\text { RACE NO.1 } \\
\text { RACE NO.3 } \\
\text { AIRPORT NO.1 } \\
\text { AIRPORT NO.3 } \\
\text { JOHNSON NO.1 }\end{array}$ \\
\hline $\begin{array}{l}\text { GROUND } \\
\text { GROUND } \\
\text { GROUND } \\
\text { GROUND } \\
\text { GROUND }\end{array}$ & $\begin{array}{l}\text { QUATERNARY ALLUVIUM } \\
\text { CLAIBORNE GROUP } \\
\text { MEMPHIS AQUIFER } \\
--- \\
--\end{array}$ & $\begin{array}{l}20 \\
50 \\
60 \\
40 \\
50\end{array}$ & $\begin{array}{l}\text { ELC } \\
\text { ELC } \\
\text { ELC } \\
\text { ELC } \\
\text { ELC }\end{array}$ & $\begin{array}{l}\text { STP } \\
\text { STP } \\
\text { STP } \\
\text { STP } \\
\text { STP }\end{array}$ & $\begin{array}{r}6 \\
8 \\
10 \\
8 \\
10\end{array}$ & $\begin{array}{l}176 \\
218 \\
210 \\
214 \\
211\end{array}$ & $\begin{array}{l}\text { ABANDONED/JOHNSON NO.5 } \\
\text { JOHNSON NO.3 } \\
\text { RACE NO. } 2 \\
\text { AIRPORT NO. } 2 \\
\text { AIRPORT NO.4 }\end{array}$ \\
\hline $\begin{array}{l}\text { GROUND } \\
\text { GROUND } \\
\text { GROUND } \\
\text { GROUND } \\
\text { GROUND }\end{array}$ & $\begin{array}{c}-- \\
\text { CLAIBORNE GROUP } \\
-- \\
-- \\
--\end{array}$ & $\begin{array}{l}30 \\
50 \\
60 \\
60 \\
60\end{array}$ & $\begin{array}{l}\text { ELC } \\
\text { ELC } \\
\text { ELC } \\
\text { ELC } \\
\text { ELC }\end{array}$ & $\begin{array}{l}\text { STP } \\
\text { STP } \\
\text { STP } \\
\text { STP } \\
\text { STP }\end{array}$ & $\begin{array}{l}6 \\
8 \\
8 \\
8 \\
8\end{array}$ & $\begin{array}{l}121 \\
144 \\
160 \\
160 \\
160\end{array}$ & $\begin{array}{l}\text { JOHNSON NO. } 2 \\
\text { JOHNSON NO.4 } \\
\text { CWL MED NO.1 } \\
\text { CWL MED NO. } \\
\text { CWL MED NO.3 }\end{array}$ \\
\hline $\begin{array}{l}\text { SURFACE } \\
\text { SURFACE } \\
\text { SURFACE } \\
\text { GROUND } \\
\text { GROUND }\end{array}$ & $\begin{array}{l}\text { ROCK CREEK } \\
\text { LAKE ALMA } \\
\text { FROG BAYOU } \\
\text { NACATOCH SAND } \\
\text { NACATOCH SAND }\end{array}$ & $\begin{array}{l}-- \\
-- \\
-- \\
30 \\
50\end{array}$ & $\begin{array}{l}-- \\
\text { ELC } \\
\text { ELC } \\
\text { ELC }\end{array}$ & $\begin{array}{l}\text { GRV } \\
\text { STP } \\
\text { STP } \\
\text { STP }\end{array}$ & $\begin{array}{l}18 \\
18 \\
36 \\
12 \\
12\end{array}$ & $\begin{array}{l}-- \\
-- \\
-- \\
501 \\
509\end{array}$ & $\begin{array}{c}-- \\
-- \\
-- \\
\text { MOCKINGBIRD WELL } \\
\text { MARTINDALE WELL }\end{array}$ \\
\hline $\begin{array}{l}\text { GROUND } \\
\text { GROUND } \\
\text { GROUND } \\
\text { GROUND } \\
\text { GROUND }\end{array}$ & $\begin{array}{l}\text { NACATOCH SAND } \\
\text { TOKIO FORMATION } \\
\text { TOKIO FORMATION } \\
\text { TOKIO FORMATION } \\
\text { TOKIO FORMATION }\end{array}$ & $\begin{array}{l}-- \\
50 \\
50 \\
40 \\
60\end{array}$ & $\begin{array}{l}\text { ELC } \\
\text { ELC } \\
\text { ELC } \\
\text { ELC } \\
\text { ELC }\end{array}$ & $\begin{array}{l}\text { STP } \\
\text { STP } \\
\text { STP } \\
\text { STP } \\
\text { STP }\end{array}$ & $\begin{array}{l}-- \\
12 \\
12 \\
12 \\
12\end{array}$ & $\begin{array}{l}1,140 \\
1,173 \\
1,134 \\
1,115\end{array}$ & $\begin{array}{l}\text { ELKS HALL WELL } \\
\text { WELL NO.1 } \\
\text { WELL NO.3 } \\
\text { WELL NO.6 } \\
\text { WELL NO.7 }\end{array}$ \\
\hline $\begin{array}{l}\text { GROUND } \\
\text { GROUND } \\
\text { GROUND } \\
\text { SURFACE } \\
\text { SURFACE }\end{array}$ & $\begin{array}{l}\text { TOKIO FORMATION } \\
\text { TOKIO FORMATION } \\
\text { TOKIO FORMATION } \\
\text { LITTLE RIVER } \\
\text { OUACHITA RIVER }\end{array}$ & $\begin{array}{r}40 \\
60 \\
50 \\
125 \\
100\end{array}$ & $\begin{array}{l}\text { ELC } \\
\text { ELC } \\
\text { ELC } \\
\text { ELC } \\
\text { ELC }\end{array}$ & $\begin{array}{l}\text { STP } \\
\text { STP } \\
\text { STP } \\
\text { STP } \\
\text { STP }\end{array}$ & $\begin{array}{l}12 \\
12 \\
12 \\
16 \\
48\end{array}$ & $\begin{array}{l}1,141 \\
1,191 \\
1,134 \\
-- \\
--\end{array}$ & $\begin{aligned} \text { WELL NO.8 } & \text { NO. } \\
\text { WELL NO.9 } & \text { WELL NO.10 } \\
\text { WEL } & --\end{aligned}$ \\
\hline $\begin{array}{l}\text { SURFACE } \\
\text { SURFACE } \\
\text { SURFACE } \\
\text { SURFACE } \\
\text { SURFACE }\end{array}$ & $\begin{array}{l}\text { SPADRA AND LUDWIG } \\
\text { LAKE MILLWOOD } \\
\text { LAKE } \text { MILLWOOD } \\
\text { LAKE MILLWOOD }\end{array}$ & $\begin{array}{l}-- \\
40 \\
-- \\
--\end{array}$ & $\begin{array}{l}-- \\
\text { ELC } \\
\text { ELC } \\
\text { ELC }\end{array}$ & $\begin{array}{l}-- \\
\text { STP } \\
\text { STP } \\
\text { STP } \\
\text { STP }\end{array}$ & $\begin{array}{l}-- \\
24 \\
-- \\
-- \\
--\end{array}$ & $\begin{array}{l}-- \\
-- \\
-- \\
--\end{array}$ & $\begin{array}{l}-- \\
-- \\
\text { DIVERTED TO NEKOOSA } \\
\text { DIVERTED TO TEXARKANA } \\
\text { DIVERTED TO HOPE }\end{array}$ \\
\hline $\begin{array}{l}\text { SURFACE } \\
\text { SURFACE } \\
\text { SURFACE } \\
\text { SURFACE } \\
\text { SURFACE }\end{array}$ & $\begin{array}{l}\text { WASHBURN CREEK } \\
\text { PETIT JEAN RIVER } \\
\text { PETIT JEAN RIVER } \\
\text { GRAY SPRINGS } \\
\text { LITTLE MISSOURI }\end{array}$ & $\begin{array}{r}75 \\
75 \\
75 \\
250 \\
25\end{array}$ & $\begin{array}{l}\text { ELC } \\
\text { ELC } \\
\text { ELC } \\
\text { ELC } \\
\text { ELC }\end{array}$ & $\begin{array}{l}\text { STP } \\
\text { STP } \\
\text { STP } \\
\text { STP } \\
\text { STP }\end{array}$ & $\begin{array}{r}8 \\
10 \\
10 \\
10 \\
24\end{array}$ & $\begin{array}{l}-- \\
-- \\
-- \\
--\end{array}$ & $\begin{array}{c}\text { LOWER LAKE/INTAKE TOWER } \\
\text { UPPER LAKE/BELOW DAM } \\
-- \\
--\end{array}$ \\
\hline $\begin{array}{l}\text { GROUND } \\
\text { SURFACE } \\
\text { SURFACE } \\
\text { SURFACE } \\
\text { SURFACE }\end{array}$ & $\begin{array}{l}\text { OUACHITA RIVER } \\
\text { LITTLE MISSOURI } \\
\text { WARD LAKE } \\
\text { ILLINOIS BAYOU }\end{array}$ & $\begin{array}{r}25 \\
50 \\
-- \\
25 \\
200\end{array}$ & $\begin{array}{l}\text { ELC } \\
\text { ELC } \\
\text { ELC } \\
\text { ELC } \\
\text { ELC }\end{array}$ & $\begin{array}{l}\text { STP } \\
\text { STP } \\
\text { STP } \\
\text { STP } \\
--\end{array}$ & $\begin{array}{l}6 \\
54 \\
18 \\
-- \\
24\end{array}$ & $\begin{array}{l}640 \\
-- \\
-- \\
-- \\
--\end{array}$ & $\begin{array}{c}\text { NEAR PUMP HOUSE } \\
-- \\
-- \\
-- \\
\text { WITHDRAWAL NO.1 }\end{array}$ \\
\hline $\begin{array}{l}\text { SURFACE } \\
\text { SURFACE } \\
\text { SURFACE } \\
\text { GROUND } \\
\text { SURFACE }\end{array}$ & $\begin{array}{l}\text { ILLINOIS BAYOU } \\
\text { GALLA CREEK } \\
\text { ILLINOIS BAY } \\
\text { TERRACE DEPOSITS } \\
\text { LAKE WINONA }\end{array}$ & $\begin{array}{l}50 \\
-- \\
-- \\
40 \\
--\end{array}$ & $\begin{array}{r}E L C \\
-- \\
-- \\
E L C \\
--\end{array}$ & $\begin{array}{l}\text { STP } \\
-- \\
\text { STP } \\
\text { STP } \\
\text { GRV }\end{array}$ & $\begin{array}{l}24 \\
-- \\
-- \\
12 \\
--\end{array}$ & $\begin{array}{l}-- \\
-- \\
-- \\
132 \\
--\end{array}$ & $\begin{array}{l}\text { WITHDRAWAL NO. } 2 / \text { IDLE } \\
\text { - } \\
52-2\end{array}$ \\
\hline $\begin{array}{l}\text { SURFACE } \\
\text { SURFACE } \\
\text { SURFACE } \\
\text { SURFACE } \\
\text { GROUND }\end{array}$ & $\begin{array}{l}\text { LAKE MAUMELLE } \\
\text { BLACK RIVER } \\
\text { LAKE WALDRON } \\
\text { CASSOTOT RIVER } \\
--\end{array}$ & $\begin{array}{r}250 \\
25 \\
200 \\
150 \\
75\end{array}$ & $\begin{array}{l}\text { ELC } \\
\text { ELC } \\
\text { ELC } \\
\text { ELC } \\
\text { ELC }\end{array}$ & $\begin{array}{l}\text { STP } \\
\text { STP } \\
\text { STP } \\
\text { STP } \\
\text { STP }\end{array}$ & $\begin{array}{r}-- \\
30 \\
14 \\
48 \\
6\end{array}$ & $\begin{array}{l}-- \\
-- \\
-- \\
-- \\
150\end{array}$ & $\begin{array}{c}-- \\
-- \\
-- \\
-- \\
\text { DIVERSION NO. } 2\end{array}$ \\
\hline
\end{tabular}


Table 2.--General description of public-supply

\begin{tabular}{|c|c|c|c|c|c|}
\hline $\begin{array}{c}\text { Water } \\
\text { user } \\
\text { ID } \\
\end{array}$ & $\begin{array}{c}\text { Site } \\
\text { ID }\end{array}$ & Water system name & $\begin{array}{r}\text { Lati- } \\
\text { tude } \\
\end{array}$ & $\begin{array}{c}\text { Longi- } \\
\text { tude }\end{array}$ & $\begin{array}{l}\text { Hydro- } \\
\text { logic } \\
\text { unit } \\
\text { code } \\
\end{array}$ \\
\hline $\begin{array}{l}20626 \\
20627 \\
20630 \\
28039 \\
28041\end{array}$ & $\begin{array}{r}3619000912900 \\
355454092061400 \\
3600000942515 \\
335430093551801 \\
340130093513001\end{array}$ & $\begin{array}{l}\text { HARDY WATERWORKS } \\
\text { MOUNTAIN VIEW WATERWORKS } \\
\text { LINCOLN WATERWORKS } \\
\text { MINERAL SPRINGS WATERWORK } \\
\text { NASHVILLE WATERWORKS }\end{array}$ & $\begin{array}{l}361900 \\
355454 \\
360004 \\
335230 \\
340130\end{array}$ & $\begin{array}{l}0912900 \\
0920614 \\
0942519 \\
0935430 \\
0935130\end{array}$ & $\begin{array}{l}11010010 \\
11010004 \\
11110103 \\
11140109 \\
08020402\end{array}$ \\
\hline $\begin{array}{l}28041 \\
28041 \\
28041 \\
28107 \\
30510\end{array}$ & $\begin{array}{r}335730093503001 \\
340300093430001 \\
340256093431401 \\
0838748 \\
3553300910515\end{array}$ & $\begin{array}{l}\text { NASHVILLE WATERWORKS } \\
\text { NASHVILLE WATERWORKS } \\
\text { NASHVILLE WATERWORKS } \\
\text { LAKE CHARLES STATE PARK } \\
\text { ALICIA WATER SYSTEM }\end{array}$ & $\begin{array}{c}335730 \\
340300 \\
340256 \\
-- \\
355330\end{array}$ & $\begin{array}{l}0935030 \\
0934300 \\
0934314 \\
0911108 \\
0910515\end{array}$ & $\begin{array}{l}11140109 \\
11140109 \\
11140109 \\
11010009 \\
11010013\end{array}$ \\
\hline $\begin{array}{l}30511 \\
30512 \\
30514 \\
30515 \\
30516\end{array}$ & $\begin{array}{r}3415200930710 \\
3432340914701 \\
342416091243701 \\
341918091504901 \\
3533450910630\end{array}$ & $\begin{array}{l}\text { ALLEN MH PARK } \\
\text { ALLPORT WATERWORKS } \\
\text { ALMYRA WATERWORKS } \\
\text { ALTHEIMER WATERWORKS } \\
\text { AMAGON WATERWORKS }\end{array}$ & $\begin{array}{l}341520 \\
343234 \\
342420 \\
341907 \\
353345\end{array}$ & $\begin{array}{l}0930710 \\
0914701 \\
0912437 \\
0915053 \\
0910630\end{array}$ & $\begin{array}{l}08040102 \\
08020401 \\
08020303 \\
08020401 \\
08020302\end{array}$ \\
\hline $\begin{array}{l}30517 \\
30518 \\
30519 \\
30520 \\
30522\end{array}$ & $\begin{array}{r}3417250932730 \\
3402300932530 \\
340345091344602 \\
342618091545501 \\
340838093032601\end{array}$ & $\begin{array}{l}\text { ANITY WATERWORKS } \\
\text { ANTOINE WATERWORKS } \\
\text { ARK DEPT OF CORREC CUMMINS } \\
\text { ARK DEPT OF CORRECTION } \\
\text { ARKADELPHIA WATERWORKS }\end{array}$ & $\begin{array}{l}341725 \\
340230 \\
340400 \\
342618 \\
340838\end{array}$ & $\begin{array}{l}0932730 \\
0932530 \\
0913400 \\
0915455 \\
0930326\end{array}$ & $\begin{array}{l}08040103 \\
08040103 \\
08050001 \\
11110207 \\
08040102\end{array}$ \\
\hline $\begin{array}{l}30523 \\
30524 \\
30525 \\
30526 \\
30526\end{array}$ & $\begin{array}{r}333624091124201 \\
3418030920336 \\
361325091363701 \\
334027094073801 \\
334027094073804\end{array}$ & $\begin{array}{l}\text { ARKANSAS CITY WATERWORKS } \\
\text { ARSENAL WATER SYSTEM } \\
\text { ASH FLAT WATERWORKS } \\
\text { ASHDOWN WATERWORKS } \\
\text { ASHDOWN WATERWORKS }\end{array}$ & $\begin{array}{l}333629 \\
341803 \\
361330 \\
334027 \\
334027\end{array}$ & $\begin{array}{l}0911158 \\
0920336 \\
0913630 \\
0940738 \\
0940738\end{array}$ & $\begin{array}{l}08050002 \\
08040205 \\
11010012 \\
11140109 \\
11140109\end{array}$ \\
\hline $\begin{array}{l}30526 \\
30528 \\
30528 \\
30530 \\
30531\end{array}$ & $\begin{array}{r}333928094065401 \\
351701091214301 \\
351701091214302 \\
3520000913515 \\
3605000910915\end{array}$ & $\begin{array}{l}\text { ASHDOWN WATERWORKS } \\
\text { AUGUSTA WATERWORKS } \\
\text { AUGUSTA WATERWORKS } \\
\text { BALD KNOB WATERWORKS } \\
\text { BALTZ DEVELOPMENT CO }\end{array}$ & $\begin{array}{l}333928 \\
351701 \\
351733 \\
352000 \\
360500\end{array}$ & $\begin{array}{l}0940654 \\
0912143 \\
0912153 \\
0913515 \\
0910915\end{array}$ & $\begin{array}{l}11140109 \\
11010013 \\
11010013 \\
11010014 \\
11010009\end{array}$ \\
\hline $\begin{array}{l}30532 \\
30533 \\
30534 \\
30535 \\
30535\end{array}$ & $\begin{array}{r}333453092160701 \\
353216090074001 \\
3545300913815 \\
330409092171301 \\
330410092171601\end{array}$ & $\begin{array}{l}\text { BANKS WATERWORKS } \\
\text { BASSETT WATERWORKS } \\
\text { BATESVILLE WATER UTILITY } \\
\text { BATTS LAPILE WATER ASSOC } \\
\text { BATTS LAPILE WATER ASSOC }\end{array}$ & $\begin{array}{l}333454 \\
353215 \\
354530 \\
330409 \\
330410\end{array}$ & $\begin{array}{l}0921600 \\
0900730 \\
0913815 \\
0921713 \\
0921716\end{array}$ & $\begin{array}{l}08040201 \\
08020203 \\
11010004 \\
08040201 \\
08040201\end{array}$ \\
\hline $\begin{array}{l}30536 \\
30536 \\
30536 \\
30537 \\
30537\end{array}$ & $\begin{array}{l}354458090333900 \\
354437090335701 \\
354459090334000 \\
334331092370301 \\
334331092370302\end{array}$ & $\begin{array}{l}\text { BAY WATERWORKS } \\
\text { BAY WATERWORKS } \\
\text { BAY WATERWORKS } \\
\text { BEARDEN WATERWORKS } \\
\text { BEARDEN WATERWORKS }\end{array}$ & $\begin{array}{l}354458 \\
354457 \\
354459 \\
334331 \\
334331\end{array}$ & $\begin{array}{l}0903339 \\
0903337 \\
0903340 \\
0923703 \\
0923703\end{array}$ & $\begin{array}{l}08020203 \\
08020203 \\
08020203 \\
08040201 \\
08040201\end{array}$ \\
\hline $\begin{array}{l}30539 \\
30539 \\
30539 \\
30539 \\
30539\end{array}$ & $\begin{array}{l}350405091524202 \\
350406091524102 \\
350406091523601 \\
350356091524401 \\
350342091513901\end{array}$ & $\begin{array}{l}\text { BEEBE WATERWORKS } \\
\text { BEEBE WATERWORKS } \\
\text { BEEBE WATERWORKS } \\
\text { BEEBE WATERWORKS } \\
\text { BEEBE WATERWORKS }\end{array}$ & $\begin{array}{l}350406 \\
350406 \\
350406 \\
350346 \\
350342\end{array}$ & $\begin{array}{l}0915241 \\
0915241 \\
0915236 \\
0915147 \\
0915139\end{array}$ & $\begin{array}{l}08020301 \\
08020301 \\
08020301 \\
08020301 \\
08020301\end{array}$ \\
\hline $\begin{array}{l}30540 \\
30541 \\
30542 \\
30544 \\
30545\end{array}$ & $\begin{array}{r}3525450910630 \\
361150093025601 \\
3350150940645 \\
362425094012101 \\
343229092363001\end{array}$ & $\begin{array}{l}\text { BEEDEVILLE WATERWORKS } \\
\text { BELLEFONTE WATER } \\
\text { BEN LOMOND WATERWORKS } \\
\text { BENTON CTY WATER/DIST } 1 \\
\text { BENTON SERVICE CENTER }\end{array}$ & $\begin{array}{l}352545 \\
361145 \\
335015 \\
362430 \\
343229\end{array}$ & $\begin{array}{l}0910630 \\
0930300 \\
0940645 \\
0940130 \\
0923630\end{array}$ & $\begin{array}{l}08020302 \\
11010003 \\
11140109 \\
11070208 \\
08040203\end{array}$ \\
\hline $\begin{array}{l}30546 \\
30547 \\
30550 \\
30550 \\
30551\end{array}$ & $\begin{array}{r}343405092364101 \\
3620000930000 \\
360007092242601 \\
360015092243001 \\
3500000923745\end{array}$ & $\begin{array}{l}\text { BENTON WATERWORKS } \\
\text { BERGMAN WATERWORKS } \\
\text { BIG FLAT WATERWORKS } \\
\text { BIG FLAT WATERWORKS } \\
\text { BIGELOW WATERWORKS }\end{array}$ & $\begin{array}{l}343405 \\
362000 \\
360015 \\
360015 \\
350000\end{array}$ & $\begin{array}{l}0923641 \\
0930000 \\
0922430 \\
0922430 \\
0923745\end{array}$ & $\begin{array}{l}08040203 \\
11010003 \\
11010004 \\
11010004 \\
11110206\end{array}$ \\
\hline $\begin{array}{l}30551 \\
30552 \\
30553 \\
30554 \\
30555\end{array}$ & $\begin{array}{r}350000092374501 \\
3620000904830 \\
352923090150301 \\
344916091241801 \\
355008090220201\end{array}$ & $\begin{array}{l}\text { BIGELOW WATERWORKS } \\
\text { BIGGERS WATERWORKS } \\
\text { BIRDSONG WHITTEN WATER } \\
\text { BISCOE WATERWORKS } \\
\text { BLACK OAK WATERWORKS }\end{array}$ & $\begin{array}{l}361959 \\
352730 \\
344915 \\
355015\end{array}$ & $\begin{array}{l}0904831 \\
0901545 \\
0912408 \\
0902200\end{array}$ & $\begin{array}{l}11110206 \\
11010008 \\
08020203 \\
08020301 \\
08020203\end{array}$ \\
\hline
\end{tabular}




\begin{tabular}{|c|c|c|c|c|c|c|c|}
\hline $\begin{array}{l}\text { Source } \\
\text { troe }\end{array}$ & Source & $\begin{array}{l}\text { Pump } \\
\text { Horse- } \\
\text { power }\end{array}$ & $\begin{array}{l}\text { descriot } \\
\text { Power } \\
\text { source }\end{array}$ & $\begin{array}{l}\text { ion } \\
\text { Pump } \\
\text { troe }\end{array}$ & $\begin{array}{l}\text { Well } \\
\text { dia- } \\
\text { meter } \\
\text { (inches }\end{array}$ & $\begin{array}{c}\text { Depth } \\
\text { of } \\
\text { well } \\
\text { s) }(\text { feet })\end{array}$ & Local descriptive ID \\
\hline $\begin{array}{l}\text { SURFACE } \\
\text { SURFACE } \\
\text { SURFACE } \\
\text { GROUND } \\
\text { SURFACE }\end{array}$ & $\begin{array}{l}\text { SPRING RIVER } \\
\text { WHITE RIVER } \\
\text { LINCOLN LAKE } \\
\text { TRINITY GROUP } \\
\text { WINE CREEK RES }\end{array}$ & $\begin{array}{r}5 \\
150 \\
60 \\
25 \\
--\end{array}$ & $\begin{array}{l}E L C \\
\text { ELC } \\
\text { ELC } \\
\text { ELC } \\
--\end{array}$ & $\begin{array}{l}\text { STP } \\
\text { STP } \\
\text { STP } \\
\text { STP } \\
\text { GRV }\end{array}$ & $\begin{array}{l}24 \\
24 \\
10 \\
10 \\
--\end{array}$ & $\begin{array}{l}-- \\
-- \\
-- \\
408 \\
--\end{array}$ & $\begin{array}{c}\text { DIVERSION NO. } 1 \\
-- \\
-- \\
\text { MINE CREEK RES }\end{array}$ \\
\hline $\begin{array}{l}\text { SURFACE } \\
\text { SURFACE } \\
\text { SURFACE } \\
\text { GROUND } \\
\text { GROUND }\end{array}$ & $\begin{array}{l}\text { LAKE NICHOLS } \\
\text { LITTLE MISSOURI } \\
\text { LITTLE MISSOURI } \\
-- \\
--\end{array}$ & $\begin{array}{r}250 \\
250 \\
3 \\
5\end{array}$ & $\begin{array}{l}E \overline{-} \\
\overline{L L} \\
E L \bar{C} \\
\text { ELC }\end{array}$ & $\begin{array}{l}\text { GRV } \\
\text { STP } \\
\text { STP } \\
\text { STP }\end{array}$ & $\begin{array}{r}-- \\
-- \\
6 \\
8\end{array}$ & $\begin{array}{l}-- \\
-- \\
-- \\
485 \\
120\end{array}$ & $\begin{array}{l}\text { LAKE NICHOLS } \\
\text { LITTLE MISSOURI RIVER } \\
\text { ABANDONED/2ND LTL MISSOURI } \\
\text { EATON } \\
\text { WELL NEAR PUMP HOUSE }\end{array}$ \\
\hline $\begin{array}{l}\text { GROUND } \\
\text { GROUND } \\
\text { GROUND } \\
\text { GROUND } \\
\text { GROUND }\end{array}$ & $\begin{array}{l}-- \\
\text { SPARTA SAND } \\
\text { SPARTA SAND }\end{array}$ & $\begin{array}{l}1 \\
-- \\
-- \\
40\end{array}$ & $\begin{array}{r}\text { ELC } \\
\overline{--} \\
\text { ELC } \\
--\end{array}$ & $\begin{array}{r}\text { STP } \\
\overline{--} \\
\text { STP } \\
--\end{array}$ & $\begin{array}{l}6 \\
-- \\
-- \\
10\end{array}$ & $\begin{array}{l}142 \\
-- \\
666 \\
1.011 \\
--\end{array}$ & $\begin{array}{l}-- \\
-- \\
-- \\
--\end{array}$ \\
\hline $\begin{array}{l}\text { SURFACE } \\
\text { GROUND } \\
\text { GROUND } \\
\text { GROUND } \\
\text { SURFACE }\end{array}$ & $\begin{array}{l}\text { CADDO RIVER } \\
\text { SPARTA SAND } \\
\text { SPARTA SAND } \\
\text { OUACHITA RIVER }\end{array}$ & $\begin{array}{l}30 \\
-- \\
30 \\
50\end{array}$ & $\begin{array}{l}\text { ELC } \\
-- \\
-\overline{-} \\
\text { ELC }\end{array}$ & $\begin{array}{l}\text { STP } \\
-- \\
\text { STP } \\
\text { STP }\end{array}$ & $\begin{array}{r}8 \\
-- \\
-- \\
--\end{array}$ & $\begin{array}{l}-- \\
-- \\
760 \\
725 \\
--\end{array}$ & 50 FT N AND 360 FT E OF PLANT \\
\hline $\begin{array}{l}\text { GROUND } \\
\text { GROUND } \\
\text { GROUND } \\
\text { GROUND } \\
\text { GROUND }\end{array}$ & $\begin{array}{l}\text { SPARTA SAND } \\
\text { SPARTA SAND } \\
\text { GUNTER SANDSTONE } \\
\text { TERRACE DEPOSITS } \\
\text { TERRACE DEPOSITS }\end{array}$ & $\begin{array}{r}15 \\
150 \\
-- \\
25 \\
15\end{array}$ & $\begin{array}{l}\text { ELC } \\
\text { ELC } \\
\text { ELC } \\
\text { ELC }\end{array}$ & $\begin{array}{l}\text { STP } \\
\text { STP } \\
-- \\
\text { STP } \\
\text { STP }\end{array}$ & $\begin{array}{r}4 \\
12 \\
-2 \\
6 \\
6\end{array}$ & $\begin{array}{r}520 \\
1,000 \\
1,525 \\
90 \\
90\end{array}$ & $\begin{aligned} 42-210 & -- \\
& -- \\
& --\end{aligned}$ \\
\hline $\begin{array}{l}\text { GROUND } \\
\text { GROUND } \\
\text { GROUND } \\
\text { SURFACE } \\
\text { GROUND }\end{array}$ & $\begin{array}{c}\text { TERRACE DEPOSITS } \\
\text { TERRACE DEPOSITS } \\
\text { TERRACE DEPOSITS } \\
-- \\
--\end{array}$ & $\begin{array}{l}25 \\
40 \\
30 \\
-- \\
--\end{array}$ & $\begin{array}{l}\text { ELC } \\
\text { ELC } \\
\text { ELC } \\
-- \\
\text { ELC }\end{array}$ & $\begin{array}{l}\text { STP } \\
\text { STP } \\
\text { STP } \\
-- \\
--\end{array}$ & $\begin{array}{l}6 \\
12 \\
-- \\
--\end{array}$ & $\begin{array}{r}95 \\
98 \\
114 \\
-- \\
--\end{array}$ & $\begin{array}{l}-- \\
-- \\
- \\
-- \\
--\end{array}$ \\
\hline $\begin{array}{l}\text { GROUND } \\
\text { GROUND } \\
\text { SURFACE } \\
\text { GROUND } \\
\text { GROUND }\end{array}$ & $\begin{array}{l}\text { SPARTA SAND } \\
\text { WILCOX GROUP } \\
\text { WHITE RIVER } \\
\text { SPARTA SAND } \\
\text { SPARTA SAND }\end{array}$ & $\begin{array}{l}-- \\
-- \\
60 \\
15 \\
--\end{array}$ & $\begin{array}{c}-- \\
\overline{-} \\
\text { ELC } \\
--\end{array}$ & $\begin{array}{c}-. \\
\text { STP } \\
-- \\
--\end{array}$ & $\begin{array}{l}-- \\
-- \\
14 \\
--\end{array}$ & $\begin{array}{r}680 \\
1.560 \\
-- \\
600 \\
339\end{array}$ & $\begin{array}{l}\text { WELL LOCATED IN PUMP HOUSE } \\
\text { WELL NO. } 1 \\
\text { WELL NO. } 2\end{array}$ \\
\hline $\begin{array}{l}\text { GROUND } \\
\text { GROUND } \\
\text { GROUND } \\
\text { GROUND } \\
\text { GROUND }\end{array}$ & $\begin{array}{l}\text { QUATERNARY ALLUVIUM } \\
\text { SPARTA SAND } \\
\text { SPARTA SAND }\end{array}$ & $\begin{array}{l}-- \\
-- \\
-- \\
40 \\
40\end{array}$ & $\begin{array}{r}-- \\
-- \\
\overline{E L C} \\
\text { ELC }\end{array}$ & $\begin{array}{r}-- \\
-- \\
\text { STP } \\
\text { STP }\end{array}$ & $\begin{array}{l}-- \\
-- \\
-- \\
10 \\
10\end{array}$ & $\begin{array}{l}-- \\
147 \\
-- \\
300 \\
300\end{array}$ & $\begin{array}{l}-- \\
-- \\
-- \\
--\end{array}$ \\
\hline $\begin{array}{l}\text { GROUND } \\
\text { GROUND } \\
\text { GROUND } \\
\text { GROUND } \\
\text { GROUND }\end{array}$ & $\begin{array}{l}\text { TERRACE DEPOSITS } \\
\text { TERRACE DEPOSITS } \\
\text { QUATERNARY ALLUVIUM } \\
\text { QUATERNARY ALLUVIUM } \\
--\end{array}$ & $\begin{array}{l}15 \\
15 \\
15 \\
15 \\
--\end{array}$ & $\begin{array}{l}\text { ELC } \\
\text { ELC } \\
\text { ELC } \\
\text { ELC } \\
--\end{array}$ & $\begin{array}{l}\text { STP } \\
\text { STP } \\
\text { STP } \\
\text { STP } \\
--\end{array}$ & $\begin{array}{r}4 \\
4 \\
6 \\
6 \\
--\end{array}$ & $\begin{array}{r}99 \\
91 \\
96 \\
96\end{array}$ & $\begin{array}{l}-- \\
-- \\
- \\
\cdots\end{array}$ \\
\hline $\begin{array}{l}\text { GROUND } \\
\text { GROUND } \\
\text { GROUND }\end{array}$ & GUNTER SANOSTONE & $\begin{array}{l}-- \\
--\end{array}$ & $\begin{array}{c}\text { ELC } \\
-- \\
--\end{array}$ & $\begin{array}{l}\cdots \\
\cdots\end{array}$ & $\begin{array}{c}4 \\
--\end{array}$ & $\begin{array}{r}130 \\
1.649 \\
-\end{array}$ & $\begin{array}{l}\cdots \\
\cdots\end{array}$ \\
\hline $\begin{array}{l}\text { GROUND } \\
\text { SURFACE }\end{array}$ & $\begin{array}{l}\text { POTOSI DOLOMITE } \\
\text { SALINE RIVER }\end{array}$ & $\begin{array}{l}60 \\
75\end{array}$ & $\begin{array}{l}\text { ELC } \\
\text { ELC }\end{array}$ & $\begin{array}{l}\text { STP } \\
\text { STP }\end{array}$ & $\begin{array}{r}8 \\
18\end{array}$ & 1,968 & WELL NEAR PUMP HOUSE AND TOWER \\
\hline $\begin{array}{l}\text { SURFACE } \\
\text { GROUND } \\
\text { GROUND } \\
\text { GROUND } \\
\text { GROUND }\end{array}$ & $\begin{array}{l}\text { SALINE RIVER } \\
\text { ROUBIDOUX FORMATION } \\
\text { ROUBIDOUX FORMATION } \\
--\end{array}$ & $\begin{array}{r}100 \\
-- \\
10 \\
50 \\
--\end{array}$ & $\begin{array}{l}\text { ELC } \\
-- \\
\text { ELC } \\
\text { ELC } \\
\text { ELC }\end{array}$ & $\begin{array}{l}\text { STP } \\
-- \\
\text { OTH } \\
\text { OTH } \\
\text { STP }\end{array}$ & $\begin{array}{r}-- \\
- \\
8 \\
8 \\
2\end{array}$ & $\begin{array}{r}-- \\
-- \\
2,603 \\
3,105 \\
400\end{array}$ & $\begin{array}{l}\text { WELL NO. } \\
\text { WELL NO. } \\
\text { W } \\
-.\end{array}$ \\
\hline $\begin{array}{l}\text { GROUND } \\
\text { GROUND } \\
\text { GROUND } \\
\text { GROUND } \\
\text { GROUND }\end{array}$ & $\begin{array}{l}\text { WILCOX GROUP } \\
\text { QUATERNARY ALLUVIUM } \\
\text { WILCOX GROUP }\end{array}$ & $\begin{array}{r}-- \\
30 \\
-- \\
20\end{array}$ & $\begin{array}{l}E L C \\
\text { ELC } \\
\text { ELC } \\
-- \\
E L C\end{array}$ & $\begin{array}{l}\text { STP } \\
\text { STP } \\
\text { STP } \\
-- \\
\text { STP }\end{array}$ & $\begin{array}{c}2 \\
6 \\
-- \\
- \\
16\end{array}$ & $\begin{array}{r}403 \\
112 \\
1.521 \\
140 \\
1,070\end{array}$ & $\begin{array}{l}\text { WELL NEAR PUMP HOUSE } \\
\text { WELL NEAR PUMP HOUSE } \\
\cdots \\
\cdots\end{array}$ \\
\hline
\end{tabular}


Table 2.--General description of public-supply

\begin{tabular}{|c|c|c|c|c|c|}
\hline $\begin{array}{c}\text { Water } \\
\text { user } \\
\text { ID } \\
\end{array}$ & $\begin{array}{c}\text { Site } \\
\text { ID } \\
\end{array}$ & Water system name & $\begin{array}{r}\text { Lati- } \\
\text { tude } \\
\end{array}$ & $\begin{array}{l}\text { Longi- } \\
\text { tude }\end{array}$ & $\begin{array}{l}\text { Hydro- } \\
\text { logic } \\
\text { unit } \\
\text { code }\end{array}$ \\
\hline $\begin{array}{l}30556 \\
30557 \\
30558 \\
30559 \\
30559\end{array}$ & 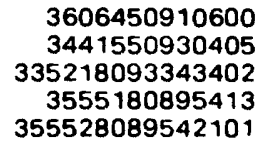 & $\begin{array}{l}\text { BLACK ROCK WATERWORKS } \\
\text { BLAKELY WATER SYSTEM } \\
\text { BLEVINS WATERWORKS } \\
\text { BLYTHEVILLE WATERWORKS } \\
\text { BLYTHEVILLE WATERWORKS }\end{array}$ & $\begin{array}{l}360645 \\
344155 \\
335220 \\
355518 \\
355524\end{array}$ & $\begin{array}{l}0910600 \\
0930405 \\
0933427 \\
0895413 \\
0895417\end{array}$ & $\begin{array}{l}11010009 \\
08040101 \\
08040103 \\
08020204 \\
08020204\end{array}$ \\
\hline $\begin{array}{l}30559 \\
30559 \\
30559 \\
30560 \\
30561\end{array}$ & $\begin{array}{l}355522089541901 \\
355521089540301 \\
355522089541801 \\
333318093412701 \\
355415090480001\end{array}$ & $\begin{array}{l}\text { BLYTHEVILLE WATERWORKS } \\
\text { BLYTHEVILLE WATERWORKS } \\
\text { BLYTHEVILLE WATERWORKS } \\
\text { BOIS D'ARC WATER SYSTEM } \\
\text { BONO WATERWORKS }\end{array}$ & $\begin{array}{l}355518 \\
355520 \\
355514 \\
333318 \\
355415\end{array}$ & $\begin{array}{l}0895419 \\
0895407 \\
0895416 \\
0934130 \\
0904800\end{array}$ & $\begin{array}{l}08020204 \\
08020204 \\
08020204 \\
11140201 \\
08020302\end{array}$ \\
\hline $\begin{array}{l}30563 \\
30564 \\
30565 \\
30565 \\
30566\end{array}$ & $\begin{array}{l}333505091491901 \\
332154091292801 \\
352457091270601 \\
352517091271101 \\
330537093391302\end{array}$ & $\begin{array}{l}\text { BOWSER WATER ASSOC } \\
\text { BOYDELL WATERWORKS } \\
\text { BRADFORD WATERWORKS } \\
\text { BRADFORD WATERWORKS } \\
\text { BRADLEY WATERWORKS }\end{array}$ & $\begin{array}{l}333515 \\
332143 \\
352519 \\
352517 \\
330540\end{array}$ & $\begin{array}{l}0915015 \\
0912905 \\
0912709 \\
0912711 \\
0933908\end{array}$ & $\begin{array}{l}08040205 \\
08040205 \\
11010013 \\
11010013 \\
11140205\end{array}$ \\
\hline $\begin{array}{l}30566 \\
30567 \\
30567 \\
30567 \\
30568\end{array}$ & $\begin{array}{r}330616093392301 \\
3527000911345 \\
352700091134501 \\
352701091134502 \\
3616300921745\end{array}$ & $\begin{array}{l}\text { BRADLEY WATERWORKS } \\
\text { BRECKENRIDGE UNION WATER } \\
\text { BRECKENRIDGE UNION WATER } \\
\text { BRECKENRIDGE UNION WATER } \\
\text { BRIAR CLIFF WATERWORKS }\end{array}$ & $\begin{array}{c}330600 \\
352700 \\
-- \\
352701 \\
361630\end{array}$ & $\begin{array}{c}0933925 \\
0911345 \\
-- \\
0911345 \\
0921745\end{array}$ & $\begin{array}{l}11140205 \\
11010013 \\
11010013 \\
11010013 \\
11010004\end{array}$ \\
\hline $\begin{array}{l}30569 \\
30569 \\
30569 \\
30570 \\
30571\end{array}$ & $\begin{array}{l}345613091145801 \\
345616091150402 \\
345616091150201 \\
355415090341300 \\
343612092293101\end{array}$ & $\begin{array}{l}\text { BRINKLEY WATERWORKS } \\
\text { BRINKLEY WATERWORKS } \\
\text { BRINKLEY WATERWORKS } \\
\text { BROOKLAND WATERWORKS } \\
\text { BRYANT WATERWORKS }\end{array}$ & $\begin{array}{l}345613 \\
345614 \\
345612 \\
355415 \\
343535\end{array}$ & $\begin{array}{l}0911458 \\
0911460 \\
0911455 \\
0903413 \\
0922927\end{array}$ & $\begin{array}{l}08020304 \\
08020304 \\
08020304 \\
08020203 \\
08040203\end{array}$ \\
\hline $\begin{array}{l}30571 \\
30571 \\
30572 \\
30572 \\
30573\end{array}$ & $\begin{array}{r}343616092293201 \\
343648092380801 \\
3321400932600 \\
332140093260001 \\
3622300923500\end{array}$ & $\begin{array}{l}\text { BRYANT WATERWORKS } \\
\text { BRYANT WATERWORKS } \\
\text { BUCKNER WATERWORKS } \\
\text { BUCKNER WATERWORKS } \\
\text { BULL SHOALS WATER }\end{array}$ & $\begin{array}{c}343525 \\
343649 \\
332140 \\
-- \\
362219\end{array}$ & $\begin{array}{c}0922929 \\
0922807 \\
0932600 \\
-- \\
0923512\end{array}$ & $\begin{array}{c}08040203 \\
08040203 \\
11140205 \\
-- \\
11010003\end{array}$ \\
\hline $\begin{array}{l}30573 \\
30574 \\
30575 \\
30576 \\
30576\end{array}$ & $\begin{array}{r}362157092362701 \\
3549000895630 \\
345926092002501 \\
360753092062601 \\
360700092081501\end{array}$ & $\begin{array}{l}\text { BULL SHOALS WATER } \\
\text { BURDETTE WATERWORKS } \\
\text { CABOT WATERWORKS } \\
\text { CALICO ROCK WATERWORKS } \\
\text { CALICO ROCK WATERWORKS }\end{array}$ & $\begin{array}{c}362157 \\
354900 \\
345800 \\
360700 \\
--\end{array}$ & $\begin{array}{l}0923627 \\
0895630 \\
0920015 \\
0920815 \\
--\end{array}$ & $\begin{array}{l}11010003 \\
08020203 \\
08020402 \\
11010004 \\
11010004\end{array}$ \\
\hline $\begin{array}{l}30576 \\
30576 \\
30576 \\
30577 \\
30577\end{array}$ & $\begin{array}{l}360700092081502 \\
360700092081503 \\
360700092081504 \\
331944092321701 \\
331948092323201\end{array}$ & $\begin{array}{l}\text { CALICO ROCK WATERWORKS } \\
\text { CALICO ROCK WATERWORKS } \\
\text { CALICO ROCK WATERWORKS } \\
\text { CALION WATERWORKS } \\
\text { CALION WATERWORKS }\end{array}$ & $\begin{array}{c}-- \\
-- \\
-- \\
331944 \\
331948\end{array}$ & $\begin{array}{c}-- \\
-- \\
0923217 \\
0923232\end{array}$ & $\begin{array}{l}11010004 \\
11010004 \\
11010004 \\
08040201 \\
08040201\end{array}$ \\
\hline $\begin{array}{l}30579 \\
30580 \\
30580 \\
30580 \\
30581\end{array}$ & $\begin{array}{r}354525090191001 \\
344705091443701 \\
344705091443702 \\
3447050914437 \\
362441093510400\end{array}$ & $\begin{array}{l}\text { CARAWAY WATERWORKS } \\
\text { CARLISLE WATERWORKS } \\
\text { CARLISLE WATERWORKS } \\
\text { CARLISLE WATERWORKS } \\
\text { CARROLL-BOONE WATER DISTRICT }\end{array}$ & $\begin{array}{c}354525 \\
344705 \\
344705 \\
362441\end{array}$ & $\begin{array}{c}0901910 \\
0914437 \\
0914437 \\
-- \\
0935104\end{array}$ & $\begin{array}{l}08020204 \\
08020402 \\
08020401 \\
08020402 \\
11010001\end{array}$ \\
\hline $\begin{array}{l}30582 \\
30583 \\
30586 \\
30587 \\
30588\end{array}$ & $\begin{array}{r}340429092333201 \\
354800090560000 \\
355631091325401 \\
3607150910845 \\
341343092140701\end{array}$ & $\begin{array}{l}\text { CARTHAGE WATERWORKS } \\
\text { CASH WATERWORKS } \\
\text { CAVE CITY WATERWORKS } \\
\text { CEDAR RAIL MOBILE HOME PK } \\
\text { CENTER GROVE WATER ASSOC }\end{array}$ & $\begin{array}{l}340415 \\
354800 \\
355631 \\
360715 \\
\mathbf{3 4 1 3 4 3}\end{array}$ & $\begin{array}{l}0923330 \\
0905600 \\
0913254 \\
0910845 \\
0921407\end{array}$ & $\begin{array}{l}08040201 \\
08020302 \\
11010004 \\
11010009 \\
08040203\end{array}$ \\
\hline $\begin{array}{l}30588 \\
30588 \\
30589 \\
30589 \\
30589\end{array}$ & $\begin{array}{l}341340092140901 \\
341345092140501 \\
362358090131400 \\
361927090354200 \\
362033090134000\end{array}$ & $\begin{array}{l}\text { CENTER GROVE WATER ASSOC } \\
\text { CENTER GROVE WATER ASSOC } \\
\text { CENTRAL CLAY REG WATER } \\
\text { CENTRAL CLAY REG WATER } \\
\text { CENTRAL CLAY REG WATER }\end{array}$ & $\begin{array}{l}341340 \\
341346 \\
362358 \\
361927 \\
362033\end{array}$ & $\begin{array}{l}0921409 \\
0921405 \\
0901314 \\
0903542 \\
0901340\end{array}$ & $\begin{array}{l}08040203 \\
08040203 \\
08020302 \\
08020302 \\
08020302\end{array}$ \\
\hline $\begin{array}{l}30590 \\
30591 \\
30591 \\
30592 \\
30593\end{array}$ & $\begin{array}{r}351704094025901 \\
352359090451401 \\
352400090450001 \\
3341550930108 \\
344145091175601\end{array}$ & $\begin{array}{l}\text { CHARLESTON WATERWORKS } \\
\text { CHERRY VALLEY WATERWORKS } \\
\text { CHERRY VALLEY WATERWORKS } \\
\text { CHIDESTER WATERWORKS } \\
\text { CLARENDON WATERWORKS }\end{array}$ & $\begin{array}{l}351704 \\
352359 \\
352400 \\
334155 \\
344143\end{array}$ & $\begin{array}{l}0940259 \\
0904514 \\
0904500 \\
0930108 \\
0911802\end{array}$ & $\begin{array}{l}11110201 \\
08020205 \\
08020205 \\
08040103 \\
08020303\end{array}$ \\
\hline
\end{tabular}




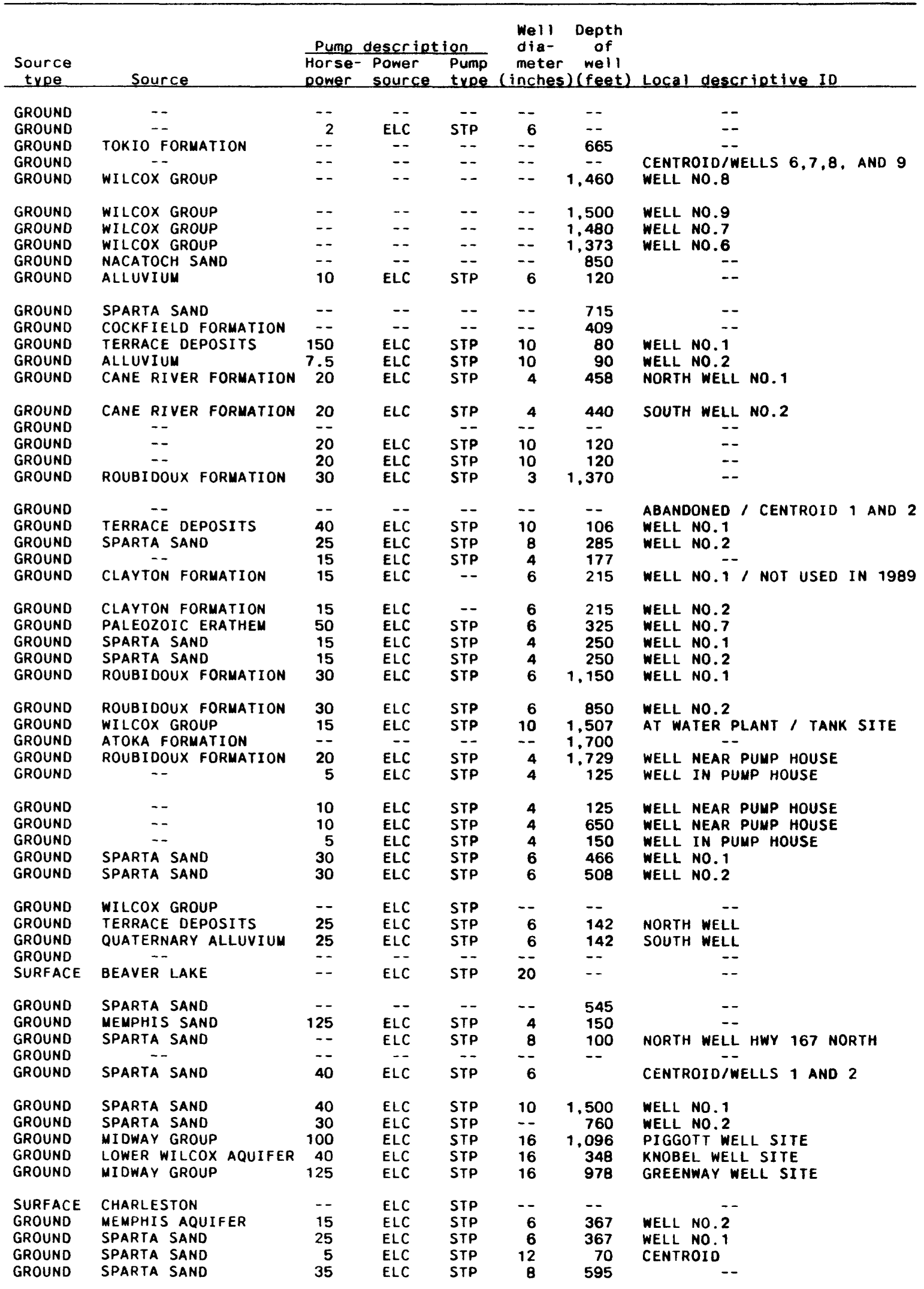


Table 2.--General description of public-supply

\begin{tabular}{|c|c|c|c|c|c|}
\hline $\begin{array}{c}\text { Water } \\
\text { user } \\
\text { ID } \\
\end{array}$ & $\begin{array}{c}\text { Site } \\
\text { ID } \\
\end{array}$ & Water system name & $\begin{array}{c}\text { Lati- } \\
\text { tude }\end{array}$ & $\begin{array}{l}\text { Longi- } \\
\text { tude } \\
\end{array}$ & $\begin{array}{l}\text { Hydro- } \\
\text { logic } \\
\text { unit } \\
\text { code } \\
\end{array}$ \\
\hline $\begin{array}{l}30595 \\
30596 \\
30597 \\
30598 \\
30599\end{array}$ & $\begin{array}{r}353235092215301 \\
333154091340401 \\
3806050904800 \\
3535300921015 \\
360607093180201\end{array}$ & $\begin{array}{l}\text { CLINTON WATERWORKS } \\
\text { COLLINS WATER ASSOC } \\
\text { COLT WATER ASSOC } \\
\text { COMMUNITY WATER ASSOC } \\
\text { COMPION WATERWORKS }\end{array}$ & $\begin{array}{l}353235 \\
330452 \\
380605 \\
353530 \\
360500\end{array}$ & $\begin{array}{l}0922153 \\
0913409 \\
0904800 \\
0921015 \\
0931815\end{array}$ & $\begin{array}{l}11010014 \\
08040205 \\
08020205 \\
11010014 \\
11010005\end{array}$ \\
\hline $\begin{array}{l}30600 \\
30600 \\
30602 \\
30602 \\
30603\end{array}$ & $\begin{array}{r}3508300924715 \\
350830092471501 \\
362441090344201 \\
362434090350901 \\
\mathbf{3 6 1 7 1 4 0 9 2 3 0 2 5 0 1}\end{array}$ & $\begin{array}{l}\text { CONWAY CO REG WATER DISTRICT } \\
\text { CONWAY CO REG WATER DISTRICT } \\
\text { CORNING WATERWORKS } \\
\text { CORNING WATERWORKS } \\
\text { COTTER WATERWORKS }\end{array}$ & $\begin{array}{l}350830 \\
- \\
362500 \\
362432 \\
361700\end{array}$ & $\begin{array}{l}0924715 \\
0 .- \\
0903000 \\
0903509 \\
0923030\end{array}$ & $\begin{array}{l}11110203 \\
11110203 \\
11010008 \\
11010008 \\
11010006\end{array}$ \\
\hline $\begin{array}{l}30604 \\
30604 \\
30605 \\
30606 \\
30606\end{array}$ & $\begin{array}{r}350028091145601 \\
350028091145401 \\
3437300925230 \\
3622000922700 \\
362200092270001\end{array}$ & $\begin{array}{l}\text { COTTON PLANT WATERWORKS } \\
\text { COTTON PLANT WATERWORKS } \\
\text { COUNTRY INN NURSING HOME } \\
\text { COUNTRY LIFE MOBILE HOME } \\
\text { COUNTRY LIFE MOBILE HOME }\end{array}$ & $\begin{array}{l}350028 \\
350028 \\
343730 \\
362200 \\
-\ldots\end{array}$ & $\begin{array}{l}0911456 \\
0911454 \\
0925230 \\
0922700 \\
--\end{array}$ & $\begin{array}{c}08020302 \\
08020302 \\
08040203 \\
11010003 \\
--\end{array}$ \\
\hline $\begin{array}{l}30607 \\
30608 \\
30610 \\
30610 \\
30611\end{array}$ & $\begin{array}{l}332310094000500 \\
343227091522701 \\
331926092321601 \\
331927092321101 \\
351312090192201\end{array}$ & $\begin{array}{l}\text { COUNTRY MOBILE ESTATES } \\
\text { COY WATERWORKS } \\
\text { CRABAPPLE POINT WATER SYSTEM } \\
\text { CRABAPPLE POINT WATER SYSTEM } \\
\text { CRAWFORDSVILLE WATERWORKS }\end{array}$ & $\begin{array}{l}332310 \\
343228 \\
331926 \\
331927 \\
351413\end{array}$ & $\begin{array}{l}0940005 \\
0915233 \\
0923216 \\
0923211 \\
0981924\end{array}$ & $\begin{array}{l}11140302 \\
08020401 \\
08040201 \\
08040201 \\
08020203\end{array}$ \\
\hline $\begin{array}{l}30612 \\
30613 \\
30613 \\
30613 \\
30613\end{array}$ & $\begin{array}{r}3340380913400 \\
330638091565001 \\
330633091564201 \\
330630091563501 \\
330630091562901\end{array}$ & $\begin{array}{l}\text { CROSS ROADS WATER ASSOC } \\
\text { CROSSETT PUBLIC UTILITIES } \\
\text { CROSSETT PUBLIC UTILITIES } \\
\text { CROSSETT PUBLIC UTILITIES } \\
\text { CROSSETT PUBLIC UTILITIES }\end{array}$ & $\begin{array}{l}334038 \\
330638 \\
330633 \\
330630 \\
330630\end{array}$ & $\begin{array}{l}0913400 \\
0915650 \\
0915642 \\
0915635 \\
0915639\end{array}$ & $\begin{array}{l}08040205 \\
08040202 \\
08040202 \\
08040202 \\
08040202\end{array}$ \\
\hline $\begin{array}{l}30615 \\
30616 \\
30617 \\
30618 \\
30619\end{array}$ & $\begin{array}{r}353330090453001 \\
\mathbf{3 4 3 2 5 0 0 9 3 2 1 3 5} \\
\mathbf{3 5 4 9 4 5 0 9 1 4 1 4 5} \\
\mathbf{3 3 5 3 3 0 0 9 3 5 2 0 0} \\
\mathbf{3 5 0 9 0 0 0 9 3 2 6 1 5}\end{array}$ & $\begin{array}{l}\text { CROWLEYS RIDGE WATER ASSOC } \\
\text { CRYSTAL SPRINGS VILLAGE } \\
\text { CUSHMAN WATER SYSTEM } \\
\text { DALTON MOBILE HOME PARK } \\
\text { DANVILLE WATERWORKS }\end{array}$ & $\begin{array}{l}353330 \\
343250 \\
354945 \\
335330 \\
350900\end{array}$ & $\begin{array}{l}0904530 \\
0932135 \\
0914145 \\
0935200 \\
0932615\end{array}$ & $\begin{array}{l}08020205 \\
08040101 \\
11010004 \\
11140109 \\
11110204\end{array}$ \\
\hline $\begin{array}{l}30620 \\
30621 \\
30621 \\
30622 \\
30623\end{array}$ & $\begin{array}{r}351352093093503 \\
3625000932500 \\
362500093250002 \\
361951094271601 \\
3603000913800\end{array}$ & $\begin{array}{l}\text { DARDANELLE WATERWORKS } \\
\text { DAVIS WATER SYSTEM } \\
\text { DAVIS WATER SYSTEM } \\
\text { DECATUR WATERWORKS } \\
\text { DEER RUN WATER CO }\end{array}$ & $\begin{array}{c}351352 \\
362500 \\
-- \\
362130 \\
360300\end{array}$ & $\begin{array}{c}0930935 \\
0932500 \\
-- \\
0942700 \\
0913800\end{array}$ & $\begin{array}{c}11110203 \\
11010001 \\
-- \\
11070209 \\
11010012\end{array}$ \\
\hline $\begin{array}{l}30626 \\
30628 \\
30629 \\
30629 \\
30629\end{array}$ & $\begin{array}{l}333141091253101 \\
344658091274701 \\
341731091200101 \\
341733091210101 \\
341734091200601\end{array}$ & $\begin{array}{l}\text { DERMOTT WATERWORKS } \\
\text { DEVALLS BLUFF WATERWORKS } \\
\text { DEWITT WATERWORKS } \\
\text { DEWITT WATERWORKS } \\
\text { DEWITT WATERWORKS }\end{array}$ & $\begin{array}{l}333207 \\
344702 \\
341731 \\
341733 \\
341734\end{array}$ & $\begin{array}{l}0912635 \\
0912747 \\
0912001 \\
0912101 \\
0912002\end{array}$ & $\begin{array}{l}08050001 \\
08020301 \\
08020303 \\
08020303 \\
08020303\end{array}$ \\
\hline $\begin{array}{l}30630 \\
30631 \\
30632 \\
30633 \\
30634\end{array}$ & $\begin{array}{r}362702092550301 \\
340839094054001 \\
3604000920700 \\
355323089552101 \\
330932093195601\end{array}$ & $\begin{array}{l}\text { DIAMOND CITY WATER } \\
\text { DIERKS WATERWORKS } \\
\text { DOGPATCH USA } \\
\text { DOGWOOD WATER ASSOC } \\
\text { DORCHEAT WATER }\end{array}$ & $\begin{array}{l}362715 \\
340839 \\
360400 \\
355330 \\
330932\end{array}$ & $\begin{array}{l}0925515 \\
0940540 \\
0920700 \\
0895530 \\
0931956\end{array}$ & $\begin{array}{l}11010003 \\
11140109 \\
11010003 \\
08020204 \\
11140203\end{array}$ \\
\hline $\begin{array}{l}30634 \\
30634 \\
30635 \\
30636 \\
30636\end{array}$ & $\begin{array}{l}331034093175801 \\
330831093215401 \\
352459093074401 \\
335215091293901 \\
335200091290001\end{array}$ & $\begin{array}{l}\text { DORCHEAT WATER } \\
\text { DORCHEAT WATER } \\
\text { DOVER WATERWORKS } \\
\text { DUMAS WATERWORKS } \\
\text { DUMAS WATERWORKS }\end{array}$ & $\begin{array}{l}331034 \\
330831 \\
352459 \\
335215 \\
335309\end{array}$ & $\begin{array}{l}0931758 \\
0932154 \\
0930744 \\
0912939 \\
0913007\end{array}$ & $\begin{array}{l}11140203 \\
11140203 \\
11110202 \\
08050001 \\
08050001\end{array}$ \\
\hline $\begin{array}{l}30636 \\
30636 \\
30637 \\
30638 \\
30639\end{array}$ & $\begin{array}{r}335202091290201 \\
335257091292201 \\
353537090125801 \\
351614090275401 \\
3442300921500\end{array}$ & $\begin{array}{l}\text { DUMAS WATERWORKS } \\
\text { DUMAS WATERWORKS } \\
\text { DYESS WATERWORKS } \\
\text { EARL WATERWORKS } \\
\text { EAST END WATER ASSOC }\end{array}$ & $\begin{array}{l}335314 \\
335257 \\
353530 \\
351614 \\
344230\end{array}$ & $\begin{array}{l}0913022 \\
0912922 \\
0901245 \\
0902717 \\
0921500\end{array}$ & $\begin{array}{l}08050001 \\
08050001 \\
08020203 \\
08020203 \\
11110207\end{array}$ \\
\hline $\begin{array}{l}30640 \\
30642 \\
30642 \\
30642 \\
30643\end{array}$ & $\begin{array}{r}3456150912603 \\
3622000923300 \\
362200092330001 \\
362200092330002 \\
331229092391700\end{array}$ & $\begin{array}{l}\text { EAST PRAIRIE CO WATER ASSOC } \\
\text { EDGEWOOD BAY WATER } \\
\text { EDGEWOOD BAY WATER } \\
\text { EDGEWOOD BAY WATER } \\
\text { EL DORADO WATERWORKS }\end{array}$ & $\begin{array}{c}345615 \\
362200 \\
- \\
- \\
331229\end{array}$ & $\begin{array}{c}0912603 \\
0923300 \\
-- \\
-- \\
0923917\end{array}$ & $\begin{array}{c}08020301 \\
11010003 \\
-- \\
-- \\
08040201\end{array}$ \\
\hline
\end{tabular}




\begin{tabular}{|c|c|c|c|c|c|c|c|}
\hline $\begin{array}{l}\text { Source } \\
\text { type }\end{array}$ & Source & $\begin{array}{l}\text { Pump } \\
\text { Horse- } \\
\text { pomer }\end{array}$ & $\begin{array}{l}\text { descript } \\
\text { Power } \\
\text { source }\end{array}$ & $\begin{array}{l}\text { ion } \\
\text { Pump } \\
\text { tyoe }\end{array}$ & $\begin{array}{l}\text { Well } \\
\text { dia- } \\
\text { meter } \\
\text { (inches) }\end{array}$ & $\begin{array}{c}\text { Depth } \\
\text { of } \\
\text { well } \\
\text { Leet) }\end{array}$ & Local descriptive ID \\
\hline \multirow{2}{*}{$\begin{array}{l}\text { SURFACE } \\
\text { GROUND } \\
\text { GROUND } \\
\text { SURFACE } \\
\text { GROUND }\end{array}$} & $\begin{array}{c}\text { GREERS FERRY } \\
\text { SPARTA SAND }\end{array}$ & $\begin{array}{l}50 \\
25 \\
20\end{array}$ & $\begin{array}{l}\text { ELC } \\
\text { ELC } \\
\text { ELC }\end{array}$ & $\begin{array}{l}\text { STP } \\
\text { STP } \\
\text { STP }\end{array}$ & $\begin{array}{r}12 \\
6 \\
6\end{array}$ & $\begin{array}{l}-2 \\
692 \\
180\end{array}$ & $\begin{array}{l}-- \\
-- \\
--\end{array}$ \\
\hline & GUNTER SANDSTONE & 40 & $\overline{E L C}$ & STP & $-\overline{8}$ & $3, \overline{215}$ & WELL NEAR PUMP HOUSE \\
\hline $\begin{array}{l}\text { GROUND } \\
\text { SURFACE } \\
\text { GROUND } \\
\text { GROUND } \\
\text { GROUND }\end{array}$ & $\begin{array}{l}\text { BREWER LAKE } \\
\text { TERRACE DEPOSITS } \\
\text { QUATERNARY ALLUVIUM } \\
\text { GUNTER SANDSTONE }\end{array}$ & $\begin{array}{r}100 \\
30 \\
30 \\
40\end{array}$ & $\begin{array}{l}\text { ELC } \\
\text { ELC } \\
\text { ELC } \\
\text { ELC }\end{array}$ & $\begin{array}{l}-- \\
\text { STP } \\
\text { STP } \\
\text { STP } \\
\text { STP }\end{array}$ & $\begin{array}{r}-- \\
-8 \\
8 \\
6\end{array}$ & $\begin{array}{l}\cdots \\
\overline{122} \\
110 \\
1,625\end{array}$ & $\begin{array}{l}\text { ABANDONED } \\
\text { IDLE } \\
\text { WELL NO. } 1 \text { / NEAR WATER PLANT } \\
\text { WELL NEAR CYPRESS DITCH }\end{array}$ \\
\hline $\begin{array}{l}\text { GROUND } \\
\text { GROUND } \\
\text { GROUND } \\
\text { GROUND } \\
\text { GROUND }\end{array}$ & $\begin{array}{l}\text { MEMPHIS AQUIFER } \\
\text { MEMPHIS AQUIFER } \\
-- \\
-- \\
--\end{array}$ & $\begin{array}{r}15 \\
15 \\
-- \\
5 \\
5\end{array}$ & $\begin{array}{l}\text { ELC } \\
\text { ELC } \\
--\overline{-} \\
\text { ELC }\end{array}$ & $\begin{array}{l}\text { STP } \\
\text { STP } \\
\text { STP } \\
\text { STP }\end{array}$ & $\begin{array}{r}8 \\
8 \\
-- \\
6 \\
6\end{array}$ & $\begin{array}{l}250 \\
268 \\
-- \\
475 \\
540\end{array}$ & $\begin{array}{r}\text { WELL NO.2 } \\
\text { WELL NO.1 } \\
-- \\
--\end{array}$ \\
\hline $\begin{array}{l}\text { GROUND } \\
\text { GROUND } \\
\text { GROUND } \\
\text { GROUND } \\
\text { GROUND }\end{array}$ & $\begin{array}{l}\text { SPARTA SAND } \\
\text { SPARTA SAND } \\
\text { SPARTA SAND } \\
\text { WILCOX GROUP }\end{array}$ & $\begin{array}{r}-- \\
25 \\
2.5 \\
2.5 \\
--\end{array}$ & $\begin{array}{l}--\overline{1} \\
E L C \\
E L C \\
E L C \\
--\end{array}$ & $\begin{array}{l}-- \\
\text { STP } \\
\text { STP } \\
\text { STP } \\
--\end{array}$ & $\begin{array}{r}-- \\
10 \\
1 \\
1 \\
--\end{array}$ & $\begin{array}{r}-- \\
542 \\
130 \\
130 \\
1,622\end{array}$ & WELL NO. $\overline{2}$ \\
\hline $\begin{array}{l}\text { GROUND } \\
\text { GROUND } \\
\text { GROUND } \\
\text { GROUND } \\
\text { GROUND }\end{array}$ & $\begin{array}{l}\text { PLIOCENE SERIES } \\
\text { PLIOCENE SERIES } \\
\text { PLIOCENE SERIES } \\
\text { PLIOCENE SERIES }\end{array}$ & $\begin{array}{l}-- \\
30 \\
20 \\
30 \\
30\end{array}$ & $\begin{array}{l}\text { ELC } \\
\text { ELC } \\
\text { ELC } \\
\text { ELC }\end{array}$ & $\begin{array}{l}\text { STP } \\
\text { STP } \\
\text { STP } \\
\text { STP }\end{array}$ & $\begin{array}{l}- \\
6 \\
6 \\
6 \\
6\end{array}$ & $\begin{array}{l}-7 \\
182 \\
124 \\
180 \\
170\end{array}$ & 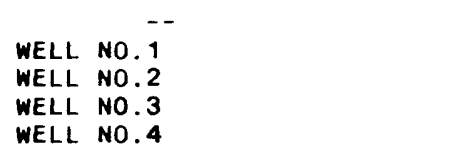 \\
\hline $\begin{array}{l}\text { GROUND } \\
\text { GROUND } \\
\text { SURFACE } \\
\text { GROUND } \\
\text { SURFACE }\end{array}$ & $\begin{array}{l}-- \\
-- \\
-- \\
--\end{array}$ & $\begin{array}{l}50 \\
-- \\
-- \\
.75 \\
--\end{array}$ & $\begin{array}{l}\text { ELC } \\
\overline{--} \\
\text { ELC } \\
--\end{array}$ & $\begin{array}{c}\text { STP } \\
-- \\
-- \\
\text { STP } \\
--\end{array}$ & $\begin{array}{r}10 \\
-- \\
- \\
2 \\
--\end{array}$ & $\begin{array}{l}209 \\
-- \\
-- \\
425 \\
--\end{array}$ & $\begin{array}{c}\text { WELL NEAR PUMP HOUSE - } 40 \mathrm{FT} \\
-- \\
-- \\
--\end{array}$ \\
\hline $\begin{array}{l}\text { GROUND } \\
\text { GROUND } \\
\text { GROUND } \\
\text { GROUND } \\
\text { GROUND }\end{array}$ & $\begin{array}{l}\text { QUATERNARY ALLUVIUM } \\
\text { GUNTER SANDSTONE } \\
---\end{array}$ & $\begin{array}{r}25 \\
1.5 \\
1.5 \\
-10\end{array}$ & $\begin{array}{l}\text { ELC } \\
\text { ELC } \\
\text { ELC } \\
-- \\
\text { ELC }\end{array}$ & $\begin{array}{l}\text { OTH } \\
\text { STP } \\
\text { STP } \\
-- \\
\text { STP }\end{array}$ & $\begin{array}{r}24 \\
6 \\
6 \\
-8\end{array}$ & $\begin{array}{r}65 \\
110 \\
110 \\
1,450 \\
236\end{array}$ & $\begin{array}{l}-- \\
-- \\
-- \\
--\end{array}$ \\
\hline $\begin{array}{l}\text { GROUND } \\
\text { GROUND } \\
\text { GROUND } \\
\text { GROUND } \\
\text { GROUND }\end{array}$ & $\begin{array}{l}\text { COCKFIELD FORMATION } \\
\text { QUATERNARY ALLUVIUM } \\
\text { SPARTA SAND } \\
\text { SPARTA SAND } \\
\text { SPARTA SAND }\end{array}$ & $\begin{array}{r}-- \\
25 \\
125 \\
125\end{array}$ & $\begin{array}{l}-- \\
-:- \\
E L C \\
E L C \\
\text { ELC }\end{array}$ & $\begin{array}{l}-- \\
\text { STP } \\
\text { STP } \\
\text { STP }\end{array}$ & $\begin{array}{l}-- \\
-- \\
8 \\
8 \\
8\end{array}$ & $\begin{array}{l}593 \\
108 \\
160 \\
802 \\
802\end{array}$ & $\begin{array}{ll} & \cdots \\
\text { WELL } & \text { NO.1/SHALLOW WELL } \\
\text { WELL NO.2 } & \\
\text { WELL NO.3 } & \end{array}$ \\
\hline $\begin{array}{l}\text { GROUND } \\
\text { SURFACE } \\
\text { GROUND } \\
\text { GROUND } \\
\text { GROUND }\end{array}$ & $\begin{array}{l}\text { POTOSI DOLOMITE } \\
\text { DIERKS LAKE } \\
-- \\
\text { WILCOX GROUP } \\
--\end{array}$ & $\begin{array}{l}40 \\
40 \\
-- \\
15 \\
--\end{array}$ & $\begin{array}{l}E L C \\
E L C \\
-- \\
E L C \\
--\end{array}$ & $\begin{array}{l}\text { STP } \\
\text { STP } \\
-- \\
\text { STP } \\
--\end{array}$ & $\begin{array}{r}8 \\
8 \\
-- \\
8 \\
--\end{array}$ & $\begin{array}{c}1,415 \\
\ldots \\
1,400 \\
--\end{array}$ & $\begin{array}{c}\text { WELL NO. } 1 \\
-- \\
-- \\
\text { PLANT METER } \\
--\end{array}$ \\
\hline $\begin{array}{l}\text { GROUND } \\
\text { GROUND } \\
\text { SURFACE } \\
\text { GROUND } \\
\text { GROUND }\end{array}$ & $\begin{array}{l}\text { SPARTA SAND } \\
\text { SPARTA SAND } \\
\text { ILLINOIS BAYOU } \\
\text { SPARTA SAND }\end{array}$ & $\begin{array}{r}30 \\
\frac{-}{10} \\
100\end{array}$ & $\begin{array}{l}E L C \\
\overline{-} \\
E L \bar{C} \\
E \bar{C}\end{array}$ & $\begin{array}{c}-- \\
\text { STP } \\
\text { STP }\end{array}$ & $\begin{array}{l}6 \\
-- \\
-- \\
- \\
8\end{array}$ & $\begin{array}{l}450 \\
-- \\
-- \\
-- \\
796\end{array}$ & $\begin{array}{l}-- \\
-- \\
-- \\
-\end{array}$ \\
\hline $\begin{array}{l}\text { GROUND } \\
\text { GROUND } \\
\text { GROUND } \\
\text { GROUND } \\
\text { GROUND }\end{array}$ & $\begin{array}{l}\text { SPARTA SAND } \\
\text { WILCOX GROUP } \\
\text { WILCOX GROUP } \\
-\end{array}$ & $\begin{array}{r}100 \\
100 \\
30 \\
-- \\
--\end{array}$ & $\begin{array}{l}\text { ELC } \\
\text { ELC } \\
\text { ELC } \\
-- \\
--\end{array}$ & $\begin{array}{l}\text { STP } \\
\text { STP } \\
\text { STP } \\
-- \\
--\end{array}$ & $\begin{array}{r}8 \\
8 \\
8 \\
-- \\
--\end{array}$ & $\begin{array}{r}809 \\
950 \\
1,400 \\
1.450 \\
--\end{array}$ & $\begin{array}{l}-- \\
-- \\
-- \\
--\end{array}$ \\
\hline $\begin{array}{l}\text { GROUND } \\
\text { GROUND } \\
\text { GROUND } \\
\text { GROUND } \\
\text { GROUND }\end{array}$ & $\begin{array}{l}\text { QUATERNARY ALLUVIUM } \\
-- \\
-- \\
\text { ROUBIDOUX FORMATION } \\
\text { SPARTA SAND }\end{array}$ & $\begin{array}{r}7.5 \\
10 \\
7.5 \\
10 \\
-.\end{array}$ & $\begin{array}{l}E L C \\
E L C \\
E L C \\
E L C \\
E L C\end{array}$ & $\begin{array}{l}\text { STP } \\
\text { STP } \\
\text { STP } \\
\text { STP } \\
\text { STP }\end{array}$ & $\begin{array}{r}2 \\
6 \\
6 \\
6 \\
--\end{array}$ & $\begin{array}{l}115 \\
380 \\
380 \\
840 \\
-\end{array}$ & $\begin{array}{l}\text { WELL NO. }{ }^{--} \\
\text {WELL NO.1 } \\
\text { WELL NO.3 } \\
\text { CENTROID/WELLS } \quad 10,11,12,15\end{array}$ \\
\hline
\end{tabular}


Table 2.--General description of public-supply

\begin{tabular}{|c|c|c|c|c|c|}
\hline $\begin{array}{c}\text { Water } \\
\text { user } \\
\text { ID } \\
\end{array}$ & $\begin{array}{l}\text { Site } \\
\text { ID }\end{array}$ & Water system name & $\begin{array}{l}\text { Lati- } \\
\text { tude }\end{array}$ & $\begin{array}{l}\text { Longi- } \\
\text { tude }\end{array}$ & $\begin{array}{l}\text { Hydro- } \\
\text { logic } \\
\text { unit } \\
\text { code } \\
\end{array}$ \\
\hline $\begin{array}{l}30643 \\
30643 \\
30643 \\
30643 \\
30643\end{array}$ & $\begin{array}{l}331358092425000 \\
331303092402201 \\
331223092393902 \\
331223092392401 \\
331235092385601\end{array}$ & $\begin{array}{l}\text { EL DORADO WATERWORKS } \\
\text { EL. DORADO WATERWORKS } \\
\text { EL. DORADO WATERWORKS } \\
\text { EL. DORADO WATERWORKS } \\
\text { EL. DORADO WATERWORKS }\end{array}$ & $\begin{array}{l}331358 \\
331303 \\
331227 \\
331223 \\
331236\end{array}$ & $\begin{array}{l}0924250 \\
0924009 \\
0923937 \\
0923923 \\
0923856\end{array}$ & $\begin{array}{l}08040201 \\
08040201 \\
08040201 \\
08040201 \\
08040201\end{array}$ \\
\hline $\begin{array}{l}30643 \\
30643 \\
30643 \\
30643 \\
30643\end{array}$ & $\begin{array}{l}331358092424301 \\
331406092430101 \\
331346092424601 \\
331228092403801 \\
331223092392201\end{array}$ & $\begin{array}{l}\text { EL. DORADO WATERWORKS } \\
\text { EL DORADO WATERWORKS } \\
\text { EL DORADO WATERWORKS } \\
\text { EL DORADO WATERWORKS } \\
\text { EL DORADO WATERWORKS }\end{array}$ & $\begin{array}{l}331358 \\
331407 \\
331349 \\
331228 \\
331237\end{array}$ & $\begin{array}{l}0924248 \\
0924256 \\
0924244 \\
0924038 \\
0923921\end{array}$ & $\begin{array}{l}08040201 \\
08040201 \\
08040201 \\
08040201 \\
08040201\end{array}$ \\
\hline $\begin{array}{l}30643 \\
30644 \\
30645 \\
30645 \\
30645\end{array}$ & $\begin{array}{r}331425092403000 \\
341831090511202 \\
3305450931150 \\
330545093115001 \\
330545093113001\end{array}$ & $\begin{array}{l}\text { EL DORADO WATERWORKS } \\
\text { ELAINE WATERWORKS } \\
\text { EMERSON WATERWORKS } \\
\text { EMERSON WATERWORKS } \\
\text { EMERSON WATERWORKS }\end{array}$ & $\begin{array}{l}331425 \\
341839 \\
330545 \\
330545 \\
330545\end{array}$ & $\begin{array}{l}0924030 \\
0905128 \\
0931150 \\
0931150 \\
0931130\end{array}$ & $\begin{array}{l}08040201 \\
08020303 \\
11140203 \\
11140203 \\
11140203\end{array}$ \\
\hline $\begin{array}{l}30645 \\
30646 \\
30647 \\
30648 \\
30649\end{array}$ & $\begin{array}{r}330545093113003 \\
3343370932812 \\
343246091582302 \\
3514450920915 \\
330645091154401\end{array}$ & $\begin{array}{l}\text { EMERSON WATERWORKS } \\
\text { EMMET WATERWORKS } \\
\text { ENGLAND WATER CO } \\
\text { ENOLA-MT VERNON WATER ASSOC } \\
\text { EUDORA WATERWORKS }\end{array}$ & $\begin{array}{l}330545 \\
334337 \\
343246 \\
351445 \\
330645\end{array}$ & $\begin{array}{l}0931130 \\
0932812 \\
0915825 \\
0920915 \\
0911544\end{array}$ & $\begin{array}{l}11140203 \\
08040103 \\
08020401 \\
11110205 \\
08050001\end{array}$ \\
\hline $\begin{array}{l}30649 \\
30649 \\
30650 \\
30651 \\
30651\end{array}$ & $\begin{array}{r}330646091154401 \\
330640091154103 \\
3603300913630 \\
330657092385901 \\
330631092370801\end{array}$ & $\begin{array}{l}\text { EUDORA WATERWORKS } \\
\text { EUDORA WATERWORKS } \\
\text { EVENING SHADE WATERWORKS } \\
\text { FAIRCREST WATER ASSOC } \\
\text { FAIRCREST WATER ASSOC }\end{array}$ & $\begin{array}{l}330646 \\
330640 \\
360330 \\
330657 \\
330631\end{array}$ & $\begin{array}{l}0911544 \\
0911541 \\
0913630 \\
0923859 \\
0923708\end{array}$ & $\begin{array}{l}08050001 \\
08050001 \\
11010012 \\
08040201 \\
08040201\end{array}$ \\
\hline $\begin{array}{l}30653 \\
30653 \\
30653 \\
30654 \\
30654\end{array}$ & $\begin{array}{l}355200090364501 \\
355234090400501 \\
355200090364503 \\
330324092084501 \\
\mathbf{3 3 0 3 2 4 0 9 2 0 8 4 5 0 2}\end{array}$ & $\begin{array}{l}\text { FARRVILLE-PHILADELPHIA } W \\
\text { FARRVILLE-PHILADELPHIA } \\
\text { FARRVILLE-PHILADELPHIA } \\
\text { FELSENTHAL WATER ASSOC } \\
\text { FELSENTHAL WATER ASSOC }\end{array}$ & $\begin{array}{c}-- \\
355234 \\
-- \\
330327 \\
330324\end{array}$ & $\begin{array}{l}091 \overline{-} \\
0 .- \\
0920905 \\
0920845\end{array}$ & $\begin{array}{l}08020302 \\
08020302 \\
08020302 \\
08040201 \\
08040201\end{array}$ \\
\hline $\begin{array}{l}30655 \\
30656 \\
30657 \\
30657 \\
30657\end{array}$ & $\begin{array}{l}355804092135201 \\
352930090582501 \\
361616092351601 \\
361630092351801 \\
361626092361301\end{array}$ & $\begin{array}{l}\text { FIFTY SIX WATERWORKS } \\
\text { FISHER WATERWORKS } \\
\text { FLIPPIN WATERWORKS } \\
\text { FLIPPIN WATERWORKS } \\
\text { FLIPPIN WATERWORKS }\end{array}$ & $\begin{array}{l}355700 \\
352930 \\
361645 \\
361630 \\
361626\end{array}$ & $\begin{array}{l}0921300 \\
0905825 \\
0923430 \\
0923518 \\
0923613\end{array}$ & $\begin{array}{l}11010004 \\
08020302 \\
11010003 \\
11010003 \\
11010003\end{array}$ \\
\hline $\begin{array}{l}30658 \\
30659 \\
30660 \\
30661 \\
30661\end{array}$ & $\begin{array}{r}3348300922445 \\
334333094235401 \\
3504000922950 \\
350208090475301 \\
350209090474901\end{array}$ & $\begin{array}{l}\text { FORDYCE WATER CO } \\
\text { FOREMAN WATERWORKS } \\
\text { FOREST LAKE ESTATES WATER } \\
\text { FORREST CITY WATERWORKS } \\
\text { FORREST CITY WATERWORKS }\end{array}$ & $\begin{array}{l}334830 \\
334415 \\
350400 \\
350209 \\
\mathbf{3 5 0 2 0 9}\end{array}$ & $\begin{array}{l}0922445 \\
0943200 \\
0922950 \\
0904749 \\
0904749\end{array}$ & $\begin{array}{l}08040201 \\
11140109 \\
11110203 \\
08020205 \\
08020205\end{array}$ \\
\hline $\begin{array}{l}30661 \\
30661 \\
30661 \\
30661 \\
30661\end{array}$ & $\begin{array}{l}350214090480201 \\
350127090472201 \\
350134090472101 \\
350125090472301 \\
350127090472101\end{array}$ & $\begin{array}{l}\text { FORREST CITY WATERWORKS } \\
\text { FORREST CITY WATERWORKS } \\
\text { FORREST CITY WATERWORKS } \\
\text { FORREST CITY WATERWORKS } \\
\text { FORREST CITY WATERWORKS }\end{array}$ & $\begin{array}{l}350214 \\
350127 \\
350134 \\
350132 \\
350214\end{array}$ & $\begin{array}{l}0904802 \\
0904721 \\
0904721 \\
0904721 \\
0904808\end{array}$ & $\begin{array}{l}08020205 \\
08020205 \\
08020205 \\
08020205 \\
08020205\end{array}$ \\
\hline $\begin{array}{l}30661 \\
30661 \\
30663 \\
30663 \\
30666\end{array}$ & $\begin{array}{r}350128090472801 \\
350148090474401 \\
331540093530401 \\
331540093530502 \\
3610000914615\end{array}$ & $\begin{array}{l}\text { FORREST CITY WATERWORKS } \\
\text { FORREST CITY WATERWORKS } \\
\text { FOUKE WATERWORKS } \\
\text { FOUKE WATERWORKS } \\
\text { FRANKLIN WATERWORKS }\end{array}$ & $\begin{array}{c}350128 \\
350208 \\
331540 \\
-- \\
361000\end{array}$ & $\begin{array}{c}0904728 \\
0904753 \\
0935305 \\
- \\
0914615\end{array}$ & $\begin{array}{l}08020205 \\
08020205 \\
11140302 \\
11140302 \\
11010012\end{array}$ \\
\hline $\begin{array}{l}30667 \\
30668 \\
30669 \\
30670 \\
30671\end{array}$ & $\begin{array}{r}3336580934837 \\
3626450935900 \\
3321450934430 \\
361715092291501 \\
3622000935815\end{array}$ & $\begin{array}{l}\text { FULTON WATERWORKS } \\
\text { GARF IELD WATERWORKS } \\
\text { GARLAND WATERWORKS } \\
\text { GASSVILLE WATERWORKS } \\
\text { GATEWAY RURAL WATER ASSOC }\end{array}$ & $\begin{array}{l}333658 \\
362645 \\
332145 \\
361715 \\
362200\end{array}$ & $\begin{array}{l}0934837 \\
0935900 \\
0934430 \\
0922915 \\
0935815\end{array}$ & $\begin{array}{l}11140201 \\
11010001 \\
11140201 \\
11010003 \\
11010001\end{array}$ \\
\hline $\begin{array}{l}30673 \\
30674 \\
30675 \\
30676 \\
30677\end{array}$ & $\begin{array}{r}3610000913700 \\
340711091224801 \\
341238094134601 \\
352450090165201 \\
341930093331501\end{array}$ & $\begin{array}{l}\text { GENTRYVILLE WATER ASSOC } \\
\text { GILLETT WATERWORKS } \\
\text { GILLHAM REG WATER DISTRICT } \\
\text { GILMORE WATERWORKS } \\
\text { GLENWOOD WATER DEPT }\end{array}$ & $\begin{array}{l}361000 \\
340700 \\
341238 \\
352439 \\
341930\end{array}$ & $\begin{array}{l}0913700 \\
0912247 \\
0941346 \\
0901644 \\
0933315\end{array}$ & $\begin{array}{l}11010012 \\
08020402 \\
11140109 \\
08020203 \\
08040102\end{array}$ \\
\hline
\end{tabular}




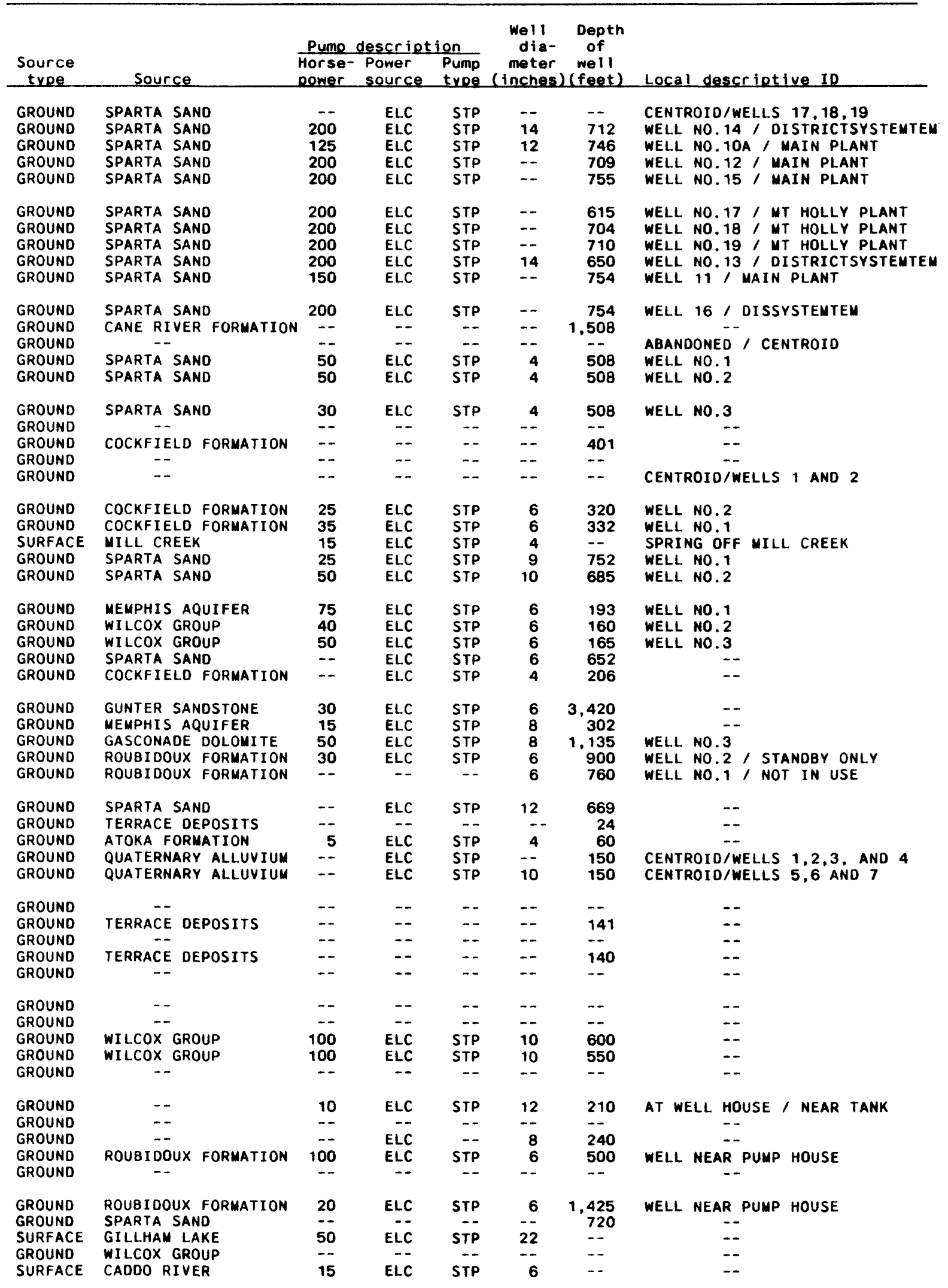


Table 2.--General description of public-supply

\begin{tabular}{|c|c|c|c|c|c|}
\hline $\begin{array}{c}\text { Water } \\
\text { user } \\
\text { ID } \\
\end{array}$ & $\begin{array}{c}\text { Site } \\
\text { ID }\end{array}$ & Water system name & $\begin{array}{l}\text { Lati- } \\
\text { tude }\end{array}$ & $\begin{array}{l}\text { Longi- } \\
\text { tude }\end{array}$ & $\begin{array}{l}\text { Hydro- } \\
\text { logic } \\
\text { unit } \\
\text { code }\end{array}$ \\
\hline $\begin{array}{l}30678 \\
30679 \\
30680 \\
30680 \\
30682\end{array}$ & $\begin{array}{r}355712089580201 \\
335908091333401 \\
340445091414001 \\
3404150914205 \\
3451520915024\end{array}$ & $\begin{array}{l}\text { GOSNELL WATER ASSOC } \\
\text { GOULD WATERWORKS } \\
\text { GRADY WATERWORKS } \\
\text { GRADY WATERWORKS } \\
\text { GRAND PRAIRIE WATER USERS }\end{array}$ & $\begin{array}{l}355715 \\
335910 \\
340445 \\
340415 \\
345152\end{array}$ & $\begin{array}{l}0895815 \\
0913315 \\
0914140 \\
0914205 \\
0915024\end{array}$ & $\begin{array}{l}08020204 \\
08050001 \\
08050001 \\
08050001 \\
08020402\end{array}$ \\
\hline $\begin{array}{l}30684 \\
30684 \\
30685 \\
30685 \\
30686\end{array}$ & $\begin{array}{r}362503094272001 \\
362527094272301 \\
3605000942000 \\
360500094200001 \\
362415093254601\end{array}$ & $\begin{array}{l}\text { GRAVETTE WATERWORKS } \\
\text { GRAVETTE WATERWORKS } \\
\text { GREEN ACRES MOBILE HOME } \\
\text { GREEN ACRES MOBILE HOWE } \\
\text { GREEN FOREST WATERWORKS }\end{array}$ & $\begin{array}{c}362503 \\
362527 \\
360500 \\
-- \\
362415\end{array}$ & $\begin{array}{c}0942720 \\
0942723 \\
0942000 \\
-- \\
0932546\end{array}$ & $\begin{array}{l}11070209 \\
11070209 \\
11010001 \\
11010001 \\
11010001\end{array}$ \\
\hline $\begin{array}{l}30687 \\
30687 \\
30687 \\
30687 \\
30687\end{array}$ & $\begin{array}{l}351413092241100 \\
351411092234300 \\
351421092214800 \\
351331092241400 \\
351031092191900\end{array}$ & $\begin{array}{l}\text { GREENBRIER WATERWORKS } \\
\text { GREENBRIER WATERWORKS } \\
\text { GREENBRIER WATERWORKS } \\
\text { GREENBRIER WATERWORKS } \\
\text { GREENBRIER WATERWORKS }\end{array}$ & $\begin{array}{l}351413 \\
351411 \\
351421 \\
351331 \\
351031\end{array}$ & $\begin{array}{l}0922411 \\
0922343 \\
0922148 \\
0922414 \\
0921919\end{array}$ & $\begin{array}{l}11110205 \\
11110205 \\
11110205 \\
11110205 \\
11110205\end{array}$ \\
\hline $\begin{array}{l}30688 \\
30689 \\
30690 \\
30691 \\
30692\end{array}$ & $\begin{array}{r}362024090132101 \\
351138094150301 \\
3539000910430 \\
3555300915645 \\
3356080930854\end{array}$ & $\begin{array}{l}\text { GREENWAY WATERWORKS } \\
\text { GREENWOOD WATERWORKS } \\
\text { GRUBBS WATERWORKS } \\
\text { GUION WATERWORKS } \\
\text { GURDON WATERWORKS }\end{array}$ & $\begin{array}{l}362030 \\
351138 \\
353900 \\
355530 \\
335608\end{array}$ & $\begin{array}{l}0901315 \\
0941503 \\
0910430 \\
0915645 \\
0930854\end{array}$ & $\begin{array}{l}08020203 \\
11110201 \\
08020302 \\
11010004 \\
08040103\end{array}$ \\
\hline $\begin{array}{l}30692 \\
30692 \\
30692 \\
30692 \\
30693\end{array}$ & $\begin{array}{r}335518093090601 \\
335455093093201 \\
335455093093202 \\
335455093093203 \\
3519000922000\end{array}$ & $\begin{array}{l}\text { GURDON WATERWORKS } \\
\text { GURDON WATERWORKS } \\
\text { GURDON WATERWORKS } \\
\text { GURDON WATERWORKS } \\
\text { GUY WATERWORKS }\end{array}$ & $\begin{array}{l}335518 \\
335440 \\
335500 \\
335445 \\
351900\end{array}$ & $\begin{array}{l}0930906 \\
0930930 \\
0930915 \\
0930855 \\
0922000\end{array}$ & $\begin{array}{l}08040103 \\
08040103 \\
08040103 \\
08040103 \\
11110205\end{array}$ \\
\hline $\begin{array}{l}30694 \\
30695 \\
30696 \\
30697 \\
30699\end{array}$ & $\begin{array}{r}331334091473901 \\
3512150904740 \\
333226092274101 \\
3434300932415 \\
333040092240301\end{array}$ & $\begin{array}{l}\text { HAMBURG WATERWORKS } \\
\text { HAMILTON MOBILE HOME PARK } \\
\text { HAMPTON WATERWORKS } \\
\text { HARBOR EAST INC WATERWORK } \\
\text { HARRELL WATERWORKS }\end{array}$ & $\begin{array}{l}331410 \\
351215 \\
333230 \\
343430 \\
333040\end{array}$ & $\begin{array}{l}0914115 \\
0904740 \\
0922740 \\
0932415 \\
0922410\end{array}$ & $\begin{array}{l}08040205 \\
08020205 \\
08040201 \\
08040101 \\
08040201\end{array}$ \\
\hline $\begin{array}{l}30700 \\
30700 \\
30700 \\
30700 \\
30701\end{array}$ & $\begin{array}{r}3533210904326 \\
353324090432101 \\
353315090432901 \\
353328090432301 \\
361030093071500\end{array}$ & $\begin{array}{l}\text { HARRISBURG WATERWORKS } \\
\text { HARRI SBURG WATERWORKS } \\
\text { HARRISBURG WATERWORKS } \\
\text { HARRISBURG WATERWORKS } \\
\text { HARRISON WATERWORKS }\end{array}$ & $\begin{array}{l}353321 \\
353324 \\
353315 \\
353328 \\
361030\end{array}$ & $\begin{array}{l}0904326 \\
0904321 \\
0904329 \\
0904323 \\
0930715\end{array}$ & $\begin{array}{l}08020205 \\
08020205 \\
08020205 \\
08020205 \\
11010003\end{array}$ \\
\hline $\begin{array}{l}30701 \\
30702 \\
30702 \\
30702 \\
30702\end{array}$ & $\begin{array}{l}361345093053001 \\
330920092451901 \\
343032092393601 \\
343131092380401 \\
343129092380301\end{array}$ & $\begin{array}{l}\text { HARRISON WATERWORKS } \\
\text { HASKELL WATER SYSTEM } \\
\text { HASKELL WATER SYSTEM } \\
\text { HASKELL WATER SYSTEM } \\
\text { HASKELL WATER SYSTEM }\end{array}$ & $\begin{array}{l}361345 \\
330920 \\
343032 \\
343131 \\
343129\end{array}$ & $\begin{array}{l}0930530 \\
0924519 \\
0923936 \\
0923804 \\
0923803\end{array}$ & $\begin{array}{l}11010003 \\
08040203 \\
08040203 \\
08040203 \\
08040203\end{array}$ \\
\hline $\begin{array}{l}30702 \\
30703 \\
30703 \\
30706 \\
30706\end{array}$ & $\begin{array}{r}343131092380402 \\
344732091335900 \\
344730091334800 \\
3431150903525 \\
343115090352501\end{array}$ & $\begin{array}{l}\text { HASKELL WATER SYSTEM } \\
\text { HAZEN WATERWORKS } \\
\text { HAZEN WATERWORKS } \\
\text { HELENA WATERWORKS } \\
\text { HELENA WATERWORKS }\end{array}$ & $\begin{array}{c}343131 \\
344732 \\
344730 \\
343115 \\
--\end{array}$ & $\begin{array}{c}0923804 \\
0913359 \\
0913348 \\
0903525 \\
--\end{array}$ & $\begin{array}{l}08040203 \\
08020303 \\
08020303 \\
08020303 \\
08020303\end{array}$ \\
\hline $\begin{array}{l}30706 \\
30706 \\
30707 \\
30708 \\
30708\end{array}$ & $\begin{array}{r}343115090352502 \\
343116090353101 \\
332658092102601 \\
3515150910615 \\
351043091470601\end{array}$ & $\begin{array}{l}\text { HELENA WATERWORKS } \\
\text { HELENA WATERWORKS } \\
\text { HERMITAGE WATERWORKS } \\
\text { HICKORY RIDGE RURAL WATER } \\
\text { HICKORY RIDGE RURAL WATER }\end{array}$ & $\begin{array}{l}343116 \\
332715 \\
351411 \\
351043\end{array}$ & $\begin{array}{l}0903531 \\
0921015 \\
0910542 \\
0914706\end{array}$ & $\begin{array}{l}08020303 \\
08020303 \\
08040204 \\
08020205 \\
08020205\end{array}$ \\
\hline $\begin{array}{l}30708 \\
30708 \\
30709 \\
30710 \\
30712\end{array}$ & $\begin{array}{r}351045091480201 \\
353243091022101 \\
352403090594901 \\
3616000913115 \\
362937093441101\end{array}$ & $\begin{array}{l}\text { HICKORY RIDGE RURAL WATER } \\
\text { HICKORY RIDGE RURAL WATER } \\
\text { HICKORY RIDGE WATERWORKS } \\
\text { HIGHLAND WATER ASSOC } \\
\text { HOLIDAY ISLAND WATERWORKS }\end{array}$ & $\begin{array}{l}351045 \\
353243 \\
352440 \\
361600 \\
362938\end{array}$ & $\begin{array}{l}0904802 \\
0910221 \\
0905930 \\
0913115 \\
0934411\end{array}$ & $\begin{array}{l}08020205 \\
08020302 \\
08020205 \\
11010010 \\
11010001\end{array}$ \\
\hline $\begin{array}{l}30712 \\
30712 \\
30712 \\
30713 \\
30715\end{array}$ & $\begin{array}{l}362757093434601 \\
362739093434901 \\
362920093463801 \\
343548091121201 \\
335607094204501\end{array}$ & $\begin{array}{l}\text { HOLIDAY ISLAND WATERWORKS } \\
\text { HOLIDAY ISLAND WATERWORKS } \\
\text { HOLIDAY ISLAND WATERWORKS } \\
\text { HOLLY GROVE WATERWORKS } \\
\text { HORATIO WATERWORKS }\end{array}$ & $\begin{array}{l}362815 \\
362739 \\
362920 \\
343548 \\
335615\end{array}$ & $\begin{array}{l}0934302 \\
0934333 \\
0934638 \\
0911208 \\
0942115\end{array}$ & $\begin{array}{l}11010001 \\
11010001 \\
11010001 \\
08020303 \\
11140109\end{array}$ \\
\hline
\end{tabular}




\begin{tabular}{|c|c|c|c|c|c|c|c|}
\hline $\begin{array}{c}\text { Source } \\
\text { tyee }\end{array}$ & Source & $\begin{array}{l}\text { Pumo } \\
\text { Horse- } \\
\text { Rower }\end{array}$ & $\begin{array}{l}\text { descriot } \\
\text { Power } \\
\text { source }\end{array}$ & $\begin{array}{l}\frac{\text { ion }}{\text { Pump }} \\
\text { tyee }\end{array}$ & $\begin{array}{l}\text { We } 11 \\
\text { dia- } \\
\text { meter } \\
\text { inches) }\end{array}$ & $\begin{array}{l}\text { Depth } \\
\text { of } \\
\text { well } \\
2 \text { (feet) }\end{array}$ & Lecal descriotive ID \\
\hline $\begin{array}{l}\text { GROUND } \\
\text { GROUND } \\
\text { GROUND } \\
\text { GROUND } \\
\text { GROUND }\end{array}$ & $\begin{array}{l}\text { WILCOX GROUP } \\
\text { SPARTA SAND } \\
\text { SPARTA SAND } \\
\text { SPARTA SAND } \\
\text { S.- }\end{array}$ & $\begin{array}{l}-- \\
-- \\
-- \\
--\end{array}$ & $\begin{array}{l}-- \\
\text { ELC } \\
\text { ELC } \\
--\end{array}$ & $\begin{array}{l}-- \\
\text { STP } \\
\text { STP } \\
--\end{array}$ & $\begin{array}{l}-- \\
-- \\
-- \\
--\end{array}$ & $\begin{array}{r}1.373 \\
827 \\
816 \\
1,200 \\
---\end{array}$ & $\begin{array}{l}-- \\
-- \\
-- \\
--\end{array}$ \\
\hline $\begin{array}{l}\text { GROUND } \\
\text { GROUND } \\
\text { GROUND } \\
\text { GROUND } \\
\text { SURFACE }\end{array}$ & $\begin{array}{l}\text { GUNTER SANDSTONE } \\
\text { GUNTER SANDSTONE } \\
-- \\
\text { ANDERSON SPRING }\end{array}$ & $\begin{array}{r}75 \\
40 \\
-- \\
-\overline{100}\end{array}$ & $\begin{array}{l}E L C \\
\text { ELC } \\
-\bar{C} \\
\text { ELC }\end{array}$ & $\begin{array}{l}\text { STP } \\
\text { STP } \\
-- \\
\text { STP }\end{array}$ & $\begin{array}{r}6 \\
8 \\
-- \\
-- \\
12\end{array}$ & $\begin{array}{c}1,603 \\
1.600 \\
-- \\
311 \\
--\end{array}$ & $\begin{array}{l}\text { WELL BY FARMER'S SUPPLY } \\
\text { BACKUP WELL ON/ WATER PLANT } \\
\text { WELL NO. } 1 \\
\text { WELL NO. } 2 \\
\text { ANDERSON SPRING }\end{array}$ \\
\hline $\begin{array}{l}\text { GROUND } \\
\text { GROUND } \\
\text { GROUND } \\
\text { GROUND } \\
\text { GROUND }\end{array}$ & $\begin{array}{l}-- \\
-- \\
-- \\
--\end{array}$ & $\begin{array}{l}-- \\
20 \\
20 \\
7.5\end{array}$ & $\begin{array}{l}\text { ELC } \\
\text { ELC } \\
\text { ELC } \\
\text { ELC } \\
\text { ELC }\end{array}$ & $\begin{array}{l}\text { OTH } \\
\text { OTH } \\
\text { OTH } \\
\text { OTH } \\
\text { OTH }\end{array}$ & $\begin{array}{l}6 \\
6 \\
6 \\
6 \\
6\end{array}$ & $\begin{array}{l}480 \\
440 \\
270 \\
615 \\
235\end{array}$ & $\begin{array}{l}\text { NO. } 2 \text { WELL } \\
\text { NO.3 WELL } \\
\text { NO.4 WELL } \\
\text { NO.6 WELL } \\
\text { NO.8 } \\
\text { WELL }\end{array}$ \\
\hline $\begin{array}{l}\text { GROUND } \\
\text { SURFACE } \\
\text { GROUND } \\
\text { GROUND } \\
\text { GROUND }\end{array}$ & $\begin{array}{c}\text { NACATOCH SAND } \\
-- \\
-- \\
-- \\
--\end{array}$ & $\begin{array}{l}-- \\
\overline{10} \\
\overline{20}\end{array}$ & $\begin{array}{l}-- \\
\overline{E L C} \\
\overline{E L C}\end{array}$ & $\begin{array}{c}-- \\
\text { STP } \\
\text { STP }\end{array}$ & $\begin{array}{l}-- \\
-- \\
--\end{array}$ & $\begin{array}{l}992 \\
-- \\
125 \\
-- \\
360\end{array}$ & 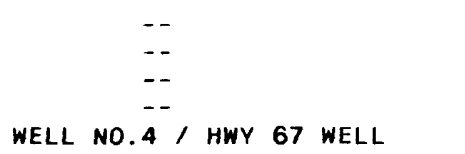 \\
\hline $\begin{array}{l}\text { GROUND } \\
\text { GROUND } \\
\text { GROUND } \\
\text { GROUND } \\
\text { GROUND }\end{array}$ & $\begin{array}{l}\text { NACATOCH SAND } \\
\text { NACATOCH SAND } \\
\text { NACATOCH SAND } \\
--\end{array}$ & $\begin{array}{l}-- \\
20 \\
-- \\
--\end{array}$ & $\begin{array}{l}-- \\
\text { ELC } \\
-- \\
--\end{array}$ & $\begin{array}{l}-- \\
\text { STP } \\
-- \\
--\end{array}$ & $\begin{array}{l}-- \\
8 \\
-- \\
--\end{array}$ & $\begin{array}{l}-- \\
245 \\
245 \\
245 \\
--\end{array}$ & $\begin{array}{l}\text { CENTROID/WELLS } 1,2,3,4 \\
\text { WELL NO.1/ HWY } 53 \text { WELL } \\
-- \\
-- \\
--\end{array}$ \\
\hline $\begin{array}{l}\text { GROUND } \\
\text { GROUND } \\
\text { GROUND } \\
\text { GROUND } \\
\text { GROUND }\end{array}$ & $\begin{array}{l}\text { TERRACE DEPOSITS } \\
\text { SPARTA SAND } \\
\text { SPARTA SAND }\end{array}$ & $\begin{array}{l}-- \\
-- \\
\overline{10} \\
20\end{array}$ & $\begin{array}{l}-- \\
-- \\
\ddot{E L C} \\
\text { ELC }\end{array}$ & $\begin{array}{l}-- \\
-- \\
\text { STP } \\
\text { STP }\end{array}$ & $\begin{array}{l}-- \\
-- \\
-- \\
6 \\
8\end{array}$ & $\begin{array}{l}115 \\
-- \\
613 \\
200 \\
615\end{array}$ & $\begin{array}{c}-- \\
-- \\
-- \\
-- \\
\text { WELL NEAR PUMP HOUSE }\end{array}$ \\
\hline $\begin{array}{l}\text { GROUND } \\
\text { GROUND } \\
\text { GROUND } \\
\text { GROUND } \\
\text { SURFACE }\end{array}$ & $\begin{array}{c}-- \\
\overline{-} \\
\overline{-} \\
\text { JENKINS SPRINGS }\end{array}$ & $\begin{array}{l}-- \\
-- \\
-- \\
50\end{array}$ & $\begin{array}{l}-- \\
-- \\
\overline{-} \\
E L C\end{array}$ & $\begin{array}{l}-- \\
-- \\
-- \\
\text { STP }\end{array}$ & $\begin{array}{l}-- \\
-- \\
-- \\
--\end{array}$ & $\begin{array}{l}-- \\
-- \\
-- \\
--\end{array}$ & $\begin{array}{l}-- \\
-- \\
-- \\
--\end{array}$ \\
\hline $\begin{array}{l}\text { SURFACE } \\
\text { GROUND } \\
\text { GROUND } \\
\text { GROUND } \\
\text { GROUND }\end{array}$ & $\begin{array}{c}\text { MITCHELL SPRGS } \\
-- \\
-- \\
-- \\
--\end{array}$ & $\begin{array}{r}100 \\
15 \\
20 \\
20 \\
20\end{array}$ & $\begin{array}{l}E L C \\
E L C \\
E L C \\
E L C \\
E L C\end{array}$ & $\begin{array}{l}\text { STP } \\
\text { STP } \\
\text { STP } \\
\text { STP } \\
\text { STP }\end{array}$ & $\begin{array}{r}-- \\
6 \\
6 \\
6 \\
6\end{array}$ & $\begin{array}{l}-- \\
200 \\
200 \\
200 \\
200\end{array}$ & $\begin{array}{l}\text { NEW } \\
\text { CENTROID } / 4 \text { WELLS } 4,6,7 \text { AND } 8 \\
\text { WELL NO.4 } \\
\text { WELL NO.6 } 6 \\
\text { ABANDONED / WELL NO.7 }\end{array}$ \\
\hline $\begin{array}{l}\text { GROUND } \\
\text { GROUND } \\
\text { GROUND } \\
\text { GROUND } \\
\text { GROUND }\end{array}$ & $\begin{array}{l}\text { CLAIBORNE GROUP } \\
\text { CLAIBORNE GROUP } \\
\text { SPARTA SAND } \\
\text { SPARTA SAND }\end{array}$ & $\begin{array}{l}15 \\
40 \\
20 \\
80 \\
40\end{array}$ & $\begin{array}{l}\text { ELC } \\
\text { ELC } \\
\text { ELC } \\
\text { ELC } \\
\text { ELC }\end{array}$ & $\begin{array}{l}\text { STP } \\
\text { STP } \\
\text { STP } \\
\text { STP } \\
\text { STP }\end{array}$ & $\begin{array}{r}6 \\
6 \\
8 \\
12 \\
8\end{array}$ & $\begin{array}{l}210 \\
209 \\
165 \\
600 \\
600\end{array}$ & $\begin{array}{l}\text { WELL NO.8 } \\
\text { WELL NO.5 } \\
\text { WELL NO.4 } \\
\text { NO.3 MISSOURI STREET WELL } \\
\text { SOUTH WELL }\end{array}$ \\
\hline $\begin{array}{l}\text { GROUND } \\
\text { GROUND } \\
\text { GROUND } \\
\text { GROUND } \\
\text { GROUND }\end{array}$ & $\begin{array}{l}\text { SPARTA SAND } \\
\text { SPARTA SAND } \\
\text { COCKFIELD FORMATION } \\
\text { QUATERNARY ALLUVIUM } \\
\text { QUATERNARY ALLUVIUM }\end{array}$ & $\begin{array}{l}80 \\
30 \\
-- \\
30 \\
50\end{array}$ & $\begin{array}{l}\text { ELC } \\
\text { ELC } \\
-- \\
\text { ELC } \\
\text { ELC }\end{array}$ & $\begin{array}{l}\text { STP } \\
\text { STP } \\
\text { STP } \\
\text { STP }\end{array}$ & $\begin{array}{r}12 \\
8 \\
-- \\
16 \\
16\end{array}$ & $\begin{array}{l}600 \\
493 \\
349 \\
108 \\
158\end{array}$ & $\begin{array}{c}\text { NO. } 2 \text { BISCOE STREET WELL } \\
\text { NO. } 4 \text { NORTHEAST WELL. } \\
\text { MORTON STATION WELL NO.5 } \\
\text { FITZGERALD CROSSING NO.1 }\end{array}$ \\
\hline $\begin{array}{l}\text { GROUND } \\
\text { GROUND } \\
\text { GROUND } \\
\text { GROUND } \\
\text { GROUND }\end{array}$ & $\begin{array}{l}\text { QUATERNARY ALLUVIUM } \\
\text { QUATERNARY ALLUVIUM } \\
\text { MEMPHIS AQUIFER } \\
-- \\
\text { GUNTER SANDSTONE }\end{array}$ & $\begin{array}{l}50 \\
25 \\
10 \\
30 \\
60\end{array}$ & $\begin{array}{l}\text { ELC } \\
\text { ELC } \\
\text { ELC } \\
\text { ELC } \\
\text { ELC }\end{array}$ & $\begin{array}{l}\text { STP } \\
\text { STP } \\
\text { STP } \\
\text { STP } \\
\text { STP }\end{array}$ & $\begin{array}{r}16 \\
16 \\
6 \\
4 \\
6\end{array}$ & $\begin{array}{r}161 \\
125 \\
400 \\
1,590 \\
1,122\end{array}$ & $\begin{array}{l}\text { FITZGERALD CROSSING NO.3 } \\
\text { PULLIUM STATION NO. } 4 \\
\text { WELL IN PUMP HOUSE } \\
\text { WELL NO. } 2\end{array}$ \\
\hline $\begin{array}{l}\text { GROUND } \\
\text { GROUND } \\
\text { GROUND } \\
\text { GROUND } \\
\text { GROUND }\end{array}$ & $\begin{array}{l}\text { ROUBIDOUX FORMATION } \\
\text { GUNTER SANDSTONE } \\
\text { ROUBIDOUX FORMATION } \\
\text { SPARTA SAND } \\
\text { WOODBINE FORMATION }\end{array}$ & $\begin{array}{r}100 \\
125 \\
75 \\
-- \\
--\end{array}$ & $\begin{array}{l}\text { ELC } \\
\text { ELC } \\
\text { ELC } \\
--\end{array}$ & $\begin{array}{l}\text { STP } \\
\text { STP } \\
\text { STP } \\
\text { STP } \\
--\end{array}$ & $\begin{array}{r}6 \\
8 \\
8 \\
--\end{array}$ & $\begin{array}{r}1,884 \\
1.800 \\
1,058 \\
625 \\
265\end{array}$ & $\begin{array}{rr}\text { WELL. NO.5 } \\
\text { WELL NO.4 } \\
\text { WELL NO.1 } \\
- \\
--\end{array}$ \\
\hline
\end{tabular}


Table 2.--General description of public-supply

\begin{tabular}{|c|c|c|c|c|c|}
\hline $\begin{array}{c}\text { Water } \\
\text { user } \\
\text { ID } \\
\end{array}$ & $\begin{array}{c}\text { Site } \\
\text { ID }\end{array}$ & Water system name & $\begin{array}{r}\text { Lati- } \\
\text { tude }\end{array}$ & $\begin{array}{l}\text { Longi- } \\
\text { tude }\end{array}$ & $\begin{array}{l}\text { Hydro- } \\
\text { logic } \\
\text { unit } \\
\text { code } \\
\end{array}$ \\
\hline $\begin{array}{l}30716 \\
30716 \\
30716 \\
30716 \\
30716\end{array}$ & $\begin{array}{l}361315091449801 \\
361459091463101 \\
361314091453101 \\
361313091454801 \\
361259091453601\end{array}$ & $\begin{array}{l}\text { HORSESHOE BEND WATERWORKS } \\
\text { HORSESHOE BEND WATERWORKS } \\
\text { HORSESHOE BEND WATERWDRKS } \\
\text { HORSESHOE BEND WATERWORKS } \\
\text { HORSESHOE BEND WATERWORKS }\end{array}$ & $\begin{array}{l}361315 \\
361459 \\
361314 \\
361313 \\
361259\end{array}$ & $\begin{array}{l}0914498 \\
0914631 \\
0914531 \\
0914548 \\
0914536\end{array}$ & $\begin{array}{l}11010012 \\
11010012 \\
11010012 \\
11010012 \\
11010012\end{array}$ \\
\hline $\begin{array}{l}30716 \\
30717 \\
30717 \\
30718 \\
30719\end{array}$ & $\begin{array}{r}361232091424201 \\
\mathbf{3 4 4 1 5 7 0 9 2 5 7 2 5 0 0} \\
\mathbf{3 4 4 0 4 0 0 9 2 5 6 4 3 0 0} \\
\mathbf{3 4 3 0 1 8 0 9 3 0 4 4 6} \\
\mathbf{3 6 0 2 5 8 0 9 0 5 7 4 5 0 1}\end{array}$ & $\begin{array}{l}\text { HORSESHOE BEND WATERWORKS } \\
\text { HOT SPRGS VILLAGE WATER } \\
\text { HOT SPRGS VILLAGE WATER } \\
\text { HOT SPRINGS WATERWORKS } \\
\text { HOXIE WATER DEPARTMENT }\end{array}$ & $\begin{array}{l}361232 \\
344157 \\
344040 \\
343018 \\
360258\end{array}$ & $\begin{array}{l}0914242 \\
0925725 \\
0925643 \\
0930446 \\
0905845\end{array}$ & $\begin{array}{l}11010012 \\
08040101 \\
08040101 \\
08040101 \\
11010013\end{array}$ \\
\hline $\begin{array}{l}30719 \\
30720 \\
30721 \\
30722 \\
30723\end{array}$ & $\begin{array}{r}360258090584502 \\
345705090284201 \\
3432370914535 \\
342515091421001 \\
3605000934230\end{array}$ & $\begin{array}{l}\text { HOXIE WATER DEPARIMENT } \\
\text { HUGHES WATERWORKS } \\
\text { HUMNOKE WATERWORKS } \\
\text { HUMPHREY WATERWORKS } \\
\text { HUNTSVILLE WATERWORKS }\end{array}$ & $\begin{array}{l}360258 \\
345715 \\
343237 \\
342518 \\
360500\end{array}$ & $\begin{array}{l}0905845 \\
0902800 \\
0914535 \\
0914217 \\
0934230\end{array}$ & $\begin{array}{l}11010013 \\
08020203 \\
08020401 \\
08020401 \\
11010001\end{array}$ \\
\hline $\begin{array}{l}30724 \\
30724 \\
30725 \\
30726 \\
30727\end{array}$ & $\begin{array}{r}330208092112101 \\
330219092111201 \\
340547092042001 \\
331226092460101 \\
3612150911015\end{array}$ & $\begin{array}{l}\text { HUTTIG WATERWORKS } \\
\text { HUTTIG WATERWORKS } \\
\text { HWY } 15 \text { WATER ASSOC } \\
\text { HWY } 82 \text { WATER ASSOC } \\
\text { IMBODEN WATERWORKS }\end{array}$ & $\begin{array}{l}\mathbf{3} 30208 \\
330219 \\
\mathbf{3 4 0 5 4 5} \\
\mathbf{3 3 1 2 2 6} \\
\mathbf{3 6 1 2 1 5}\end{array}$ & $\begin{array}{l}0921121 \\
0921112 \\
0920423 \\
0924601 \\
0911015\end{array}$ & $\begin{array}{l}08040202 \\
08040202 \\
08040204 \\
08040201 \\
11010010\end{array}$ \\
\hline $\begin{array}{l}30728 \\
30728 \\
30730 \\
30730 \\
30730\end{array}$ & $\begin{array}{r}3437150922845 \\
\mathbf{3 4 3 7 0 9 0 9 2 2 8 5 2 0 1} \\
\mathbf{3 4 4 7 5 5 0 9 2 5 0 4 5 0 1} \\
\mathbf{3 4 4 7 5 5 0 9 2 5 0 4 5 0 2} \\
\mathbf{3 4 4 7 5 5 0 9 2 5 0 4 5 0 3}\end{array}$ & $\begin{array}{l}\text { INOIAN SPRINGS MHP WATERWORKS } \\
\text { INDIAN SPRINGS MHP WATERWORKS } \\
\text { JACKSONVILLE WATER DEPT } \\
\text { JACKSONVILLE WATER DEPT } \\
\text { JACKSONVILLE WATER DEPT }\end{array}$ & $\begin{array}{c}343716 \\
343709 \\
-- \\
-- \\
--\end{array}$ & $\begin{array}{c}0922915 \\
0922852 \\
-- \\
-- \\
--\end{array}$ & $\begin{array}{l}11110207 \\
11110207 \\
08020402 \\
08020402 \\
08020402\end{array}$ \\
\hline $\begin{array}{l}30730 \\
30730 \\
30730 \\
30730 \\
30730\end{array}$ & $\begin{array}{l}344755092504504 \\
344755092504505 \\
344755092504506 \\
344755092504507 \\
344755092504508\end{array}$ & $\begin{array}{l}\text { JACKSONVILLE WATER DEPT } \\
\text { JACKSONVILLE WATER DEPT } \\
\text { JACKSONVILLE WATER DEPT } \\
\text { JACKSONVILLE WATER DEPT } \\
\text { JACKSONVILLE WATER DEPT }\end{array}$ & $\begin{array}{l}-- \\
-- \\
-- \\
--\end{array}$ & $\begin{array}{l}-- \\
-- \\
-- \\
--\end{array}$ & $\begin{array}{l}08020402 \\
08020402 \\
08020402 \\
08020402 \\
08020402\end{array}$ \\
\hline $\begin{array}{l}30730 \\
30730 \\
30730 \\
30731 \\
30732\end{array}$ & $\begin{array}{r}\mathbf{3 4 4 7 5 5 0 9 2 5 0 4 5 0 9} \\
\mathbf{3 4 4 7 5 5 0 9 2 5 0 4 5 1 0} \\
\mathbf{3 4 4 7 5 5 0 9 2 5 0 4 5} \\
\mathbf{3 6 0 0 1 4 0 9 3 1 1 2 9 0 1} \\
\mathbf{3 4 2 3 4 0 0 9 2 1 0 3 5}\end{array}$ & $\begin{array}{l}\text { JACKSONVILLE WATER DEPT } \\
\text { JACKSONVILLE WATER DEPT } \\
\text { JACKSONVILLE WATER DEPT } \\
\text { JASPER WATERWORKS } \\
\text { JEFFERSON-SAMPLES-DEXTER }\end{array}$ & $\begin{array}{c}-- \\
-- \\
-\overline{360015} \\
\mathbf{3 4 2 3 4 0}\end{array}$ & $\begin{array}{c}-- \\
-- \\
0931130 \\
0921035\end{array}$ & $\begin{array}{l}08020402 \\
08020402 \\
08020402 \\
11010005 \\
08040203\end{array}$ \\
\hline $\begin{array}{l}30732 \\
30732 \\
30733 \\
30735 \\
30735\end{array}$ & $\begin{array}{l}342218092095701 \\
342340092103502 \\
353030090090001 \\
330059092430601 \\
330107092432301\end{array}$ & $\begin{array}{l}\text { JEFFERSON-SAMPLES-DEXTER } \\
\text { JEFFERSON-SAMPLES-DEXTER } \\
\text { JOINER WATERWORKS } \\
\text { JUNCTION CITY WATERWORKS } \\
\text { JUNCTION CITY WATERWORKS }\end{array}$ & $\begin{array}{c}342218 \\
-- \\
353030 \\
330110 \\
330057\end{array}$ & $\begin{array}{l}0920957 \\
0900900 \\
0924321 \\
0924327\end{array}$ & $\begin{array}{l}08040203 \\
08040203 \\
08020203 \\
08040206 \\
08040206\end{array}$ \\
\hline $\begin{array}{l}30736 \\
30736 \\
30737 \\
30738 \\
30739\end{array}$ & $\begin{array}{r}354034090055301 \\
354033090055201 \\
334630091170901 \\
3435580920045 \\
335133092174901\end{array}$ & $\begin{array}{l}\text { KEISER WATERWORKS } \\
\text { KEISER WATERWORKS } \\
\text { KELSO-ROHWER WATER ASSOC } \\
\text { KEO WATERWORKS } \\
\text { KINGSLAND WATERWORKS }\end{array}$ & $\begin{array}{c}354015 \\
-- \\
334638 \\
343558 \\
335130\end{array}$ & $\begin{array}{l}0900545 \\
0911715 \\
0920045 \\
0921740\end{array}$ & $\begin{array}{l}08020203 \\
08050002 \\
08020401 \\
08040204\end{array}$ \\
\hline $\begin{array}{l}30741 \\
30742 \\
30743 \\
30744 \\
30744\end{array}$ & $\begin{array}{r}3619150903615 \\
3341450933300 \\
3608300931000 \\
340444091504201 \\
340510091505901\end{array}$ & $\begin{array}{l}\text { KNOBEL WATERWORKS } \\
\text { KOUNTRY KOURTS MHP } \\
\text { KROOKED KREEK WATER ASSOC } \\
\text { LADD WATER ASSOC } \\
\text { LADD WATER ASSOC }\end{array}$ & $\begin{array}{l}361915 \\
334145 \\
360830 \\
340452 \\
340510\end{array}$ & $\begin{array}{l}0903615 \\
0933300 \\
0931000 \\
0915039 \\
0915059\end{array}$ & $\begin{array}{l}08020302 \\
08040103 \\
11010003 \\
08040205 \\
08040205\end{array}$ \\
\hline $\begin{array}{l}30744 \\
30744 \\
30745 \\
30746 \\
30747\end{array}$ & $\begin{array}{r}340631091452001 \\
340354091433501 \\
361118090242201 \\
3625000925245 \\
354857090261201\end{array}$ & $\begin{array}{l}\text { LADD WATER ASSOC } \\
\text { LADD WATER ASSOC } \\
\text { LAFE RURAL WATER ASSOC } \\
\text { LAKE BULL SHOALS EST } \\
\text { LAKE CITY WATERWORKS }\end{array}$ & $\begin{array}{l}340605 \\
340354 \\
361118 \\
362500 \\
354900\end{array}$ & $\begin{array}{l}0914523 \\
0914335 \\
0902422 \\
0925245 \\
0912600\end{array}$ & $\begin{array}{l}08040205 \\
08050001 \\
08020302 \\
11010003 \\
08020203\end{array}$ \\
\hline $\begin{array}{l}30748 \\
30749 \\
30749 \\
30750 \\
30751\end{array}$ & $\begin{array}{r}3622450935145 \\
331953091163801 \\
332104091185701 \\
\mathbf{3 4 2 4 0 3 0 9 0 4 9 1 4 0 1} \\
\mathbf{3 3 4 1 2 9 0 9 3 3 2 5 0}\end{array}$ & $\begin{array}{l}\text { LAKE FOREST SUBORDINATE } \\
\text { LAKE VILLAGE WATERWORKS } \\
\text { LAKE VILLAGE WATERWORKS } \\
\text { LAKEVIEW-WABASH WATER } \\
\text { LAKEWOOD ESTATES MHP }\end{array}$ & $\begin{array}{l}362245 \\
332030 \\
332104 \\
342403 \\
334129\end{array}$ & $\begin{array}{l}0935145 \\
0911855 \\
0911857 \\
0904915 \\
0933250\end{array}$ & $\begin{array}{l}11010001 \\
08050002 \\
08050002 \\
08020303 \\
08040103\end{array}$ \\
\hline
\end{tabular}




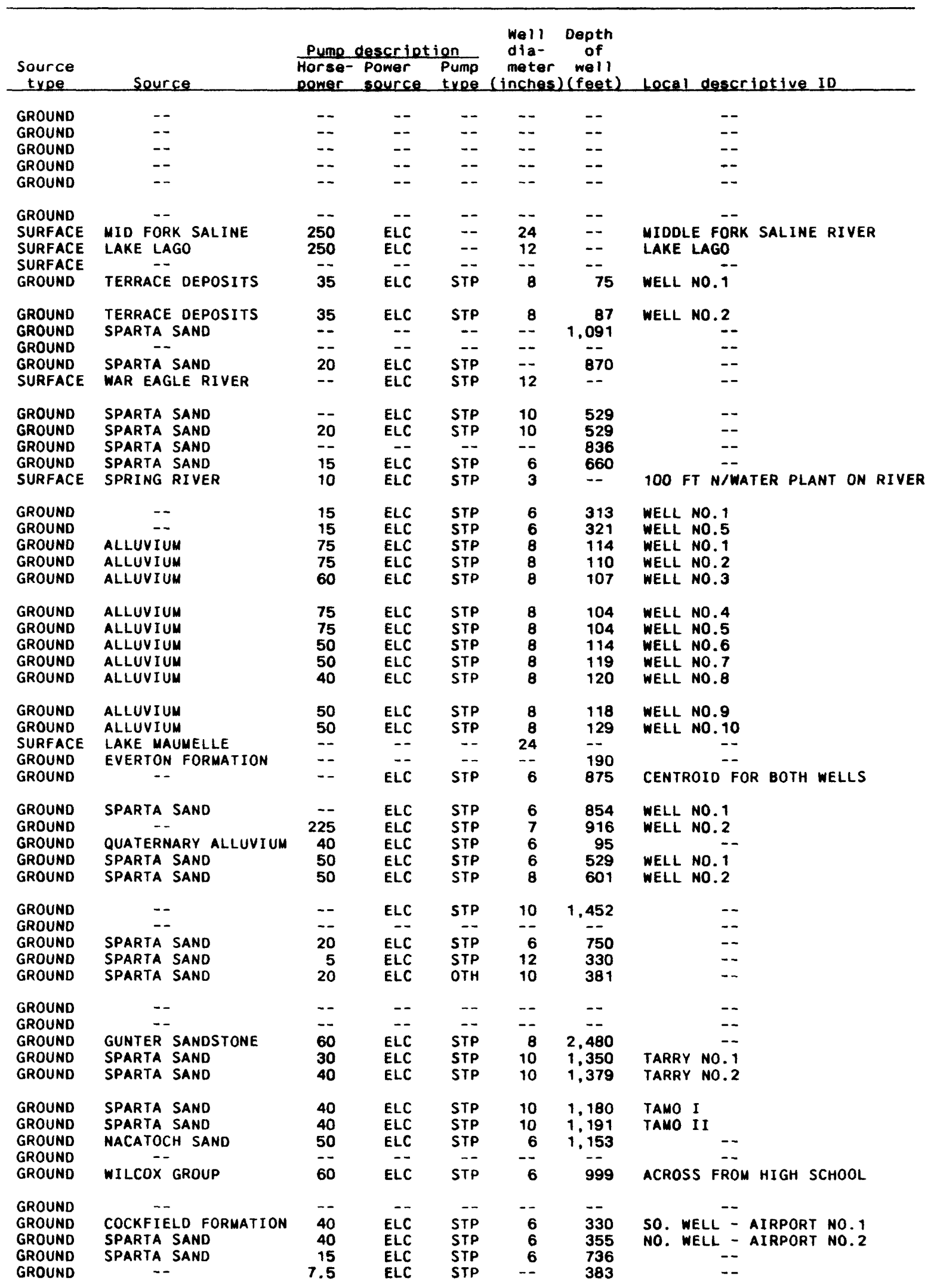


Table 2.--General description of public-supply

\begin{tabular}{|c|c|c|c|c|c|}
\hline $\begin{array}{c}\text { Water } \\
\text { user } \\
\text { ID }\end{array}$ & $\begin{array}{c}\text { Site } \\
\text { ID } \\
\end{array}$ & Water system name & $\begin{array}{l}\text { Lati- } \\
\text { tude } \\
\end{array}$ & $\begin{array}{l}\text { Longi- } \\
\text { tude }\end{array}$ & $\begin{array}{l}\text { Hydro- } \\
\text { logic } \\
\text { unit } \\
\text { code } \\
\end{array}$ \\
\hline $\begin{array}{l}30752 \\
30752 \\
30753 \\
30754 \\
30755\end{array}$ & $\begin{array}{r}331205092291601 \\
331203092290801 \\
355606090152601 \\
362507092545301 \\
3142000941714\end{array}$ & $\begin{array}{l}\text { LAWSON-URBANA WATER ASSOC } \\
\text { LAWSON-URBANA WATER ASSOC } \\
\text { LEACHVILLE WATERWORKS } \\
\text { LEAD HILL WATERWORKS } \\
\text { LEISURE HILLS MHP }\end{array}$ & $\begin{array}{l}331205 \\
331203 \\
355600 \\
362515 \\
314200\end{array}$ & $\begin{array}{l}0922916 \\
0922908 \\
0901515 \\
0925445 \\
0941714\end{array}$ & $\begin{array}{l}08040201 \\
08040201 \\
08020204 \\
11010003 \\
11070208\end{array}$ \\
\hline $\begin{array}{l}30756 \\
30756 \\
30757 \\
30758 \\
30759\end{array}$ & $\begin{array}{r}3623150923100 \\
362315092310001 \\
341024092354501 \\
353629090195501 \\
354924092325401\end{array}$ & $\begin{array}{l}\text { LEISURE HILLS WATERWORKS } \\
\text { LEISURE HILLS WATERWORKS } \\
\text { LEOLA WATERWORKS } \\
\text { LEPANTO WATERWORKS } \\
\text { LESLIE WATERWORKS }\end{array}$ & $\begin{array}{c}362315 \\
-- \\
341025 \\
353630 \\
354930\end{array}$ & $\begin{array}{l}0923100 \\
0923540 \\
0901945 \\
0923330\end{array}$ & $\begin{array}{c}11010003 \\
-- \\
08040203 \\
08020204 \\
11010014\end{array}$ \\
\hline $\begin{array}{l}30760 \\
30760 \\
30762 \\
30763 \\
30765\end{array}$ & $\begin{array}{r}332119093345201 \\
332121093344901 \\
0443630 \\
3541450901400 \\
335806094100102\end{array}$ & $\begin{array}{l}\text { LEWISVILLE WATERWORKS } \\
\text { LEWISVILLE WATERWORKS } \\
\text { LITTLE RIVER COUNTRY CLUB } \\
\text { LITTLE RIVER WATER ASSOC } \\
\text { LOCKESBURG WATERWORKS }\end{array}$ & $\begin{array}{c}332119 \\
332121 \\
-- \\
354145 \\
335806\end{array}$ & $\begin{array}{c}0933452 \\
0933449 \\
-- \\
0901400 \\
0941001\end{array}$ & $\begin{array}{l}11140205 \\
11140205 \\
11140109 \\
08020204 \\
11140109\end{array}$ \\
\hline $\begin{array}{l}30765 \\
30766 \\
30767 \\
30768 \\
30769\end{array}$ & $\begin{array}{r}335804094101101 \\
\mathbf{3 4 4 7 0 1 0 9 1 5 3 5 8 0 2} \\
3624000935430 \\
332330092471701 \\
\mathbf{3 5 4 5 0 0 0 9 3 0 4 3 0}\end{array}$ & $\begin{array}{l}\text { LOCKESBURG WATERWORKS } \\
\text { LONOKE WATERWORKS } \\
\text { LOST BRIDGE VILLAGE WATER } \\
\text { LOUANN WATERWORKS } \\
\text { LURTON-PELSOR WATER ASSOC }\end{array}$ & $\begin{array}{l}335804 \\
344701 \\
362400 \\
332330 \\
354500\end{array}$ & $\begin{array}{l}0941011 \\
0915358 \\
0935430 \\
0924356 \\
0930430\end{array}$ & $\begin{array}{l}11140109 \\
08020402 \\
11010001 \\
08040201 \\
11110202\end{array}$ \\
\hline $\begin{array}{l}30770 \\
30770 \\
30771 \\
30772 \\
30773\end{array}$ & $\begin{array}{r}354527089554901 \\
354528089554701 \\
3000300911500 \\
361041093432601 \\
3316070931200\end{array}$ & $\begin{array}{l}\text { LUXORA WATERWORKS } \\
\text { LUXORA WATERWORKS } \\
\text { LYWN WATERWORKS } \\
\text { MADISON CO WATER ASSOC } \\
\text { MAGNOLIA WATERWORKS }\end{array}$ & $\begin{array}{c}354527 \\
354528 \\
300030 \\
361041 \\
--\end{array}$ & $\begin{array}{c}0895549 \\
0895547 \\
0911500 \\
0934326 \\
--\end{array}$ & $\begin{array}{c}08020203 \\
08020203 \\
11010012 \\
11010001 \\
--\end{array}$ \\
\hline $\begin{array}{l}30773 \\
30773 \\
30773 \\
30773 \\
30773\end{array}$ & $\begin{array}{l}331640093134101 \\
331607093144301 \\
331559093151801 \\
331519093115801 \\
331756093151301\end{array}$ & $\begin{array}{l}\text { MAGNOLIA WATERWORKS } \\
\text { MAGNOLIA WATERWORKS } \\
\text { MAGNOLIA WATERWORKS } \\
\text { MAGNOLIA WATERWORKS } \\
\text { MAGNOLIA WATERWORKS }\end{array}$ & $\begin{array}{l}331640 \\
331607 \\
331559 \\
331519 \\
331756\end{array}$ & $\begin{array}{l}0931341 \\
0931443 \\
0931518 \\
0931158 \\
0931513\end{array}$ & $\begin{array}{l}11140203 \\
11140203 \\
11140203 \\
11140203 \\
11140203\end{array}$ \\
\hline $\begin{array}{l}30773 \\
30773 \\
30773 \\
30775 \\
30776\end{array}$ & $\begin{array}{r}331518093065401 \\
331538093053601 \\
331530093080601 \\
362841091323501 \\
3328550935750\end{array}$ & $\begin{array}{l}\text { MAGNOLIA WATERWORKS } \\
\text { MAGNOLIA WATERWORKS } \\
\text { MAGNOLIA WATERWORKS } \\
\text { MAMMOTH SPRGS WATERWORKS } \\
\text { MANDEVILLE WATER }\end{array}$ & $\begin{array}{l}331518 \\
331538 \\
331530 \\
362900 \\
332855\end{array}$ & $\begin{array}{l}0930654 \\
0930536 \\
0930806 \\
0913200 \\
0935750\end{array}$ & $\begin{array}{l}11140203 \\
11140203 \\
11140203 \\
11010010 \\
11140302\end{array}$ \\
\hline $\begin{array}{l}30776 \\
30777 \\
30778 \\
30779 \\
30779\end{array}$ & $\begin{array}{l}332855093575002 \\
355252090095701 \\
344629090455802 \\
351257090114001 \\
351238090114701\end{array}$ & $\begin{array}{l}\text { MANDEVILLE WATER } \\
\text { MANILA WATERWORKS } \\
\text { MARIANNA WATERWORKS } \\
\text { MARION WATERWORKS } \\
\text { MARI ON WATERWORKS }\end{array}$ & $\begin{array}{l}332855 \\
355300 \\
344629 \\
351257 \\
351238\end{array}$ & $\begin{array}{l}0935750 \\
0901000 \\
0904558 \\
0901140 \\
0901147\end{array}$ & $\begin{array}{l}11140302 \\
08020204 \\
08020304 \\
08020203 \\
08020203\end{array}$ \\
\hline $\begin{array}{l}30780 \\
30781 \\
30781 \\
30782 \\
30784\end{array}$ & $\begin{array}{r}353146090252201 \\
3611150902300 \\
361112090225601 \\
355434092375601 \\
343324090544601\end{array}$ & $\begin{array}{l}\text { MARKED TREE WATERWORKS } \\
\text { MARMADUKE WATERWORKS } \\
\text { MARMADUKE WATERWORKS } \\
\text { MARSHALL WATERWORKS } \\
\text { MARVELL WATERWORKS }\end{array}$ & $\begin{array}{l}353145 \\
361058 \\
361112 \\
355434 \\
343321\end{array}$ & $\begin{array}{l}0902530 \\
0902303 \\
0902256 \\
0923756 \\
0905436\end{array}$ & $\begin{array}{l}08020203 \\
08020203 \\
08020203 \\
11010005 \\
08020304\end{array}$ \\
\hline $\begin{array}{l}30785 \\
30786 \\
30787 \\
30787 \\
30788\end{array}$ & $\begin{array}{r}331351092572701 \\
3450320922600 \\
345409092301201 \\
345413092295101 \\
3457150922745\end{array}$ & $\begin{array}{l}\text { MARYSVILLE WATER ASSOC } \\
\text { MAUMELLE SID NO.5O0 } \\
\text { MAUMELLE WATER CORP } \\
\text { MAUMELLE WATER CORP } \\
\text { MAYFLOWER WATERWORKS }\end{array}$ & $\begin{array}{l}331351 \\
345032 \\
345409 \\
345413 \\
\mathbf{3 4 5 7 1 5}\end{array}$ & $\begin{array}{l}0925727 \\
0922600 \\
0923012 \\
0922951 \\
0922745\end{array}$ & $\begin{array}{l}08040201 \\
11110207 \\
11110207 \\
11110207 \\
11110203\end{array}$ \\
\hline $\begin{array}{l}30789 \\
30790 \\
30791 \\
30792 \\
30793\end{array}$ & $\begin{array}{r}362440090535101 \\
3355080933830 \\
351526091120301 \\
3626150902330 \\
3338030912359\end{array}$ & $\begin{array}{l}\text { MAYNARD WATERWORKS } \\
\text { MCCASKILL WATERWORKS } \\
\text { MCCRORY WATERWORKS } \\
\text { MCDOUGAL WATERWORKS } \\
\text { MCGEHEE WATERWORKS }\end{array}$ & $\begin{array}{l}362445 \\
335508 \\
351510 \\
362615 \\
333803\end{array}$ & $\begin{array}{l}0905345 \\
0933830 \\
0911215 \\
0902330 \\
0912359\end{array}$ & $\begin{array}{l}11010009 \\
08040103 \\
08020302 \\
08020302 \\
08050001\end{array}$ \\
\hline $\begin{array}{l}30793 \\
30793 \\
30794 \\
30795 \\
30795\end{array}$ & $\begin{array}{r}333636091230401 \\
333740091225501 \\
3356000924900 \\
3320500931236 \\
332040093123301\end{array}$ & $\begin{array}{l}\text { MCGEHEE WATERWORKS } \\
\text { MCGEHEE WATERWORKS } \\
\text { MCLAUGHLIN VILLAGE MHP } \\
\text { MCNEIL WATERWORKS } \\
\text { MCNEIL WATERWORKS }\end{array}$ & $\begin{array}{l}333642 \\
333748 \\
335600 \\
332050 \\
332040\end{array}$ & $\begin{array}{l}0912308 \\
0912258 \\
0924900 \\
0931236 \\
0931233\end{array}$ & $\begin{array}{l}08050001 \\
08050001 \\
11140109 \\
11140203 \\
11140203\end{array}$ \\
\hline
\end{tabular}




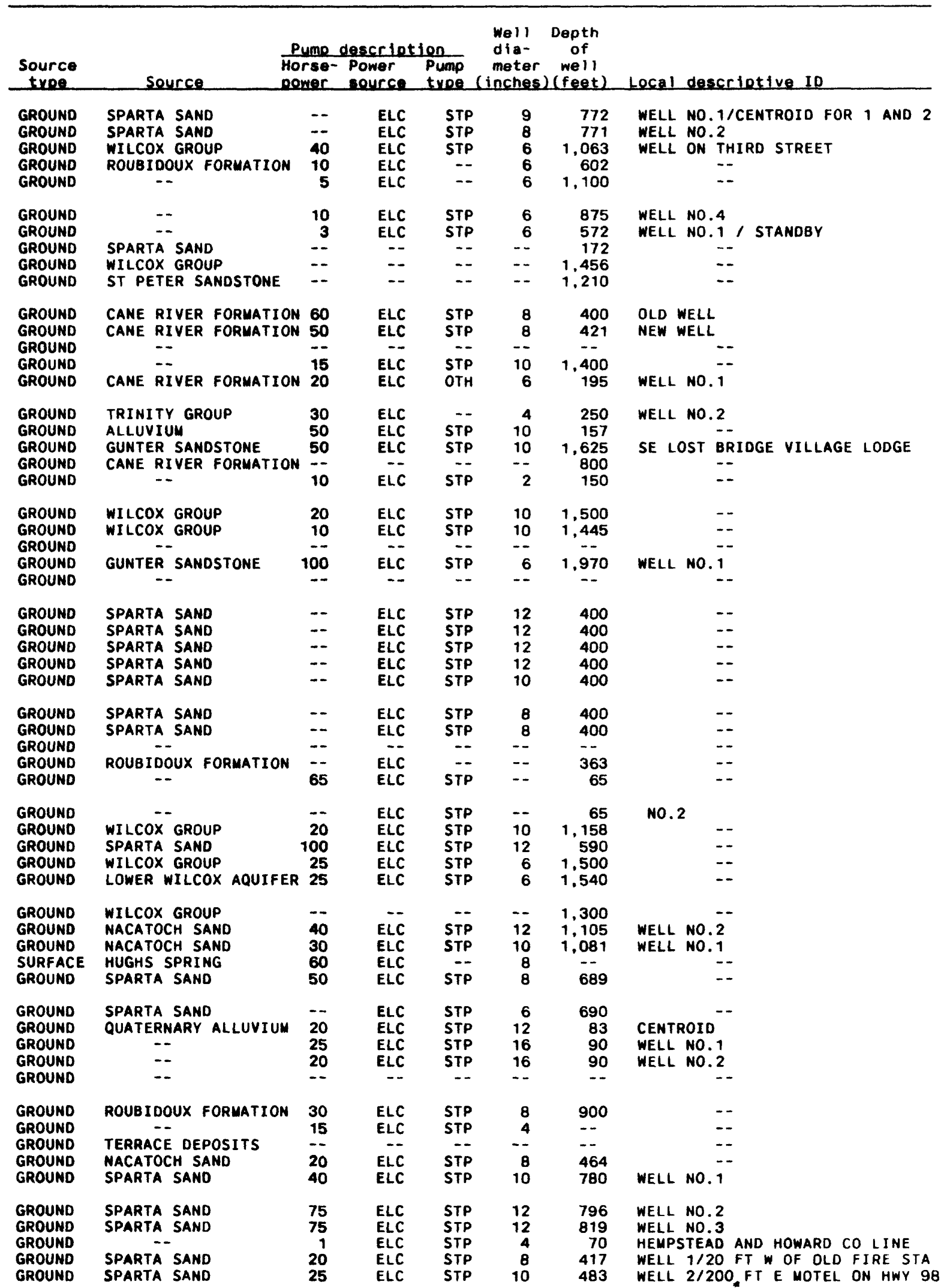


Table 2.--General description of public-supply

\begin{tabular}{|c|c|c|c|c|c|}
\hline $\begin{array}{c}\text { Water } \\
\text { user } \\
\text { ID } \\
\end{array}$ & $\begin{array}{l}\text { Site } \\
\text { ID }\end{array}$ & Water system name & $\begin{array}{l}\text { Lati- } \\
\text { tude }\end{array}$ & $\begin{array}{l}\text { Longi- } \\
\text { tude }\end{array}$ & $\begin{array}{l}\text { Hydro- } \\
\text { logic } \\
\text { unit } \\
\text { code } \\
\end{array}$ \\
\hline $\begin{array}{l}30796 \\
30797 \\
30798 \\
30798 \\
30800\end{array}$ & $\begin{array}{r}350641091492801 \\
3321080931138 \\
360333091535501 \\
360338091531701 \\
3508360923300\end{array}$ & $\begin{array}{l}\text { MCRAE WATERWORKS } \\
\text { MCNEIL RURAL WATER SYSTEM } \\
\text { MELBOURNE WATERWORKS } \\
\text { MELBOURME WATERWORKS } \\
\text { MENIFEE WATER DEPARTMENT }\end{array}$ & $\begin{array}{l}360645 \\
332108 \\
360333 \\
360338 \\
350836\end{array}$ & $\begin{array}{l}0904900 \\
0931138 \\
0915355 \\
0915317 \\
0923300\end{array}$ & $\begin{array}{l}08020301 \\
11140203 \\
11010004 \\
11010004 \\
11110203\end{array}$ \\
\hline $\begin{array}{l}30801 \\
30802 \\
30804 \\
30805 \\
30806\end{array}$ & $\begin{array}{r}350519090181001 \\
3615450913915 \\
3354200912953 \\
355722093093401 \\
355314090010801\end{array}$ & $\begin{array}{l}\text { MIOWAY WATER ASSOC } \\
\text { MIMOSA EST S/D WATERWORKS } \\
\text { MITCHELLVILLE WATERWORKS } \\
\text { MOCKINGBIRD HILL WATER } \\
\text { MONETTE WATERWORKS }\end{array}$ & $\begin{array}{l}350522 \\
361545 \\
335420 \\
355600 \\
355314\end{array}$ & $\begin{array}{l}0901801 \\
0913915 \\
0912953 \\
0931000 \\
0902108\end{array}$ & $\begin{array}{l}08020203 \\
11010012 \\
08050001 \\
11110202 \\
08020203\end{array}$ \\
\hline $\begin{array}{l}30807 \\
30807 \\
30807 \\
30807 \\
30807\end{array}$ & $\begin{array}{l}333808091454200 \\
333700091444800 \\
333751091470902 \\
333743091473000 \\
333534091485201\end{array}$ & $\begin{array}{l}\text { MONTICELLO WATER DEPT } \\
\text { MONTICELLO WATER DEPT } \\
\text { MONTICELLO WATER DEPT } \\
\text { MONTICELLO WATER DEPT } \\
\text { MONTICELLO WATER DEPT }\end{array}$ & $\begin{array}{l}333808 \\
333700 \\
333758 \\
333743 \\
333534\end{array}$ & $\begin{array}{l}0914542 \\
0914448 \\
0914659 \\
0914730 \\
0914852\end{array}$ & $\begin{array}{l}08040205 \\
08040205 \\
08040205 \\
08040205 \\
08040205\end{array}$ \\
\hline $\begin{array}{l}30808 \\
30809 \\
30809 \\
30810 \\
30811\end{array}$ & $\begin{array}{r}3559000923445 \\
331808092563801 \\
331805092570902 \\
3434000933915 \\
3556300930415\end{array}$ & $\begin{array}{l}\text { MORNING STAR WATER ASSOC } \\
\text { MOUNT HOLLY WATERWORKS } \\
\text { MOUNT HOLLY WATERWORKS } \\
\text { MOUNT IDA WATERWORKS } \\
\text { MOUNT JUDEA WATERWORKS }\end{array}$ & $\begin{array}{l}355900 \\
331808 \\
\mathbf{3 3 1 8 0 5} \\
\mathbf{3 4 3 4 0 0} \\
\mathbf{3 5 5 6 3 0}\end{array}$ & $\begin{array}{l}0923445 \\
0925638 \\
0925709 \\
0933915 \\
0930415\end{array}$ & $\begin{array}{l}11010005 \\
08040201 \\
08040201 \\
08040101 \\
11010005\end{array}$ \\
\hline $\begin{array}{l}30812 \\
30813 \\
30815 \\
30816 \\
30818\end{array}$ & $\begin{array}{r}362402092194400 \\
3434400931035 \\
3557450912630 \\
3605000932500 \\
3623150935145\end{array}$ & $\begin{array}{l}\text { MOUNTAIN HOME WATERWORKS } \\
\text { MOUNTAIN PINE WATERWORKS } \\
\text { MT PLEASANT WATERWORKS } \\
\text { MT SHERMAN WATER ASSOC } \\
\text { MUNDELL HGTS WATERWORKS }\end{array}$ & $\begin{array}{l}362402 \\
343440 \\
355745 \\
360500 \\
362315\end{array}$ & $\begin{array}{l}0921944 \\
0931035 \\
0912630 \\
0932500 \\
0935145\end{array}$ & $\begin{array}{l}11010006 \\
08040101 \\
11010004 \\
11010005 \\
11010001\end{array}$ \\
\hline $\begin{array}{l}30820 \\
30822 \\
30822 \\
30823 \\
30823\end{array}$ & 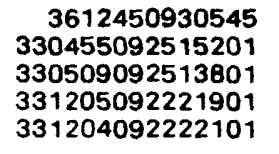 & $\begin{array}{l}\text { MYERS WATER SYSTEM } \\
\text { NEW HOPE WATER ASSOC } \\
\text { NEW HOPE WATER ASSOC } \\
\text { NEW LONDON WATER ASSOC } \\
\text { NEW LONDON WATER ASSOC }\end{array}$ & $\begin{array}{l}361245 \\
330455 \\
330509 \\
331203 \\
331204\end{array}$ & $\begin{array}{l}0930545 \\
0925152 \\
0925138 \\
0922218 \\
0922221\end{array}$ & $\begin{array}{l}11010003 \\
08040201 \\
08040201 \\
08040201 \\
08040201\end{array}$ \\
\hline $\begin{array}{l}30824 \\
30825 \\
30825 \\
30825 \\
30826\end{array}$ & $\begin{array}{r}354118091263701 \\
3539000911145 \\
353900091114501 \\
353900091114502 \\
353601091165401\end{array}$ & $\begin{array}{l}\text { NEWARK WATERWORKS } \\
\text { NEWPORT IND PARK WATERWORKS } \\
\text { NEWPORT IND PARK WATERWORKS } \\
\text { NEWPORT IND PARK WATERWORKS } \\
\text { NEWPORT WATERWORKS }\end{array}$ & $\begin{array}{c}354145 \\
353900 \\
-- \\
-- \\
353601\end{array}$ & $\begin{array}{c}0912630 \\
0911145 \\
-- \\
-- \\
0911654\end{array}$ & $\begin{array}{c}11010004 \\
11010013 \\
-- \\
-- \\
11010013\end{array}$ \\
\hline $\begin{array}{l}30826 \\
30826 \\
30826 \\
30826 \\
30826\end{array}$ & $\begin{array}{l}353617091162801 \\
353617091162802 \\
353617091162803 \\
353608091164101 \\
353616091162801\end{array}$ & $\begin{array}{l}\text { NEWPORT WATERWORKS } \\
\text { NEWPORT WATERWORKS } \\
\text { NEWPORT WATERWORKS } \\
\text { NEWPORT WATERWORKS } \\
\text { NEWPORT WATERWORKS }\end{array}$ & $\begin{array}{l}353617 \\
353617 \\
353617 \\
353608 \\
353616\end{array}$ & $\begin{array}{l}0911628 \\
0911628 \\
0911628 \\
0911641 \\
0911628\end{array}$ & $\begin{array}{l}11010013 \\
11010013 \\
11010013 \\
11010013 \\
11010013\end{array}$ \\
\hline $\begin{array}{l}30827 \\
30827 \\
30828 \\
30829 \\
30830\end{array}$ & $\begin{array}{r}3613000921800 \\
361300092180001 \\
361237092164501 \\
3428150934045 \\
331900092395602\end{array}$ & $\begin{array}{l}\text { MORFORK VILLAGE WATERWORKS } \\
\text { NORFORK VILLAGE WATERWORKS } \\
\text { NORFORK WATERWORKS } \\
\text { NORWAN WATERWORKS } \\
\text { NORPHLET WATERWORKS }\end{array}$ & $\begin{array}{l}361300 \\
-- \\
361230 \\
342815 \\
331900\end{array}$ & $\begin{array}{l}0921800 \\
0921700 \\
0934045 \\
0923956\end{array}$ & $\begin{array}{l}11010004 \\
-- \\
11010004 \\
08040102 \\
08040201\end{array}$ \\
\hline $\begin{array}{l}30830 \\
30831 \\
30832 \\
30834 \\
30835\end{array}$ & $\begin{array}{r}331842092395001 \\
3310000925627 \\
3510150904830 \\
096074 \\
3437500934700\end{array}$ & $\begin{array}{l}\text { NORPHLET WATERWORKS } \\
\text { NORTH CROSSETT WATERWORKS } \\
\text { O'KEAN WATERWORKS } \\
\text { OAKWOOD MHP WATERWORKS } \\
\text { ODEN-PENCIL BLUFF WATER }\end{array}$ & $\begin{array}{c}331842 \\
331000 \\
351015 \\
-- \\
343750\end{array}$ & $\begin{array}{c}0923950 \\
0925627 \\
0904830 \\
-- \\
0934700\end{array}$ & $\begin{array}{l}08040201 \\
08040202 \\
08020302 \\
08020402 \\
08040101\end{array}$ \\
\hline $\begin{array}{l}30836 \\
30836 \\
30836 \\
30837 \\
30838\end{array}$ & $\begin{array}{r}3335150940230 \\
333511094024001 \\
333507094025101 \\
3538000912745 \\
335910093195301\end{array}$ & $\begin{array}{l}\text { OGDEN WATERWORKS } \\
\text { OGDEN WATERWORKS } \\
\text { OGDEN WATERWORKS } \\
\text { OIL TROUGH WATERWORKS } \\
\text { OKOLONA WATERWORKS }\end{array}$ & $\begin{array}{l}333515 \\
-- \\
333507 \\
353800 \\
340100\end{array}$ & $\begin{array}{c}0940230 \\
-- \\
0940251 \\
0912745 \\
0932208\end{array}$ & $\begin{array}{c}11140106 \\
-- \\
11140106 \\
11010004 \\
08040103\end{array}$ \\
\hline $\begin{array}{l}30839 \\
30840 \\
30841 \\
30842 \\
30842\end{array}$ & $\begin{array}{r}3501450931215 \\
362708093111301 \\
3507000924600 \\
3542190895805 \\
354220089580901\end{array}$ & $\begin{array}{l}\text { OLA WATERWORKS } \\
\text { OMAHA WATERWORKS } \\
\text { OPPELO WATER DEPT } \\
\text { OSCEOLA WATERWORKS } \\
\text { OSCEOLA WATERWORKS }\end{array}$ & $\begin{array}{l}350145 \\
362700 \\
350700 \\
354219 \\
354218\end{array}$ & $\begin{array}{l}0931215 \\
0931130 \\
0924600 \\
0895805 \\
0895808\end{array}$ & $\begin{array}{l}11110204 \\
11010003 \\
11110203 \\
08020203 \\
08020203\end{array}$ \\
\hline
\end{tabular}




\begin{tabular}{|c|c|c|c|c|c|c|c|}
\hline $\begin{array}{c}\text { Source } \\
\text { tyoe }\end{array}$ & Source & $\begin{array}{l}\text { Pumo d } \\
\text { Horse- } \\
\text { Domer }\end{array}$ & $\begin{array}{l}\text { escrioti } \\
\text { Power } \\
\text { source }\end{array}$ & $\begin{array}{l}\text { on } \\
\text { Pump } \\
\text { tyoe }\end{array}$ & $\begin{array}{l}\text { Well } \\
\text { dia- } \\
\text { meter } \\
\text { (inches) }\end{array}$ & $\begin{array}{c}\text { Depth } \\
\text { of } \\
\text { well } \\
\mathcal{L} \text { (feet) }\end{array}$ & Local descrietive ID \\
\hline $\begin{array}{l}\text { GROUND } \\
\text { GROUND } \\
\text { GROUND } \\
\text { SURFACE } \\
\text { GROUND }\end{array}$ & $\begin{array}{l}\text { QUATERMARY ALLUVIUM } \\
-- \\
\text { ARNOLD AND TATE SPG } \\
--\end{array}$ & $\begin{array}{l}-- \\
-- \\
40 \\
--\end{array}$ & $\begin{array}{r}-- \\
\text { ELC } \\
\text { ELC } \\
--\end{array}$ & $\begin{array}{r}-- \\
-- \\
\text { STP } \\
--\end{array}$ & $\begin{array}{r}-- \\
-- \\
4 \\
--\end{array}$ & $\begin{array}{c}89 \\
1,400 \\
---\end{array}$ & $\begin{array}{ll} & -- \\
\text { WELL } & -- \\
\text { ARNOLD AND TATE SPRING }\end{array}$ \\
\hline $\begin{array}{l}\text { GROUND } \\
\text { GROUND } \\
\text { GROUND } \\
\text { GROUND } \\
\text { GROUND }\end{array}$ & $\begin{array}{l}\text { WILCOX GROUP } \\
\text { ROUBIDOUX FORMATION } \\
\text { WILCOX GROUP }\end{array}$ & $\begin{array}{r}-- \\
150 \\
-- \\
25\end{array}$ & $\begin{array}{l}--\overline{-} \\
E L C \\
E L C \\
E L C\end{array}$ & $\begin{array}{r}-- \\
\text { STP } \\
-- \\
\text { STP }\end{array}$ & $\begin{array}{l}-- \\
-- \\
-- \\
10\end{array}$ & $\begin{array}{r}1,541 \\
240 \\
850 \\
3,142 \\
1,034\end{array}$ & $\begin{array}{l}\because- \\
\because- \\
\cdots\end{array}$ \\
\hline $\begin{array}{l}\text { GROUND } \\
\text { GROUND } \\
\text { GROUND } \\
\text { GROUND } \\
\text { GROUND }\end{array}$ & $\begin{array}{l}\text { SPARTA SAND } \\
\text { SPARTA SAND } \\
\text { SPARTA SAND } \\
\text { SPARTA SAND }\end{array}$ & $\begin{array}{r}150 \\
150 \\
150 \\
150 \\
75\end{array}$ & $\begin{array}{l}\text { ELC } \\
\text { ELC } \\
\text { ELC } \\
\text { ELC }\end{array}$ & $\begin{array}{l}\text { STP } \\
\text { STP } \\
\text { STP } \\
\text { STP } \\
\text { STP }\end{array}$ & $\begin{array}{r}10 \\
8 \\
8 \\
8 \\
6\end{array}$ & $\begin{array}{l}779 \\
844 \\
880 \\
842 \\
718\end{array}$ & $\begin{array}{l}\text { WELL NO.6 } \\
\text { WELL NO.5 } \\
\text { WELL NO.4 } \\
\text { WELL NO.3 } \\
\text { WELL NO.7 }\end{array}$ \\
\hline $\begin{array}{l}\text { GROUND } \\
\text { GROUND } \\
\text { GROUND } \\
\text { SURFACE } \\
\text { GROUND }\end{array}$ & $\begin{array}{l}\text { SPARTA SAND } \\
\text { SPARTA SAND } \\
\text { OUACHITA RIVER }\end{array}$ & $\begin{array}{l}140 \\
-- \\
30 \\
--\end{array}$ & $\begin{array}{l}\text { ELC } \\
\text { ELC } \\
\text { ELC } \\
\text { ELC } \\
--\end{array}$ & $\begin{array}{l}-2 \\
\text { STP } \\
\text { STP } \\
\text { STP } \\
--\end{array}$ & $\begin{array}{r}8 \\
8 \\
4 \\
6 \\
--\end{array}$ & $\begin{array}{r}2,900 \\
480 \\
465 \\
-\therefore\end{array}$ & $\begin{array}{c}61 / 2 \text { MI N MARSHALL, ARK } \\
-- \\
\text { NEAR SO FORK } 270 \text { BRIDGE } \\
\text {-- }\end{array}$ \\
\hline $\begin{array}{l}\text { SURFACE } \\
\text { GROUND } \\
\text { GROUND } \\
\text { GROUND } \\
\text { GROUND }\end{array}$ & $\begin{array}{c}\text { LAKE NORFORK } \\
-- \\
-- \\
-- \\
--\end{array}$ & $\begin{array}{r}400 \\
-- \\
20 \\
--\end{array}$ & $\begin{array}{l}\text { ELC } \\
-\overline{-} \\
\text { ELC } \\
--\end{array}$ & $\begin{array}{c}\text { STP } \\
\text { STP } \\
-- \\
--\end{array}$ & $\begin{array}{r}8 \\
-7 \\
-- \\
--\end{array}$ & $\begin{array}{l}-- \\
- \\
35 \\
--\end{array}$ & $\begin{array}{l}-- \\
-- \\
-- \\
--\end{array}$ \\
\hline $\begin{array}{l}\text { GROUND } \\
\text { GROUND } \\
\text { GROUND } \\
\text { GROUND } \\
\text { GROUND }\end{array}$ & $\begin{array}{l}\text { SPARTA SAND } \\
\text { SPARTA SAND } \\
\text { SPARTA SAND } \\
\text { SPARTA SAND }\end{array}$ & $\begin{array}{l}-- \\
-- \\
-- \\
--\end{array}$ & $\begin{array}{l}\text { ELC } \\
\text { ELC } \\
\text { ELC } \\
\text { ELC }\end{array}$ & $\begin{array}{l}\text { STP } \\
\text { STP } \\
\text { STP } \\
\text { STP }\end{array}$ & $\begin{array}{r}-- \\
6 \\
6 \\
9 \\
9\end{array}$ & $\begin{array}{l}-- \\
595 \\
586 \\
822 \\
828\end{array}$ & $\begin{array}{l}\text { WELL NO.1/CENTROIO FOR } 1 \text { AND } \\
\text { WELL NO. } 2 \\
\text { WELL. NO.1/CENTROIO FOR } 1 \text { AND } \\
\text { WELL NO.2 }\end{array}$ \\
\hline $\begin{array}{l}\text { GROUND } \\
\text { GROUND } \\
\text { GROUND } \\
\text { GROUND } \\
\text { GROUND }\end{array}$ & $\begin{array}{l}\text { ALLUVIUM } \\
\text { ALLUVIUM } \\
\text { ALLUVIUM } \\
\text { QUATERNARY ALLUVIUM }\end{array}$ & $\begin{array}{l}-- \\
-- \\
25 \\
10 \\
20\end{array}$ & $\begin{array}{l}-- \\
\text { ELC } \\
\text { ELC } \\
\text { ELC }\end{array}$ & $\begin{array}{l}-- \\
\text { STP } \\
\text { STP } \\
\text { STP } \\
\text { STP }\end{array}$ & $\begin{array}{l}-- \\
10 \\
10 \\
10 \\
10\end{array}$ & $\begin{array}{l}45 \\
90 \\
90 \\
90 \\
90\end{array}$ & $\begin{array}{l}\text { CENTROID/WELLS } 1 \text { AND } 2 \\
-- \\
\text { WELL NO. } 1\end{array}$ \\
\hline $\begin{array}{l}\text { GROUND } \\
\text { GROUND } \\
\text { GROUND } \\
\text { GROUND } \\
\text { GROUND }\end{array}$ & $\begin{array}{l}\text { QUATERNARY ALLUVIUM } \\
\text { QUATERNARY ALLUVIUM } \\
\text { QUATERNARY ALLUVIUM } \\
-- \\
\text { QUATERNARY ALLUVIUM }\end{array}$ & $\begin{array}{l}30 \\
30 \\
30 \\
- \\
40\end{array}$ & $\begin{array}{l}\text { ELC } \\
\text { ELC } \\
\text { ELC } \\
\text { ELC }\end{array}$ & $\begin{array}{l}\text { STP } \\
\text { STP } \\
\text { STP } \\
\text { STP }\end{array}$ & $\begin{array}{l}10 \\
\overline{12} \\
-- \\
--\end{array}$ & $\begin{array}{r}103 \\
100 \\
90 \\
-- \\
94\end{array}$ & $\begin{array}{l}\text { WELL NO.3 } \\
\text { WELL. NO.4 } \\
\text { WELL. NO.5 } \\
\text { CENTROID/WELLS } 1,2,3,4,5 \\
\text { WELL NO.2 }\end{array}$ \\
\hline $\begin{array}{l}\text { GROUND } \\
\text { GROUND } \\
\text { GROUND } \\
\text { SURFACE } \\
\text { GROUND }\end{array}$ & $\begin{array}{l}\text { ROUBIDOUX FORMATION } \\
\text { ROUBIDOUX FORMATION } \\
\text { GUNTER SANDSTONE } \\
\text { CADDO RIVER } \\
\text { SPARTA SAND }\end{array}$ & $\begin{array}{r}3 \\
3 \\
15 \\
-- \\
40\end{array}$ & $\begin{array}{l}\text { ELC } \\
\text { ELC } \\
\text { ELC } \\
\text { ELC } \\
\text { ELC }\end{array}$ & $\begin{array}{l}\text { STP } \\
\text { STP } \\
\text { STP } \\
\text { STP } \\
\text { STP }\end{array}$ & $\begin{array}{r}6 \\
6 \\
8 \\
-6 \\
6\end{array}$ & $\begin{array}{r}240 \\
178 \\
1.507 \\
- \\
603\end{array}$ & $\begin{array}{l}\text { NORTH WELL } \\
\text { SOUTH WELL } \\
-\overline{--} \\
\text { WELL NO. }\end{array}$ \\
\hline $\begin{array}{l}\text { GROUND } \\
\text { GROUND } \\
\text { GROUND } \\
\text { GROUND } \\
\text { GROUND }\end{array}$ & $\begin{array}{c}\text { SPARTA SAND } \\
-- \\
-- \\
-- \\
--\end{array}$ & $\begin{array}{r}40 \\
-- \\
-- \\
-1\end{array}$ & $\begin{aligned} E L C \\
-- \\
-- \\
E L C\end{aligned}$ & $\begin{array}{r}\text { STP } \\
-- \\
-- \\
\text { STP } \\
--\end{array}$ & $\begin{array}{r}6 \\
-- \\
-- \\
--\end{array}$ & $\begin{array}{l}665 \\
-- \\
-- \\
--\end{array}$ & $\begin{array}{l}\text { WELL NO.3 } \\
30 \text { FT FROM PUMP HOUSE } \\
\text {-- }\end{array}$ \\
\hline $\begin{array}{l}\text { GROUND } \\
\text { GROUND } \\
\text { GROUND } \\
\text { GROUND } \\
\text { GROUND }\end{array}$ & $\begin{array}{c}-- \\
\overline{--} \\
\text { TOKIO FORMATION }\end{array}$ & $\begin{array}{r}7.5 \\
7.5 \\
7.5 \\
--\end{array}$ & $\begin{array}{l}E L C \\
E L C \\
E L C\end{array}$ & $\begin{array}{r}\text { STP } \\
-- \\
\text { STP } \\
-- \\
--\end{array}$ & $\begin{array}{r}12 \\
-- \\
10 \\
6 \\
--\end{array}$ & $\begin{array}{r}80 \\
-- \\
150 \\
50 \\
500\end{array}$ & $\begin{array}{l}\text { WELL NO.1 } \\
\text { CENTROID/WELLS } 1 \text { AND } 2 \\
\text { WELL NO.2 } \\
--\end{array}$ \\
\hline $\begin{array}{l}\text { SURFACE } \\
\text { GROUND } \\
\text { GROUND } \\
\text { GROUND } \\
\text { GROUND }\end{array}$ & $\begin{array}{l}\text { ROUBIDOUX FORMATION } \\
-- \\
\text { WILCOX GROUP }\end{array}$ & $\begin{array}{l}-- \\
-- \\
-- \\
50\end{array}$ & $\begin{array}{l}-- \\
\overline{-} \\
\text { ELC }\end{array}$ & $\begin{array}{l}-- \\
-- \\
-- \\
\text { STP }\end{array}$ & $\begin{array}{l}-- \\
-- \\
-- \\
--\end{array}$ & $\begin{array}{c}\overline{-} \\
1,315 \\
-\overline{-} \\
1,400\end{array}$ & $\begin{array}{l}-- \\
-- \\
-- \\
\text { CENTROID/WELLS } 1,2,3 \\
\text { WELL NO.1 / FIRE STATION }\end{array}$ \\
\hline
\end{tabular}


Table 2.--General description of public-supply

\begin{tabular}{|c|c|c|c|c|c|}
\hline $\begin{array}{c}\text { Water } \\
\text { user } \\
\text { ID }\end{array}$ & $\begin{array}{c}\text { Site } \\
\text { ID }\end{array}$ & Water system name & $\begin{array}{r}\text { Lati- } \\
\text { tude }\end{array}$ & $\begin{array}{c}\text { Longi- } \\
\text { tude }\end{array}$ & $\begin{array}{l}\text { Hydro- } \\
\text { logic } \\
\text { unit } \\
\text { code } \\
\end{array}$ \\
\hline $\begin{array}{l}30842 \\
30842 \\
30843 \\
30844 \\
30844\end{array}$ & $\begin{array}{l}354223089580701 \\
354223089581001 \\
361324091555001 \\
335047093431601 \\
335100093431501\end{array}$ & $\begin{array}{l}\text { OSCEOLA WATERWORKS } \\
\text { OSCEOLA WATERWORKS } \\
\text { OXFORD WATERWORKS } \\
\text { OZAN WATERWORKS } \\
\text { OZAN WATERWORKS }\end{array}$ & $\begin{array}{c}354223 \\
354215 \\
361200 \\
335100 \\
-\end{array}$ & $\begin{array}{c}0895807 \\
0895803 \\
0915500 \\
0934315 \\
-.\end{array}$ & $\begin{array}{c}08020203 \\
08020203 \\
11010012 \\
08040103 \\
-.\end{array}$ \\
\hline $\begin{array}{l}30845 \\
30845 \\
30846 \\
30847 \\
30848\end{array}$ & $\begin{array}{r}361814091233501 \\
361720091230401 \\
353138093503700 \\
3525000911500 \\
360322090290401\end{array}$ & $\begin{array}{l}\text { OZARK ACRES WATER CO } \\
\text { OZARK ACRES WATER CO } \\
\text { OZARK WATERWORKS } \\
\text { PANGBURN WATERWORKS } \\
\text { PARAGOULD CITY LIGHT/WATER }\end{array}$ & $\begin{array}{l}361800 \\
361814 \\
353138 \\
352530 \\
360330\end{array}$ & $\begin{array}{l}0912315 \\
0912335 \\
0935037 \\
0915000 \\
0902900\end{array}$ & $\begin{array}{l}11010010 \\
11010010 \\
11110201 \\
11010014 \\
08020203\end{array}$ \\
\hline $\begin{array}{l}30848 \\
30848 \\
30848 \\
30848 \\
30848\end{array}$ & $\begin{array}{l}360330090290201 \\
360330090290003 \\
360437090291201 \\
360330090290005 \\
360330090290006\end{array}$ & $\begin{array}{l}\text { PARAGOULD CITY LIGHT/WATER } \\
\text { PARAGOULD CITY LIGHT/WATER } \\
\text { PARAGOULD CITY LIGHT/WATER } \\
\text { PARAGOULD CITY LIGHT/WATER } \\
\text { PARAGOULD CITY LIGHT/WATER }\end{array}$ & $\begin{array}{c}360330 \\
-- \\
360437 \\
-- \\
--\end{array}$ & $\begin{array}{c}0902902 \\
-- \\
0902912 \\
-- \\
--\end{array}$ & $\begin{array}{l}08020203 \\
08020203 \\
08020203 \\
08020203 \\
08020203\end{array}$ \\
\hline $\begin{array}{l}30848 \\
30849 \\
30850 \\
30851 \\
30851\end{array}$ & $\begin{array}{r}360330090290007 \\
3516300934330 \\
330713091324801 \\
331011092431701 \\
331024092422901\end{array}$ & $\begin{array}{l}\text { PARAGOULD CITY LIGHT/WATER } \\
\text { PARIS WATERWORKS } \\
\text { PARKDALE WATERWORKS } \\
\text { PARKERS CHAPEL WATER ASSOC } \\
\text { PARKERS CHAPEL WATER ASSOC }\end{array}$ & $\begin{array}{l}351630 \\
336715 \\
331011 \\
331024\end{array}$ & $\begin{array}{l}0934330 \\
0913300 \\
0924317 \\
0924229\end{array}$ & $\begin{array}{l}08020203 \\
11110202 \\
08020203 \\
08040201 \\
08040201\end{array}$ \\
\hline $\begin{array}{l}30852 \\
30853 \\
30854 \\
30855 \\
30855\end{array}$ & $\begin{array}{r}351546090335502 \\
3515260911347 \\
3532450903000 \\
341158092014801 \\
341332092010901\end{array}$ & $\begin{array}{l}\text { PARKIN WATERWORKS } \\
\text { PATTERSON WATERWORKS } \\
\text { PAYNEWAY WATER ASSOC } \\
\text { PB/GENERAL WATERWORKS CO } \\
\text { PB/GENERAL WATERWORKS CO }\end{array}$ & $\begin{array}{l}351600 \\
351526 \\
353245 \\
341158 \\
341332\end{array}$ & $\begin{array}{l}0903403 \\
0911347 \\
0903000 \\
0920148 \\
0920100\end{array}$ & $\begin{array}{l}08020203 \\
08020302 \\
08020203 \\
08040205 \\
08040205\end{array}$ \\
\hline $\begin{array}{l}30855 \\
30855 \\
30855 \\
30855 \\
30855\end{array}$ & $\begin{array}{l}341147092022301 \\
341337092005201 \\
341331092010801 \\
341142092022801 \\
341331092005501\end{array}$ & $\begin{array}{l}\text { PB/GENERAL WATERWORKS CO } \\
\text { PB/GENERAL WATERWORKS CO } \\
\text { PB/GENERAL WATERWORKS CO } \\
\text { PB/GENERAL WATERWORKS CO } \\
\text { PB/GENERAL WATERWORKS CO }\end{array}$ & $\begin{array}{l}341147 \\
341337 \\
341328 \\
341140 \\
341331\end{array}$ & $\begin{array}{l}0920201 \\
0920052 \\
0920050 \\
0920207 \\
0920055\end{array}$ & $\begin{array}{l}08040205 \\
08040205 \\
08040205 \\
08040205 \\
08040205\end{array}$ \\
\hline $\begin{array}{l}30855 \\
30855 \\
30857 \\
30858 \\
30859\end{array}$ & $\begin{array}{r}340112092014201 \\
341332092010904 \\
3502450924015 \\
3342260933136 \\
350026092485201\end{array}$ & $\begin{array}{l}\text { PB/GENERAL WATERWORKS CO } \\
\text { PB/GENERAL WATERWORKS CO } \\
\text { PERRY WATERWORKS } \\
\text { PERRYTOWN WATERWORKS } \\
\text { PERRYVILLE WATERWORKS }\end{array}$ & $\begin{array}{l}340112 \\
341342 \\
350245 \\
334226 \\
350026\end{array}$ & $\begin{array}{l}0920142 \\
0920103 \\
0924015 \\
0933136 \\
0924852\end{array}$ & $\begin{array}{l}08040205 \\
08040205 \\
11110203 \\
08040103 \\
11110206\end{array}$ \\
\hline $\begin{array}{l}30860 \\
30861 \\
30861 \\
30862 \\
30863\end{array}$ & 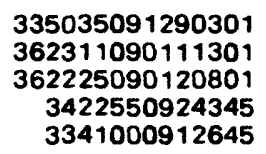 & $\begin{array}{l}\text { PICKENS WATERWORKS } \\
\text { PIGGOTT WATERWORKS } \\
\text { PIGGOTT WATERWORKS } \\
\text { PINE REST MOBILE HOME PARK } \\
\text { PINEHAVEN MOBILE HOME PARK }\end{array}$ & $\begin{array}{l}335035 \\
362236 \\
362236 \\
342255 \\
334100\end{array}$ & $\begin{array}{l}0912855 \\
0901216 \\
0901216 \\
0924345 \\
0912645\end{array}$ & $\begin{array}{l}08050001 \\
08020203 \\
08020203 \\
08040102 \\
08040205\end{array}$ \\
\hline $\begin{array}{l}30864 \\
30865 \\
30867 \\
30868 \\
30869\end{array}$ & $\begin{array}{r}3559300931745 \\
350936092381302 \\
3625450901615 \\
3605150910400 \\
331415091302901\end{array}$ & $\begin{array}{l}\text { PLAINVIEW WATER DEPT } \\
\text { PLUMERVILLE WATERWORKS } \\
\text { POLLARD WATERWORKS } \\
\text { PORTIA WATERWORKS } \\
\text { PORTLAND WATERWORKS }\end{array}$ & $\begin{array}{l}345930 \\
350915 \\
362545 \\
360515 \\
331415\end{array}$ & $\begin{array}{l}0931745 \\
0923815 \\
0901615 \\
0910400 \\
0913029\end{array}$ & $\begin{array}{l}11110206 \\
11110203 \\
08020302 \\
11010009 \\
08050001\end{array}$ \\
\hline $\begin{array}{l}30870 \\
30871 \\
30872 \\
30872 \\
30874\end{array}$ & $\begin{array}{r}341923092382501 \\
3556000942000 \\
341841092332001 \\
341840092333002 \\
361512092504501\end{array}$ & $\begin{array}{l}\text { POYEN WATERWORKS } \\
\text { PRAIRIE GROVE WATERWORKS } \\
\text { PRATTSVILLE WATERWORKS } \\
\text { PRATTSVILLE WATERWORKS } \\
\text { PYATT WATERWORKS }\end{array}$ & $\begin{array}{c}341915 \\
355600 \\
341840 \\
-- \\
361700\end{array}$ & $\begin{array}{c}0923830 \\
0942000 \\
0923330 \\
-- \\
0924800\end{array}$ & $\begin{array}{l}08040203 \\
11110103 \\
08040203 \\
08040203 \\
11010002\end{array}$ \\
\hline $\begin{array}{l}30875 \\
30876 \\
30876 \\
30876 \\
30876\end{array}$ & $\begin{array}{l}361818091304601 \\
352245092131601 \\
352249092132301 \\
352243092132701 \\
352244092130501\end{array}$ & $\begin{array}{l}\text { CHEROKEE VILLAGE WATER } \\
\text { QUITMAN WATERWORKS } \\
\text { QUITMAN WATERWORKS } \\
\text { QUITMAN WATERWORKS } \\
\text { QUITMAN WATERWORKS }\end{array}$ & $\begin{array}{l}361900 \\
352245 \\
352249 \\
352243 \\
352244\end{array}$ & $\begin{array}{l}0913230 \\
0921316 \\
0921323 \\
0921327 \\
0921305\end{array}$ & $\begin{array}{l}11010010 \\
11110205 \\
11110205 \\
11110205 \\
11110205\end{array}$ \\
\hline $\begin{array}{l}30877 \\
30877 \\
30879 \\
30880 \\
30881\end{array}$ & $\begin{array}{r}3620000941000 \\
362000094100001 \\
3619000911330 \\
3614000911515 \\
3617000922200\end{array}$ & $\begin{array}{l}\text { RAMBO RIVIERA S/D WATER } \\
\text { RAMBO RIVIERA S/D WATER } \\
\text { RAVENDEN SPRGS WATERWORKS } \\
\text { RAVENDEN WATERWORKS } \\
\text { RAY MARTIN MOBILE HOME PARK }\end{array}$ & $\begin{array}{c}362000 \\
361900 \\
361400 \\
361700\end{array}$ & $\begin{array}{c}0941000 \\
-- \\
0911330 \\
0911515 \\
0922200\end{array}$ & $\begin{array}{c}11110103 \\
- \\
11010010 \\
11010010 \\
11010004\end{array}$ \\
\hline
\end{tabular}




\begin{tabular}{|c|c|c|c|c|c|c|c|}
\hline $\begin{array}{c}\text { Source } \\
\text { type }\end{array}$ & Source & $\begin{array}{l}\text { Pump } \\
\text { Horse- } \\
\text { power }\end{array}$ & $\begin{array}{l}\text { descriot } \\
\text { Power } \\
\text { source }\end{array}$ & $\begin{array}{l}\text { ien } \\
\text { Pump } \\
\text { tyoe }\end{array}$ & $\begin{array}{l}\text { Well } \\
\text { dia- } \\
\text { meter } \\
\text { Ginches }\end{array}$ & $\begin{array}{c}\text { Depth } \\
\text { of } \\
\text { well } \\
\text { s) }(\text { feet })\end{array}$ & Local descriotive ID \\
\hline $\begin{array}{l}\text { GROUND } \\
\text { GROUND } \\
\text { GROUND } \\
\text { GROUND } \\
\text { GROUND }\end{array}$ & $\begin{array}{l}\text { SPARTA SAND } \\
\text { SPARTA SAND } \\
\text { COTTER DOLOMITE } \\
\text { TOKIO FORMATION } \\
\text { TOKIO FORMATION }\end{array}$ & $\begin{array}{r}20 \\
50 \\
-- \\
2.5 \\
2\end{array}$ & $\begin{array}{l}\text { ELC } \\
\text { ELC } \\
-- \\
\text { ELC } \\
\text { ELC }\end{array}$ & $\begin{array}{l}\text { STP } \\
\text { STP } \\
\text { STP } \\
\text { STP }\end{array}$ & $\begin{array}{l}-- \\
-- \\
- \\
4\end{array}$ & $\begin{array}{r}1,500 \\
1,500 \\
600 \\
555 \\
500\end{array}$ & $\begin{array}{l}\text { WELL. NO.3 I BEHIND POWER PLANT } \\
\text { WELL. NO. } 2 \text { /BEHINO PLANT OFFICE } \\
\text { WELL NEAR PUMP HOUSE } \\
\text { WELL NEAR PUMP HOUSE }\end{array}$ \\
\hline $\begin{array}{l}\text { GROUND } \\
\text { GROUND } \\
\text { SURFACE } \\
\text { SURFACE } \\
\text { GROUND }\end{array}$ & $\begin{array}{l}\text { ROUBIDOUX FORMATION } \\
\text { ROUBIDOUX FORMATION } \\
\text { WHITE OAK } \\
\text { LITTLE RED } \\
\text { WILCOX GROUP }\end{array}$ & $\begin{array}{r}30 \\
20 \\
125 \\
-- \\
125\end{array}$ & $\begin{array}{l}E L C \\
\text { ELC } \\
\text { ELC } \\
\text { ELC } \\
\text { ELC }\end{array}$ & $\begin{array}{l}\text { STP } \\
\text { STP } \\
\text { GRV } \\
\text { STP } \\
\text { STP }\end{array}$ & $\begin{array}{r}6 \\
6 \\
18 \\
10 \\
12\end{array}$ & $\begin{array}{l}611 \\
940 \\
\cdots \\
-- \\
507\end{array}$ & $\begin{array}{lr}\text { WELL } & \text { NO.5 } \\
\text { WELL } & \text { NO.3 } \\
& -.- \\
\text { NO.1 } & -\end{array}$ \\
\hline $\begin{array}{l}\text { GROUND } \\
\text { GROUND } \\
\text { GROUND } \\
\text { GROUND } \\
\text { GROUND }\end{array}$ & $\begin{array}{l}\text { WILCOX GROUP } \\
\text { WILCOX GROUP } \\
\text { WILCOX GROUP } \\
\text { WILCOX GROUP } \\
\text { WILCOX GROUP }\end{array}$ & $\begin{array}{r}75 \\
50 \\
100 \\
150 \\
200\end{array}$ & $\begin{array}{l}\text { ELC } \\
\text { ELC } \\
\text { ELC } \\
\text { ELC }\end{array}$ & $\begin{array}{l}\text { STP } \\
\text { STP } \\
\text { STP } \\
\text { STP } \\
\text { STP }\end{array}$ & $\begin{array}{l}12 \\
12 \\
12 \\
12 \\
12\end{array}$ & $\begin{array}{l}501 \\
500 \\
446 \\
500 \\
500\end{array}$ & $\begin{array}{l}\text { NO. } \\
\text { NO.3 } \\
\text { NO. } \\
\text { NO.5 } \\
\text { NO. }\end{array}$ \\
\hline $\begin{array}{l}\text { GROUND } \\
\text { SURFACE } \\
\text { GROUND } \\
\text { GROUND } \\
\text { GROUND }\end{array}$ & $\begin{array}{l}\text { WILCOX GROUP } \\
\text { CITY LAKE RES } \\
\text { COCKFIELD FORMATION } \\
\text { SPARTA SAND } \\
\text { SPARTA SAND }\end{array}$ & $\begin{array}{l}200 \\
125 \\
-- \\
40 \\
40\end{array}$ & $\begin{array}{l}E L C \\
E L C \\
-- \\
E L C \\
E L C\end{array}$ & $\begin{array}{l}\text { STP } \\
\text { STP } \\
\text { STP } \\
\text { STP }\end{array}$ & $\begin{array}{r}12 \\
8 \\
-- \\
8 \\
8\end{array}$ & $\begin{array}{l}500 \\
-- \\
400 \\
767 \\
797\end{array}$ & $\begin{array}{l}\text { NO.7 } \\
\text { RWMMR-I } \\
\text { WELL NO. } 2 \\
\text { WELL NO.1 }\end{array}$ \\
\hline $\begin{array}{l}\text { GROUND } \\
\text { GROUND } \\
\text { GROUND } \\
\text { GROUND } \\
\text { GROUND }\end{array}$ & $\begin{array}{l}\text { CLAIBORNE GROUP } \\
-- \\
-- \\
\text { SPARTA SAND } \\
\text { SPARTA SAND }\end{array}$ & $\begin{array}{r}7 .- \\
7.5 \\
200 \\
150\end{array}$ & $\begin{array}{l}-\overline{-} \\
E L C \\
-\bar{C} \\
E L C\end{array}$ & $\begin{array}{l}--\overline{S T P} \\
--\overline{-} \\
\text { STP } \\
\text { STP }\end{array}$ & $\begin{array}{l}-- \\
-- \\
-- \\
16 \\
18\end{array}$ & $\begin{array}{l}491 \\
196 \\
-- \\
847 \\
850\end{array}$ & $\begin{array}{l}-- \\
-- \\
-- \\
--\end{array}$ \\
\hline $\begin{array}{l}\text { GROUND } \\
\text { GROUND } \\
\text { GROUND } \\
\text { GROUND } \\
\text { GROUND }\end{array}$ & $\begin{array}{l}\text { SPARTA SAND } \\
\text { SPARTA SAND } \\
\text { SPARTA SAND } \\
\text { SPARTA SAND } \\
\text { SPARTA SAND }\end{array}$ & $\begin{array}{l}210 \\
210 \\
200 \\
200 \\
200\end{array}$ & $\begin{array}{l}\text { ELC } \\
\text { ELC } \\
\text { ELC } \\
\text { ELC } \\
\text { ELC }\end{array}$ & $\begin{array}{l}\text { STP } \\
\text { STP } \\
\text { STP } \\
\text { STP } \\
\text { STP }\end{array}$ & $\begin{array}{l}16 \\
18 \\
18 \\
18 \\
18\end{array}$ & $\begin{array}{l}863 \\
886 \\
904 \\
876 \\
864\end{array}$ & $\begin{array}{l}-- \\
-- \\
-- \\
--\end{array}$ \\
\hline $\begin{array}{l}\text { GROUND } \\
\text { GROUND } \\
\text { GROUND } \\
\text { GROUND } \\
\text { SURFACE }\end{array}$ & $\begin{array}{c}\text { SPARTA SAND } \\
\text { SPARTA SAND } \\
--- \\
-- \\
\text { CEDAR CREEK }\end{array}$ & $\begin{array}{r}200 \\
250 \\
7.5 \\
-- \\
40\end{array}$ & $\begin{array}{l}\text { ELC } \\
-- \\
E L C \\
E L C \\
\text { ELC }\end{array}$ & $\begin{array}{l}\text { STP } \\
-- \\
\text { STP } \\
\text { STP } \\
\text { STP }\end{array}$ & $\begin{array}{l}18 \\
-- \\
14 \\
-- \\
--\end{array}$ & $\begin{array}{r}876 \\
840 \\
21 \\
284 \\
--\end{array}$ & $\begin{array}{l}-- \\
-- \\
-- \\
--\end{array}$ \\
\hline $\begin{array}{l}\text { GROUND } \\
\text { GROUND } \\
\text { GROUND } \\
\text { GROUND } \\
\text { GROUND }\end{array}$ & $\begin{array}{c}\text { SPARTA SAND } \\
\text { NACATOCH SAND } \\
\text { NACATOCH SAND } \\
-- \\
--\end{array}$ & $\begin{array}{l}10 \\
60 \\
60 \\
-- \\
--\end{array}$ & $\begin{array}{l}E L C \\
\text { ELC } \\
\text { ELC } \\
-- \\
--\end{array}$ & $\begin{array}{l}\text { STP } \\
\text { STP } \\
\text { STP } \\
-- \\
--\end{array}$ & $\begin{array}{r}4 \\
12 \\
12 \\
-- \\
--\end{array}$ & $\begin{array}{r}830 \\
976 \\
1,062 \\
-- \\
--\end{array}$ & $\begin{aligned} & \text { WELL NO. }- \\
& \text { WELL NO.4 } \\
&-- \\
&--\end{aligned}$ \\
\hline $\begin{array}{l}\text { SURFACE } \\
\text { GROUND } \\
\text { GROUND } \\
\text { GROUND } \\
\text { GROUND }\end{array}$ & $\begin{array}{l}\text { LAKE NIMROD } \\
\text { QUATERNARY ALLUVIUM } \\
-- \\
\text { COCKFIELD FORMATION }\end{array}$ & $\begin{array}{r}15 \\
5 \\
-2 \\
5 \\
10\end{array}$ & $\begin{array}{l}E L C \\
E L C \\
--\bar{C} \\
E L C \\
\text { ELC }\end{array}$ & $\begin{array}{l}\text { STP } \\
\text { STP } \\
-- \\
\text { STP } \\
\text { STP }\end{array}$ & $\begin{array}{r}6 \\
13 \\
-- \\
-- \\
4\end{array}$ & $\begin{array}{l}-- \\
38 \\
-- \\
-- \\
332\end{array}$ & $\begin{array}{l}-- \\
-- \\
-- \\
--\end{array}$ \\
\hline $\begin{array}{l}\text { GROUND } \\
\text { SURFACE } \\
\text { GROUND } \\
\text { GROUND } \\
\text { GROUND }\end{array}$ & $\begin{array}{l}\text { SPARTA SAND } \\
\text { PRAIRIE GROVE } \\
\text { SPARTA SAND } \\
\text { SPARTA SAND } \\
\text { ROUBIDOUX FORMATION }\end{array}$ & $\begin{array}{l}-- \\
15 \\
20 \\
20 \\
20\end{array}$ & $\begin{array}{l}--\overline{1} \\
\text { ELC } \\
\text { ELC } \\
\text { ELC }\end{array}$ & $\begin{array}{l}-- \\
\text { STP } \\
\text { STP } \\
\text { STP } \\
\text { STP }\end{array}$ & $\begin{array}{l}-- \\
10 \\
10 \\
10 \\
--\end{array}$ & $\begin{array}{r}-- \\
-- \\
370 \\
360 \\
1,392\end{array}$ & $\begin{array}{l}-- \\
\text { WELL NO. } \\
\text { WELL NO. } \\
\text {--- }\end{array}$ \\
\hline $\begin{array}{l}\text { SURFACE } \\
\text { GROUND } \\
\text { GROUND } \\
\text { GROUND } \\
\text { GROUND }\end{array}$ & $\begin{array}{c}\text { ROARING SPRINGS } \\
-- \\
-- \\
-- \\
--\end{array}$ & $\begin{array}{r}200 \\
-15 \\
15 \\
15 \\
15\end{array}$ & $\begin{array}{l}E L C \\
-- \\
E L C \\
\text { ELC }\end{array}$ & $\begin{array}{c}\text { STP } \\
-- \\
-- \\
-- \\
--\end{array}$ & $\begin{array}{r}-- \\
-- \\
2 \\
2 \\
2\end{array}$ & $\begin{array}{l}-- \\
-- \\
180 \\
180 \\
180\end{array}$ & $\begin{array}{l}-- \\
-- \\
-- \\
--\end{array}$ \\
\hline $\begin{array}{l}\text { GROUND } \\
\text { GROUND } \\
\text { GROUND } \\
\text { GROUND } \\
\text { GROUND }\end{array}$ & $\begin{array}{c}\text { ROUBIDOUX FORMATION } \\
-- \\
-- \\
-- \\
--\end{array}$ & $\begin{array}{r}10 \\
3 \\
-- \\
20 \\
--\end{array}$ & $\begin{array}{l}\text { ELC } \\
\text { ELC } \\
-- \\
\text { ELC } \\
--\end{array}$ & $\begin{array}{l}\text { STP } \\
\text { STP } \\
-- \\
\text { STP } \\
--\end{array}$ & $\begin{array}{r}6 \\
6 \\
-- \\
--\end{array}$ & $\begin{array}{c}1.170 \\
700 \\
-- \\
700 \\
--\end{array}$ & $\begin{array}{l}\text { WELL NEAR PUMP HOUSE } \\
\text { BACK UP WELL ONLY } \\
\text { 2ND ST CITY }\end{array}$ \\
\hline
\end{tabular}


Table 2.--General description of public-supply

\begin{tabular}{|c|c|c|c|c|c|}
\hline $\begin{array}{c}\text { Water } \\
\text { user } \\
\text { ID } \\
\end{array}$ & $\begin{array}{c}\text { Site } \\
\text { ID }\end{array}$ & Water system name & $\begin{array}{r}\text { Lati- } \\
\text { tude } \\
\end{array}$ & $\begin{array}{c}\text { Longi- } \\
\text { tude }\end{array}$ & $\begin{array}{l}\text { Hydro- } \\
\text { logic } \\
\text { unit } \\
\text { code }\end{array}$ \\
\hline $\begin{array}{l}30882 \\
30883 \\
30883 \\
30883 \\
30884\end{array}$ & $\begin{array}{r}3625000932500 \\
361601090175001 \\
361601090175101 \\
361600090171503 \\
342644092105501\end{array}$ & $\begin{array}{l}\text { REAVIS WATERSYSTEM } \\
\text { RECTOR WATERWORKS } \\
\text { RECTOR WATERWORKS } \\
\text { RECTOR WATERWORKS } \\
\text { REDFIELD WATERWORKS }\end{array}$ & $\begin{array}{c}362500 \\
361600 \\
361601 \\
-- \\
342649\end{array}$ & $\begin{array}{c}0932500 \\
0901715 \\
0901751 \\
-- \\
0921056\end{array}$ & $\begin{array}{l}11010001 \\
08020203 \\
08020203 \\
08020203 \\
11110207\end{array}$ \\
\hline $\begin{array}{l}30885 \\
30886 \\
30886 \\
30889 \\
30889\end{array}$ & $\begin{array}{r}3426080925330 \\
3621450904515 \\
362145090451501 \\
335728092113301 \\
335729092112001\end{array}$ & $\begin{array}{l}\text { REMMEL DAM LANDING WATER } \\
\text { REYNO WATERWORKS } \\
\text { REYNO WATERWORKS } \\
\text { RISON WATERWORKS } \\
\text { RISON WATERWORKS }\end{array}$ & $\begin{array}{c}342608 \\
362145 \\
-- \\
335730 \\
335729\end{array}$ & $\begin{array}{c}0925330 \\
0904515 \\
0 .- \\
0921120 \\
0921133\end{array}$ & $\begin{array}{c}08040102 \\
11010007 \\
-- \\
08040204 \\
08040204\end{array}$ \\
\hline $\begin{array}{l}30890 \\
30891 \\
30892 \\
30892 \\
30892\end{array}$ & $\begin{array}{r}3505250922945 \\
3624000922800 \\
342553092574501 \\
342553092574502 \\
342553092574503\end{array}$ & $\begin{array}{l}\text { RIVER DR MOBILE HOME PARK } \\
\text { RIVERSIDE MOBILE HOME PARK } \\
\text { RIVIERA UTILITIES } \\
\text { RIVIERA UTILITIES } \\
\text { RIVIERA UTILITIES }\end{array}$ & $\begin{array}{c}350525 \\
362400 \\
342553 \\
\ldots- \\
--\end{array}$ & $\begin{array}{c}0922945 \\
0922800 \\
0925745 \\
-- \\
--\end{array}$ & $\begin{array}{l}11110203 \\
11010003 \\
08040101 \\
08040101 \\
08040101\end{array}$ \\
\hline $\begin{array}{l}30892 \\
30892 \\
30892 \\
30893 \\
30893\end{array}$ & $\begin{array}{r}342553092574504 \\
342553092574505 \\
342553092574506 \\
3545300913815 \\
354530091381501\end{array}$ & $\begin{array}{l}\text { RIVIERA UTILITIES } \\
\text { RIVIERA UTILITIES } \\
\text { RIVIERA UTILITIES } \\
\text { ROCK MOORE WATER ASSOC } \\
\text { ROCK MOORE WATER ASSOC }\end{array}$ & $\begin{array}{l}-- \\
-- \\
-- \\
--\end{array}$ & $\begin{array}{l}-- \\
-- \\
-- \\
-\end{array}$ & $\begin{array}{c}08040101 \\
08040101 \\
08040101 \\
11010004\end{array}$ \\
\hline $\begin{array}{l}30893 \\
30893 \\
30894 \\
30895 \\
30896\end{array}$ & $\begin{array}{r}354530091381502 \\
354530091381503 \\
3438000912400 \\
333529093163801 \\
3521450913030\end{array}$ & $\begin{array}{l}\text { ROCK MOORE WATER ASSOC } \\
\text { ROCK MOORE WATER ASSOC } \\
\text { ROE WATERWORKS } \\
\text { ROSSTON WATER DEPT } \\
\text { RUSSELL WATERWORKS }\end{array}$ & $\begin{array}{c}-- \\
34 \\
33800 \\
352145\end{array}$ & $\begin{array}{c}\cdots \\
0912400 \\
0931645 \\
0913030\end{array}$ & $\begin{array}{l}11010004 \\
11010004 \\
08020303 \\
08040103 \\
11010013\end{array}$ \\
\hline $\begin{array}{l}30898 \\
30899 \\
30900 \\
30901 \\
30901\end{array}$ & $\begin{array}{r}3602300925130 \\
361132093113501 \\
362712090083901 \\
362207091492401 \\
362245091490701\end{array}$ & $\begin{array}{l}\text { S P G WATER ASSOC } \\
\text { S W BOONE COUNTY WATER } \\
\text { SAINT FRANCIS WATERWORKS } \\
\text { SALEM WATERWORKS } \\
\text { SALEM WATERWORKS }\end{array}$ & $\begin{array}{l}360230 \\
361130 \\
362715 \\
362210 \\
362245\end{array}$ & $\begin{array}{l}0925130 \\
0931145 \\
0900830 \\
0914923 \\
0914907\end{array}$ & $\begin{array}{l}11010005 \\
11010003 \\
08020203 \\
11010010 \\
11010010\end{array}$ \\
\hline $\begin{array}{l}30902 \\
30903 \\
30903 \\
30903 \\
30903\end{array}$ & $\begin{array}{l}361437092163201 \\
343141092230901 \\
343129092225201 \\
343127092230901 \\
343119092230601\end{array}$ & $\begin{array}{l}\text { SALESVILLE WATERWORKS } \\
\text { SARDIS WATER ASSOC } \\
\text { SARDIS WATER ASSOC } \\
\text { SARDIS WATER ASSOC } \\
\text { SARDIS WATER ASSOC }\end{array}$ & $\begin{array}{l}361430 \\
343141 \\
343129 \\
343127 \\
343119\end{array}$ & $\begin{array}{l}0921645 \\
0922309 \\
0922253 \\
0922309 \\
0922306\end{array}$ & $\begin{array}{l}11010006 \\
08040203 \\
08040203 \\
08040203 \\
08040203\end{array}$ \\
\hline $\begin{array}{l}30904 \\
30906 \\
30907 \\
30908 \\
30909\end{array}$ & $\begin{array}{r}339450935815 \\
351657091430601 \\
3558300915145 \\
3322350940025 \\
3427200931000\end{array}$ & $\begin{array}{l}\text { SCHALL SUBDIVISION } \\
\text { SEARCY WATERWORKS } \\
\text { SEDGWICK WATERWORKS } \\
\text { SHADY ACRES MOBILE HOME } \\
\text { SHADY HILLS MOBILE HOME }\end{array}$ & $\begin{array}{l}334945 \\
351657 \\
355830 \\
332235 \\
342720\end{array}$ & $\begin{array}{l}0935815 \\
0914306 \\
0915145 \\
0940025 \\
0931000\end{array}$ & $\begin{array}{l}11140109 \\
11010014 \\
08020302 \\
11140302 \\
08040101\end{array}$ \\
\hline $\begin{array}{l}30910 \\
30910 \\
30911 \\
30911 \\
30911\end{array}$ & $\begin{array}{r}355345090480001 \\
355345090480002 \\
3437250922328 \\
343729092232802 \\
343725092272803\end{array}$ & $\begin{array}{l}\text { SHADY PARK WATER ASSOC } \\
\text { SHADY PARK WATER ASSOC } \\
\text { SHANNON HILLS WATER ASSOC } \\
\text { SHANMON HILLS WATER ASSOC } \\
\text { SHANMON HILLS WATER ASSOC }\end{array}$ & $\begin{array}{c}-- \\
343725 \\
343728 \\
343723\end{array}$ & $\begin{array}{c}-- \\
0922328 \\
0922327 \\
0922415\end{array}$ & $\begin{array}{l}08020302 \\
08020302 \\
11110207 \\
11110207 \\
11110207\end{array}$ \\
\hline $\begin{array}{l}30911 \\
30911 \\
30911 \\
30912 \\
30914\end{array}$ & $\begin{array}{r}343725092232804 \\
343725092232805 \\
343725092232806 \\
341836092240302 \\
3539300921815\end{array}$ & $\begin{array}{l}\text { SHANNON HILLS WATER ASSOC } \\
\text { SHANNON HILLS WATER ASSOC } \\
\text { SHANNON HILLS WATER ASSOC } \\
\text { SHERIDAN WATERWORKS } \\
\text { SHIRLEY WATERWORKS }\end{array}$ & $\begin{array}{c}\cdots \\
\cdots \\
341835 \\
353930\end{array}$ & $\begin{array}{c}-- \\
\overline{-} \\
0922400 \\
0921815\end{array}$ & $\begin{array}{l}11110207 \\
11110207 \\
11110207 \\
08040203 \\
11010014\end{array}$ \\
\hline $\begin{array}{l}30915 \\
30915 \\
30915 \\
30915 \\
30915\end{array}$ & $\begin{array}{l}333937092424101 \\
333929092421101 \\
333944092424001 \\
333944092425201 \\
333944092430401\end{array}$ & $\begin{array}{ll}\text { SHUMAKER PUBLIC } & \text { SERV } \\
\text { SHO } \\
\text { SHUMAKER PUBLIC SERV CO } \\
\text { SHUMAKER PUBLIC SERV CO } \\
\text { SHUMAKER PUBLIC SERV CO } \\
\text { SHUMAKER PUBLIC SERV CO }\end{array}$ & $\begin{array}{l}\mathbf{3 3 3 9 3 7} \\
333929 \\
333944 \\
333944 \\
\mathbf{3 3 3 9 4 4}\end{array}$ & $\begin{array}{l}0924241 \\
0924211 \\
0924240 \\
0924252 \\
0924304\end{array}$ & $\begin{array}{l}08040201 \\
08040201 \\
08040201 \\
08040201 \\
08040201\end{array}$ \\
\hline $\begin{array}{l}30915 \\
30916 \\
30917 \\
30918 \\
30918\end{array}$ & $\begin{array}{r}333936092430601 \\
3559450913915 \\
3607450943345 \\
332211092433701 \\
332205092433001\end{array}$ & $\begin{array}{l}\text { SHUMAKER PUBLIC SERV CO } \\
\text { SIDNEY WATER SYSTEM } \\
\text { SILOAM SPRINGS WATERWORKS } \\
\text { SMACKOVER WATERWORKS } \\
\text { SMACKOVER WATERWORKS }\end{array}$ & $\begin{array}{l}333936 \\
355945 \\
360745 \\
332211 \\
332205\end{array}$ & $\begin{array}{l}0924306 \\
0913915 \\
0943345 \\
0924337 \\
0924330\end{array}$ & $\begin{array}{l}08040201 \\
11010004 \\
11110103 \\
08040201 \\
08040201\end{array}$ \\
\hline
\end{tabular}




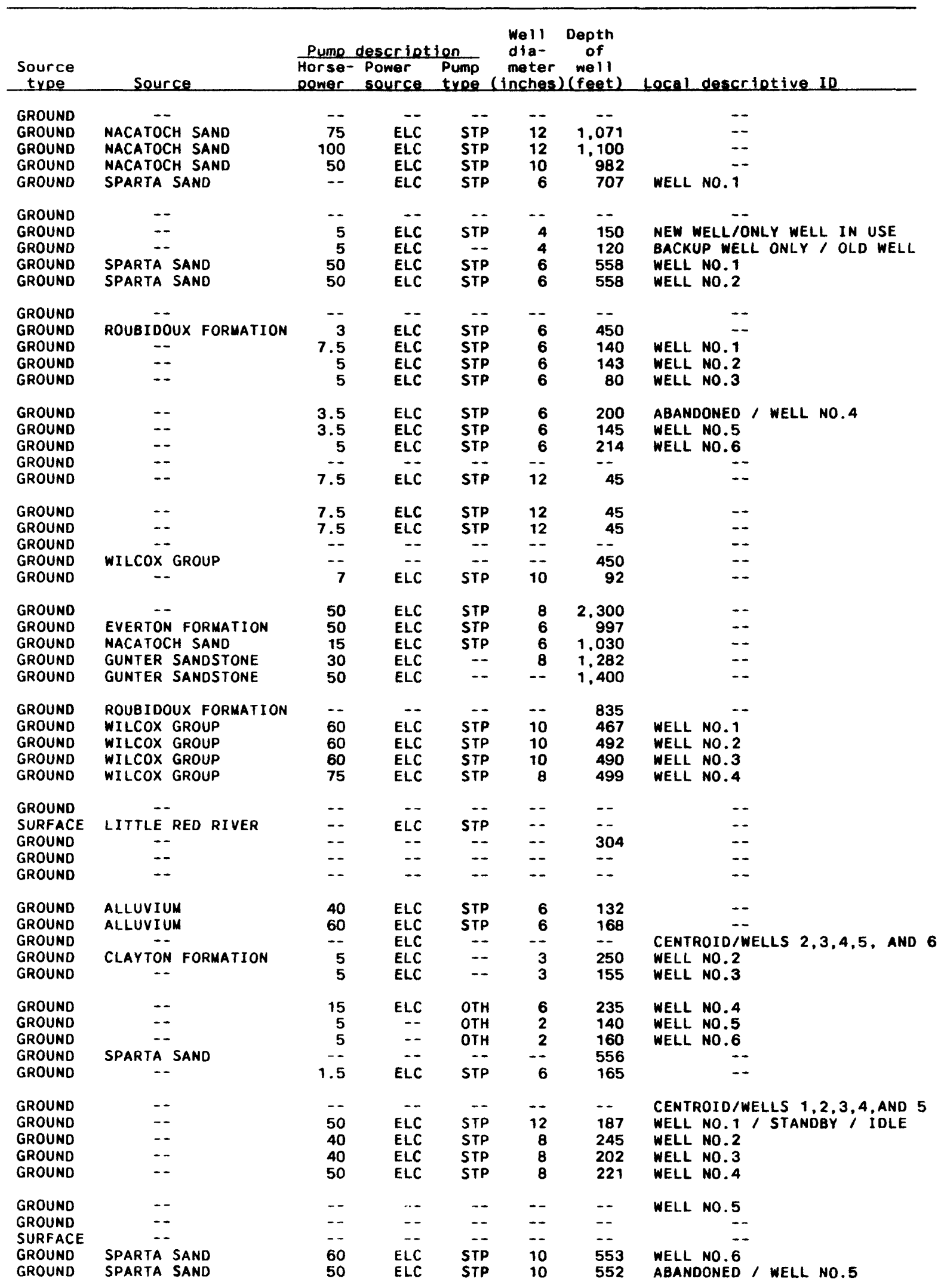


Table 2.--General description of public-supply

\begin{tabular}{|c|c|c|c|c|c|}
\hline $\begin{array}{c}\text { Water } \\
\text { User } \\
\text { ID } \\
\end{array}$ & $\begin{array}{c}\text { Site } \\
\text { ID } \\
\end{array}$ & Water system name & $\begin{array}{r}\text { Lati- } \\
\text { tude }\end{array}$ & $\begin{array}{c}\text { Longi- } \\
\text { tude }\end{array}$ & $\begin{array}{l}\text { Hydro- } \\
\text { logic } \\
\text { unit } \\
\text { code } \\
\end{array}$ \\
\hline $\begin{array}{l}30918 \\
30918 \\
30918 \\
30918 \\
30918\end{array}$ & $\begin{array}{l}332113092421001 \\
332131092423001 \\
332171092428301 \\
332211092433700 \\
332116092420500\end{array}$ & $\begin{array}{l}\text { SMACKOVER WATERWORKS } \\
\text { SMACKOVER WATERWORKS } \\
\text { SMACKOVER WATERWORKS } \\
\text { SMACKOVER WATERWORKS } \\
\text { SMACKOVER WATERWORKS }\end{array}$ & $\begin{array}{c}332115 \\
332131 \\
332171 \\
-- \\
--\end{array}$ & $\begin{array}{c}0924213 \\
0924230 \\
0924283 \\
-- \\
--\end{array}$ & $\begin{array}{l}08040201 \\
08040201 \\
08040201 \\
-- \\
--\end{array}$ \\
\hline $\begin{array}{l}30918 \\
30919 \\
30920 \\
30921 \\
30921\end{array}$ & $\begin{array}{r}332133092423400 \\
3625000932500 \\
331750092143001 \\
354530091381501 \\
354500091410001\end{array}$ & $\begin{array}{l}\text { SMACKOVER WATERWORKS } \\
\text { SOOTER WATER SYSTEM } \\
\text { SOUTHERN ARK UNIV WATER } \\
\text { SOUTHSIDE WATER ASSOC } \\
\text { SOUTHSIDE WATER ASSOC }\end{array}$ & $\begin{array}{l}362500 \\
331750 \\
354530 \\
354500\end{array}$ & $\begin{array}{l}-- \\
0932500 \\
0921430 \\
0913815 \\
0914100\end{array}$ & $\begin{array}{l}11010001 \\
11140203 \\
11010004 \\
11010004\end{array}$ \\
\hline $\begin{array}{l}30922 \\
30922 \\
30922 \\
30923 \\
30923\end{array}$ & $\begin{array}{r}325455092494501 \\
335457092505001 \\
335500092505002 \\
3437380923055 \\
343742092305201\end{array}$ & $\begin{array}{l}\text { SPARKMAN WATERWORKS } \\
\text { SPARKMAN WATERWORKS } \\
\text { SPARKMAN WATERWORKS } \\
\text { SPRINGHILL WATERWORKS } \\
\text { SPRINGHILL WATERWORKS }\end{array}$ & $\begin{array}{c}-- \\
335500 \\
-- \\
343738 \\
343742\end{array}$ & $\begin{array}{c}0924945 \\
0925050 \\
-- \\
0923055 \\
0923052\end{array}$ & $\begin{array}{l}08040102 \\
08040102 \\
08040102 \\
08040203 \\
08040203\end{array}$ \\
\hline $\begin{array}{l}30923 \\
30923 \\
30923 \\
30923 \\
30924\end{array}$ & $\begin{array}{r}343735092302801 \\
343739092310501 \\
343736092310701 \\
343740092312001 \\
\mathbf{3 4 2 2 2 6 0 9 1 0 8 0 8}\end{array}$ & $\begin{array}{l}\text { SPRINGHILL WATERWORKS } \\
\text { SPRINGHILL WATERWORKS } \\
\text { SPRINGHILL WATERWORKS } \\
\text { SPRINGHILL WATERWORKS } \\
\text { ST CHARLES WATERWORKS }\end{array}$ & $\begin{array}{l}\mathbf{3 4 3 7 3 5} \\
\mathbf{3 4 3 7 3 9} \\
\mathbf{3 4 3 7 3 6} \\
\mathbf{3 4 3 7 4 0} \\
\mathbf{3 4 2 2 2 6}\end{array}$ & $\begin{array}{l}0923028 \\
0923105 \\
0923107 \\
0923120 \\
0910808\end{array}$ & $\begin{array}{l}08040203 \\
08040203 \\
08040203 \\
08040203 \\
08020303\end{array}$ \\
\hline $\begin{array}{l}30925 \\
30925 \\
30926 \\
30926 \\
30926\end{array}$ & $\begin{array}{r}3549150934615 \\
354915093461501 \\
332130093294501 \\
332129093294502 \\
332133093294401\end{array}$ & $\begin{array}{l}\text { ST PAUL WATERWORKS } \\
\text { ST PAUL WATERWORKS } \\
\text { STAMPS WATERWORKS } \\
\text { STAMPS WATERWORKS } \\
\text { STAMPS WATERWORKS }\end{array}$ & $\begin{array}{c}354915 \\
-- \\
332130 \\
332129 \\
332133\end{array}$ & $\begin{array}{c}0934615 \\
-- \\
0932945 \\
0932945 \\
0932944\end{array}$ & $\begin{array}{l}11010001 \\
11010001 \\
11140205 \\
11140205 \\
11140205\end{array}$ \\
\hline $\begin{array}{l}30927 \\
30927 \\
30929 \\
30930 \\
30931\end{array}$ & $\begin{array}{l}335631091512101 \\
335643091511701 \\
332437093043102 \\
355758091190501 \\
330627092213500\end{array}$ & $\begin{array}{l}\text { STAR CITY WATER CO } \\
\text { STAR CITY WATER CO } \\
\text { STEPHENS WATERWORKS } \\
\text { STRAWBERRY WATERWORKS } \\
\text { STRONG WATERWORKS }\end{array}$ & $\begin{array}{l}335632 \\
335643 \\
332420 \\
355800 \\
330627\end{array}$ & $\begin{array}{l}0915127 \\
0915117 \\
0930400 \\
0911900 \\
0922135\end{array}$ & $\begin{array}{l}08040205 \\
08040205 \\
08040201 \\
11010012 \\
08040202\end{array}$ \\
\hline $\begin{array}{l}30931 \\
30931 \\
30931 \\
30931 \\
30931\end{array}$ & $\begin{array}{l}330644092212601 \\
330643092214801 \\
330651092212001 \\
330607092212101 \\
330619092221401\end{array}$ & $\begin{array}{l}\text { STRONG WATERWORKS } \\
\text { STRONG WATERWORKS } \\
\text { STRONG WATERWORKS } \\
\text { STRONG WATERWORKS } \\
\text { STRONG WATERWORKS }\end{array}$ & $\begin{array}{l}330642 \\
330643 \\
330652 \\
330607 \\
330619\end{array}$ & $\begin{array}{l}0922125 \\
0922153 \\
0922119 \\
0922121 \\
0922214\end{array}$ & $\begin{array}{l}08040202 \\
08040202 \\
08040202 \\
08040202 \\
08040202\end{array}$ \\
\hline $\begin{array}{l}30931 \\
30932 \\
30932 \\
30932 \\
30932\end{array}$ & $\begin{array}{l}330633092213901 \\
342855091331101 \\
342818091332101 \\
343016091325401 \\
343015091325401\end{array}$ & $\begin{array}{l}\text { STRONG WATERWORKS } \\
\text { STUTTGART WATERWORKS } \\
\text { STUTTGART WATERWORKS } \\
\text { STUTTGART WATERWORKS } \\
\text { STUTTGART WATERWORKS }\end{array}$ & $\begin{array}{l}330633 \\
342855 \\
342818 \\
343016 \\
343015\end{array}$ & $\begin{array}{l}0922139 \\
0913311 \\
0913321 \\
0913254 \\
0913254\end{array}$ & $\begin{array}{l}08040202 \\
08020402 \\
08020402 \\
08020402 \\
08020402\end{array}$ \\
\hline $\begin{array}{l}30932 \\
30932 \\
30932 \\
30933 \\
30934\end{array}$ & $\begin{array}{r}343018091325201 \\
343013091325401 \\
343013091325201 \\
3517000933815 \\
362715090433001\end{array}$ & $\begin{array}{l}\text { STUTTGART WATERWORKS } \\
\text { STUTTGART WATERWORKS } \\
\text { STUTTGART WATERWORKS } \\
\text { SUBIACO ACADEWY WATERWORKS } \\
\text { SUCCESS WATERWORKS }\end{array}$ & $\begin{array}{l}343014 \\
343013 \\
343013 \\
351700 \\
362715\end{array}$ & $\begin{array}{l}0913252 \\
0913254 \\
0913252 \\
0933815 \\
0904330\end{array}$ & $\begin{array}{l}08020402 \\
08020402 \\
08020402 \\
11110202 \\
11010008\end{array}$ \\
\hline $\begin{array}{l}30934 \\
30935 \\
30937 \\
30938 \\
30939\end{array}$ & $\begin{array}{r}362715090433002 \\
362913094273101 \\
361511092413801 \\
3549300910800 \\
345445092114501\end{array}$ & $\begin{array}{l}\text { SUCCESS WATERWORKS } \\
\text { SULPHUR SPRGS WATERWORKS } \\
\text { SUMMIT WATERWORKS } \\
\text { SWIFTON WATERWORKS } \\
\text { SYCAMORE VALLEY MHP }\end{array}$ & $\begin{array}{l}362230 \\
361600 \\
354930 \\
\mathbf{3 4 5 4 4 5}\end{array}$ & $\begin{array}{l}-- \\
0935230 \\
0944200 \\
0910800 \\
0921145\end{array}$ & $\begin{array}{l}11010008 \\
11070208 \\
11010003 \\
11010013 \\
08020402\end{array}$ \\
\hline $\begin{array}{l}30940 \\
30941 \\
30944 \\
30945 \\
30946\end{array}$ & $\begin{array}{r}3622300935230 \\
330609093274302 \\
3432450931040 \\
334633092292901 \\
334248091270801\end{array}$ & $\begin{array}{l}\text { SYLVAN SHORES S/D WATER } \\
\text { TAYLOR WATERWORKS } \\
\text { THOMPSON MHP WATER SYSTEM } \\
\text { THORNTON WATERWORKS } \\
\text { TILLAR WATERWORKS }\end{array}$ & $\begin{array}{l}362230 \\
330645 \\
343245 \\
334630 \\
\mathbf{3 3 4 2 4 8}\end{array}$ & $\begin{array}{l}0935230 \\
0932740 \\
0931040 \\
0922930 \\
0912707\end{array}$ & $\begin{array}{l}11010001 \\
11140205 \\
08040101 \\
08040201 \\
08050001\end{array}$ \\
\hline $\begin{array}{l}30947 \\
30948 \\
30949 \\
30950 \\
30950\end{array}$ & $\begin{array}{r}3442000924115 \\
333745092212201 \\
334815093524500 \\
354000090301201 \\
354038090305901\end{array}$ & $\begin{array}{l}\text { TIMBER RIDGE RANCH WATER } \\
\text { TINSWAN WATERWORKS } \\
\text { TOLLETTE WATER } \\
\text { TRUMAN WATERWORKS } \\
\text { TRUMAN WATERWORKS }\end{array}$ & $\begin{array}{l}344200 \\
333745 \\
334815 \\
354015 \\
354030\end{array}$ & $\begin{array}{l}0924115 \\
0922123 \\
0935245 \\
0903015 \\
0903045\end{array}$ & $\begin{array}{l}08040203 \\
08040201 \\
11140109 \\
08020203 \\
08020203\end{array}$ \\
\hline
\end{tabular}




\begin{tabular}{|c|c|c|c|c|c|c|c|}
\hline $\begin{array}{c}\text { Source } \\
\text { type }\end{array}$ & Source & $\begin{array}{l}\text { Pump } \\
\text { Horse- } \\
\text { eomer }\end{array}$ & $\begin{array}{l}\text { descript } \\
\text { Power } \\
\text { source }\end{array}$ & $\begin{array}{l}\text { ion } \\
\text { Pump } \\
\text { type }\end{array}$ & $\begin{array}{l}\text { Well } \\
\text { dia- } \\
\text { meter } \\
\text { inches }\end{array}$ & $\begin{array}{c}\text { Depth } \\
\text { of } \\
\text { well } \\
\text { s) (feet) }\end{array}$ & Local descriotive ID \\
\hline $\begin{array}{l}\text { GROUND } \\
\text { GROUND } \\
\text { GROUND } \\
\text { GROUND } \\
\text { GROUND }\end{array}$ & $\begin{array}{c}\text { SPARTA SAND } \\
\text { SPARTA SAND } \\
-- \\
-- \\
--\end{array}$ & $\begin{array}{l}-- \\
-- \\
-- \\
50\end{array}$ & $\begin{array}{l}-- \\
-- \\
-- \\
\text { ELC } \\
\text { ELC }\end{array}$ & $\begin{array}{l}-- \\
-- \\
\text { STP } \\
\text { STP }\end{array}$ & $\begin{array}{r}13 \\
13 \\
-- \\
8 \\
6\end{array}$ & $\begin{array}{l}470 \\
473 \\
-- \\
496 \\
473\end{array}$ & $\begin{array}{l}\text { WELL } 7 / \text { PUMP ONLY WHEN NEEDED } \\
\text { WELL NO.8 } \\
\text { CENTROID FOR WELLS } 6 \text { AND } 8 \\
\text { WELL NO. } 6 \\
\text { WELL NO.8 }\end{array}$ \\
\hline $\begin{array}{l}\text { GROUND } \\
\text { GROUND } \\
\text { GROUND } \\
\text { GROUND } \\
\text { GROUND }\end{array}$ & $\begin{array}{l}\text { SPARTA SAND } \\
\text { ROUBIDOUX FORMATION } \\
\text { ROUBIDOUX FORMATION }\end{array}$ & $\begin{array}{r}50 \\
-2 \\
125 \\
50 \\
75\end{array}$ & $\begin{array}{l}\text { ELC } \\
--\overline{1} \\
\text { ELC } \\
\text { ELC } \\
\text { ELC }\end{array}$ & $\begin{array}{l}\text { STP } \\
\text { STP } \\
\text { STP } \\
\text { STP }\end{array}$ & $\begin{array}{r}6 \\
-- \\
6 \\
6 \\
6\end{array}$ & $\begin{array}{l}470 \\
-- \\
451 \\
53 \\
43\end{array}$ & $\begin{array}{l}\text { WELL NO. } 7 \\
-- \\
\text { WELL NO. } \\
\text { WELL NO.3 }\end{array}$ \\
\hline $\begin{array}{l}\text { GROUND } \\
\text { GROUND } \\
\text { GROUND } \\
\text { GROUND } \\
\text { GROUND }\end{array}$ & $\begin{array}{c}\text { CANE RIVER FORMATION } \\
\text { CANE RIVER FORMATION } \\
\text { CANE RIVER FORMATION } \\
-- \\
--\end{array}$ & $\begin{array}{r}\text { N } \\
\text { N } 10 \\
\text { N7.5 } \\
-- \\
--\end{array}$ & $\begin{array}{l}\text { ELC } \\
\text { ELC } \\
\text { ELC } \\
-- \\
\text { ELC }\end{array}$ & $\begin{array}{l}\text { STP } \\
\text { STP } \\
\text { STP } \\
-- \\
--\end{array}$ & $\begin{array}{r}6 \\
2 \\
2 \\
-2 \\
6\end{array}$ & $\begin{array}{l}239 \\
244 \\
210 \\
-- \\
300\end{array}$ & $\begin{array}{l}\text { WELL NO. } 1 \\
\text { WELL NO. } \\
\text { WELL NO.3 } \\
\text { CENTROID/WELLS } 1,2,3,4,5 \\
\text { WELL NO.1 }\end{array}$ \\
\hline $\begin{array}{l}\text { GROUND } \\
\text { GROUND } \\
\text { GROUND } \\
\text { GROUND } \\
\text { GROUND }\end{array}$ & $\begin{array}{l}-- \\
-- \\
-- \\
--\end{array}$ & $\begin{array}{l}7 .- \\
-- \\
-- \\
10\end{array}$ & $\begin{array}{l}\text { ELC } \\
\text { ELC } \\
\text { ELC } \\
\text { ELC } \\
\text { ELC }\end{array}$ & $\begin{array}{l}-- \\
-- \\
-- \\
\text { STP }\end{array}$ & $\begin{array}{l}6 \\
6 \\
6 \\
6 \\
6\end{array}$ & $\begin{array}{l}200 \\
374 \\
300 \\
300 \\
750\end{array}$ & $\begin{array}{l}\text { WELL NO. } 2 \\
\text { WELL NO.3 } \\
\text { WELL NO.4 } \\
\text { WELL NO.5 } \\
\text { WELL NEAR PUMP HOUSE }\end{array}$ \\
\hline $\begin{array}{l}\text { GROUND } \\
\text { GROUND } \\
\text { GROUND } \\
\text { GROUND } \\
\text { GROUND }\end{array}$ & $\begin{array}{lll} & -- & \\
\text { CANE } & \text { RIVER FORMATION } \\
\text { CANE } & \text { RIVER FORMATION } \\
\text { CANE RIVER FORMATION }\end{array}$ & $\begin{array}{r}7.5 \\
1 \\
40 \\
40 \\
--\end{array}$ & $\begin{array}{c}\text { ELC } \\
\text { ELC } \\
-- \\
-- \\
--\end{array}$ & $\begin{array}{l}\text { STP } \\
\text { STP } \\
\text { STP } \\
\text { STP } \\
--\end{array}$ & $\begin{array}{r}2 \\
6 \\
14 \\
14 \\
--\end{array}$ & $\begin{array}{l}148 \\
120 \\
349 \\
378 \\
378\end{array}$ & $\begin{array}{l}\text { WELL NO.1 , SMALL NEW WELL } \\
\text { WELL NO.2 / SMLL } \\
-- \\
--\end{array}$ \\
\hline $\begin{array}{l}\text { GROUND } \\
\text { GROUND } \\
\text { GROUND } \\
\text { GROUND } \\
\text { GROUND }\end{array}$ & $\begin{array}{l}\text { SPARTA SAND } \\
\text { SPARTA SAND } \\
\text { SPARTA SAND } \\
\text { POWELL DOLOMITE } \\
\text { SPARTA SAND }\end{array}$ & $\begin{array}{l}50 \\
75 \\
-- \\
-- \\
--\end{array}$ & $\begin{array}{l}\text { ELC } \\
\text { ELC } \\
-- \\
\text { ELC } \\
--\end{array}$ & $\begin{array}{l}\text { STP } \\
\text { STP } \\
-- \\
\text { STP } \\
--\end{array}$ & $\begin{array}{r}10 \\
12 \\
-- \\
--\end{array}$ & $\begin{array}{r}1,052 \\
900 \\
300 \\
527 \\
--\end{array}$ & $\begin{array}{c}-- \\
-- \\
-- \\
-- \\
\text { ABANDONED / CENTROID }\end{array}$ \\
\hline $\begin{array}{l}\text { GROUND } \\
\text { GROUND } \\
\text { GROUND } \\
\text { GROUND } \\
\text { GROUND }\end{array}$ & $\begin{array}{l}\text { SPARTA SAND } \\
\text { SPARTA SAND } \\
\text { SPARTA SAND } \\
\text { SPARTA SAND } \\
\text { SPARTA SAND }\end{array}$ & $\begin{array}{r}5 \\
10 \\
10 \\
10 \\
10\end{array}$ & $\begin{array}{l}\text { ELC } \\
\text { ELC } \\
\text { ELC } \\
\text { ELC } \\
\text { ELC }\end{array}$ & $\begin{array}{l}\text { STP } \\
\text { STP } \\
\text { STP } \\
\text { STP } \\
\text { STP }\end{array}$ & $\begin{array}{r}18 \\
6 \\
10 \\
6 \\
6\end{array}$ & $\begin{array}{l}430 \\
400 \\
466 \\
290 \\
310\end{array}$ & $\begin{array}{l}\text { WELL NO. } 1 \\
\text { WELL NO.2 } \\
\text { WELL NO.3/NOT PUMPED IN } 2 \text { YRS } \\
\text { WELL NO.5 } \\
\text { WELL NO.6 }\end{array}$ \\
\hline $\begin{array}{l}\text { GROUND } \\
\text { GROUND } \\
\text { GROUND } \\
\text { GROUND } \\
\text { GROUND }\end{array}$ & $\begin{array}{c}\text { SPARTA SAND } \\
-- \\
-- \\
-- \\
--\end{array}$ & $\begin{array}{l}3 \\
-- \\
-- \\
--\end{array}$ & $\begin{array}{l}\text { ELC } \\
-- \\
\text { ELC } \\
\text { ELC } \\
--\end{array}$ & $\begin{array}{l}\text { STP } \\
-- \\
\text { STP } \\
\text { STP } \\
--\end{array}$ & $\begin{array}{l}4 \\
-- \\
12 \\
-- \\
--\end{array}$ & $\begin{array}{l}312 \\
-- \\
-- \\
-- \\
--\end{array}$ & $\begin{array}{l}\text { WELL NO.4 } \\
\text { ABANDONED , CENTROID } \\
\text { WELL NO.1, 760 FT DEEP } \\
\text { WELL NO.2, 760 FT DEEP } \\
\text { WELL NO.3, 760 FT DEEP }\end{array}$ \\
\hline $\begin{array}{l}\text { GROUND } \\
\text { GROUND } \\
\text { GROUND } \\
\text { SURFACE } \\
\text { GROUND }\end{array}$ & $\begin{array}{l}\text { QUATERNARY ALLUVIUM } \\
\text { QUATERNARY ALLUVIUM } \\
\text { QUATERNARY ALLUVIUM } \\
\text { SUBIACO RES } \\
--\end{array}$ & $\begin{array}{l}-- \\
-- \\
-- \\
15 \\
15\end{array}$ & $\begin{array}{l}-- \\
-- \\
\text { ELC } \\
\text { ELC }\end{array}$ & $\begin{array}{l}-- \\
-- \\
\text { STP } \\
\text { STP }\end{array}$ & $\begin{array}{l}-- \\
-- \\
- \\
8 \\
4\end{array}$ & $\begin{array}{l}-- \\
-- \\
-- \\
-- \\
165\end{array}$ & $\begin{array}{l}\text { WELL NO.4，150 FT DEEP／IDLE } \\
\text { WELL NO.5，150 FT DEEP； IDLE } \\
\text { WELL NO.6 } 6 \text { 150 FT DEEP / IDLE } \\
\text { INTAKE TOWER IN LAKE } \\
\text { WELL NO.1 }\end{array}$ \\
\hline $\begin{array}{l}\text { GROUND } \\
\text { GROUND } \\
\text { GROUND } \\
\text { GROUND } \\
\text { GROUND }\end{array}$ & $\begin{array}{l}\text { ROUBIDOUX FORMATION } \\
\text { GUNTER SANDSTONE } \\
\text { QUATERNARY ALLUVIUM } \\
--\end{array}$ & $\begin{array}{r}15 \\
--- \\
7.5 \\
--\end{array}$ & $\begin{array}{l}E L C \\
E L C \\
E L C \\
--\end{array}$ & $\begin{array}{c}\text { STP } \\
-- \\
\text { OTH } \\
-- \\
--\end{array}$ & $\begin{array}{r}4 \\
- \\
4 \\
4 \\
--\end{array}$ & $\begin{array}{r}300 \\
1,442 \\
1,524 \\
120 \\
--\end{array}$ & $\begin{array}{c}\text { WELL NO. } 2 \text { / IDLE } \\
-- \\
-- \\
-- \\
--\end{array}$ \\
\hline $\begin{array}{l}\text { GROUND } \\
\text { GROUND } \\
\text { GROUND } \\
\text { GROUND } \\
\text { GROUND }\end{array}$ & $\begin{array}{l}\text { SPARTA SAND } \\
\text { SPARTA SAND } \\
\text { SPARTA SAND }\end{array}$ & $\begin{array}{r}3 \\
15 \\
-- \\
-- \\
20\end{array}$ & $\begin{array}{l}\text { ELC } \\
\text { ELC } \\
\overline{--} \\
\text { ELC }\end{array}$ & $\begin{array}{l}\text { OTH } \\
\text { STP } \\
-- \\
\text { STP }\end{array}$ & $\begin{array}{l}6 \\
-- \\
-- \\
-- \\
4\end{array}$ & $\begin{array}{l}500 \\
385 \\
-- \\
650 \\
622\end{array}$ & $\begin{array}{r}\text { WELL NO. } 1 \\
-- \\
-- \\
-- \\
--\end{array}$ \\
\hline $\begin{array}{l}\text { GROUND } \\
\text { GROUND } \\
\text { GROUND } \\
\text { GROUND } \\
\text { GROUND }\end{array}$ & $\begin{array}{l}\text { SPARTA SAND } \\
\text { WILCOX GROUP } \\
\text { WILCOX GROUP }\end{array}$ & $\begin{array}{l}-- \\
-- \\
20 \\
25 \\
60\end{array}$ & $\begin{array}{l}-- \\
-- \\
\text { ELC } \\
\text { ELC } \\
\text { ELC }\end{array}$ & $\begin{array}{l}-- \\
\text { STP } \\
\text { STP } \\
\text { STP }\end{array}$ & $\begin{array}{r}-- \\
-- \\
11 \\
8 \\
6\end{array}$ & $\begin{array}{r}-- \\
723 \\
480 \\
1,071 \\
1,071\end{array}$ & $\begin{array}{l}-- \\
-- \\
-- \\
\text { DAVIS ST WELL/WELL NO. } 1 \\
\text { OAK ST WELL/STANDBY WELL }\end{array}$ \\
\hline
\end{tabular}


Table 2.--General description of public-supply

\begin{tabular}{|c|c|c|c|c|c|}
\hline $\begin{array}{c}\text { Water } \\
\text { user } \\
\text { ID } \\
\end{array}$ & $\begin{array}{c}\text { Site } \\
\text { ID } \\
\end{array}$ & Water system name & $\begin{array}{c}\text { Lati- } \\
\text { tude }\end{array}$ & $\begin{array}{l}\text { Longl- } \\
\text { tude }\end{array}$ & $\begin{array}{l}\text { Hydro- } \\
\text { logic } \\
\text { unit } \\
\text { code }\end{array}$ \\
\hline $\begin{array}{l}30950 \\
30951 \\
30952 \\
30952 \\
30953\end{array}$ & $\begin{array}{r}354005090302401 \\
3426250915714 \\
354340091115701 \\
354340091115702 \\
352225090151001\end{array}$ & $\begin{array}{l}\text { TRUMAN WATERWORKS } \\
\text { TUCKER WATERWORKS } \\
\text { TUCKERMAN WATERWORKS } \\
\text { TUCKERMAN WATERWORKS } \\
\text { TURRELL WATERWORKS }\end{array}$ & $\begin{array}{l}354005 \\
342625 \\
354340 \\
354340 \\
352228\end{array}$ & $\begin{array}{l}0903024 \\
0915714 \\
0911157 \\
0911157 \\
0951520\end{array}$ & $\begin{array}{l}08020203 \\
08020401 \\
11010013 \\
11010013 \\
08020203\end{array}$ \\
\hline $\begin{array}{l}30954 \\
30955 \\
30956 \\
30957 \\
30957\end{array}$ & $\begin{array}{r}352924090213001 \\
3434400912748 \\
3557150895745 \\
361025093005101 \\
361130093015801\end{array}$ & $\begin{array}{l}\text { TYRONZA WATERWORKS } \\
\text { ULM WATERWORKS } \\
\text { USAF HOSPITAL/SGPB } \\
\text { VALLEY SPRINGS WATERWORKS } \\
\text { VALLEY SPRINGS WATERWORKS }\end{array}$ & $\begin{array}{l}352915 \\
343440 \\
355715 \\
361025 \\
361130\end{array}$ & $\begin{array}{l}0902130 \\
0912748 \\
0895745 \\
0930051 \\
0930158\end{array}$ & $\begin{array}{l}08020203 \\
08020303 \\
08020204 \\
11010003 \\
11010003\end{array}$ \\
\hline $\begin{array}{l}30958 \\
30958 \\
30959 \\
30959 \\
30960\end{array}$ & $\begin{array}{r}354715090420000 \\
354715090420001 \\
352231090421501 \\
352215090411001 \\
3545150900345\end{array}$ & $\begin{array}{l}\text { VALLEY VIEW WATER ASSOC } \\
\text { VALLEY VIEW WATER ASSOC } \\
\text { VANNDALE-BIRDEYE WATER } \\
\text { VANNDALE-BIRDEYE WATER } \\
\text { VICTORIA WATER ASSOC }\end{array}$ & $\begin{array}{c}354715 \\
-- \\
352215 \\
-- \\
354515\end{array}$ & $\begin{array}{c}0904200 \\
-- \\
0904110 \\
-- \\
0900345\end{array}$ & $\begin{array}{l}08020203 \\
08020203 \\
08020205 \\
08020205 \\
08020203\end{array}$ \\
\hline $\begin{array}{l}30961 \\
30963 \\
30964 \\
30965 \\
30965\end{array}$ & $\begin{array}{r}3315450930310 \\
362359091590001 \\
342140091474101 \\
331956093173601 \\
332050093152501\end{array}$ & $\begin{array}{l}\text { VILLAGE WATER ASSOC } \\
\text { VIOLA WATERWORKS } \\
\text { WABBASEKA WATERWORKS } \\
\text { WALDO WATERWORKS } \\
\text { WALOO WATERWORKS }\end{array}$ & $\begin{array}{l}331545 \\
362400 \\
342138 \\
331956 \\
332050\end{array}$ & $\begin{array}{l}0930310 \\
0915900 \\
0914741 \\
0931736 \\
0931525\end{array}$ & $\begin{array}{l}11140203 \\
11010006 \\
08020401 \\
11140203 \\
11140203\end{array}$ \\
\hline $\begin{array}{l}30965 \\
30965 \\
30967 \\
30968 \\
30968\end{array}$ & $\begin{array}{r}332042093162301 \\
332819093174101 \\
3305250931945 \\
3604080905740 \\
360407090574201\end{array}$ & $\begin{array}{l}\text { WALDO WATERWORKS } \\
\text { WALDO WATERWORKS } \\
\text { WALKERVILLE WATER ASSOC } \\
\text { WALNUT RIDGE WATERWORKS } \\
\text { WALNUT RIDGE WATERWORKS }\end{array}$ & $\begin{array}{l}332042 \\
332819 \\
330525 \\
360408 \\
360407\end{array}$ & $\begin{array}{l}0931623 \\
0931741 \\
0931945 \\
0905740 \\
0905742\end{array}$ & $\begin{array}{l}11140203 \\
11140203 \\
11140203 \\
11010013 \\
11010013\end{array}$ \\
\hline $\begin{array}{l}30968 \\
30969 \\
30969 \\
30969 \\
30970\end{array}$ & $\begin{array}{r}360410090573701 \\
3456300915820 \\
345538091590201 \\
350021091523501 \\
333647092042501\end{array}$ & $\begin{array}{l}\text { WALNUT RIDGE WATERWORKS } \\
\text { WARD WATERWORKS } \\
\text { WARD WATERWORKS } \\
\text { WARD WATERWORKS } \\
\text { WARREN WATERWORKS }\end{array}$ & $\begin{array}{l}360408 \\
345630 \\
345538 \\
350021 \\
333642\end{array}$ & $\begin{array}{l}0905738 \\
0915820 \\
0915902 \\
0915235 \\
0920425\end{array}$ & $\begin{array}{l}11010013 \\
08020301 \\
08020301 \\
08020301 \\
08040204\end{array}$ \\
\hline $\begin{array}{l}30970 \\
30970 \\
30970 \\
30971 \\
30972\end{array}$ & $\begin{array}{l}333647092040701 \\
333648092041101 \\
333647092040602 \\
334640093412101 \\
341117092050401\end{array}$ & $\begin{array}{l}\text { WARREN WATERWORKS } \\
\text { WARREN WATERWORKS } \\
\text { WARREN WATERWORKS } \\
\text { WASHINGTON WATER SYSTEM } \\
\text { WATSON CHAPEL WATER ASSOC }\end{array}$ & $\begin{array}{l}333647 \\
333648 \\
333648 \\
334642 \\
341117\end{array}$ & $\begin{array}{l}0920407 \\
0920411 \\
0920416 \\
0934113 \\
0920458\end{array}$ & $\begin{array}{l}08040204 \\
08040204 \\
08040204 \\
11140201 \\
08040205\end{array}$ \\
\hline $\begin{array}{l}30972 \\
30973 \\
30974 \\
30974 \\
30975\end{array}$ & $\begin{array}{l}341105092050201 \\
335341091152201 \\
353704090540501 \\
353658090540801 \\
330809092461101\end{array}$ & $\begin{array}{l}\text { WATSON CHAPEL WATER ASSOC } \\
\text { WATSON WATERWORKS } \\
\text { WEINER WATERWORKS } \\
\text { WEINER WATERWORKS } \\
\text { WESSON-NEWELL WATER ASSOC }\end{array}$ & $\begin{array}{l}341107 \\
335345 \\
353704 \\
353658 \\
330807\end{array}$ & $\begin{array}{l}0920453 \\
0911520 \\
0905405 \\
0905408 \\
0924613\end{array}$ & $\begin{array}{l}08040205 \\
08050002 \\
08020302 \\
08020302 \\
08040201\end{array}$ \\
\hline $\begin{array}{l}30977 \\
30977 \\
30977 \\
30977 \\
30977\end{array}$ & $\begin{array}{r}3432420903853 \\
343243090384601 \\
343245090385301 \\
343242090390201 \\
343243090384401\end{array}$ & $\begin{array}{l}\text { WEST HELENA WATERWORKS } \\
\text { WEST HELENA WATERWORKS } \\
\text { WEST HELENA WATERWORKS } \\
\text { WEST HELENA WATERWORKS } \\
\text { WEST HELENA WATERWORKS }\end{array}$ & $\begin{array}{l}343242 \\
343240 \\
343245 \\
343242 \\
343243\end{array}$ & $\begin{array}{l}0903853 \\
0903846 \\
0903853 \\
0903902 \\
0903844\end{array}$ & $\begin{array}{l}08020304 \\
08020304 \\
08020304 \\
08020304 \\
08020304\end{array}$ \\
\hline $\begin{array}{l}30978 \\
30979 \\
30980 \\
30981 \\
30981\end{array}$ & $\begin{array}{r}350906090104201 \\
3345350921345 \\
360350090365801 \\
341639092053901 \\
341634092053401\end{array}$ & $\begin{array}{l}\text { WEST MEMPHIS WATERWORKS } \\
\text { WEST SALINE WATER ASSOC } \\
\text { WESTERN GREENE CTY RWD } \\
\text { WHITE HALL WATERWORKS } \\
\text { WHITE HALL WATERWORKS }\end{array}$ & $\begin{array}{l}350910 \\
334535 \\
360445 \\
341639 \\
341634\end{array}$ & $\begin{array}{l}0901049 \\
0921345 \\
0903645 \\
0920539 \\
0920534\end{array}$ & $\begin{array}{l}08020203 \\
08040204 \\
08020302 \\
11110207 \\
11110207\end{array}$ \\
\hline $\begin{array}{l}30983 \\
30985 \\
30986 \\
30987 \\
30988\end{array}$ & $\begin{array}{r}3341150912700 \\
3530000915230 \\
361517091212001 \\
3330500931720 \\
333749091554201\end{array}$ & $\begin{array}{l}\text { WHITE MOBILE HOME PARK } \\
\text { WILBURN WATER ASSOC } \\
\text { WILLI IFORD WATERWORKS } \\
\text { WILLISVILLE WATERWORKS } \\
\text { WILMAR WATERWORKS }\end{array}$ & $\begin{array}{l}334115 \\
353000 \\
361515 \\
333050 \\
333749\end{array}$ & $\begin{array}{l}0912700 \\
0915230 \\
0912145 \\
0931720 \\
0915549\end{array}$ & $\begin{array}{l}08040205 \\
11010014 \\
11010010 \\
08040103 \\
08040204\end{array}$ \\
\hline $\begin{array}{l}30989 \\
30990 \\
30991 \\
30992 \\
30993\end{array}$ & $\begin{array}{r}330335091341301 \\
353344090021002 \\
3333300940845 \\
334610091290401 \\
350745092571501\end{array}$ & $\begin{array}{l}\text { WILMOT WATERWORKS } \\
\text { WILSON WATER SYSIEM } \\
\text { WILTON WATERWORKS } \\
\text { WINCHESTER WATERWORKS } \\
\text { WINROCK INTERNATIONAL WATER }\end{array}$ & $\begin{array}{l}330335 \\
353344 \\
333330 \\
334636 \\
350745\end{array}$ & $\begin{array}{l}0913414 \\
0900210 \\
0940845 \\
0912832 \\
0925715\end{array}$ & $\begin{array}{l}08040205 \\
08020203 \\
11140109 \\
08050001 \\
11110203\end{array}$ \\
\hline
\end{tabular}




\begin{tabular}{|c|c|c|c|c|c|c|c|}
\hline $\begin{array}{l}\text { Source } \\
\text { troe }\end{array}$ & Source & $\begin{array}{l}\text { Pumo } \\
\text { Horse- } \\
\text { pomer }\end{array}$ & $\begin{array}{l}\text { descriot } \\
\text { Power } \\
\text { source }\end{array}$ & $\begin{array}{l}\text { ion } \\
\text { Pump } \\
\text { tyoe }\end{array}$ & $\begin{array}{l}\text { Well } \\
\text { dia- } \\
\text { meter } \\
\text { (inches) }\end{array}$ & $\begin{array}{c}\text { Depth } \\
\text { of } \\
\text { well } \\
\text { (feet) }\end{array}$ & Lecal descriotive ID \\
\hline $\begin{array}{l}\text { GROUND } \\
\text { GROUND } \\
\text { GROUND } \\
\text { GROUND } \\
\text { GROUND }\end{array}$ & $\begin{array}{l}\text { WILCOX GROUP } \\
\text { SPARTA SAND } \\
\text { TERRACE DEPOSITS } \\
\text { TERRACE DEPOSITS } \\
\text { WILCOX GROUP }\end{array}$ & $\begin{array}{l}35 \\
15 \\
20 \\
20 \\
--\end{array}$ & $\begin{array}{l}\text { ELC } \\
\text { ELC } \\
\text { ELC } \\
\text { ELC } \\
--\end{array}$ & $\begin{array}{l}\text { STP } \\
\text { STP } \\
\text { STP } \\
\text { STP } \\
--\end{array}$ & $\begin{array}{r}-- \\
10 \\
8 \\
6 \\
--\end{array}$ & $\begin{array}{r}1,090 \\
643 \\
104 \\
104 \\
1,510\end{array}$ & $\begin{array}{l}\text { CITY HALL WELL } \\
\text { WELL NO.1 } \\
\text { WELL NO.2 } \\
\text {-. }\end{array}$ \\
\hline $\begin{array}{l}\text { GROUND } \\
\text { GROUND } \\
\text { GROUND } \\
\text { GROUND } \\
\text { GROUND }\end{array}$ & $\begin{array}{l}-- \\
-- \\
-- \\
\text { GUNTER SANDSTONE } \\
\text { GUNTER SANDSTONE }\end{array}$ & $\begin{array}{l}-- \\
-- \\
-- \\
60\end{array}$ & $\begin{array}{l}--\overline{-} \\
E L C \\
E L C \\
E L C\end{array}$ & $\begin{array}{l}-- \\
\text { STP } \\
\text { STP } \\
\text { STP }\end{array}$ & $\begin{array}{c}4 \\
-- \\
8 \\
8\end{array}$ & $\begin{array}{c}-- \\
-- \\
2,055 \\
2,109\end{array}$ & $\begin{array}{l}-- \\
-- \\
\text { WELL NO.1 } \\
\text { WELL NO.2 }\end{array}$ \\
\hline $\begin{array}{l}\text { GROUND } \\
\text { GROUND } \\
\text { GROUND } \\
\text { GROUND } \\
\text { GROUND }\end{array}$ & $\begin{array}{l}-- \\
\text { MEMPHIS AQUIFER } \\
\text { MEMPHIS AQUIFER } \\
--\end{array}$ & $\begin{array}{l}-- \\
-- \\
75 \\
60 \\
7.5\end{array}$ & $\begin{array}{l}-- \\
\text { ELC } \\
\text { ELC } \\
\text { ELC }\end{array}$ & $\begin{array}{l}-- \\
\text { STP } \\
\text { STP } \\
\text { STP }\end{array}$ & $\begin{array}{r}-- \\
10 \\
10 \\
6\end{array}$ & $\begin{array}{c}-- \\
-\overline{-} \\
1,132 \\
1,082 \\
1,427\end{array}$ & $\begin{array}{l}\text { STADIUM NO. } 1 \\
\text { HARRISBURG NO.2 } \\
\text { WELL NO.1 } \\
\text { WELL NO.2 } \\
\end{array}$ \\
\hline $\begin{array}{l}\text { GROUND } \\
\text { GROUND } \\
\text { GROUND } \\
\text { GROUND } \\
\text { GROUND }\end{array}$ & $\begin{array}{l}\text { ROUBIDOUX FORMATION } \\
\text { SPARTA SAND } \\
\text { SPARTA SAND } \\
\text { SPARTA SAND }\end{array}$ & $\begin{array}{l}-- \\
25 \\
-- \\
50 \\
50\end{array}$ & $\begin{array}{l}--\overline{-} \\
E L C \\
-\bar{C} \\
\text { ELC }\end{array}$ & $\begin{array}{l}-- \\
\text { STP } \\
-- \\
\text { STP } \\
\text { STP }\end{array}$ & $\begin{array}{r}-- \\
-8 \\
--\end{array}$ & $\begin{array}{l}-- \\
950 \\
756 \\
411 \\
585\end{array}$ & $\begin{array}{l}-- \\
-- \\
\text { GENESITE WELL } \\
\text { COTTON BELT WELL }\end{array}$ \\
\hline $\begin{array}{l}\text { GROUND } \\
\text { GROUND } \\
\text { GROUND } \\
\text { GROUND } \\
\text { GROUND }\end{array}$ & $\begin{array}{c}\text { SPARTA SAND } \\
\text { SPARTA SAND } \\
-- \\
-- \\
--\end{array}$ & $\begin{array}{r}30 \\
30 \\
5 \\
--\end{array}$ & $\begin{array}{l}\text { ELC } \\
\text { ELC } \\
\text { ELC } \\
-- \\
--\end{array}$ & $\begin{array}{l}\text { STP } \\
\text { STP } \\
-- \\
-- \\
--\end{array}$ & $\begin{array}{r}- \\
6 \\
2 \\
--\end{array}$ & $\begin{array}{l}385 \\
385 \\
450 \\
-- \\
--\end{array}$ & $\begin{array}{l}\text { SIMUS WELL } \\
-- \\
-- \\
\text { CENTROID/WELLS } 1 \text { AND } 2 \\
\text { WELL NO.1 }\end{array}$ \\
\hline $\begin{array}{l}\text { GROUND } \\
\text { GROUND } \\
\text { GROUND } \\
\text { GROUND } \\
\text { GROUND }\end{array}$ & $\begin{array}{l}\text { TERRACE DEPOSITS } \\
\text { SPARTA SAND } \\
\text { SPARTA SAND } \\
\text { COCKFIELD FORMATION } \\
\text { SPARTA SAND }\end{array}$ & $\begin{array}{l}-- \\
20 \\
20 \\
30 \\
--\end{array}$ & $\begin{array}{l}--\overline{1} \\
\text { ELC } \\
\text { ELC } \\
--\end{array}$ & $\begin{array}{l}-- \\
\text { STP } \\
\text { STP } \\
\text { STP } \\
--\end{array}$ & $\begin{array}{l}-- \\
24 \\
12 \\
16 \\
--\end{array}$ & $\begin{array}{l}140 \\
-- \\
106 \\
114 \\
--\end{array}$ & $\begin{array}{l}\text { WELL NO.2 } \\
\text { WELL NO.1 } \\
\text { WELL NO.2 } \\
\text { WELL NO.4 } \\
\text { CENTROID/WELLS } 3,4 \text { AND } 5\end{array}$ \\
\hline $\begin{array}{l}\text { GROUND } \\
\text { GROUND } \\
\text { GROUND } \\
\text { GROUND } \\
\text { GROUND }\end{array}$ & $\begin{array}{l}\text { SPARTA SAND } \\
\text { SPARTA SAND } \\
\text { SPARTA SAND } \\
\text { TOKIO FORMATION } \\
\text { SPARTA SAND }\end{array}$ & $\begin{array}{r}-- \\
-- \\
-- \\
100\end{array}$ & $\begin{array}{l}E L C \\
\text { ELC } \\
\text { ELC } \\
-\overline{-}\end{array}$ & $\begin{array}{l}\text { STP } \\
\text { STP } \\
\text { STP } \\
-- \\
\text { STP }\end{array}$ & $\begin{array}{r}16 \\
8 \\
10 \\
-- \\
8\end{array}$ & $\begin{array}{r}1,022 \\
1,070 \\
1,050 \\
953 \\
865\end{array}$ & $\begin{array}{l}\text { WELL NO.5 } \\
\text { WELL NO.3 , IN CITY PARK } \\
\text { WELL NO.4, IN CITY PARK } \\
\text { WELL NO.1 }\end{array}$ \\
\hline $\begin{array}{l}\text { GROUND } \\
\text { GROUND } \\
\text { GROUND } \\
\text { GROUND } \\
\text { GROUND }\end{array}$ & $\begin{array}{l}\text { SPARTA SAND } \\
\text { SPARTA SAND } \\
\text { ALLUVIUM } \\
\text { ALLUVIUM } \\
\text { SPARTA SAND }\end{array}$ & $\begin{array}{r}100 \\
-- \\
30 \\
15 \\
--\end{array}$ & $\begin{array}{l}E L C \\
-- \\
\text { ELC } \\
--\end{array}$ & $\begin{array}{l}-- \\
-\overline{-} \\
\text { STP } \\
\text { STP }\end{array}$ & $\begin{array}{r}8 \\
-- \\
6 \\
6 \\
8\end{array}$ & $\begin{array}{l}850 \\
626 \\
175 \\
180 \\
636\end{array}$ & $\begin{array}{l}\text { WELL NO. } 2 \\
\text { OLD STANDBY WELL/SW OF CH } \\
\text { INSIDE FENCE AT WATER PLANT }\end{array}$ \\
\hline $\begin{array}{l}\text { GROUND } \\
\text { GROUND } \\
\text { GROUND } \\
\text { GROUND } \\
\text { GROUND }\end{array}$ & $\begin{array}{l}\text { SPARTA SAND } \\
-- \\
\text { SPARTA SAND }\end{array}$ & $\begin{array}{r}-- \\
-- \\
100\end{array}$ & $\begin{array}{r}\text { ELC } \\
-- \\
\overline{--} \\
\text { ELC }\end{array}$ & $\begin{array}{c}\text { STP } \\
-- \\
-- \\
\text { STP }\end{array}$ & $\begin{array}{l}-- \\
-- \\
-- \\
-- \\
16\end{array}$ & $\begin{array}{l}-- \\
-- \\
-- \\
-- \\
525\end{array}$ & $\begin{array}{l}\text { CENTROID/WELLS } 7,8,9.10 \\
\text { WELL NO.7 } \\
\text { WELL NO.8 } \\
\text { WELL NO.9 } \\
\text { WELL NO.10 }\end{array}$ \\
\hline $\begin{array}{l}\text { GROUND } \\
\text { GROUND } \\
\text { GROUND } \\
\text { GROUND } \\
\text { GROUND }\end{array}$ & $\begin{array}{l}\text { WILCOX GROUP } \\
\text { WILCOX GROUP } \\
\text { SPARTA SAND } \\
\text { SPARTA SAND }\end{array}$ & $\begin{array}{l}-- \\
-- \\
30 \\
30\end{array}$ & $\begin{array}{l}-- \\
-- \\
\text { ELC } \\
\text { ELC }\end{array}$ & $\begin{array}{r}-- \\
-- \\
\text { STP } \\
\text { STP }\end{array}$ & $\begin{array}{l}-- \\
-- \\
-- \\
10 \\
10\end{array}$ & $\begin{array}{c}1.470 \\
-311 \\
904 \\
865\end{array}$ & $\begin{array}{ll} & -- \\
\text { WELL NO. } & -- \\
\text { WELL NO.1 } & \end{array}$ \\
\hline $\begin{array}{l}\text { GROUND } \\
\text { GROUND } \\
\text { GROUND } \\
\text { GROUND } \\
\text { GROUND }\end{array}$ & $\begin{array}{l}-- \\
\text { ROUBIDOUX FORMATION } \\
\text { SPARTA SAND }\end{array}$ & $\begin{array}{r}-- \\
2 \\
20 \\
--\end{array}$ & $\begin{array}{l}--\overline{1} \\
\text { ELC } \\
\overline{E L C}\end{array}$ & $\begin{array}{l}-- \\
\text { STP } \\
\text { STP } \\
\text { STP }\end{array}$ & $\begin{array}{r}-- \\
8 \\
-8 \\
8\end{array}$ & $\begin{array}{c}1, \overline{800} \\
670 \\
-- \\
543\end{array}$ & WELL MEAR PUMP HOUSE/ HWY 19 \\
\hline $\begin{array}{l}\text { GROUND } \\
\text { GROUND } \\
\text { GROUND } \\
\text { GROUND } \\
\text { SURFACE }\end{array}$ & $\begin{array}{l}\text { COCKFIELD FORMATION } \\
\text { WILCOX GROUP } \\
-- \\
\text { COCKFIELD FORMATION } \\
\text { LAKE GEORGE }\end{array}$ & $\begin{array}{r}40 \\
50 \\
-- \\
5 \\
20\end{array}$ & $\begin{array}{l}\text { ELC } \\
\text { ELC } \\
\text { ELC } \\
\text { ELC }\end{array}$ & $\begin{array}{l}-- \\
\text { STP } \\
-- \\
\text { STP } \\
\text { STP }\end{array}$ & $\begin{array}{r}6 \\
-- \\
-- \\
4 \\
6\end{array}$ & $\begin{array}{c}360 \\
1,542 \\
-- \\
556 \\
--\end{array}$ & $\begin{array}{c}-- \\
-- \\
-- \\
\overline{-} \\
\text { LAKE NEAR PUMP HOUSE }\end{array}$ \\
\hline
\end{tabular}


Table 2.--General description of public-supply

\begin{tabular}{|c|c|c|c|c|c|}
\hline $\begin{array}{c}\text { Water } \\
\text { user } \\
\text { ID } \\
\end{array}$ & $\begin{array}{l}\text { Site } \\
\text { ID }\end{array}$ & water sustem name & $\begin{array}{l}\text { Lati- } \\
\text { tude }\end{array}$ & $\begin{array}{l}\text { Longi- } \\
\text { tude }\end{array}$ & $\begin{array}{l}\text { Hydro- } \\
\text { logic } \\
\text { unit } \\
\text { code }\end{array}$ \\
\hline $\begin{array}{l}30995 \\
30996 \\
30996 \\
30996 \\
30997\end{array}$ & $\begin{array}{r}3555300942300 \\
343635092283001 \\
343635092283002 \\
343635092283003 \\
343118092123401\end{array}$ & $\begin{array}{l}\text { WINTHROP WATER ASSOC } \\
\text { WOODLAND HILLS WATER ASSOC } \\
\text { WOODLAND HILLS WATER ASSOC } \\
\text { WOODLAND HILLS WATER ASSOC } \\
\text { WOODSON-HENSLEY WATER }\end{array}$ & $\begin{array}{c}355530 \\
-- \\
-- \\
\overline{-} \\
343118\end{array}$ & $\begin{array}{c}0942300 \\
-- \\
-- \\
0 \\
0921234\end{array}$ & $\begin{array}{l}11140109 \\
11110207 \\
11110207 \\
11110207 \\
11110207\end{array}$ \\
\hline $\begin{array}{l}30997 \\
30997 \\
30998 \\
30999 \\
31000\end{array}$ & $\begin{array}{r}343118092123701 \\
3431180921236 \\
3512000922715 \\
3425020940432 \\
351216090472201\end{array}$ & $\begin{array}{l}\text { WOODSON-HENSLEY WATER } \\
\text { WOODSON-HENSLEY WATER } \\
\text { WOOSTER WATERWORKS } \\
\text { WRIGHT-PASTORIA WATER } \\
\text { WYNNE WATERWORKS }\end{array}$ & $\begin{array}{r}343118 \\
-1 \\
351200 \\
342502 \\
351216\end{array}$ & $\begin{array}{l}0921237 \\
-- \\
0922715 \\
0940432 \\
0904722\end{array}$ & $\begin{array}{l}11110207 \\
1110205 \\
11110207 \\
08020205\end{array}$ \\
\hline $\begin{array}{l}31000 \\
31000 \\
31000 \\
31001 \\
31002\end{array}$ & $\begin{array}{r}351203090466001 \\
351211090464901 \\
351230090474501 \\
3342150941930 \\
355848089542201\end{array}$ & $\begin{array}{l}\text { WYNNE WATERWORKS } \\
\text { WYNNE WATERWORKS } \\
\text { WYNNE WATERWORKS } \\
\text { YARBOROUGH LANDING WATER } \\
\text { YARBRO WATERWORKS }\end{array}$ & $\begin{array}{l}351203 \\
351211 \\
351230 \\
334215 \\
355848\end{array}$ & $\begin{array}{l}0904660 \\
0904649 \\
0904745 \\
0941930 \\
0895422\end{array}$ & $\begin{array}{l}08020205 \\
08020205 \\
08020205 \\
11140109 \\
08020204\end{array}$ \\
\hline $\begin{array}{l}31004 \\
31004 \\
31004 \\
31004 \\
31004\end{array}$ & $\begin{array}{l}335135091504301 \\
334444091491501 \\
335851091522201 \\
335858091521701 \\
335608091525601\end{array}$ & $\begin{array}{l}\text { YORKTOWN WATER ASSOC } \\
\text { YORKTOWN WATER ASSOC } \\
\text { YORKTOWN WATER ASSOC } \\
\text { YORKTOWN WATER ASSOC } \\
\text { YORKTOWN WATER ASSOC }\end{array}$ & $\begin{array}{l}335135 \\
334450 \\
335851 \\
335858 \\
335608\end{array}$ & $\begin{array}{l}0915043 \\
0914905 \\
0915222 \\
0915217 \\
0915256\end{array}$ & $\begin{array}{l}08040205 \\
08040205 \\
08040205 \\
08040205 \\
08040205\end{array}$ \\
\hline $\begin{array}{l}31005 \\
31015 \\
31034 \\
31058 \\
31059\end{array}$ & $\begin{array}{r}3447100914500 \\
331040092353101 \\
1376753 \\
1376767 \\
1376766\end{array}$ & $\begin{array}{l}\text { ZIMMERMAN NURSING HOME } \\
\text { JOHNSON TOWNSHIP WATER } \\
\text { AUSTIN WATERWORKS } \\
\text { J W SWANSON INC } \\
\text { HATTIEVILLE WATER }\end{array}$ & $\begin{array}{c}344710 \\
331040 \\
-- \\
-- \\
--\end{array}$ & $\begin{array}{c}0914500 \\
0923531 \\
-- \\
-- \\
--\end{array}$ & $\begin{array}{l}08200402 \\
08040201 \\
08020301 \\
11010006 \\
11110203\end{array}$ \\
\hline $\begin{array}{l}31060 \\
31065 \\
31074 \\
31127 \\
31139\end{array}$ & $\begin{array}{l}1376804 \\
1376809 \\
1376818 \\
1376871 \\
1376883\end{array}$ & $\begin{array}{l}\text { BEULAH GROVE WATER } \\
\text { PLEASURE ENTERPRISE } \\
\text { NORTH EAST CROSSETT WATER } \\
\text { LUDWIG WATER ASSOC } \\
\text { NORTH CARBON CITY WATER }\end{array}$ & $\begin{array}{l}-- \\
-- \\
-- \\
--\end{array}$ & $\begin{array}{l}-- \\
-- \\
-- \\
--\end{array}$ & $\begin{array}{l}08040201 \\
08040101 \\
08040202 \\
11110202 \\
11110202\end{array}$ \\
\hline $\begin{array}{l}31150 \\
31150 \\
31157 \\
31185 \\
47455\end{array}$ & $\begin{array}{r}345614091146001 \\
345612091145501 \\
343322090505701 \\
331421092333201 \\
47455059\end{array}$ & $\begin{array}{l}\text { UNITED WATER ASSOC } \\
\text { UNITED WATER ASSOC } \\
\text { BARTON LEXA WATER ASSOC } \\
\text { OLD UNION WATER ASSOC } \\
\text { KIMZEY WATER DISTRICT }\end{array}$ & $\begin{array}{c}-- \\
-- \\
343322 \\
331421 \\
--\end{array}$ & $\begin{array}{c}-- \\
0905057 \\
0923332 \\
--\end{array}$ & $\begin{array}{c}-- \\
08020304 \\
08040201 \\
08040102\end{array}$ \\
\hline $\begin{array}{l}50543 \\
50543\end{array}$ & $\begin{array}{r}3435150922630 \\
343515092263001\end{array}$ & $\begin{array}{l}\text { BENNETT ACRES MH P } \\
\text { BENNETT ACRES MH P }\end{array}$ & 343515 & 0922630 & $\begin{array}{l}11110207 \\
11110207\end{array}$ \\
\hline
\end{tabular}


withdrawal sites in Arkansas--Continued

\begin{tabular}{|c|c|c|c|c|c|c|c|}
\hline $\begin{array}{c}\text { Source } \\
\text { tyoe }\end{array}$ & Source & $\begin{array}{l}\text { Pumo } \\
\text { Horse- } \\
\text { pomer }\end{array}$ & $\begin{array}{l}\text { descript } \\
\text { Power } \\
\text { source }\end{array}$ & $\begin{array}{l}\text { ion } \\
\text { Pump } \\
\text { type }\end{array}$ & $\begin{array}{l}\text { Well } \\
\text { dia- } \\
\text { meter } \\
\text { (inches) }\end{array}$ & $\begin{array}{c}\text { Depth } \\
\text { of } \\
\text { well } \\
\text { (feet) }\end{array}$ & Local descriptive ID \\
\hline $\begin{array}{l}\text { GROUND } \\
\text { GROUND } \\
\text { GROUND } \\
\text { GROUND } \\
\text { GROUND }\end{array}$ & $\begin{array}{c}-- \\
\overline{--} \\
\overline{--} \\
\text { SPARTA SAND }\end{array}$ & $\begin{array}{r}1.5 \\
3 \\
5 \\
7.5 \\
--\end{array}$ & $\begin{array}{l}\text { ELC } \\
\text { ELC } \\
\text { ELC } \\
\text { ELC } \\
\text { ELC }\end{array}$ & $\begin{array}{l}\text { STP } \\
\text { STP } \\
\text { STP } \\
\text { STP } \\
\text { STP }\end{array}$ & $\begin{array}{r}4 \\
-- \\
-- \\
6 \\
8\end{array}$ & $\begin{array}{l}150 \\
360 \\
360 \\
360 \\
250\end{array}$ & $\begin{array}{c}-- \\
\overline{-} \\
\overline{-} \\
\text { WELL NO. } 1 / \text { AMTS FOR BOTH WELLS }\end{array}$ \\
\hline $\begin{array}{l}\text { GROUND } \\
\text { GROUND } \\
\text { GROUND } \\
\text { GROUND } \\
\text { GROUND }\end{array}$ & $\begin{array}{l}\text { SPARTA SAND } \\
\text { SPARTA SANO } \\
\text {-- }\end{array}$ & $\begin{array}{r}-- \\
7.5 \\
25 \\
--\end{array}$ & $\begin{array}{l}E L C \\
-- \\
E L C \\
\text { ELC } \\
--\end{array}$ & $\begin{array}{l}\text { STP } \\
\text { STP } \\
\text { STP } \\
--\end{array}$ & $\begin{array}{r}8 \\
-- \\
6 \\
10 \\
--\end{array}$ & $\begin{array}{l}255 \\
-- \\
-- \\
679 \\
--\end{array}$ & $\begin{array}{c}\text { WELL NO.2 } \\
-- \\
-- \\
\text { CENTROIO/WELLS } 1,2,3\end{array}$ \\
\hline $\begin{array}{l}\text { GROUND } \\
\text { GROUND } \\
\text { GROUND } \\
\text { GROUND } \\
\text { GROUND }\end{array}$ & WILCOX GROUP & $\begin{array}{r}75 \\
75 \\
75 \\
3 \\
--\end{array}$ & $\begin{array}{l}\text { ELC } \\
\text { ELC } \\
\text { ELC } \\
\text { ELC } \\
\text { ELC }\end{array}$ & $\begin{array}{l}\text { STP } \\
\text { STP } \\
\text { STP } \\
\text { STP } \\
\text { STP }\end{array}$ & $\begin{array}{r}12 \\
12 \\
- \\
6 \\
8\end{array}$ & $\begin{array}{r}169 \\
158 \\
157 \\
70 \\
1,360\end{array}$ & $\begin{aligned} & \text { WELL NO. } 1 \\
& \text { WELL NO. } \\
& \text { WELL NO.3 }- \\
&--\end{aligned}$ \\
\hline $\begin{array}{l}\text { GROUND } \\
\text { GROUND } \\
\text { GROUND } \\
\text { GROUND } \\
\text { GROUND }\end{array}$ & $\begin{array}{l}\text { SPARTA SAND } \\
\text { SPARTA SAND } \\
\text { SPARTA SAND } \\
\text { SPARTA SAND }\end{array}$ & $\begin{array}{l}-- \\
50 \\
75 \\
60 \\
60\end{array}$ & $\begin{array}{l}-- \\
\text { ELC } \\
\text { ELC } \\
\text { ELC } \\
\text { ELC }\end{array}$ & $\begin{array}{l}-- \\
\text { STP } \\
\text { STP } \\
\text { STP } \\
\text { STP }\end{array}$ & $\begin{array}{r}- \\
6 \\
6 \\
6 \\
6\end{array}$ & $\begin{array}{r}850 \\
900 \\
1,000 \\
887\end{array}$ & $\begin{array}{l}\text { ABANDONED } \\
\text { WELL NO.3 } \\
\text { WELL NO.1 AND NO. } 2 \\
\text { ABANDONED } \\
\text { WELL NO.5 }\end{array}$ \\
\hline $\begin{array}{l}\text { GROUND } \\
\text { GROUND } \\
\text { GROUND } \\
\text { GROUND } \\
\text { GROUND }\end{array}$ & $\begin{array}{c}-- \\
\text { SPARTA SANO } \\
-- \\
-- \\
--\end{array}$ & $\begin{array}{l}-- \\
-- \\
--\end{array}$ & $\begin{array}{r}E \bar{C} \\
\overline{E L C} \\
--\end{array}$ & $\begin{array}{r}-- \\
\text { STP } \\
\text { STP } \\
--\end{array}$ & $\begin{array}{r}-- \\
10 \\
--\end{array}$ & $\begin{array}{l}-- \\
783 \\
-- \\
-- \\
--\end{array}$ & $\begin{array}{r}-- \\
-- \\
-- \\
- \\
\text { ABANDONED }\end{array}$ \\
\hline $\begin{array}{l}\text { GROUND } \\
\text { SURFACE } \\
\text { GROUND } \\
\text { SURFACE } \\
\text { SURFACE }\end{array}$ & $\begin{array}{l}-- \\
-- \\
-- \\
--\end{array}$ & $\begin{array}{l}-- \\
-- \\
-- \\
--\end{array}$ & $\begin{array}{l}-- \\
-- \\
-- \\
--\end{array}$ & $\begin{array}{l}-- \\
-- \\
-- \\
--\end{array}$ & $\begin{array}{l}-- \\
-- \\
-- \\
--\end{array}$ & $\begin{array}{l}-- \\
-- \\
-- \\
--\end{array}$ & $\begin{array}{l}-- \\
-- \\
-- \\
--\end{array}$ \\
\hline $\begin{array}{l}\text { GROUND } \\
\text { GROUND } \\
\text { GROUND } \\
\text { GROUND } \\
\text { SURFACE }\end{array}$ & $\begin{array}{l}-- \\
-- \\
\text { SPARTA SAND } \\
\text { SPARTA SAND } \\
\text { OUACHITA RIVER }\end{array}$ & $\begin{array}{l}40 \\
25 \\
75 \\
50 \\
--\end{array}$ & $\begin{array}{l}\text { ELC } \\
\text { ELC } \\
\text { ELC } \\
\text { ELC } \\
--\end{array}$ & $\begin{array}{l}\text { STP } \\
\text { STP } \\
\text { STP } \\
\text { STP } \\
--\end{array}$ & $\begin{array}{r}10 \\
8 \\
8 \\
10 \\
-\cdots\end{array}$ & $\begin{array}{l}101 \\
110 \\
672 \\
700 \\
--\end{array}$ & $\begin{array}{c}-- \\
-- \\
\text { PROWELL AND WILLI }- \text { CHURCH } \\
-- \\
--\end{array}$ \\
\hline $\begin{array}{l}\text { GROUND } \\
\text { GROUND }\end{array}$ & -- & $\begin{array}{l}.75 \\
1.5\end{array}$ & $\begin{array}{l}\text { ELC } \\
\text { ELC }\end{array}$ & $\overline{\text { STP }}$ & $\begin{array}{l}3 \\
4\end{array}$ & $\begin{array}{r}85 \\
448\end{array}$ & $\begin{array}{l}\text { WELL NO. } 1 \\
\text { WELL NO. } 2\end{array}$ \\
\hline
\end{tabular}


Table 3.--Supplemental public-supply well information for [Logs available: C, Caliper; D, driller's; E, electrical; G, geologist's;

\begin{tabular}{|c|c|c|c|c|c|c|}
\hline $\begin{array}{l}\text { Water } \\
\text { user } \\
\text { ID } \\
\end{array}$ & $\begin{array}{c}\text { Site } \\
\text { ID } \\
\end{array}$ & Water system name & $\begin{array}{c}\text { Principal } \\
\text { aquifer } \\
\text { geohydrologic } \\
\text { unit }\end{array}$ & $\begin{array}{l}\text { Alt itude } \\
\text { of land } \\
\text { surface } \\
\text { (feet) }\end{array}$ & $\begin{array}{c}\text { Depth } \\
\text { of well } \\
\text { (feet) }\end{array}$ & $\begin{array}{l}\text { Depth to } \\
\text { first } \\
\text { opening } \\
\text { (inches) }\end{array}$ \\
\hline $\begin{array}{l}20560 \\
20560 \\
20560 \\
20560 \\
20560\end{array}$ & $\begin{array}{l}354844090394501 \\
354929090391801 \\
355019090375301 \\
355037090422701 \\
355107090422801\end{array}$ & $\begin{array}{l}\text { JONESBORO WATER SYSTEM } \\
\text { JONESBORO WATER SYSTEM } \\
\text { JONESBORO WATER SYSTEM } \\
\text { JONESBORO WATER SYSTEM } \\
\text { JONESBORO WATER SYSTEM }\end{array}$ & $\begin{array}{l}\text { CLAIBORNE GROUP } \\
\text { QUATERNARY ALLUVIUM } \\
\text { MEMPHIS SAND } \\
\text { CLAIBORNE GROUP } \\
\text { CLAIBORNE GROUP }\end{array}$ & $\begin{array}{l}250 \\
255 \\
245 \\
300 \\
290\end{array}$ & $\begin{array}{l}218 \\
216 \\
244 \\
132 \\
218\end{array}$ & $\begin{array}{r}158 \\
176 \\
204 \\
-- \\
168\end{array}$ \\
\hline $\begin{array}{l}20560 \\
20560 \\
20560 \\
20560 \\
20580\end{array}$ & $\begin{array}{l}355116090422901 \\
355041090422301 \\
354929090392201 \\
354839090403301 \\
334331093381901\end{array}$ & $\begin{array}{l}\text { JONESBORO WATER SYSTEM } \\
\text { JONESBORO WATER SYSTEM } \\
\text { JONESBORO WATER SYSTEM } \\
\text { JONESBORO WATER SYSTEM } \\
\text { HOPE WATERWORKS }\end{array}$ & $\begin{array}{l}\text { CLAIBORNE GROUP } \\
\text { QUATERNARY ALLUVIUM } \\
\text { MEMPHIS SAND } \\
\text { MEMPHIS SAND } \\
\text { TOKIO FORMATION }\end{array}$ & $\begin{array}{l}290 \\
299 \\
256 \\
254 \\
345\end{array}$ & $\begin{array}{r}144 \\
176 \\
240 \\
210 \\
1.191\end{array}$ & $\begin{array}{r}134 \\
126 \\
190 \\
152 \\
1,096\end{array}$ \\
\hline $\begin{array}{l}20580 \\
20580 \\
20580 \\
20580 \\
20609\end{array}$ & $\begin{array}{l}334358093370101 \\
334405093363801 \\
334412093362001 \\
334337093380001 \\
345838091295001\end{array}$ & $\begin{array}{l}\text { HOPE WATERWORKS } \\
\text { HOPE WATERWORKS } \\
\text { HOPE WATERWORKS } \\
\text { HOPE WATERWORKS } \\
\text { DES ARC WATERWORKS }\end{array}$ & $\begin{array}{l}\text { TOKIO FORMATION } \\
\text { TOKIO FORMATION } \\
\text { TOKIO FORMATION } \\
\text { TOKIO FORMATION } \\
\text { TERRACE DEPOSITS }\end{array}$ & $\begin{array}{l}355 \\
335 \\
360 \\
368 \\
200\end{array}$ & $\begin{array}{l}1.140 \\
1.115 \\
1,141 \\
1.134 \\
132\end{array}$ & $\begin{array}{r}1,106 \\
1,100 \\
1,134 \\
\ldots\end{array}$ \\
\hline $\begin{array}{l}20839 \\
30514 \\
30515 \\
30519 \\
30520\end{array}$ & $\begin{array}{l}335430093551801 \\
342416091243701 \\
341918091504901 \\
340345091344602 \\
\mathbf{3 4 2 6 1 8 0 9 1 5 4 5 5 0 1}\end{array}$ & $\begin{array}{l}\text { MINERAL SPRINGS WATERWORK } \\
\text { ALMYRA WATERWORKS } \\
\text { ALTHEIMER WATERWORKS } \\
\text { AR DEPT OF CORREC CUMMINS } \\
\text { ARK DEPT OF CORRECTION }\end{array}$ & $\begin{array}{l}\text { TRINITY GROUP } \\
\text { SPARTA SAND } \\
\text { SPARTA SAND } \\
\text { SPARTA SAND } \\
\text { SPARTA SAND }\end{array}$ & $\begin{array}{l}345 \\
203 \\
200 \\
180 \\
215\end{array}$ & $\begin{array}{r}408 \\
666 \\
1.011 \\
760 \\
725\end{array}$ & $\begin{array}{r}-- \\
606 \\
981 \\
--\end{array}$ \\
\hline $\begin{array}{l}30523 \\
30525 \\
30526 \\
30526 \\
30526\end{array}$ & $\begin{array}{l}333624091124201 \\
361325091363701 \\
334027094073801 \\
334027094073804 \\
333928094065401\end{array}$ & $\begin{array}{l}\text { ARKANSAS CITY WATERWORKS } \\
\text { ASH FLAT WATERWORKS } \\
\text { ASHDOWN WATERWORKS } \\
\text { ASHDOWN WATERWORKS } \\
\text { ASHDOWN WATERWORKS }\end{array}$ & $\begin{array}{l}\text { SPARTA SAND } \\
\text { GUNTER SANDSTONE } \\
\text { TERRACE DEPOSITS } \\
\text { TERRACE DEPOSITS } \\
\text { TERRACE DEPOSITS }\end{array}$ & $\begin{array}{l}136 \\
655 \\
330 \\
330 \\
327\end{array}$ & $\begin{array}{r}520 \\
1,525 \\
90 \\
90 \\
95\end{array}$ & $\begin{array}{r}434 \\
500 \\
-- \\
70 \\
65\end{array}$ \\
\hline $\begin{array}{l}30528 \\
30528 \\
30532 \\
30533 \\
30535\end{array}$ & $\begin{array}{l}351701091214301 \\
351701091214302 \\
333453092160701 \\
353216090074001 \\
330409092171301\end{array}$ & $\begin{array}{l}\text { AUGUSTA WATERWORKS } \\
\text { AUGUSTA WATERWORKS } \\
\text { BANKS WATERWORKS } \\
\text { BASSETT WATERWORKS } \\
\text { BATTS-LAPILE WATER ASSOC }\end{array}$ & $\begin{array}{l}\text { TERRACE DEPOSITS } \\
\text { TERRACE DEPOSITS } \\
\text { SPARTA SAND } \\
\text { WILCOX GROUP } \\
\text { SPARTA SAND }\end{array}$ & $\begin{array}{l}210 \\
210 \\
250 \\
237 \\
190\end{array}$ & $\begin{array}{r}98 \\
114 \\
680 \\
1,560 \\
600\end{array}$ & $\begin{array}{l}-- \\
-- \\
476\end{array}$ \\
\hline $\begin{array}{l}30535 \\
30536 \\
30537 \\
30537 \\
30539\end{array}$ & $\begin{array}{l}330410092171601 \\
354437090335701 \\
334331092370301 \\
334331092370302 \\
350356091524401\end{array}$ & $\begin{array}{l}\text { BATTS-LAPILE WATER ASSOC } \\
\text { BAY WATERWORKS } \\
\text { BEARDEN WATERWORKS } \\
\text { BEARDEN WATERWORKS } \\
\text { BEEBE WATERWORKS }\end{array}$ & $\begin{array}{l}\text { SPARTA SAND } \\
\text { QUATERNARY ALLUVIUM } \\
\text { SPARTA SAND } \\
\text { SPARTA SAND } \\
\text { QUATERNARY ALLUVIUM }\end{array}$ & $\begin{array}{l}180 \\
226 \\
230 \\
230 \\
237\end{array}$ & $\begin{array}{r}339 \\
147 \\
300 \\
350 \\
96\end{array}$ & $\begin{array}{r}309 \\
97 \\
-- \\
310 \\
--\end{array}$ \\
\hline $\begin{array}{l}30539 \\
30541 \\
30544 \\
30550 \\
30553\end{array}$ & $\begin{array}{l}350405091524202 \\
361150093025601 \\
362425094012101 \\
360007092242601 \\
352923090150301\end{array}$ & $\begin{array}{l}\text { BEEBE WATERWORKS } \\
\text { BELLEFONTE WATER } \\
\text { BENTON CTY WATER-DIST } 1 \\
\text { BIG FLAT WATERWORKS } \\
\text { BIRDSONG WHITTEN WATER }\end{array}$ & $\begin{array}{l}\text { TERRACE DEPOSITS } \\
\text { GUNTER SANDSTONE } \\
\text { POTOSI DOLOMITE } \\
\text { ROUBIDOUX FORMATION } \\
\text { WILCOX GROUP }\end{array}$ & $\begin{array}{r}237 \\
1.150 \\
1,430 \\
1,300 \\
225\end{array}$ & $\begin{array}{r}99 \\
1,649 \\
1,968 \\
2,603 \\
1,521\end{array}$ & $\begin{array}{r}-- \\
490 \\
--\end{array}$ \\
\hline $\begin{array}{l}30554 \\
30555 \\
30558 \\
30559 \\
30559\end{array}$ & $\begin{array}{l}344916091241801 \\
355008090220201 \\
335218093343402 \\
355521089540301 \\
355522089541801\end{array}$ & $\begin{array}{l}\text { BISCOE WATERWORKS } \\
\text { BLACK OAK WATERWORKS } \\
\text { BLEVINS WATERWORKS } \\
\text { BLYTHEVILLE WATERWORKS } \\
\text { BLYTHEVILLE WATERWORKS }\end{array}$ & $\begin{array}{l}\text { QUATERNARY ALLUVIUM } \\
\text { WILCOX GROUP } \\
\text { TOKIO FORMATION } \\
\text { WILCOX GROUP } \\
\text { WILCOX GROUP }\end{array}$ & $\begin{array}{l}188 \\
232 \\
425 \\
255 \\
255\end{array}$ & $\begin{array}{r}140 \\
1,070 \\
665 \\
1,480 \\
1,373\end{array}$ & $\begin{array}{r}120 \\
987 \\
602 \\
-- \\
--\end{array}$ \\
\hline $\begin{array}{l}30559 \\
30559 \\
30560 \\
30563 \\
30564\end{array}$ & $\begin{array}{l}355528089542101 \\
355522089541901 \\
333318093412701 \\
333505091491901 \\
332154091292801\end{array}$ & $\begin{array}{l}\text { BLYTHEVILLE WATERWORKS } \\
\text { BLYTHEVILLE WATERWORKS } \\
\text { BOIS D'ARC WATER SYSTEM } \\
\text { BOWSER WATER ASSOCIATION } \\
\text { BOYDELL WATERWORKS }\end{array}$ & $\begin{array}{l}\text { WILCOX GROUP } \\
\text { WILCOX GROUP } \\
\text { NACATOCH SAND } \\
\text { SPARTA SAND } \\
\text { COCKFIELD FORMATION }\end{array}$ & $\begin{array}{l}255 \\
255 \\
270 \\
270 \\
128\end{array}$ & $\begin{array}{r}1,460 \\
1,500 \\
850 \\
715 \\
409\end{array}$ & $\begin{array}{l}-- \\
-- \\
-- \\
380\end{array}$ \\
\hline $\begin{array}{l}30565 \\
30566 \\
30566 \\
30569 \\
30569\end{array}$ & $\begin{array}{l}352457091270601 \\
330537093391302 \\
330616093392301 \\
345616091150201 \\
345616091150402\end{array}$ & $\begin{array}{l}\text { BRADFORD WATERWORKS } \\
\text { BRADLEY WATERWORKS } \\
\text { BRADLEY WATERWORKS } \\
\text { BRINKLEY WATERWORKS } \\
\text { BRINKLEY WATERWORKS }\end{array}$ & $\begin{array}{l}\text { TERRACE DEPOSITS } \\
\text { CANE RIVER FORMATION } \\
\text { CANE RIVER FORMATION } \\
\text { SPARTA SANO } \\
\text { TERRACE DEPOSITS }\end{array}$ & $\begin{array}{l}230 \\
255 \\
253 \\
182 \\
185\end{array}$ & $\begin{array}{r}80 \\
458 \\
440 \\
285 \\
106\end{array}$ & $\begin{array}{r}-- \\
408 \\
-- \\
--\end{array}$ \\
\hline
\end{tabular}


sites investigated by the U.S. Geological Survey

$J$, gamma ray; $T$, temperature; and U, gamma-gamma]

\begin{tabular}{|c|c|c|c|c|c|}
\hline $\begin{array}{c}\text { Casing } \\
\text { diameter } \\
\text { (inches) }\end{array}$ & $\begin{array}{r}\text { Water } \\
\text { level } \\
\text { (feet) }\end{array}$ & $\begin{array}{l}\text { Date } \\
\text { water } \\
\text { level } \\
\text { measured } \\
\end{array}$ & $\begin{array}{c}\text { Discharge } \\
\text { (gallons } \\
\text { per } \\
\text { minute) }\end{array}$ & $\begin{array}{l}\text { Specific } \\
\text { capacity } \\
\text { (gallons per } \\
\text { minute per foot) }\end{array}$ & $\begin{array}{l}\text { Types } \\
\text { of logs } \\
\text { available }\end{array}$ \\
\hline $\begin{array}{r}6.00 \\
6.00 \\
2.00 \\
10.00 \\
2.00\end{array}$ & $\begin{array}{r}38.71 \\
-.- \\
24.85 \\
-\overline{37}\end{array}$ & $\begin{array}{c}10-30-79 \\
-- \\
10-30-79 \\
-- \\
10-15-79\end{array}$ & $\begin{array}{c}-- \\
- \\
1,000 \\
--\end{array}$ & $\begin{array}{l}-- \\
\cdots \\
\cdots \\
\cdots\end{array}$ & $\begin{array}{l}D, E, J \\
D, E, J \\
D, E, J \\
D, E, J\end{array}$ \\
\hline $\begin{array}{r}2.00 \\
8.00 \\
10.00 \\
24.00 \\
12.00\end{array}$ & $\begin{array}{r}21.62 \\
48.16 \\
-- \\
95.22\end{array}$ & $\begin{array}{c}10-23-79 \\
12-09-76 \\
-- \\
-- \\
04-16-72\end{array}$ & $\begin{array}{r}550 \\
1,200 \\
--\end{array}$ & $\begin{array}{l}-- \\
\because- \\
-\end{array}$ & $\begin{array}{r}D, J \\
-\mathcal{G} \\
D, E, J\end{array}$ \\
\hline $\begin{array}{r}12.00 \\
1.0 \\
12.00 \\
20.00 \\
8.00\end{array}$ & $\begin{array}{r}79.44 \\
-- \\
177.21 \\
-- \\
--\end{array}$ & $\begin{array}{c}04-05-71 \\
-- \\
04-04-78 \\
-- \\
--\end{array}$ & $\begin{array}{r}200 \\
-- \\
200 \\
400 \\
--\end{array}$ & 2.30 & $\begin{array}{r}0 \\
0 . J \\
0 . J \\
-.-\end{array}$ \\
\hline $\begin{array}{r}6.00 \\
10.00 \\
6.00 \\
-- \\
10.00\end{array}$ & $\begin{array}{r}-\overline{-} \\
86.38 \\
18.67 \\
95.60 \\
110.25\end{array}$ & $\begin{array}{l}-- \\
02-23-65 \\
12-02-48 \\
11-05-73 \\
08-16-66\end{array}$ & \begin{tabular}{r}
50 \\
200 \\
$\cdots-$ \\
\hdashline- \\
316
\end{tabular} & $\begin{array}{l}-- \\
-- \\
-- \\
--\end{array}$ & $\begin{array}{r}-- \\
\cdots, E, J \\
D\end{array}$ \\
\hline $\begin{array}{r}8.62 \\
8.00 \\
10.00 \\
8.00 \\
12.00\end{array}$ & $\begin{array}{r}128.00 \\
-- \\
-- \\
24.75\end{array}$ & $\begin{array}{c}05-10-66 \\
- \\
-\overline{16}-70\end{array}$ & $\begin{array}{r}300 \\
180 \\
80 \\
-- \\
1,100\end{array}$ & $\begin{array}{c}-. \\
5.40 \\
-- \\
--\end{array}$ & $\begin{array}{r}D \\
G \\
-- \\
--\end{array}$ \\
\hline $\begin{array}{r}10.00 \\
12.00 \\
14.00 \\
8.00 \\
8.00\end{array}$ & $\begin{array}{r}-- \\
-\overline{ } \\
149.52 \\
25.80 \\
177.00\end{array}$ & $\begin{array}{c}-- \\
-- \\
11-24-64 \\
08-02-67 \\
09-01-79\end{array}$ & $\begin{array}{r}-- \\
-- \\
225 \\
160 \\
82\end{array}$ & $\begin{array}{l}-- \\
-- \\
-- \\
.66\end{array}$ & $\begin{array}{l}-- \\
-- \\
-- \\
E\end{array}$ \\
\hline $\begin{array}{r}8.00 \\
16.00 \\
10.00 \\
10.00 \\
10.00\end{array}$ & $\begin{array}{r}131.80 \\
8.96 \\
-- \\
--\end{array}$ & $\begin{array}{c}06-21-89 \\
03-07-68 \\
\cdots \\
-\end{array}$ & $\begin{array}{r}100 \\
465 \\
75 \\
300 \\
1,250\end{array}$ & $\begin{array}{l}-- \\
-- \\
--\end{array}$ & $\begin{array}{l}D \\
-- \\
-- \\
--\end{array}$ \\
\hline $\begin{array}{r}-- \\
-- \\
8.00 \\
8.00 \\
10.00\end{array}$ & $\begin{array}{r}139.82 \\
130.10 \\
640.00 \\
26.08\end{array}$ & $\begin{array}{c}-- \\
06-06-67 \\
08-20-74 \\
06-28-72 \\
08-11-77\end{array}$ & $\begin{array}{r}1,850 \\
95 \\
230 \\
54 \\
-.\end{array}$ & $\begin{array}{r}--\overline{-} \\
.83 \\
-\overline{.23} \\
.-\end{array}$ & $\begin{array}{r}- \\
D \\
D, E, J \\
D, E, J, T \\
D, J\end{array}$ \\
\hline $\begin{array}{l}10.00 \\
10.00 \\
10.00 \\
16.00 \\
18.00\end{array}$ & $\begin{array}{r}21.49 \\
8.85 \\
-0 \\
33.00 \\
--\end{array}$ & $\begin{array}{c}01-26-70 \\
03-07-68 \\
- \\
11-16-66 \\
-\end{array}$ & $\begin{array}{r}425 \\
2,200 \\
-\end{array}$ & $\begin{array}{l}- \\
- \\
- \\
-\end{array}$ & D,E,J \\
\hline $\begin{array}{r}12.00 \\
12.00 \\
4.00 \\
8.00 \\
10.00\end{array}$ & $\begin{array}{r}-- \\
37.45 \\
53.73 \\
177.10 \\
26.12\end{array}$ & $\begin{array}{c}-- \\
03-14-73 \\
04-02-71 \\
03-28-68 \\
11-06-75\end{array}$ & $\begin{array}{r}1.800 \\
-- \\
200 \\
- \\
120\end{array}$ & $\begin{array}{l}-- \\
-- \\
-- \\
--\end{array}$ & $\begin{array}{l}-- \\
-- \\
-\bar{E} \\
E, J\end{array}$ \\
\hline $\begin{array}{l}10.00 \\
10.00\end{array}$ & $69 . \overline{60}$ & $04-\overline{-02-68}$ & -- & -- & - \\
\hline 16.00 & $\begin{array}{l}14.15 \\
16.14\end{array}$ & $\begin{array}{l}05-18-76 \\
10-14-83\end{array}$ & 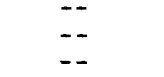 & $\overline{-}$ & $E, \bar{J}$ \\
\hline
\end{tabular}


Table 3.--Supplemental public-supply well information for

\begin{tabular}{|c|c|c|c|c|c|c|}
\hline $\begin{array}{l}\text { Water } \\
\text { user } \\
\text { ID } \\
\end{array}$ & $\begin{array}{c}\text { Site } \\
\text { ID } \\
\end{array}$ & Water system name & $\begin{array}{c}\text { Principal } \\
\text { aquifer } \\
\text { geohydrologic } \\
\text { unit }\end{array}$ & $\begin{array}{l}\text { Altitude } \\
\text { of land } \\
\text { surface } \\
\text { (feet) }\end{array}$ & $\begin{array}{c}\text { Depth } \\
\text { of well } \\
(\text { feet })\end{array}$ & $\begin{array}{l}\text { Depth to } \\
\text { first } \\
\text { opening } \\
\text { (inches) }\end{array}$ \\
\hline $\begin{array}{l}30571 \\
30571 \\
30571 \\
30575 \\
30576\end{array}$ & $\begin{array}{l}343612092293101 \\
343616092293201 \\
343648092380801 \\
345926092002501 \\
360753092062601\end{array}$ & $\begin{array}{l}\text { BRYANT WATERWORKS } \\
\text { BRYANT WATERWORKS } \\
\text { BRYANT WATERWORKS } \\
\text { CABOT WATERWORKS } \\
\text { CALICO ROCK WATERWORKS }\end{array}$ & $\begin{array}{l}\text { CLAYTON FORMATION } \\
\text { CLAYTON FORMATION } \\
\text { PALEOZOIC ERATHEM } \\
\text { ATOKA FORMATION } \\
\text { ROUBIDOUX FORMATION }\end{array}$ & $\begin{array}{l}400 \\
400 \\
345 \\
280 \\
541\end{array}$ & $\begin{array}{r}215 \\
215 \\
325 \\
1,700 \\
1,729\end{array}$ & $\begin{array}{r}-- \\
176 \\
-- \\
-- \\
553\end{array}$ \\
\hline $\begin{array}{l}30577 \\
30577 \\
30579 \\
30580 \\
30580\end{array}$ & $\begin{array}{l}331944092321701 \\
331948092323201 \\
354525090191001 \\
344705091443701 \\
344705091443702\end{array}$ & $\begin{array}{l}\text { CALION WATERWORKS } \\
\text { CALION WATERWORKS } \\
\text { CARAWAY WATERWORKS } \\
\text { CARLISLE WATERWORKS } \\
\text { CARLISLE WATERWORKS }\end{array}$ & $\begin{array}{l}\text { SPARTA SAND } \\
\text { SPARTA SAND } \\
\text { WILCOX GROUP } \\
\text { TERRACE DEPOSITS } \\
\text { QUATERNARY ALLUVIUM }\end{array}$ & $\begin{array}{r}94 \\
95 \\
221 \\
235 \\
235\end{array}$ & $\begin{array}{r}466 \\
508 \\
-- \\
142 \\
142\end{array}$ & $\begin{array}{r}-- \\
438 \\
-- \\
112 \\
--\end{array}$ \\
\hline $\begin{array}{l}30582 \\
30586 \\
30588 \\
30591 \\
30593\end{array}$ & $\begin{array}{l}340429092333201 \\
355631091325401 \\
341345092140501 \\
352359090451401 \\
344145091175601\end{array}$ & $\begin{array}{l}\text { CARTHAGE WATERWORKS } \\
\text { CAVE CITY WATERWORKS } \\
\text { CENTER GROVE WATER ASSOC } \\
\text { CHERRY VALLEY WATERWORKS } \\
\text { CLARENDON WATERWORKS }\end{array}$ & $\begin{array}{l}\text { SPARTA SAND } \\
\text { ST PETER SANDSTONE } \\
\text { SPARTA SAND } \\
\text { MEMPHIS SAND } \\
\text { SPARTA SAND }\end{array}$ & $\begin{array}{l}330 \\
675 \\
260 \\
278 \\
172\end{array}$ & $\begin{array}{l}545 \\
360 \\
760 \\
367 \\
595\end{array}$ & $\begin{array}{c}-- \\
-- \\
-- \\
347 \\
--\end{array}$ \\
\hline $\begin{array}{l}30596 \\
30599 \\
30602 \\
30602 \\
30603\end{array}$ & $\begin{array}{l}333154091340401 \\
360607093180201 \\
362441090344201 \\
362434090350901 \\
361714092302501\end{array}$ & $\begin{array}{l}\text { COLLINS WATER ASSOC } \\
\text { COMPTON WATERWORKS } \\
\text { CORNING WATERWORKS } \\
\text { CORNING WATERWORKS } \\
\text { COTTER WATERWORKS }\end{array}$ & $\begin{array}{l}\text { SPARTA SAND } \\
\text { GUNTER SANDSTONE } \\
\text { TERRACE DEPOSITS } \\
\text { QUATERNARY ALLUVIUM } \\
\text { GUNTER SANDSTONE }\end{array}$ & $\begin{array}{r}169 \\
2.110 \\
292 \\
285 \\
720\end{array}$ & $\begin{array}{r}692 \\
3,215 \\
122 \\
110 \\
1.625\end{array}$ & $\begin{array}{r}635 \\
902 \\
-- \\
-- \\
--\end{array}$ \\
\hline $\begin{array}{l}30604 \\
30604 \\
30608 \\
30610 \\
30610\end{array}$ & $\begin{array}{l}350028091145401 \\
350028091145601 \\
343227091522701 \\
331926092321601 \\
331927092321101\end{array}$ & $\begin{array}{l}\text { COTTON PLANT WATERWORKS } \\
\text { COTTON PLANT WATERWORKS } \\
\text { COY WATERWORKS } \\
\text { CRABAPPLE POINT WATER SYS } \\
\text { CRABAPPLE POINT WATER SYS }\end{array}$ & $\begin{array}{l}\text { MEMPHIS SAND } \\
\text { MEMPHIS SAND } \\
\text { SPARTA SAND } \\
\text { SPARTA SAND } \\
\text { SPARTA SAND }\end{array}$ & $\begin{array}{r}193 \\
193 \\
216 \\
90 \\
90\end{array}$ & $\begin{array}{l}268 \\
250 \\
542 \\
130 \\
130\end{array}$ & $\begin{array}{r}-- \\
-- \\
500 \\
--\end{array}$ \\
\hline $\begin{array}{l}30611 \\
30620 \\
30622 \\
30626 \\
30628\end{array}$ & $\begin{array}{l}351312090192201 \\
351352093093503 \\
361951094271601 \\
333141091253101 \\
344658091274701\end{array}$ & $\begin{array}{l}\text { CRAWFORDSVILLE WATERWORKS } \\
\text { DARDANELLE WATERWORKS } \\
\text { DECATUR WATERWORKS } \\
\text { DERWOTT WATERWORKS } \\
\text { DEVALLS BLUFF WATERWORKS }\end{array}$ & $\begin{array}{l}\text { WILCOX GROUP } \\
\text { QUATERNARY ALLUVIUM } \\
\text { GUNTER SANDSTONE } \\
\text { COCKFIELD FORMATION } \\
\text { QUATERNARY ALLUVIUM }\end{array}$ & $\begin{array}{r}220 \\
330 \\
1,275 \\
135 \\
208\end{array}$ & $\begin{array}{r}1.622 \\
65 \\
1.450 \\
593 \\
108\end{array}$ & $\begin{array}{r}-- \\
-- \\
22 \\
527 \\
92\end{array}$ \\
\hline $\begin{array}{l}30629 \\
30630 \\
30633 \\
30636 \\
30636\end{array}$ & $\begin{array}{l}341734091200601 \\
362702092550301 \\
355323089552101 \\
335200091290001 \\
335202091290201\end{array}$ & $\begin{array}{l}\text { DEWITT WATERWORKS } \\
\text { DIAMOND CITY WATER } \\
\text { DOGWOOD WATER ASSOC } \\
\text { DUMAS WATERWORKS } \\
\text { DUMAS WATERWORKS }\end{array}$ & $\begin{array}{l}\text { SPARTA SAND } \\
\text { POTOSI DOLOMITE } \\
\text { WILCOX GROUP } \\
\text { SPARTA SAND } \\
\text { SPARTA SAND }\end{array}$ & $\begin{array}{l}187 \\
882 \\
250 \\
150 \\
150\end{array}$ & $\begin{array}{r}802 \\
1,415 \\
1,400 \\
796 \\
809\end{array}$ & $\begin{array}{r}500 \\
1,337 \\
-- \\
--\end{array}$ \\
\hline $\begin{array}{l}30637 \\
30638 \\
30643 \\
30643 \\
30643\end{array}$ & $\begin{array}{l}353537090125801 \\
351614090275401 \\
331228092403801 \\
331223092393902 \\
331223092392201\end{array}$ & $\begin{array}{l}\text { DYESS WATERWORKS } \\
\text { EARLE WATERWORKS } \\
\text { EL DORADO WATERWORKS } \\
\text { EL DORADO WATERWORKS } \\
\text { EL DORADO WATERWORKS }\end{array}$ & $\begin{array}{l}\text { WILCOX GROUP } \\
\text { WILCOX GROUP } \\
\text { SPARTA SAND } \\
\text { SPARTA SAND } \\
\text { SPARTA SAND }\end{array}$ & $\begin{array}{l}220 \\
215 \\
220 \\
265 \\
285\end{array}$ & $\begin{array}{r}1.400 \\
1.530 \\
650 \\
746 \\
754\end{array}$ & $\begin{array}{r}1,450 \\
-- \\
--\end{array}$ \\
\hline $\begin{array}{l}30643 \\
30643 \\
30643 \\
30643 \\
30643\end{array}$ & $\begin{array}{l}331223092392401 \\
331303092402201 \\
331235092385601 \\
331358092424301 \\
331406092430101\end{array}$ & $\begin{array}{l}\text { EL DORADO WATERWORKS } \\
\text { EL DORADO WATERWORKS } \\
\text { EL DORADO WATERWORKS } \\
\text { EL DORADO WATERWORKS } \\
\text { EL DORADO WATERWORKS }\end{array}$ & $\begin{array}{l}\text { SPARTA } \\
\text { SPAND } \\
\text { SPARTA SAND } \\
\text { SPARTA SAND } \\
\text { SPARTA SAND } \\
\text { SPARTA SAND }\end{array}$ & $\begin{array}{l}267 \\
255 \\
275 \\
205 \\
220\end{array}$ & $\begin{array}{l}709 \\
712 \\
712 \\
615 \\
704\end{array}$ & $\begin{array}{r}609 \\
-- \\
-\overline{4} \\
603\end{array}$ \\
\hline $\begin{array}{l}30643 \\
30644 \\
30647 \\
30649 \\
30649\end{array}$ & $\begin{array}{l}331346092424601 \\
341831090511202 \\
343246091582302 \\
330640091154103 \\
330646091154401\end{array}$ & $\begin{array}{l}\text { EL DORADO WATERWORKS } \\
\text { ELAINE WATERWORKS } \\
\text { ENGLAND WATER CO } \\
\text { EUDORA WATERWORKS } \\
\text { EUDORA WATERWORKS }\end{array}$ & $\begin{array}{l}\text { SPARTA SAND } \\
\text { CANE RIVER FORMATION } \\
\text { COCKFIELD FORMATION } \\
\text { COCKFIELD FORMATION } \\
\text { COCKFIELD FORMATION }\end{array}$ & $\begin{array}{l}207 \\
173 \\
226 \\
135 \\
135\end{array}$ & $\begin{array}{r}710 \\
1.508 \\
401 \\
332 \\
320\end{array}$ & $\begin{array}{r}605 \\
-- \\
-- \\
280 \\
--\end{array}$ \\
\hline $\begin{array}{l}30651 \\
30651 \\
30653 \\
30654 \\
30654\end{array}$ & $\begin{array}{l}330631092370801 \\
330657092385901 \\
355234090400501 \\
330324092084501 \\
330324092084502\end{array}$ & $\begin{array}{l}\text { FAIRCREST WATER ASSOC } \\
\text { FAIRCREST WATER ASSOC } \\
\text { FARRVILLE-PHILADELPHIA } \\
\text { FELSENTHAL WATER ASSOC } \\
\text { FELSENTHAL WATER ASSOC }\end{array}$ & $\begin{array}{l}\text { SPARTA SAND } \\
\text { SPARTA SAND } \\
\text { WILCOX GROUP } \\
\text { SPARTA SAND } \\
\text { COCKFIELD FORMATION }\end{array}$ & $\begin{array}{r}201 \\
253 \\
330 \\
82 \\
82\end{array}$ & $\begin{array}{l}685 \\
752 \\
160 \\
652 \\
206\end{array}$ & $\begin{array}{l}605 \\
706 \\
130 \\
596 \\
169\end{array}$ \\
\hline
\end{tabular}


sites investigated by the U.S. Geological Survey--Continued

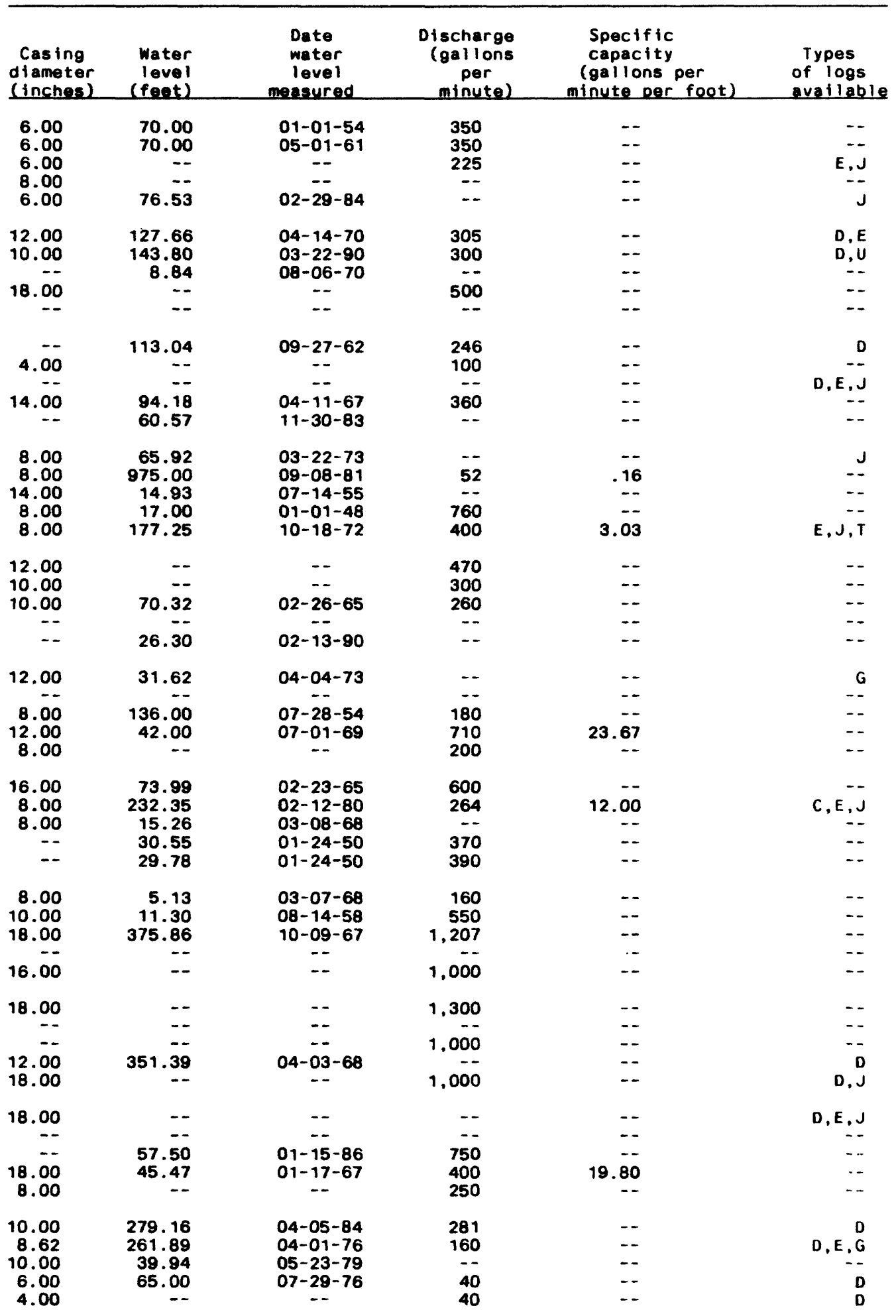


Table 3.--Supplemental public-supply well information for

\begin{tabular}{|c|c|c|c|c|c|c|}
\hline $\begin{array}{l}\text { Water } \\
\text { user } \\
\text { ID } \\
\end{array}$ & $\begin{array}{c}\text { Site } \\
10 \\
\end{array}$ & Water system name & $\begin{array}{c}\text { Principal } \\
\text { aquifer } \\
\text { geohydrologic } \\
\text { unit } \\
\end{array}$ & $\begin{array}{l}\text { Altitude } \\
\text { of land } \\
\text { surface } \\
\text { (feet) } \\
\end{array}$ & $\begin{array}{c}\text { Depth } \\
\text { of wel) } \\
\text { (feet) }\end{array}$ & $\begin{array}{l}\text { Depth to } \\
\text { first } \\
\text { opening } \\
\text { (inches) }\end{array}$ \\
\hline $\begin{array}{l}30655 \\
30656 \\
30657 \\
30657 \\
30657\end{array}$ & $\begin{array}{l}355804092135201 \\
352930090582501 \\
361616092351601 \\
361626092361301 \\
361630092351801\end{array}$ & $\begin{array}{l}\text { FIFTY SIX WATERWORKS } \\
\text { FISHER WATERWORKS } \\
\text { FLIPPIN WATERWORKS } \\
\text { FLIPPIN WATERWORKS } \\
\text { FLIPPIN WATERWORKS }\end{array}$ & $\begin{array}{l}\text { GUNTER SANDSTONE } \\
\text { MEMPHIS SAND } \\
\text { GASCONADE DOLOMITE } \\
\text { ROUBIDOUX FORMATION } \\
\text { ROUBIDOUX FORWATION }\end{array}$ & $\begin{array}{l}985 \\
232 \\
800 \\
675 \\
630\end{array}$ & $\begin{array}{r}3,420 \\
302 \\
1,135 \\
760 \\
900\end{array}$ & $\begin{array}{c}-- \\
260 \\
-- \\
50\end{array}$ \\
\hline $\begin{array}{l}30659 \\
30661 \\
30661 \\
30663 \\
30674\end{array}$ & $\begin{array}{l}334333094235401 \\
350125090472301 \\
350127090472201 \\
33154009350401 \\
340711091224801\end{array}$ & $\begin{array}{l}\text { FOREMAN WATERWORKS } \\
\text { FORREST CITY WATERWORKS } \\
\text { FORREST CITY WATERWORKS } \\
\text { FOUKE WATERWORKS } \\
\text { GILLETT WATERWORKS }\end{array}$ & $\begin{array}{l}\text { TERRACE DEPOSITS } \\
\text { TERRACE DEPOSITS } \\
\text { TERRACE DEPOSITS } \\
\text { WILCOX GROUP } \\
\text { SPARTA SAND }\end{array}$ & $\begin{array}{l}425 \\
260 \\
260 \\
320 \\
185\end{array}$ & $\begin{array}{r}24 \\
140 \\
141 \\
600 \\
720\end{array}$ & $\begin{array}{r}-- \\
100 \\
81 \\
-- \\
--\end{array}$ \\
\hline $\begin{array}{l}30676 \\
30678 \\
30679 \\
30680 \\
30684\end{array}$ & $\begin{array}{l}352450090165201 \\
355712089580201 \\
335908091333401 \\
340445091414001 \\
362503094272001\end{array}$ & $\begin{array}{l}\text { GILMORE WATERWORKS } \\
\text { GOSNELL WATER ASSOCIATION } \\
\text { GOULD WATERWORKS } \\
\text { GRADY WATERWORKS } \\
\text { GRAVETTE WATERWORKS }\end{array}$ & $\begin{array}{l}\text { WILCOX GROUP } \\
\text { WILCOX GROUP } \\
\text { SPARTA SAND } \\
\text { SPARTA SAND } \\
\text { GUNTER SANDSTONE }\end{array}$ & $\begin{array}{r}222 \\
248 \\
167 \\
185 \\
1.200\end{array}$ & $\begin{array}{r}1.373 \\
827 \\
816 \\
1.603\end{array}$ & $\begin{array}{c}\cdots \\
\cdots \\
400\end{array}$ \\
\hline $\begin{array}{l}30684 \\
30688 \\
30692 \\
30692 \\
30692\end{array}$ & $\begin{array}{l}362527094272301 \\
362024090132101 \\
335455093093201 \\
335455093093202 \\
335455093093203\end{array}$ & $\begin{array}{l}\text { GRAVETTE WATERWORKS } \\
\text { GREENWAY WATERWORKS } \\
\text { GURDON WATERWORKS } \\
\text { GURDON WATERWORKS } \\
\text { GURDON WATERWORKS }\end{array}$ & $\begin{array}{l}\text { GUNTER SANDSTONE } \\
\text { NACATOCH SAND } \\
\text { NACATOCH SAND } \\
\text { NACATOCH SAND } \\
\text { NACATOCH SAND }\end{array}$ & $\begin{array}{r}1.210 \\
286 \\
202 \\
202 \\
200\end{array}$ & $\begin{array}{r}1,600 \\
992 \\
245 \\
245 \\
245\end{array}$ & $\begin{array}{c}400 \\
-- \\
-- \\
--\end{array}$ \\
\hline $\begin{array}{l}30694 \\
30696 \\
30699 \\
30706 \\
30707\end{array}$ & $\begin{array}{l}331334091473901 \\
333226092274101 \\
333040092240301 \\
343116090353101 \\
332658092102601\end{array}$ & $\begin{array}{l}\text { HAMBURG WATERWORKS } \\
\text { HAMPTON WATERWORKS } \\
\text { HARRELL WATERWORKS } \\
\text { HELENA WATERWORKS } \\
\text { HERMI TAGE WATERWORKS }\end{array}$ & $\begin{array}{l}\text { TERRACE DEPOSITS } \\
\text { SPARTA SAND } \\
\text { SPARTA SAND } \\
\text { SPARTA SAND } \\
\text { COCKFIELD FORMATION }\end{array}$ & $\begin{array}{l}170 \\
208 \\
205 \\
185 \\
193\end{array}$ & $\begin{array}{l}115 \\
450 \\
613 \\
493 \\
349\end{array}$ & $\begin{array}{c}-- \\
560 \\
-- \\
300\end{array}$ \\
\hline $\begin{array}{l}30109 \\
30712 \\
30712 \\
30712 \\
30712\end{array}$ & $\begin{array}{l}352403090594901 \\
362739093434901 \\
362757093434601 \\
362920093463801 \\
362937093441101\end{array}$ & $\begin{array}{l}\text { HICKORY RIDGE WATERWORKS } \\
\text { HOLIOAY ISLAND WAIERWORKS } \\
\text { HOLIDAY I ISLAND WATERWORKS } \\
\text { HOLIDAY ISLAND WATERWORKS } \\
\text { HOLIDAY I SLAND WATERWORKS }\end{array}$ & $\begin{array}{l}\text { MEMPHIS SAND } \\
\text { GUNTER SANDSTONE } \\
\text { ROUBIDOUX FORMATION } \\
\text { ROUBIDOUX FORMATION } \\
\text { GUNTER SANDSTONE }\end{array}$ & $\begin{array}{r}234 \\
1.520 \\
1.500 \\
1.010 \\
1.102\end{array}$ & $\begin{array}{r}400 \\
1.800 \\
1.184 \\
1.058 \\
1.122\end{array}$ & $\begin{array}{l}- \\
515 \\
500 \\
500 \\
500\end{array}$ \\
\hline $\begin{array}{l}30713 \\
30715 \\
30719 \\
30719 \\
30720\end{array}$ & $\begin{array}{l}343548091121201 \\
335607094204501 \\
360258090574501 \\
360258090584502 \\
345705090284201\end{array}$ & $\begin{array}{l}\text { HOLIY GROVE WATERWORKS } \\
\text { HORATIO WATERWORKS } \\
\text { HOXIE WATER DEPT } \\
\text { HOXIE WATER DEPI } \\
\text { HUGHES WATERWORKS }\end{array}$ & $\begin{array}{l}\text { SPARIA SAND } \\
\text { WOOOHINE FORMAIIION } \\
\text { TERRACE DEPOSIIS } \\
\text { TERRACE DEPOSITS } \\
\text { SPARIA SAND }\end{array}$ & $\begin{array}{l}181 \\
362 \\
265 \\
265 \\
198\end{array}$ & $\begin{array}{r}623 \\
265 \\
75 \\
87 \\
1.091\end{array}$ & $\begin{array}{r}563 \\
-- \\
-- \\
-- \\
--\end{array}$ \\
\hline $\begin{array}{l}30722 \\
30724 \\
30724 \\
30725 \\
30726\end{array}$ & $\begin{array}{l}342515091421001 \\
330219092111201 \\
330208092112101 \\
340547092042001 \\
331226092460101\end{array}$ & $\begin{array}{l}\text { HUMPHREY WATERWORKS } \\
\text { HUTIIG WATERWORKS } \\
\text { HUIIIG WATERWORKS } \\
\text { HWY } 15 \text { WAIER ASSOC } \\
\text { HWY O2 WAIER ASSOC }\end{array}$ & $\begin{array}{l}\text { SPARIA SAND } \\
\text { SPARIA SAND } \\
\text { SPARIA SAND } \\
\text { SPARIA SAND } \\
\text { SPARIA SAND }\end{array}$ & $\begin{array}{l}191 \\
135 \\
120 \\
311 \\
232\end{array}$ & $\begin{array}{l}870 \\
529 \\
528 \\
836 \\
660\end{array}$ & $\begin{array}{r}-- \\
-- \\
448 \\
773 \\
--\end{array}$ \\
\hline $\begin{array}{l}30731 \\
30732 \\
30733 \\
30735 \\
30735\end{array}$ & $\begin{array}{l}360014093112901 \\
342218092095701 \\
353030090090001 \\
330059092430601 \\
330107092432301\end{array}$ & $\begin{array}{l}\text { JASPER WATERWORKS } \\
\text { JEFHERSON-SAMPIES OEXILR } \\
\text { JOINER WATERWORKS } \\
\text { JUNCTION CIIY WATERWORKS } \\
\text { JUNCIION CIIY WAIERWOKKS }\end{array}$ & $\begin{array}{l}\text { EVERIUN FORMAIION } \\
\text { SPARIA SAND } \\
\text { QUAIERNARY ALI UVIUM } \\
\text { SPARIA SANO } \\
\text { SPARIA SAND }\end{array}$ & $\begin{array}{l}880 \\
400 \\
230 \\
185 \\
175\end{array}$ & $\begin{array}{l}190 \\
854 \\
95 \\
529 \\
\text { (20) }\end{array}$ & $\begin{array}{r}40 \\
801 \\
\cdots \\
546\end{array}$ \\
\hline $\begin{array}{l}30737 \\
30739 \\
30744 \\
30744 \\
30745 \\
30741 \\
30149 \\
30750 \\
30752 \\
30753\end{array}$ & $\begin{array}{l}334630091170901 \\
3351330921 / 4901 \\
340444091504201 \\
340631091452001 \\
361118090242201 \\
354851090261201 \\
331943091163801 \\
342403050491401 \\
33120,092291601 \\
355606090152601\end{array}$ & 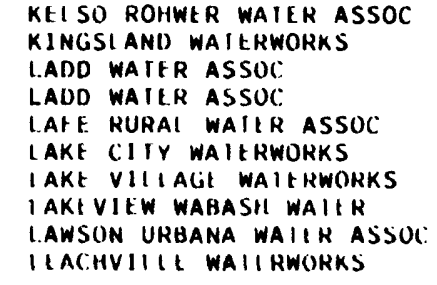 & 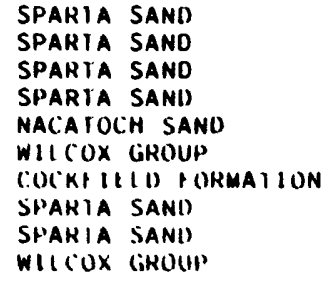 & $\begin{array}{l}141 \\
220 \\
204 \\
180 \\
292 \\
221 \\
110 \\
112 \\
216 \\
2310\end{array}$ & $\begin{array}{r}150 \\
381 \\
1.350 \\
1.180 \\
1.153 \\
909 \\
350 \\
736 \\
172 \\
1.063\end{array}$ & $\begin{array}{r}670 \\
\cdots \\
1.120\end{array}$ \\
\hline
\end{tabular}


sites investigated by the U.S. Geological Survey--Continued

\begin{tabular}{|c|c|c|c|c|c|}
\hline $\begin{array}{c}\text { Casing } \\
\text { diameter } \\
\text { (inches) }\end{array}$ & $\begin{array}{r}\text { Water } \\
\text { level } \\
\text { (feet) }\end{array}$ & $\begin{array}{c}\text { Date } \\
\text { water } \\
\text { level } \\
\text { measured }\end{array}$ & $\begin{array}{c}\text { Discharge } \\
\text { (gal lons } \\
\text { per } \\
\text { minute) }\end{array}$ & $\begin{array}{l}\text { Specific } \\
\text { capacity } \\
\text { (gallons per } \\
\text { minute oer foot) }\end{array}$ & $\begin{array}{c}\text { Types } \\
\text { of logs } \\
\text { availoble }\end{array}$ \\
\hline $\begin{array}{l}6.00^{--} \\
8.00 \\
--\end{array}$ & $\begin{array}{r}399.03 \\
50.11 \\
346.50 \\
\ldots\end{array}$ & $\begin{array}{c}02-09-77 \\
03-04-68 \\
09-28-77 \\
--\end{array}$ & $\begin{array}{r}40 \\
260 \\
250 \\
--\end{array}$ & $\begin{array}{l}18 \\
-- \\
--\end{array}$ & $\begin{array}{l}C, D, E, J \\
C, E, G, J \\
G\end{array}$ \\
\hline 8.00 & 85.62 & $08-14-66$ & 80 & -- & -- \\
\hline $\begin{array}{l}20.00 \\
16.00 \\
16.00 \\
10.00\end{array}$ & $\begin{array}{r}20.33 \\
75.62 \\
75.44 \\
112.77 \\
70.07\end{array}$ & $\begin{array}{l}11-16-67 \\
07-20-60 \\
07-20-60 \\
01-01-66 \\
02-23-65\end{array}$ & $\begin{array}{r}38 \\
825 \\
825 \\
-- \\
475\end{array}$ & $\begin{array}{l}-- \\
-- \\
-- \\
--\end{array}$ & $\begin{array}{l}-- \\
-- \\
D, F \\
--\end{array}$ \\
\hline $\begin{array}{l}-- \\
4.00 \\
4.00 \\
8.00\end{array}$ & $\begin{array}{r}17.80 \\
\overline{-0} \\
170.00\end{array}$ & $\begin{array}{c}-\overline{-} \\
06-01-67 \\
-- \\
-\overline{0} \\
12-01-54\end{array}$ & $\begin{array}{r}-- \\
-- \\
350 \\
550 \\
125\end{array}$ & $\begin{array}{l}-- \\
-- \\
-- \\
--\end{array}$ & $\begin{array}{r}--\bar{G} \\
E, J \\
E \\
D\end{array}$ \\
\hline $\begin{array}{l}8.00 \\
8.00 \\
4.00 \\
4.00 \\
6.00\end{array}$ & $\begin{array}{r}250.00 \\
-5.65 \\
33.40 \\
-. \\
37.47\end{array}$ & $\begin{array}{c}01-01-64 \\
03-02-67 \\
02-00-75 \\
-- \\
07-26-50\end{array}$ & $\begin{array}{r}-- \\
-- \\
60 \\
50 \\
100\end{array}$ & $\begin{array}{l}-- \\
-- \\
-- \\
--\end{array}$ & $\begin{array}{r}D \\
-- \\
-- \\
--\end{array}$ \\
\hline $\begin{array}{r}20.00 \\
18.00 \\
6.00 \\
14.00 \\
10.00\end{array}$ & $\begin{array}{r}41.27 \\
134.49 \\
145.11 \\
25.00 \\
74.83\end{array}$ & $\begin{array}{l}06-26-58 \\
07-06-64 \\
10-20-69 \\
01-01-50 \\
04-28-66\end{array}$ & $\begin{array}{r}250 \\
100 \\
-- \\
1,000 \\
285\end{array}$ & $\begin{array}{l}-- \\
-- \\
-- \\
--\end{array}$ & $\begin{array}{l}D \\
-- \\
-- \\
--\end{array}$ \\
\hline $\begin{array}{r}6.00 \\
8.00 \\
10.00 \\
8.00 \\
12.00\end{array}$ & $\begin{array}{r}50.69 \\
520.00 \\
146.00 \\
86.40 \\
135.24\end{array}$ & $\begin{array}{l}03-04-68 \\
07-07-72 \\
06-30-77 \\
11-00-70 \\
11-00-70\end{array}$ & $\begin{array}{r}33 \\
502 \\
500 \\
600 \\
500\end{array}$ & $\begin{array}{l}-- \\
5.91 \\
7.73 \\
8.82 \\
8.33\end{array}$ & $\begin{array}{r}E, J, T \\
E . \bar{J} \\
E, J\end{array}$ \\
\hline 8.00 & 42.95 & $01-24-67$ & -- & -- & -- \\
\hline-- & - & -- & $\begin{array}{l}10 \\
--\end{array}$ & -- & D.E \\
\hline 24.00 & -- & -- & $\begin{array}{r}550 \\
--\end{array}$ & -- & -- \\
\hline $\begin{array}{r}10.00 \\
10.00 \\
10.00 \\
10.00 \\
6.00\end{array}$ & $\begin{array}{r}93.15 \\
110.19 \\
115.00 \\
261.02 \\
--\end{array}$ & $\begin{array}{c}03-21-67 \\
12-10-64 \\
04-05-84 \\
04-27-82 \\
--\end{array}$ & $\begin{array}{r}300 \\
400 \\
350 \\
--\end{array}$ & $\begin{array}{l}-- \\
-- \\
-- \\
--\end{array}$ & $\begin{array}{r}-- \\
-- \\
D \\
--\end{array}$ \\
\hline $\begin{array}{r}8.00 \\
6.00 \\
12.00 \\
10.00\end{array}$ & $\begin{array}{r}66.28 \\
267.72 \\
-- \\
240.50 \\
182.33\end{array}$ & $\begin{array}{c}06-07-67 \\
11-15-65 \\
-- \\
01-01-80 \\
10-11-67\end{array}$ & $\begin{array}{l}150 \\
200 \\
250 \\
225 \\
412\end{array}$ & $\begin{array}{l}-- \\
-- \\
-- \\
--\end{array}$ & $\begin{array}{l}D \\
-- \\
-- \\
--\end{array}$ \\
\hline $\begin{array}{r}12.00 \\
10.00 \\
-0 \\
15.00 \\
12.00\end{array}$ & $\begin{array}{r}39.56 \\
85.35 \\
119.24 \\
113.60 \\
15.98\end{array}$ & $\begin{array}{l}02-23-76 \\
10-31-67 \\
03-27-68 \\
06-23-69 \\
04-18-78\end{array}$ & $\begin{array}{r}-- \\
300 \\
-- \\
--\end{array}$ & $\begin{array}{l}-- \\
-- \\
-- \\
--\end{array}$ & $\begin{array}{r}J \\
-.- \\
0, J \\
0, J\end{array}$ \\
\hline $\begin{array}{r}10.00 \\
14.00 \\
10.00 \\
8.62 \\
6.00\end{array}$ & $\begin{array}{r}10.88 \\
22.00 \\
29.25 \\
272.00 \\
9.34\end{array}$ & $\begin{array}{l}07-03-75 \\
02-01-51 \\
07-29-70 \\
11-13-75 \\
04-11-72\end{array}$ & $\begin{array}{r}638 \\
410 \\
-- \\
150 \\
300\end{array}$ & $\begin{array}{r}24.54 \\
-. \\
-- \\
5.17 \\
--\end{array}$ & $\begin{array}{r}D \\
D \\
E, J \\
D \\
--\end{array}$ \\
\hline
\end{tabular}


Table 3.--Supplemental public-supply well information for

\begin{tabular}{|c|c|c|c|c|c|c|}
\hline $\begin{array}{l}\text { Water } \\
\text { user } \\
\text { ID } \\
\end{array}$ & $\begin{array}{c}\text { Site } \\
\text { I0 }\end{array}$ & Water system name & $\begin{array}{c}\text { Principal } \\
\text { aquifer } \\
\text { geohydrologic } \\
\text { unit }\end{array}$ & $\begin{array}{l}\text { Altitude } \\
\text { of land } \\
\text { surface } \\
\text { (feet) }\end{array}$ & $\begin{array}{c}\text { Depth } \\
\text { of well } \\
\text { (feet) }\end{array}$ & $\begin{array}{l}\text { Depth to } \\
\text { first } \\
\text { opening } \\
\text { (inches) }\end{array}$ \\
\hline $\begin{array}{l}30754 \\
30757 \\
30758 \\
30759 \\
30760\end{array}$ & $\begin{array}{l}362507092545301 \\
341024092354501 \\
353629090195501 \\
354924092325401 \\
332119093345201\end{array}$ & $\begin{array}{l}\text { LEAD HILL WATERWORKS } \\
\text { LEOLA WATERWORKS } \\
\text { LEPANTO WATERWORKS } \\
\text { LESLIE WATERWORKS } \\
\text { LEWISVILLE WATERWORKS }\end{array}$ & $\begin{array}{l}\text { ROUBIDOUX FORMATION } \\
\text { SPARTA SAND } \\
\text { WILCOX GROUP } \\
\text { ST PETER SANDSTONE } \\
\text { CANE RIVER FORMATION }\end{array}$ & $\begin{array}{l}840 \\
280 \\
216 \\
978 \\
270\end{array}$ & $\begin{array}{r}602 \\
172 \\
1,456 \\
1,210 \\
400\end{array}$ & $\begin{array}{r}90 \\
142 \\
1,376 \\
380\end{array}$ \\
\hline $\begin{array}{l}30760 \\
30765 \\
30765 \\
30766 \\
30768\end{array}$ & $\begin{array}{l}332121093344901 \\
335806094100102 \\
335804094101101 \\
344701091535802 \\
332330092471701\end{array}$ & $\begin{array}{l}\text { LEWISVILLE WATERWORKS } \\
\text { LOCKESBURG WATERWORKS } \\
\text { LOCKESBURG WATERWORKS } \\
\text { LONOKE WATERWORKS } \\
\text { LOUANN WATERWORKS }\end{array}$ & $\begin{array}{l}\text { CANE RIVER FORMATION } \\
\text { TRINITY GROUP } \\
\text { TRINITY GROUP } \\
\text { QUATERNARY ALLUVIUM } \\
\text { CANE RIVER FORMATION }\end{array}$ & $\begin{array}{l}260 \\
440 \\
432 \\
238 \\
152\end{array}$ & $\begin{array}{l}421 \\
195 \\
250 \\
157 \\
600\end{array}$ & $\begin{array}{r}-- \\
175 \\
-- \\
--\end{array}$ \\
\hline $\begin{array}{l}30770 \\
30770 \\
30772 \\
30775 \\
30777\end{array}$ & $\begin{array}{l}354527089554901 \\
354528089554701 \\
361041093432601 \\
362841091323501 \\
355252090095701\end{array}$ & $\begin{array}{l}\text { LUXORA WATERWORKS } \\
\text { LUXORA WATERWORKS } \\
\text { MADISON CO WATER ASSN } \\
\text { MAMMOTH SPRINGS WATERWORK } \\
\text { MANILA WATERWORKS }\end{array}$ & $\begin{array}{l}\text { WILCOX GROUP } \\
\text { WILCOX GROUP } \\
\text { GUNTER SANDSTONE } \\
\text { ROUBIDOUX FORMATION } \\
\text { WILCOX GROUP }\end{array}$ & $\begin{array}{r}245 \\
245 \\
1,558 \\
580 \\
240\end{array}$ & $\begin{array}{r}1,500 \\
1,445 \\
1,970 \\
363 \\
1,158\end{array}$ & $\begin{array}{r}-- \\
1,367 \\
490 \\
-- \\
--\end{array}$ \\
\hline $\begin{array}{l}30778 \\
30779 \\
30779 \\
30780 \\
30781\end{array}$ & $\begin{array}{l}344629090455802 \\
351257090114001 \\
351238090114701 \\
353146090252201 \\
361112090225601\end{array}$ & $\begin{array}{l}\text { MARIANNA WATERWORKS } \\
\text { MARION WATERWORKS } \\
\text { MARION WATERWORKS } \\
\text { MARKED TREE WATERWORKS } \\
\text { MARMADUKE WATERWORKS }\end{array}$ & $\begin{array}{l}\text { SPARTA SAND } \\
\text { WILCOX GROUP } \\
\text { LOWER WILCOX AQUIFER } \\
\text { WILCOX GROUP } \\
\text { NACATOCH SAND }\end{array}$ & $\begin{array}{l}225 \\
222 \\
221 \\
215 \\
270\end{array}$ & $\begin{array}{r}590 \\
1,500 \\
1,540 \\
1,300 \\
1,081\end{array}$ & $\begin{array}{r}-- \\
1,415 \\
1,470 \\
-- \\
--\end{array}$ \\
\hline $\begin{array}{l}30784 \\
30785 \\
30789 \\
30791 \\
30793\end{array}$ & $\begin{array}{l}343324090544601 \\
331351092572701 \\
362440090535101 \\
351526091120301 \\
333636091230401\end{array}$ & $\begin{array}{l}\text { MARVELL WATERWORKS } \\
\text { MARYSVILLE WATER ASSOC } \\
\text { MAYNARD WATERWORKS } \\
\text { MCCRORY WATERWORKS } \\
\text { MCGEHEE WATERWORKS }\end{array}$ & $\begin{array}{l}\text { SPARTA SAND } \\
\text { SPARTA SAND } \\
\text { ROUBIDOUX FORMATION } \\
\text { TERRACE DEPOSITS } \\
\text { SPARTA SAND }\end{array}$ & $\begin{array}{l}211 \\
280 \\
485 \\
210 \\
147\end{array}$ & $\begin{array}{r}689 \\
690 \\
900 \\
-- \\
796\end{array}$ & $\begin{array}{r}629 \\
660 \\
350 \\
-- \\
736\end{array}$ \\
\hline $\begin{array}{l}30793 \\
30796 \\
30801 \\
30805 \\
30806\end{array}$ & $\begin{array}{l}333740091225501 \\
350641091492801 \\
350519090181001 \\
355722093093401 \\
355314090010801\end{array}$ & $\begin{array}{l}\text { MCGEHEE WATERWORKS } \\
\text { MCRAE WATERWORKS } \\
\text { MIDWAY WATER ASSOC } \\
\text { MOCKINGBIRD HILL WATER } \\
\text { MONETTE WATERWORKS }\end{array}$ & $\begin{array}{l}\text { SPARTA SAND } \\
\text { QUATERNARY ALLUVIUM } \\
\text { WILCOX GROUP } \\
\text { ROUBIDOUX FORMATION } \\
\text { WILCOX GROUP }\end{array}$ & $\begin{array}{r}138 \\
230 \\
207 \\
2,135 \\
232\end{array}$ & $\begin{array}{r}819 \\
89 \\
1,541 \\
3,142 \\
1,034\end{array}$ & $\begin{array}{r}-- \\
-- \\
-- \\
-- \\
896\end{array}$ \\
\hline $\begin{array}{l}30807 \\
30809 \\
30809 \\
30822 \\
30822\end{array}$ & $\begin{array}{l}333751091470902 \\
331805092570902 \\
331808092563801 \\
330455092515201 \\
330509092513801\end{array}$ & $\begin{array}{l}\text { MONTICELLO WATER DEPT } \\
\text { MOUNT HOLLY WATERWORKS } \\
\text { MOUNT HOLLY WATERWORKS } \\
\text { NEW HOPE WATER ASSOC } \\
\text { NEW HOPE WATER ASSOC }\end{array}$ & $\begin{array}{l}\text { SPARTA SAND } \\
\text { SPARTA SAND } \\
\text { SPARTA SAND } \\
\text { SPARTA SAND } \\
\text { SPARTA SAND }\end{array}$ & $\begin{array}{l}273 \\
248 \\
235 \\
240 \\
220\end{array}$ & $\begin{array}{l}880 \\
465 \\
480 \\
595 \\
586\end{array}$ & $\begin{array}{r}-- \\
-- \\
440 \\
525 \\
546\end{array}$ \\
\hline $\begin{array}{l}30823 \\
30823 \\
30824 \\
30826 \\
30826\end{array}$ & $\begin{array}{l}331204092222101 \\
331205092221901 \\
354118091263701 \\
353601091165401 \\
353616091162801\end{array}$ & $\begin{array}{l}\text { NEW LONDON WATER ASSOC } \\
\text { NEW LONDON WATER ASSOC } \\
\text { NEWARK WATERWORKS } \\
\text { NEWPORT WATERWORKS } \\
\text { NEWPORT WATERWORKS }\end{array}$ & $\begin{array}{l}\text { SPARTA SAND } \\
\text { SPARTA SAND } \\
\text { QUATERNARY ALLUVIUM } \\
\text { QUATERNARY ALLUVIUM } \\
\text { QUATERNARY ALLUVIUM }\end{array}$ & $\begin{array}{l}220 \\
230 \\
236 \\
223 \\
222\end{array}$ & $\begin{array}{r}828 \\
822 \\
45 \\
90 \\
94\end{array}$ & $\begin{array}{r}770 \\
772 \\
-- \\
56 \\
--\end{array}$ \\
\hline $\begin{array}{l}30826 \\
30826 \\
30826 \\
30828 \\
30830\end{array}$ & $\begin{array}{l}353617091162801 \\
353617091162802 \\
353617091162803 \\
361237092164501 \\
331900092395602\end{array}$ & $\begin{array}{l}\text { NEWPORT WATERWORKS } \\
\text { NEWPORT WATERWORKS } \\
\text { NEWPORT WATERWORKS } \\
\text { NORFORK WATERWORKS } \\
\text { NORPHLET WATERWORKS }\end{array}$ & $\begin{array}{l}\text { QUATERNARY ALLUVIUM } \\
\text { QUATERNARY ALLUVIUM } \\
\text { QUATERNARY ALLUVIUM } \\
\text { GUNTER SANDSTONE } \\
\text { SPARTA SAND }\end{array}$ & $\begin{array}{l}222 \\
222 \\
222 \\
500 \\
190\end{array}$ & $\begin{array}{r}103 \\
100 \\
90 \\
1,507 \\
603\end{array}$ & $\begin{array}{l}-- \\
-- \\
-- \\
550\end{array}$ \\
\hline $\begin{array}{l}30830 \\
30838 \\
30840 \\
30842 \\
30842\end{array}$ & $\begin{array}{l}331842092395001 \\
335910093195301 \\
362708093111301 \\
354220089580901 \\
354223089581001\end{array}$ & $\begin{array}{l}\text { NORPHELT WATERWORKS } \\
\text { OKOLONA WATERWORKS } \\
\text { OMAHA WATERWORKS } \\
\text { OSCEOLA WATERWORKS } \\
\text { OSCEOLA WATERWORKS }\end{array}$ & $\begin{array}{l}\text { SPARTA SAND } \\
\text { TOKIO FORMATION } \\
\text { ROUBIDOUX FORMATION } \\
\text { WILCOX GROUP } \\
\text { WILCOX GROUP }\end{array}$ & $\begin{array}{r}140 \\
430 \\
1,357 \\
246 \\
246\end{array}$ & $\begin{array}{r}665 \\
500 \\
1,315 \\
1,400 \\
1,500\end{array}$ & $\begin{array}{r}488 \\
- \\
64 \\
1,320 \\
1,420\end{array}$ \\
\hline $\begin{array}{l}30843 \\
30844 \\
30845 \\
30845 \\
30848\end{array}$ & $\begin{array}{l}361324091555001 \\
335047093431601 \\
361720091230401 \\
361814091233501 \\
360322090290401\end{array}$ & $\begin{array}{l}\text { OXFORD WATERWORKS } \\
\text { OZAN WATERWORKS } \\
\text { OZARK ACRES WATER CO } \\
\text { OZARK ACRES WATER CO } \\
\text { PARAGOULD CITY }\end{array}$ & $\begin{array}{l}\text { COTTER DOLOMITE } \\
\text { TOKIO FORMATION } \\
\text { ROUBIDOUX FORMATION } \\
\text { ROUBIDOUX FORMATION } \\
\text { WILCOX GROUP }\end{array}$ & $\begin{array}{l}820 \\
390 \\
473 \\
590 \\
285\end{array}$ & $\begin{array}{l}600 \\
555 \\
940 \\
611 \\
507\end{array}$ & $\begin{array}{r}-- \\
\overline{27} \\
-- \\
467\end{array}$ \\
\hline
\end{tabular}


sites investigated by the U.S. Geological Survey--Continued

\begin{tabular}{|c|c|c|c|c|c|}
\hline $\begin{array}{r}\text { Casing } \\
\text { diameter } \\
\text { (inches) } \\
\end{array}$ & $\begin{array}{r}\text { Water } \\
\text { level } \\
\text { (feet) }\end{array}$ & $\begin{array}{l}\text { Date } \\
\text { water } \\
\text { level } \\
\text { measured } \\
\end{array}$ & $\begin{array}{c}\text { Discharge } \\
\text { (gallons } \\
\text { per } \\
\text { minute) }\end{array}$ & $\begin{array}{l}\text { Specific } \\
\text { capacity } \\
\text { (gallons per } \\
\text { minute per foot) }\end{array}$ & $\begin{array}{c}\text { Types } \\
\text { of logs } \\
\text { available } \\
\end{array}$ \\
\hline $\begin{array}{r}8.00 \\
10.00 \\
10.00 \\
6.25 \\
16.00\end{array}$ & $\begin{array}{r}98.80 \\
55.52 \\
11.27 \\
240.00 \\
--\end{array}$ & $\begin{array}{c}05-22-68 \\
05-07-64 \\
03-07-67 \\
10-18-57 \\
--\end{array}$ & $\begin{array}{r}-- \\
250 \\
-- \\
30 \\
--\end{array}$ & $\begin{array}{l}-- \\
-- \\
-- \\
--\end{array}$ & $\begin{array}{l}-- \\
-- \\
-- \\
--\end{array}$ \\
\hline $\begin{array}{r}12.00 \\
12.00 \\
4.00 \\
--\end{array}$ & $\begin{array}{r}-- \\
78.54 \\
55.00 \\
--\end{array}$ & $\begin{array}{c}-- \\
-\overline{-} \\
02-00-71 \\
01-01-46\end{array}$ & $\begin{array}{c}135 \\
-- \\
300 \\
--\end{array}$ & $\begin{array}{l}-- \\
-- \\
-- \\
--\end{array}$ & $\begin{array}{l}-- \\
-- \\
-- \\
--\end{array}$ \\
\hline $\begin{array}{r}10.00 \\
8.00 \\
8.00 \\
--\end{array}$ & $\begin{array}{r}20.72 \\
31.59 \\
362.48 \\
74.00 \\
12.69\end{array}$ & $\begin{array}{l}11-16-66 \\
07-25-78 \\
04-09-75 \\
09-07-67 \\
03-07-68\end{array}$ & $\begin{array}{l}-- \\
500 \\
219 \\
445 \\
505\end{array}$ & $\begin{array}{l}-- \\
-\overline{-} \\
.94 \\
--\end{array}$ & $\begin{array}{r}G, D, E, G, J \\
D \\
D \\
D\end{array}$ \\
\hline $\begin{array}{r}6.00 \\
10.00 \\
10.00 \\
10.00 \\
--\end{array}$ & $\begin{array}{r}30 . \overline{--} \\
31.25 \\
--- \\
-3.12\end{array}$ & $\begin{array}{c}07-21-67 \\
12-13-74 \\
-- \\
03-05-68\end{array}$ & $\begin{array}{r}350 \\
300 \\
656 \\
-- \\
373\end{array}$ & $\begin{array}{l}-- \\
-- \\
-- \\
--\end{array}$ & $\begin{array}{l}-- \\
D \\
-- \\
--\end{array}$ \\
\hline $\begin{array}{r}10.00 \\
6.00 \\
4.00 \\
--\end{array}$ & $\begin{array}{r}56.08 \\
283.60 \\
89.57 \\
-\end{array}$ & $\begin{array}{c}12-01-66 \\
04-14-77 \\
09-04-79 \\
--\end{array}$ & $\begin{array}{r}524 \\
100 \\
-- \\
--\end{array}$ & $\begin{array}{r}26.20 \\
-- \\
-- \\
--\end{array}$ & $C, D, E, J$ \\
\hline 12.00 & 46.20 & $03-26-69$ & 919 & -- & D \\
\hline $\begin{array}{r}-- \\
-\overline{-} \\
8.00 \\
10.00\end{array}$ & $\begin{array}{r}25 . \overline{-} \\
. \overline{-} \\
1,296.20 \\
14.05\end{array}$ & $\begin{array}{c}\overline{--} \\
08-22-83 \\
\overline{-} \\
11-22-76 \\
03-08-78\end{array}$ & $\begin{array}{r}-- \\
-- \\
515 \\
62 \\
--\end{array}$ & $\begin{array}{l}-- \\
-- \\
.49 \\
--\end{array}$ & \begin{tabular}{r}
$D, E, J$ \\
\hdashline$G$ \\
$C, D, E, J$ \\
$J$
\end{tabular} \\
\hline $\begin{array}{r}12.00 \\
4.00 \\
8.00 \\
6.00 \\
6.00\end{array}$ & $\begin{array}{r}-- \\
201.00 \\
186.05 \\
272.00 \\
274.00\end{array}$ & $\begin{array}{c}-- \\
05-12-80 \\
06-08-82 \\
03-07-83 \\
02-00-87\end{array}$ & $\begin{array}{r}900 \\
60 \\
100 \\
150 \\
185\end{array}$ & $\begin{array}{r}-- \\
-- \\
2.90 \\
--\end{array}$ & $\begin{array}{r}D \\
D \\
- \\
D \\
D\end{array}$ \\
\hline $\begin{array}{r}18.62 \\
8.62 \\
-\end{array}$ & $\begin{array}{l}226.00 \\
225.00\end{array}$ & $\begin{array}{c}10-31-86 \\
12-05-78 \\
-\end{array}$ & $\begin{array}{l}175 \\
150 \\
400\end{array}$ & $\begin{array}{l}-- \\
--\end{array}$ & $\begin{array}{r}D \\
D, E, J\end{array}$ \\
\hline 16.00 & -- & -- & 692 & -- & -- \\
\hline $\begin{array}{r}8.00 \\
8.00\end{array}$ & -- & -- & $\begin{array}{l}550 \\
500\end{array}$ & -- & -- \\
\hline $\begin{array}{r}8.50 \\
18.00\end{array}$ & $\begin{array}{r}91.00 \\
269.04\end{array}$ & $\begin{array}{l}07-30-69 \\
12-09-64\end{array}$ & $\begin{array}{l}122 \\
340\end{array}$ & -- & $E, J$ \\
\hline $\begin{array}{r}10.75 \\
-.- \\
6.00 \\
8.00 \\
10.00\end{array}$ & $\begin{array}{r}240.00 \\
450.00 \\
=- \\
13.80\end{array}$ & $\begin{array}{c}05-12-83 \\
0 \\
05-01-67 \\
11-05-58\end{array}$ & $\begin{array}{r}235 \\
-- \\
-- \\
440 \\
640\end{array}$ & $\begin{array}{l}-- \\
-- \\
-- \\
--\end{array}$ & $\begin{array}{l}\text { D } \\
-- \\
-- \\
-- \\
--\end{array}$ \\
\hline $\begin{array}{r}4 .-\overline{0} \\
6.00 \\
6.00 \\
20.00\end{array}$ & $\begin{array}{r}100.42 \\
-- \\
66.00 \\
16.50 \\
77.03\end{array}$ & $\begin{array}{c}08-23-66 \\
-- \\
05-20-68 \\
05-20-68 \\
03-02-67\end{array}$ & $\begin{array}{r}86 \\
-- \\
125 \\
120 \\
1,641\end{array}$ & $\begin{array}{l}-- \\
-- \\
-- \\
--\end{array}$ & $\begin{array}{l}\text { D } \\
-- \\
-- \\
--\end{array}$ \\
\hline
\end{tabular}


Table 3.--Supplemental public-supply well information

\begin{tabular}{|c|c|c|c|c|c|c|}
\hline $\begin{array}{l}\text { Water } \\
\text { user } \\
\text { ID } \\
\end{array}$ & $\begin{array}{l}\text { Site } \\
\text { ID }\end{array}$ & Water system name & $\begin{array}{c}\text { Principal } \\
\text { aquifer } \\
\text { geohydrologic } \\
\text { unit }\end{array}$ & $\begin{array}{l}\text { Altitude } \\
\text { of land } \\
\text { surface } \\
\text { (feet) }\end{array}$ & $\begin{array}{l}\text { Depth } \\
\text { of we } 11 \\
\text { (feet) }\end{array}$ & $\begin{array}{l}\text { Depth to } \\
\text { first } \\
\text { opening } \\
\text { (inches) }\end{array}$ \\
\hline 30848 & 360330090290201 & $\begin{array}{l}\text { PARAGOULD CITY } \\
\text { LIGHT-WATER }\end{array}$ & WILCOX GROUP & 280 & 501 & -- \\
\hline 30848 & 360437090291201 & $\begin{array}{l}\text { PARAGOULD CITY } \\
\text { LIGHT-WATER }\end{array}$ & WILCOX GROUP & 278 & 446 & 391 \\
\hline $\begin{array}{l}30850 \\
30851 \\
30851\end{array}$ & $\begin{array}{l}330713091324801 \\
331011092431701 \\
331024092422901\end{array}$ & $\begin{array}{l}\text { PARKDALE WATERWORKS } \\
\text { PARKERS CHAPEL WATER ASSN } \\
\text { PARKERS CHAPEL WATER ASSN }\end{array}$ & $\begin{array}{l}\text { COCKFIELD FORMATION } \\
\text { SPARTA SAND } \\
\text { SPARTA SAND }\end{array}$ & $\begin{array}{l}117 \\
270 \\
303\end{array}$ & $\begin{array}{l}400 \\
767 \\
797\end{array}$ & $\begin{array}{l}704 \\
464\end{array}$ \\
\hline $\begin{array}{l}30852 \\
30855 \\
30855 \\
30855 \\
30855\end{array}$ & $\begin{array}{l}351546090335502 \\
341142092022801 \\
341147092022301 \\
341331092010801 \\
341332092010901\end{array}$ & $\begin{array}{l}\text { PARKIN WATERWORKS } \\
\text { PB-GENERAL WATERWORKS CO } \\
\text { PB-GENERAL WATERWORKS CO } \\
\text { PB-GENERAL WATERWORKS CO } \\
\text { PB-GENERAL WATERWORKS CO }\end{array}$ & $\begin{array}{l}\text { CLAIBORNE GROUP } \\
\text { SPARTA SAND } \\
\text { SPARTA SAND } \\
\text { SPARTA SAND } \\
\text { SPARTA SAND }\end{array}$ & $\begin{array}{l}208 \\
230 \\
231 \\
230 \\
229\end{array}$ & $\begin{array}{l}491 \\
847 \\
863 \\
904 \\
850\end{array}$ & $\begin{array}{r}42 \\
-- \\
783 \\
-- \\
740\end{array}$ \\
\hline $\begin{array}{l}30855 \\
30860 \\
30861 \\
30861 \\
30865\end{array}$ & $\begin{array}{l}341332092010904 \\
335035091290301 \\
362225090120801 \\
362311090111301 \\
350936092381302\end{array}$ & $\begin{array}{l}\text { PB-GENERAL WATERWORKS CO } \\
\text { PICKENS WATERWORKS } \\
\text { PIGGOTT WATERWORKS } \\
\text { PIGGOTT WATERWORKS } \\
\text { PLUMERVILLE WATERWORKS }\end{array}$ & $\begin{array}{l}\text { SPARTA SAND } \\
\text { SPARTA SAND } \\
\text { NACATOCH SAND } \\
\text { NACATOCH SAND } \\
\text { QUATERNARY ALLUVIUM }\end{array}$ & $\begin{array}{l}230 \\
161 \\
360 \\
290 \\
310\end{array}$ & $\begin{array}{r}840 \\
830 \\
1,062 \\
976 \\
38\end{array}$ & $\begin{array}{r}755 \\
790 \\
981 \\
900 \\
--\end{array}$ \\
\hline $\begin{array}{l}30869 \\
30870 \\
30872 \\
30874 \\
30883\end{array}$ & $\begin{array}{l}331415091302901 \\
341923092382501 \\
341841092332001 \\
361512092504501 \\
361601090175101\end{array}$ & $\begin{array}{l}\text { PORTLAND WATERWORKS } \\
\text { POYEN WATERWORKS } \\
\text { PRATTSVILLE WATERWORKS } \\
\text { PYATT WATERWORKS } \\
\text { RECTOR WATERWORKS }\end{array}$ & $\begin{array}{l}\text { COCKFIELD FORMATION } \\
\text { SPARTA SAND } \\
\text { SPARTA SAND } \\
\text { ROUBIDOUX FORMATION } \\
\text { NACATOCH SAND }\end{array}$ & $\begin{array}{l}125 \\
232 \\
293 \\
758 \\
345\end{array}$ & $\begin{array}{r}332 \\
-- \\
370 \\
1.392 \\
1.100\end{array}$ & $\begin{array}{c}302 \\
-- \\
-\overline{--} \\
--\end{array}$ \\
\hline $\begin{array}{l}30883 \\
30884 \\
30889 \\
30889 \\
30895\end{array}$ & $\begin{array}{l}361601090175001 \\
342644092105501 \\
335728092113301 \\
335729092112001 \\
333529093163801\end{array}$ & $\begin{array}{l}\text { RECTOR WATERWORKS } \\
\text { REDFIELD WATERWORKS } \\
\text { RISON WATERWORKS } \\
\text { RISON WATERWORKS } \\
\text { ROSSTON WATER DEPT }\end{array}$ & $\begin{array}{l}\text { NACATOCH SAND } \\
\text { SPARTA SAND } \\
\text { SPARTA SAND } \\
\text { SPARTA SAND } \\
\text { WILCOX GROUP }\end{array}$ & $\begin{array}{l}330 \\
310 \\
230 \\
266 \\
360\end{array}$ & $\begin{array}{r}1.071 \\
707 \\
558 \\
558 \\
450\end{array}$ & $\begin{array}{r}1.011 \\
671 \\
-- \\
--\end{array}$ \\
\hline $\begin{array}{l}30899 \\
30900 \\
30901 \\
30902 \\
30903\end{array}$ & $\begin{array}{l}361132093113501 \\
362712090083901 \\
362207091492401 \\
361437092163201 \\
\mathbf{3 4 3 1 2 9 0 9 2 2 2 5 2 0 1}\end{array}$ & $\begin{array}{l}\text { S W BOONE COUNTY WATER } \\
\text { SAINT FRANCIS WATERWORKS } \\
\text { SALEM WATERWORKS } \\
\text { SALESVILLE WATERWORKS } \\
\text { SARDIS WATER ASSOCIATION }\end{array}$ & $\begin{array}{l}\text { EVERTON FORMATION } \\
\text { NACATOCH SAND } \\
\text { GUNTER SANDSTONE } \\
\text { ROUBIDOUX FORMATION } \\
\text { WILCOX GROUP }\end{array}$ & $\begin{array}{r}1.550 \\
295 \\
660 \\
725 \\
318\end{array}$ & $\begin{array}{r}997 \\
1,030 \\
1,282 \\
835 \\
492\end{array}$ & $\begin{array}{r}500 \\
-- \\
-- \\
--\end{array}$ \\
\hline $\begin{array}{l}30911 \\
30912 \\
30918 \\
30918 \\
30918\end{array}$ & 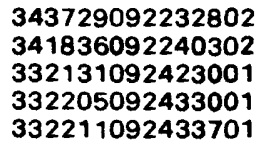 & $\begin{array}{l}\text { SHANNON HILLS WATER ASSOC } \\
\text { SHERIDAN WATERWORKS } \\
\text { SMACKOVER WATERWORKS } \\
\text { SMACKOVER WATERWORKS } \\
\text { SMACKOVER WATERWORKS }\end{array}$ & $\begin{array}{l}\text { CLAYTON FORMATION } \\
\text { SPARTA SAND } \\
\text { SPARTA SAND } \\
\text { SPARTA SAND } \\
\text { SPARTA SAND }\end{array}$ & $\begin{array}{l}360 \\
281 \\
119 \\
116 \\
117\end{array}$ & $\begin{array}{l}250 \\
556 \\
473 \\
552 \\
553\end{array}$ & $\begin{array}{r}-- \\
494 \\
433 \\
480 \\
--\end{array}$ \\
\hline $\begin{array}{l}30918 \\
30922 \\
30926 \\
30926 \\
30926\end{array}$ & $\begin{array}{l}332113092421001 \\
335457092505001 \\
332129093294502 \\
332130093294501 \\
332133093294401\end{array}$ & $\begin{array}{l}\text { SMACKOVER WATERWORKS } \\
\text { SPARKMAN WATERWORKS } \\
\text { STAMPS WATERWORKS } \\
\text { STAMPS WATERWORKS } \\
\text { STAMPS WATERWORKS }\end{array}$ & $\begin{array}{l}\text { SPARTA SAND } \\
\text { CANE RIVER FORMATION } \\
\text { CANE RIVER FORMATION } \\
\text { CANE RIVER FORMATION } \\
\text { CANE RIVER FORMATION }\end{array}$ & $\begin{array}{l}112 \\
244 \\
265 \\
265 \\
266\end{array}$ & $\begin{array}{l}470 \\
244 \\
378 \\
349 \\
378\end{array}$ & $\begin{array}{l}430 \\
-- \\
-- \\
--\end{array}$ \\
\hline $\begin{array}{l}30927 \\
30929 \\
30930 \\
30931 \\
30931\end{array}$ & $\begin{array}{l}335631091512101 \\
332437093043102 \\
355758091190501 \\
330633092213901 \\
330619092221401\end{array}$ & $\begin{array}{l}\text { STAR CITY WATER CO } \\
\text { STEPHENS WATERWORKS } \\
\text { STRAWBERRY WATERWORKS } \\
\text { STRONG WATERWORKS } \\
\text { STRONG WATERWORKS }\end{array}$ & $\begin{array}{l}\text { SPARTA SAND } \\
\text { SPARTA SAND } \\
\text { POWELL DOLOMITE } \\
\text { SPARTA SAND } \\
\text { SPARTA SAND }\end{array}$ & $\begin{array}{l}300 \\
280 \\
320 \\
145 \\
150\end{array}$ & $\begin{array}{r}1,052 \\
300 \\
\mathbf{5 2 7} \\
\mathbf{3 1 2} \\
\mathbf{3 1 0}\end{array}$ & $\begin{array}{r}-- \\
40 \\
280 \\
260\end{array}$ \\
\hline $\begin{array}{l}30931 \\
30931 \\
30931 \\
30931 \\
30932\end{array}$ & $\begin{array}{l}330607092212101 \\
330644092212601 \\
330643092214801 \\
330651092212001 \\
343018091325201\end{array}$ & $\begin{array}{l}\text { STRONG WATERWORKS } \\
\text { STRONG WATERWORKS } \\
\text { STRONG WATERWORKS } \\
\text { STRONG WATERWORKS } \\
\text { STUTTGART WATERWORKS }\end{array}$ & $\begin{array}{l}\text { SPARTA SAND } \\
\text { SPARTA SAND } \\
\text { SPARTA SAND } \\
\text { SPARTA SAND } \\
\text { QUATERNARY ALLUVIUM }\end{array}$ & $\begin{array}{l}135 \\
115 \\
112 \\
112 \\
210\end{array}$ & $\begin{array}{l}290 \\
430 \\
400 \\
466 \\
135\end{array}$ & $\begin{array}{l}250 \\
144 \\
202 \\
436 \\
--\end{array}$ \\
\hline $\begin{array}{l}30935 \\
30937 \\
30941 \\
30945 \\
30946\end{array}$ & $\begin{array}{l}362913094273101 \\
361511092413801 \\
330609093274302 \\
334633092292901 \\
334248091270801\end{array}$ & $\begin{array}{l}\text { SULPHUR SPRINGS WATERWORK } \\
\text { SUMMIT WATERWORKS } \\
\text { TAYLOR WATERWORKS } \\
\text { THORNTON WATERWORKS } \\
\text { TILLAR WATERWORKS }\end{array}$ & $\begin{array}{l}\text { ROUBIDOUX FORMATION } \\
\text { GUNTER SANDSTONE } \\
\text { SPARTA SAND } \\
\text { SPARTA SAND } \\
\text { SPARTA SAND }\end{array}$ & $\begin{array}{r}1.036 \\
950 \\
248 \\
310 \\
148\end{array}$ & $\begin{array}{r}1,442 \\
1,524 \\
385 \\
650 \\
622\end{array}$ & $\begin{array}{r}600 \\
-- \\
-- \\
640 \\
580\end{array}$ \\
\hline
\end{tabular}


sites investigated by the U.S. Geological Survey--Continued

\begin{tabular}{|c|c|c|c|c|c|}
\hline $\begin{array}{l}\text { Casing } \\
\text { diameter } \\
\text { (inches) }\end{array}$ & $\begin{array}{r}\text { Water } \\
\text { level } \\
\text { (feet) }\end{array}$ & $\begin{array}{c}\text { Date } \\
\text { water } \\
\text { level } \\
\text { measured } \\
\end{array}$ & $\begin{array}{c}\text { Discharge } \\
\text { (gallons } \\
\text { per } \\
\text { minute) }\end{array}$ & $\begin{array}{l}\text { Specific } \\
\text { capacity } \\
\text { (gallons per } \\
\text { minute per foot) }\end{array}$ & $\begin{array}{l}\text { Types } \\
\text { of logs } \\
\text { available }\end{array}$ \\
\hline 12.00 & -- & -- & 1,000 & -- & -. \\
\hline 21.00 & 58.48 & $03-06-68$ & 18 & -- & -- \\
\hline $\begin{array}{r}4.00 \\
8.00 \\
10.00\end{array}$ & $\begin{array}{r}416 . \overline{66} \\
415.00\end{array}$ & $\begin{array}{l}12-\overline{-06}-78 \\
03-22-68\end{array}$ & $\begin{array}{l}115 \\
110 \\
155\end{array}$ & $\begin{array}{l}-- \\
--\end{array}$ & D,E,J \\
\hline $\begin{array}{l}10.00 \\
18.00 \\
16.00 \\
18.00 \\
16.00\end{array}$ & $\begin{array}{r}26.00 \\
224.00 \\
-- \\
-- \\
78.50\end{array}$ & $\begin{array}{c}02-08-55 \\
07-22-71 \\
\cdots- \\
10-27-48\end{array}$ & $\begin{array}{r}\cdots \\
1,500 \\
1,250\end{array}$ & $\begin{array}{l}-- \\
-- \\
--\end{array}$ & $\begin{array}{l}-- \\
D \\
-- \\
--\end{array}$ \\
\hline $\begin{array}{r}16.00 \\
8.00 \\
12.00 \\
12.00 \\
8.00\end{array}$ & $\begin{array}{r}94.00 \\
77.01 \\
108.05 \\
-- \\
4.52\end{array}$ & $\begin{array}{c}09-01-52 \\
02-14-80 \\
05-31-78 \\
-- \\
01-31-59\end{array}$ & $\begin{array}{r}1,980 \\
300 \\
80 \\
390 \\
120\end{array}$ & $\begin{array}{r}23.09 \\
2.97 \\
-- \\
--\end{array}$ & $\begin{array}{l}-- \\
0 . j \\
0 . j \\
--\end{array}$ \\
\hline $\begin{array}{r}16.00 \\
-\overline{0} \\
10.00 \\
12.00 \\
6.00\end{array}$ & $\begin{array}{l}26.96 \\
12.59 \\
80.72 \\
26.18 \\
64.13\end{array}$ & $\begin{array}{l}04-26-66 \\
04-10-68 \\
04-11-68 \\
11-00-75 \\
04-02-70\end{array}$ & $\begin{array}{r}350 \\
-- \\
250 \\
236 \\
--\end{array}$ & 1.09 & $\begin{array}{r}-- \\
- \\
\text { G.J } \\
--\end{array}$ \\
\hline $\begin{array}{r}12.75 \\
8.00 \\
\ldots\end{array}$ & $144 . \overline{95}$ & $01-22-65$ & $\begin{array}{r}600 \\
313 \\
-.\end{array}$ & 14.23 & D,E \\
\hline $\begin{array}{l}4.00 \\
6.00\end{array}$ & $\begin{array}{l}150.51 \\
125.00\end{array}$ & $\begin{array}{l}11-23-64 \\
01-01-45\end{array}$ & 104 & -- & -- \\
\hline $\begin{array}{r}8.25 \\
10.00 \\
8.00 \\
8.00 \\
.-\end{array}$ & $\begin{array}{r}393.31 \\
-7.25 \\
27.60 \\
130.90\end{array}$ & $\begin{array}{c}12-12-78 \\
03-03-67 \\
10-15-65 \\
08-22-74 \\
--\end{array}$ & $\begin{array}{r}140 \\
-- \\
170 \\
--\end{array}$ & $\begin{array}{l}-- \\
-- \\
-- \\
--\end{array}$ & $\begin{array}{r}C, E, J \\
\overline{-} \\
0, J \\
E, J\end{array}$ \\
\hline $\begin{array}{l}10.00 \\
10.00 \\
12.00 \\
10.00 \\
.-\end{array}$ & $\begin{array}{r}86.13 \\
62.40 \\
154.00 \\
148.85 \\
130.00\end{array}$ & $\begin{array}{l}04-17-63 \\
03-02-50 \\
06-21-74 \\
12-09-64 \\
07-16-58\end{array}$ & $\begin{array}{r}60 \\
450 \\
300 \\
-- \\
440\end{array}$ & $\begin{array}{l}-- \\
-- \\
-- \\
--\end{array}$ & $\begin{array}{r}-- \\
D \\
D \\
-- \\
D\end{array}$ \\
\hline $\begin{array}{r}12.00 \\
8.00 \\
-0 \\
-- \\
10.00\end{array}$ & $\begin{array}{r}154.00 \\
-- \\
-- \\
49.25 \\
--\end{array}$ & $\begin{array}{c}06-03-74 \\
- \\
- \\
06-17-75 \\
\cdots\end{array}$ & $\begin{array}{c}300 \\
-- \\
-- \\
-- \\
550\end{array}$ & $\begin{array}{l}= \\
-- \\
-\end{array}$ & $\begin{array}{r}D \\
-- \\
-\overline{0} \\
--\end{array}$ \\
\hline $\begin{array}{r}10.00 \\
8.00 \\
6.00 \\
4.00 \\
6.00\end{array}$ & $\begin{array}{r}222.85 \\
137.04 \\
41.32 \\
88.00 \\
97.00\end{array}$ & $\begin{array}{l}11-06-73 \\
11-16-49 \\
07-25-75 \\
04-14-78 \\
05-14-85\end{array}$ & $\begin{array}{r}140 \\
150 \\
35 \\
85\end{array}$ & $\begin{array}{r}280.00 \\
-- \\
--\end{array}$ & $\begin{array}{r}G \\
C, E, J \\
D \\
D\end{array}$ \\
\hline $\begin{array}{r}6.00 \\
18.00 \\
6.00 \\
10.00 \\
11.00\end{array}$ & $\begin{array}{r}85.00 \\
8.60 \\
57.56 \\
100.00 \\
--\end{array}$ & $\begin{array}{c}07-09-83 \\
01-01-47 \\
06-09-82 \\
07-28-71 \\
\ldots-\end{array}$ & $\begin{array}{r}85 \\
80 \\
-- \\
185 \\
600\end{array}$ & $\begin{array}{l}-- \\
=- \\
-- \\
--\end{array}$ & $\begin{array}{r}0 \\
-\therefore \\
- \\
--\end{array}$ \\
\hline $\begin{array}{r}10.00 \\
6.00 \\
12.00 \\
6.00 \\
12.00\end{array}$ & $\begin{array}{r}118.54 \\
-- \\
47.51 \\
142.14 \\
39.48\end{array}$ & $\begin{array}{c}02-01-77 \\
-- \\
04-11-67 \\
11-07-84 \\
03-30-65\end{array}$ & $\begin{array}{r}\cdots 1 \\
-\cdots \\
200 \\
250\end{array}$ & $\begin{array}{l}-- \\
.30 \\
-- \\
--\end{array}$ & $\begin{array}{r}C, E, J \\
E, J \\
-\bar{D} \\
D\end{array}$ \\
\hline
\end{tabular}


Table 3.--Supplemental public-supply well information for

\begin{tabular}{|c|c|c|c|c|c|c|}
\hline $\begin{array}{l}\text { Water } \\
\text { user } \\
\text { ID } \\
\end{array}$ & $\begin{array}{c}\text { Site } \\
\text { ID }\end{array}$ & Water system name & $\begin{array}{c}\text { Principal } \\
\text { aquifer } \\
\text { geohydrologic } \\
\text { unit }\end{array}$ & $\begin{array}{l}\text { Altitude } \\
\text { of land } \\
\text { surface } \\
\text { (feet) }\end{array}$ & $\begin{array}{c}\text { Depth } \\
\text { of well } \\
\text { (feet) }\end{array}$ & $\begin{array}{l}\text { Depth to } \\
\text { first } \\
\text { opening } \\
\text { (inches) }\end{array}$ \\
\hline $\begin{array}{l}30948 \\
30950 \\
30950 \\
30952 \\
30952\end{array}$ & $\begin{array}{l}333745092212201 \\
354000090301201 \\
354038090305901 \\
354340091115701 \\
354340091115702\end{array}$ & $\begin{array}{l}\text { TINSWAN WATERWORKS } \\
\text { TRUMANN WATERWORKS } \\
\text { TRUMANN WATERWORKS } \\
\text { TUCKERMAN WATERWORKS } \\
\text { TUCKERMAN WATERWORKS }\end{array}$ & $\begin{array}{l}\text { SPARTA SAND } \\
\text { WILCOX GROUP } \\
\text { WILCOX GROUP } \\
\text { TERRACE DEPOSITS } \\
\text { TERRACE DEPOSITS }\end{array}$ & $\begin{array}{l}176 \\
220 \\
222 \\
241 \\
241\end{array}$ & $\begin{array}{r}723 \\
1,071 \\
1,071 \\
104 \\
104\end{array}$ & $\begin{array}{r}690 \\
991 \\
991 \\
-- \\
--\end{array}$ \\
\hline $\begin{array}{l}30953 \\
30957 \\
30959 \\
30963 \\
30964\end{array}$ & $\begin{array}{l}352225090151001 \\
361025093005101 \\
352231090421501 \\
362359091590001 \\
342140091474101\end{array}$ & $\begin{array}{l}\text { TURRELL WATERWORKS } \\
\text { VALLEY SPGS WATERWORKS } \\
\text { VANWDALE-BIRDEYE WATER } \\
\text { VIOLA WATERWORKS } \\
\text { WABBASEKA WATERWORKS }\end{array}$ & $\begin{array}{l}\text { WILCOX GROUP } \\
\text { GUNTER SANDSTONE } \\
\text { MEMPHIS SAND } \\
\text { ROUBIDOUX FORMATION } \\
\text { SPARTA SAND }\end{array}$ & $\begin{array}{r}220 \\
1,300 \\
429 \\
860 \\
200\end{array}$ & $\begin{array}{r}1,510 \\
2,055 \\
1,148 \\
950 \\
756\end{array}$ & $\begin{array}{r}1,430 \\
500 \\
1.038 \\
510 \\
-\ldots\end{array}$ \\
\hline $\begin{array}{l}30968 \\
30970 \\
30970 \\
30971 \\
30972\end{array}$ & $\begin{array}{l}360410090573701 \\
333647092040602 \\
333647092040701 \\
334640093412101 \\
341105092050201\end{array}$ & $\begin{array}{l}\text { WALNUT RIDGE WATERWORKS } \\
\text { WARREN WATERWORKS } \\
\text { WARREN WATERWORKS } \\
\text { WASHINGTON WATER SYSTEW } \\
\text { WATSON CHAPEL WATER ASSN }\end{array}$ & $\begin{array}{l}\text { TERRACE DEPOSITS } \\
\text { SPARTA SAND } \\
\text { SPARTA SAND } \\
\text { TOKIO FORMATION } \\
\text { SPARTA SAND }\end{array}$ & $\begin{array}{l}270 \\
212 \\
208 \\
460 \\
230\end{array}$ & $\begin{array}{r}140 \\
1,050 \\
1,022 \\
953 \\
850\end{array}$ & $\begin{array}{r}-- \\
-\overline{9} \\
-- \\
--\end{array}$ \\
\hline $\begin{array}{l}30972 \\
30973 \\
30974 \\
30975 \\
30977\end{array}$ & $\begin{array}{l}341117092050401 \\
335341091152201 \\
353704090540501 \\
330809092461101 \\
343243090384601\end{array}$ & $\begin{array}{l}\text { WATSON CHAPEL WATER ASSN } \\
\text { WATSON WATERWORKS } \\
\text { WEINER WATERWORKS } \\
\text { WESSON-NEWELL WATER ASSN } \\
\text { WEST HELENA WATERWORKS }\end{array}$ & $\begin{array}{l}\text { SPARTA SAND } \\
\text { SPARTA SAND } \\
\text { QUATERNARY ALLUVIUM } \\
\text { SPARTA SAND } \\
\text { SPARTA SAND }\end{array}$ & $\begin{array}{l}232 \\
153 \\
245 \\
225 \\
252\end{array}$ & $\begin{array}{r}865 \\
626 \\
175 \\
636 \\
--\end{array}$ & $\begin{array}{r}-- \\
-- \\
596 \\
--\end{array}$ \\
\hline $\begin{array}{l}30978 \\
30980 \\
30981 \\
30981 \\
30986\end{array}$ & $\begin{array}{l}350906090104201 \\
360350090365801 \\
341634092053401 \\
341639092053901 \\
361517091212001\end{array}$ & $\begin{array}{l}\text { WEST MEMPHIS WATERWORKS } \\
\text { WESTERN GREENE CITY RWD } \\
\text { WHITE HALL WATERWORKS } \\
\text { WHITE HALL WATERWORKS } \\
\text { WILLIFORD WATERWORKS }\end{array}$ & $\begin{array}{l}\text { WILCOX GROUP } \\
\text { WILCOX GROUP } \\
\text { SPARTA SAND } \\
\text { SPARTA SAND } \\
\text { ROUBIDOUX FORMATION }\end{array}$ & $\begin{array}{l}210 \\
505 \\
300 \\
310 \\
400\end{array}$ & $\begin{array}{r}1,470 \\
311 \\
865 \\
904 \\
670\end{array}$ & $\begin{array}{r}1,380 \\
-- \\
- \\
- \\
-\end{array}$ \\
\hline $\begin{array}{l}30988 \\
30989 \\
30990 \\
30992 \\
30997\end{array}$ & $\begin{array}{l}333749091554201 \\
330335091341301 \\
353344090021002 \\
334610091290401 \\
343118092123401\end{array}$ & $\begin{array}{l}\text { WILMAR WATERWORKS } \\
\text { WILMOT WATERWORKS } \\
\text { WILSON WATERWORKS } \\
\text { WINCHESTER WATERWORKS } \\
\text { WOODSON-HENSLEY WATER }\end{array}$ & $\begin{array}{l}\text { SPARTA SAND } \\
\text { COCKFIELD FORMATION } \\
\text { WILCOX GROUP } \\
\text { COCKFIELD FORMATION } \\
\text { SPARTA SAND }\end{array}$ & $\begin{array}{l}173 \\
115 \\
235 \\
152 \\
245\end{array}$ & $\begin{array}{r}543 \\
360 \\
1.542 \\
556 \\
250\end{array}$ & $\begin{array}{r}503 \\
-- \\
-- \\
-- \\
218\end{array}$ \\
\hline $\begin{array}{l}30997 \\
31002 \\
31004 \\
31015\end{array}$ & $\begin{array}{l}343118092123701 \\
355848089542201 \\
334444091491501 \\
331040092353101\end{array}$ & $\begin{array}{l}\text { WOODSON-HENSLEY WATER } \\
\text { YARBO WATERWORKS } \\
\text { YORKTOWN WATER ASSN } \\
\text { JOHNSON TOWNSHIP WATER }\end{array}$ & $\begin{array}{l}\text { SPARTA SAND } \\
\text { WILCOX GROUP } \\
\text { SPARTA SAND } \\
\text { SPARTA SAND }\end{array}$ & $\begin{array}{l}244 \\
251 \\
395 \\
225\end{array}$ & $\begin{array}{r}255 \\
1,360 \\
850 \\
783\end{array}$ & $\begin{array}{r}215 \\
1,282 \\
-- \\
720\end{array}$ \\
\hline
\end{tabular}


sites investigated by the U.S. Geological Survey--Continued

\begin{tabular}{|c|c|c|c|c|c|}
\hline $\begin{array}{c}\text { Casing } \\
\text { diameter } \\
\text { (inches) }\end{array}$ & $\begin{array}{r}\text { Water } \\
\text { level } \\
\text { (feet) }\end{array}$ & $\begin{array}{l}\text { Date } \\
\text { water } \\
\text { level } \\
\text { mesured }\end{array}$ & $\begin{array}{c}\text { Discharge } \\
\text { (gallons } \\
\text { per } \\
\text { minute) }\end{array}$ & $\begin{array}{c}\text { Specific } \\
\text { capacity } \\
\text { (gallons per } \\
\text { minute oer foot) }\end{array}$ & $\begin{array}{c}\text { Types } \\
\text { of logs } \\
\text { available }\end{array}$ \\
\hline $\begin{array}{r}10.00 \\
12.00 \\
10.00 \\
8.00 \\
8.00\end{array}$ & $\begin{array}{r}84.50 \\
11.96 \\
9.04 \\
-- \\
--\end{array}$ & $\begin{array}{c}09-15-78 \\
03-26-68 \\
03-07-68 \\
-- \\
--\end{array}$ & $\begin{array}{r}-- \\
1.214 \\
725 \\
160 \\
--\end{array}$ & $\begin{array}{l}-- \\
-- \\
-- \\
--\end{array}$ & $\begin{array}{c}0 . J \\
-.- \\
-- \\
--\end{array}$ \\
\hline $\begin{array}{r}10.00 \\
6.00 \\
10.00 \\
8.00 \\
10.00\end{array}$ & $\begin{array}{r}25.60 \\
333.29 \\
242.34 \\
92.60 \\
107.28\end{array}$ & $\begin{array}{l}06-15-66 \\
07-01-74 \\
07-26-73 \\
07-24-78 \\
03-26-65\end{array}$ & $\begin{array}{l}-- \\
200 \\
500 \\
300 \\
320\end{array}$ & $\begin{array}{l}-- \\
1.27 \\
-- \\
--\end{array}$ & $\begin{array}{r}E,- \\
\text { D, E,J } \\
\text { E,J } \\
--\end{array}$ \\
\hline $\begin{array}{r}8.00 \\
10.00 \\
10.00\end{array}$ & $\begin{array}{r}-- \\
87.02 \\
173 . \overline{-}\end{array}$ & $\begin{array}{c}\overline{-} \\
12-\overline{17-49} \\
0-\overline{-0}-84\end{array}$ & $\begin{array}{r}280 \\
500 \\
500 \\
-- \\
--\end{array}$ & $\begin{array}{l}-- \\
-- \\
-- \\
--\end{array}$ & $\begin{array}{l}-- \\
-- \\
-- \\
--\end{array}$ \\
\hline 8.00 & $\begin{array}{r}198.66 \\
34.79 \\
60.43 \\
306.21 \\
116.53\end{array}$ & $\begin{array}{l}05-05-86 \\
03-30-65 \\
03-06-68 \\
01-08-77 \\
07-12-66\end{array}$ & $\begin{array}{l}-- \\
-- \\
-- \\
750\end{array}$ & $\begin{array}{l}-- \\
-- \\
-- \\
--\end{array}$ & $\begin{array}{r}-- \\
- \\
D, E, J\end{array}$ \\
\hline $\begin{array}{r}16.00 \\
- \\
6.00 \\
-- \\
--\end{array}$ & $\begin{array}{r}60.93 \\
149.95 \\
226.66 \\
234.40 \\
--\end{array}$ & $\begin{array}{c}03-16-83 \\
03-23-83 \\
03-31-72 \\
04-06-68 \\
--\end{array}$ & $\begin{array}{r}-- \\
60 \\
305 \\
-- \\
100\end{array}$ & $\begin{array}{r}-- \\
15.00 \\
-- \\
--\end{array}$ & $\begin{array}{r}- \\
\mathbf{D} \\
\mathbf{D} \\
-- \\
--\end{array}$ \\
\hline 8.00 & 40.00 & 04-01-58 & -- & -- & $=$ \\
\hline $\begin{array}{l}6.00 \\
8.00\end{array}$ & $\begin{array}{l}16 . \overline{00} \\
29.15\end{array}$ & $\begin{array}{c}01-\overline{-} \\
01-16 \\
03-18-66\end{array}$ & $\begin{array}{r}-\overline{80} \\
250\end{array}$ & $\begin{array}{l}-- \\
--\end{array}$ & $\begin{array}{c}\text { E,G } \\
--\end{array}$ \\
\hline $\begin{array}{r}8.00 \\
8.00 \\
-- \\
10.00\end{array}$ & $\begin{array}{r}13.00 \\
361.00\end{array}$ & $\begin{array}{c}0-\overline{0}-68 \\
\overline{0} \\
08-22-89\end{array}$ & $\begin{array}{r}-- \\
150 \\
\overline{100}\end{array}$ & $\begin{array}{l}-- \\
-- \\
--\end{array}$ & $\begin{array}{r}\text { E.J } \\
\text { D } \\
J \\
\text { D }\end{array}$ \\
\hline
\end{tabular}


Table 4.--Supplemental public-supply ground-water quality information

[deg C, degrees Celsius; $\mu \mathrm{S} / \mathrm{cm}$, microsiemens per centimeter; mg/L, milligrams per Iiter; used for computer storage of

\begin{tabular}{|c|c|c|c|c|c|c|c|c|}
\hline $\begin{array}{l}\text { Water } \\
\text { user } \\
\text { ID } \\
\end{array}$ & $\begin{array}{c}\text { Site } \\
\text { ID }\end{array}$ & Water system name & Date & $\begin{array}{l}\text { Depth } \\
\text { below } \\
\text { land } \\
\text { surface } \\
\text { (water } \\
\text { level) } \\
\text { (feet) } \\
(72019)\end{array}$ & $\begin{array}{l}\text { Spe- } \\
\text { cific } \\
\text { con- } \\
\text { duct- } \\
\text { ance } \\
(\mu s / \mathrm{cm}) \\
(00095)\end{array}$ & $\begin{array}{l}\text { PH } \\
\text { (stand- } \\
\text { ard } \\
\text { units) } \\
(00400)\end{array}$ & $\begin{array}{l}\text { Temper- } \\
\text { ature } \\
\text { water } \\
\text { (deg C) } \\
(00010)\end{array}$ & $\begin{array}{l}\text { Hard- } \\
\text { ness } \\
\text { total } \\
\text { (mg/L } \\
\text { as } \\
\text { Caco3) } \\
(00900)\end{array}$ \\
\hline $\begin{array}{l}20580 \\
30526 \\
30535 \\
30536 \\
30577\end{array}$ & 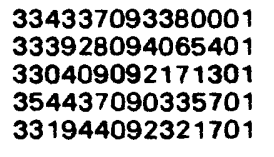 & $\begin{array}{l}\text { HOPE WATERWORKS } \\
\text { ASHDOWN WATERWORKS } \\
\text { BATTS LAPILE WATER ASSOC } \\
\text { BAY WATERWORKS } \\
\text { CALION WATER WORKS }\end{array}$ & $\begin{array}{l}09-05-90 \\
06-09-86 \\
10-26-82 \\
06-02-81 \\
10-26-82\end{array}$ & $\begin{array}{l}-- \\
-- \\
-- \\
--\end{array}$ & $\begin{array}{r}480 \\
799 \\
1.170 \\
328 \\
540\end{array}$ & $\begin{array}{r}7.5 \\
7.3 \\
8.3 \\
7.7 \\
--\end{array}$ & $\begin{array}{l}20.5 \\
18.5 \\
23.0 \\
18.0 \\
--\end{array}$ & $\begin{array}{l}220 \\
320 \\
-- \\
-180 \\
--\end{array}$ \\
\hline $\begin{array}{l}30599 \\
30630 \\
30633 \\
30643 \\
30643\end{array}$ & 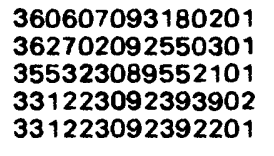 & $\begin{array}{l}\text { COMPTON WATERWORKS } \\
\text { DIAMOND CITY WATER } \\
\text { DOGWOOD WATER ASSOC } \\
\text { EL DORADO WATERWORKS } \\
\text { EL DORADO WATERWORKS }\end{array}$ & $\begin{array}{l}10-09-81 \\
02-04-80 \\
07-30-87 \\
05-26-82 \\
03-31-82\end{array}$ & $\begin{array}{c}-- \\
232.35 \\
-- \\
-- \\
--\end{array}$ & $\begin{array}{l}-- \\
-- \\
220 \\
468 \\
465\end{array}$ & $\begin{array}{l}-- \\
-\overline{7.2} \\
8.6 \\
8.8\end{array}$ & $\begin{array}{l}-- \\
-\overline{26.0} \\
23.5 \\
23.5\end{array}$ & $\begin{array}{r}130 \\
120 \\
28 \\
-\quad 6\end{array}$ \\
\hline $\begin{array}{l}30643 \\
30643 \\
30643 \\
30643 \\
30649\end{array}$ & $\begin{array}{l}331223092392401 \\
331235092385601 \\
331358092424301 \\
331406092430101 \\
330640091154103\end{array}$ & $\begin{array}{l}\text { EL DORADO WATERWORKS } \\
\text { EL DORADO WATERWORKS } \\
\text { EL DORADO WATERWORKS } \\
\text { EL DORADO WATERWORKS } \\
\text { EUDORA WATERWORKS }\end{array}$ & $\begin{array}{l}03-31-82 \\
03-31-82 \\
08-21-89 \\
03-31-82 \\
06-14-88\end{array}$ & $\begin{array}{l}-- \\
-- \\
-- \\
-- \\
47.65\end{array}$ & $\begin{array}{l}650 \\
500 \\
496 \\
475 \\
770\end{array}$ & $\begin{array}{r}-- \\
8.3 \\
8.4 \\
-7.1\end{array}$ & $\begin{array}{l}23.5 \\
23.5 \\
26.5 \\
23.0 \\
21.5\end{array}$ & $\begin{array}{l}-- \\
-- \\
-300\end{array}$ \\
\hline $\begin{array}{l}30651 \\
306541 \\
30654 \\
30656 \\
30724\end{array}$ & $\begin{array}{l}330657092385901 \\
330324092084501 \\
330324092084502 \\
352930090582501 \\
330219092111201\end{array}$ & $\begin{array}{l}\text { FAIRCREST WATER ASSOC } \\
\text { FELSENTHAL WATER ASSOC } \\
\text { FELSENTHAL WATER ASSOC } \\
\text { FISHER WATERWORKS } \\
\text { HUTTIG WATERWORKS }\end{array}$ & $\begin{array}{l}04-30-82 \\
06-15-82 \\
06-15-82 \\
08-08-90 \\
06-10-82\end{array}$ & $\begin{array}{c}-- \\
-- \\
-- \\
76.24 \\
--\end{array}$ & $\begin{array}{r}660 \\
1,390 \\
1,000 \\
517 \\
1,050\end{array}$ & $\begin{array}{l}9.0 \\
-- \\
-- \\
--\end{array}$ & $\begin{array}{l}24.0 \\
24.0 \\
20.0 \\
18.0 \\
22.5\end{array}$ & $\begin{array}{l}-- \\
-- \\
-- \\
--\end{array}$ \\
\hline $\begin{array}{l}30726 \\
30735 \\
30745 \\
30765 \\
30779\end{array}$ & $\begin{array}{l}331226092460101 \\
330107092432301 \\
361118090242201 \\
335806094100102 \\
351238090114701\end{array}$ & $\begin{array}{l}\text { HWY } 82 \text { WATER ASSOC } \\
\text { JUNCTION CITY WATERWORKS } \\
\text { LAFE RURAL WATER ASSOC } \\
\text { LOCKESBURG WATERWORKS } \\
\text { WARION WATERWORKS }\end{array}$ & $\begin{array}{l}04-29-82 \\
08-04-82 \\
11-03-83 \\
08-21-89 \\
10-01-81\end{array}$ & $\begin{array}{l}-- \\
-- \\
-- \\
--\end{array}$ & $\begin{array}{r}328 \\
510 \\
845 \\
60 \\
200\end{array}$ & $\begin{array}{r}8.6 \\
--8.5 \\
6.4 \\
7.4\end{array}$ & $\begin{array}{l}23.0 \\
23.5 \\
24.5 \\
25.0 \\
24.5\end{array}$ & $\begin{array}{r}-- \\
-- \\
11 \\
--\end{array}$ \\
\hline $\begin{array}{l}30785 \\
30805 \\
30809 \\
30809 \\
30823\end{array}$ & $\begin{array}{l}331351092572701 \\
355722093093401 \\
331805092570902 \\
331808092563801 \\
331205092221901\end{array}$ & $\begin{array}{l}\text { MARYSVILLE WATER ASSOC } \\
\text { MOCKINGBIRD HILL WATER } \\
\text { MOUNT HOLLY WATERWORKS } \\
\text { MOUNT HOLLY WATERWORKS } \\
\text { NEW LONDON WATER ASSOC }\end{array}$ & $\begin{array}{l}04-29-82 \\
06-02-80 \\
08-01-80 \\
06-08-82 \\
08-11-82\end{array}$ & $\begin{array}{l}-- \\
-- \\
-- \\
--\end{array}$ & $\begin{array}{l}310 \\
-- \\
-- \\
360 \\
950\end{array}$ & $\begin{array}{l}8.5 \\
-- \\
-- \\
-- \\
--\end{array}$ & $\begin{array}{l}25.0 \\
-- \\
21.5 \\
--\end{array}$ & $\begin{array}{l}-- \\
110 \\
-- \\
--\end{array}$ \\
\hline $\begin{array}{l}30830 \\
30848 \\
30851 \\
30855 \\
30861\end{array}$ & 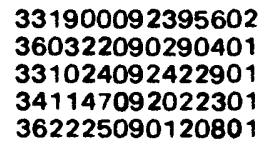 & $\begin{array}{l}\text { NORPHLET WATERWORKS } \\
\text { PARAGOULD CITY LGHT/WATER } \\
\text { PARKERS CHAPEL WATER ASSOC } \\
\text { PB/GENERAL WATERWORKS CO } \\
\text { PIGGOTT WATERWORKS }\end{array}$ & $\begin{array}{l}08-10-82 \\
08-07-90 \\
05-27-82 \\
06-13-88 \\
07-30-87\end{array}$ & $\begin{array}{l}-- \\
-- \\
-- \\
--\end{array}$ & $\begin{array}{l}300 \\
221 \\
546 \\
160 \\
825\end{array}$ & $\begin{array}{l}6.9 \\
8.5 \\
6.7 \\
8.5\end{array}$ & $\begin{array}{l}24.0 \\
20.0 \\
-- \\
26.5 \\
24.0\end{array}$ & $\begin{array}{r}10 \\
6 \\
30 \\
23\end{array}$ \\
\hline $\begin{array}{l}30861 \\
30931 \\
30931 \\
30931 \\
30952\end{array}$ & $\begin{array}{l}362311090111301 \\
330633092213901 \\
330643092214801 \\
330651092212001 \\
354340091115702\end{array}$ & $\begin{array}{l}\text { PIGGOTT WATERWORKS } \\
\text { STRONG WATERWORKS } \\
\text { STRONG WATERWORKS } \\
\text { STRONG WATERWORKS } \\
\text { TUCKERMAN WATERWORKS }\end{array}$ & $\begin{array}{l}06-21-82 \\
06-09-82 \\
06-09-82 \\
06-15-82 \\
08-29-80\end{array}$ & $\begin{array}{l}-- \\
-- \\
-- \\
--\end{array}$ & $\begin{array}{l}841 \\
500 \\
415 \\
980 \\
480\end{array}$ & $\begin{array}{l}8.4 \\
-- \\
-- \\
-\overline{7.5}\end{array}$ & $\begin{array}{l}23.5 \\
20.0 \\
19.5 \\
21.0 \\
18.0\end{array}$ & $\begin{array}{l}25 \\
-- \\
-- \\
-- \\
230\end{array}$ \\
\hline $\begin{array}{l}30959 \\
30963 \\
30975 \\
30978\end{array}$ & $\begin{array}{l}352231090421501 \\
362359091590001 \\
330809092461101 \\
350906090104201\end{array}$ & $\begin{array}{l}\text { VANNDALE-BIRDEYE WATER } \\
\text { VIOLA WATERWORKS } \\
\text { WESSON-NEWELL WATER ASSOC } \\
\text { WEST MEMPHIS WATERWORKS }\end{array}$ & $\begin{array}{l}08-22-89 \\
07-29-87 \\
04-30-82 \\
08-23-89\end{array}$ & $\begin{array}{l}-- \\
-- \\
-- \\
75.00\end{array}$ & $\begin{array}{l}550 \\
370 \\
575 \\
208\end{array}$ & $\begin{array}{l}7.3 \\
7.8 \\
8.8 \\
7.2\end{array}$ & $\begin{array}{l}23.0 \\
21.0 \\
24.0 \\
25.5\end{array}$ & $\begin{array}{r}230 \\
190 \\
--\quad \\
7\end{array}$ \\
\hline
\end{tabular}


for sites investigated by the U.S. Geological Survey

Hg/L. micrograms per liter; five digit numbers in parentheses are STORET parameter codes data; --, no data available]

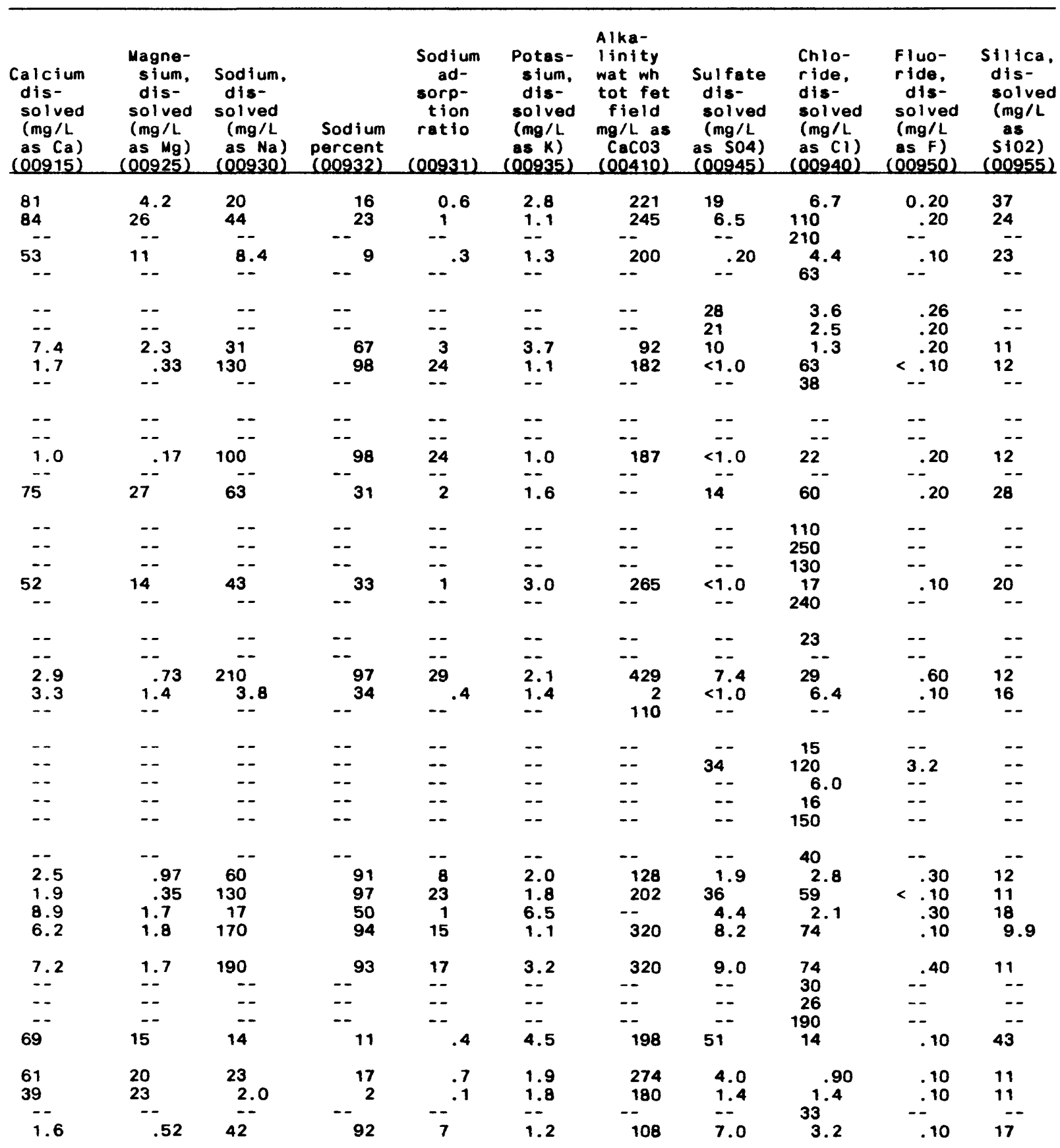


Table 4.--Supplemental public-supply ground-water quality information

\begin{tabular}{|c|c|c|c|c|c|c|c|c|}
\hline $\begin{array}{l}\text { Water } \\
\text { user } \\
\text { I0 } \\
\end{array}$ & $\begin{array}{c}\text { Site } \\
\text { ID }\end{array}$ & Water system name & $\begin{array}{l}\text { Solids, } \\
\text { residue } \\
\text { at } 180 \\
\text { deg. C } \\
\text { dis- } \\
\text { solved } \\
(\mathrm{mg} / \mathrm{L}) \\
(70300)\end{array}$ & $\begin{array}{l}\text { Solids, } \\
\text { sum of } \\
\text { consti- } \\
\text { tuents, } \\
\text { dis- } \\
\text { solved } \\
\text { (mg/l) } \\
(70301)\end{array}$ & $\begin{array}{l}\text { Nitro- } \\
\text { gen, } \\
\text { NO2+NO3 } \\
\text { dis- } \\
\text { solved } \\
\text { (mg/L } \\
\text { as N) } \\
(00631)\end{array}$ & $\begin{array}{l}\text { Phos- } \\
\text { phorus } \\
\text { or tho, } \\
\text { dis- } \\
\text { solved } \\
\text { (mg/L } \\
\text { as P) } \\
(00671)\end{array}$ & $\begin{array}{c}\text { Barium, } \\
\text { dis- } \\
\text { solved } \\
(\mu \mathrm{g} / \mathrm{L} \\
\text { as Ba) } \\
(01005)\end{array}$ & $\begin{array}{c}\text { Boron, } \\
\text { dis- } \\
\text { solved } \\
(\mu g / L \\
\text { As B) } \\
(01020)\end{array}$ \\
\hline $\begin{array}{l}20580 \\
30526 \\
30535 \\
30536 \\
30577\end{array}$ & 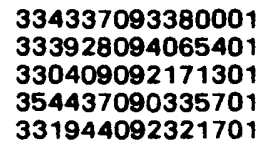 & $\begin{array}{l}\text { HOPE WATERWORKS } \\
\text { ASHDOWN WATERWORKS } \\
\text { BATTS LAPILE WATER ASSOC } \\
\text { BAY WATERWORKS } \\
\text { CALION WATER WORKS }\end{array}$ & $\begin{array}{c}304 \\
479 \\
-- \\
208 \\
--\end{array}$ & $\begin{array}{c}304 \\
447 \\
-- \\
222 \\
--\end{array}$ & $\begin{array}{l}<0.100 \\
<.100 \\
-- \\
.060 \\
--\end{array}$ & $\begin{array}{l}0.060 \\
.020 \\
-- \\
-- \\
--\end{array}$ & $\begin{array}{l}19 \\
350 \\
-- \\
--\end{array}$ & $\begin{array}{r}40 \\
--\quad \\
--\end{array}$ \\
\hline $\begin{array}{l}30599 \\
30630 \\
30633 \\
30643 \\
30643\end{array}$ & $\begin{array}{l}360607093180201 \\
362702092550301 \\
355323089552101 \\
331223092393902 \\
331223092392201\end{array}$ & $\begin{array}{l}\text { COMPTON WATERWORKS } \\
\text { DIAMOND CITY WATER } \\
\text { DOGWOOD WATER ASSOC } \\
\text { EL DORADO WATERWORKS } \\
\text { EL DORADO WATERWORKS }\end{array}$ & $\begin{array}{l}-- \\
- \\
124 \\
--\end{array}$ & $\begin{array}{l}121 \\
190 \\
126 \\
-- \\
--\end{array}$ & $\begin{array}{l}-- \\
-- \\
<.100 \\
.010 \\
--\end{array}$ & $\begin{array}{l}-- \\
- \\
.010 \\
--\end{array}$ & $\begin{array}{r}100 \\
500 \\
110 \\
- \\
-\end{array}$ & $\begin{array}{r}70 \\
200 \\
--\end{array}$ \\
\hline $\begin{array}{l}30643 \\
30643 \\
30643 \\
30643 \\
30649\end{array}$ & $\begin{array}{l}331223092392401 \\
331235092385601 \\
331358092424301 \\
331406092430101 \\
330640091154103\end{array}$ & $\begin{array}{l}\text { EL DORADO WATERWORKS } \\
\text { EL DORADO WATERWORKS } \\
\text { EL DORADO WATERWORKS } \\
\text { EL DORADO WATERWORKS } \\
\text { EUDORA WATERWORKS }\end{array}$ & $\begin{array}{l}-- \\
-- \\
254 \\
-- \\
449\end{array}$ & $\begin{array}{l}-- \\
-- \\
-- \\
482\end{array}$ & $\begin{array}{l}-- \\
-- \\
<.100 \\
-- \\
<.100\end{array}$ & $\begin{array}{l}-- \\
-\overline{1} \\
.220 \\
- \\
.020\end{array}$ & $\begin{array}{l}-- \\
- \\
- \\
230\end{array}$ & $\begin{array}{l}-- \\
-- \\
120 \\
-- \\
120\end{array}$ \\
\hline $\begin{array}{l}30651 \\
30654 \\
30654 \\
30656 \\
30724\end{array}$ & $\begin{array}{l}330657092385901 \\
330324092084501 \\
330324092084502 \\
352930090582501 \\
330219092111201\end{array}$ & $\begin{array}{l}\text { FAIRCREST WATER ASSOC } \\
\text { FELSENTHAL WATER ASSOC } \\
\text { FELSENTHAL WATER ASSOC } \\
\text { FISHER WATERWORKS } \\
\text { HUTTIG WATERWORKS }\end{array}$ & $\begin{array}{l}-- \\
-- \\
-- \\
291\end{array}$ & $\begin{array}{l}-- \\
-- \\
-- \\
--\end{array}$ & $\begin{array}{l}-- \\
-- \\
-- \\
-100\end{array}$ & $\begin{array}{l}-- \\
-- \\
-- \\
.110\end{array}$ & $\begin{array}{l}-- \\
-- \\
-- \\
220\end{array}$ & $\begin{array}{l}-- \\
-- \\
--\end{array}$ \\
\hline $\begin{array}{l}30726 \\
30735 \\
30745 \\
30765 \\
30779\end{array}$ & $\begin{array}{l}331226092460101 \\
330107092432301 \\
361118090242201 \\
335806094100102 \\
351238090114701\end{array}$ & $\begin{array}{l}\text { HWY } 82 \text { WATER ASSOC } \\
\text { JUNCTION CITY WATERWORKS } \\
\text { LAFE RURAL WATER ASSOC } \\
\text { LOCKESBURG WATERWORKS } \\
\text { MARION WATERWORKS }\end{array}$ & $\begin{array}{r}-- \\
-- \\
539 \\
48 \\
104\end{array}$ & $\begin{array}{l}-- \\
-- \\
523 \\
--\end{array}$ & $\begin{array}{l}-- \\
-- \\
.100 \\
.880 \\
--\end{array}$ & $\begin{array}{l}-- \\
- \\
- \\
.010 \\
--\end{array}$ & $\begin{array}{l}-- \\
- \\
160 \\
--\end{array}$ & $\begin{array}{l}-- \\
-- \\
870 \\
<10 \\
--\end{array}$ \\
\hline $\begin{array}{l}30785 \\
30805 \\
30809 \\
30809 \\
30823\end{array}$ & $\begin{array}{l}331351092572701 \\
355722093093401 \\
331805092570902 \\
331808092563801 \\
331205092221901\end{array}$ & $\begin{array}{l}\text { MARYSVILLE WATER ASSOC } \\
\text { MOCKINGBIRD HILL WATER } \\
\text { MOUNT HOLLY WATERWORKS } \\
\text { MOUNT HOLLY WATERWORKS } \\
\text { NEW LONDON WATER ASSOC }\end{array}$ & $\begin{array}{l}-- \\
-- \\
210 \\
--\end{array}$ & $\begin{array}{l}-- \\
418 \\
-- \\
--\end{array}$ & $\begin{array}{l}-- \\
-- \\
-- \\
--\end{array}$ & $\begin{array}{l}-- \\
-- \\
-- \\
--\end{array}$ & $\begin{array}{l}-- \\
100 \\
-- \\
--\end{array}$ & $\begin{array}{l}-- \\
-- \\
-- \\
--\end{array}$ \\
\hline $\begin{array}{l}30830 \\
30848 \\
30851 \\
30855 \\
30861\end{array}$ & $\begin{array}{l}331900092395602 \\
360322090290401 \\
331024092422901 \\
341147092022301 \\
362225090120801\end{array}$ & $\begin{array}{l}\text { NORPHLET WATERWORKS } \\
\text { PARAGOULD CITY LGHT/WATER } \\
\text { PARKERS CHAPEL WATER ASSO } \\
\text { PB/GENERAL WATERWORKS CO } \\
\text { PIGGOTT WATERWORKS }\end{array}$ & $\begin{array}{l}-- \\
161 \\
-- \\
100 \\
218\end{array}$ & $\begin{array}{l}161 \\
363 \\
107 \\
464\end{array}$ & $\begin{array}{r}-. \\
<.100 \\
<.010 \\
.100 \\
.110\end{array}$ & $\begin{array}{r}-. \\
.360 \\
-.070 \\
.010\end{array}$ & $\begin{array}{r}19 \\
17 \\
100 \\
28\end{array}$ & $\begin{array}{r}- \\
210 \\
210 \\
40 \\
<10\end{array}$ \\
\hline $\begin{array}{l}30861 \\
30931 \\
30931 \\
30931 \\
30952\end{array}$ & $\begin{array}{l}362311090111301 \\
330633092213901 \\
330643092214801 \\
330651092212001 \\
354340091115702\end{array}$ & $\begin{array}{l}\text { PIGGOTT WATERWORKS } \\
\text { STRONG WATERWORKS } \\
\text { STRONG WATERWORKS } \\
\text { STRONG WATERWORKS } \\
\text { TUCKERMAN WATERWORKS }\end{array}$ & $\begin{array}{l}462 \\
-- \\
-- \\
-- \\
324\end{array}$ & $\begin{array}{l}489 \\
-- \\
-- \\
-- \\
329\end{array}$ & $\begin{array}{l}-- \\
-- \\
-- \\
--\end{array}$ & $\begin{array}{l}-- \\
-- \\
-- \\
--\end{array}$ & $\begin{array}{l}-- \\
-- \\
-- \\
--\end{array}$ & $\begin{array}{l}310 \\
-- \\
-- \\
-- \\
--\end{array}$ \\
\hline $\begin{array}{l}30959 \\
30963 \\
30975 \\
30978\end{array}$ & $\begin{array}{l}352231090421501 \\
362359091590001 \\
330809092461101 \\
350906090104201\end{array}$ & $\begin{array}{l}\text { VANNDALE-BIRDEYE WATER } \\
\text { VIOLA WATERWORKS } \\
\text { WESSON-NEWELL WATER ASSOC } \\
\text { WEST MEMPHIS WATERWORKS }\end{array}$ & $\begin{array}{r}120 \\
181 \\
-- \\
286\end{array}$ & $\begin{array}{r}287 \\
188 \\
-- \\
139\end{array}$ & $\begin{array}{l}<.100 \\
<.100 \\
-. \\
<.100\end{array}$ & $\begin{array}{l}.010 \\
.010 \\
-0 \\
.070\end{array}$ & $\begin{array}{r}30 \\
34 \\
-- \\
130\end{array}$ & $\begin{array}{r}50 \\
<10 \\
--\quad 30\end{array}$ \\
\hline
\end{tabular}


for sites investigated by the U.S. Geological Survey

\begin{tabular}{|c|c|c|c|c|c|c|c|c|c|}
\hline $\begin{array}{l}\text { Beryl- } \\
\text { lium, } \\
\text { dis- } \\
\text { solved } \\
\text { ( } \mu \mathrm{g} / \mathrm{L} \\
\text { as Be) } \\
(01010)\end{array}$ & $\begin{array}{l}\text { Cadmium } \\
\text { dis- } \\
\text { solved } \\
\text { ( } \mu \text { l / } / \mathrm{L} \\
\text { as Cd) } \\
(01025) \\
\end{array}$ & $\begin{array}{l}\text { Chro } \\
\text { mlum, } \\
\text { dis- } \\
\text { solved } \\
\text { ( } \mu 1 \text { ged } \\
\text { (s Cr) } \\
(01030)\end{array}$ & $\begin{array}{l}\text { Cobalt, } \\
\text { dis- } \\
\text { solved } \\
\text { ( } \mu g / L \\
\text { as Co } \\
\text { Co1035 } \\
\end{array}$ & 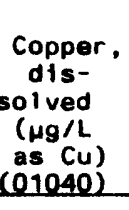 & $\begin{array}{c}\text { Iron, } \\
\text { dis- } \\
\text { solved } \\
\text { ( } \mathrm{l} \text { gol } \\
\text { as Fe) } \\
(01046) \\
\end{array}$ & $\begin{array}{c}\text { Lead, } \\
\text { dis- } \\
\text { solved } \\
\text { ( } \mu g / L \\
\text { as } \mathrm{Pb}) \\
(01049) \\
\end{array}$ & $\begin{array}{l}\text { Lithium, } \\
\text { dis- } \\
\text { solved } \\
\text { ( } \mu g / L \\
\text { as } L i) \\
(01130) \\
(01130)\end{array}$ & $\begin{array}{c}\text { Manga- } \\
\text { nese, } \\
\text { dis- } \\
\text { solved } \\
\text { ( } \mu g / L \\
\text { as Mn) } \\
(01056)\end{array}$ & $\begin{array}{c}\text { Mol yb- } \\
\text { denum, } \\
\text { dis- } \\
\text { solved } \\
\text { ( } \mu 9 / \mathrm{L} \\
\text { as Mo) } \\
(01060) \\
\end{array}$ \\
\hline $\begin{array}{l}<0.5 \\
<.5\end{array}$ & $\begin{array}{l}<1.0 \\
<1.0\end{array}$ & $\ldots<5$ & $\begin{array}{l}<3 \\
<3\end{array}$ & $\begin{array}{l}<10 \\
<10\end{array}$ & $\begin{array}{r}220 \\
2,400\end{array}$ & $\begin{array}{l}<10 \\
<10\end{array}$ & $\begin{array}{l}22 \\
33\end{array}$ & $\begin{array}{r}15 \\
190\end{array}$ & $\begin{array}{l}<10 \\
<10\end{array}$ \\
\hline 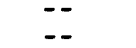 & -- & $\because$ & $=-$ & $=-$ & 40 & $=$ & -- & 60 & $\begin{array}{l}-- \\
--\end{array}$ \\
\hline-- & -- & -- & -- & -- & --4 & -- & -- & -. & $\cdots$ \\
\hline-- & 1.0 & 5 & -- & 10 & 70 & 5 & -- & -- & -- \\
\hline $\bar{c} 5$ & $\begin{array}{r}4.0 \\
410\end{array}$ & $\begin{array}{r}5 \\
\leq 5\end{array}$ & -- & $\begin{array}{r}10 \\
<10\end{array}$ & $\begin{array}{r}40 \\
300\end{array}$ & $1^{1}$ & -- & $-\overline{120}$ & $\bar{s}$ \\
\hline$\leq .5$ & $<1.0$ & $\ldots<5$ & $\ldots$ & _- & $\begin{array}{l}3,100 \\
11\end{array}$ & $\begin{array}{r}<10 \\
--\end{array}$ & 30 & $\begin{array}{r}120 \\
<1\end{array}$ & $\leq 10$ \\
\hline-- & -- & -- & -- & -- & -- & -- & -- & -- & -- \\
\hline-- & -- & -- & -- & -- & -- & -- & -- & -- & -- \\
\hline$<.5$ & $\begin{array}{l}-\overline{1} \\
<1.0\end{array}$ & $<5$ & $-<3$ & $<10$ & 19 & $<10$ & $-{ }_{5}$ & -10 & - \\
\hline.- & --.0 & $\ldots$ & $--\infty$ & -- & -. & -- & -. & .- & .. \\
\hline$<.5$ & $<1.0$ & $<5$ & $<3$ & $<10$ & 1,200 & $<10$ & 14 & 190 & $<10$ \\
\hline-- & -- & -- & -- & -- & -- & -- & -- & -- & -. \\
\hline-- & -- & -- & -- & -- & -- & -- & -- & -- & -- \\
\hline$\leq .5$ & 2.0 & $-_{-}^{<5}$ & - $^{<3}$ & $\begin{array}{l}<10 \\
-\end{array}$ & 1,800 & $\begin{array}{l}<10 \\
--\end{array}$ & $--^{8}$ & $\begin{array}{r}170 \\
--\end{array}$ & $\begin{array}{l}<10 \\
--\end{array}$ \\
\hline-- & -- & -- & -- & -- & -- & -- & -- & -- & -- \\
\hline $\begin{array}{l}<.5 \\
<. .5\end{array}$ & $\begin{array}{l}<1.0 \\
<1.0 \\
--\end{array}$ & $\begin{array}{r}<10 \\
-<5\end{array}$ & $-->3$ & $\begin{array}{r}2 \\
-2\end{array}$ & $\begin{array}{r}27 \\
-\quad 20\end{array}$ & $\begin{array}{r}2 \\
<10 \\
--\quad\end{array}$ & $\begin{array}{r}38 \\
-\quad 8\end{array}$ & $\begin{array}{r}1 \\
-\quad 4\end{array}$ & $\begin{array}{r}3 \\
<10 \\
---\end{array}$ \\
\hline-- & -- & -- & -- & -- & -- & -- & -. & -- & -- \\
\hline-- & 1.0 & 5 & -- & 30 & 1 & 5 & -- & -- & -- \\
\hline$=$ & $\because$ & -- & -- & $=$ & -- & -- & -- & -- & -- \\
\hline-- & -- & -- & -- & -- & -- & -- & -- & -- & - \\
\hline -- & -- & -. & -- & -- & -- & -- & -. & -- & -- \\
\hline$<.5$ & 2.0 & $<5$ & $<3$ & $<10$ & 380 & $<10$ & 20 & 23 & $<10$ \\
\hline$<.5$ & $<1.0$ & $<5$ & $<3$ & $<10$ & 1,500 & $<10$ & 23 & $\begin{array}{l}0 \\
76\end{array}$ & $<10$ \\
\hline & $<1.0$ & $<5$ & $<3$ & $<10$ & & $<10$ & 9 & $<1$ & $<10$ \\
\hline-- & -- & -- & -- & -- & 55 & -- & -- & 2 & -- \\
\hline -. & -- & -- & -- & -- & -- & -- & -- & - & -- \\
\hline-- & -- & -- & & - & -- & 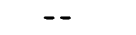 & 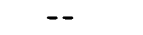 & -- & - \\
\hline-- & -- & -- & -- & -- & 30 & -- & -- & 40 & -- \\
\hline $\begin{array}{l}<.5 \\
<\quad .5\end{array}$ & $\begin{array}{l}<1.0 \\
<1.0\end{array}$ & $\begin{array}{l}<5 \\
<5\end{array}$ & $\begin{array}{l}<3 \\
<3\end{array}$ & $\begin{array}{l}<10 \\
<10\end{array}$ & $\begin{array}{r}280 \\
4\end{array}$ & $\begin{array}{l}<10 \\
<10\end{array}$ & $\begin{array}{r}10 \\
9\end{array}$ & $\begin{array}{l}22 \\
<1\end{array}$ & $\begin{array}{l}<10 \\
<10\end{array}$ \\
\hline$<.5$ & $<1.0$ & $<5$ & $<3$ & $<10$ & 990 & $<10$ & 5 & 110 & $<10$ \\
\hline
\end{tabular}


152 
Table 4.--Supplemental public-supply ground-water quality information for sites investigated by the U.S. Geological Survey--Continued

\begin{tabular}{|c|c|c|c|c|c|c|}
\hline $\begin{array}{l}\text { Water } \\
\text { user } \\
\text { ID } \\
\end{array}$ & $\begin{array}{c}\text { Site } \\
\text { ID }\end{array}$ & Water sxstem name & $\begin{array}{c}\text { Nickel, } \\
\text { dis- } \\
\text { solved } \\
(\mu g / L \\
\text { as Ni) } \\
(01065)\end{array}$ & $\begin{array}{l}\text { Silver, } \\
\text { dis- } \\
\text { solved } \\
\text { ( } \mu g / L \\
\text { as Ag) } \\
(01075)\end{array}$ & $\begin{array}{c}\text { Stron- } \\
\text { tium, } \\
\text { dis- } \\
\text { solved } \\
\text { ( } \mu g / L / L \\
\text { as } S r \text { ) } \\
(01080 \\
(01080\end{array}$ & $\begin{array}{r}\text { Zinc, } \\
\text { dis- } \\
\text { solved } \\
(\mu g / L \\
\text { as } 2 n \\
(01090)\end{array}$ \\
\hline $\begin{array}{l}20580 \\
30526 \\
30535 \\
30536 \\
30577\end{array}$ & $\begin{array}{l}334337093380001 \\
333928094065401 \\
330409092171301 \\
354437090335701 \\
331944092321701\end{array}$ & $\begin{array}{l}\text { HOPE WATERWORKS } \\
\text { ASHDOWN WATERWORKS } \\
\text { BATTS LAPILE WATER ASSOC } \\
\text { BAY WATERWORKS } \\
\text { CALION WATER WORKS }\end{array}$ & $\begin{array}{l}<10 \\
-- \\
-- \\
--\end{array}$ & $\begin{array}{l}<1.0 \\
-- \\
-- \\
--\end{array}$ & $\begin{array}{l}470 \\
620 \\
-- \\
-- \\
--\end{array}$ & $\begin{array}{l}<3 \\
8 \\
-- \\
--\end{array}$ \\
\hline $\begin{array}{l}30599 \\
30630 \\
30633 \\
30643 \\
30643\end{array}$ & $\begin{array}{l}360607093180201 \\
362702092550301 \\
355323089552101 \\
331223092393902 \\
331223092392201\end{array}$ & $\begin{array}{l}\text { COMPTON WATERWORKS } \\
\text { DIAMOND CITY WATER } \\
\text { DOGWOOD WATER ASSOC } \\
\text { EL DORADO WATERWORKS } \\
\text { EL OORADO WATERWORKS }\end{array}$ & $\begin{array}{l}-- \\
-- \\
<10 \\
<100\end{array}$ & $\begin{aligned} & 5.0 \\
& 5.0 \\
&<1.0 \\
&-- \\
&--\end{aligned}$ & $\begin{array}{l}-- \\
- \\
340 \\
--\end{array}$ & $\begin{array}{r}10 \\
10 \\
25 \\
-- \\
--\end{array}$ \\
\hline $\begin{array}{l}30643 \\
30643 \\
30643 \\
30643 \\
30649\end{array}$ & $\begin{array}{l}331223092392401 \\
331235092385601 \\
331358092424301 \\
331406092430101 \\
330640091154103\end{array}$ & $\begin{array}{l}\text { EL DORADO WATERWORKS } \\
\text { EL DORADO WATERWORKS } \\
\text { EL DORADO WATERWORKS } \\
\text { EL DORADO WATERWORKS } \\
\text { EUDORA WATERWORKS }\end{array}$ & $\begin{array}{l}-- \\
- \\
<10 \\
-- \\
<10\end{array}$ & $\begin{array}{l}-- \\
-\overline{1} .0 \\
-\overline{1} .0\end{array}$ & $\begin{array}{c}-- \\
-- \\
-- \\
440\end{array}$ & $\begin{array}{c}-- \\
-- \\
-7 \\
-7\end{array}$ \\
\hline $\begin{array}{l}30651 \\
306541 \\
30654 \\
30656 \\
30724\end{array}$ & $\begin{array}{l}330657092385901 \\
330324092084501 \\
330324092084502 \\
352930090582501 \\
330219092111201\end{array}$ & $\begin{array}{l}\text { FAIRCREST WATER ASSOC } \\
\text { FELSENTHAL WATER ASSOC } \\
\text { FELSENTHAL WATER ASSOC } \\
\text { FISHER WATERWORKS } \\
\text { HUTTIG WATERWORKS }\end{array}$ & $\begin{array}{l}-- \\
-- \\
-- \\
--\end{array}$ & $\begin{array}{l}-- \\
-- \\
-\overline{1} .0 \\
--\end{array}$ & $\begin{array}{l}-- \\
-- \\
-- \\
400\end{array}$ & $\begin{array}{l}-- \\
-- \\
-- \\
--\end{array}$ \\
\hline $\begin{array}{l}30726 \\
30735 \\
30745 \\
30765 \\
30779\end{array}$ & $\begin{array}{l}331226092460101 \\
330107092432301 \\
361118090242201 \\
335806094100102 \\
351238090114701\end{array}$ & $\begin{array}{l}\text { HWY } 82 \text { WATER ASSOC } \\
\text { JUNCTION CITY WATERWORKS } \\
\text { LAFE RURAL WATER ASSOC } \\
\text { LOCKESBURG WATERWORKS } \\
\text { MARION WATERWORKS }\end{array}$ & $\begin{array}{l}-- \\
-- \\
- \\
-\end{array}$ & $\begin{array}{l}-- \\
-- \\
<1.0 \\
<1.0\end{array}$ & $\begin{array}{l}-- \\
-- \\
120 \\
--\end{array}$ & $\begin{array}{r}-- \\
-- \\
55 \\
--\end{array}$ \\
\hline $\begin{array}{l}30785 \\
30805 \\
30809 \\
30809 \\
30823\end{array}$ & $\begin{array}{l}331351092572701 \\
355722093093401 \\
331805092570902 \\
331808092563801 \\
331205092221901\end{array}$ & $\begin{array}{l}\text { MARYSVILLE WATER ASSOC } \\
\text { MOCKINGBIRO HILL WATER } \\
\text { MOUNT HOLLY WATERWORKS } \\
\text { MOUNT HOLLY WATERWORKS } \\
\text { NEW LONOON WATER ASSOC }\end{array}$ & $\begin{array}{l}-- \\
-- \\
-- \\
--\end{array}$ & $\begin{array}{l}-- \\
5.0 \\
-- \\
--\end{array}$ & $\begin{array}{l}-- \\
-- \\
-- \\
--\end{array}$ & $\begin{array}{l}-- \\
40 \\
-- \\
--\end{array}$ \\
\hline $\begin{array}{l}30830 \\
30848 \\
30851 \\
30855 \\
30861\end{array}$ & $\begin{array}{l}331900092395602 \\
360322090290401 \\
331024092422901 \\
341147092022301 \\
362225090120801\end{array}$ & $\begin{array}{l}\text { NORPHLET WATERWORKS } \\
\text { PARAGOULD CITY LGHT/WATER } \\
\text { PARKERS CHAPEL WATER ASSOC } \\
\text { PB/GENERAL WATERWORKS CO } \\
\text { PIGGOTT WATERWORKS }\end{array}$ & $\begin{array}{r}- \\
<10 \\
<100 \\
<10 \\
<10\end{array}$ & $\begin{array}{l}-- \\
<1.0 \\
-- \\
<1.0 \\
1.0\end{array}$ & $\begin{array}{r}-- \\
-- \\
300 \\
35\end{array}$ & $\begin{array}{r}-- \\
-- \\
300 \\
22\end{array}$ \\
\hline $\begin{array}{l}30861 \\
30931 \\
30931 \\
30931 \\
30952\end{array}$ & $\begin{array}{l}362311090111301 \\
330633092213901 \\
330643092214801 \\
330651092212001 \\
354340091115702\end{array}$ & $\begin{array}{l}\text { PIGGOTT WATERWORKS } \\
\text { STRONG WATERWORKS } \\
\text { STRONG WATERWORKS } \\
\text { STRONG WATERWORKS } \\
\text { TUCKERMAN WATERWORKS }\end{array}$ & $\begin{array}{l}-- \\
-- \\
-- \\
--\end{array}$ & $\begin{array}{l}-- \\
- \\
-- \\
--\end{array}$ & $\begin{array}{l}-- \\
-- \\
-- \\
--\end{array}$ & $\begin{array}{l}-- \\
-- \\
-- \\
--\end{array}$ \\
\hline $\begin{array}{l}30959 \\
30963 \\
30975 \\
30978\end{array}$ & $\begin{array}{l}352231090421501 \\
362359091590001 \\
330809092461101 \\
350906090104201\end{array}$ & $\begin{array}{l}\text { VANNOALE-BIRDEYE WATER } \\
\text { VIOLA WATERWORKS } \\
\text { WESSON-NEWELL WATER ASSOC } \\
\text { WEST MEMPHIS WATERWORKS }\end{array}$ & $\begin{array}{r}<10 \\
<10 \\
-- \\
<10\end{array}$ & $\begin{array}{l}<1.0 \\
<1.0 \\
-- \\
<1.0\end{array}$ & $\begin{array}{r}67 \\
32 \\
-- \\
260\end{array}$ & $\begin{array}{r}7 \\
39 \\
-24\end{array}$ \\
\hline
\end{tabular}


Table 5.--Supplemental surface-water-qual ity information

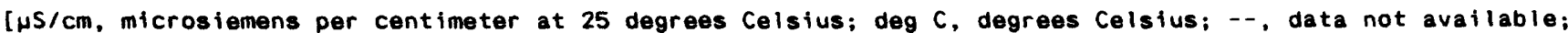
ideal range; $\mu g / L$. micrograms per liter; five digit numbers in parentheses are

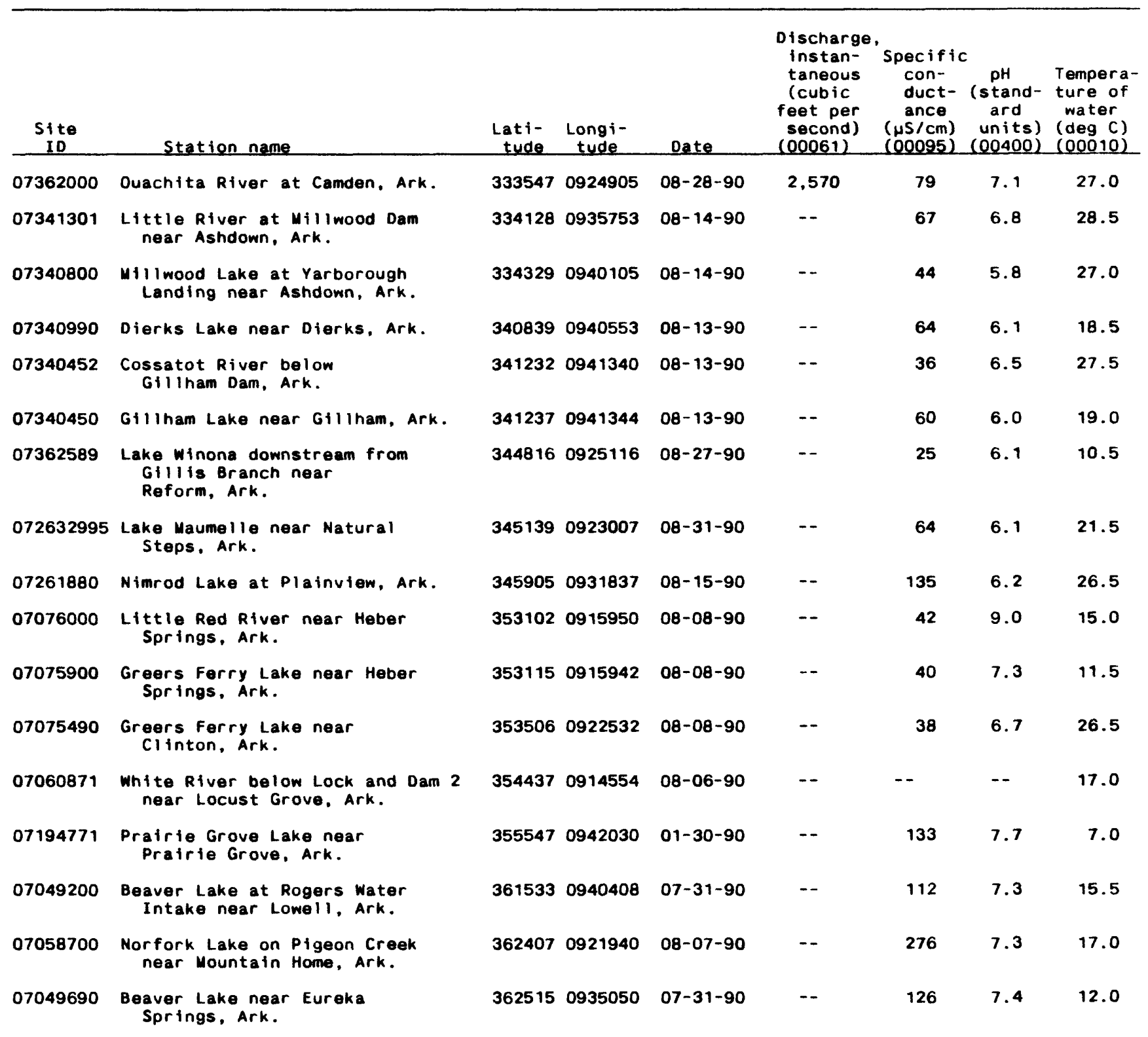


for sites near public-supply intakes--Continued

ntu, nephelometric turbidity units; $\mathrm{mg} / \mathrm{L}$, milligrams per liter; mL, milliliter; K, plate count outside STORET parameter codes used for computer storage of data]

\begin{tabular}{|c|c|c|c|c|c|c|c|c|c|c|}
\hline $\begin{array}{c}\text { Tur- } \\
\text { bid- } \\
\text { ity } \\
\text { (ntu) } \\
(00076)\end{array}$ & $\begin{array}{c}\text { Oxygen, } \\
\text { dis- } \\
\text { solved } \\
\text { (mg/l) } \\
(00300)\end{array}$ & $\begin{array}{l}\text { Oxygen, } \\
\text { dis- } \\
\text { solved } \\
\text { (per- } \\
\text { cent } \\
\text { satur- } \\
\text { ation) } \\
\text { (oos01) }\end{array}$ & $\begin{array}{l}\text { Baro- } \\
\text { metric } \\
\text { pressure } \\
\text { (milli- } \\
\text { meter } \\
\text { of } \\
\text { Hg) } \\
(0.0025)\end{array}$ & $\begin{array}{c}\text { Coli- } \\
\text { form, } \\
\text { fecal, } \\
\text { mf agar } \\
\text { (colonies } \\
\text { per } \\
100 \mathrm{~mL}) \\
(31625)\end{array}$ & $\begin{array}{c}\text { Strep- } \\
\text { tococct } \\
\text { fecal, } \\
\text { kf agar } \\
\text { (colonies } \\
\text { per } \\
100 \mathrm{~mL}) \\
(31673)\end{array}$ & $\begin{array}{l}\text { Hard- } \\
\text { ness } \\
\text { total } \\
\text { (mg/L } \\
\text { as } \\
\text { Caco3) } \\
(00900)\end{array}$ & $\begin{array}{c}\text { Calcium } \\
\text { dis- } \\
\text { solved } \\
\text { (mg/L } \\
\text { as Ca) } \\
(00915)\end{array}$ & $\begin{array}{l}\text { Magne- } \\
\text { s lum, } \\
\text { dis- } \\
\text { solved } \\
\text { (mg/L } \\
\text { as Mg) } \\
(00925)\end{array}$ & $\begin{array}{c}\text { Sodium, } \\
\text { dis- } \\
\text { solved } \\
\text { (mg/L } \\
\text { As Na) } \\
(00930)\end{array}$ & $\begin{array}{l}\text { Sodium } \\
\text { percent } \\
\text { (00932) }\end{array}$ \\
\hline 5.6 & 6.4 & 81 & 761 & 83 & 150 & 21 & 6.2 & 8. 1 & 44 & 0.8 \\
\hline 4.5 & 7.8 & 101 & 760 & 57 & -- & 22 & 6.5 & -- & -- & - \\
\hline 8.1 & 6.4 & 81 & 758 & -- & -- & 13 & 3.4 & -- & -- & -- \\
\hline 2.5 & .4 & 4 & 756 & -. & -. & 14 & 3.2 & -- & -- & -- \\
\hline 1.5 & 7.8 & 100 & 752 & 20 & -- & 11 & 2.7 & -. & -- & -- \\
\hline 6.6 & .5 & 5 & 750 & -- & -- & 13 & 3.3 & -- & -- & -. \\
\hline 3.8 & 2.4 & 22 & 747 & 0 & 41 & 8 & 1.6 & .90 & 19 & .1 \\
\hline 2.3 & .1 & 1 & 757 & 8 & 39 & 10 & 1.6 & 1.2 & 20 & .2 \\
\hline 3.4 & 3.0 & 38 & 756 & -- & -- & 38 & 7.1 & -- & -. & -- \\
\hline 1.8 & 13.1 & 130 & 760 & k6 & -. & 15 & 4.4 & - & -- & -. \\
\hline 1.1 & 7.1 & 66 & 756 & -. & -. & 16 & 4.7 & -. & -- & -- \\
\hline 2.3 & 7.2 & 90 & 755 & - & -- & 16 & 4.1 & -- & -- & -- \\
\hline - & 9.9 & -- & 761 & -. & -- & -. & -- & -- & -- & - \\
\hline -. & 12.6 & 109 & 728 & -- & -- & -. & -. & -. & -- & -- \\
\hline 41 & .4 & 4 & 734 & -. & -. & 54 & 19 & -. & -. & -- \\
\hline 1.5 & .6 & 6 & 753 & -- & -- & 160 & 34 & -- & -. & -- \\
\hline 1.8 & 6.5 & 62 & 745 & -- & -- & 60 & 21 & - & -- & -- \\
\hline
\end{tabular}


Table 5.--Supplemental surface-water quality information

\begin{tabular}{|c|c|c|c|c|c|c|c|c|}
\hline $\begin{array}{l}\text { Site } \\
\text { ID }\end{array}$ & Station name & $\begin{array}{l}\text { Lati- } \\
\text { tude }\end{array}$ & $\begin{array}{l}\text { Longi- } \\
\text { tude }\end{array}$ & $\begin{array}{c}\text { Sodium } \\
\text { ad- } \\
\text { sorp- } \\
\text { tion } \\
\text { ratio } \\
\text { (00931) }\end{array}$ & $\begin{array}{l}\text { Potas- } \\
\text { sium, } \\
\text { dis- } \\
\text { solved } \\
\text { (mg/L } \\
\text { As K) } \\
(00935)\end{array}$ & $\begin{array}{l}\text { Chlo- } \\
\text { ride, } \\
\text { dis- } \\
\text { solved } \\
\text { (mg/L } \\
\text { As Cl) } \\
(00940)\end{array}$ & $\begin{array}{l}\text { Sulfate } \\
\text { dis- } \\
\text { solved } \\
\text { (mg/L } \\
\text { As S04) } \\
(00945)\end{array}$ & $\begin{array}{l}\text { Fluo- } \\
\text { ride, } \\
\text { dis- } \\
\text { solved } \\
\text { (mg/L } \\
\text { As F) } \\
(00950)\end{array}$ \\
\hline 07362000 & Ouachita River at Camden, Ark. & 333547 & 0924905 & 1.0 & 8.8 & 12 & $<0.10$ & 1.4 \\
\hline 07341301 & $\begin{array}{l}\text { Little River at Milliwood Dam } \\
\text { near Ashdown. Ark. }\end{array}$ & 334128 & 0935753 & -- & 4.8 & 3.0 & -- & 1.4 \\
\hline 07340800 & $\begin{array}{l}\text { Mill wood Lake at Yarborough } \\
\text { Landing near Ashdown. Ark. }\end{array}$ & 334329 & 0940105 & -- & 3.4 & 2.8 & -- & 1.2 \\
\hline 07340990 & Dierks Lake near Dierks, Ark. & 340839 & 0940553 & -- & 1.9 & 1.7 & -- & 1.5 \\
\hline 07340452 & $\begin{array}{c}\text { Cossatot River below } \\
\text { Gilliham Dam, Ark. }\end{array}$ & 341232 & 0941340 & -- & 1.5 & 2.2 & $\cdots$ & 1.0 \\
\hline 07340450 & Gillham Lake near Gillham, Ark. & 341237 & 0941344 & -- & 1.5 & 1.5 & -- & 1.2 \\
\hline 07362589 & $\begin{array}{l}\text { Lake Winona downstream from } \\
\text { Gillis Branch near } \\
\text { Reform, Ark. }\end{array}$ & 344816 & 0925116 & .50 & 1.3 & 2.5 & $<.10$ & .95 \\
\hline 072632995 & $\begin{array}{l}\text { Lake Maumelle near Natural } \\
\text { Steps. Ark. }\end{array}$ & 345139 & 0923007 & .70 & 1.7 & 2.2 & $<.10$ & 1.4 \\
\hline 07261880 & Nimrod Lake at Plainview, Ark. & 345905 & 0931837 & -- & $\cdots$ & -- & -- & 4.9 \\
\hline 07076000 & $\begin{array}{l}\text { Little Red River near Heber } \\
\text { Springs, Ark. }\end{array}$ & 353102 & 0915950 & -- & -- & -- & -- & 1.0 \\
\hline 07075900 & $\begin{array}{l}\text { Greers Ferry Lake near Heber } \\
\text { Springs. Ark. }\end{array}$ & 353115 & 0915942 & -- & -- & -- & -- & 1.0 \\
\hline 07075490 & $\begin{array}{l}\text { Greers Ferry Lake near } \\
\text { Clinton. Ark. }\end{array}$ & 353506 & 0922532 & -- & -- & -- & -- & 1.3 \\
\hline 07060871 & $\begin{array}{l}\text { White River below Lock and Dam } 2 \\
\text { near Locust Grove, Ark. }\end{array}$ & 354437 & 0914554 & -- & -- & -- & -- & -- \\
\hline 07194771 & $\begin{array}{l}\text { Prairie Grove Lake near } \\
\text { Prairie Grove, Ark. }\end{array}$ & 355547 & 0942030 & -- & -- & -- & -- & -- \\
\hline 07049200 & $\begin{array}{l}\text { Beaver Lake at Rogers Water } \\
\text { Intake near Lowell. Ark. }\end{array}$ & 361533 & 0940408 & -- & -- & -- & -- & 1.6 \\
\hline 07058700 & $\begin{array}{l}\text { Norfork Lake on Pigeon Creek } \\
\text { near Mountain Home, Ark. }\end{array}$ & 362407 & 0921940 & -- & -- & -- & -- & 18 \\
\hline 07049690 & $\begin{array}{l}\text { Beaver Lake near Eureka } \\
\text { Springs. Ark. }\end{array}$ & 362515 & 0935050 & -- & -- & -- & -- & 1.8 \\
\hline
\end{tabular}


for sites near public-supply intakes--Continued

\begin{tabular}{|c|c|c|c|c|c|c|c|}
\hline $\begin{array}{l}\text { Silica, } \\
\text { dis- } \\
\text { solved } \\
\text { (mg/l } \\
\text { as } \\
\text { sio2) } \\
(00955)\end{array}$ & $\begin{array}{l}\text { Solids, } \\
\text { Residue } \\
\text { at } 180 \\
\text { deg. C } \\
\text { dis- } \\
\text { solved } \\
(\mathrm{mg} / \mathrm{L}) \\
(70300)\end{array}$ & $\begin{array}{l}\text { Solids, } \\
\text { sum of } \\
\text { consti- } \\
\text { tuents, } \\
\text { dis- } \\
\text { solved } \\
(\mathrm{mg} / \mathrm{L}) \\
(70301)\end{array}$ & $\begin{array}{c}\text { Nitro- } \\
\text { gen, } \\
\text { NO2+NO3 } \\
\text { dis- } \\
\text { solved } \\
\text { (mg/L } \\
\text { as N) } \\
(00631)\end{array}$ & $\begin{array}{l}\text { Nitro- } \\
\text { gen, } \\
\text { ammonia } \\
\text { total } \\
(\mathrm{mg} / \mathrm{L} \\
\text { as N) } \\
(00610)\end{array}$ & $\begin{array}{l}\text { Nitrogen, } \\
\text { ammonia + } \\
\text { organic } \\
\text { total } \\
(\mathrm{mg} / \mathrm{L} \\
\text { as } \mathrm{N}) \\
(00625)\end{array}$ & $\begin{array}{l}\text { Phos- } \\
\text { phorus } \\
\text { total } \\
(\mathrm{mg} / \mathrm{L} \\
\text { as P) } \\
(00665)\end{array}$ & $\begin{array}{r}\text { Iron, } \\
\text { dis- } \\
\text { solved } \\
(\mu g / L \\
\text { as } F e) \\
(01046)\end{array}$ \\
\hline 4.8 & 61 & 54 & 0.700 & 0.040 & 0.20 & 0.040 & 73 \\
\hline-- & -- & -- & -- & -- & -- & .050 & -- \\
\hline-- & -- & -- & -- & -- & -- & .040 & -- \\
\hline-- & -- & -- & -- & -- & - & .090 & -- \\
\hline-- & -- & -- & -- & -- & - & .020 & -- \\
\hline-- & -- & -- & -- & -- & -- & .090 & -- \\
\hline 4.9 & 32 & 17 & .100 & $<.010$ & .30 & .010 & 250 \\
\hline 4.4 & 24 & 19 & $<.100$ & $<.010$ & .40 & .020 & 92 \\
\hline-- & - & -- & -- & -- & -- & .080 & -- \\
\hline-- & -- & -- & -- & -- & -- & .030 & -- \\
\hline-- & -- & -- & -- & -- & -- & $<.020$ & -- \\
\hline-- & -- & -- & -- & -- & -- & .020 & -- \\
\hline -- & -- & -- & -- & -- & -- & -- & -- \\
\hline-- & -- & -- & -- & -- & -- & -- & -- \\
\hline-- & -- & -- & -- & -- & -- & .060 & -- \\
\hline-- & -- & -- & -- & -- & - & $<.010$ & -- \\
\hline-- & -- & -- & -- & -- & - & $<.020$ & -- \\
\hline
\end{tabular}


1: ALICLA WATER SYSTEM

2. ALLEN MIH PARK

3: ALLPORT WATER WORKS

4: ALMA WATERWORKS

6: ALMYRA WATERWORKS

6: ALPENA WATERWORKS

7: ALPINE WATER ASSOCIATION

B: ALTHEIMIOR WATERWORKS

9. ALTUS WATERWORKS

10: AMAGON WATERWORKS

11: AMITY WATERWORKS

12: ANTOINE WATFRWORKS

13: AR DGPT OF CORREC CUMMINS

14: ARBOR INC

15: ARK DEPT OF CORRECTION

16: ARKADELPHIA WATERWORKS

17: ARKANSAS CITY WATERWORKS

18: ARKOMA WATHR CO

19: ARSENAL WATER SYSTEM

20: ASH FLAT WATERWORKS

21: ASHDOWN HOMESITES

22: ASHDOWN WATERWORKS

23: ATKINS WATHRWORKS

24: AUGUSTA WATERWORKS

25: AURMILE WATER SYSTEM

26: AUSTIN WATERWORKS

27: BALD KNOB NORTH WATHR ASN

28: BALD KNOB WATERWORK

29: BALTZ DEVELOPMENT CO

30: BANKS WATERWORKS

31: BARKADA WATER ASSOCIATION

32: BARLING WATERWORKS

33: BARTON IEXA WATISR ASSN

34: BASSETT WATERWORKS

35: BATESVILLE WATER UTILTY

96: BATTS LAPILE WATER ASSOC

97: BAY WATERWORKS

38: BAYOU TWO WATER USERS

39: BEARDEN WATERWORKS

40. BEAVER WATER DIST

41: BEE BRANCH WATER

42: BEFBE WATERWORKS

43: BEEDEVILLS WATGR WORKS

4. BELLA VISTA POA

45. BELLFONTE WATER

46: BEHLEVILE WATER

47: BEN LOMOND WATHRWORKS

48: BENNETT ACRES MH P

49: BENTON CTY WATERDIST 1

50: BENTON SFRVICE CENTKR

61: BENTON WATERWORKS

62: BENTONVILLE WATERWORKS

E3: BYRGMAN WATERWORKS

54: BGRRYVILLE WATERWORKS

55: BETHESDA WATER ASSOC

56: BLULAH GROVE WATER

67: BIG FLAT WATERWORKS

68: BIGELOW WATERWORKS

60: BICGERS WATERWORKS

60: BIRDSONG WHITTEN WATER

61: BISCOE WATERWORKS

62: BLACK OAK WATERWORKS

63: BLACK ROCK WATERWORKS

64: BLAKMLY WATGR SYSTYM

65: BLEVINS WATERWORKS

66: BLYTHEVILLE WATHRWORKS

67: BOIS D'ARC WATER SYSTEY

68: BONO WATERWORKS

69: BOONEVIILE WATERWORKS

70: BOWSER WATER ASSOCIATION

71: BOYDELL WATERWORKS

72: BRADFORD RURAL WATER ASSN

73: BRADFORD WATERWORKS

74: BRADLEY CO WATER

75: BRADLEY WATYRWORKS

76: BRECKENRIDGE UNION WATER

77: BRIAR CLFF WATHRWORKS

78: BRINKLEY WATERWORKS

\begin{tabular}{ll} 
Water user & \multicolumn{1}{c}{ System name } \\
ID & \multicolumn{1}{c}{} \\
30510 & 79: BROOKIAND WATERWORKS \\
30511 & 80: BRYANT WATERWORKS \\
30512 & 81: BUCKNGR WATERWORKS \\
20574 & 82: BUENA VISTA-OGEMAW WATER \\
30514 & 83: BUFFALO ISLAND REG WATER \\
31014 & 84: BUIE-SWEET HOME WATER
\end{tabular}

Water user

ID

30570

30572

31152

31087

31112

85: BULL SHOALS WATER

86: BURDETTE WATERWORKS

87: BURNT RIDGE WATER ASSN

88: CABOT WATERWORKS

89: CADDO VALLEY WATERWORKS

90: CALDWELL WATER

91: CALICO ROCK WATERWORKS

92: CALION WATER WORKS

93: CAMDEN WATERWORKS

94: CARAWAY WATERWORKS

95: CARBON CITY WATER

96: CARLISLE WATERWORKS

97: CARROLL-BOONE WATER DIST

98: CARTHAGE WATERWORKS

99: CASA WATER DEPT

100: CASH WATERWORKS

101: CAVE CITY WATERWORKS

102: CAVE SPRINGS WATGRWORKS

103: CEDAR RAIL MOBILE HOME PK

104: CEDARVILLE WATER USERS

105: CENTER GROVE WATER ASSOC

106: CENTIRTTON WATERWORKS

107: CENTRAL AR WATER ASSN.

108: CENTRAL CLAY REG WATER

109: CHARLESTON WATERWORKS

110: CHEROKEE VILLAGE WATER

111: CHERRY VALLEY WATERWORKS

112: CHICOT JUNCTION

113: CHIDESTER WATER WORKS

114: CLARENDON WATTHWORKS

115: CLARKDALE-JERICHO WATER

116: CLARKSVILLE WATERWORKS

117: CLEVELAND CO WATER ASSN

118: CLINTON WATERWORKS

119: COAL HILL WATER DEPT

120: COLLINS WATER ASSOC

121: COLT WATER ASSOCLATION

122: COMMUNITY WATER ASSOC

123: COMPTON WATERWORKS

124: CONWAY CO REG WATER DIST

125: CONWAY WATER CO

126: CORNING WATERWORKS

127: COTTYR WATERWORKS

128: COTTON PLANT WATERWORKS

129: COUNTRY INN NURSING HOME

130: COUNTRY LIFE MOBILE HOME

131: COUNTRY MOBILE ESTATES

132: COUNTRY WATER ASSOC

139: COVE WATERWORKS

134: COY WATERWORKS

135: CRABAPPLE POINT WATER SYS

136: CRAINE WATER CO

137: CRAWFORDSVILE WATERWORKS

138: CROSS ROADS WATER ASSOC

139: CROSSETT PUBLIC UTILITIES

140: CROWLEYS RIDGE COLLEGE

141: CROWLEYS RDGE WATER ASSN

142: CRYSTAL SPRINGS VILLAGE

143: CUSHMAN WATER SYSTEM

144: DALTON MOBILE HOME PARK

145: DAMASCUS WATER WORKS

146: DANVILLE WATERWORKS

147: DARDANELLE WATERWORKS

148: DAVIS WATER SYSTEM

149: DECATUR WATERWORKS

150: DEER RUN WATER CO

151: DEER WATER ASSN

162: DEILAPLAINE WATERWORKS

153: DELIGHT WATERWORKS

154: DELL WATER ASSOCIATION

155: DENNARD WATER ASSN
System name

156: DENNDNG WATERWORKS

Water user

31106

31121

20624

158. DEQUEEN WATER WORK

159: DERMOTT WATERWORKS 30626

160: DES ARC WATERWORKS 20609

161: DEVALIS BLUFF WATERWORKS 30628

162: DEWITT WATERWORKS 30629

163: DIAMOND CITY WATER

164: DIAZ WATERWORKS 31122

165: DIFRKS WATER WORKS 30631

166: DOGPATCH USA 30632

167: DOGWOOD WATER ASSOC 30633

168: DORA WATER ASSN 31172

169. DORCHEAT WATER

170: DOTA WATER ASSN 31119

171: DOVER WATERWORKS

172: DRIVER GRIDER WATER ASSN 31146

173: DUMAS WATERWORKS 30636

174: DYER WATERWORKS 31089

175: DYESS RURAL WATER 31147

176: DYESS WATERWORKS 30637

177: EARL WATERWORKS

178: EAST END WATER ASSOC $\quad 30639$

179: EAST MONROE COUNTY RWA 31149

160: EAST PRAIRIE CO WATER ASN 30640

181: EASTERN CLAY COUNTY WATER 31204

182: EDGEWOOD BAY WATER 30642

183: EL DORADO WATERWORKS 30643

184: ELAINE WATERWORKS 3064

185: ELKINS WATERWORKS 31209

186: EMORSON WATERWORKS 3064

187: EMMET WATERWORKS 30646

188: ENGLAND WATER CO

189. ENOLA-MT VERNON WATER ASN 30648

190: ENON WATER ASSN 31103

191: EUDORA WATERWORKS

192: EUREKA SPRINGS WATERWORKS 31203

193: EVENING SHADE WATERWORKS 30650

194: FAIRCREST WATER ASSN

195: FARRVIILIS-PHILADELPHIA $W \quad 30653$

196: FAYETTHYILLE WATERWORKS 31076

197. FILSENTHAL WATER ASSOC

198: FIFTY SIX WATERWORKS 3065

199: FISHBR WATERWORKS

200: FLIPPIN WATERWORKS 3065

201: FORDYCE RURAL, WATER ASSN 31100

202: FORDYCE WATER CO

203: FOREMAN WATERWORKS 30659

204: FOREST LAKE ESTATES WATER 30660

205: FORREST CITY WATERWORKS 30661

206: FORT CHAFFEE WATERWORKS 3117

207: FORT SMTTH WATMRWORKS 20575

208: FOUKE WATERWORKS

209: FOUR MILE HILL WATER 31007

210: FRANKLIN WATTRWORKS

211: FREE HOPE WATER ASSN

212: FRENCHPORT WATER ASSOC $\quad 31026$

213: FULTON WATTRWORKS 30667

214: FURLOW WATGR ASSN 31166

216: GARFIELD WATERWORKS 30668

216. CARLAND WATERWORKS

217: GARRETT BRDGE WATER 31102

218: GARRETT GROVE WATER ASSN 3113

219: GASSVILLE WATERWORKS 30670

220: GATEWAY RURAL WATER ASSN 3067

221: GENTRY WATERWORKS

202: GENTRYVILLE WATER ASSOC 30673

223: GILLETT WATERWORKS 30674

224: GLLHAM RכG WATMR DIST 30675

225: GILLHAM WATERWORKS 31200

226: GILMORE WATERWORKS 30676

227: GLENWOOD WATER DEPT

228: GOSNELL WATER ASSOCLATION 30678

229: GOUTD WATERWORKS

230: GRADY WATERWORKS 30680

231: GRAND PRAIRIE REG WATER 30681

232: GRAND PRAIRIS WATER USERS 30682 
Water uner

\section{DD}

239: GRANNIS WATERWORKS

234: GRAVETTE WATERWORKS

235: GRAY ROCK WATER ASSN

236: GREASY VALLEY WATER ASSN

237: GREEN ACRES MOBILE HOME

296: GREEN FOREST WATERWORKS

239: GREEN HILL-BROOKS CHAPEL

240: GREENBRIFR WATERWORKS

241: GREENWAY WATERWORKS

242: GREENWOOD WA TERWORKS

243: GRUBBS WATERWORKS

244: GUION WATERWORKS

245: GUM SPRINGS WATER ASSOC

246: GURDON WATERWORKS

247: GUY WATERWORKS

248: HACKETT WATERWORKS

249: HAMBURG WATERWORKS

250: HAMILTON MOBILE HOME PARK

251: HAMPTON WATERWORKS

25ARBOR EAST INC WATERWORK 30697

253: HARDIN WATER ASSOCIATION

254: HARDY WATERWORKS

255: HARMONY GROVE WATER ASSN

256: HARMONY WATER ASSOC

257: HARRELL WATERWORKS

256: HARRISBURG WATERWORKS

259: HARRISON WATERWORKS

260: HARTTORD WATERWORKS

261: HARTMAN WATERWORKS

262: HASKKLLL WATER SYSTEM

263: HATFIELD WATERWORKS

264: HATTIEVILLE WATER

265: HAVANNA WATERWORKS

266: HATEN WATERWORKS

267: HEAFER-BLACK OAK WATER

268: HEBER SPRGS WATER \& SEWER

260: HECTOR WATERWORKS

270: HIMLENA WATER WORKS

271: HERMITAGE WATERWORKS

272: HICKORY RIDGE RURAL WATER

273: HICKORY RIDGE WATERWORKS

274: HIGHIAND PARK WATER

275: HIGHLAND WATER ASSOC

277: HOLLY GROVE WATERWORKS 30713

278: HOPE WATERWORKS

279: HORATIO WATERWORKS

280: HORSEHEAD WATER ASSN

20580

30715

31190

281: HORSESHOE BEND WATERWORKS 30716

282: HOT SPRGS VILLAGE WATER $\mathbf{3 0 7 1 7}$

299. HOT SPRDNG COUNTY WATER

284: HOT SPRNGS WATERWORKS 30718

285: HOUSTON WATERWORKS

286: HOXIE WATER DEPARTMENT 30718

287: HUGHES COMMUNITY WATER 31180

288: HUGHES WATERWORKS 30720

289: HUMAN DEVILOPMENT CENTER 20687

290: HUMNOKE WATERWORKS 3072

291: HUMTPREY WATERWORKS

292: HUNTINGTON WATERWORKS 31178

298: HUNTSVILLE WATERWORKS 30729

29: HUTTIG WATERWORKS

205. HWY 16

296: HWY 64 WATER ASSN

297: HWY 64-71 WATER USTERS

296: HWY 77 WATER ASSN

299: HWY 62 WATER ASSOC

900: HWY 9 WATER ASSN

301: DMBODEN WATERWORKS

302: INDIAN SPRGS MHP WATERWKS

303: IVARD CO RURAL WATER ASSN

304: J W SWANSON INC

306: JACKSONPORT WATERWORKS

506: JACKSONVILE WATER DEPT

307: JASPER WATERWORKS

306: JDPFERSON-SAMPLES-DEXTER

309: JOHNSON TOWNSHIP WATER

310: JONNER WATERWORKS

311: JONES MILL SEWER \& WATER
System name

312: JONESBORO WATER SYSTEM

313: JUDSONIA WATERWORKS

314: JUNCTION CITY WA TERWORKS

315: KEISER WATERWORKS

316: KELSO-ROHWER WATER ASSOC

317: KENSETT WATERWORKS

318: KEO WATER WORKS

319: KIBLER WATERWORKS

320: KIMYAEY WATER DISTRICT

321: KINGSLAND WATERWORKS

322: KNOBEL WATERWORKS

323: KNOXVILLE WATERWORKS

324: KOUNTRY KOURTS MHP

325: KROOKED KREEK WATER ASSN

326: LACEY-LADELLE WATER ASSN

327: LADD WATER ASSOCIATION

328: LAFE RURAL WATER ASSOC

329: LAKE BULL SHOALS EST

330: LAKE CHARLES STATE PARK

331: LAKE CHICOT WATER ASSOC

332: LAKE CITY WATERWORKS

333: LAKE FOREST SUBORDINATE

334: LAKE VILLAGE WATERWORKS

335: LAKESHORE ESTATES WATER

336: LAKEVIEW-MIDWAY WATER

337: LAKEVIEW-WABASH WATER

338: LAKEWOOD ESTATES MHP

339: LAMAR WATERWORKS

340: LAVACA WATERWORKS

341: LAWRENCE COUNTY REG WATER

342: LAWSON-URBANA WATER ASS'N

343: LEACHVILLE WATERWORKS

344: LEAD HILL WATERWORKS

345: LEE COUNTY WATER ASSOC

346: LEF CREEK RURAL WATER DIS

347: LEISURE HILLS MHP

348: LEISURE HILLS WATERWORKS

349: LEOLA WATERWORKS

350: LEPANTO WATERWORKS

351: LESLIE WATERWORK

952: LEWISVILLE. WATERWORKS

353: LINCOLN WATERWORKS

354. LISBON WATER ASSN

365: LITTLE CREEK WATER ASSN

356: LITTLE RIVGR COUNTRY CLUB

957: LITTLE RIVER WATER ASSOC

358: LITTLE ROCK MUN WATERWORK 2061

959. LOCKESBURG WATERWORKS

360. LOCUST BAYOU WATER

361: LONDON WATERWORKS

362: LONG LAKE WATER ASSN

363: LONOKE WATERWORKS

364: LOST BRIDGE VILLAGE WATER

365: LOUANN WATERWORKS

366: LUDWIG WATER ASSOCIATION

367: LURTON-PELSOR WATER ASSN

368: LUXORA WATERWORKS

369: LYDESDALE WATER ASSN

370: LYNN WATERWORKS

371: MADISON CO WATER ASSN

372: MAGAZINE WATERWORKS

373: MAGNESS WATTR ASSN

374: MAGNET-BUTTERFIELD WATER

375: MAGNOLIA WATERWORKS

376: MALVERN WATERWORKS

377: MAMMOTH SPRGS WATERWORKS

378: MANDEVIILE WATER

379: MANTA WATERWORKS

380: MANSFIELD WATERWORKS

381: MARIANNA WATERWORKS

382: MARIE WATER SYSTEM

383: MARION WATERWORKS

384: MARKED TREE WATERWORKS

385: MARMADUKE WATERWORKS

386: MARSHALL WATERWORKS

887: MARVELL RURAL WATER ASSN

388: MARVELL WATERWORKS

389: MARYSVILLE WATER ASSOC

390: MAUMELLE SID $\# 500$
Water user

ID

20560

6115

30735

30736

30737

46131

30738

31090

47455

30739

30741

31125

30742

30743

31105

9074

30746

30746

28107

46121

30747

30748

30749

31097

31038

30760

20751

31126

31175

4613

30752

30754

31011

46125

30755

30756

30757

30758

30759

30760

20630

31184

31110

30762

30763

30765

31155

31163

31158

30766

$\mathbf{3 0 7 6 7}$

30768

31127

30769

30770

31085

30771

30772

31136

31120

31116

30773

20581

30775

30776

30777

31176

30778

31145

30779

30780

30761

30782

30783

30784

30785

30786

System name

Water user

391: MAUMELLE WATER CORP

ID

393: MAYNARD WATERWORKS 3078

394: MCCASKILL WATERWORKS

95: MCCRORY WATERWORKS 3079

396: MCDOUGAL WATERWORKS 30792

997: MCGEHEE WATERWORKS

398: MCLAUGHLIN VILLAGE MHP 3079

399: MCNEIL RURAL WATER SYSTEM 30797

400: MCNEIL WATERWORKS

401: MCRAE WATERWORKS 30796

402. MIALOURNE WATERWORKS 3079

403: MENA WATER DEPT 20605

404: MENIFEE WATER DEPARTMENT 30800

405: MIDWAY WATER ASSOCIATION 3080

406: MILTOWN-WASHBURN WATER 47458

407: MIMOSA EST SD WATERWORKS 30802

408: MINERAL SPRINGS WATERWORK 28039

409: MITCHELLVILLE WATERWORKS 3080

410: MOCKINGBIRD HILL WATER 30805

411: MONETTE WATERWORKS 3080

412: MONTICELLO WATER DEPT 30807

413: MONTROSE WATERWORKS 31073

114: MORNING STAR WATER ASSN 30808

415: MORO WATERWORKS 31135

416: MORRISON BLUFF WATER SYS 31029

417: MOUNT HOLLY WATERWORKS 30809

418: MOUNT IDA WATERWORKS 30810

419: MOUNT JUDEA WATERWORKS 30811

420: MOUNTAIN HOME WATERWORKS 30812

421: MOUNTAIN PINE WATERWORKS 30813

422: MOUNTAIN TOP WATER ASSN 31083

423: MOUNTAIN VIEW WATERWORKS 20627

424: MOUNTAINBURG WATERWORKS 31044

425: MT PLEASANT WATERWORKS 30815

426: MT SHERMAN WATER ASSOC 30816

427: MT ZION WATER ASSOCIATION 31108

428: MULBERRY WATERWORKS 20573

429: MUNDELL HGTS WATERWORKS $\mathbf{3 0 8 1 8}$

430: MURFREESBORO WATERWORKS 20602 
Water user

ID

468: OLA WATERWORKS 469: OLD UNION WATER ASSN 470: OMAHA WATERWORKS 471: OPPELO WATER DEPT 472: OSCEOLA WATERWORKS 473: OXFORD WATERWORKS 474: OZAN WATERWORKS 475: OZARK ACRES WATER CO 476: OZARK WATERWORKS 477: PALESTINE WATER UTILITY 478: PANGBURN WATERWORKS 479: PARAGOULD CITY LGHT/WATER 480: PARIS WATERWORKS 481: PARKDALE WATERWORKS 482: PARKERS CHAPIEL WATER ASSN 483. PARKIN RURAL WATER ASSN 484: PARKIN WATERWORKS 485: PARTHENON WATER ASSN 488: PATTERSON WATERWORK 487: PAYNEWAY WATER ASSN 488: PB/GENERAL WATERWORKS CO 489: PEA RIDGE WATGRWORKS 491: PENTLTON PIOATCF WATER 192: Pant 493: PERRY WATERWORKS 494: PERRYTOWN WATERWORKS 495: PERRYVILLE WATERWORKS 496: PFEIFFER WATER ASSOC 197: PICKENS WATERWORKS 496: PIGGOTT WATERWORKS 499: PINE REST MOBILE HOME PRK 500: PINEHAVEN MOBIIE HOME PRK 601: PLAINVIEW WATER DEPT 502: PLEASANT GROVE

508: PLEASANT VIEW WATGR 504: PLEASURE ENTERPRISE 605: PLUMERVILLE WATERWORKS 506: POCAHONTAS WATERWORKS 607: POLLARD WATHRWORKS 609: PORTLA WATERWORKS 609: PORTLAND WATERWORKS 610: POTTSVILLE WATHR

511: POYEN WATERWORKS 612: PRAIRIE GROVE WATERWORKS 613: PRATTSVILLE WATERWORKS 514: PRESCOTT WATERWORKS 516: PYATT WATERWORKS 516: QUINN WATER ASSOCLATION 517: QUTTMAN WATERWORKS E18: RAMBO RIVIBRA SD WATER 519: RANKWN TOWN WATERWORKS 620: RATCLIFP WATERWORKS 621: RAVENDEN SPRGS WATERWORKS 309 622. RAVENDEN WATERWORKS 523: RAY MARTIN MOBILE HONE PK S088 524: READLAND-GRANDLAKE WATER 31080 625: REAVIS WATER SYS

626: RBCTOR WATERWORKS

627: REDFIELD WATERWORKS

528: REDHILL WATKR ASSN

529: REED WATERWORKS

690: REMMEL DAM LANDING WATER 691: REYNO WATERWORKS

532: RISON WATERWORKS

533: RIVER DR MOBILE HOME PK 684: RIVERSIDE MOBILE HOME PK 635: RIVIGRA UTILITLES 596: ROCK MOORE WATER ASSOC 537: ROE WATERWORKS 538: ROGERS WATERWORKS 639: ROSSTON WATER DEPT 540: RUDDLEHILL WATER ASSN 611: RUSSELL WATERWORKS 642: RUSSELLVILE DMPROVEMENT 543: RUSSELLVILLE WATERWORKS 544: S P G WATER ASSOC
System name

545: S SEBASTIAN WATER USERS 546: S W BOONE COUNTY WATER 547: $\$$ W WHITE COUNTY WATGR 548: S.E. WHITE COUNTY WATER 549: SAINT FRANCIS WATERWORKS 550: SALEM WATER USERS ASSOC 551: SALEM WATERWORKS

652. SALESVILLE WATERWORKS 553: SAND RIDGE-BARDSTOWN WTR 564: SARDIS WATER ASSOCIATION

555: SCHALL SUBDIVISION

656: SCRANTON WATERWORKS 657: SEARCY VALLEY WATER ASSN 658: SEARCY WATERWORKS 559: SEBASTIAN LAKE UTILFTY CO 660: SEDGWICK WATERWORKS 561: SELMA WATER ASSOCLATION 562: SHADY ACRES MOBILE HOME 663: SHADY GROVE WATER ASSN 564: SHADY HILLS MOBILE HOME 665: SHADY PARK WATER ASSOC 566: SHANNON HILLS WATER ASSN 667: SHANNON WATER USERS ASSN 568: SHIFRIDAN WATERWORKS 569: SHERRIILL WATERWORKS 570: SHIRLEY WATERWORKS 571: SHUMAKFR PUBLIC SERV CO 572: SIDNEY WATER SYSTEM 573: SILOAM SPRINGS WATERWORKS 674: SMACKOVER WATERWORKS 575: SOOTER WATER SYSTEM 576: SOUTH OZARK WATER USERS 577: SOUTH SHERIDAN WATER 678: SOUTHERN ARK UNIV WATER 579: SOUTHSIDE WATER ASSN 680: SOUTHWEST WATER ASSOC 561: SPADRA-GOOSE CAMP WATER 582: SPARKMAN WATERWORKS 583: SPRINGDALE WATERWORKS 584: SPRINGHILL WATERWORKS 585: ST CHARLES WATERWORKS 586: ST FRANCIS WATER ASSOC 587: ST PAUL WATERWORKS

688: STAMPS WATERWORKS 589: STANDARD-UMIPSTEAD WATER 590: STAR CITY WATER CO 691: STEPHENS WATERWORKS 692: STRAWBERRY WATERWORKS 593: STRONG WATERWORKS 594: STUTTGART WATERWORKS 595: SUBLACO ACADEMY WATERWORK 3093 596: SUBLACO WATGRWORKS 697: SUBURBAN WATER \& SEWER 698: SUCCESS WATERWORKS 699. SULPHUR SPRGS WATERWORKS 600: SUMAIT WATERWORKS 601: SUNSIDT WATER ASSN 602: SW ARKANSAS WATER DIST 603: SW ATKINS WATER USERS 604: SWIFTON WATERWORKS 605: SYCAMORE VALLEY MHP 606: SYLVAN SHORES SD WATER 607: TAYLOR WATERWORKS 608: TEXARKANA WATER UTHITTES 609: THOMPSON MHP WATER SYSTEM 610: THORNTON WATERWORKS 611: THLAR WATERWORKS 612: TMMBER RIDGE RANCH WATER 613: TINSMAN WATERWORKS 614: TOLLETTE WATER 615: TONTTTOWN WATER DEPT 616: TRI-COUNTY WATER DISTR 617: TRUMAN WATERWORKS 618. TRUMANN RURAL WATER ASSN 619. TUCKER WATERWORKS 620: TUCKERMAN WATERWORKS 621: TULL WATER USERS ASSOC
ID

31042

30899

31195

31009

30900

31048

30901

30902

31148

30903

30004

31137

31196

30906

31006

46128

30909

90910

30911

31170

30912

30914

30915

30916

30917

30918

30919

31054

31111

30920

30921

31047

31131

31077

30923

30924

31020

30925

30926

91186

30929

30930

30932

31143

46127

30934

30985

30937

31096

20685

47466

5008

30940

30941

47139

30944

30945

30946

3094

30948

30949

46133

$\$ 0960$

30951

30952

91050
30908

30913

30939

Water user

622: TUMBLING SHOALS WATER SYS 31040 623: TURREILL WATERWORKS 30953 624: TYRONZA WATERWORKS 3095 625: U S ARR FORCE BASE WATER 31167 626: ULM WATERWORKS 627: UNTTED WATER ASSN 31150 628: USAF HOSPITALAGPB 30956 20. VALENTINE-DICK JETER WTR 31169 630: VALLEY SPGS WATERWORKS 30957 631: VALLEY VIEW WATER ASSN 30958 632: VAN BUREN COUNTY W.UA. 31188 633: VAN BUREN WATERWORKS 46124 634: VANDERVOORT WATERWORKS 31202 635: VANNDALE-BIRDEYE WATER 30959 636: VICTORIA WATER ASSOC 30960 637: VILAGE WATER ASSOCIATION 30961 638: VILONIA WATERWORKS 30962 639: VIOLA WATERWORKS 30969 640: W K M M RURAL WATER ASSN 31101 641: WABBASEKA WATERWORKS 30064 642: WALDENBURG WATER ASSN 31161 643: WALDO WATERWORKS 644: WALDRON WATERWORKS 20622 615: WALKGR WATER ASSN 31086 646: WALKGRVILLE WATER ASSOC 30967 647: WALNUT HILL WATER ASSN 31132 648: WALNUT RIDGE WATERWORKS 30968 649: WARD WATERWORKS 30969 650: WARREN WATERWORKS 30970 651: WASHINGTON WATER AUTHOR 31008 652: WASHINGTON WATER SYSTEM 30971 653: WATALULA WATER ASSN 654: WATSON CHAPEL WATER ASSN 30972 655: WATSON WATERWORKS 30973 656: WEINER WATERWORKS 30974 657: WESSON-NEWELL WATER ASSN 3097 658: WEST BAUXITE WATER USERS 31049 659: WEST FORK WATER ASSN 20629 660. WEST HELENA WATER WORKS 30977 661: WEST MEMPHIS WATERWORKS 30978 662: WEST SALINE WATER ASSN $\quad 30979$ 663: WEST STONE COUNTY WATER 31182 664: WEST WOODRUTF WATER DIST 53275 665: WESTERN GREENE CTY RWD 30980 666: WESTKRN GROVE WATER ASSN. 31024 667: WHEATLEY WATERWORKS 31032 668: WHITE HALL WATERWORKS 30981 669: WHITE MOBLEE HOME PARK 30983 670: WHITE RIVIR RURAL WATER 30884 671: WICKES WATERWORKS 31199 672. WIDENER WATER WORKS 31021 673: WILBURN WATER ASSOCLATION 30985 
Hydrologic unit code Basin name

$\begin{array}{ll}08010100 & \text { Lower Mississippi-Memphis } \\ 08020100 & \text { Lower Mississippi-Helena } \\ 08020203 & \text { Lower St. Francis } \\ 08020204 & \text { Little River Ditches } \\ 08020205 & \text { L'Anguille }\end{array}$

08020301 Lower White-Bayou Des Arc

08020302 Cache

08020303 Lower White

08020304 Big

08020401 Lower Arkansas

08020402 Bayou Meto

08030100 Lower Mississippi-Greenville

08040101 Ouachita Headwaters

08040102 Upper Ouachita

08040103 Little Missouri

08040201 Lower Ouachita-Smackover

08040202

08040203

08040204

08040205

Lower Ouachita-Bayou De Loutre

Upper Saline

Lower Saline

Bayou Bartholomew

08040206

08040207

08050001

08050002

11010001

Bayou D'Arbonne

Lower Ouachita

Boeuf

Bayou Macon

Beaver Reservoir

11010003

11010004

11010005

11010006

11010007

Bull Shoals Lake

Middle White

Buffalo

North Fork White

11010008

11010009

11010010

11010011

11010012

Upper Black

11010013

11010014

11070206

11070208

11070209

Current

Lower Black

Spring

Eleven Point

Strawberry

11110103

11110104

11110105

11110201

Upper White-Village

Little Red

Lake O'The Cherokees

Elk

Lower Neosho

11110202

Illinois

Robert S. Kerr Reservoir

Poteau

Frog-Mulberry

Dardanelle Reservoir

11110203

11110204

11110205

11110206

Lake Conway-Point Remove

Petit Jean

Cadron

Fourche La Fave

Lower Arkansas-Maumelle

11140105

11140106

11140108

11140109

Kiamichi

Pecan-Waterhole

Mountain Fork

Lower Little

Mckinney-Posten Bayous

11140203 Loggy Bayou

11140205

11140302

11140304

Bodcau Bayou

Lower Sulphur

Cross Bavou

B-1 